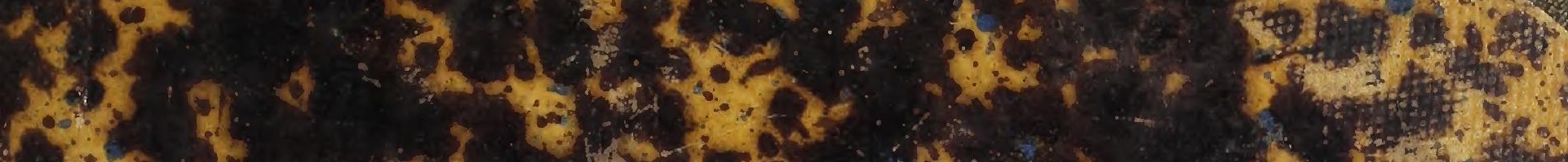

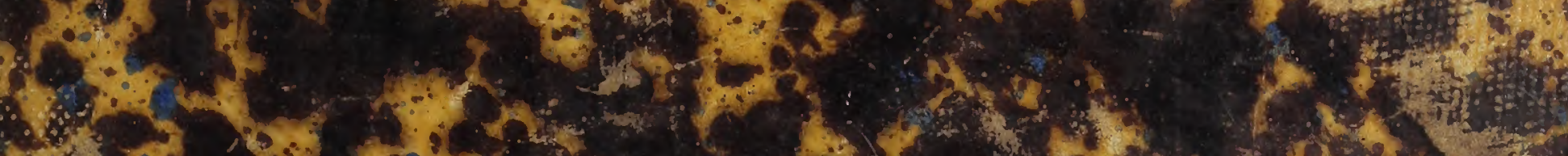

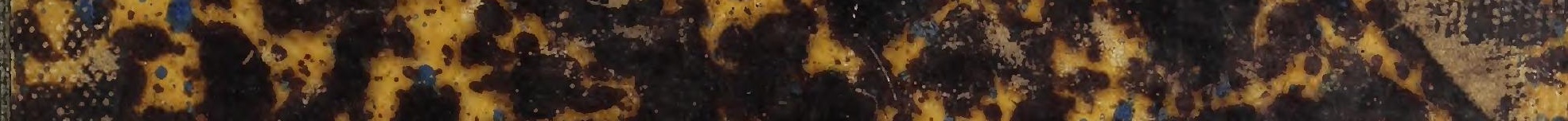

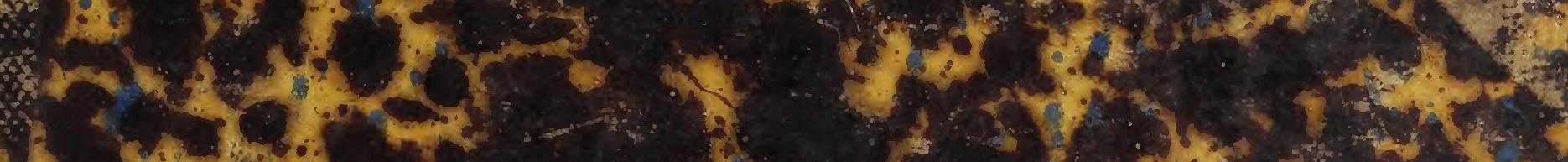
1)

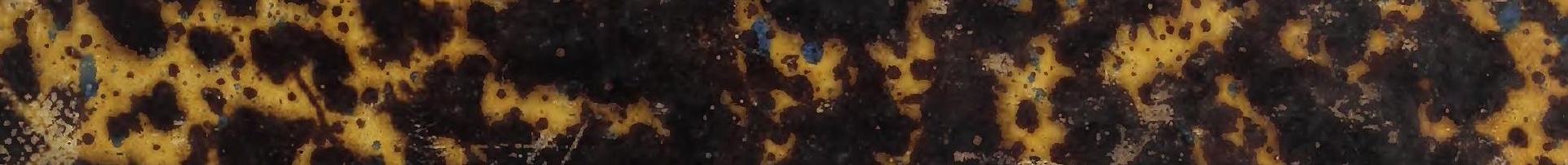

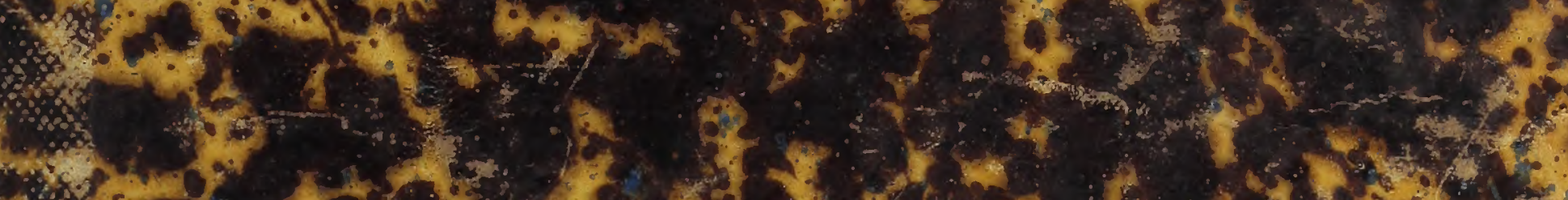

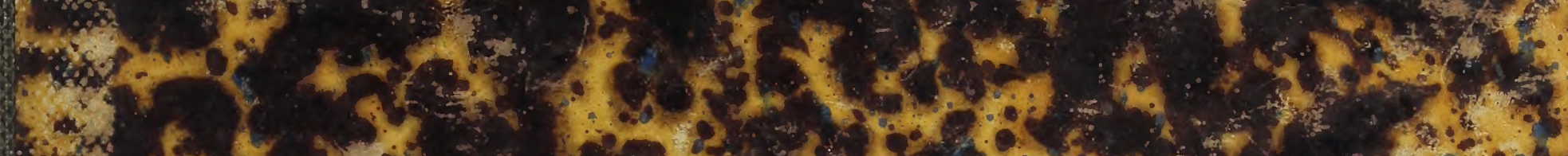

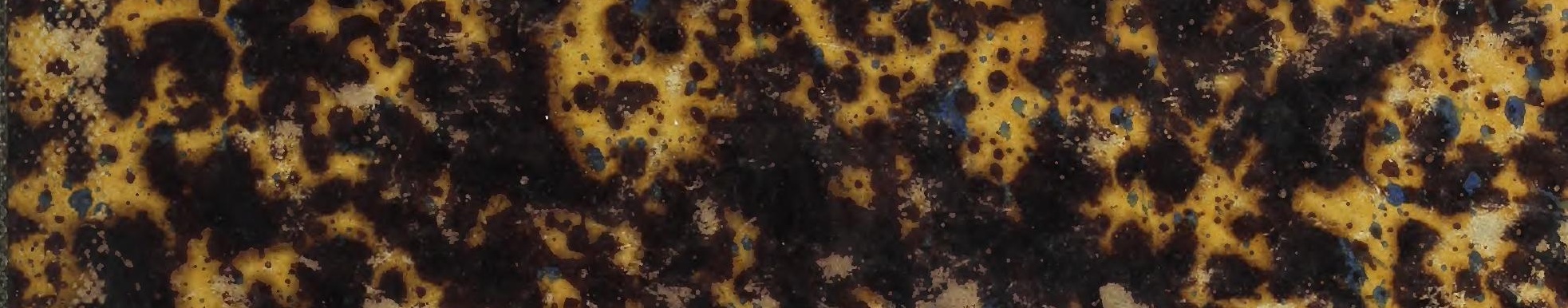

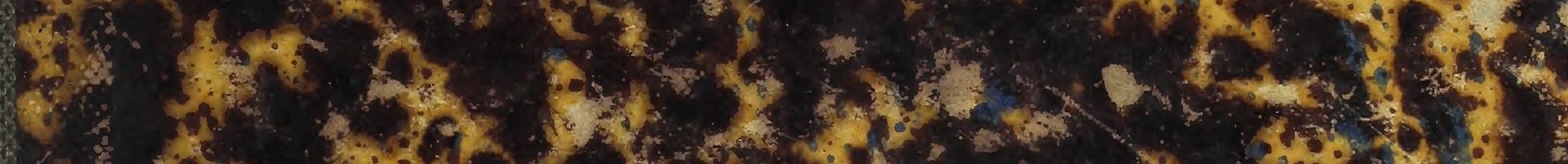

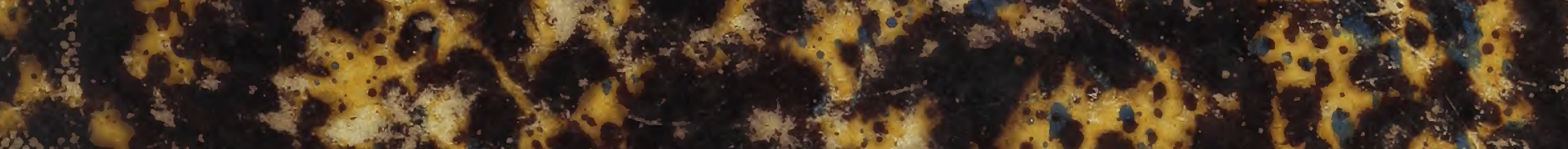

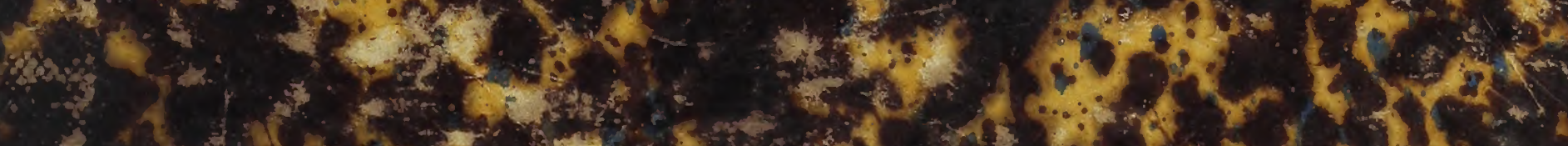

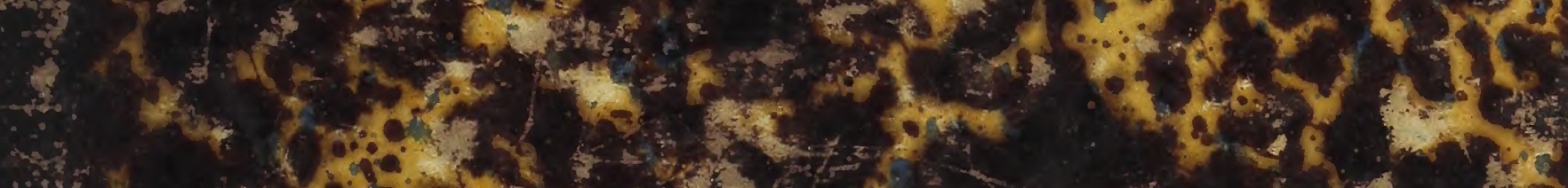

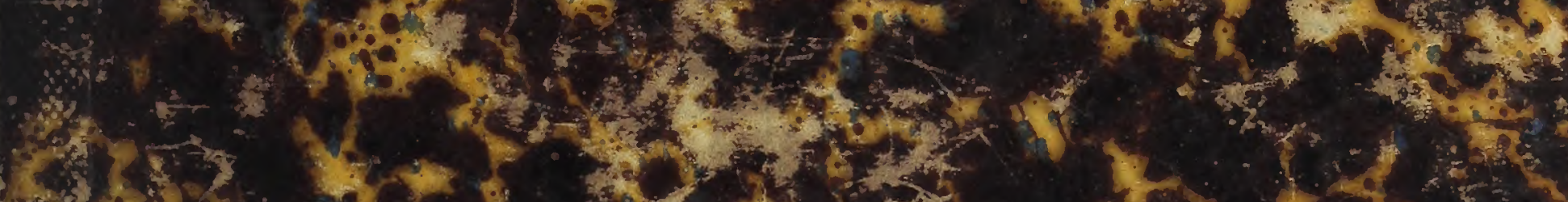

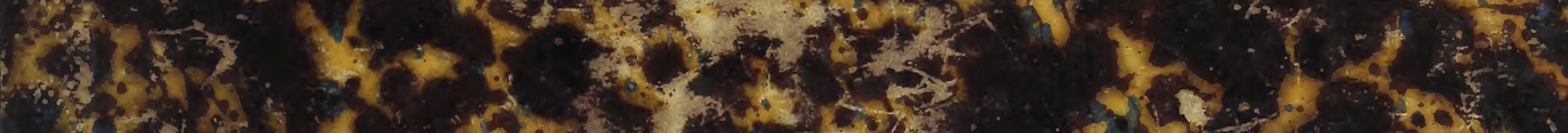

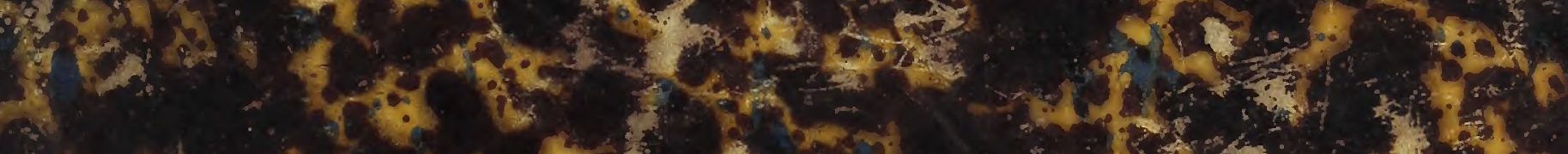
=

\footnotetext{
xis 8

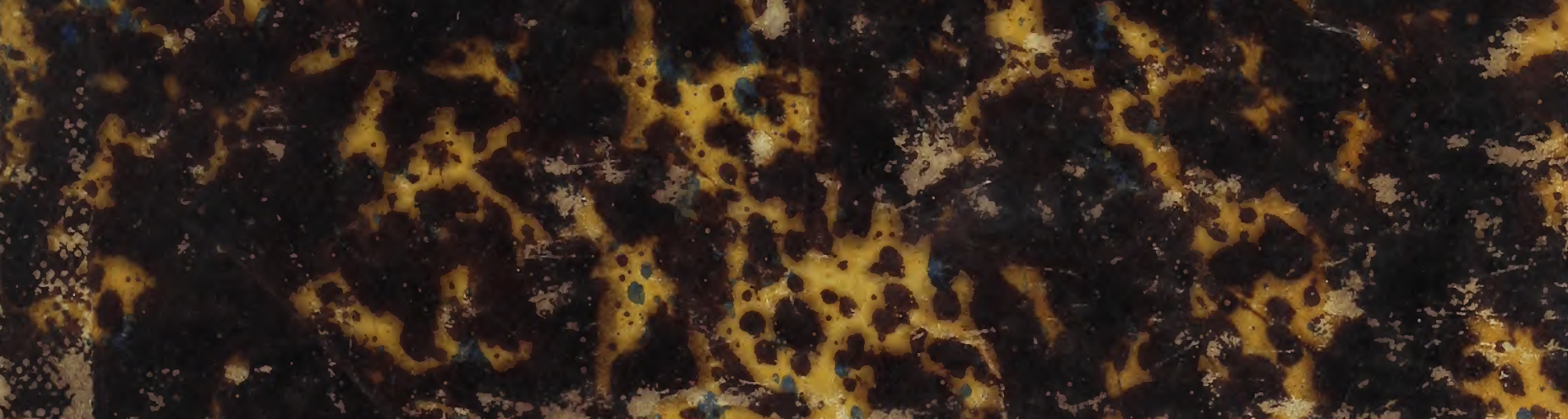

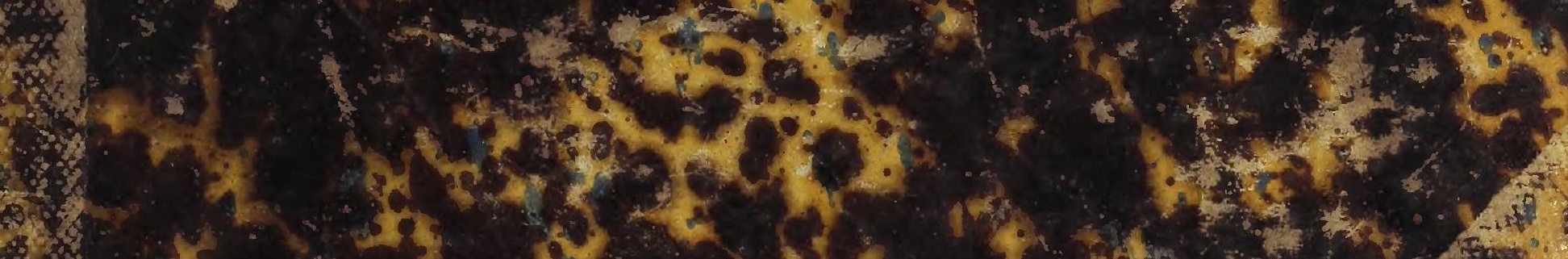

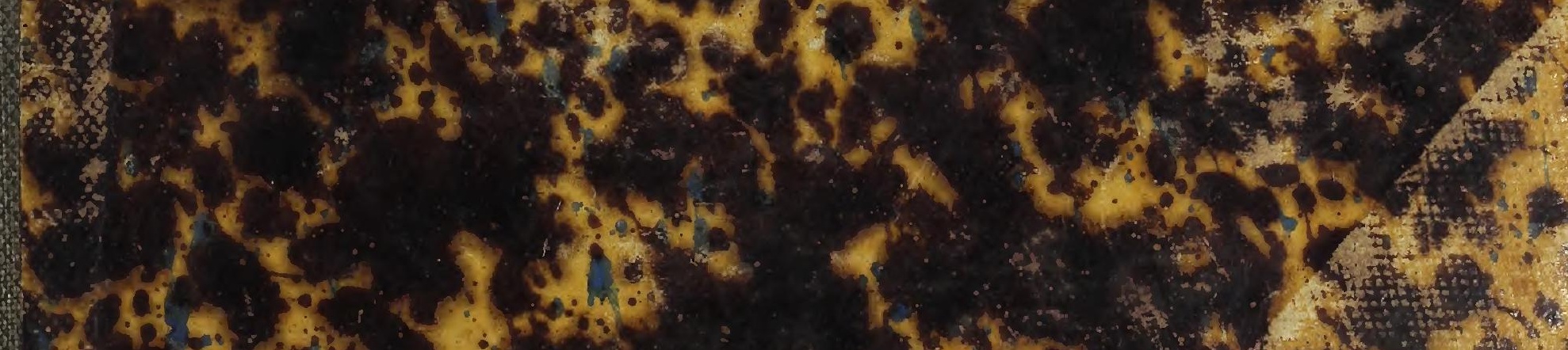



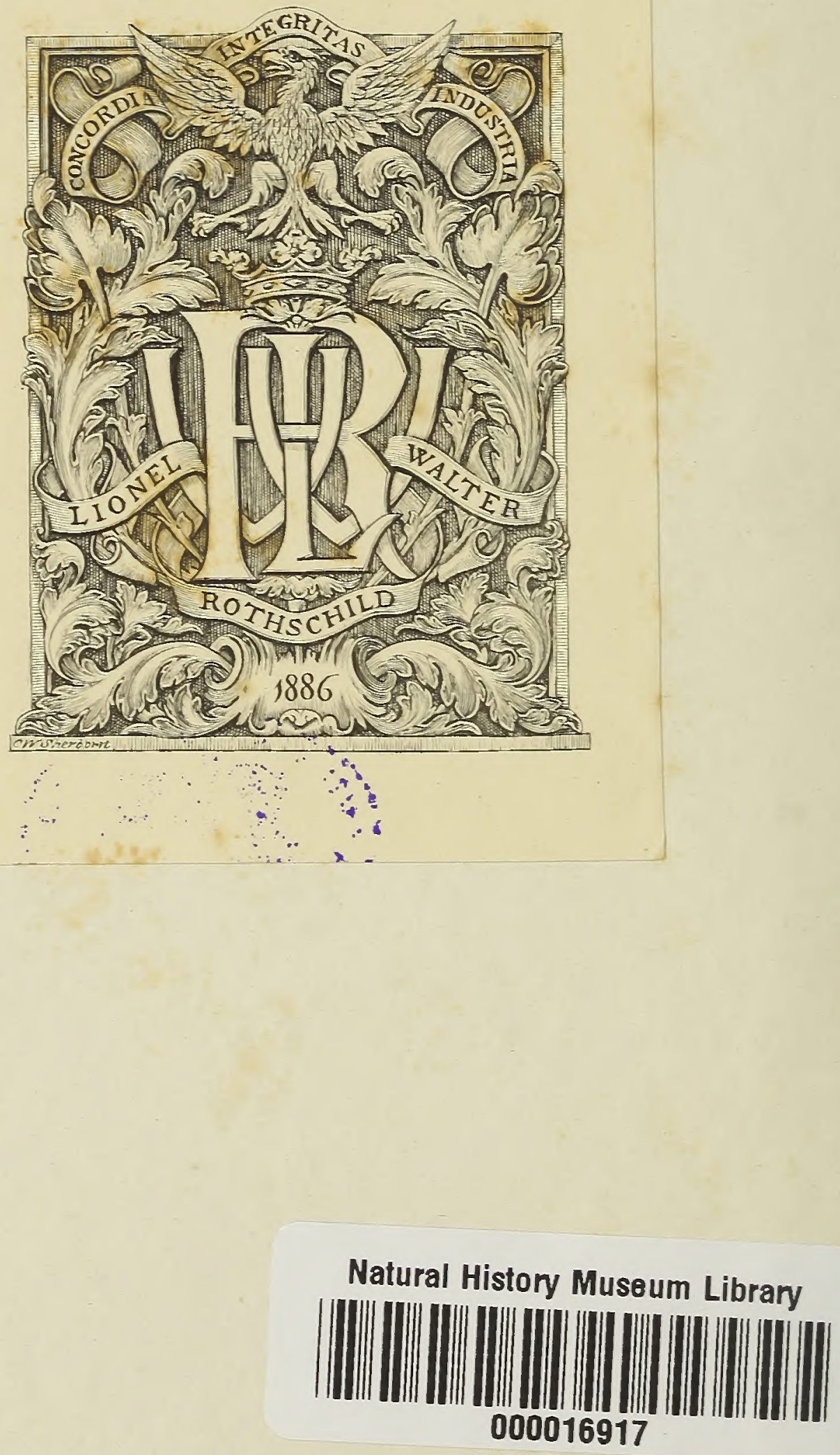

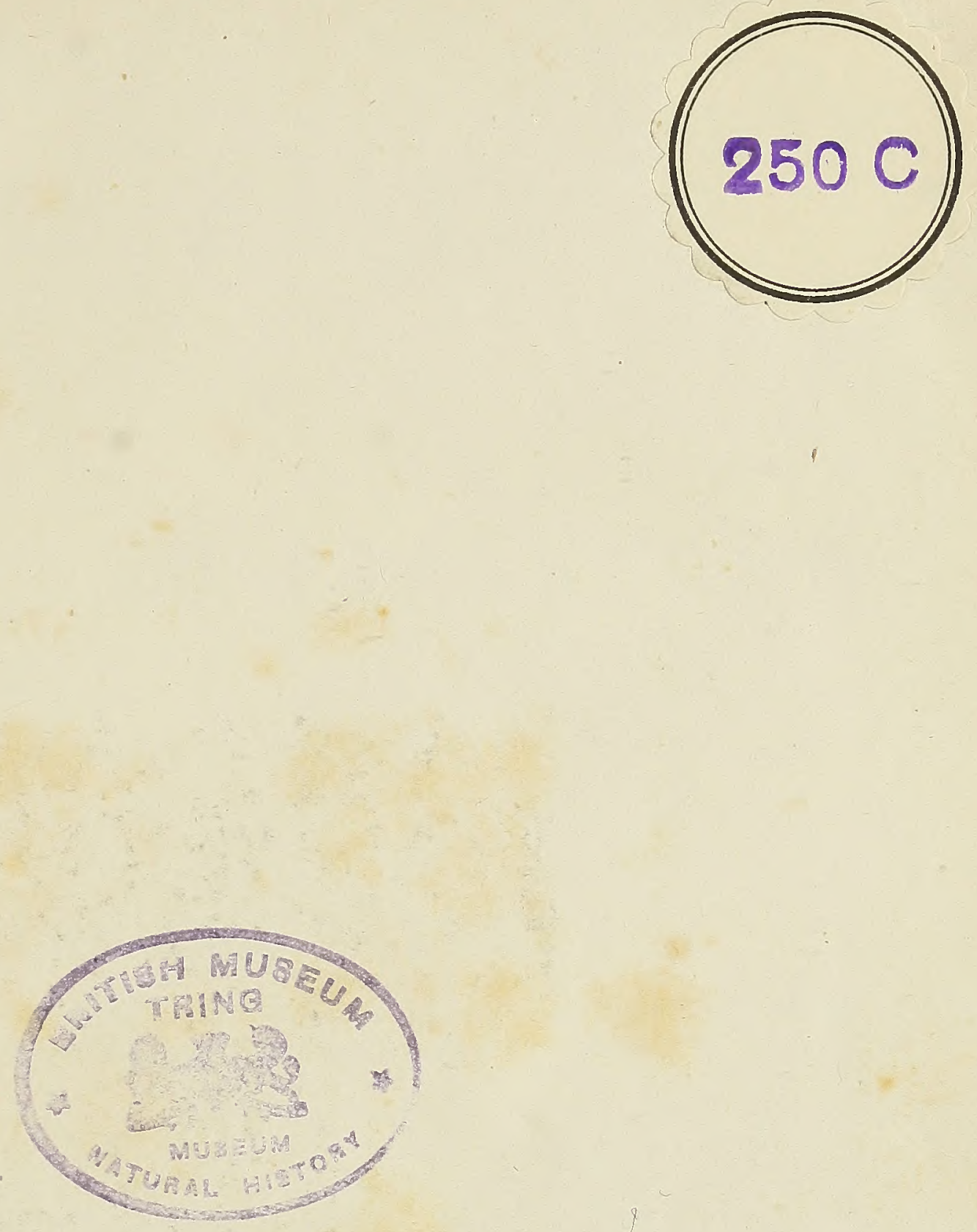

cos

H. 17 a

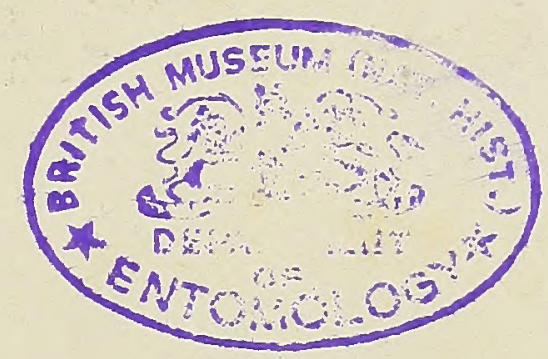





\title{
DIE KÄFER
}

\author{
VON
}

\section{NASSAU UND FRANKFURT.}

\section{ZUSAMMENGESTELLT}

VON

\section{LUCAS VON HEYDEN}

Dr. philos, honor. c. - K. Hauptmann z. D. - Corresp. Mitglied des Nassauischen Vereins für Naturkunde. - Ehrenmitglied des Vereins für Insectenkunde am Niederrhein in Düsseldorf; des Naturhist. Vereins zu Anhalt; der Mannheimer und Offenbacher Vereine für Naturkunde. - Mitglied der kais. Leopold. Carolin. Academie deutscher Naturforscher. Wirkliches ordentliches oder correspond. Mitglied der Entomologischen Vereine zu München, Berlin, Paris, St. Petersburg, der Società entomologica italiana di Firenze, der Société Linnéenne de Lyon, der Sociedad española de Historia natural de Madrid; der schweizerischen entomol. Gesellschaft, der Senckenberg'schen naturf. Gesellschaft zu Frankfurt a.M., der Wetterau'schen naturf. Gesellschaft in Hanau, der naturf. Gesellschaft Graubündtens, der Oberhessischen Gesellschaft für Natur- und Heilkunde in Giessen, des zoolog. mineral. Vereins in Regensburg, der zoolog. botan. Gesellschaft in Wien, der k. ostpreuss. physik. ökonom. Gesellschaft in Königsherg, der naturf. Gesellschaft in Danzig maturhist. Vereins der preuss. Rheinlande und Westphaleus und des Vereins rumbaturwissensch. Unterhaltung in Hamburg etc.

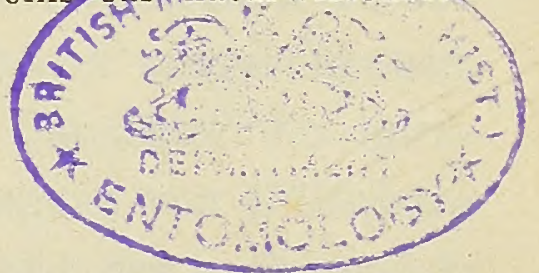

AUS DEN JAHRPÜCHERN DES NASSAUISCHEN VFREINS FÜR NATURKUNDE, JAHRG. XXIX UND XXX. S. $55 \mathrm{ff}$.

\section{WIESBADEN.}

JULIUS NIEDNER, VERLAGSHANDLUNG. 

Ueber einen grösseren Theil dieses Gebietes von Mitteldeutschland besitzen wir zwar schon Namensverzeichnisse mit Angabe der Fundorte, allein es fehlte noch immer an einer genanen, kritisch bestimmten $\mathrm{Zu}$ sammenstellung der Käferarten des ganzen Gebietes. Vor allen andern sei hier erwähnt die genane Arbeit von Decan Scriba in Wimpfen: „Die Käfer im Grossherzogthum Hessen und seiner nächsten Umgebung“", in den Berichten der Oberhessischen Ges. für Natur- und Heilkunde. Giessen 1863. -

Das Gebiet, welches hier behandelt wird, umfasst das Taunusgebirge (in seiner höchsten Erhebung, dem grossen Feldberg, 2711 par. Fuss über Meer) und ist begrenzt im Süden durch den Lauf des Mains von Offenbach über Frankfurt nach Mainz und den Rhein herunter bis Bingen, im Westen weiter bis zur Mündung der Lahn. Die Ostgrenze bildet eine Linie Offenbach-Friedberg-Giessen, so dass die eigentliche Wetterau und die Umgegend von Hanau ausserhalb des Gebietes fällt, weil sie schon zu sehr von den letzten Ausläufern des Vogelsbergs und des Spessartgebirges beeinflusst werden. Im Norden begrenzt es im Allgemeinen der Lauf der Lahn von Giessen bis zu ihrer Mündung bei Lahnstein in den Rhein. Es wird dadurch der nördlichste Theil Nassaus ausgeschlossen, denn die Fauna des Westerwaldes trägt schon den Charakter des norddeutschen-westfälischen Gebirgslandes. Nur die Umgegend von Dillenburg und Hadamar wurde noch mithereingezogen, weil von hier das umfassendste Sammelmaterial der HH. Professoren Dr. Schenck und Dr. Kirschb a u m, sowie des Kgl. Landesgeologen Dr. Carl Koch vorlag. Diese Gebiete bilden somit die nördlichste Grenze unserer Fauna. Eine Gebietsüberschreitung fand ferner statt im Süden; - hier wurde die Main- und Rheinlinie nicht genau eingehalten und besonders die Mainzer und Mombacher Umgebung in grösserer Ausdehnung hinzugezogen. Auch der Frankfurter und Schwanheimer Wald gehören, als auf dem linken Mainufer gelegen, mehr den letzten Ausläufern der Bergstrasse an.

Als Grundlage dieser Arbeit diente meine Sammlung, von meinem Vater, Senator Dr. Carl von Heyden in Frankfurt, schon zu Anfang 
dieses Jahrhunderts begründet und bis zu seinem Tode 1866, also nach 60 jähriger Thätigkeit, von ihm und von da an von mir allein fortgeführt. Wohl wenige Forscher verstanden es so wie mein Vater, die Natur in ihrem innersten Treiben zu belauschen; fast unter jedem Stück seiner Sammlung aus allen Insectenordnungen ist das Datum des Fundes, die Futterpflanze und Lebensweise angegeben. Ich selbst sammelte seit meiner frühesten Jugend unter seiner Anleitung und war sein steter Begleiter an den wöchentlich einmal unternommenen Excursionen.

In der Umgegend von Frankfurt sammelten ferner sehr eifrig in der Neuzeit die HH. Dr. jur. Georg Hag $\mathrm{H}$ - Rutenberg, besonders in der Gegend von Isenburg, wo er längere Zeit wohnte. - Dr. phil. Oscar Böttger sammelte höchst gewissenhaft mit steter Angabe der Fundorte und Erscheinungszeit. - Dr. med. Moritz Schmidt war eine Reihe von Jahren hindurch mein Begleiter im Frankfurter Wald und im Taunus. - Die HH. A u gust und Carl Ster n sammelten fleissig, erzogen aber in letzter Zeit mehr die Käfer, indem sie, wie früher schon mein Vater, kränkelnde Holzstämme in ein besonders dazu hergerichtetes Zimmer stellten und dann mit Bequemlichkeit die sich daraus entwickelnden Käfer, oft die seltensten, die im Freien nur des Nachts zum Vorschein kommen oder in den Gipfeln der Bäume leben, in grosser Menge von den Fensterscheiben ablesen konnten.

Weitere Frankfurter Sammler sind die HH. Dr. phil. Steitz, der verstorbene Rentier Harer und G. Gremmers, Seb. Alex. Scheidel, Bahnmeister Gräf und Oberrevisor Jännicke, beide jetzt in Mainz, Heinr. von Twa rdowski, jetzt preussischer Hauptmann, und Dr. med. Gerlach, jetzt in Hongkong.

Bei Friedberg sammelten Decan Scriba (damals in Oberlais), die verstorbenen HH. Dr. Renner und Hauptmann Fuhr, bei Giessen der gleichfalls verstorbene Baurath Laubenheimer, dessen Sammlung ich durch die Güte des Herrn Professor Schneider und Conservator Zinszer Gelegenheit hatte, auf der Universität Giessen, wo sie nun aufgestellt ist, zu studiren.

Herr Prälat Dr. Schmitt, ein Zeitgenosse meines Vaters, sammelt noch seit mehr denn 50 Jahren bei Mainz und Mombach.

In Nassau selbst sammelten oder sammeln noch jetzt von 1844-46 um Weilburg, 1846 um Hadamar und von da ab um Wiesbaden Professor Dr. Kirs chb a um, mein hochverehrter Freund, welcher zur Bearbeitung dieses Verzeichnisses den entscheidenden Anstoss gab. Professor Dr. Schenck sammelte 1845 bei Hadamar und Dillenburg, 
von da an bei Weilburg; bei Dillenburg auch Dr. C. Ko ch. - Bei der Irrenanstalt Eichberg im Rheingau sammelte Dr. Richter, jetzt Arzt an der Irrenanstalt in Göttingen.

Allen diesen Herren, besonders aber Dr. Böttger, Professor Schenck und Prälat Schmitt, welche meinem Manuscripte die werthvollsten Notizen selbst beifügten und mir zweifelhafte Arten zur Untersuchung einschickten, - die anderen Sammlungen durfte ich durch die Freundlichkeit ihrer Besitzer oft mehrfach selbst durchsehen - meinen besonderen Dank.

Von früheren Bearbeitungen verdienen Erwähnung das schon oben angeführte Verzeichniss von Scriba, das unstreitig die erste Stelle einnimmt und worin auch die meisten der Frankfurter Funde schon erwähnt sind. Es umfasst aber ein mehr wie doppelt so grosses Gebiet und sind desshalb auch viel mehr Arten verzeichnet, weil der Vogelsberg und die rheinhessische Ebene, sowie der Odenwald, eine ganze Anzahl von Arten beherbergen, die unserem Gebiet fehlen.

Das Junker'sche ,Verzeichniss der Käfer, welche drei Meilen in der Umgegend von Hanau gefunden werden", erwähnte ich desshalb nicht, weil es zu viele Irrthümer enthält, die auf Unkenntniss der Arten und falschen Bestimmungen beruhen. Derartige Werke bringen direkten Schaden, weil sie Unrichtiges verbreiten. Leider wurde dieses Verzeichniss in Zebe's Synopsis benutzt und sind Notizen daraus in die besten Werke, z. B. Red ten b a c he r's Fauna austriaca übergegangen. - Nach dem heutigen Stande der Wissenschaft kann man fest behaupten, dass z. B. Calathus punctipennis, Hydroporus 12-pustulatus, Ipidia 4-notata, Cyllodes ater, Anomala vitis und Julii, Cetonia florentina, Dircaea ferruginea, Boros corticalis, Helops testaceus, Trachyphloeus ventricosus, Liophloeus Herbstii und gibbus, Tyloderes chrysops, Larinus Cynarae, Hammaticherus velutinus, Labidostomis taxicornis, Nundina discimacula u. s. w. einfach nicht vorkommen ,können", zum Theil der deutschen Fauna überhaupt nicht angehören. Derartige Verzeichnisse mit einfacher Namensangabe, ohne Nachweis des Finders und ohne jegliche Garantie der richtigen Bestimmung haben gar keinen Werth.

In den Programmen der Realschule von Bingen publicirte Lehrer M üh r ein Verzeichniss der dortigen Käfer. Ich konnte mir dieses Werkchen nicht verschaffen, doch wurde mir von anderer Seite nichts Günstiges darüber mitgetheilt; scheint auch über den Anfang (die ersten Familien bis Lucaniden als leichtesten Theil) nicht weit hinauszukommen, wie bei so vielen derartigen Arbeiten. 
Zu Ende des vorigen Jahrhunderts war in Mainz besonders thätig: Nicolaus Joseph Brahm. Er publicirte:

1. Insektenkalender in 2 Theilen, 1790 und 91.

2. In Borkhausens Rheinischem Magazin 1793: „Versuch einer Fauna entomologica der Gegend um Mainz."

3. In Hoppe's entomologischem Taschenbuch 1797: „Verzeichniss der von der Gattung Clerus in den Gegenden von Mainz und Aschaffenburg einheimischen Arten."

4. Im Naturforscher 1802 :

a) „Bemerkungen über die von der Gattung Coccinella bei Mainz einheimischen Arten."

b) Desgleichen der Gattung Cassida.

5. Ich besitze handschriftliche Manuscripte, ,zwei Bände entomologische Taschenbücher von $1785-1830$ ", die nie publicirt wurden.

Wegen der alten Nomenclatur und weil nahe ver"wandte Arten damals noch nicht unterschieden waren, ist es oft unmöglich, sich klar zu machen, welche Art Brahm vor Augen hatte; nur wo gar kein Zweifel obwaltet, habe ich daher von einzelnen Arten Notiz genommen.

Was das System betrifft, nach welchem sich hier die Familien und Gattungen aneinander reihen, so folgte ich der neuesten Arbeit von Seidlitz in seiner „Fauna baltica. Die Käfer $1875^{\prime \prime}$. Das Werk meines lieben Freundes hat den grossen Vorzug, dass es auf dem Boden der neuesten Forschung, mit Benutzung der von Harold'schen Nomenclaturprincipien, steht und überragt die meisten Handbücher, die letzte Ausgabe von Redtenbacher nicht ausgeschlossen, durch gründliche erneute Untersuchung auch der häufigsten Thiere, die denn auch eine ganz neue Reihenfolge, nach der Verwandtschaft der einzelnen Gattungen, bedingte.

Einer besonders interessanten Lokalität sei hier noch Erwähnung gethan, es ist der "Mombacher Sand" oder die "Mombacher Haide“ eine sehr ausgedehnte Sandfläche zwischen Mainz, Mombach und Gonsenheim. Der Hauptgrund, warum so viele meist sonst mehr südlich lebende Thiere hier vorkommen, liegt wohl in den Anschwemmungen des Rheins, welche viele fremde Gäste mitbringen, die, wenn die lokalen Verhältnisse nur einigermassen entsprechen, dann gerne dableiben. Im Hochsommer entwickelt sich auf der grossen Sandfäche eine intensive Hitze, die umsäumenden Waldränder sind dann eine wahre Fundgrube der seltensten 
südlichen Insekten. Ich erinnere nur an Anoxia, Anisoplia, Phytoecia Jourdani, Cassida filaginis u. s. w.

Die Umgebung von Frankfurt war früher reich an grösseren und kleineren Sümpfen und Wüsteneien, jetzt sind sie fast alle der Cultur gewichen.

Der Schmitterhof bei Giessen ist ein Landgut bei Rodheim an der Bieber, am Fusse der Ruine Vetzberg; ich sammelte dort öfter recht fleissig, er ist zugleich die nordöstlichste Grenze unseres Gebietes.

Zur Vermeidung zahlreicher Wiederholungen führte ich folgende Abkürzungen ein:

Fr. = Frankfurt.

Dlb. = Dillenburg.

G. $=$ Giessen.

Had. = Hadamar.

Mo. = Mombach.

Mz. = Mainz.

M. = Umgegend von Mainz, namentlich die waldigen Theile von Mombach bis Heidesheim und der Wald nach Oberolm.

Wlb. = Weilburg.

Wsb. = Wiesbaden.

B. $=$ Dr. Böttger.

C. H. = Dr. Carl von Heyden.

L. H. = Dr. Lucas von Heyden.

Hg. = Dr. Ha ag.

K. = Prof. Dr. Kirschbaum.

Ko. = Dr. Carl Koch.

L. = Baurath La ubenheimer.

M. S. $=$ Dr. med. Moritz Schmidt.

S. = Prälat Dr. Schmitt.

Sch. = Prof. Dr. Schenck.

St. = August und Carl Stern. 
„wir" und ,von uns" bedeutet von meinem Vater und mir gefunden.

s. $=$ selten.

s. s. $=$ sehr selten.

n. s. $=$ nicht seltenl.

n.s.s. $=$ nicht sehr selten.

h. = häufig.

s. h. = sehr häufig.

g. $=$ gemein.

S. V. = das in der Vorrede erwähnte Verzeichniss von Scriba.

$<$ Anfang eines Monats.

$=$ Mitte $\gg \gg$

$>$ Ende $\gg \quad 》$

Eine Zahl hinter diesen Zeichen bedeutet den Monat, z. B. $=6$ heisst Mitte Juni. 


\section{CARABICIDAE.}

\section{Cicindela L.}

1. C. campestris L. - Fr. Im ganzen Gebiet. - Bei Wlb. und Dlb. kommt nur diese Art vor (Sch.). - Mz. Mo. (S.) - G. (L.) - Bei Soden schon 2. April 1876. (L. H.) Im Nadelholzwald zwischen Holzhausen und Allendorf in Nassau im Mai (K.). -

2. C. hybrida L. - Im Gebiet kommt nur die Normalform vor, mit ungetheiltem Mondfleck an der Schulter, der Seitenfleck durch eine sehr kurze Linie mit dem etwas rückwärts neben der Naht stehenden Punkt verbunden. $>4$ in Begattung gef. Friedberg (Scriba). - Mz. Mo. (S.) - G. (L.) - Bei Mo. auf Blössen im Kiefernwald, besonders im Juli und August (K.). -

3. C. sylvatica L. - Im Frankfurter Wald an Sandstellen in Kiefernbeständen, z. B. am Sandhof. - Mo. von Brahm schon am 13. April 1786, später von S. s. gef. -

4. C. germanica L. - C. H. fand diese Art vor langen Jahren unter geschnittenern Hafer auf dem Wege von Soden nach Cronthal. G. (L.) -

\section{Omophron Latreille.}

1. O. limbatus F. - Im ganzen Gebiet am Rande von Tümpeln. Von $=5$ bis $>10$ z. B. bei Bockenheim an den ehemaligen Basaltbrüchen. - Friedberg an den Ufern der Us (Scriba). - Bei Biebrich am Rhein. und am Lahnufer bei Wlb. von K. gef. - Am Rhein und den Lachen, die in den Rhein münden h. (S.) -

\section{Notiophilus Duméril.}

1. N. aquaticus $L$. - Fr. von L. H. oft gef. und von St. in Sandbrüchen. - Dlb. und Wlb. (Sch.) - Mz. Mo. (S.) - G. (L.) Schierstein am Rhein 15. Mai (K.). - 
2. N. palustris Duft. - Fr. - Schlangenbad, Dlb. und Wlb. (Sch.) - Mz. Mo. (S.) - G. (L.) - Mo. auf Waldblössen 18. Juli (K.) -

3. N. biguttatus F. - Fr. - Taunus. - Die 3 Arten n. s. an feuchten Stellen Dlb. and Wlb. (Sch.) - G. (L.) -

4. N. substriatus Waterh. = punctulatus Wesm. - Ich besitze ein bei Fr. gef. Stück. -

\section{Elaphrus Fabricius.}

1. E. uliginosus F. - Fr. - s. - Wetzlar (Bach.). - Mo. s. (S.) -

2. E. cupreus Dft. $-\mathrm{Fr} .-\mathrm{s}$. -

3. E. riparius L. - Fr. - Flörsheim am Main. Ueberall h. auch am Rhein (S.). - G. (L.) - Am Ufer der Mainspitze 22. April 1852 (K.). -

\section{Blethisa Bonelli.}

1. B. multipunctata L. - Fr. früher häufiger. - Unter Steinen auf Sandboden in der Nähe des Wassers am Königsbrünnchen, 2 Ex. 1861 (B.). - Mz. Mo. (S.) -

\section{Cychrus Fabricius.}

1. C. rostratus L. (Form a. Schaum=elongatus Hoppe.) Die bei Fr. gef. Stücke meiner Sammlung gehören entschieden dieser gestreckteren Form mit längeren Flügeldecken an, welche nach Schaum in Mitteldeutschland fehlen, aber wieder im Norden (Dänemark, Schweden) vorkommen soll. Auch ein Stück der Uebergangsform zu der Form b. (dem ächten rostr., den ich nicht aus dem Gebiet kenne). - Bei Wlb. (Sch.). - Nach demselben fehlt er bei Dlb. - Im Niederhadamarer Wald unter Moos im Frühjahr (K.). -

2. C. attenuatus F. - Schlangenbad (C. H.) - Rumberg bei Königstein, Juli 1872 (L. H.). - Bei Dlb. fehlt er (Sch.). - Wlb. am Odersbacherweg einmal im Frühjahr gef. (K.) -

\section{Carabus Linne.}

1. (Procrustes Bon.) coriaceus F. - Fr. (C. und L. H., St., Gräf). - Elsheim bei Ingelheim 1874 (B.). - Unter gefällten Baumstämmen zwischen Sandhof und Forsthaus von St. gef. - Nach Sch. s. h. bei Dlb. auf Waldwegen, ebenda auch von Ko. gef. - Mr. auf den Spaziergängen am oberen Theile der Stadt n. s. (S.) - Wsb., Wlb., Had. unter Moos im Frühjahr. - 
2. C. intricatus $\mathbf{L}$. = cyaneus $\mathbf{F}$. - In Wäldern unter Laub und Moos am Fuss von Bäumen: Fr., Schlangenbad. - Friedberg (Scriba). - Lorsbacher Thal (B.). - Buchrainweiher bei Offenbach (B.). - Von St. wie der vorige gef. - Had. n. s. (K.) - In Wäldern bei Dlb. in alten Eichenstrünken h. (Sch., Ko.) -

3. C. auratus L. - Fr. Auf Feldwegen in der Ebene h. Auch im Taunus g. (B.). - Wsb. (K.) - Bei Dlb. and Wlb. g. (Sch.). Mz. Mo. h. (S.) -

4. C. auronitens F. - Gebirgsthier. Im Taunus n. s. s.: Grosse Feldberg (doch nicht auf dem Plateau) mit intensiv kupfergoldenem Halsschild; die Form mit grüngoldenem Halsschild ist häufiger: Falkenstein. - Wsb. zwischen Neroberg und Platte h. unter Moos im Winter von K. gef. - Ziemlich h. bei Obershausen und Wlb. (Sch.) - Haiger bei Dlb. (Ko.) - Weg über den Trompeter nach Bellingen (K.). -

5. C. morbillosus Panz. - Fr. Früher n. s. s., sonst in der ganzen Mainebene. - Wsb. (K.) - Dlb. auf Wegen (Sch.). - Mo. n. s. (S.) -

6. C. granulatus L. - Fr. h. - Bei Had. roth- und schwarzbeinige Stücke von K. gef. - h. bei Dlb. und Wlb. mit schwarzer, grüner und broncener Oberseite, mit schwarzen und rothen Schenkeln (Sch.). - Mz. Mo. h. (S.) -

7. C. cancellatus F. - Form mit rothen Schenkeln bei Fr. n. s. - Dlb. nur mit rothen Schenkeln (Sch.). - Mz. Mo. h. (S.) - G. (L.) Varietät mit schwarzen Schenkeln Fr. s. (St.) - G. (L.) -

\section{C. monilis $F$.}

Form b. Suffrian. (Zwischen zwei Rippen mit einer Reihe länglicher Körner, welche von je einer oft unterbrochenen Längslinie begleitet sind. Die Rippe höher.) Bei Fr. - Mz. auf den Höhen um die Stadt und hinter Mo. (S.) -

Form d. Suffr. = affinis Panz. (Die zwei ganzen Linien, welche die Rippen begleiten, so hoch wie diese, so dass zwischen zwei Tubercelreihen sich drei gleich hohe Längslinien befinden. Grösse 22 Millim.) Auf den Wiesen bei Hausen, also an den äussersten Ausläufern des Taunus.

Auf dem Plateau des grossen Feldberg, doch nur da, und zwar unter Steinen in der Umgebung des Feldberghauses, findet sich eine kleine var. der Form d. von nur 16-18 Millim., welche ich unter dem Namen var. Taunicus in 12. Bericht des Offenbacher Vereins für Natur- 
kunde 1871, p. 44 beschrieben habe. - Sonst findet sich diese kleine Form nur noch auf dem höchsten Punkt des Vogelsberges, dem Hohenrothskopf und in der Rhön. Doch sind die 'Taunusstücke stets kleiner. -

9. C. arvensis F. - Hellbronceglänzende Stïcke mit schwarzen Schenkeln auf dem Feldbergplateau n. s. unter Steinen. Von St. wie coriaceus gef. - Im Niederhadamarer Wald s. h. (K.) - Wlb.; fehlt bei Dlb. (Sch.) - Mz. Mo. (S.) - G. (L.) -

Ein Ex. mit ganz dunkler Oberseite im Frankfurter Wald bei der unteren Schweinstiege tief im Wald $>5$ von C. H. gef. - Auch am Buchrainweiher bei Offenbach von B. im Vorfrühling gef. -

10. C. catenualatus F. - Fr. - Nördlicher Abhang des grossen Feldbergs am 3. Sept. 1871. - Schwanheimer Wald oberhalb des Goldsteins in faulen Baumstrünken im April h. von St. wie coriaceus gef. - Dlb. (Ko. und K.) - Am grossen Mannstein, Staufen im Taunus (L. H. 2. April 1876). -

\section{C. violaceus $L$.}

Bei Form $\alpha$. Suffr. ist die ganze Oberfläche der Flügeldecken mit scharf von einander getrennten Köruchen dicht besät. - Fr. s. (C. H.) -

Bei Form $\beta$. Suffr. ordnen sich die Körnchen hie und da meist zu drei Längslinien, indem 2 oder 3 der Länge nach in eins zusammenfliessen.

Diese Form ist im Frankfurtel Unterwald $=6$ n. s. $^{\circ}$ Mr. im Oberolmer Wald (S.) - Dlb. s. (Sch.) -

Form $\delta$. Suffr. (Einzelne Körnerreihen șind zu vollständigen Längslinien vereinigt.)

Frankfurter Wald. - Mz. (Bach.) - Dlb. h. (Sch.) - Die häufigste Form. -

12. C. purpurascens F. - Ausser der Sculptur durch andere Penisbildung des Männchens von violaceus unterschieden. Die Spitze der forceps ist schmäler und länger, leicht gebogen, oben in der Mitte fast eckig. -

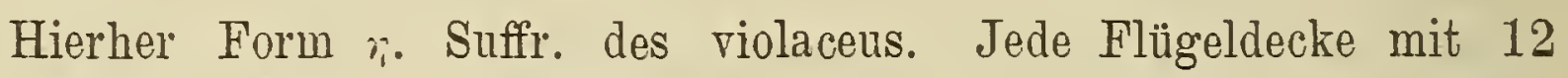
regelmässigen Längsrippen, nur die 2., 8., 12. durch Grübchen unterbrochen. - Auf dem Westerwald im Nassauischen bis gegen Mainz (Bach). - Mz. (S.) - G. (L.) - Wsb., Wlb., Had. im Frühjahr unter Moos (K.). - Fr. - Hochstadt $<9$ (C. und L. H.) -

Var. exasperatus Duft. (= Form 3. Suffr. des violaceus.) Der $4 ., 8 ., 12$. Streifen sind die regelmässigsten, sie sind kielförmig erhaben, 
aber durch eingestochene Pünktchen getrennt. Fr. Wald (Gräf). Dlb. h. (Sch.) -

Var. crenatus Sturm. (= Form จ. Suffr. des violaceus.) Die Rippen meist nicht durch Grübchen unterbrochen, die Zwischenräume durch grosse, oft in die Quere gezogene Grübchen ausgefüllt, die beiderseits in die Rippen hineinragen. - Fr. von uns und Gräf gef. Schlangenbad, Rüdesheim $=5$. Schwalbach (St.). -

13. C. glabratus Payk. - Im Frankfurter Wald h. Von St. wie coriaceus gef. -

14. C. Convexus F. - Fr., Schlangenbad, Bingen $=4$, grosser Feldberg 14. April 1865 ein breites Weibchen; - Wetzlar (Bach). Isenburg (B.). - Dlb. unter Steinen (Sch.). - G. (L.) - Zwischen Dotzheim und Frauenstein im Rheingau im April (K.). -

15. C. nemoralis Illig. - Fr. sehr intensiv gefärbte Stücke fand St. - Grosser Feldberg 4. September 1870 ein Männchen mit dunkel purpurrothem Halsschild und Flügeldeckenrand, Oberseite dunkelbronce. Auch grüngefärbte Stücke von St. h. in Gärten in eingegrabenen Töpfen gef. - Nach K. bei Had. häufiger als bei Wsb. - s. h. bei Dlb. und Wlb. in Gärten, Feldern, Wäldern auf Wegen (Sch., Ko.). — G. (L.) -

\section{Calosoma Weber.}

1. C. sycophanta L. - Fr. in Waldungen, doch h. nur in Jahren, in denen auch die Bombyx monacha und die Processionsraupe h. sind, denen er eifrig nachstellt. - Station Schwanheim an jungen Eichen h. und im Rödelheimer Wäldchen von St. gef. - Bei Mo. einmal von K. beim Forsthaus Lenaberg gef. - Mz. und Mo. n. s. (S.) - G. (L.) -

2. C. inquisitor F. - Fr. (C. H.) - In neuerer Zeit im Rebstockwald besonders von H. Gräf und St. gef. - Soden an alten Eichen $>$ 5. Bei Hochstadt im Hartigwäldchen am Boden 1862 (B.). - Bei Wsb: von K. gesammelt, bei Wlb. von Sch. - Dlb. (Ko.) - Mz. im Wald bei Oberolm und hinter Mo. (S.). - G. (L.) -

\section{Nebria Latreille.}

1. N. livida L. - Das einzig bekannte Exemplar aus hiesiger Gegend fand C. H. am Obermain bei Fr., an der sogen. Kaiserley. Nach Bach „Im August unter Ufergenist bei Bingen" (Klingelhöffer).

2. N. brevicollis F. - Ueberall n. s. - Soden unter Rinden an den Wiesen. - Dlb. auf Waldwiesen (Sch.). - In einem alten Tage- 
buch meines Vaters finde ich die Notiz ,die Eier sind fast eine Linie lang, länglich, wachsgelb, etwas glänzend, sehr dünnhäutig. Das Weibchen legte 24-30 Eier im September und October 1822." - Mz. Mo. (S.) -

\section{Leïstus Fröhlich.}

1. L. spinibarbis F. - Ein Ex. meiner Sammlung von Renner bei Friedberg gesammelt. - Dlb. z. h. auf Waldwiesen (Sch. Ko.). Mz. im Wald bei Oberolm (S.). -

2. L. ferrugineus L. - Fr., Bergen n. s. gesellig unter Steinen. - Dlb. (Sch.) - G. (L.) -

3. L. rufescens F. - Im kleinen Feldbergthal zweimal mit dem Streifnetz von L. H. gef. -

4. L. piceus Fröhl. - Ein Stück bei Schlangenbad von C. H. gef. - In S. V. durch einen Irrthum meinerseits als rufomarginatus aufgeführt. -

\section{Dyschirius Bonelli.}

(Die Exemplare meiner Sammlung vom Monographen Putzeys bestimmt.)

1. D. globosus Hbst. - Fr. - Ueberall h. an feuchten Stellen. - Ems. - Wlb. unter Pappelrinde (Sch.). - Mz. Mo. (S.) -

2. D. angustatus Putz. - Ein Originalexemplar von Fr. (C. H.). - Einmal bei Mo. auf dem Sand, weit von Wasser entfernt von L. H., auch von S. gef. -

3. D. nitidus Dej. - Fr. $=6$ am Mainufer. - Ems. -

4. D. politus Dej. - Offenbach am Mainufer $<8$. -

5. D. intermedius Putz. - Das einzig bekannte Ex. aus dem Gebiet, zugleich eines der Typen von Putzeys fand C. H. bei Fr. -

6. D. aeneus Dej. - Fr. h. - Soden im August. - Mz. Mo. (S.) -

\section{Clivina Latreille.}

1. C. fossor L. - Fr., Ems, Bingen überall h. - Wlb. (Sch.) Mz. Mo. (S.) - Bei Mo. 19. Juni (K.). -

Var. collaris Hbst. - Fr. ebenso h. - Mz. Mo. (S.) -

\section{Brachinus Weber.}

1. B. crepitans L. - Bei Fr. z. s., doch nur auf Basaltboden z. B. am neuen Irrenhaus. - B. fand ein Exemplar unter einem Stein 
am Main. - Fr. von St. mehrfach gef. - Wsb. n. s. (K.). - Dlb. (Sch., Ko.) - Wlb. h. (Sch.) - Mz. Mo. h. (S.). -

2. B. explodens Dft. - Im ganzen Gebiet s. h. unter Steinen. Fr. - Wsb. (K.) - Wlb. seltener als der vorige (Sch.). - Mz. Mo. h. (S.) - G. (L.) -

\section{Odacantha Payk.}

1. O. melanura L. - Früher h. im Röhricht um Fr., z. B. am Kettenhof, Hellerhof, Metzgerbruch, Enkheimer Torf, jetzt sind die Lokalitäten zum Theil angebaut und das schöne Thierchen recht selten geworden. Das ganze Jahr hindurch, überwintert in den hohlen Rohrstängeln. - Bei Mz. in Genist, welches der Rhein auswirft n. s. (S.). -

\section{Aëtophorus Schmidt-Göbel.}

1. A. imperialis Germ. - Dasselbe Vorkommen wie Odacantha. - Von St. am Main unter Platanenrinde einmal gef. - Mz. wie die vorige Gattung (S.). - Fr. 8. April 1860 (B.). -

\section{Demetrias Bonelli.}

1. D. unipunctatus Germ. - Lebensweise und. Fundorte wie Odacantha und Aëtophorus. - Mz. wie die vorige Gattung (S.). -

2. D. atricapillus L. - Ueberall h. Fr. - Soden. Mz. wie die vorige Art (S.). - Auf den Blössen bei Mo. 22. Sept. und an der Wellritz bei Wsb. 6. Mai (K.). -

\section{Dromius Bonelli.}

1. D. linearis $\mathbf{0 l}$. - Im ganzen Gebiet verbreitet, unter Rinden, Fr. - Soden. - Wlb. (Sch.) - G. (L.) -

2. D. marginellus F. - Fr. s., Wsb. im Dezember unter Platanenrinde (C. H.). - G. (L.) -

3. D. testaceus Er. - Wsb. unter Platanenrinde, noch im November in Begattung (C. H.). - Fr. ebenso in den-Promenaden. Hierher meridionalis in S. V.

4. D. agilis F. - Im Winter unter Platanenrinde bei Wsb., im Fr. Wald unter Fichtenrinde im Februar. - Wlb. (Sch.) - Mz. Mo. (S.) - G. (L.) -

5. D. quadrimaculatus Panz. - Fr. - Wsb. in Gesellschaft der vorigen Arten. - Wlb. (Sch.) - Mz. Mo. (S.) - G. (L.) - 
6. D. quadrinotatus Duft. - Fr. - Wsb., wie die vorigen. Im Fr. Wald auch unter Fichtenrinde. - Mz. Mo. (S.) - G. (L.) -

Var. biplagiatus Heyd. - Diese von Schaum aus der Mark Brandenburg erwähnte Varietät mit zusammenfliessenden Flecken besitze ich einmal von Fr. -

7. D. notatus Steph. - Fr. - Soden, Falkenstein. Ueberall h. Mz. Mo. (S.) -

Var. nigriventris Thoms. - Fr., Soden. Bei Wlb. 4 Exempl. von Sch. gef. (in seiner Sammlung als fasciatus). - Mz. Mo. (S.) -

Var. melanocephalus Dej. - Fr. Alle drei Formen überall h. unter Rinden und Laub. - Mz. Mo. (S.) -

8. D. sigma Rossi. - Fr. - Enkheim an feuchten Stellen. Wlb. (Sch.) -

\section{Blechrus Motschulsky.}

1. B. glabratus Dft. = maurus Stm. - Wurden stets für zwei Arten gehalten, doch bei reichlichem Material nicht zu trennen, da alle Uebergänge in der Form des Halsschildes vorkommen. Fr. - Soden, Ems an trockenen, sterilen Orten unter Steinen. - Wlb. (Sch.) -

\section{Metabletus Schmidt-Göbel.}

1. M. truncatellus F. - Fr., Wsb. im Febr. unter Platanenrinde. Wlb. (Sch.) - Mz. Mo. (S.) - G. (L.) - Eichberg im Rheing. (Richter). -

2. M. foveola Gyll. = punctatellus Dft. - Fr. - Seltener wie die vorige Art. - Wlb. (Sch.) - Mz. Mo. (S.) - G. (L.) -

\section{Lionychus Wissmann.}

1. L. quadrillum Dft. - Bei Ems am Ufer der Lahn von C. H. gef., ebenda auch fulgende Varietäten:

ß. Die hinteren Flecken sehr klein.

$\gamma$. Die hinteren Flecken fehlen ganz (= bipunctatus Heer). -

$\delta$. Auch die vorderen Flecken fehlen. - Die letzte Varietät s., wird auch sonst nicht angeführt.

\section{Lamprias Bonelli.}

1. L. cyanocephala L. - Fr. Nach B. früher (1861) auf der jetzt angebauten Bornheimer Haide; Bieberer Höhe auf Kalkboden (B.). - An der Mainschanze noch jetzt (B.). - Mz. (S.) - Mo. (K., S.) - 
2. L. chlorocephala Ent. Hfte. - Fr. Beide Arten waren an jetzt angebauten sumpfigen mit Rohr bewachsenen Stellen, z. B. am Kettenhof n. s. - Bieberer Höhe bei Offenbach. - Im Genist des Metzgerbruchs (B.). Nach Demselben häufiger als die vorige. - Im Rebstockwald n. s. von St. gef. - Wlb. oft an Pflanzen hinauflaufend gef. (Sch.). - Mz. Mo. (S.) - G. (L.) - Had. (K.) -

\section{Lebia Latreille.}

1. L. crux-minor L. - Fr. - Bieberer Höhe bei Offenbach $=7$. - Mo. $>4$ einzeln mit dem Streifnetz gef. - Friedberg (Fuhr). Auf einem sandigen mit Gras bewachsenen Wege oberhalb des Goldsteins im Schwanheimer Wald im Sommer 1860 gestreift (B.). - Bei Wlb. mehrmals mit dem Streifnetz gef. (Sch.). - Dlb. (K.) - S. fing ein Exemplar in Mz. im Zimmer, in welches es durchs offene Fenster hereinflog, ein anderes Stück in den Steinbrüchen bei Mo. - G. am 15. Mai 1862 (L.). - Oberzeuzheim bei Had. (K.). -

2. L. haemorrhoidalis F. - Fr. - Hofheim und Soden im Taunus von $>5$ bis $=9$ von niedrigen Gesträuchen geklopft. Das vorzugsweise Vorkommen nach Schaum auf Kalkboden bestätigt sich nach den Hochlieimer Exemplaren. - Wsb. Abhänge des Dambachthales, wo kein Kalk, sondern Taunusschiefer, h. von K. gef. -

\section{Cymindis Latreille.}

1. C. humeralis F. $->7$ im Fr. Goldsteinforst in Nadelholzwaldungen. - Soden $=7$. - Ems. - Nach B. h. auf Kalkboden auf der Bieberer Höhe bei Offenbach. - Wlb. (Sch.) - Mo. (S.) - G. (I.) Auf dem grossen Feldberg von Scheidel gef. -

\section{Masoreus Dejean.}

1. M. Wetterhali Gyll. - Dieses seltene Thier fand C. H. einmal bei Fr. - B. fand am 3. April 1860 ein Stück im Maingenist. -

\section{Loricera Latreille.}

1. L. pilicornis F. - Fr. an feuchten Stellen vereinzelt. Von St. am Main unter Steinen gef. - Wlb. z. h. (Sch.). - Mz. Mo. (S.) G. (L.) - 


\section{Panagaeus Latreille.}

1. P. crux-major L. - Fr. im Gebiet verbreitet; unter Steinen. Had., Wsb., am Rhein von Weiden geklopft (K.). - Wlb. (Sch.) Mz. Mo. (S.) - G. (L.) -

Var. trimaculatus Dej. - Einmal am ehemaligen Rüstersee am Fr. Forsthause von C. H. gef. - Mz. Mo. (S.) -

Auch die von Schaum aufgeführte seltene Var. (bei welcher die schwarze Querbinde am Seitenrand der Flügeldecken mit der schwarzen Spitze zusammenhängt, so dass hinten ein völlig von Schwarz eingeschlossener, rother runder Fleck gebildet wird), fanden Gräf und L. H. je einmal bei Fr.; L. H. auch einmal bei Falkenstein. Bei dieser Var. wird die Querbinde bei zwei Ex. so schmal in der Mitte, dass die drei Flecken der Var. trimaculatus nur durch eine feine Linie verbunden sind. Von Sch. auch einmal bei Wlb. gef.

2. P. quadripustulatus Sturm. - Fr. seltener wie der rorige. - Had. (K.) - Mo. (S.) - G. (L.) -

\section{Callistus Latreille.}

1. C. Iunatus F. - An trockenen sonnigen Orten s. - Fr. Einmal ein Exemplar auf dem Rossmarkt in Fr. von L. H. gef. - Schmitter Hof bei G. - Röderberg bei Fr. (B.). - Im Wisperthal (B.). - Bei Eltville am Rhein unter Steinen (St.). - Wlb. h. (K., Sch.). - Einmal bei Hechtsheim bei $M \%$. (S.). - Had. am Waldrand, - Geisberg bei Wsb. (K.). -

\section{Chlaenius Bonelli.}

1. C. caelatus Web. - Bei Seckbach von v. Twardowski gef., in Sammlung L. H. - Durch einen Fehler meinerseits in S. V. als sulcicollis bezeichnet, den ich nicht aus unserem Gebiet besitze. - B. fand die seltene Art auch am Frankfurter Röderberg.

2. C. holosericeus F. - Von C. H. sehr einzeln am Obermainufer, gegenüber der Gerbermühle gef. — Am Lenaberg hinter Mo. s. (S.) -

3. C. variegatus Fourcr. = agrorum OI. - Am Mainufer bei Fr. n. s. unter Steinen. - Ems (Bach). -

4. C. vestitus Payk. - Am Mainufer und an feuchten Stellen h. - Wlb. (Sch.) - Mz. Mo. (S.) - G. (L.) - Fr. 8. April 1860 (B.). - Am Rheinufer im Frühjahr (K.). - 
5. C. Schrankii Dft. - Fr. - Ems. - Von St. am Mainufer unter Steinen gef. - Bei Wsb. an einer jetzt eingegangenen Pfütze am Kirchhof 1847 (K.). - Wlb., Dlb. (Sch.) - G. (L.) -

6. C. nigricornis Sturm. (Stammform mit schwarzen Beinen). Von Harer bei Fr. mit vestitus zusammengefunden. - Von St. am Main gef. - Wlb., Dlb. (Sch.) - Mz. Mo. (S.) -

Var. melanocornis Dej. (mit rostrothen Beinen) am Mainufer n. s. - Auf dem Röderberg unter Steinen (B.). - Wsb. in Gesellschaft der vorigen Art (K.). - Wlb. Dlb. (Sch.) - G. (L.) -

\section{Oodes Bonelli.}

1. O. helopioides F. - Fr. an trockenen sandigen Orten, z. B. an der Salpeterhütte, am Königsbrunnen n. s.; auch von St. gef. Mo. (S.) - Bei Cronthal von Scheidel gef. -

\section{Licinus Latreille.}

1. L. cassideus F. - Früher auf dem jetzt zum Theil urbar gemachten Lerchesberg über Sachsenhausen von C. H. n. s. s. gef. - Von Dr. Steitz einmal bei Fr. - Von Hg. auf der Grüneburg in der Schener gefangen, wahrscheinlich mit Frucht eingebracht. -

2. L. depressus Payk. $->6$ und $<71831$ von C. H. gef., wie die vorige Art. -

\section{Badister Clairville.}

1. B. unipustulatus Bon. - Fr. s. - Mz. Mo. (S.) -

2. B. bipustulatus F. - Fr. - Im Taunus, z. B. Festung Königstein unter Steinen im Mai. - Wlb. (Sch.) Mz. Mo. (S.) - G. (L.) --

Var. binotatus Fisch. Die schwarze Zeichnung der Flügeldecken besteht nur in einem kleinen, mittleren Fleck zu beiden Seiten der Naht und in einer die Spitze einnehmenden Binde. - Fr. s. s. -

3. B. humeralis Bon. - Fr. s. In den Enkheimer Torfgruben, liebt Schilfgegenden. Bei Fr. auch von B. einmal gef.

4. B. peitatus Panz. - Fr. n. h. - Mo. (S.) -

\section{Broscus Panzer.}

1. B. cephalotes L. - In sandigen Gegenden unter Steinen in fast 3 Zoll tiefen Löchern lebend, z. B. am Königsbrunnen, auf den Maininseln an der alten Frankfurter Brücke. 
B. beobachtete, als er den Käfer fing, wie er die Kiefer öffnete, die 4 Vorderbeine an sich zog, aber die Hinterbeine weit von sich streckte.

Auf dem Sachsenhäuser Berg von St. oft gef. - Dlb. Wlb. (Sch.) Mz. bei Gonsenheim n. s. (S.). - Wsb. am Schiessstand 10. Mai (K.). -

\section{Patrobus Dejean.}

1. P. excavatus Payk. - Im Taunus (C. H.). - Fr. zwei Stück (B.). - G. (L.) -

\section{Sphodrus Clairville.}

1. S. leucophthalmus L. - Fr. in dumpfen Kellern, Pferdeställen. $>8$ auch von St. gef. - Wsb. (K.) - Wlb. in Kellern (Sch.). Mz. Mo. n. s. (S.). -

2. S. (Pristonychus) inaequalis Panz. = subcyaneus III. = terricola Hbst. - In den verlassenen Kellern der Ziegelhütte am Sachsenhäıser Berg. - Wsb. in Kellern h. (K.). - Mz. Mo. (S.) - Rheingau (K.). -

Var. lithuanicus Motsch. (kleiner, mit geraden Mittelschienen) fand C. H. zweimal mit der Stammart bei Fr. $<8$.

\section{Calathus Bonelli.}

1. C. cisteloides Illig. - Ueberall h. Fr. - Dlb. (Sch.) - Mz. Mo. h. (S.) - G. (L.) - Had. unter Moos (K.). - Neroberg bei Wsb. 6. August (K.). -

Var. frigidus F. (mit dunkeln Beinen). 'Seltener. -

2. C. flavipes Fourc. $=$ fulvipes Gyll. - Ueberall h. - Fr. - Dlb. (Sch.) - Mz. Mo. (S.) - G. (L.) - Mo. am Ufer und vordere Blösse gegenüber Schierstein 13. September 1851 (K.). -

3. C. ambiguus Payk. = fuscus F. - Fr. weniger h. - Von St. n. s. auf dem Mombacher Sand gef.; Mz. MIo. (S.) -

4. C. melanocephalus L. - Fr. Besonders h. im Frühjahr (B.). Dlb. (Sch.) - Mz. Mo. (S.) - G. (L.) - Had. (K.) -

5. C. micropterus Dft. - Schlangenbad $=7$ C. H. - Von Fresenius bei Fr. ein Stück gef., ebenso von St. - Mz. Mo. (S.) -

Taphria Bonelli.

1. T. nivalis Panz. - Fr. n. h. - G. (L.) - 


\section{Anchomenus Erichson.}

1. A. angusticollis F. - Im Fr. Wald unter Moos an alten Bäumen. - Wlb. (Sch. Ko.) - Had. (K.) - Mz. Mo. (S.) - G. (L.) Fr. 1. Mai 1860 (B.). -

2. A. livens Gyll. - Ein von Becker bei Offenbach gefundenes Exemplar in Sammlung v. Heyden. -

3. A. dorsalis Müll. = prasinus F. - Im ersten Frühjahr gesellschaftlich oft in grosser Menge unter Steinen, überall. - Dlb. Wlb. mit Brachinus crepitans unter Steinen (Sch.). - Mz. Mo. h. (S.). Soden im Taunus 2. April 1876 (L. H.). - Had. Wsb. (K.) -

4. A. albipes III. - Im ganzen Gebiet s. h, unter Steinen. Fr. - Ems. - Mz. Mo. h. (S.). - G. (L). - An einer Pfütze zwischen Schwalbacher Chanssee und Wellritzmühle 18. April (K.). -

5. A. oblongus $\mathbf{F}$. - Seltener als der vorige, mehr in schilfigem Terrain von St. und B. gef. - Miz. Mo. am Rhein (S.) - G. (L.) Rheinufer bei Schierstein 16. April (K.). -

6. A. quadripunctatus Deg. var. cupratus Sturm. - Diese Seltenheit fand C. H. einmal in àer Fr. Promenade unter Platanenrinde = 10. - Einmal bei Wlb. ein schwarzes Exemplar von Sch. gef. -

7. (Agonum) marginatus L. - Fr. am Mainufer und anderen feuchten Stellen h. - Mo, am Rhein, häufiger bei Had. und Wlb. (K.). Mz. Mo. (S.) - Rheingau, Wsb. (K.) - G. (L.) -

8. (Ag.) impressus Panz. - Von C. H. bei Fr. s. s. gesammelt. - Mz. Mo. unter Steinen am Rheinufer (S.). -

9. (Ag.) sexpunctatus F. - Fr. Ueberall n. s. - Am Hellelhof von St. gef. - b. bei Had. (K.). - Wlb. (K., Sch.) - Dlb. (Sch.) - Mz. Mo. (S.) - G. (L.) - Wsb. an der Wellritz (K.). -

10. (Ag.) parumpunctatus F. - Ueberall h. - Fr. - Ems. - Mz. Mo. (S.) - G. (L.) -

11. (Ag.) austriacus Dft. Race a. modestus Sturm. - Fr. B. 1859 am Main der Gerbermühle gegenüber n. s. unter Steinen gesammelt. - Mz. am Rheinufer (S.). - G. (L.) - Eltville, 26. Mai (K.). -

12. (Ag.) viduus Panz. (mit grünlichem Metallglanz). - Fr. h. - Mz. am Rheinufer (S.). - G. (L.) -

Var. moestus Dft. (schwarz ohne Metallglanz). - Am Mainufer. - Mz. am Rheinufer (S.). - G. (L.) - Wsb. an der Wellritz 5. Juni 1851 (K.). - 
Var. emarginatus Gyll. (Die Flügeldecken um das Schildchen herum gemeinschaftlich niedergedrückt; kommt auch bei anderen Agonum vor, z. B. viduum und versutum.) - Fr. s. - MLo. in den Steinbrüchen (S.). -

13. (Ag.) versutus Sturm. - Fr. s. - Mo. in den Steinbrüchen (S.). - G. (L.) -

14. (Ag.) micans Nicol. - Bei Fr. von St. gef. - Wlb. (Sch.) - G. (L.) - An der Wellritz bei Wsb. (K.). -

15. (Ag.) piceus $L$. = picipes F. - An schilfigen Stellen n. s. - Fr. - Mz. am Rheinufer (S.). -

16. (Ag.) gracilis Gyll. - Fr. s., in der sogen. Schwarzen Steinkaut an der "Louisa“ am Fr. Wald im März. - Wlb. (Sch.) -

17. (Ag.) Thoreyi Dej. - s. s. um Fr., früher an schilfigen Stellen am Kettenhof.

[NB. (Ag.) fuliginosus Panz. Besitze ich nicht aus hiesigem Gebiet, die in S. V. erwähnten, dafür gehaltene Stücke, aus Fr. waren Thoreyi. Ich besitze ihn nur aus Erlangen. - Ebenso ist puellus Dej. nicht im Gebiet von mir gef.]

\section{Olisthopus Dejean.}

1. 0. rotundatus Payk. - Fr. sehr einzeln; auch von St. gef. Mo. (S.) - Von v. Harold im Juni 1871 bei Nauheim gesammelt. -

Stomis Clairville.

1. S. pumicatus Panz. - Im ganzen Gebiet. Königstein. - Bingen. Bei Frr. von C. H., I. H., St. gef. - Wlb. (Sch.) - Mo. (S.) - G. (L.) -

\section{Pterostichus Erichson.}

1. (Poecilus) punctulatus F. - Hofheim $>6$. - Nicht häufig. Fr. von C. H., L. H. und St. gef. - Mz. Mo. (S.) - Wsb. am Schiessstand 17. Mai (K.). -

2. (P.) cupreus $\mathbf{L}_{0}=$ punctiçeps Thoms. - Fr. $=5$. Mehr in der Ebene. - Auch von St. im Gebiet gef. - Dlb. Wlb. h. auf Wegen (Sch.). - Mz. Mo. (S.) - G. (L.) -

Var. erythropus Stev. (mit rothen Schenkeln) Fr. -

3. (P.) versicolor Sturm = pauciseta Thoms. - Fr. Taunus. - < 7. - Mehr im Gebirg. - Ebenfalls von St. gef. G. (L.) - 
4. (P.) dimidiatus Oliv. - Fr. - Mainkur. s. - Bei Oberursel von St. gef. - Had. (K.) - Mo. (S.) - G. 24. September 1860 (L.). -

Var. (mit grünem Halsschild) fand L. H. auf dem Schmitterhof bei Giessen $>8$. - Soden (Scheidel). -

5. (P.) lepidus F. - Fr. - Im ganzen Gebiet auch von St. gef. - Had. (K.) - Mz. Mo. (S.) - G. (L.) - Ernsthausen in Nassau (Dörr bei K.). -

6. (Adelosia) picimanus Duft. - Ám Feldberg von M. S. und Gräf gesammelt. - G. (Zimmer, in Sammlung v. Heyden). - Friedberg (Scriba). - Auf den Höhen hinter Mz. (S.). - Von Hg. an der Grüneburg bei Fr. im Juni 1876 gesammelt. Liebt Basaltboden, so auch im Vogelsberg von L. H. beobachtet. -

7. (Lagarus) inaequalis Mrsh. - Fr. s. - G. (L.) - Bei Fr. auch von B. und St. gesammelt.

8. (Lyperus Chaud.) aterrimus Payk. - Von St. einmal bei Fr. gef. - Wlb. (Sch.) -

9. (Omaseus) niger Schaller. - Fr. Bei der Oede. - Im ganzen Gebiet, doch n. h. - Mz. Mo. (S.) -

10. (0.) vulgaris L. - Fr. s. h. - Auf dem grossen Feldberg $=5$. - Wlb. (Sch.) - Mz. Mo. (S.) - G. (L.) -

Var. pennatus Dej. - Die geflügelte Form s. - Fr. einmal von C. H. gef.

11. (0.) melas Creutz. - Friedberg (Scriba). - Wlb. (Sch.) -

12. (0.) anthracinus III. - Im ganzen Gebiet h. - Fr. - Mz. Mo. (S.) -

13. (0.) nigrita F. - Ueberall h. - Fr. - Wlb. (Sch.) - Mz. Mo. (S.) - G. (L.) -

14. (0.) gracilis Dej. - Fr. z. s. - Mz. Mo. (S.) - G. (L.) 16. Februar 1862.

15. (0.) minor Gyll. - Ueberall s. h. - Fr. - Mz. Mo. (S.) -

16. (Glyptodactylus) vernalis Panz. - Im ganzen Gebiet $h$. - Fr. - Ems. - Mr. Mo. (S.) -

17. (Argutor) intérstinctus Sturm. - Fr.n. h. - Wlb. (Sch.) -

18. (A.) strenuus Panz. = erythropus Marsh. - Fr. h. auch von B. gef. - 
[(A.) diligens Sturm = pullus Gyll. - Kommt im Gebiet nach Scriba vor, doch besitze ich keine Stücke daher.]

19. (Platysma) oblongopunctatus F. - Fr. - In Wälderu unter Moos. Schlangenbad. - Besonders h. im Taunus von B. gef. Im Wald hinter $\mathrm{N}$ [o. (S.). - G. (L.) -

20. (P.) angustatus Dft. - Im Röder- und im Rebstockwald bei Fr. S. - Wlb. (Sch.) -

21. (Steropus) madidus F. var. concinnus F. - Bei uns kommt nur die schwarz-schenkelige Varietät vor. Im Gebirg häufiger als in der Ebene: Soden, Falkenstein = 7. - Had. (K.) - G. (L.) - Die rothschenkelige Form soll in Deutschland auf dem linken Rheinufer vorkommen; ich besitze nur französische Stücke. -

22. (S.) aethiops Panz. Gebirgsthier: Schlangenbad, Feldberg = 5. - Dlb. Wlb. (Sch.) -

23. (Pterostichus) cristatus Dufour. = parumpunctatus Germ. - Ein ausschliessliches Gebirgsthier. - Im Taunus bis jetzt nur in der Schlucht bei der Königsteiner Kaltwasseranstalt von L. H. und St. Goarshausen von C. H. gef. - Had. (K.) - Wlb. (Sch.) -

24. (P.) metallicus F. - Nur im Gebirge, doch da n. s. unter Steinen im Laub. - Auf dem Feldberg (B.). - Wsb. auf der Platte n. s. (K.). - Dlb. Wlb. (Sch.) -

25. (Haptoderus) spadiceus Dej. = pumilio Dej. - Eigentlich Gebirgsthier, doch vereinzelt auch. bei Fr. und Darmstadt gef. Im Schwarzwald und den Alpen h. -

26. (Abax) striola F. - Ueberall h., doch in der Ebene seltener. - Soden, Fr. (St.) - Wlb. (Sch. K.) - Had. (K.) - Dlb. (Sch.) Mz. Mo. (S.) - G. (L.) -

27. (A.) ovalis Dft. - Im Taunus: Schlangenbad. - Im Fr. Wald, auch bei Offenbach am Buchrain im Frühjahr 1861 unter Steinen von B. gef. - Wlb. Had. (K.) - Mr. Mro. (S.) - G. (L.) -

28. (A.) parallelus Dft. - Fr. h. - Wlb. (Sch. K.) - Had. (K.) - Dlb. (Sch.) - Mz. Mo. (S.) - G. (L.) -

\section{Molops Bonelli.}

1. M. elatus F. - Im Gebirge weit verbreitet: Schlangenbad, Falkenstein im Juli. - Hofheimer Kapelle April 1861, auch mit terri- 
cola zusammen im Rehstöcker Wald bei Fr. (B.). - Dlb. Wlb. (Sch.) - G. (L.) - Gimbacher Hof am Staufen im Taunus (L. H. und B.). -

2. M. terricola F. - Ebenso verbreitet: Fr. - Schlangenbad, Niederwald und Bingen = 4. - Lorsbach April 1861 und Rebstöcker Wald (B.). - Im Walde hinter Mo. unter Steinen (S.). - Dlb. Wlb. (Sch.) - G. (L.) -

\section{Zabrus Clairville.}

1. Z. gibbus Clairv. - Lebt in Getreidefeldern, wo, wenn er in Masse auftritt, Schaden anrichtet: die Larven zerstören die Wurzeln und der Käfer klettert Nachts an den Halmen in die Höhe, um die Körner zu fressen. Bei Mainz und Mombach von Prälat Dr. Schmitt gef. - Fehlt bei Fr. -

\section{Amara Bonelli.}

(Die Arten der Sammlung v. Heyden sind von den Monographen Schaum und Putzeys revidirt.)

1. (Triaena) tricuspidata Dej. - Fr. öfter gef. (Hg.). -

2. (Amara) similata Gyll. - Im Gebiet weit verbreitet. - Fr. im Genist von B. gesammelt. - Wlb. (Sch.) - In Genist, welches der Rhein auswirft (S.). - G. (L.) -

G. (L.) -

3. (A.) ovata F. = obsoleta Dej. - Er. n. h. - Wlb. (Sch.)

4. (A.) montivaga Sturm. - Fr. s. - G. (L.) - Fr. im Genist (B.). - Taunus (Hg.). -

5. (A.) communis Illig. Sturm. - Früher mit anderen Arten oft verwechselt. - Wlb. einmal (Sch.). - Mo. (S.) - G. (L.) -

6. (A.) continua Thoms. - (Deutsche Entom. Zeitschr. 1876, p. 183 und 397.) L. H. fand ein Stück bei Königstein im Taunus. Von communis dadurch unterschieden, dass die Reihe Punkte neben dem Seitenrande in der Mitte nicht breit unterbrochen, sondern ähnlich wie bei similata ziemlich gleichmässig aus vielen Punkten zusammengesetzt ist. -

7. (A.) Iunicollis Schiödte $=$ vulgaris Panz. - Fr. - Auf dem grossen Feldberg einmal gef. - Verbreitet, aber s. - G. (L.) -

8. (A.) trivialis Gyll. - Im ganzen Gebiet h., auch bei Fr. (B.). - Wlb. (Sch.) - Mz. Mo. (S.) -

9. (A.) spreta Dej. - Fr. s. - 
10. (A.) curta Dej. - Friedberg nicht gerade s. (Scriba). Wlb. drei Mämnchen und ein Weibchen (Sch.). -

11. (A.) familiaris Duft. - Fr. - Ems. - Häufige Art. Wlb. (Sch.) - Mz. Mo. (S.) - Gimbacher Hof am Staufen im Taunus (L. H. und B.). -

12. (A.) lucida Dft. = gemina Zmrm. - Fr. s. -

13. (Celia) ingenua Dft. - Fr. (Hg.) -

14. (C.) fusca Dej. - Diese seltene Art fing C. H. bei Fr. -

15. (C.) cursitans Zmrm. - Diese s. s. Art fand C. H. im Taumns. - Von St. einmal bei Fr. gef. -

[(C.) erratica Duft. - Kommt nach Scriba unter Steinen auf den Gipfeln des Taunus vor; wohl falsche Bestimmung. Ich kenne sie nur als hochalpines Thier; doch findet sie sich auch im Harz.] —

16. (C.) livida F. = bifrons Gyll. - Fr. z. s., auch ron St. gef. - G. (L.) - Auf den Mo. Blössen 18. Juli 1851 (K.). -

17. (C.) infima Duft. - Fr. s. -

[NB. Dass die hochalpine C. rufocincta Sahlb. nach Bach in der Wetterau, bei Ortenberg" und bei Darmstadt rorkommt, ist höchst zweifelhaft; wahrscheinlich liegt eine Verwechselung zu Grunde.]

18. (Curtonotus) aulica Panz. - Im ganzen Gebiet s. - Ein Stück im Genist bei Fr. (B.), auch von L. H. und St. gef. - G. (I.) -

19. (Bradytus) fulva Deg. - Fr. in Sandg'egenden unter Steinen; Biebererhöhe $>4$ - Mo. (S.) - G. (L.) - Bockenheim 17. April 1876 (L. H.). -

20. (B.) consularis Dft. - Fr. - Falkenstein. - Auf der Bornheimer Haide 1 Stück (B.). - Wlb. (Sch.) - Mo. (S.) -

21. (B.) apricaria Payk. - Fr. - In Neuenhain bei Soden einmal Abends $>7$. in Menge in das erleuchtete Zimmer geflogen. Mo. (S.) - G. (L.) -

22. (Percosia) patricia Duft. - Fr. s. -

Var. dilatata Heer. - G. (I.)

\section{Diachromus Erichson.}

1. D. germanus L. - Fr. - Cronthal im Taunus unter Steinen n. h. - Zrischen Eschersheim und Bonames auf Wiesen unter Steinen. (B.) - Mz. Mo. h. (S.) - 


\section{$-27-$ \\ Anisodactylus Dejean.}

1. A. signatus Panz. - Fr. s. - Friedberg (Renner). - Mz. Mo. (S., Bach). - G. (L.) -

2. A. binotatus F. - s. h. - Fr. - Dlb. Wlb. (Sch.) - Mr. Mo. (S.) - G. (L.) - Wsb. an einer Pfütze zwischen Schwalbacher Chaussee und Wellritzmühle (K.). -

Var. spurcaticornis Dej. (mit rothen Beinen) s. - Bei Fr. von L. H. und Hg. gef. - Dlb. Wlb. (Sch.) - G. (L.) - Wsb. Weg nach Kehlheim 18. Mai 1851 (K.). -

3. A. nemorivagus Dft. - s. - Ems. - Mo. (S.) -

4. A. pseudoaeneus Dej. - Ein Salzbodenbewohner. - Von Scriba einigemal bei Friedberg (Saline Nauheim) gesammelt. -

\section{Harpalus Latreille.}

1. (Ophonus) sabulicola Panz. - Friedberg (L. H. und Scriba). Fr. (C. H. und Hg.). -

2. (0.) obscurus Dej. - Auf dem Lerchesberg über Sachsenhausen von C. H. gef. - Auch von St. und Hg. bei Fr. gesammelt. -

3. (0.) punctulatus Dft. - Fr. - Auch von Hg. gef. - G. (L.) Var. laticollis Mhm. - Fr. - G. (L.) -

4. (0.) azureus $\mathbf{F}_{\text {. }}=$ chlorophanus Panz. - Fr. - Ems. Auf dem Mühlberg über Sachsenhausen s., unter Steinen (B., Hg.). Wlb. (Sch.) - Mo. (S.) -

5. (0.) cordatus Dft. - Von Hg. bei Fr. ges. - Wlb. (Sch.) -

6. (0.) rupicola Sturm $=$ subcordatus Dej. - Von uns und Hg. bei Fr., von L. bei G. gesammelt. -

7. (0.) rufibarbis $\mathbf{F}$. = brevicollis Dej. - Fr. von L. H. einzeln, von Hg. h. gef. - Mz. Mo. (S.) -

8. (O.) puncticollis Payk. - Fr. s. (C. H., L. H., B.) - Wlb. (Sch.) -

9. (O.) signaticornis Duft. - Friedberg (Fuhr). - Fr. (Hg.) -

10. H. pubescens Müll. = ruficornis F. - Ueberall g. $-\mathrm{Fr}$. Wlb. Dlb. (Sch.) - Mz. Mo. (S.) - G. (L.) -

11. H. griseus Panz. - Ueberall h. - Fr. - Mz. Mo. (S.) G. (L.) - 
12. H. aeneus F. $-\mathrm{Fr}$. - Wlb. Dlb. (Sch.) - Mz. Mo. (S.) - G. (L.) Sodeu im Taunus (L. H.). -

Var. confusus Dej. - Fr. - Wlb. Dlb. (Sch.) - G. (L.) -

13. H. distinguendus Dft. - Soden. - Fr. von Hg. h. gef. Wlb. (Sch.) - Mz. Mo. (S.) - G. (L.) -

14. H. honestus Dft. - Fr. - Ems. - Taunus. - Schwalbach. - Wlb. Dlb. (Sch.) - Miz. Mo. (S.) -

Var. ignavus Dft. - Fr. - Ems. - Wlb. Dlb. (Sch.) -

15. H. neglectus Dej. - Bei Wlb. ron Sch. gef. -

16. H. discoideus F. - Fr. - Wlb. (Sch.) - Mo. (S.) -

17. H. calceatus Dft. - Fr. - Friedberg (Scriba). - Soden. -

18. H. ferrugineus F. - Fr. in Sandgegenden. - Mo. am Rande der grossen Sandfläche unter Steinen. - Dlb. (Sch.) - Mo. (S.) -

19. H. Hottentotta Dft. - Fr. - Tamus. - Friedberg (Scriba). - Wlb. (Sch.) - Mo. (S.) -

20. H. seriepunctatus Gyll. = quadripunctatus Dej. - Taunus am grossen Feldberg. Gebirgsthier. -

21. H. latus $\mathrm{L}$. = fulvipes F. - Fr. - Auf den Bergen bei Bingen. - Wlb. (Sch.) - G. (L.) -

22. H. luteicornis Dft. - Fr. h. - Im Lorsbacher Thal. Wlb. (Sch.) - G. (L.) -

23. H. laevicollis Dft. = satyrus Sturm. - Feldberg. - Fr. ron C. H. und Hg. s. gef. - Wlb. (Sch.) -

Var. nitens Heer. - Taunus einmal. -

24. H. rubripes Dft. - Fr. - Ems. - Wlb. Dlb. (Sch.) Mo. (S.) - G. (L.) -

Var. fulvipes Dft. - Kleine Feldberg. - Mo. (S.) -

Var. erythrocephalus F. - Fr. -

25. H. hirtipes Panz. - Fr. - Mo. von S. und L. H. ges. -

26. H. caspius Stev. = semiviolaceus Dej. = melampus Dft. - Fr. - Taunus. - Ueberall verbreitet und h. - Wlb. (Sch.) -

27. H. impiger Dft. - Fr. - In Sandgegenden h. -

28. H. tenebrosus Dej. - Fr. zweimal. -

29. H. melancholicus Dej. - Zwei Männchen und ein Weibchen bei Wlb. von Sch. gef. - 
30. H. tardus Panz. - Fr. - Auf Sandboden h. - Mo. (S.) - G. (L.) - Fr. 21. März 1860 (B.). -

31. H. flavicornis Dej. - Fr. -

32. H. serripes Dft. - Fr. h. - Rüdesheim. - Mio. (S.) -

33. H. fuscipalpis Sturm. - Fr. s. s. (C. H.) -

34. H. anxius Dft. - Fr. h. - Mz. Mo. (S.) -

Var. pumilus Dej. - Ems. - Fr. -

35. H. servus Dft. - Fr. s. - Bei der Ruine Ehrenfels am Rhein. - Mz. Mo. (S.) -

36. H. flavitarsis Dej. - Offenbach von L. H. gef. - Fr. im April. - Wlb. (Sch.) -

37. H. picipennis Dft. - Fr. - Bieberer Höhe bei Offenbach. - Sehr früh im Jahre, 1860 schon im März von B. gef. - Wlb. (Sch.) - Mz. Mo. (S.) -

\section{Stenolophus Dejean.}

1. S. Teutonus Schrk. - Fr. - Ueberall n. s. - Fr. auch von Hg. h. gesammelt.

2. S. Skrimshiranus Steph. - Bei Fr. von C. H. und Hg. gef. - s. s. -

[Var. affinis Bach (mit einem schwarzen blauschillernden Fleck vor der Spitze der Flügeldecken) bei Boppard entdeckt, findet sich vielleicht auch auf der nassauischen Rheinseite.] -

3. S. vespertinus Illig. - Von C. H. früher bei Fr. öfter gef. Von B. und Hg. später s. gesammelt. - Mo. (S.) -

4. S. elegans Dej. - Ein ächtes Salzthier. C. H. fand ein Stück bei den Nauheimer (Yrradierhäusern.

5. (Acupalpus) dorsalis F. - Bei Fr. s.

Var. flavicollis Sturm. - Fr. - Ebenso s. - G. (L.) -

6. (A.) brunnipes Sturm. - Fr. s. -

7. (A.) meridianus L. - Im ganzen Gebiet g. Fr. - Bei Wlb. s. h. (Sch.) - Mz. Mo. (S.) - G. (L.) -

8. (A.) exiguus Dej. - n. h. Fr. Mo. (S.) - G. (L.) -

Var. luridus Dej. - Fr. einmal.

9. (Balius Schiödte) consputus Dft. - Fr. n. s. - G. (L.) - 


\section{Bradycellus Erichson.}

1. B. Verbasci Dft. - Von Mr. S. bei Fr. gef., das Ex. in Sammlung v. Heyden. -

2. B. harpalinus Dej. = fulvus Mrsh. - Fr. n. h. - G. (L.) -

3. B. collaris Payk. - Fr. n. s. - G. (L.) -

\section{Trachycellus Morawitz.}

1. T. similis Dej. - Bingen (C. H.) - Fr. -

Trechus Clairville.

1. T. discus F. - Am Mainufer von C. H. gef. s. s. - Ebenso:

2. T. micros Hbst. - Fr. -

3. T. longicornis Sturm. - 3 Stück im April 1860 im Genist des Mains bei Fr. von B. gef., eines davon in der Sammlung Hg. Mz. (S.) -

4. T. rubens $\mathbf{F}$. - Fr. von $\mathrm{Hg}$. einmal gef. -

5. T. palpalis Dej. - Fr. von Hg. 1 St. gef. -

6. T. minutus F. - Im ganzen Gebiet g. unter nassem Laub. Dlb. Wlb. (Sch.) - Mz. Mo. (S.) - G. (L.) -

Var. obtusus Er. - Nur ein sicher bestimmtes Ex., trächtiges Weibchen, mit Flügelstummeln, die für diese Art characteristisch sind, aus der Fr. Gegend $=9$ von C. H. gef.

7. (Epaphius) secalis Payk. - Bockenheim, nach Ueberschwemmung unter zurückgebliebenen Pflanzentheilen, doch s.; bei Fr. auch von C. H. und Hg. gesammelt. - Wlb. (Sch.) - Im Genist am Rheinufer (S.). -

\section{Perileptus Schaum.}

1. P. areolatus Cr. - Ems am Ufer der Lahn im Sande von C. H. gef. - G. (L.) -

\section{Tachyta Kirby.}

1. T. nana Gyll. = quadristriata Illig. - Im Gebiet weit verbreitet, unter Baumrinden. - Fr. - Falkenstein. - Wlb. (Sch.) - 


\section{$-31-$ \\ Tachys Schaum.}

1. T. Fockii Hummel. - C. Stern fing ein Ex. dieses seltenen Thieres bei Fr. - (In Sammlung v. Heyden sind Stücke aus Darmstadt und Kreuznach.) -

2. T. bistriatus Dft. - Fr. h. unter Steinen. -- Wlb. (Sch.) Mz. Mo. (S.) -

Var. gregarius Chaud. - (Hell mit dunklem Kopf.) - Fr. Ems seltener. -

3. T. sexstriatus Dft. var. quadrisignatus Dft. - Ems am Ufer der Lahn (C. H.). - Die Stammart, obne Flecken, im Gebiet noch nicht gef. Stellen. -

4. T. parvulus Dej. = pulicarius Dej. - Ems an trockenen

\section{Bembidlium Latreille.}

1. (Ocys) quinquestriatum Gyll. = pumilio Dft. - Fr. Griesheim s. s. unter Baumrinden. - Wlb. (Sch.) -

2. (Philochthus) obtusum Sturm. - Fr. - Biebrich, Bingen. Wlb. (Sch.) -

3. (Ph.) Mannerheimi Sahlbg. - Einmal bei Wlb. von Prof. Schenk gef. - (Ich habe das Ex. gesehen.) -

4. (Ph.) guttulum F. = bipustulatum Rdtb. - Fr. - Biebrich. Mo. (Sch.) -

5. $(\mathrm{Ph}$.) vulneratum Dej. = inoptatum Schaum $=$ biguttulum Redtb. - Fr. - Biebrich. - Königstein. - G. (L.) -

6. (Ph.) biguttatum F. Seidl. Krtz. = riparium Payk.=guttulum Redtb. - Mo. (Sch.) - Hg. fand diese Art dreimal bei Fr.; die vorige gar nicht. Die erste Art hat ganz abgerundete Hinterecken des Halsschildes, bei bigutt. bilden sie kleine Zähnchen.

7. (Ph.) assimile Gyll. - Fr. s. -

8. (Lopha) quadrimaculatum L. - Biebrich, Soden, Königstein. Fr: 3 Stück 1860 von B. gef. - Wlb. (Sch.) - Mo. (S.) - G. (L.) -

9. (L.) quadriguttatum $F_{\text {. }}$ - Die Lopha-Arten finden sich an Fluss- und Bachufern zwischen den Steinen umberlaufend. - Fr. Wlb. (Sch.) - Mo. (S.) -

10. (Leja) articulatum Panz. - Fr. h. am Wasser. - Soden. - Wlb. (Seh.) - Mo. (S.) - 
11. (L.) Sturmii Panz. - Fr. - Rumpenheim, Soden. - Wlb. (Sch.) -

12. (L.) Doris Panz. - Fr. s. s. von C. H. gef. -

13. (L.) aspericolle Germ. - Lebt auf Salzboden bei der Saline Nauheim. (Auch in der Wetterau an den salzigen Lokalitäten von Salzliausen, Traishorloff und Wisselsheim gef.). -

[(L.) minimum F. = pusillum Gyll. - Lebt ebenso, doch besitze ich ihn nur von den Wetterauer Salinen.] -

14. (L.) lampros Hbst. = celere F. - Fr. h. an feuchten Stellen unter Laub. - Wlb. (Sch.) - Mz. Mo. (S.) -

Var. velox Er. - Fr. seltener. - Wlb. (Sch.) -

15. (Peryphus) modestum F. - Fr. am Mainufer. - Ems am Lahnufer von C. H. gef. - Friedberg (Scriba). - Mz. Mo. (S.) G. (L.) -

16. (P.) decorum Panz. - Fr. - Ems nicht gerade s. (C. H.) - Wlb. (Sch.) - Mz. Mo. (S.) -

17. (P.) nitidulum Mrsh. = rufipes Gyll. Dej. = brunnipes Sturm. - Fr. - Ehrenbreitstein am Rhein. -

Var. deletum Dej. - Schlangenbad (C. H.). -

18. (P.) fasciolatum Dft. - Einmal bei Ems am Lahnufer von C. H. gef. - G. (Klingelhöffer). -

19. (P.) conforme Dej. - Ein sicher bestimmtes Ex. fand Hg. bei Fr. -

20. (P.) atrocoeruleum Steph. = cyanescens Wesm. = cumatile Schiödte. - Ems an der Lahn s. -- G. (L.) - Fr. (Hg.) -

21. (P.) complanatum Heer. - In hiesigen Sammlungen irrthümlich als cumatile bestimmt. - Fr. — s. s.; mehr Gebirgsthier. -

22. (P.) obsoletum Dej. - Ems am Lahnufer. s. -

23. (P.) femoratum Sturm. - Ems h. an Flussufern. - Fr. von L. H. und Hg. ges. - Mz. am Rheinufer (S.). - G. (L.) Wlb. (Sch.) -

24. (P.) lunatum Dft. - Mainz am Rhein von S. und L. H. gef. - Fr. 1 Ex. (Hg.). -

25. (P.) litorale 01. = rupestre Illig. Dej. = Andreae Er. Eins h. - Fr. von C. H. and Hg. gef. - Wlb. (Sch.) - Mr. am Rheinufer (S.). - 
26. (P.) elongatum Dej. - Diese s. s. Art fing C. H. früher bei Fr. -

27. (Notaphus) flammulatum Clairv. = undulatum Sturm. $=$ majus Gyll. - Fr. s. von L. H., von Hg. einmal h. gef. - G. (L.) -

28. (N.) varium 01 . = ustulatum Dej. - Fr. von Hg. gef. -

29. (N.) adustum Schaum = fumigatum Dej. - Ems. Von L. H. und Hg. bei Fr. gef. - Am Ufer der Mainspitze bei Mz. 22. April (K.). -

30. B. prasinum Dft. = olivaceum Gyll. - Ems von C. H. an der L Lahn gef. - s. - Ich vermuthe, dass die seither nicht gedeutete Art B. Eichhoffi Bach. (Käferfauna I, p. 388) hierhergehört. Eichhoff fing das Thier ziemlich h. bei Wetzlar an der Lahn vor dem Heuserthor.

31. B. punctulatum Drapiez $=$ aerosum Er. = striatum Dej. - Im ganzen Gebiet an Bachufern; Hg. fand einige, B. im April 1860 zwei Ex. bei Fr. - G. (L.) - Am Ufer der Mainspitze bei Mz. 22. April (K.). -

32. B. bipunctatum L. -- Von Hg. einmal bei Fr. gef. -

33. B. impressum Panz. - Von St. am Mainufer gef. -

34. B. paludosum Panz. - Bingen an der Nahe (C. H.). Bei Fr. von St. und Hg. gef. - Wlb. (Sch.) -

\section{Tachypus Lacordaire.}

1. T. flavipes L. - Fr. l. an Bächen und in feuchtem Laub, anch von Hg. gef. - Wlb. (Sch.) - G. (L.) -

2. T. pallipes Dft. - Wsb. (K.) - 


\section{DYTISCIDAE.}

Die sămmtlichen Wasserkäfer der Sammlung v. Heyden sind fon dem Specialisten E. If ehncke revidirt.)

A. HALIPLOIDAE.

\section{Cnemid otus nlliger.}

1. C. caesus Dft. - Fr. in Tümpeln s. von T. H. und Hg. gef. - Mr. Mo. (S.) - G. (L.) -

\section{Haliplus Latreille.}

1. H. elevatus Panz. - Nur ein Stück aus dem Magen einer Bachstelze von Bonames (B.). - Bei Mo. einmal von K., öfter und auch bei Mz. von S. gef. - In der Wellritz bei Wsb. 10. Mai (K.). -

2. H. lineatocollis Mrsh. - Ueberall n. s. in stehenden Gewässern. Bockenheim 2 Stück (B.). -

3. H. ruficollis Deg. - Fr. s. - Früher mit dem folgenden verwechselt. - Von B. h. bei Bockenheim an den ehemal. Tümpeln am Bahnhof gef. - Wlb. (Sch.) - Mz. Mo. (S.) - G. (L.) -

4. H. fluviatilis Aubé. - Lebt in fliessendem Wasser. - Fr. am Ufer des Mains unter Wasserpflanzen n. s. s. an der ehemal. Militärschwimmanstalt. G. (L.) - Mr. Mo. (S.) -

5. H. fulvicollis Er. - Fr. s. s. -

6. H. variegatus Sturm. - Bei Mo. von K. und S. ges. -

7. H. apicalis Thoms. = striatus Sharp. - Fr. ein Stück, sonst nur aus Schweden bekannt.

8. H. fulvus F. - Fr. S. In den früheren Tümpeln am Bahnhof bei Bockenheim von B. gef. - Am Rheinufer in Tümpeln bei Mo. (S.). -

9. H. impressus F. = flavicollis Sturm. - Fr. - Von B. mit dem vorigen ges. - Wlb. (Sch.) -

[H. badius Aubé. Soll nach Scriba von Katheder bei Fr. einmal gefunden sein, doch zweifle ich an dem Fundort; das Ex. dieser süd- 
europäischen Art stanımt möglicherweise aus Malaga, woher Katheder viele Käfer erhielt.]

10. H. affinis Steph. = cinereus Aubé. - Fr. s. - G. (L.) -

11. H. obliquus Gyll. - Fr., besonders hei Ginheim in Tümpeln gef. - Langgöns (L.). - Bockenheim (B.). - Wsb. (K.) - Mz. In Lachen und Tümpeln am Main (S.). -

\section{Hydrachna F. = Pelobius Sch.}

1. H. tarda Hbst. = Hermanni F. - In stehendem Wasser auf dem Weg von der Brücke am Untermainthor nach dem Sandhof von B. und von Twardowski s. s. gef. - Friedberg (Renner). Grüneburg von $\mathrm{Hg}$. n. s. in Wassertümpeln ges. - In dem Weiher bei Mo. aber selten (S.). - Bei Enkheim von Ko. gef. -

\section{Hyphydrus Illiger.}

1. H. ovatus L. - Fr. n. s. in stehenden Gewässern. - In den ehemaligen Steinbrüchen bei Bockenheim (B.). - Mo. 2. Mai (K.). Wlb. (Sch.) - - G. (L.) -

\section{Hygrotus Stephens.}

1. H. inaequalis F. - Fr. von I. H. und Hg. ges. - Wlb. (Sch.) -

2. H. reticulatus F. - Mo. im Tümpel, gegenüber Schierstein 2. Mai (K.). - G. (I..) -

\section{Coelambus Thomson.}

1. C. confluens F. - Fr. -

2. C. impressopunctatus Schaller $=$ picipes F. - Fr. Bockenheimer Tümpel. - Fr. (Hg.) - Mo. (K.) - Wlb. (Sch.) -

Var. des Weibchens mit matten Flügeldecken = lineellus Gyll. G. (L.) -

\section{Hydroporus Clairville.}

1. H. geminus F. - Fr. (L. H. und Hg.) - In den ehemaligen Bockenheimer Tümpeln in den. alten Steinbrüchen h. (B.) - Wlb. 2 Stück (Sch.). — In Tümpeln längs des Rheins bei Mo. (S.) - G. (L.) -

2. H. unistriatus Schrk. - Fr. - B. fand ihn einmal bei Fr.; Sch. zweimal bei Wlb. - Mo. (S.) wie die vorige Art. - 
3. H. pictus F. - G. (L.) - Bockenheimer Tümpel (B.), Fr. von C. H. und Hg. ges., bei Mo. (K., S.) - Wlb. einmal (Sch.).

4. H. bilineatus Sturm. - G. (L.) - Bockenheim (B.). -

5. H. latus Steph. = ovatus Stm. - Von K. zweimal in der Wellritzbach bei Wsb. gef. - Mo. (S.) -

6. H. granularis L. - Im Gebiet weit verbreitet. Bockenheimer Tümpel einmal gef. (B.) - Fr. (Hg.) - Mo. 2. Mai (K.). -

Var. suturalis Müll. - Fr. s. -

7. H. Halensis F. - Ueber das ganze Gebiet verbreitet. - Ginheimer Tümpel. - Bockenheim einmal (B.). - Fr. (Hg.) - Mo. (S.) Limburg im Weiher 6. Juni (K.). -

8. H. depressus F. = elegans Panz. - Ein Salzwasserbewohner. - Von Scriba bei Friedberg, ron Scheidel bei Nauheim gef. -

9. H. erythrocephalus L. - Im Gebiet h., offter mit H. rufifrons Dft. verwechselt. - Er. von Eg. und L. H. gef., bei MLo. (K., S.) -

10. H. rufifrons Dft. - Mo. (S.) - G. (L.) -

11. H. planus F. - Im ganzen Gebiet s. h. - Bockenheim (B.) Fr. (Hg.) - Wlb. (Sch.) - Mo. (S.) -

12. H. marginatus Dft. - Einmal hei Wlb. von Sch. gef. (ich habe das Ex. gesehell). - MIo. (S.) -

13. H. pubescens Gyll. Thoms. = discretus Fairm. Schaum = nigrita Sturm Redth. - Fr. ron Hg. gef. -

14. H. nigrita Gyll. Thoms. = pubescens Schaum. - Königstein ron L. H. ges. - Fr. (Hg.) - Wlb. (Sch.) -

15. H. memnonius Nicol. - Bei Offenbach einmal gef. - Fr. ein Stück (Hg.) -

16. H. tristis Payk. - Fr. s. von Hg. gef. - Mo. (S.) -

17. H. neglectus Schaum. - Fr. einmal gef. (In S. V. als Frankfurter umbrosus angeführt.)

18. H. palustris L. = sexpustulatus F. - Im ganzen Gebiet h. - Fr. - Bockenheim (B.) - Mo. (K., S.) -

19. H. angustatus Sturm. - Fr. s. - Mo. (S.) -

20. H. lineatus F. - Fr. von Hg. ges. - Mo. (K.) -

[Die Hydroporus del Sammlungen v. Heyden und Haag sind gründlich ron Wehncke, dem besten Kenner der Gattung, revidirt. - Bei genauerer Durchforschung des Gebietes würden sich vielleicht noch manche 
der 32 von Scriba aufgeführten Arten der schwierigen Gattung auffinden lassen. Die mit tristis und planus verwandten Arten werden immer noch oft verwechselt.]

\section{Noterus Clairville.}

1. N. clavicornis Deg. = crassicornis F. - Fr. - h. in den chemaligen Bockenheimer Tümpeln (B.). — Limburg (K.). - Wlb. (Sch.) - Mo. (S.) - G. (L.) -

2. N. semipunctatus F. = sparsus Mrsh. - Fr. Beide Arten in stehendem Wasser n. s. - Mo. (S.) - G. (L.) -

\section{Laccophilus Leach.}

1. L. minutus L. = interruptus Panz. = hyalinus Marsh. Thoms. - Fr. - g. in den ehemaligen Bockenneimer Tümpelı (B.). - Mo. (S.) -

2. L. hyalinus Deg. Er. = obscurus Panz, = minutus Mrsh. - Im ganzen Gebiet h. - Fr. (B.) - Beide Arten bei Mo. in Tümpeln am Rhein gef. (K., S.) - Wlb. (Sch.) -

\section{Acilius Leach.}

1. A. sulcatus L. - Im ganzen Gebiet in stehenden Gewässern. Einmal in der Stadt im botanischen Garten im Bassin gef. - Bei Wassernoth fliegen die Wasserkäfer oft sehr weit, und werden dann durch blendende Flächen leicht verleitet, sich an Orten niederzulassen, die gar nicht für ihre Lebensweise passen. -- Dlb. Wlb. (Sch.) - Fr. (Ko.) -

\section{Graphoderes Eschscholtz.}

1. G. bilineatus Deg. - G. (L.) - Bei Bockenheim 5 St. von B. und von Harer in der Fr. Gegend gef. -

2. G. zonatus Hoppe. - Fr. s. - Friedberg (Klingelhöffer) , -

3. G. cinereus L. - Fr. s. - Bockenheim (B.). - Mo. (S.) -

4. G. Austriacus L. - Fr. häufiger auch von St. gef. - Friedberg (Renner). - Alle vier Arten bei Fr. auch von Scheidel gef. -

\section{Hydaticus Leach.}

1. H. seminiger Deg. = Hybneri $\mathbf{F}$. - Fr. - In den Wasserbehältern der Sachsenhäuser Gärtnerei h. - Bockenheim 1 St. (B.) Mo. (S.) - 
2. H. transversalis Bergstr. = punctipennis Thoms. - Fr. seltener. - Bockenheim in den ehemaligen Tümpeln nicht gerade s. (B.) - Wsb. (K.) - Mo. (S.) - G. (L.) -

3. H. stagnalis $\mathbf{F}$. - s. s., immer nur einzeln bei Fr. von L. H. und r. Twardowski gef. — Rebstöcker Wald einmal (B.). -

\section{Cymatopterus Eschscholtz.}

1. C. fuscus F. - Im ganzen Gebiet h. in Tümpeln. - Fr. Wsb., Had., Wlb. (K., Sch.) - Dlb. (Sch.) - Mo. (S.) - G. (L.) -

\section{Colymbetes Clairville.}

1. C. Grapii Gyll. - Fr. einmal von C. H. gef. -

Rantus Eschscholtz.

1. R. pulverosus Steph. Sturm. - Fr. h. - Wsb. s. h. (K.) - Wlb. (Sch.) - Mo. (S.) - G. (L.) - Limburg 6. Juni (K.). -

2. R. notatus F. - Fr. s. -

3. R. aberratus Gem. Har. = adspersus F. - Fr. - Hierher das in S. V. als bistriatus (von M. S. gef.) bezeichnete Fr. Exemplar, welches ich jetzt besitze. Die Art ist nicht gerade s. - G. (L.) Bockenheimer Tümpel (B.). - Mo. (S.) -

4. R. exoletus Forst. = adspersus Panz. = collaris Payk. - Fr. n. s. -

\section{Ilybius Erichson.}

1. I. fuliginosus $\boldsymbol{F}$. $=$ uliginosus $\mathrm{L}$. (pars.) - Fr. von L. H., Hg. und St. ges. - Wlb. (S.) - Mo. (K., S.) - G. (I.) -

2. I. ater Degeer. - Sachsenhäuser Gärtnerei in Tümpeln n. s. - Bockenheim (B.). - Fr. von L. H. und Hg. ges. - Mo. (S.) G. (L.) -

3. I. obscurus Mrsh. - Fr. von L. H. und Hg. gef. -

4. I. subaeneus Er. - Seltene Art, mehr in Nord-Europa. Fr, einmal ron C. H. und einmal von B. ges. -- Mro. (S.) -

5. I. guttiger Gyll. - Seltene Art. - Fr. ein Ex. - Ebenfalls mehr im Norden. - 
6. I. fenestratus F. - In ganzen Gebiet verbreitet. - Fr. von L. H. und Hg. gef. - Mo. (S.) - Mo. in einem Graben 20. Sept. (K.) - G. (L.) -

\section{Liopterus Eschscholtz.}

1. L. agilis F. - Fr. - Sachsenhäuser Gärtnerei n. s. - Bockenheimer Tümpel (B.). - Mo. (S.) -

\section{Platambus Thomson.}

1. P. maculatus L. - In klarem fliessendem Wasser. - n. s. in Waldteichen, z. B. der Grastränke von B. gef. - Fr. von L. H. und Hg. ges. - In dem Mühlbach hinter der Hartmühle bei Mz. h. (S.) - Mo. (K.) - Wlb. (Sch.) - G. (L.) - Ernsthausen in Nassau (Dörr bei K.). -

\section{Eriglenus Thomson.}

1. E. abbreviatus F. - Fr. n. s. in stehenden Gewässern. Mo. (S.) -

2. E. femoralis Payk. - Fr. n. h. - Beide Arten auch von Hg. ges. - Wlb. (Sch.) -

\section{Gaurodytes Thomson.}

1. G. paludosus F. - Fr. von Hg. gef. - n. s. - Friedberg (Scriba). - Wlb. (Sch.) -

2. G. uliginosus L. - Früher h. im Fr. Stadtgraben (B., Hg.). - Mo. (S.) - G. (L.) -

3. G. didymus 0I. - Friedberg (Fuhr). - Fr. (Hg.) -

4. G. Sturmii Gyll. -- Bei Wlb. von Sch. gef. (ich habe das Ex. verglichen). -

5. G. nebulosus Forst. = bipunctatus F. - Bei Fr. von Hg. h. gesammelt. - G. (L.) -

6. G. guttatus Payk. - Königstein, Fr. von L. H. und Hg. gef. - Wlb. (Sch.) -

7. G. bipustulatus L. - In klaren Quelien und Bächen h. Fr. (Hg.) - Offenbach, Königstein. - Bockenheimer Tümpel 2 Stück (B.) - Wlb., Dlb. (Sch.) - In dem Mühlbach hinter der Hartenmühle bei Mz. (S.) - - G. (L.) - 
8. G. chalconotus Panz. - Fr. (Hg.) - Königstein in klaren Bächen. - Bockenheim 1 Stück (B.) - Wlb. (Sch.) - In dem Mühlbach hinter der Hartenmühle bei $\mathrm{Mz}$. (S.) - G. (L.) -

\section{Dytiscus Linné.}

1. D. latissimus L. - In der Stern'schen Sammlung befinden sich Stücke, welche H. Baier in Gräben bei Rödelheim fand. - Von Scheidel früher sicher bei Fr. gef. - Prof. K. besitzt ein in einem Brunnen in Wsb. gef. Weibchen dieser Art. - Fehlt nach Sch. bei Dlb. u. Wlb. - Prälat Schmitt fand ein Weibchen in den Lachen an dem rechten Rheinufer bei Kostheim.

\section{Macrodytes Thomson.}

1. M. marginalis L. - Fr. in Teichen, z. B. in der Grastränke im Fr. Wald von Ko. oft gef.; auch sonst in grösseren Tümpeln h., z. B. Bockenheim (B.) - h. bei Dlb. und Wlb., mehrmals in Wasserfässern im Garten gef. (Sch.) - G. (L.) - Im Weiher bei Mo. und in den Festungsgräben bei Mz. h. (S.) -

Var. conformis Kunze (Weibchen mit glatten Flügeldecken), unter den normalen ebenso h. von Ko. gef. - G. (L.) - Dlb. (Sch.) -

2. M. circumcinctus Ahr. - Fr. s. - Bockenheim einmal (B.). -

3. M. circumflexus F. - Im Weiher bei Mu. s. s. (S.) -

4. M. punctulatus F. - Fr. n. s. - Früher n. s. im Stadtgraben (B.). - Dlb. (Sch.) - Fr. (Ko.) - Mo. (S.) -

5. M. dimidiatus Bergsträsser. - Fr. 7. S. von L. H. und Ko. gef. -

\section{Trogus Leach $=$ Cybister Curtis.}

1. T. Virens Müll. $=$ Roeseli F. - Er. s. - In den Bockenheimer Steinbruchstümpeln einmal von B. gef. - Fr. $\left(\mathrm{Ko}_{0}\right)$ - Bei Mz. in den Lachen rechts und links des Rheins n. s. (S.) - Im Absperrungswasser bei der Wsb. Gasfabrik (K.). - 


\section{GYRINIDAE.}

(Th der Sammlung $\nabla$. Heyden vom Monographen Suffrian revidirt.)

\section{Gyrinus Geoffiroy.}

1. G. minutus F. - Fr. s. s., früher von C. H. gef. - Bei Mo. am Rhein (K., S.) - Auch bei Mz. (S.) -

2. G. natator L. = mergus Ahrens. - Fr. - Im Hengster bei Offenbach s. h. - Mo. (S.) - G. (L.) - In einer Pfütze bei Wsb. zwischen Schwalbacher Chaussee und Wellritzmühle 18. April (K.). -

3. G. marinus Gyll. - Fr. je einmal von C. H. und M. S. gef. - Mo. (S.) -

4. G. opacus Sahlb. - Einmal von M. S. bei Offenbach gef. (Das Ex. in v. Heyden's Sammlung.) - Mo. (S.) -

5. G. bicolor. - Nach B. von Harer einmal im Rebstöcker Wald gef. -

\section{Orectochilus Eschscholtz.}

1. 0. villosus Müll. - C. H. fand $=5.1832$ im Main am Ausflusse eines Baches $\mathrm{zwischen} \mathrm{Niederrad} \mathrm{und} \mathrm{Schwanheim} \mathrm{klumpenweise}$ diese Art.unter Steinen. Aufgestört schwammen sie so behende auf der Wasseroberfläche wie Gyrinus. - L. H. fand einmal ein Dutzend Ex. unter einem Steine im Main an der Main-Neckar-Eisenbahnbrücke. Ems in der Lahn von C. H. gef. - 


\section{N. PALPICORNIA.}

\section{A. HYDROPHILINI.}

\section{Hydrophilus Geoffroy.}

1. H. piceus L. - Fr. In grossen Tümpeln h. - Bockenheim (B.). - Dlb. Wlb. (Sch.) - Bei Mz. in den Lachen rechts und links des Rheins h. (S.). - G. (L.) -

2. H. aterrimus Eschscholtz. - Fr. Wie der vorige. Bockenheim (B.) - Bei Mz. wie die vorige Art s. (S.) -

\section{Hydrous Brullé.}

1. H. caraboides L. - Fr. - In der Sachsenhäuser Gärtnerei in Wassertümpeln h. - Bockenheim (B.) - Mz. Mo. (S.) - G. (L.) - Münster bei Soden im Tannus (L. H.). - In der Wellritz bei Wsb. (K.) -

Var. (mit gelbem Hinterleib). Fr. zweimal gef. -

2. H. (Limoxenus Motsch.) oblongus Hbst. - Fr. s. -

\section{Hydrobius Leach.}

1. H. fuscipes L. - Ueberall h. Fr. - Königstein. - Bockenheim (B.) - Bei Wsb. und Mo. von S. und K. gef. - Wlb. Dlb. (Sch.) - Mz. (S.) -

Var. chalconotus Curtis. = aeneus Sol. - Fr. - Sachsenhäuser Gärtnerei seltener.

\section{Philydrus Solier.}

(Die Gattung von L. v. Heyden revidirt. Deutsche Entom. Zeitschr. 1875, p. 394.)

1. P. testaceus F. - In Salzwasser der Saline Nauheim (Scriba). - C. H. fand ihn auch an den Wetterauer Salzquellen. - Bei Mo. von K. gef. -

2. P. melanocephalus 0I. - Fr. - Sachsenhäuser Gärtnerei. Rumpenheim in dem Teich an den Schiessständen. - Mo. (S.) - 
3. P. nigricans Zett. Thoms. = frontalis Er. - s. s. - Fr. einmal. -

4. P. coarctatus Gredler $=$ suturalis Sharp. - Fr. 3 Ex., früher mit marginellus vermengt. -

5. P. marginellus F. = ovalis Thoms. - Fr. 3 Ex. - Wlb. (Sch.) - Mo. (S.) - G. (L.) -

\section{Helochares Mulsant.}

(Die Gattung von L. v. Heyden revidirt. Deutsche Entom. Zeitschr. 1875, p. 396.)

1. H. lividus Forst. $=$ griseus F. - Fr. - Bockenheimer Steinbrüche h. (B.) - Mo. (K.) - Wlb. (Sch.) -

2. H. punctatus Sharp. - Einmal bei Fr. gef. s. s. -

\section{Enochrus Thomson.}

1. E. bicolor Payk. - Fr. s. im April. - Bockenheim (B.) Wlb. Nlb. (Sch.) - G. (L.) -

\section{Anacaena Thomson.}

1. A. limbata $\mathbf{F}$. = variabilis Sharp. = carinata Thoms. Fr. - Soden in stehenden Gewässern. - Wlb. Dlb. (Sch.) - G. (L.) -

2. A. bipustulata Mrsh. - Fr. s. -

3. A. globulus Thoms. - Wlb. (Sch.) - Gebirgskäfer. - Diese Gattung wurde von v. Kiesenwetter, Dentsche Entom. Zeitschr. 1875, p. 230 besprochen. -

[NB. globulus Payk. ist ein Gemenge dieser drei Arten.]

\section{Laccobius Erichson.}

(Die Stücke der Sammlung $\nabla$. Heyden vom Monographen $\nabla$. Rotten berg revidirt.)

[Bis zum Erscheinen der Monographie kannte man bei uns nur, eine Art, welche man für minutus linne hielt, doch ist gerade diese Art s. s. und in unserem Gebiet noch nicht beobachtet.]

1. L. nigriceps Thoms. (Hierher die meisten minutus der Sammlungen.) - Fr. n. s. - Wlb. (Sch.) - Mo. (S.) -

Var. maculiceps Rttbg. - Fr. s. - Mo. am Rhein n. s. von K. gef. - Wlb. (Sch.) -

Var. minor Rttbg. - Wsb. einmal von K. ges. -

2. L. alutacius Thoms. - Fr. einigemal gef. - Cronthal. - 


\section{$-44-$ \\ Limnebius Leach.}

1. L. truncatellus Thunbg. - Fr. (B.) - Wlb. Dlb. (Sch.) Mo. (S.) -

2. L. papposus Mls. - Fr. zweimal gef. - Mo. (S.) -

3. L. truncatulus Thoms. - Von uns und Hg. einzeln bei Fr. gesammelt. -

4. L. picinus Mrsh. = sericans Muls. Gerh. Germ. - Fr. von C. H. ges., im Januar $1821 \mathrm{im}$ ehemaligen Rüstersee bei Oberrad unter Steinen gesellschaftlich. - Von Gerhardt in Deutsche Entomol. Zeitschr. 1876, p. 163 neu beschrieben. - L. atomus Duft. ist eine verwandte grössere, schwarze Art, während picinus braune Flügeldecken hat. -

\section{Chaetarthria Stephens.}

1. C. seminulum Payk. - Fr. n. s. - Rumpenheim im Tümpel an den Schiessständen. - Wlb. Dlb. (Sch.) -

\section{Berosus Leach.}

1. B. spinosus Stev. - Bei Mz. und Mo. von S., an letzterem Fundorte auch von $K$. gesammelt. -

2. B. aericeps Curtis = signaticollis Charp. - Fr. z. s. Mz. Mo. (S.) - G. (L.) - Wsb. Pfütze neben der Schwalbacher Chaussee 20. April (K.). -

3. B. luridus L. - Fr. häufiger. - Mz. Mo. (S.) - G. (L.) - Wsb. (K.) -

\section{B. SPERCHEİNI.}

\section{Sperchëus Kugelann.}

1. S. emarginatus Schaller. - Mz. (Dr. Zitz.) - Ein Ex. in Sammlung v. Heyden. - Im April 1872 fand L. H. in einem Tümpel in der Sachsenhäuser Gärtnerei zwei Exemplare. C. H. hatte das Thier in der langen Zeit, welche er auf die Durchforschung unserer Gegend verwandte, nie gefunden. B. fand 4. April 1860 ein einzelnes Stück im Geniste des Metzgerbruches bei Fr. - Bei Limburg in einer Pfütze vor dem Schafberg von K. 5. Juni 1852 ges. - Nz. Mo. (S.) - 


\section{HELOPHORINI.}

\section{Helophorus Fabricius.}

1. H. rugosus 01. = fennicus Payk. - Auf dem grossen Sand bei Gonsenheim bei Mz. von Ludwig gef.; ein Ex. in Sammlung v. Heyden. - Sonst lebt die Art mehr auf den Dünen des Meeres. -

2. H. nubilus F. - Fr. - Saline Nauheim. Die Art findet sich oft ganz im Trocknen, weit von allem Wasser; so fand sie C. H. einmal in Menge in Gesellschaft der Formica rufa in einer alten Eiche bei Soden < 7. - Offenbach. - Fr. s. h. auch von B. gef. - Wlb. (Sch.) - Mz. Mo. (S.) -

3. H. aquaticus L. = grandis llig. - Fr. - Rumpenheim, Hofheim, im Taunus überall h. in Pfützen. - Bornheimer Haide, Königswiese im April; im Genist der Nied bei Bonames (B.). - Dlb. (Sch.) - Mz. Mo. (S.) - Münster bei Soden im Taunus (I. H.). -

4. H. griseus Hbst. = granularis Thoms. - Fr. s. (B.) Wlb. einmal von Sch. gef. - Mz. Mo. (S.) -

5. H. granularis Er. - In der hohen Mark im Taunus. - Bei Fr. h. (B.) - Dlb. Wlb. (Sch.) - Mz. Mo. (S.) -

6. H. aeneipennis Thoms. = aquaticus Er. - Dlb. von Sch. gef.; auch von Wlb. -

7. H. dorsalis Er. = griseus Thoms. - Soden im Taunus s. - Dlb. (Sch.) - Mz. Mo. (S.) -

8. H. nanus Sturm. - G. (L.) -

[Der in S. V. erwälnte von Laubenheimer im Lahn-Anspülicht g'osammelte glacialis Heer ist sicher nicht dieses o u r alpine Thier; in seiner Sammlung auch kein Stück mehr unter diesem Namen.]

\section{Hydrochus Leach.}

1. H. carinatus Germ. - Fr. - Im ganzen Gebiet. - Bockenheim im April (B.). -

2. H. elongatus Schaller. - Fr. - Bockenheim ein Stück (B.). - G. (L.) - 


\section{Ochthebius Leach.}

1. 0. margipallens Latr. - Bei Rumpenheim in dem Tümpel an den Schiessständen $3 \mathrm{Ex}$. von C. H. gef.

2. O. bicolon Germ. - Bei Fr. von Hg. gef. -

3. (Asiobates Thoms.) pygmaeus F. - Fr.n. s. - Mo. (S.) -

\section{Hydraena Kugelann.}

1. H. riparia Kug. - Fr. - Wlb. (Sch.) - Mz. Mo. (S.) $<5$ Epstein in der Schwarzbach.

2. H. gracilis Germ. - Fr. 2 Ex. von C. H. gesammelt. Mo. (S.) -

3. H. pulchella Germ. - In der Schwarzbach bei Hattersheim von C. H. gesammelt. -

\section{SPHAERIDIINI.}

Cyclonotum Erichson.

1. C. orbiculare F. - Fr. - Wlb. (Sch.) - Mo. (S.) -

Sphaeridium Fabricius.

1. S. scarabaeoides L. - Im ganzen Gebiet h. in Kuhdünger. - Früher auf der Bornheimer Haide (B.) - Dlb. Wlb. s. h. (Sch.)

- Mz. Mo. (S.) -

2. S. bipustulatum F. - Die Stammart ohne rothen Fleck an der Schulter ist nach Sch. bei Dlb. und Wlb. n. s. - Mz. Mo. (S.) -

Var. B. Muls. = quadrimaculatum Mrsh. (ausser dem Fleck an der Flügeldeckenspitze mit einem rothen Schulterfleck). - Fr. n. s. - Dlb. Wlb. (Sch.) -

Var. C. Mulsant (nur mit rothen Schulterfleck.) - Fr. s. -

Var. D. Muls. = marginatum Scriba (ohne Flecken, nur mit feinem gelbem Saum um die Flügeldecken, welcher bei den anderen Varietäten nur theilweise vorhanden ist). - Fr. sehr einzeln. - Wlb. Dlb. (Sch.) -

\section{Cercyon Leach.}

1. C. haemorrhous Gyll. - Unter faulenden Pflanzenresten n. s. - Wlb. (K.), nach Sch. S. - Mr. Mo. (S.) - Nach einer alten Notiz 
von C. H. aus dem Jahre 181.7 bei Fr. am Rüstersee bei Oberrad (existirt nicht mehr) unter Steinen und im Sande, wo er an der Oberfläche lange Gänge gräbt, im Frühling; nie im Dung. - Fr. (B.) 2 Stück. -

2. C. obsoletus Gyll. - Bei Fr. einmal von C. H. gef. G. (L.) -

3. C. minutus F. - Fr. einmal. -

4. C. granarius Er. - Fr. S. -

5. C. flavipes F. - Ueberall in Kuhdünger. - Fr. - Eppstein im Taunus. - Wlb. h. (Sch.) - Mz. Mo. (S.) -

6. C. melanocephalus L. - Fr. - Offenbach. - Wlb. (Sch.) -

7. C. haemorrhoidalis F. - Fr. h. - Ebenso bei Wlb. (Sch.) - Mz. Mo. (S.) -

8. C. lateralis Mrsh. - Fr. s. s. - Wlb. (Sch.) -

9. C. pygmaeus Illig. - Bei Offenbach von C. H., bei Wlb. von Sch. gef. -

10. C. nigriceps Mrsh. $=$ centrimaculatus Sturm. - Fr. Wilb. (Sch.) -

11. C. quisquilius L. - Fr. h. - Wlb. (Sch.) -

12. C. unipunctatus L. - Fr. h. im Mist. - Wlb. (Sch.) -

13. C. analis Payk. - Fr. - Wlb. (Sch.) -

\section{Megasternum Mulsant.}

1. M. obscurum Mrsh. = bolitophagum Steph. - Fr. h. unter faulenden Vegetabilien; im Walde hinter Mo. und in den Gemuisefeldern von Mo. und Gonsenheim (S.). - Fr. 3. April 1860 (B.) -

\section{Cryptopleurum Mulsant.}

1. C. atomarium F. - Fr. g. an Dunghaufen; ebenso bei Dlb. and Wlb. (Sch.) - Bei Mo. wie die vorige Gattung (S.). - 


\section{HETEROCERIDAE.}

\section{Heterocerus Fabricius.}

1. H. salinus Ksw. = parallelus Ksw. nec Gebl. - Einmal von K. bei Wsb. gef. -

2. H. marginatus F. - Fr. an sandigen Bach- und Flussufern, wo er Gänge gräbt und kleine Erdhäufchen aufwirft. - Auch von Hg. gef. - Mo. (S.) -

3. H. obsoletus Curtis. - Wsb. (K.) - Wlb. (Sch.) -

4. H. hispidulus Ksw. - C. H. fing $=6$ ein Ex. bei Oherliederbach im Fluge. - Friedberg (Fuhr). - Fr. (Hg.) -

5. H. laevigatus Panz. - Fr. h. - Rödelheim $>5$ am Rande von Tümpeln; auch von Hg. gef. - Wlb. (Sch.) - Mo. (S.) - Nauheim $=5$.

6. H. crinitus Ksw. - Bei Fr. von Hg. gesammelt. -

7. H. fusculus Ksw. - Fr. je einmal von C. H. und Hg. gef. -

\section{PAR NIDAE.}

\section{Parnus Fabricius.}

1. P. prolifericornis F. - In stehendem Wasser h. - Fr. - Taunus. - Wsb. (K.) - Dlb. (Sch.) - Mo. (S.) - Fr. 3. April 1860 (B.). -

2. P. luridus Er. - Rumpenheimer Schiessstände an dem grossen Tümpel. - Soden, Cronthal in der Mineralquelle in der Wiese. -

3. P. striatopunctatus Heer. - Hg. fand ein Stück bei Fr. -

4. P. auriculatus Illig. - Fr. am Mainufer. - Cronthal in Wiesenbächen h. - Am Lahnufer bei Ems. - Wsb. (K.) - Mo. (S.) - 


\section{Macronychus Müller.}

1. M. quadrituberculatus Müll. - Die Sammlung v. Heyden besitzt ein von H. Lindheimer bei Fr. gefangenes Ex. -

\section{Elmis Latreille.}

1. E. aeneus Müll. - Fr. (Hg.) - Wsb. (K.) - Unter Steinen im Wasser der Urselbach $>7$ h. - Ems (C. H.) - Wlb. (Sch.) -

2. E. Kirschii Cl. Müller n. sp. - Seither mit aeneus, sogar vom alten Pfarrer Müller, verwechselt; erst von Clemens Müller in Dresden, der die Arten in Sammlung v. Heyden revidirte und die Gruppen monographisch bearbeiten will, unterschieden. - Ems zweimal von C. H. gef. (Hierher die in S. V. erwähnten Stücke aus Eins.) - Königstein $>8$ einmal. - Siehe Berlin. Ent. Zeit. XIII., p. 261.

3. E. Maugeti Latr. - An dem Bach im kleinen Feldbergthal = 5. - In der Erlenbach in der hohen Mark im Taunus $>6$. -

4. E. Mülleri Er. $=6$ am Mainufer unter Weiden (C. H.)

5. E. Volkmari Panz. - Von K. bei Wsb. an der Wellritzbach 25. Juli mit dem Streifnetz Mittags gef. -

6. E. parallelepipedus Müll. - Wsb. (K.) - Unter Steinen in der Erlenbach einmal von C. H. gef. -

7. E. angustatus Müll. - Im Taunus bei Königstein an der Oelmühle $>6$ und bei Falkenstein in dem Wiesenbach an der Nordseite $=5$ je einmal gef. -

8. (Limnius Er.) tuberculatus Müll. - Von K. bei Wsb. in der Wellritzbach im Mai gef. - Wlb. (Sch.) -

\section{GEORYSSIDAE.}

\section{Georyssus Latreille.}

1. G. pygmaeus F. - Offenbach in einem nassen Graben h. - Soden. Das Thierchen ist oben ganz mit einer Erdkruste überzogen, so dass es wie ein wandelndes Erdklümpchen aussieht. - Mz. Mo. (S.) - 


\section{L U CA N IDAE.}

\section{Lucanus Linne.}

1. L. cervus L. - Im ganzen Gebiet in Wäldern an alten Eichen, da diese aber immer seltener werden, so verschwindet mit diesen auch unser grösster europäischer Käfer. - Im Schwanheimer Wald, an der Mainkur noch stets zu finden. - Nach Prof. Sch. früher bei Dlb. einer der häufigsten Käfer. - In Holzmagazinen in Mz. h. (S.) - Wsb., Wlb., Had. (K.) - Auch im Taunus h. (B.) -

Var. capra Oliv., aus kümmerlich ernährten Larven entwickelt, findet sich mit der Stammart.

Dorcus Mac Leay.

1. D. parallelepipedus L. - Im ganzen Gebiet s. h. an alten Bäumen. - Fr. an Buchen. - Wlb., Wsb., Had. (K.) - Auch an Weiden und Eichen von B. gef. - Bei Dlb. h. an alten Pappeln (Sch.). - In und an alten Weidenstämmen h. Mz. Mo. (S.) -

\section{Platycerus Geoffroy.}

1. P. caraboides L. - In Wäldern im ganzen Gebiet h., doch mehr im Gebirge. - Fr. - Taunus. - Wsb. Had. (K.) - Hofheim (B.). - Wlb. Dlb. s. h. (Sch.) - Im Oberolmer Wald bei Mz. (S.) -

Var. rufipes Hbst. - Diese rothbeinige Varietät fand L. H. einmal n. s. auf dem Wege laufend bei Cronthal im Taunus. - Bei Dlb. und Wlb. öfter gef. (Sch.) - Ernsthausen in Nassau (Dörr bei K.). -

\section{Sinodendron Fabricius.}

1. S. cylindricum L. - Im Taunusgebirge z. S. - Königstein $>7$ unter Eichenrinde, bei Cronthal im Holz von zahmen Kastanien (Castanea vesca). - In Schlangenbad und Wsb. von C. H. gef. Wsb. am Chausseehaus in Buchenstrünken (K.). - Nach Sch. bei Dlb. an alten Buchenstämmen, bei Wlb. auf Zimmerplätzen, auch auf Stämmen ron Nadelholz. - 


\section{SCARABAEIDAE.}

A. COPRINI.

\section{Si is y hus Latreille.}

1. S. Schäfferi L. -- Dieses interessante Thier kommt bei Herborn h. vor, wo es von Sch., s. auch bei Wlb. gef. wurde. - Einmal zwei Stück auf einem Kothhaufen bei Mo. gef. (S.) - Auch K. fand die Art bei Dlb. in Weinbergen im Mai an Kuhmist. - Sonst mehr im Süden, aber auch im Hunsrück. -

\section{Gymnopleurus Illiger.}

1. G. cantharus Er. - Nach Bach bei Bingen. - [Sonst bei Grünstadt in der Pfalz, Stücke in meiner Sammlung. - Auch bei dem Emmerichshof bei Hanau von Heynemann und Apotheker Dr. Hille gef.] - Im Oberolmer Wald bei Mz. (S.) -

\section{Copris Geoffroy.}

1. C. Iunaris L. - Im ganzen Gebiet n. s. in Kuhdünger. Früher bei Fr. h., auf den Viehtriften am Grindbrunnen, Bornheimer Haide. - Auf Bergwiesen nach der Platte bei Wsb. (K.) - Fehlt nach Sch. bei Dlb. und Wlb. - Bingen (Bach). - Im Oberolmer Wald bei Mz. (S.) -

\section{Caccobius Thomson.}

1. C. Schreberi L. -- Da das Vieh in unserer Gegend wenig mehr hinausgetrieben wird, so sind fast alle Mistkäferarten, die von den thierischen Abfällen leben, seltener geworden. - Bei Fr. und Mz. von C. H. früher h. in Kuhdung gef. -

\section{Onthophagus Latreille.}

1. O. Taurus L. - Das Männchen variirt, wie alle Arten der Gattung, sehr in der Grösse der Kopfhörner. - Im ganzen Gebiet n. s. 
in Kuhmist. - Fr. - Bergwiese nach der Platte bei Wsb. (K.) Im Rebstockwäldchen bei Fr. von B. gef. - Fehlt nach Sch. bei Dlb. und Wlb. -

2. O: nutans F. - Zwischen Cronthal und Cronberg 3 Stück von $\mathrm{Hg}$, , bei Wsb. von K. ges. - Auch B. fand ihn einmal in hiesiger Gegend. - Dlb. und Wlb. (Sch.) - Mz. Mo. (S.) -

3. O. semicornis Panz. - Von B. bei Fr. einmal und von K. bei Wsb. gef. -

4. O. vacca L. - Bei Fr. n. s. - Wsb. (K.) - Mz. Mo. (S.) -

5. O. Coenobita Hbst. - Im Gebiet h. - Fr. - Wsb. (K.) - In Kuhmist von B. bei Fr. gef. - Dlb. und Wlb. (Sch.) - Mr. Mo. (S.) - Staufen an Fuchslosung 2. April 1876 (B.). -

6. 0. fracticornis Preyssl. - Bei uns die gemeinste Art. Fr. - Wsb. (K.) - Dlb. und Wlb. (Sch.) - Mz. Mo. (S.) - Soden im Taunus 2. April 1876 (L. H.). -

7. O. nuchicornis F. - Im Gebiet s. h. - Fr. - Dlb. Wlb. (Sch.) - Wsb. (K.) - Mz. Mo. (S.) - Am Staufen wie coenobita (B.). -

8. O. Lemur F. - Fr. s. von L. H. ges. - Taunus (Hg. und B.). - Dlb. Wlb. (Sch.) - Mo. und Gonsenheim bei Mz. (S.) -

9. O. ovatus L. - In Mist s. h. - Fr. - Ems. - Wsb. (K.) - Dlb. Wlb. (Sch.) - Mo. (S.) - Fr. 3. April 1860 (B.). -

\section{Oniticellus Lepelletier.}

1. O. flavipes F. - Von C. H. früher am Grindbrunnen bei Fr. n. s. s., später noch von Scheidel gef. -

\section{B. APHODIINI.}

\section{Aphodius Illiger.}

1. (Colobopterus Muls.) erraticus L. - Im ganzen Gebiet h. an Kuhmist. - Fr. - Wsb. 3. October (K.). - Dlb. (Sch.) Mz. (S.) -

2. (Eupleurus Muls.) subterraneus L. - Im Gebiet verbreitet. Fr. - Rumpenheim. - Mo. (K.) - Wlb. ein Ex. mit rothem Schulterfleck (Sch.) - Mz. Mo. (S.) - G. (L.) - Mo. 20. Juli (K.). - 
3. (Teuchestes Muls.) fossor L. - Im Kuhdünger h. Fr. Mz. (S.) - Dlb. und Wlb. (Sch.) - G. (L.) -

Var. sylvaticus Ahr. (mit braunen Flügeldecken). - Fr. (Hg., B., Scheidel). - Wsb. (K.) - Wlb. zweimal (Sch.). - G. (L.) -

4. (Otophorus Muls.) haemorrhoidalis L. - Fr. (Hg.) -

5. A. scybalarius F. - Stücke mit ganz gelben Flügeldecken $=$ argillicolor Mls. sammelte C. H. zweimal bei Fr. und Sch. bei Wlb. - Normale Ex. fand Hg. - Mz. (S.) -

6. A. foetens F. - Von C. H. bei Ems, bei Fr. s. von L. H. und Hg., Wsb. von K. gesammelt. -

7. A. fimetarius L. - In ganz Europa in Kuh- und Pferdemist g. - Fr. - Ems. - Bei Dlb. und Wlb. s. g. (Sch.) - Mz. (S.) Wsb. 3. October (K.). -

Var. orophilus Charp. (mit rothem Halsschild und viel stärkerer Punktirung) s. s. bei Fr. und Soden.

8. A. ater De Geer. - Hg. fand vier Stück bei Fr. -

9. A. granarius L. - In ganz Europa h. - Fr. - Wlb. (Sch.) - Mz. (S.) -

10. A. sordidus F. - Im Gebiet n. s. - Fr. - Rumpenheim. - Auch von Hg. gesammelt. - Mz. (S.) -

11. A. rufescens F. - Ein Ex., mit einem schwarzen Wisch auf den Decken, von C. H. im Fr. Wald (Gehren) $>9$ gef. - Fr. (Hg.) - Auch von B. einmal bei Fr. gef. -

12. A. nitidulus F. - z. S. - Fr. - Soden. - Auch von Hg. im Gebiet bei Fr. gef. -

13. A. immundus Creutz. - Fr. sehr einzeln von C. H. gef. - Mz. (S.) -

14. A. varians Dft. - Bei Fr. einmal von Hg. und bei Wsb. von K. gef. -

Var. bimaculatus F. - Fr. an Gänsekoth. - Bieberer Höhe bei Offenbach. - Von B. bei Fr. einmal am 4. April 1860 gef. Mz. (L. H. und S.) - G. (L.) - Soden (Scheidel). -

15. A. niger Panz. Illig. $-<6$ auf Wiesen bei Cronberg, gräbt Gänge in fetter Erde, nach der Beobachtung von C. H. -

16. A. lividus Oliv. - Von Hg. einmal bei Fr. gef. - 
17. A. inquinatus Herbst. - Im ganzen Gebiet g. in Mist. - Variirt ausserordentlich in der Zeichnung der Flügeldecken. - Fr., Enkheim, meist in Pferdemist; auch von Hg. gef. - Wlb. und Dlb. (Sch.) - Mz. (S.) -

18. A. melanostictus Schmidt. - n. s. s. bei Fr. auch von Hg. gef. - Bürgel am Main, Rumpenheim. - Wsb. (K.) - Wlb. einmal (Sch.). - Mz. (S.) -

19. A. sticticus Panz. - An Kuhkoth am Fr. Forsthaus, Mainkur und am Feldberg. - Auch ron Hg. ges. - Wlb. (Sch.) - Mz. (S.) -

20. A. pictus Sturm. - Fr. s. im ersten Frühjahr auf Aeckern fliegend. - Wsb. (K.) - Mz. (S.) -

21. A. tessulatus Payk. - Fr. von C. H. im Flng gef. MIz. (S.) -

22. A. maculatus Sturm. - C. H. fing bei Ems ein Ex. $=8$ an Eselskoth. - K. ein Stück bei Wsb. an der Rentmauer. -

23. A. porcus $\mathbf{F}$. - Von $\mathrm{Hg}$. dreimal bei Fr. gef. -

24. A. scrofa F. - Von B. s. bei Fr. gef. -

25. A. tristis Panz. verus. - Wlb. (Sch.) -

26. A. pusillus Hbst. - Fr. n. s. - Offenbach. [Hierher die Fr. tristis in S. V.] - Auch von B. h. gef. - Wlb. (Sch.) -

27. A. quadriguttatus Hbst. - Fr. von C. H. und $\mathrm{Hg}$, bei Offenbach von Scheidel gef. - Mz. (S.) -

28. A. quadrimaculatus L. - Friedberg (Fuhr), - Bei Fr. s. von C. H. und $\mathrm{Hg}$. gef. - Wlb. einmal (Sch.). -

29. A. sanguinolentus Panz. - Von B. einmal bei Fr. gef., das Exemplar in Sammlung r. Heyden = Er. Var. b. (wie c., aber auch ein Schulterfleck). -

Var. c. Erichs. (elyttris nigris, macula ante apicem rufa) bei Wlb. einmal (Sch:). - Mz. (S.) -

30. A. biguttatus Germ. - Von Hg. einmal bei Fr. gef. -

31. A. merdarius F. - Bei Offenbach; Soden und am Feldberg in Kihkoth ges. - Bei Fr. von Hg., von B. h. am Forsthaus gef. - Wlb. Dlb. (Sch.) - Mz. (S.) -

32. (Melinopterus Mls.) prodromus Brahm. - Im Fr. Gebiet an Kuhkoth s. h.; auch von Hg. gef. - Wsb. (K.) - Wlb. und Dlb. s. h. (Sch.) - 
33. (M.) punctatosulcatus Sturm. - Fr. weniger h.; auch von Hg. gef. - Wlb. (Sch.) -

[Dass A. limbatus Germ. nach einer Mittheil. Scriba's bei Soden gefangen sein soll, beruht sicher auf falscher Bestimmung; die Art findet sich nur in Oesterreich. - Ebenso habe ich kein verbürgtes Vorkommen im Gebiet von consputus Creutz.]

34. A. contaminatus Hbst. - Bei Rumpenheim in Nenge an Menschenkoth gef. - Von Hg. bei Fr. ges. - Mz. (S.) -

35. A. obliteratus (Heyd. i. I.) Panz. - Von C. H. beim Fr. Forsthaus entdeckt und ron Panzer 1823 nach solchen Exemplaren beschrieben und abgebildet. -

36. (Acrossus Muls.) rufipes L. - Fr. z. s. - Mz. von uns gef. -

37. (A.) Iuridus Payk. - Bei Gronau (Kreis Vilbel) in Menge von C. H. an Menschenkoth $<5$ gef. (Flügeldecken gelb mit schwarzen Längsflecken). - Zweimal bei Fr. am 8. und 29. April 1861 von B. gef. - Wlb. (Sch.) - Mrz. (S.) - G. (L.) -

Var. gagates Müll. = gagatinus Fourc. (mit schwarzen Flingeldecken) mit dem vorigen bei Gronau. - Feldberg an Kuhkoth. Wlb. (Sch.) - G. (L.) -

38. A. depressus Kug. - [Die Stammart mit rothen Flüge]decken kenne ich nicht aus dem Gebiet.]

Var. nigripes Dft. (mit schwarzen Flügeldecken) von $\mathrm{Hg}$. bei Fr. gef. -

39. A. pecari F. - Früher von C. H. n. s. bei Fr. im Frühjahr auf der Viehweide am Grindbrunnen, später noch von Scheidel bei Fr. gef. - Mz. (S.) -

[A. arenarius 0 . in $\mathrm{S} . \mathrm{V}$. von uns gef, beruht auf falscher Bestimmung.]

40. (Oxyomus Cast.) Sus. F. - L. H. fand diese Art einmal in Menge an Menschenkoth auf dem Mombacher Sand. - Miz. (S.) -

41. (0.) testudinarius F. - Bei Fr. $<4$ s. auf Aeckern fliegend; am Forsthaus s. von B. 18. April 1860 gef. - Mz. h. (S.) - Mo. 4. Mai (K.) -

42. (0.) porcatus F. - Im ganzen Gebiet in regetabilischen Dunghaufen s. g. - Fr. - Soden. - Dlb. und Wlb. s. g. (Sch.) Mz. (S.) - 


\section{Rhyssemus Mulsant.}

1. R. germanus $\mathbf{L}$. = asper F. - Bei Fr. sehr einzeln ron C. H. und B. gef. - Wsb. (K.) -

\section{Psammobius Heer.}

1. P. caesus Panz. - Fr. sehr einzeln, auch von B. 8. April 1860 gef. - Wsb. (K.) -

2. P. cruciatosulcatus Preyss!. = sulcicollis Illig. - An der Ziegellütte am Fr. Wald auf Sandboden unter Steinen, ebenso bei Mo. von L. H. s. s. gef. - Wsb. (K.) - Mz. (S.) - Fr. 1: Mai 1860 (B.). -

3. P. vulneratus Sturm. - Bei Wsb. von K. gef. - Wlb. (Sch.) -

\section{ORPHNINI.}

\section{Ochodaeus Lepelletier.}

1. O. chrysomelinus F. - Wir fingen diese seltene Art sehr einzeln auf der Bieberer Höhe in der Dämmerung fliegend am 1. Juni und $=7.1856$; C. H. auch einmal $=5$ bei Fr. auf dem sogen. Diebsweg. - Auch im Fr. Wald (Babenhäuser Weg) = 5. 1831. -

\section{GEOTRUPINI.}

\section{Odontaeus Klug.}

1. O. mobilicornis F. - Von C. H. öfter bei Oberheckstadt gef. - Ein Männchen mit langem Horn fing Steitz bei Fr. und St. bei Homburg, L. H. ein Männchen mit kurzem Horn $<7$ an den Planken bei der "Louisa" im Fr. Wald. - Friedberg (Fuhr). - Wsb. je ein Männchen mit grossem und kleinem Horn (K.). - v. Harold fand ein langhorniges Männchen bei Nauheim im Juni 1871. - B. fand ihn einmal im Flug bei Königstein Abends, zur Zeit der Heuernte. - Ein Männchen mit sehr langem Horn und eins mit kleinem Horn, bei welchem die seitlichen Eindrücke des Thorax fehlen und keine Seitenhöcker, sondern nur vorn 4 Höcker Forhanden sind, von Sch. bei Wlb. gef. Ein Männchen und ein Weibchen auf frisch geackertem Feld oberhalb Mr. (S.). - 
Var. a. fulvus Mls. (hellbraun). Fr. - M. S. fand ein langhorniges Männchen. -

Var. b. testaceus F. (gelb). - Ein Männchen bei Wsb. von K. gef. - Von Scheidel bei Fr. ein Pärchen gef. -

\section{Geotrupes Latreille.}

1. (Minotaurus Mls.) Typhöeus L. - Fr. - Bei Rumpenheim in Sandgegenden h. gef. Er gräbt fusstiefe Löcher, in welche er Schaf- und Lapin-Koth hineinzieht, um daran seine Eier zu legen. Wsb. im April und Mai (K.). - Bei Bieber (bei Offenbach) h. 1872 und dreimal im Rebstöcker Wald von B. gef. - Von Sch. ein Männchen bei Wlb. gef., das nach einem Kahdüngerhaufen lief. - Oberhalb Mo. bis nach Heidesheim (S.). -

2. G. spiniger Mrsh. Harold = stercorarius Er. = mesolaius Thoms. - Fr. sehr einzeln von L. H. und Hg. gef. - Wsb. fünfmal von K. gesammelt. - Bei Wlb. von Sch. 3 sehr grosse Männchen und 3 kleinere Weibchen gef. [Siehe Kraatz. Entomol. Monatsblätter. I, No. 6,1876, p. 81.] - Sch. beobachtete von No. 2 und 3 eine grosse und eine kleine Form. -

3. G. stercorarius L. = putridarius Er. - Fr, von L. H. und Hg. gef. - Einen sehr kleinen Mann und 3 Weiber bei Wlb. von Sch. gef. -

4. G. mutator Marsh. - Fr. h. - Wsb. s. h. (K.) - Bei Wlb. die häufigste Art, Sch. fand viele Männchen und Weibchen. Mo. (S.) -

5. (Anoplotrupes Jekel) sylvaticus Panz. - Im Fr. Wald s. h. an faulen Schwämmen und auf dem Weg laufend. - Wsb. (K.) - s. h. bei Wlb. (Sch.) - Mo. (S.) - G. (L.) - Gimbacher Hof am Fuss des Staufen 2. April 1876 (L. H.). -

6. (Sternotrupes Jekel) vernalis L. - Fr. 1.. s. - Bei Dlb. in Wäldern (Sch.). - Wsb. (K.) - Mz. Mo. (S.) - G. (L.) -

\section{E. TROGINI.}

\section{T r $0 \mathbf{x}$ Fabricius.}

1. T. perlatus Goeze. - Am Rossert, dem zweithöchsten GipfeI des Taunus, $<5$ gef. - Neuenhain bei Soden. - Wsb. h. (K.) - 
Nach B. in Cyrenenmergelgegenden im Rheingau und Rheinhessen h. Wlb. (Sch.) - Mz. Mo. (S.) - In Menge auf dem Staufen, dem dritten höchsten Berge des Taunus, unter Fuchslosung an alten Säugethierehaaren am 2. April 1876 (L. H. und B.). - Ebenso an Hundekoth im Mo. Wald 27. Mai 1876 (L. H.). -

2. T. hispidus Laich. - Fr. sehr einzeln gef. - Wsb. (K.) -

3. T. sabulosus L. - Fr. - Offenbach. - Wsb. (K.) - Bei Fr. h. nach B. - Dlb. Wlb. (Sch.) - Mz. Mo. (S.) -

4. T. scaber L. - Fr. - Wlb. (Sch.) - Wsb. (K.) - Mz. Mo. (S.) - G. (L.) -

Die Trox-Arten finden sich in Sandgegenden unter Steinen und öfter am Leder alter Schuhe und an Lumpen. -

\section{F. DYNASTINI.}

\section{Oryctes llliger.}

1. 0. nasicornis L. - Herr S. A. Scheidel fand eine Anzahl Exemplare in der Lohe einer Gerberei in Höchst am Main; Stücke davon sind im Senckenberg'schen Museum. -

\section{G. MELOLONTHINI.}

\section{Polyphylla Harris.}

1. P. fullo L. - In Sandgegenden. - Im Fr. Wald am Sandhof fand L. H. eine Flügeldecke. - Dreimal im Offenbacher Wald ron B. gef. - Fehlt nach Sch. bei Wlb. und Dlb. - In manchen Jahren im Kiefernwald zwischen Mo. und Gonsenheim h. (S. K.) - Der Käfer (ein Weibchen) bringt einen zirpenden Ton dadurch hervor, dass das Thier die Innenseite der Flügeldeckenspitze auf der quergefältelten umgebogenen Hauptader der Unterflügel hin- und herstreifen lässt und zwar durch Ein- und Ausziehen des Hinterleibes (I. v. H.). -

\section{Anoxia Laporte.}

1. A. villosa F. - Vor Jahren in grösserer Menge an Obstbäumen zwischen Castel und Biebrich ron Sch. gesammelt. - 


\section{Melolontha Fabricius.}

1. M. vulgaris L. - Ueberall s. h. - Mz. h. (S.) -

Var. ruficollis Muls. (rothes Halsschild) seltener, Z. B. an der Mainkur, am Fr. Forsthaus. -

Var. lugubris Muls. (der ganze Körper mehr ader weniger dunkelbraun) fand C. H. = 5. 1847 zwei Ex. auf dem Röderberg bei Fr. - Auch von B. Männchen und Weibchen bei Offenbach gef. Dlb. Wlb. (Sch.) -

Var. albida Redtb. nec Er. (auf schön weiss bestäubte Weibchen gegründet) besitzt die Sammlung r. Heyden mehrere aus dem Gebiet. Dlb. und Wlb. (Sch.) -

2. M. Hippocastani F. - Im ganzen Gebiet h., mehr in Sandgegenden. - Mo. 19.-21. Juni h. (K.) - Na.ch B. besonders auf Eichen und Buchen. - Fehlt nach Sch. bei Dlb. und Wlb. -

Var. nigripes Porro. (mit schwarzen Beinen) etwas seltener. -

Var. coronata Muls. (Halsschild nur in der Mitte röthlich) selten unter den anderen. -

Ueber die Maikäferflugjahre (d. h. die periodisch wiederkehrenden Jahre, in welchen der Käfer massenhaft auftritt, um dann in den nächsten Jahren nur weniger zahlieich zu erscheinen) sind nur unzulängliche Beobachtungen an dem häufigen Thiere gemacht, obgleich doch die Sache für den praktischen Landwirth von grösster Wichtigkeit ist.

Schon 1841 im 2. Band Stettiner Entomol. Zeitschr. richtet Prof. Heer in Zürich eine Anfrage an die Entomologen, ihm Nachrichten über die Flugjahre der Maikäfer zukommen zu lassen und bearbeitete in demselben Jahre die gewonnenen Mittheilungen in einem besonderen Schriftchen „Ueber die geographische Verbreitung und periodisches Auftreten del Maikäfer".

Heer glaubt für Nitteleuropa, wie überhaupt eine dreijäbrige Entwicklungszeit (sodass zwei volle Jahre zwischen dem Ei und dem entwickelten Thier liegen) annehmen $z 11$ müssen und hält die Angaben Ratzeburg's für die Mark Brandenburg und Bechstein's für Franken, die eine vierjährige Flugzeit annehmen, für irrthümlich, „da sie wohl Gegenden mit verschiedenen Flugjahren zusammenstellten und wurden dadurch zu irrigen Schlüssen verleitet".

Doch das steht fest, dass nicht alle Gegenden in den gleichen Jahren Flugjahre haber. 
1. In den Umgebungen von Basel fällt das Flugjahr nach Heer auf die Jahre, welche sich durch 3 dividiren lassen, also 1836, 1839, $1842 \ldots$. . es wären danach auch 1872 und 75 ebenfalls Flugjahre gewesen und 1878 würden sie wieder in Menge bei Basel erscheinen. Heer nennt dies das Baseler Flugjahr.

2. Im Canton Bern, Berner Flugjahr, auf die Jahre, welche durch 3 dividirt 1 zum Rest geben, also: 1834, 37, 40, ..., 1873, 1876,79 .

3. Im Canton Uri, Urner Flugjahr, auf die Jahre, welche durch 3 dividirt 2 zum Rest geben, also $1835,38,41, \ldots, 74,77$, 1880 .

Bei Basel lässt sich die Flugperiode bis zum Jahr 1755 zurückverfolgen.

In der Schweiz hat das Berner einen viel grösseren Umfang als das Baseler Flugjahr.

Ueber das Urner Flugjahr gehen die Beobachtungen zurück bis 1644, doch ist es nur im Canton Uri am schärfsten ausgesprochen.

Nach den Erfahrungen, die $\mathrm{Heer}$ sammelte, ist ausserhalb der Schweiz das Baseler Flugjahr das am meisten verbreitete, wenigstens gehören hierzu: Frankreich, Elsass, Schwaben, Main- und Neckar-Gegenden, Rheinlande, Berlin (1820 und 36)*), Sachsen (1860 nach v. Kiesenwetter), Oesterreich (1767), Polen (1812), Venedig (1806, 1812), Russland (1770).

Innerhalb dieses grossen Bezirks gehen nach Heer einzelne Landstriche durch, in welchen die Maikäfer im Berner Jahr erscheinen, z. B. in den Donaugegenden Würtembergs, bei Heidenheim und auf der Mittleren Alp ebendaselbst; ein anderer Strich geht durch Nordbaiern (Anspach), ein weiterer bei Braunschweig.

Vom Urner Jahr zieht ein Strich zwischen Neustadt und Berlin quer durch, während diese Städte selbst ausserhalb liegen. Ebenso gehört nach $\mathrm{Heer}$ hierher Frankfurt a. M. (wo sie 1769 alle Bäume kahl gefressen hatten). Dies stimmt auch mit den mehrjährigen Beobachtungen, welche Dr. Steitz in der Zeitschrift ,Der Zoologische Garten,

*) Ich muss gestehen, dass ich es nicht für das Richtige halte, eine Gegend, nach ein em constatirten Maikäferjahr, schon gleich in ein Schema einzureihen und zu sagen, weil in diesem Jahr ein Flugjahr war, ist nun in dieser Gegend auch in den schematisch folgenden Jahren ein Maikäferjahr.

v. Heyden. 
Band III, 1862, p. 81 " mittheilte und noch kürzlich in einem Briefe an mich bestätigte, in dem er schrieb: „Ich hatte von 1850 an mehrere Jahre das Erscheinen der beiden Melolontha-Arten beobachtet und glaubte als Gesetz für un sere Gegend gefunden zu haben, dass M. vulgaris mit ihrer dreijährigen Flugperiode $1850,53,56$ u. s. W. erscheinen müsse. Bis 1862 setzte ich diese Beobachtungen fort, fand sie immer bestätigt und wunderte mich sehr, als für 1870 von Oekonomen ein Erscheinen in Masse vorausgesagt und darauf hin von der Regierung Mittel zur Vertilgung bewilligt wurden, während ich berechnet hatte, der Käfer würde erst 1871 wieder ein Flugjahr haben. Damit behielt ich Recht. Nun sollte er aber 1874 wieder in Masse erscheinen - und so viel ich weiss, fehlte er doch in diesem Jahre fast ganz. Sollte die Vertilgung eine so radikale gewesen sein?" -

Auch 1876 sollte nach Angabe der Oekonomen ein Flugjahr sein, doch flog er nicht in Menge, während er nach den Frankfurter Beobachtungen erst 1877 wieder ein Flugjahr haben soll.

$\mathrm{Zu}$ vermuthen ist, dass die Regierung zu Wiesbaden bei ihrer Bewilligung der Mittel zur Vertilgung im Jahre 1870 sich nach Angaben dortiger Oekonomen richtete. Ueber die Flugjahre bei Wiesbaden liegen mir zwar keine Notizen vor, doch gibt $\mathrm{Bach}$ in seiner ,Käferfauna für Nord- und Mitteldeutschland Band I, 1851, p. 332" für die Gegend von Boppard am Rhein das Baseler Jahr an, also 1842, 45, $48, \ldots$, folglich hätte er dort zu er'scheinen gehabt 1869, 72, 75.

Ich glaube, dass Dr. Steitz Recht hat, und dass wir bei Frankfurt seit einer Reihe von Jahren Urner Flugjahre haben, welches Verhältniss sich aber von jedem Jahr an durch Dazwischentreten verschiedener Umstände ändern kann. Eine gewisse Schwankung trat z. B. 1874 deutlich hervor; die Zukunft wird es lehren, ob wir in ein anderes Flugjahr für eine Reihe von Jahren versetzt werden, oder ob wir auf längere Zeit hinaus gar keine besonders hervortretende Maikäferjahre haben werden.

In Giessen war 1874 ein entschiedenes Flugjahr, also Urner Jahr.

Ueber die $2^{\text {te }}$ Maikäferart (Melolontha Hippocastani), (die in frühel'er Zeit mit vulgaris vermengt wurde), sind die Notizen recht spärlich. - Nach Dr. Steitz hat sie eine vierjährige Flugzeit und trat in Frankfurt in Masse (doch nur in Sandgegenden) auf: 1850, 54, 58, würde also 1870, 74 erschienen sein, worüber mir jedoch Angaben fehlen. Ich selbst beobachtete sie 1867 in zahlloser Menge (also ein 
Jahr früher wie bei Frankfurt) auf dem Gaualgesheimerkopf, eine Stunde oberhalb Bingen am 19. Mai.

Gemeinsame Flugjahre für beide Arten bei Frankfurt wa r e n 1850 , 62, 74, also immer nach 13 Jahren. Eine Regel kann meiner Meinung nach hier noch weniger aufgestellt werden, da in der Erscheinungszeit beider Arten die grössten Modifikationen eintreten können, noch mehr als wenn wir es nur mit einer Art zu thun hätten.

Für M. Hippocastani gibt Heer auch eine dreijährige Flugperiode an und sagt: „Nur um Nürnberg möchte dies vielleicht nicht der Fall sein, da nach Rösel's Insektenbelustigungen II, p. 7, dort die rothund schwarzschildigen mit einander abwechseln sollen. Rösel gibt für beide Arten (die rothschildigen und schwarzschildigen, wie er sie nennt und nach der Schwanzspitze wohl unterscheidet = rothsch. ist hippoc. die schwarzsch. vulgaris) eine vierjährige Entwicklungszeit an. - Heer bestreitet dieses und sagt, dass $R \ddot{\text { se }} \mathrm{l}$ und $\mathrm{Kleemann}$ die Larven in Medien auferzogen, die für ihre Entwicklung sehr ungünstig waren und sie sich dadurch später entwickelten. - Für die rothschildigen führt Rösel an, dass sie im Jahre 1740 zahlreich auftraten, aher in demselben Jahre auch die schwarzschildigen; möglicherweise war dies aber eins der schon oben erwähnten Jahre, in welchen beide Arten zusammen zahlreich vorkamen.

Diese Notizen waren bereits im Manuscript aufgenommen, als ich Gelegenheit fand, mich längere Zeit mit meinem Vetter $\mathrm{Adolph}$ von Harnier zu Echzell in der Wetterau, dem Bruder des Afrikareisenden, einem erfahrenen Landwirth und gewissenhaften Beobachter der Vorkommnisse in der freien Natur, über diesen Gegenstand zu unterhalten. Da seine Theorie über das zeitweise häufigere Auftreten der Maikäfer zu dem Schlusse gelangt, dass die Maikäferjahre, d. h. ein so zahlreiches Aufreten der Maikäfer, dass sie zur Plage werden, gerade das Unregelmässig sind, während sonst meist angenommen wird, sie seien die Regel, so theile ich hier das Hauptsächlichste aus einem Briefe an mich mit.

A. von Harnier schreíbt: „Jedes Jahr finden sich allerorts einige Maikäfer; ich nenne diese den eisernen Bestand. Wenn nun die Lebens- und Entwicklungsbedingungen ein Jahr besonders günstig sind, so wird der eiserne Bestand dieses Jahres - [der Maikäfer ist Ende des 
dritten Jahres vom Ei aus entwickelt und erscheint erst über der Erde im vierten Jahre; die im Jahre 1870 z. B. gelegten Eier liefern den Käfer 1873: v. He yden] — durch

1. seine Eier im Jahre I;

2. seine sich entwickelnden Larven im Jahre II;

3. durch die Weiterentwicklung im Jahre III und

4. durch ihre Metamorphose zu Maikäfer im Jahre IV

sich einer starken Nachkommenschaft zu erfreuen haben und das Jahr IV ein Maikäferjahr sein.

Ist diese zweite Generation der A-Familie (aus dem Jahr I) in dem Jahre IV und den drei folgenden Jahren mit gleich günstigen Vorbedingungen zur Vermehrung und weiteren Entwicklung gesegnet wie ihre Väter und Mütter, so wird im Jahre VII diese zweite Generation wiederum als starkes Contingent Maikäfer - als dritte Generation - erscheinen und erfreut sich diese Familie gleich günstiger Umstände innerhalb der nächsten Periode, so wird, wenn nicht eine theilweise Reduction durch feindliche Mächte stattfindet, diese Familie A die Weltmacht erlangt haben und die Existenz selbst der Menschen bedrohen. Glücklicherweise ist diese regelmässige Wiederkehr ganz gleich günstiger Umstände in der Natur nicht der Fall, denn sonst würde ebenso der ,eiserne Bestand" aus dem Jahre II und III u. s. f. sich einer gleich starken Vermehrung zu erfreuen haben und wo bliebe da die übrige Schöpfung? Wir würden keine Bäume mehr haben, ausser Tannen und Kiefern, keine Wiesen und Kulturpflanzen, infolge dessen unsere Lebensbedingungen auf das feuchte Element (die Fischerei?!) beschränkt sein.

Nehmen wir daher diese besonders günstigen Verhältnisse nur für die Familie A aus Jahr I und B aus Jahr II an, während wir annehmen, dass Familie $\mathrm{C}$ aus Jahr III u. s. w. durch ungünstige Verhältnisse auf die Minorität „des eisernen Bestandes" reducirt blieben. Es würden dann Maikäferjahre werden :

Jahr IV durch Familie A II. Generation repräsentirt;

$» \mathrm{~V} \gg$ Familie B II. Generation;

» VI keine Maikäfer;

»VII Maikäferjahr der Familie A III. Generation;

» VIII 》 der Familie B III. Generation;

» IX kein Maikäferjahr ;

» X Maikäferjahr der Familie A IV. Generation;

* XI $\gg$ der Familie B IV. Generation u. s. w. 
Da unzweifelhaft also die Bedingungen für je de n Maikäfer günstig sein müssen, wir aber wissen, dass die „eisernen Bestände“ aus gewissen Jahren, also hier z. B. die Familie C, die ihr Flugjahr in den Jahren VI, IX u. s. w. haben würde, sich dieser günstigen Umstände nicht zu erfreuen hatten, so würde man an einen Maikäfergeburtsadel glauben müssen, wenn man annehmen wollte, dass die Familien A und $\mathrm{B}$ sich allein solcher Vorzüge zu erfreuen hätten, während die Familie C ewig in der Minorität zu bleiben hätte.

Der Wechsel des Glücks ist aber für die $3 \times 365$ Tage der Lebensperiode des Maikäfers nicht geringer als die eines Menschenalters von $30 \times 365$ Tagen, resp. einer menschlichen Generation im Durchschnitt, denn nicht nur alle 3 Jahre kann sein Geschlecht verderben, sondern jedes Jahr drohen ihm die feindseligen Elemente in Gestalt von Frost, Hitze, Wasser, Maulwürfe und Parasiten u. s. w.

Selbst eine zahlreiche Familie kann ihren Feinden unterliegen, resp. auf den ,eisernen Bestand“ reducirt werden. Da diese Umstände allerorts mehr oder weniger wechselnd sind, so unterliegt also nicht nur Familie A und B, sondern auch C denselben bald zu ihren Gunsten, bald zu ihren Nachtheilen. Ebenso halten diese Umstände, die $\nabla 0 \mathrm{r}-$ wi egend (zum Heil des Menschen) ungünstige sind, eine Vermehrung ad infinitum im Zaum und verhindern einen Weltuntergang in Folge der Maikäfer!

Meiner Ansicht nach kann also das regelmässige Auftreten der 3resp. 4 jährigen Periode, mit anderen. Worten die regelmässig im 4. Jahre sich wiederholenden Maikäferflugjahre nicht als allgemeine Regel, soudern als $\mathrm{A}$ us nahme für eine bestimmte Lokalität bezeichnet werden, die ihren Grund hat:

1. in relativ sehr geringem eisernem Bestand dieser Lokalität, und

2. in, dem Allgemeingedeihen des Maikäfers ungünstigen Verhältnissen, so dass ein einmaliges, ausnahmsweise bes onders g ü n stig e s Zusammentreffen der Umstände in einem bestimmten Jahr, auf eine längere Reihe von Jahren hinaus das Ueberwiegen dieser Familie, also das regelmässig im 4. Jahr erscheinende Flugjahr dieser einen Familie A zur Folge hat, während der ,eiserne Bestand" der B- und C-Familien aus dem 2. und 3. Jahr durch die, im Allgemeinen an diesem Orte, vorwiegend ungünstigen Verhältnisse auf ihr Minimum beschränkt geblieben sind. 
Wo hingegen diese Bedingungen lokal öfter günstig sind, wird eine Unregelmässigkeit statthaben, die darin besteht, dass bald Familie A, bald B oder C ein- oder zweimal hintereinander ein Maikäferjahr repräsentiren.

Wenn es einen Ort gäbe, wo diese Bedingungen immer giinstig sind, dann würde und müsste es nothwendig jedes Jahr Maikäfer in Masse geben.

Hier in unserem Theil der Wetteran, Echzell und Nachbarschaft war \%. B. das Jahr 1865 Maikäferjahr mit kolossaler Verheerung in Garten, Feld, Wiesen und Wald (in den Larvenjahren dieser Familie 1866 und 67 wurden ganze Wiesenflächen durch die Engerlinge zerstört); 1868 erschien die Familie wieder als Maikäfer, aber lange nicht so zalılreich als 1865. Dann war anno 70 ein Maikäferjahr geringer Art, also waren dies entweder die Nachkommen des ,eisernen Bestandes“" aus dem nicht als Maikäferjahr sich auszeichnenden Jahre 1867, die aber durch die günstigen Umstände von 1868 ebenso begünstigt wurden wie die 65er und infolge dessen 1870 sich als Maikäferjahr bemerkbar machte. Im Jahre 1871 gab es wieder ziemlich Maikäfer, also war dies die dritte Generation der Familie von 1865.

Seit 1871 bis heute, Juli 1876, haben wir dagegen im Frühjahr kaum einen Maikäfer zu sehen bekommen, Engerlingverwüstungen haben auch scit 1867 keine mehr bemerkbar stattgefunden.

Ich erinnere mich ferntr ganz gut, dass in den Jahren, wo wir sehr starke Flugjahre hatten, ich von der Nachbarschaft auf 1-2 Stunden öfter gehört habe, dass dort gar keine Maikäfer waren; umgekehrt habe 'ich von starkem Maikäferfrass in Gärten etc. in der Nachbarschaft gehört, während wir gar keine Maikäfer hatten. Im Jahre 1865 war die Verbreitung eine weit ausgedehnte, wenigstens 2 Meilen von hier gleichartig auftrétende." (v. Harnier.)

Ich, für meinen Theil, kann den Ansichten des Hrn. v. Harnier nur beistimmen, dass Maikäferflugjahre, die sich an bestimmten Lokalitäten scheinbar regelmässig wiederholen, nur die Ausnahme bilden und dass sich allgemein durchgefülrte Regeln nicht aufstellen lassen. Eine Zeit lang kann eine solche Regelmässigkeit, die man als Gesetz annehmen könnte, eintreten, aber zum Glück für den Menschen sind häufig auftretende schädliche Thiere, gerade wenn sie in Massen erscheinen, so vielen Unbillen der Natur ausgesetzt, dass dadurch die richtige Schranke schon eingehalten wird. Aber gerade in diesem Dazwischentreten feindlicher Elemente liegt auch der Feind der Regelmässigkrit. (v. Heyden.) 


\section{Rhizotrogus Latreille.}

1. (Amphimallus Muls.) solstitialis L. - Im ganzen Gebiet auf Grasplätzen g.; bekannt unter dem Namen „Junikäfer“. — Nach B. Ảbends besonders an Robinien schwärmend. - Wlb. Dlb. h. (Sch.) - Mz. (S.) -

2. (A.) ochraceus Knoch. - Bei Fr. von M. S. einzeln gef.; dreimal von $\mathrm{Hg}$. -

3. (A.) ruficornis F. - L. H. fand ein Ex. auf der Landstrasse zwischen Fr. und Höchst und einmal bei Nenenhain über Soden unter einem Stein. -

4. R. aestivus Oliv. - Eichberg im Rheingau (Richter). Auf dem Niederwald; bei Bingen auf den Bergen; Mo. am Rhein im Sand unter Steinen im April. - Fr. von C. H. und Hg. gef. Wsb. (K.) - Bei Bergen in April von B. gef. - Mz. Mo. (S.) Wlb. und Dlb. h. (Sch.) - G. (L.) - Schierstein am Rhein 24. April 1851 (K.). -

5. R. marginipes MIs. = foveolatus Bach. - Cronthal $=5$ unter Steinen, ebenso im Juli 1871. - Zwei Weibchen in Neuenhain bei Soden. - Fr. - Wsb. (K.) - Mehrmal bei Wlb. beide Geschlechter unter Steinen gef. (Sch.) -

6. R. aequinoctialis Hbst. - Fr. von B. gef. - Sehr dunkel pechbraun. -

\section{Serica Mac Leay.}

1. S. holosericea Scop. = variabilis F. - In Sandgegenden unter Steinen h. im Herbst. - Mo. (S.) - Von K. 20. Juli 1851 gef. -

2. S. brunnea L. - Fr. in Sandgegenden viel seltener. - Im Rödelheimer Wald öfter in Spimnenweben gef., worin sie Nachts beim Schwärmen hängen blieb. - Mo. (S., K.) - Wlb. (Sch.) -

\section{Homaloplia Stephens.}

1. H. ruricola $\mathbf{F}$. $>6$ auf der Bieberer Höhe s. - Auf Wiesen bei Griesheim am Main $=6$. - 


\section{Hoplia Illiger.}

1. H. philanthus Sulz. = argentea OI. - Auf Wiesen im Hengster bei Offenbach $>6$. - Bei Friedberg von Renner gef. in Sammlung v. Heyden. - Fr. (Hg.) - Mo. (S., K.) - Wsb. auf Weiden an der Wellritz 14. Juli (K.). -

2. H. praticola Duft. - Früher von C. H. bei Fr. drei Stücke; später auch von Hg. und B. s. gef. - Wlb. (Sch.) -

3. H. farinosa L. = squamosa F. - Früher von C. H. oft auf Wiesen hinter den Kettenhöfen, von neueren Frankfurter Sammlern nicht mehr gef. - Friedberg (Renner). -

4. H. graminicola F. - Diese kleine Art ist nach Sch. bei Dlh. h. auf Wiesen an Grashalmen. -

\section{G. RUTELINI.}

\section{Anisoplia Laporte.}

1. A. segetum Hbst. = fruticola F. - Von M. S. einigemal bei Fr., auch von B. nicht gerade h. gef. - Mo. (S.) -

2. A. agricola F. - Bei Mo. an Gras anhängend von S., St., Hg. und K. 2.-19. Juni gef. - Fr, (Hg.) -

\section{Phyllopertha Kirby.}

1. P. horticola F. - n. s. bei Fr. auf Blüthen in Wiesen, in Gärten auf Blumen, im Juni. - Wsb., Mo., Wlb. und Had. (K.) Nach B. besonders auf Rosen und Brombeeren im Wald. - Bei Dlb. und Wlb. auf Haselstauden gef. (Sch.) - Mz. Mo. (S.) -

\section{Anomala Samouelle.}

1. A. aena Deg. = Frischii F. - Im Gebiet auf Sandboden h. bei Isenburg im Fr. Wald. - Mo. $>8$. - Mit braunen und mit blaugrünen Flügeldecken. - Mo. auf Kief rnn 20. Juli 1851 (K.). Auf Weiden bei Königstein zweimal von B. gef. - Fehlt nach Sch. bri Dlb. und Wlb. - G. (L.) - 


\section{H. CETONIINI.}

\section{Ox yth yr e i Mulsant.}

1. O. stictica L. - Im ganzen Gebiet g. auf Blüthen. - Im Nai in Bonames eiust den Aepfelblüthen durch ihre Menge schädlich. - Fr. h. in Gärten, schon im April. - Zwischen Walluf und Eltville 1871 s. h. (K.) - Bei Wlb. h., besonders auf Campanula glomerata und Leontodon taraxacum von Sch. gef. - Mz. MIo. (S.) -

\section{Tropinota Mulsant.}

1. T. hirtella L. - s. h. im Gebiet auf den Blüthen des Lömenzahn (Leontodon taraxacum) im Mai, Mo. 30. Juni (K.). - Fr. (B.) Nach Sch. bei Wlb. einer der häufigsten Käfer. - Mr. Mo. (S.) G. (L.) -

\section{Cetonia Fabricius.}

1. C. aeruginosa Drury $=$ speciosissima Scop. $-1830 \mathrm{im}$ Fr. Wald in alten Eichen, in welchen sich Wildtanben-Nester befanden, von C. H. gesammelt, s. s.; im Januar war der Käfer schon ganz entwickelt, aber noch weich in einem braunen eirunden Cocon aus Holzerde und Sand eingeschlossen. Im Rebstockwald bei Fr. Herbst 1875 von Hg. einmal gef. - Im Offenbacher Wald mehrmals gef. (Samml. des Offenbacher Ver. f. Naturk.) und im Taunus im Gensingau bei Eppstein ron B. gef. - Fehlt nach Sch. bei Dlb. und Wlb. - Bei Mro. an żwei alten Eichen in der Nähe des Rheins jedes Jahr gef. (S.) - Auf dem Geisberg bei Wsb. voul Dr. v. Canstein gef. -

2. C. affinis Andersch. - Je eimmal im Fr. Wald bei Niederrad ron C. H. und Hg. gef. -

3. C. marmarota F. - Im Fr. Wald im Mulm alter Eichen s. - Wsb. (K.) - Fr. auch auf Blumen (B.). - Nach Sch. früher h. bei Dlb. und Wlb. an dem Saft alter Eichen, jetzt wohl ausgegangen wegen des Weghanens aller alten Eichen und Entfernung der Strünke. - An Weiden unterhalb, Mainz angeflogen, Saft saugend, gef. (S.) -

4. C. floricola Hbst. = aenea Gyll. - Im Gebiet auf Waldwiesen n. s., besonders auf Spiraea ulmaria, Fr. Wald im Jumi. Wsb. (K.) - Bei Wlb. h. auf Sambucus nigra, auch bei Dlb. (Sch.) - Mz. Mo. (S.) - G. (L.) - Bockenheim in alten Weiden 2. Juli 1876.

- Wsb. 17. Juni in Ameisenhanfen (K.). - 
Var. obscura Andersch (mit schwächerer weissgesprenkelter Zeichnung) von Anton Schmid bei Fr. gef. - Wsb. (K.) -

5. C. aurata L. - Im ganzen Gebiet g. in Gärten, besonders in Rosen; $>6$ in Begattung. - In Königstein auch s. h: auf Brombeerblüthen. April bis September. - Bei Dlb. und Wlb. vom Frühjahr bis Ende des Sommers s. h. (Sch.) - Mz. MIo. (S.) - G. (L.) -

\section{VALGINI. \\ V a 1 g u s Scriba.}

1. V. hemipterus L. - Im ganzen Gebiet 1. s. in alten Bäumen, auf Wegen laufend und in Blüthen. - Fr. im Mai an Buchenstämmen. Bei Dlb. und Wlb. h. an Apfelbäumen (Sch.). — G. (L.) - Had., Wsb., Schierstein 18. Mai, Mo. 23. Mai (K.). -

\section{K. TRICHIINI.}

\section{Osmoderma Lepelletier.}

1. O. eremita L. - Im ganzen Gebiet in aiten Bäumew. - Fr. - Am Grindbrumnen an den alten Linden von Hg. h. gef. - Wsb. (K.) - An Eichen bei Schwanheim 1874 einmal von B. gef. - Bei Wlb. h. an Linden, daselbst auch seine Larre (Sch.). -

\section{Gnorimus Serrille.}

1. G. variabilis L. - Im Fr. Gebiet s. - Im Schwanheimer Wald in einer alten Eiche ron $\nabla$. Twardorski gef.; auch ron Gerlach und B. gesammelt. -

2. G. nobilis L. - Im Fr. Wald auf Blüthen von Spiraea ulmaria und Rubus im Juni n. s. - Wsb. (K.) - Nach B. gelegentlich im Tauuus s. h. auf Blüthen. - Bei Wlb. auf Rosen mit Cetonia aurata, auch bei Dlb. (Sch.) - Im Wald hinter $\mathrm{M}$ o. (S.) -

\section{Trichins Fabricius.}

1. T. fasciatus L. - Fr. h. auf Blüthen in Wald und Garten. - Im Taunus in der hohen Mark im Juli h. - Wsb. (K.) - Bei Dlb. und Wlb. auf Blüthen s. h., z. B. Leontodon, Ligustrum, Sambucus, Schirmpflanzen (Sch.). - G. (L.) - 
2. T. abdominalis Menetr. = gallicus Muls. $-\mathrm{Fr}$. - Hofheim in Juni. Seltener, anch ron $\mathrm{Hg}$. wie die rorige Art bei Fr. gef. - Wsb. (K.) -

\section{BUPRESTIDAE.}

\section{Chalcophora Solier.}

1. C. Mariana L. - Schon rom alten Brahm bei Mz. gesammelt. - In Holzhơfen bei Mz. noch jetzt (S.). -

\section{Dicerca Eschscholtz.}

1. D. Berolinensis Herbst. - Im ganzen Gebiet an alten Buchen, z. B. im Fr. Wald n. s.; auch h. aus Buchenholz gezogen, als der fast zuletzt erscheinende Kăfer in der Holzkammer $>7$ und Angust. Neroberg bei Wsb. an Buchen im Juni (K.). - In den Holzhöfen bei Nz. (S.) - G. ein Stück 20, August 1858 (L.). -

2. D. Alni Fisch. - Ton C. H. $=8$ eimmal aus Fr. Waldholz erzogen. -

Poecilonota Eschscholtz.

1. P. conspersa Gyll. = variolosa Payk. - L. H. besitzt ein ron r. Trardowski in Fr. gefangenes Stück. - Von St. öfter erzogen aus Fr. Waldholz. -

2. (Lampra Sol.) rutilans F. - Bei Bockenheim rom rerstorhenen Lehrer Berntheusel gesammelt. [L. H. sah seiner Zeit das Exemplar.] -

[Ancylocheira punctata F. - Nach S. V. von C. H. bei Fr. gef.; ich kenne kein Exemplar daher.] -

\section{Eurythyrea Solier.}

1. E. scutellaris Ol. = carniolica Hbst. - Bei Bockenheim rom rerstorbenen Lehrer Berntheusel gef. - Mainkur (Heynemanu). Friedberg (Scriba). - Ueberall s. s. - 


\section{Phaenops Lacord.}

1. P. cyanea F. = tarda F. - Im Fr. Wald an Buchenholz angeflogen, anch an Kiefern (C. H.). - B. sammelte die Art am Lantersborn bei Offenbach. -

\section{Anthaxia Eschscholtz.}

1. A. Cichorii Oliv. - Bei Ems von C. H. gef. - Bei Wlb. 2 Exemplare grün und bronzefarben ron Sch. gef. -

2. A. umbellatarum $\mathbf{F}$. $=$ Millefolii F. - Bei Wsb. zmeimal gef. (K.) -

3. A. candens Panz. - Lebt an alten Kirschbäumen, fliegt in der Mittagshitze. - Schon ron C. H. 1823 in den Băumen zwischen Mainkur und Enkheim, ebenda später 28. April 1864 Ton St. und 1. Juni 1860 ron B. gef. - Ein Ex. auf den ehemaligen Zimmerwiesen bei Fr. auf Blumen ron C. H. gef., auch am Rande des Röderwäldchens in Kirschłăumen ron B. gesammelt. - NIz. (Bach). -

4. A. salicis F. - Fr. s. auf Wiesenblumen = 5. - Wsb. (K.) - Auf Weidenstrünken am Metzgerbruch 11. Juni 1860 ron B. gef. - G. (L.) - Wsb. an hölzernen Pfosten $>5$ auf dem Bleichplatz (K.). -

5. A. nitidula L. (Weib = laeta F. mit kupfergoldenem Halsschild). - Im ganzen Gebiet auf Wiesenblumen n. s.; bei Bergen im Mai, bei Soden und Cronberg im Juni und August. - 1875 zmischen Cronthal und Mammolshain ron B. gef. - Wlb. Dlb. (Sch.) - G. (L.) - Wsb. an der Platter Chaussee (K.). -

Var. cyanipennis Gory (mit riolettblauen Flitgeldecken) s. s. bei Fr. und Enkheim auf Tmbellen $<8$. - Wlb. Dlb. (Sch.) -

6. A. nitida Rossi. - Wlb. oztreimal (Sch.). - Ich besitze auch ein Stück aus Heidelberg. -

7. A. sepulchralis $\mathbf{F}$. = umbellatarum Ol. - Wsb. (K.) -

8. A. quadripunctata L. - Im Fr. Wald $=6$ auf Blumen in Kiefernmaldungen s. h.; auch aus dürrem Kiefernholz in Mrenge gezogen. - G. (L.) - Wlb. (Sch.) - Mo. im Wald (S.). -

Var. Godeti Lap. (mit schwachem Bronzeglanz) einmal bei Fr. ron C. H. gef. - Wlb. (Sch.) - 


\section{Ptosima Solier.}

1. P. undecimmaculata Hbst. = flavoguttata Illig. - Von Wagner unterhalb des Rüdesheimer Bergs, gegenüber von Bingen in Ulmen gef. - (Nach Bach bei Boppard in Prunus Mahaleb.) -

\section{Acmaeodera Eschscholtz.}

1. A. taeniata F. - In Sammlung v. Heyden befindet sich ein von $\mathrm{Dr}$. Zitz bei $\mathrm{Mz}$. gefangenes Exemplar. - Auch S. sammelte die Art dort. -

\section{Chrysobothrys Eschschoitz.}

1. C. affinis F. - Schon von Brahm aus Fr. erwähnt (als chrysostigma) ron Aspen im August s. - An altem Buchenholz im Fr. Wald $=6$ und $\mathrm{im}$ Lorsbacher Thal $=5$ je einmal von C. H. ges. Wsb. (K.) - An der Götheruhe im Fr. Wald n. s. s. und am Lautersborn bei Offenbach von B. gef. - Wlb. auf Zimmerplätzen nebst Larve (Sch.). -

\section{Coraebus Laporte.}

1. C. undatus F. - Im Fr. Wald, besonders an der Babenhänser Strasse öfter, aber stets einzeln auf Buchengestränch im Juni von uns gef. - Ein Stück von Brombeeren am Forsthans 1874 gestreift von B. - Nz. (Bach). -

\section{Agrilus Solier.}

1. A. biguttatus F. - Fr. in grosser Menge von L. H. und St. ans Waldholz gezogen. $-<6$ unter Eichenrinde, mit deren Puppen bei Falkenstein; bei Soden mit Puppen und Larven $<10$ von C. H. gesammelt. - Wlb. (Sch.) - Aus Rinde von dicken Eichenstrünken hinter der Walkmühle bei" Wsb. im April erzogen (K.). -

2. A. sexguttatus Hbst. - Von St. aus Waldholz erzogen. Bei Mo. an Pappeln von K. gef. - Fr. zweimal (B.). - Mz. Mo. (S.) -

3. A. sinuatus Oliv. - Bei Fr. von Jännicke, bei Bingen von Wagner in Birnbäumen, bei Friedberg von Fuhr gesammelt. — 2 Stück auf Weiden an der Grüneburg, einmal auf einem Kohlfeld bei Bornheim gef. von B. - 
4. A. subauratus Gebl. = coryli Ratzebg. - Auf Aspen an der Bieberer Höhe bei Offenbach $=7$ von L. H. und im Rothen Graben bei der Mainkur von Gremmers gef. - Friedberg (Scriba). — Einmal bei Fr. von B. gesammelt. -

5. A. tenuis Ratzbg. - An Eichenholz im Fr. Wald $>6$ von C. H. in Begattung gef. - Auch schon $=5$ aus Eichenholz entwickelt. - 6 Ex. von Fr. (B.) - Wlb. und Dlb. (Sch.) -

6. A. angustulus Illig. - Besonders auf Birken $=6$, Hainbuchen und Aspern. - Mo. - Ems. - Fr. von L. H. und n. s. von B. am 2. Juni 1860 gef. - Wlb. (Sch.) -

Dr. Böttg e r fand im Rothen Graben bei der Mainkur einen kleinen Agrilus von nur 3 Millim. Länge, den ich vor kurzem an Herrn Geheime Regierungsrath v. Kiesenwetter zur Begutachtung schickte. Derselbe schrieb mir darüber:....., ,bin ich zu der Ansicht gelangt, dass A. angustulus namentlich in der Grösse sehr variirt. Ich würde daher nicht anstehen, den Käfer für eine sehr kleine, vielleicht verkümmerte Form dieser Art zu betrachten, wenn ich ihn nicht für ein Männchen hielte, der schlanken Gestalt wegen, und wenn ihm nicht als Männchen die beiden Körnchen des ersten Hinterleibsringes fehlten. Nun scheint es mir aber sehr wahrscheinlich, dass diese Körnchen, die in dem Grade ihrer Entwicklung variiren, bei schwächlichen Exemplaren ganz verschwinden können und ich habe mich zu der Meinung entschlossen, auf Grund reichlichen Materials, die beiden mir nach einzelnen Ratzeburg'schen Exemplaren bekannt gewordenen Stïcke von rugicollis und scaberrimus für Weiber von angustulus und laticornis zu betrachten, beziehendlich was rugicollis betrifft, für verkümmerte Männchen von angustulus. Damit würde es übereinstimmen, wenn man $\mathrm{A}$. tenuis umgekehrt als sehr kräftig entwickelte Stücke von angustulus ansieht, was mir richtig scheint. -

Ich habe aus Rosen einmal eine Zahl sehr kleiner Exemplare von A. angustulus erhalten, die wohl, weil die Rosenstämmchen wenig Raum zum Ausbreiten der Frassgänge der Larven geboten hatten, so dürftig geblieben waren. Vielleicht hat etwas Aehnliches bei Ihrem Stück stattgefunden. Es wäre recht interessant, wenn man von diesen kleinen Agrilen mehr Material erlangte, um über diese Fragen, die jetzt im Grunde nur hypothetische Antworten zulassen, zu bestimmteren Ansichten zu gelangen. - Bis auf Weiteres entspricht es aber dem Stande unserer Artenkenntniss, Ihren Agrilus als verkümmerte Zwergform von Agr. rugicollis zu bestimmen." - 
7. A. olivicolor Ksw. = olivaceus Rtzb. - Auf Haseln $>6$ von C. H.; bei Fr. ron B. gef., n. s. am 3. und 9. Juni 1860. Wlb. (Sch.) -

8. A. graminis Lap. - Im Rothen Graben bei der Mainkur von C. H. $>7$ ron Eichen geklopft. -

9. A. litura Kiesw. - Von B. bei Fr. gef. (er hatte ihn seiner Zeit derasofasciatus benamnt). -

10. A. convexicollis Redtb. - Von B. bei Fr. ges. (1 Ex. in Sammlung r. Hejden), n. s. auf Eschen an der Gerbermühle 2. Juni 1860.-

11. A. caeruleus Rossi = cyanescens Rtzbg. - Bei Bingen ron Wag̀ner gef. (1 Stück in Sammlung r. Heyden). - Einmal ron B. am 1. Juni 1860 gef. bei Fr. und ebendaher von Mühlig 6 Stück gezogen. - Wlb. (Sch.) - G. (L.) -

12. A. Iaticornis Illig. - Auf Eichen im Gebiet im Juni und Juli n. s. im Fr. Wald und Soden. - Von B. h. von jungen Eichentrieben bei Fr. gestreift, z. B. im Goldsteinforst. -

13. A. Roberti Chevr. = pratensis Ratzbg. - Auf Aspen bei Fr. und No. mehrfach gef, auch von S. im Mai und Juni. - Von Aspenschossen h. am Goldstein 3. Juni 1860 von B. gestreift. - G. (L.) -

14. A. auricollis Ksw. - Auf Birken an der Bieberer Höhe und Mo. = 5 auf Pappeln gesammelt. - Wlb. (Sch.) -

15. A. betuleti Ratzbg. - Aus dürrem Fr. Waldholz $<6$ von C. H. erzogen. - Von B. 6 Stück bei Fr. gesammelt. - Wlb. (Sch.) -

16. A. viridis L. - Variirt in solchem Grade, dass man keine Form als Grundtypus annehmen kann.

Var. nocivus Ratzbg. = bicolor. Redtb. (grünlichblau). - Auf der Bieberer Höhe $=7$ an Birken. -

Var. linearis Panz (mit kupfer-röthlichem Halsschild). - Im Sodener Wald $<6$ an Salix caprea. -

Var. fagi Ratzbg. = quercinus Rdtb. (ganz kupferig). - Aus Fr. Waldholz im Juli s. h. erzogen. -

Var. ater Rdtb. (oben mattschwarz). - Einmal im Fr. Wald an aufgeschichtetem Kieferholz von C. H. gef. -

Sollte die verschiedene Farbe mit der Nahrung im verschiedenen Holze zusammenhängen!

17. A. Hyperici Creutz. - Bei Soden an dürren Stellen im Wald von C. H. einst auf Hypericum n. s. gef. - Im Wald hinter Mo. h. (S.) - 
18. A. cinctus Ol. - Von C. H. bei Ems auf Siartium scoparium in Anzahl gef. [Ich sammelte die Art ebenso in Portugal 1868.] —

19. A. aurichalceus Redtb. - Bei Hofheim, im Lorsbacher Thal und bei Falkenstein im Mai und Juni auf Himbeeren (Rubus idaeus) öfter gef. - B. fand 1875 ein Weibchen bei Fr. - Wlb. (Sch.) -

20. A. integerrimus Ratzb. = cupreus Redtb. - Auf Aspen bei Fr. $>$ 6. s. - Bei Ems von C. H. gesammelt, auch von Bach. G. (L.) - Wlb. (Sch.) -

\section{Cylindromorphus Kiesenwetter.}

1. C. filum Schh. - Von B. am Goldstein auf Wiesen il. S. gestreift 1860 .

\section{Aphanisticus Latreille.}

1. A. pusillus OI. - An der Försterwiese im Fr. Wald $>7$ von C. H. gesammelt. - Wsb. (K.) -

2. A. emarginatus F. - Auf den Wiesen bei Falkenstein ges. - Von B. an der Louisa und hinter dem Forsthaus nach Schwanheim zu 1874 mit dem Streifnetz gef. - Wlb. 3 Ex. auf Blüthen (Sch.). Mz. (Bach). - Mo. (S.) -

\section{Trachys Fabricius.}

1. T. minuta L. - Auf Weiden, besonders Salix caprea h. Die Entwickelung von C. H. genau beschrieben in Berl. Entom. Zeitschr. 1862, p. 61. - Dlb. Wlb. (Sch.) - Mo. (S.) -

2. T. pumila Illig. var. scrobiculata Marsl. - Von B. bei Fr. 1875 gef. (1 Ex. in Sammlung v. Heyden). -

3. T. troglodytes Schh. = aenea Mnhm. - Bei $\mathrm{ML}_{0}>5$ von C. H. und S. gesammelt, auch von K. (in seiner Sammlung als pygmaea). - Dlb. und Wlb. (Sch.) -

4. T. nana Payk. - Von S. bei Mz. gesammelt. - Von C. H. im Fr. Unterwald und bei Eppstein auf Geranium sanguineum. - Auch bei Mo. von L. H. am 7. Mai 1867 und $>8$ gef. - Wsb. (K.) Fr. einmal gef. (B.) - Dlb. Wlb. (Sch.) - 


\section{EUCNEMIDAE.}

Drapetes Redtenbacher.

1. D. mordelloides Host. = cinctus Panz. = equestris F. Von Jämnicke in Fr. Wald bei Schwanheim einmal gef. - Dlb. zweimal von Sch. gef. -

\section{Trixagus Kugel. = Throscus Latreille.}

1. T. dermestoides L. = adstrictor F. - Von C. H. bei Fr. $>6$ auf Salix caprea gef., die Blätter benagend. — Soden. — Schmitterhof bei G. - Mo. (S.) -

2. T. carinifrons Bonvoul. - Von C. H. im Juni an Dornzäunen bei Soden, von L. H. in Neuenhain bei Soden n. s. s. am Fusse alter Kastanien gesammelt. -

3. T. exul Bonvoul. - Einmal von C. H. bei Soden mit dem vorigen gef. -

[Das Vorkommen von elateroides Heer in S. V. bei Fr. ist falsch; die Stücke waren carinifrons.]

4. T. brevicollis Bonyoul. (Augen nicht gekielt, Stirn mit zwei Kielen, Zwischenräume der Decken mit einer Punktreihe.) - Wlb. einmal (Sch.). -

\section{Cerophytum Latreille.}

1. C. elateroides Latr. - Zuerst im Gebiet ron H. Scheidel im April 1852 auf der ehemaligen Zimmerwiese gesammelt. - L. H. fand in den Enkheimer Torfbrüchen am Fusse einer alten Weide 19. April 1856 ein Weibchen und Tags darauf an demselben Baume eine grössere Anzahl Männchen. Das Weibchen schnellt sich, auf den Rücken gelegt, nach Elateren-Art schwach in die Höhe, springt auch auf dem Bauch sitzend, selten aber weit (11 Linien). - Diese Beobachtung finde ich nirgends erwähnt. - 


\section{Melasis Olivier.}

1. M. buprestoides L. - Aus Fr. Waldholz (Buchen, aber auch Birken) n. s. von uns und St. erzogen. - Bei Dlb. s. h. unter Buchenrinde und in altem Buchenholz (Sch.). - G. (L.) -

\section{Tharops Laporte.}

1. T. melasoides Lap. - Wie die, vorige Gattung bei Fr, aus Buchenholz h. erzogen. Auch von B. 1874 an der Louisa an einer Buche gef. -

\section{Dromaeolus Kiesenwetter.}

1. D. barnabita Villa $=$ Heydeni Bach $=$ currax Heyd. i. I. - In Italien entdeckt im Jahre 1831; von C. H. für Deutschland nẹu aufgefunden. - Im Juni aus dürrem Buchenholz aus dem Fr. Wald einmal öfter, später von St. in grösserer Anzahl erzogen. - Das Thierchen läuft sehr rasch, schnellt sich in die Höhe und fliegt sehr schnell weg. -

\section{Eucnemis Ahrens.}

1. E. capucinus Ahr. - An alten dürren Bäumen: Buchen, Linden, Pappeln n. s. s. im Mai und Juni, z. B. in den alten Linden im Zoologischen Garten. - In Apfelholz an der Louisa (Fr. Wald) 21. April 1861 von B. gef. - Dlh. h. in alten Pappeln (Sch.). - Ebenso bei Mz. längs des Rheins (S.) und Wsb. (K.) -

\section{Microrhagus Eschscholtz.}

1. M. nov. spec. - Von St. aus Fr. Waldholz eine Anzahl Exemplare 1870 erzogen. - 


\section{ELATERIDAE.}

\section{Adelocera Latreille.}

1. A. quercea Hbst. = varia Oliv. - Bei Soden im Taunus und bei Fr. im Schwanheimer Wald unter Eichenrinde $=5$ von C. H. und M. S. gesammelt. — Neroberg bei Wsb. an Eichen 9. Juni (K.). -

\section{Lacon Laporte.}

1. L. murinus L. - Im Gebiet auf Wegen unter Steinen s. h. - Fr. - Wlb. Dlb. (Sch.) - Mz. (S.) - G. (L.) -

\section{Elater Linné.}

1. E. sanguineus L. - Lebt in Kiefernstöcken im Fr. Wald und im Taunus bei Königstein vom April bis Juni. - Dlb. Wlb. (Sch.) -

2. E. lythropterus Germ. - Im Taunus bei Falkenstein und Soden unter Eichenrinde. - Auch in Buchenstrünken von B. gesammelt. - Wlb. (Sch.) - Ernsthausen in Nassau (Dörr bei K.). - Wsb. Baumstrunk unter der Rontmauer 31. Nai und Pappelstrunk an der Wellritzmühle 18. April (K.). -

3. E. sanguinolentus Schrk. - Im Taunus von L. H. gef. 1 Stück bei H'r. 25. April 1860 von B. gef. - Wlb. einmal (Sch.). -

Var. ephippium F. - Bei Fr. an Kettenhof früher an den alten Weiden. - Wlb. (Sch.) — Im Fr. Wald am Sandhof von Jännicke und Gräf öfter gef. - G. (L.) -

4. E. Pomonae Steph. - Von St. aus Fr. Waldholz erzogen. -

5. E. ferrugatus Lac. = Pomorum Hbst. - Bei Fr. n. s. in Holz ron Weiden und Pappeln; am Feldberg in Buchenstümpfen und bei der Mainkur in Hainbuchen. - Dib. Wlb. (Sch.) - G. (L.) -

6. E. erubescens Esch. = crocatus Geoffr. - Aus Fr. Buchenwaldholz erzogen. - Auf der ehemaligen Bornheimer Haide in Pappeln. - Auf Weiden h. von B. gef. - Wsb. (K.) - Dlb. an Pappeln (Sch.). - G. (L.) - 
7. E. balteatus L. - Fr. Wald an Kiefern bei Schwanheim n. s. auch auf blühenden Kiefern von B. gef. - Mo. (S.) -

8. E. elongatulus $\mathbf{F}$. - Wlb. einmal von Suh. gef. -

9. E. elegantulus Schh. - Im Fr. Gebiet aus altem Weidenholz herausgeschnitten, z. B. schon im Februar bei Bockenheim, Rödelheimer Damm. Auch von Jännicke und St. gef. - Mo. (S.) -

10. E. erythrogonus Müll. - Von Scheidel am 17. October im Fr. Wald an Buchen gef. -

11. E. Megerlei Lacord. - An altem Eichenholz s. s. im Fr. Wald von L. H. und auf dem Niederwald bei Rüdesheim im April von C. H. gef. -

12. E. nigerrimus Lacord. = obsidianus Germ. - Einmal von C. H. bei Soden an einer alten Eiche gef. $=6$. - Wlb. (Sch.) -

13. E. nigrinus Hbst. - Ein von v. Twardowski im Schwanheimer Wald gefundenes (30. Juni 1860) Exemplar in Sammlung v. Heyden. -

\section{Ischnodes Germar.}

1. I. sanguinicollis Panz. - Grosse Seltenheit. Von C. H. zweimal an einer alten Buche $=6$. und einmal von B. bei Fr. gef. -

\section{Megapenthes Kiesenwetter.}

1. M. tibialis Boisd. = subcarinatus Germ. - Am 20. Mai 1856 von L. H. an alten Eichen bei Königstein gesammelt, von C. H. aus dürrem Waldholz erzogen. erzogen. -

2. M. lugens Redtb. - Von C. H. einzeln aus Fr. Waldholz

\section{Cryptohypnus Eschscholtz.}

1. C. pulchellus L. - Am Ufer der Lahn im nassen Sand bei Ems von C. H. gef., auch bei Fr. - Mo. (S.) -

2. C. sabulicola Bohem. - Früher mit dem vorigen vermengt, aber oft noch einmal so gross, Hinterecken des Halsschilds mit kürzerem Längskiel, stärker gestreifte Flügeldecken. - Lebt auf ganz trockenen Stellen im Mai bei Fr. in Sandgruben. - Auch von B. auf Sandflächen bei Fr. s. am 25. Mai 1860 gef. - Mo. und Gonsenheim (S.). -

3. C. quadripustulatus F. - Wlb. zweimal (Sch.). - 
4. C. tetragraphus Germ. - Bei Ems an trockenen Kiesstellen der Lahn ron C. H., bei Friedberg ron Fuhr gef. - Bei Fr. nach B. n. S. - Wlb. (Sch.) - G. (L.) -

Var. dermestoides Hbst. - Bai Friedberg von Fuhr gesammelt. - G. (L.) -

5. C. meridionalis Cast. $=$ C. lapidicola Germ. - Bei Ems mit C. tetragr. von C. H. im Juli gef. -

6. C. minutissimus Germ. - Bei Fr. ron Haselgesträuch geklopft. - Wlb. Dlb. h. (Sch.) - Auf dem Neroberg bei Wsb. auf jungen Birken 25. Juni (K.). -

\section{Cardiophorns Eschscholtz.}

1. C. thoracicus L. - Im Fr. Wald, z. B. bei Schwanheim, n. s. von Nadelholz geklopft. - In morschem Eichenholz bei Hofheim 7. April 1861 ron B. gef. - Ernsthausen in Nassau (Dörr bei K.). -

2. C. ruficollis L. - An denselben Orten wie der vorige, auch öfter bei Formica rufa gef, doch wohl nur zufällig mit den Nadeln in das Nest gelangt. - Am 18. und 24. April 1860 von B. gef. -

3. C. rufipes Fourcr. - Bei Königstein mnter Eichenrinde von C. H. und hei Bingen von L. H. gef. -

4. C. nigerrimus Er. - Von C. H. einmal bei Fr., 2 Stück bei Fr. 22. Nai 1860 von B. gef. -

5. C. musculus Er. - Von C. H. $<7$, ans faulem Holzmehl ans dem Fr. Wald erzogen. Einmal am 19. Mai 1860 bei Fr. von B. gef. -

6. C. asellus Er. - Bei Fr. von L. H. und bei Bürgel am Mainufer $>4$ ron C. H. gesammelt.

7. C. ebeninus Germ. - Am Fr. Forsthaus einmal von C. H. $<12$. unter Rinde gef. -

8. C. cinereus Hbst. - Bei Fr. auf Waldwiesen n. s. im Frühjahr. - Oefter, so am 14. Mai 1860, von B. mit dem Streifnetz gef. -

9. C. equiseti Hbst. - Bei Ho. am Rande der grossen Sandflächen auf Blumen $>4$ einigemal von S. und L. H. gesammelt. -

Melanotus Eschscholtz.

1. M. punctolineatus Pelerin = niger F. - Fr. s. im Mai. -

2. M. brunnipes Germ. - Fr. Wald $=6 .-$ Friedberg (Renner). - 
3. M. castanipes Payk. - Ein Pärchen 25. Mai 1861 von $T_{\text {t. }}$. bei G. gef. -

4. M. rufipes Hbst. - Fr. n. s. - Niederwald bei Rüdesheim von C. H. gef. - Wlb. (Sch.) - G. (L.) -

\section{Limonius Eschscholtz.}

1. L. violaceus Müll. - Ein Exemplar dieses seltenen Thieres fand C. H. vor langen Jahren an einer Eiche an der Babenhäuser Chaussee im Fr. Wald. -

2. L. pilosus Leske = nigripes Gyll. -- Im Geiviet auf Wiesen s. h. - Fr. - Bieberer Höhe, Cronthal, Elörsheim, Ems. - Von B. = 5. g(f. - Wlb. (Sih.) - Mz. Mo. (S) -

3. L. aeruginosus 0 I. = cylindricus Gyll. - Fr. z. s. - Wie der vorige (B.). - Mz. Mo. (S.) - G. (L.) -

4. L. minutus L. - Auf Kiefern im Fr. Wald und bei Soden. - Fr. h. von B. gef. -

5. L. parvulus Panz. = mus Illig. - Fr. Wald. - Johannisberg bei Friedberg. — Wlb. (Sch.) -

6. L. lythrodes Germ. - Fr. s. von C. H. gesammelt. -

7. (Pheletes Kiesw.) aeneoniger Deg. = Bructeri F. - Ein Gebirgsthier. - Im Wald von Königstein, Falkenstein im April und Mai; am grossen Feldberg $<6$ gef. - Wlb. (Sch.) -

\section{Athous Eschscholtz.}

1. A. niger $\mathbf{L}$. = aterrimus $\mathbf{F}_{\text {. }}=$ hirtus Hbst. - Im (rebiet h. - Fr. - Sodun. - Wlb. Dlb. (Sch.) - Mz. (S.) - G. (L.) -

Var. scrutator Hbst. (mit braunen Flïgeldecken) sehr einzeln. bei Fr. von M. S. gef., 2 Stück von B. --

2. A. mutilatus Roshr. = foveolatus Hampe. - Aus dürrem Buchenholz aus dem Fr. Wald $>5$ zweimal erzogen voì C. H. -

3. A. haemorrhoidalis F. - Im ganzen Gebiet g. - Fr. Im Hengster bei Offenbach. - Wlb. (Sch.) - Mz. (S.) -

4. A. vittatus F. - Variirt in der Färbung von einfarbigen gelbbraunen Fligeldecken, mit schwarzer Naht und Randbinde bis ganz schwarzbrauner Oberseite. Im Gehiet s. h. - Fr. - Falkenstein, Soden. 
WIb. (Sch.) - Die Weil, chen lehen rarsteckter und werden, wie überhatupt in dieser Gattung, seltener gef. - Sie sind breiter und gleichen oft dom Mämmchen fast gar nicht in der Crestalt. -

5. A. Iongicollis OI. - Das Männchen n. s. - Fr. - Hofheim. - Weihchen besitze ich nicht aus dem Gebiet. - Wlb. (Sch.) G. (L.) -

6. A. subfuscus Müll. $=$ analis F. - Im Fr. Gebiet n. s. Von B. = 5. gef. - G. (L.) -

7. A. Zebei Bach = biformis Redtb. = leucophaeus Ksw. - Wih. (Sch.) -

\section{Corymbites Latreille.}

1. C. pectinicornis L. - Gebirgsthier. - Taunus bei Cronthal $=5$. - Wlb. Dlb. (Sch.) - Im Wald hinter Mo. (S.) -

2. C. cupreus F. (die vordere Flügeldeckenhälfte gelbbraun). Am Feldberg $<7$. s. -

Var. aeruginosus F. (ganz grüne Flügeldecken) viel häufiger wie die Stammart. Im Taunus n. s., z. B. am Feldberg 12. Juni 1856. Dlb. (Sch.) -

3. C. Heyeri Saxes. - Fin Gebirgsthier, seither meist aus dem Harz bekannt. - Sch. fand die Art bei Dlb. -

4. C. castaneus L. - Bei Mo. von v. Twardowski gesammelt. Dlb. (Sch.) - Von Scheidel im Taunus gef. -

5. C. purpureus Poda $=$ haematodes F. - Im Taunus n. s. - Königstein. - Frirdberg (Renner). - Bei Lorsbach von B. 1872 h. gef. -.. Wlb. (Sch.) - G. (L.) -

6. (Actenicerus Ksw.) sjaelandicus Müll. = tesselatus F. Auf den Wiesen bei Falkenstein $=5$. - Hengster bei Offenbach. Auf der Königswiese im Fr. Wald von B. h. gef. - Wlb. (Sch.) G. (L.) - Ermstlıausen in Nassau (Dörr bei $\mathrm{K}_{\text {.) }}$ ).

Var. assimilis Gyll. (Flügeldecken gleichmässig, nicht scheckig: wie bei der Stammart, behaart). - Mit dem vorigen bei Soden auf Waldwiesen. - Fr. 1 Stück (B.). -

7. (Orithales Ksw.) serraticornis Gyll. - L. H. fand 2 Weibchen (= longulus Gyll.) bei Fr. -

8. (Diacanthus Latr.) impressus F. -- Am Gimbacher Hof am Fusse des Staufen 2. April 1876 von L. H. gef, auch sonst im Taunus. - 
9. (D.) nigricornis Panz. = metallicus Payk. - Von C. H. einmal am Feldberg $<6$. gef. - Einmal am 19. Mai 1860 bei Fr. von B. gef. -

10. (D.) aeneus L. - Oberseite grün oder dunkelbronze, Beine roth. - In der Ebene und im Gebirg s. h. - Fr. auf Wegen in den Feldern oder unter Steinen. - Wlb. Dlb. (Sch.) - Mz. (S.) - G. (L.) -

Var. aeneus 0l. (Oberseite violett, Beine roth) mit der Stammart s. -

Var. nitens Scop. (wie die Stammart, aber Beine schwarz). Fr. Wald, Schwanheim, Königstein im April. - G. (L.) -

Var. germanus L. (wie Var. aenens Ol., aber Beine schwarz). Fr. seltener = 5. - Mz. (S.) -

11. (D.) latus F. - Fr. in der Ebene unter Steinen n. s., auch auf Feldwegen. - Von 29. April bis 16. Mai 1860 von B. bei Fr. gef. - Wlb. Dlb. (Sch.) - Mz. (S.) -

Var. gravidus Germ. (glänzender, sparsamer punktirt). - Bei Fr. und im kleinen Feldbergthal $=6$. gesammelt. -

Var. milo Germ. (kleiner, dichter punktirt). - L. H. fand diest Form bei Offenbach. -

12. (D.) cruciatus F. - Im Fr. Wald an Sandstellen, z. B. am Sandhof an Waldrande unter Steinen n. s. s. im April und Mai. Auf jungen Buchen 12. Mai 1860 von B. bei Fr. gesammelt. - Mz. Mo. (S.) -

13. (D.) bipustulatus L. - Einzeln im Fr. Wald unter Eichenrinde im Frühjahr; auch unter Apfelrinde von uns gef. - Wlb. (Sch.) - 2 Stück auf jungem Waizen am Sandhof, 1 Stück am Königsbrunnen von Roggen abgestreift (B.). - G. (L.) -

14. (Liotrichus Kiesw.) angustulus Kiesw. - Wlb. (Sch.) -

[(Liotrichus Ksw.) affinis Payk. soll nach Scriba im Taunus vorkommen, ich kenne ihn nicht aus unserer Gegend.]

15. (Tactocomus Ksw.) tesselatus L. = holosericeus Oliv. - Im Gebiet h. - Fr. - Hofleim im Juni auf Wiesenblumen. Wlb. (Sch.) - G. (L.) -

16. (Hypoganus Ksw.) cinctus Payk. - In alten Weiden im Gebiet n. s., meist in Winter tief im Holz gef., am Hellerhof, bei Bockenheim und Ginheim. - Voun B. anf der früheren Ziminerwiese vom 18. -23. Mai 1860 in Weiden gef. - Mo. (S.) - 


\section{Ludius Latreille.}

1. L. ferrugineus L. - Am Fusse von alten Weiden zur Zeit der Heuernte von M. S., Gräf und St. s. gesammelt, besonders an den Rödelheimer Dämmen. - Bei Wlb. zweimal von Sch. gef. Mo. (S.) -

Var. occitanicus Villers (mit schwarzem Halsschild). - St. fand bei Soden und C. H. je 1 Ex. dieser seltenen Varietät bei Ems. -

\section{Agriotes Eschschoitz.}

1. A. pilosellus Schh. = pilosus Panz. - Im Gebiet n. s. Falkenstein, kleine Feldbergthal, Rüdesheim, Eins, Soden im Mai und Juni. - Wlb. (Sch.) - Mz. (S.) - G. (L.) -

2. A. ustulatus Schall. - Selhr veränđerlich in der Farbe; die Flügeldecken meist gelb, ferner gelb mit brauner Spitze (= sputator Redtb.) und ganz brann. - Im Gebiet s. h. - Fr. - Soden, - Feldberg. - Wlb. h. (Sch.) - Nz. (S.) -

3. A. sputator L. = graminicola Redtb. - Im ganzen Gebiet h. - Fr. - Tamuns. - Auch in Naingenist 1. April 1860 von B. gef. - Wlb. (Sch.) - Mr. (S.) _- Münster bei Soden 2. April 1876 (B.). -

4. A. lineatus $\mathrm{L}$. = segetis Bjerkander. - Im ganzen Gebiet g. und die Larve oft dem Getreide durch Abfressen der Wurzeln schädlich. - Fr. h. inuf Feldwegen. - Wlb. (Sch.) - Mz. (S.) - G. (L.) -

5. A. obscurus $\mathbf{L}$. = variabilis F. - Bei Fr. seltener. Enkheimer Torfbrüche im April an Gras. - Wlb. (Sch.) - G. (L.) -

6. A. aterrimus L. - Im Fr. Wald z. s. - Von B. z. s. $=5$. gef. - Wlb. (Sch.) -

7. A. sobrinus Ksw. = pallidulus Redtb. - Fr. 7. s. Wsb. (Bach). -

8. A. pallidulus Illig. = umbrinus Germ. - Fr. Wald. Feldberg. - L Lrsbacher Thal. - Wlb. (Sch.) -

9. A. gallicus Lap. - Fr. s. - Soden. - Ems. - Wlb. (Sch.) -

10. A. picipennis Bach = styriacus Redtb. - Fr. s. $\mathrm{inn}$ C. H. gesammelt. - Wlb. (Sch.) - 
Sericus Eschsch. $=$ Sericosomms Stephens.

1. S. brunneus L. (Mann = fugax F.) - Am Feldberg $<6$. s. von C. H. gesammelt. - Wlb. beide Geschlechter s. (Sch.) - G. (L.) -

2. (Dolopius Esch.) marginatus L. - Fr. h. - Cronberg. Wlb. s. h. (Sch.) - Mz. (S.) - G. (L.) -

\section{Synaptus Eschsch. = Ctenonychus Stephens.}

1. S. filiformis F. - Fr. n. s. - Soden $>$ 5. auf Blüthen. Dlb. Wlb. (Sch.) - Mz. (S.) - G. (L.) -

\section{Arrastus Eschscholtz.}

1. A. limbatus $\mathbf{F}$. $=$ pusillus $\mathbf{F}$. - Fr. n. s. - Wlb. (Sch.) - Mr. (S.) -

2. A. axillaris Er. - Bei Fr. h. von B. gef. -

3. A. pallens Er. - Fr. - Mainkur < 8. - Wlb. h. (Sch.) -

4. A. lacertosus Er. - Einmal bei Fr. von B. gef. -

5. A. humilis Er. - Fr. - Rüdesheim. - Wlb. h. (Sch.) -

\section{Leptruroilles Hbst. = Campylus Fischer.}

1. L. rubens Pill. et Mitt. = denticollis F. - Am Altkönig $<6$. von Eichen geklopft. - Am Königsbrünnchen im Fr. Wald n. h. von B. gestreift. - Wsb. (Bach). - Dlb. Wlb. (Sch.) -

2. L. linearis L. - Fr. - Wsb. - Feldberg. - Dlb. Wlb. (Sch.) - G. (L.) - Obersaustiege im Fr. Wald = 5. -

Var. mesomelas L. (Form des Weilchens mit schwarzen rothgerandeten Flügeldecken) fand L. einmal bei G. — [Auf dem hohen Vogelsherg fand L. H. diese Varietät öfter.] 


\section{DERMESTIDAE.}

\section{Dermestes Linné.}

1. D. vulpinus F. - Durch Thierhäutehandel über die ganze Erde verbreitet. - Fr. einmal von C. H. in der Stadt gef. - Mr. (S.) - -

2. D. Frischii Kugel. - Wie die vorige Art zweimal gef. Nach B. s. bei Fr. 4. April 1860. - Mz. (S.) - .

3. D. murinus L. - An todten Maulwürfen, kleinen Vögeln bei Fr. h. -- Königswiese bei Fr. und Rödêrwald im April (B.). Dlb. (Śch.) - Mz. (S.) - Wsb. im Wald an der Tränke mit Larven 17. Juni (K.). -

4. D. undulatus Brahm. - Fr. - Bergen n. s. - Von B. gef. $>$ 3. - Wsb. 5. Juni (K.). -

5. D. Ianiarius Illig. = affinis Gyll. - Bei Fr. auf trockenen Aeckern = 5. h. - Mo. (L. H.) - Mz: (S.) -

6. D. Iardarius L. - Der Speckkäfer. Im Gebiet h., die Larve oft an getrocknetem Fleisch; aber auch den Insektensammlungen schädlich, doch nur wenn einzelne Kasten stark vernachlässigt sind, nicht gut schliessen und selten nachgesehen werden. Das häufige Nachsehen und Stör:n der Raubinsekten halte ich überhaupt sowie erwärmte Zimmer für das beste Präservativmittel für Insektensammlungen. - C. H. fand die Art aber auch im Wald unter alter Buchenrinde $<5$, wo sich die Larve wolll von todten trockenen Insekten nährte. - Von Sch. h. als Zerstörer alter Wespennester bei Wlb. beobachtet. -

7. D. bicolor F. - Von L. H. und St. öfter in Häuser in Fr. gef., da wo 'Taubenschläg'e in der Nähe waren. -

\section{Attagenus Latreille.}

1. A. pellio L. - Unter dem Namen Pelzkäfer überall bekannt. Häufig in Häusern unter Teppichen, wo die Larve vermuthlich in den Fussbodenritzen ihrer Nahrung nachgeht. Besonders im Frühjahr; aber auch im Freien auf Blüthen bei Nauheim gef. und aus altem Waldholz erzogen. Von Sch. oft in seinen Insektenkasten in Wlb. als Hauptzer- 
störer gef, geht auch besonders den alten Wespennestern nach. Auf. Blïthen bei Wlb. h., z. B. Ligustrum vulgare, Crataegus oxyacantha, Aegopodium podagraria etc. - In seinem alten Tagebuch schreibt mein Vater 1827: „Im März, April hauptsächlich in Zimmern; der Käfer sucht in warmen Zimmern begierig Wasser auf und ersäuft hier häufig. $\mathrm{N}$ ie an Insekten gefunden (d. h. bei Frankfurt)." - Mz. (S.) Einmal 2. April 1876 in einem Neste der Formica rufa bei Münster im Taunus (L. H.). -

2. A. piceus Oliv. = megatoma F. - Fr. auf Blïthen n. s. s. im Juni und Juli, auch von Oberrevisor Jännicke gef. - 2 Stück bei Fr. von B. gef. - Wlb. h. (Sch.) - Mz. (S.) - Fr. am Fenster $<6$. -

3. A. vigintiguttata F. - Im Schwanheimer Wald unter Eichenrinde s. s. von uns gef. -

\section{Megatoma Herbst.}

1. M. undata L. - Ans dürrem Buchen- und Pappelholz aus dem Fr. Wald n. s. im Juni erzogen. - Schmitterhof bei Giessen aus Carpinusholz nocli $=10$. - An Häusern angefiogen s. gef. von B. Wlb. (Sch.) -

\section{Hadrotoma Erichson.}

1. H. marginata Payk. - C. H. fand 1 Ex. bei Fr. - Wlb. (Sch.) -

2. H. nigripes F. - Im Fr. Wald im Gehren in den Blitthen von Crataegus oxyacantha $>5$ l. gef., auch von B. gesammelt. - Bei Bockenheim 3. Juni 1876 in Blüthen von Cornus. -

\section{Trogoderma Latreille.}

1. T. glabrum Hbst. = elongatulum F. (Grundton der Flügeldecken schwarz, Schenkel dunkel). — Wlb. Dlb. (Sch.) -

2. T. nigrum Hbst. (Flügeldecken mit röthlicher Spitze, Beine rothbraun). - Fr. an den Mauern der Häuser und in den Zimmern s. s.; in Bürgel am Main flog das Thierchen Mittags an die weissen Hauswände im Juli. - Wlb. (Sch.) -

\section{Tiresians Stephens.}

1. T. serra F. - Unter Rinden von Weiden, Pappeln, Buchen bei Fr. n. s. Die Larve $>3$ unter Kiefernrinde, der Käfer entwickelte sich $<$ 6. - Mz. Mo. (S.) - 


\section{Anthrenus Geoffroy.}

1. A. Scrophulariae F. - Fr. auf Blüthen h. C. H. fand die Larve $>7$.; sie häutete sich $=9$.; verpuppte sich $\langle 10$. ; $\rangle$ 10. war der Käfer ausgefärbt, blieb aber unbeweglich in der geborstenen Hülle bis $<4$. $>4$. starb der Käfer. Findet sich s. h. in Häusern, in Gesellschaft des Attagenus pellio. - Nach Sch. n. s. an wollenen Zeugen, s. in Insektensammlungen, h. auf Blüthen hei Wlb. - Mz. (S.) - G. (L.) -

2. A. Pimpinellae F. - Auf Blüthen h. - Ueberall. - Fr. Wlb. auf Blüthen (Sch.). - Mz. (S.) - G. (L.) -

3. A. varius $\mathbf{F}$. = tricolor Hbst. - Der bekannte Zerstörer der Insektensammlungen. Nach einer Notiz von C. H. ișt die Hauptentwicklungsperiode $=5$. Das Weibchen setzt sich um diese Zeit gern an die Ränder der Insektenschachteln mit gesenktem Kopfe und emporgerichtetem Hinterleib und erwartet so das Bännchen. Um diese Zeit muss man fleissig nachsehen und die Käfer tödten. In geringerer Anzahl erscheint er das ganze Jahr, selbst zuweilen im Winter. In einem geheizten Zimmer scheint er nicht so zu gedeihen, wie in einem kalten. Sch. schrieb mir über diese Art: „Dieses Käferchen habe ich nie in meinen Insektenkasten gef., fehlt bei Dlb. und Wlb.; ich habe es in Wsb. auf Blüthen im Garton des ,Adler" gef. - Mz. (S.) -

4. A. museorum L. - Aus dürrem Fr. Waldholz $>6$. elzogen. Ist bei Dlb. und Wlb. der Zerstörer aller' Thiersammlungen, der gefährlichste Feind der Insektensammlungen, oft an den Fenstern (Sch.). - Mz. (S.) -

5. A. claviger Er. - Fr. Wald im Juni unter loser Rinde und aus alten Spinuennestern entwickelt. - Auch am Feldberg gef. - Nach Sch. mit dem vorigen oft verwechselt, s. in Naturaliensammlungen, oft an Fenstern; h. in Wlb. Die Larven länger und viel weniger behaart als bei dem vorigen, die Haarbüschel am Ende kürzer. -

\section{Trinodles Latreille.}

1. T. hirtus F. - In hohlen Eichen und Linden in der Nähe von Spinnenweken, wo sich die Larve von Insektenresten nährt, beim Fr. Forsthaus, Soden, Hofheim im Juni s. s. - In Eichen am Neroberg bei Wsb. im Juli (K.). -

[Orphilus glabratus F. kommt nicht bei Fr. vor, die in S. V. erwähnten Stücke sind abgeriebene Anthrenus, doch besitze ich ein sicher bestimmtes Stück aus Mannheim.] 


\section{BYRRHIDAE.}

\section{Limmichus Latreille.}

1. L. sericeus Dft. - Fr. einmal von C. H. und öfter bei Ems am Lahnufer gef. - Läuft schnell für einen Byrrhiden. -

\section{Simplocaria Marsham.}

1. S. semistriata $\boldsymbol{F}$. - An den Wurzeln von Aepfelbäumen am Hellerhof $>6$. von C. H. gef. - Bei Soden auf Salzboden $>10$. einmal, im Dezember 1831 in den Sandgruben bei der Ziegelhütte am Fr. Wald von C. H. gef. - Wlb. (Sch.) - Fr. 3. April 1860 (B.). - [S. metallica in S. V. gehört hierher.] -

\section{Pedillophorms Stefiahny = Morychus Erichs.}

1. P. nitens Panz. - Auf thonigen Schaftriften bei Fr. h. $=5$. von C. H. gef. - Bingen im April. - Auf trockenen Grasplätzen h., z. B. in den Fr. Gärten von B. gef. - Wlb. (Sch.) - M\%. (S.) -

\section{Cytilus Erichson.}

1. C. varius F. - Fr. n. s. an trockenen Stellen unter Steinen. - Mo. bei der Cantine am Schiessstand. - Im Sodener Wald $>6$. - Wlb. (Sch.) - Mz. Mo. (S.) -

\section{Pryrohnus Linné.}

1. B. ornatus Panz. - Fr. von C. H. gesammelt, ebenso wie bei Schlangenbad unter Moos s. s. -

2. B. Iuniger Germ. - C. H. fand =5. 1 Ex. auf dem grossen Feldberg-Plateau. - 
3. B. pilula L. - Ueberall h. auf Feldwegen, unter Steinen Fr. - Homburg. Auch im Genist. - Bei Dlb. Wlb. s. h. unter Steines und Moos, auch die Larve daselbst (Sch.). -

Uebergänge bis zur Var. arietinus Steff. n. s. bei Fr. Die Varietät selbst (mit grau eingefasster Querbinde über die Flügeldecken) bei Fr. s. und $=5$. auf dem Feldberg. In der Mainschanze unter einem Stein von B. gef. -

4. B. fasciatus Oliv. - Von L. H. und Twardowski s. S. in Sandgruben im Röderspiesswald bei Fr. gef. - Wlb. Dlb. (Sch.) Mr. (S.) -

5. B. dorsalis F. - Fr. - Schlangenbad. -

6. B. murinus Illig. - Im Wald zwischen Soden und Königstein von L. H. gesammelt. -

\section{Syncalypta Dillwyn.}

1. S. setigera Illig. - L. H. siebte einst diese bei uns seltene Art aus Mulm in einer Colonie der Formica fuliginosa am Fusse alter Pappeln bei Ginheim. -

2. S. spinosa Rossi. - In Anzahl am Ufer der Lahn bei Ems ron C. H. im Juli gesammelt, ebenso bei Fr. am Mainufer. -

\section{Nosodendron Latreille.}

1. N. fasciculare Oliv. - An ausfliessendem Saft von Olmen (1817) an den Kettenhöfen, der Eichen im Fr. Wald, der Rosskastanien (Aesculus) bei Biebrich im Mai und Juni von uns gesammelt. - Lebt gesellschaftlich. - Am Fusse alter Ulmen, nahe der Königswiese bei Fr. von B. gef. - Ebenso bei Wlb. Käfer und Larven s. h. (Sch.) Mr. (S.) - 


\section{HISTERIDAE.}

\section{Platysoma Leach.}

1. P. frontale Payk, - Fr. - Falkenstein, Schlangenbad, Ems s. unter Baumrinden. - Wlb. (Sch.) -

2. P. compressum Hbst. = depressum Fabr. - Häufiger. Fr. - Schlangenbad. - Unter Pappelrinde bei Fr. von B. gef. Wlb. h. (Sch.) - Mz. (S.) - G. (L.) - In Baumstrünken bei Wsb. Platter Chanssee im Mai (K.). -

3. P. oblongum F. - Fr. 2 Stiick. - Wlb. (Sch.) - Wsb. mit der vorigen Art 31. Mai (K.). -

4. P. angustatum E. H. - Fr. wie die vorige Art. -

\section{Hister Linné.}

1. H. quadrimaculatus L. - Fr. in Kuhmist h. - Dlb. Wlb. (Sch.) - Mz. (S.) -

Var. Durch Fehlen des mittleren Theiles der Binde bleiben auf jeder Flügeldecke nur zwei kleine runde rothe Flecken stehen. Bingen einmal.

Ganz schwarze Stücke Var. aethiops Heer = gagates Illig. sammelte Sch. bei Wlb. -

2. H. helluo Truqui = Silesiacus Roger. -- Einmal von C. H. bei Hofheim auf einem Erlenblatt gef., wo sich das seltene Thier von den Larven der Agelastica alni nährte. - [Hierher das in S. V. als bissexstr. erwähnte Exemplar.]

3. H. unicolor L. - Fr. n. s. An todten Krähen im Röderwald = 4. von B. gef. - Wlb. (Sch.) - Mz. (S.) - G. (L.) -

4. H. cadaverinus Ent. Hefte. - Fr. An Aas h. - Wlb. (Sch.) - Mz. (S.) -

5. H. succicola Thoms. - Fr. 3 Stïck an ausfliessendem Saft von Büumen. -

6. H. terricola Germ. - C. H. fand einmal diese seltene Art bei Fr. - Bei G. von L. öfter gef. - 
7. H. merdarius Ent. Hefte. - Fr, an Aborten und anderen unreinen Stellen n. s. - Schon >3. bei Fr. von B. gef. - Wlb. (Sch.) - Miz. (S.) -

8. H. fimetarius Hbst. = sinuatus $F_{\text {. }}-\mathrm{Fr}$. s.; häufiger bei Mo. in Sandgegenden von S. und L. H. gef. -

9. H. neglectus Germ. - Fr. einigemal. - Wlb. z. h. (Sch.) - Mz. (S.) - G. (L.) -

10. H. carbonarius Illig. - Fr. - Schlangenbad. - Von B. s. gef. -

11. H. ventralis Marsl. - Fr. 11. s. s. -

12. H. ruficornis Grimm. = myrmecophilus Mls. - Fr. Enkheimer Torfbrïche an alten Weiden in den Nestern der Formica fuliginosa s. -

13. H. purpurascens Hbst. - Fr. n. s. - Wlb. (Sch.) IIz. (S.) - G. (L.) -

14. H. marginatus Er. - Er. einmal. -

15. H. stercorarius Ent. Hefte. - Fr. h., auch am Feldberg $=5 .-$ Wlb. (Sch.) - $\mathrm{L} \%$. (S.) - G. (L.) -

16. H. sinuatus Illig. = uncinatus Illig. - Von St. und L. H. h. bei IIn. gesammelt. - Wlb. (Sch.) - Mo. 2. Mai (K.) -

17. H. quadrinotatus Scriba. - Fr. 11. s. - Wlb. Dlb. (Scl.) - Mz. (S.) - G. (L.) -

18. H. funestus Er. - C. H. fing die seltene Art einmal bei Bingen und einmal auf dem Nieilerwald im Rheingau. - 3 Stück bei Wlb. (Sch.) -

19. H. bissexstriatus F. - Fr. einmal. - Wlb. (Sch.) -

20. (Atholus Thoms,) bimaculatus L. - Fr. seltener, häufiger bei MIo. auf Sandboden. - Wlb. (Sch.) - Mz. Mo. (S.) - G. (L.) -

21. (A.) duodecimstriatus Schrk. - Fr. n. s. $->3$ und 29. April ron B. gef. - Mz. (S.) - Mz. (S.) - G. (L.) -

Var. quatuordecimstriatus Gyll. - Fr. einmal. -

22. H. Corvinus Germ. - Fr. z. s. in der Nähe von Aborten. Von B. wie der vorige gef. -- Wlb. (Sch.) - G. (L.) - Eichberg: im Rheingan (Richter). - 


\section{Paromalus Erichson.}

1. P. parallelopipedus Hbst. - Fr. s. an feuchtem Holz; anch von B. 1875 gef. -

2. P. flavicornis Hbst. - Fr. h. in faulem Holz, besonders Pappeln. - Wlb. (Sch.) - Mo. (S.) -

\section{Hotaerius Erichson.}

1. H. ferrugineus Oliv. = quadratus Kug. = sesquicornis Preyssl. - Leht ausschliesslich bei Ameisen und zwar bri Formica fuliginosa, rufa, fusca und flava im ganzen Gebiet. - Fr. - Offenbach. - Wlb. bei Formica fusca (Sch.). - Mz. Mo. (S.) - Im Lehen bei Oberrad 14. Mai 1876 (b.). -

\section{Dendrophilus Leach.}

1. D. punctatus Hbst. - Bei Formica rufa und fuliginosa, aber anch im Mulm alter Bäume. Fr. Mainkur. - Soden. - Im Mulm eines Apfelbaumes von B. gef. - Neroberg bei Wsb. (K.) -

2. D. pygmaeus $L$. = formicetorum Aubé. - Bis jetzt nur in Ameisennestern gef. und zwar nur bei F. rufa Fr. Wald. - Am 10. März 1860 h. von B. beobachtet. -

\section{Saprinus Erichson.}

1. S. nitidulus Payk. - Ueberall h. an unreinen Orten. Ueber ganz Europa und den Orient verbreitet. An Aas bei Fr. von L. H. und B. gef. — Sch. fand l:ei Wlb. Exemplare mit ausgeprägter Querlinie hinter dem Epistom; die Art variirt in dieser Beziehung. - Mz. (S.) - G. (L.) - Schiersteiner Weg bei Ws1. 23. Mai an todten Maulwürfen (K.). -

2. S. speculifer Payk. - Fr. von C. H. gef. - Wsb. mit der vorigen Art (K.). -

3. S. aeneus F. - Fr. h. an Aas. - Wlb. (Sch.) - Wsb. mit dem vorigen $(\mathrm{K}$.$) . -$

4. S. virescens Payk. - Bei Fr. zweimal und bei Wsb. 1 Stück von C. H. gef.; am 4. Mai 1860 am Ohermainthor an einer toitten Katze 1 Ex. von B. grf. - 
5. S. conjungens Payk. - Fr. Mainkur roin C. H. gef. Frieşberg (Fuhr). -

6. S. quadristriatus Ent. Hefte. - Bei Fr. 4 Ex. von C. H. gef. -

7. S. rugifrons Payk, - Fr., Rumpenheim, Grieshein am Main an Aas kieiner Thiere n. s. -

\section{Gnathoncus Duval.}

1. G. rotundatus Kugel. - Fr. s. an Aborten. - Wlb. (Sch.) - Eichberg in Rheingau (Richter). — Wsb. (K.) -

2. G. punctulatus Thoms. - Fr. einmal. -

\section{Myl'metes Marseul.}

1. M. piceus Payk. - Bei Formica rufa im Schwanheimer Wald einmal ron B. gef. -

\section{Teretrius Erichson.}

1. T. picipes F. - Fr. einmal. - Wlb. einmal (Sch.). Seltene Art. -

\section{Plegaderus Erichson.}

1. P. saucius Er. - Einmal unter Rinde bei Fr. am 6. April 1860 ron B. gef. -

2. P. caesus Illig. - Fr. in morschem Buchen- und Pappelholz n. S. - Mo. (S.) -

3. P. dissectus Er. - Fr. einmal. -

\section{Onthophilus Leach.}

1. O. striatus $\mathbf{F}$. - Fr. unter faulenden Vegetabilien n. s. In Kuhmist, gelegentlich auch $>3$. gestreift von B. - Nz. (S.) -

\section{Abraeus Leach.}

1. A. globulus Creutz. - Fr. einmal von L. H., auch von B. gef. -

2. A. globosus Ent. Hefte. - Fr. Wald an altem Eichenholz. - Schmitterhof bei Giessen. - Unter Eichenrinde bei Schwanheim 27. März 1861 ron B. grf. - Wlb. eimmal (Sch.). - 
3. A. parvulus Aubé. - Fr. 2 Ex. - Hierher die in S. V. erwähnten granulum Er. aus Fr. -

\section{Acritus Le Conte.}

1. A. fulvus Mars. - Einmal bei Fr. - Hierher eins der in S. V. als punctum Aubé erwähnten Exemplare (von Marseul revidirt), das andere ist wegen schlechter Conservirung unbestimmbar. -

2. A. nigricornis Ent. Hefte. - - Hierher die in S. V. erwähnten Bacanius rhombophorus Aubé in Bockenheim von M. S. bei Formica flava gef. Die Stücke sind nun in Sammlung v. Heyden. -

3. A. atomarius Aubé. - Von B. gef., s. h. an Steinen unter verrottetem Mist am Röderberg 21. März 1860. - Mz. (S.) -

4. A. minutus F. - Fr. s. - Wlb. (Sch.) - Eichberg im Rheingau (Richter). -

\section{MICROPEPLIDAE.}

\section{Micropeplus Latreille.}

1. M. porcatus Payk. - Im ganzen Gebiet n. s. - Fr. - In Neuenhain bei Soden nnter einer alten Kastanie in grosser Anzahl Abends mit dem Streifnetz gesammelt. Auf feuchten Stellen, so auf der Königswiese von B. gestreift, aber auch in Kuhmist h. im April gef. - Wlb. s. h. mit dem Streifnetz im Grase gef. (Sch.) - Mz. (S.) - 


\section{NITIDULIDAE.}

(Sammlung v. Heyden, Stern und Schenck von dem Monographen Reitter revidirt.)

\section{Cercus Latreille.}

1. (Anomaeocera Shukard) pedicularius L. - Bieberer Höhe bei Offenbach. - Fr. unter Riedgräsern; nach Hg., B. und St. h.; ebenso bei Dlb. (Sch.) -

2. C. dalmatinus Sturm. - Fr. einmal von C. H. gef., auch von Hg. gesammelt. -

3. C. rufilabris Latr. - Je 1 Stück fanden C. H. bei Fr. und L. H. an der Gerbcrmühle bei Oberrad. - Auch Hg. fand die Art bei Fr, und nach Sch. ist sie bei Wlb. h. - M. (S.) -

\section{Amartus Le Conte.}

1. A. affinis Heer. = rubiginosus Er. $=$ Rhenanus Bach. $=$ Spiraeae Märkel. - Von Hg. und St. bei Fr. gef. -

2. A. Sambuci Er. - Bei Bingen h. von C. H. auf Blüthen von Sambucus racemosa $=4$. gef. - Taunus. - M. (S.) - Wlb. (Sch.) Königstein $=5$. -

\section{Brachypterus Kugelann.}

1. B. pubescens Er. - Fr. zweimal. - M. (S.) - G. (L.) -

2. B. Urticae Kug. - Im ganzen Gebiet auf Nesseln h. M. (S.) - G. (L.) - Wlb. (Sch.) -

3. (Heterostomus Duval.) gravidus Illig. - Im ganzen Gebiet auf Antirrhinum Linaria h. - Offenhach. — Falkenstein. - Rüdesheim. - Bei Fr. von C. H. und Hg., bei Wlb. von Sch. gef. - M. (S.) - G. (L.) -

4. (H.) Linariae Cornel. - Fr. einmal. -

5. (H.) cinereus Er. - Falkenstein und Soden im Taunus. Fr. (Hg.) - Wlb. (Sch.) - 


\section{Carpophilus Leach.}

1. C. hemipterus L. - Von Hg. in Fr. in Droguen gef. -

2. C. sexpustulatus Er. - Unter saftiger Birkenrinde in Fr. Wald mehrfach gef. - Dlb. (Sch.) - Auch von St. bei Fr. gesammelt. -

\section{Epuraea Erichson.}

1. (Dadopora Thoms.) decemguttara F. - Fr. Wald und Soden an ausfliessendem Eichensaft; auch von B. und St. s. gesammelt. Bei Wlb. nach Sch. h. - M. (S.) -

2. E. neglecta Heer. - Mit der vorigen s. -

3. E. terminalis Mnhm. = immunda Sturm. - Am Fr. Forsthaus im August; aus Baumschwämmen entwickelt s. $<8$. - M. (S.) -

4. E. deleta Sturm. - Fr. zweimal von C. H. gef. - Neu für das Gebiet. -

5. E. aestiva L. = ochracea Er. - Fr. - Soden auf Wiesenblumen < 10. - Mainkur. - Röderberg in Kirschblüthen; auch auf Viburnum opulus. - s. h. - Wlb. (Sch.) - M. (S.) - G. (L.) -

Var. bisignata Sturm. - Fr. s., zweimal auch von B. ges. M. (S.) - G. (L.) -

6. E. melina Er. - Fr. Wald auf Blüthen - Schlangenbad. Wlb. (Sch.) -

7. E. castanea Duft. - Von St. bei Er. gesammelt [Reitt. vid.]. -

8. E. parvula Sturm. - Fr. an ausfliessendem Eichensaft, auch auf Salix caprea Blüthen. Fr. Wald an =der Unteren Saustipge. M. (S.) -

9. E. nana Reitt. var. binotata Reitt. - Von Sch. einmal bei Wlb. gef. [Reitt. vid.] -

10. E. variegata Hbst. - Fr. von $\mathrm{Hg}$. und B. s. gesammelt. -

11. E. obsoleta F. - Fr. n. s. - Königstein. - Bingen im April auf Blüthen. - Wlb. (Sch.) [Reitt. vid.] - Fr. 8. April 1860 (B.) - M. (S.) -

12. E. Fagi Bris. - Bei Wlb. viermal von Sch. gef. [Reitt. vid.]; 1 Ex. davon in Sammlung v. Heyden. -

13. E. longula Er. - Rödelheimer Wald. - Fr. - Taunus. Wlb. (Sch.) [Reitt. vid.] - 
14. E. pygmaea Gyll. = rubromarginata Reitter. - Einmal bei Griesheim am Main im August von C. H. gef. - Seltene Art. -

15. E. pusilla Er. - Fr. n. s. - Wlb. (Sch.) [Reitt. vid.] M. (S.) -

16. E. oblonga Hbst. - Fr. zweimal. - Bingen 1 Stück. -

17. E. Fussii Reitt. - Seither nur aus Prag und Siebenbürgen bekannt. - Von Sch. zweimal bei Wlk. gesammelt [Reitt. vid.]. 1 Ex. nun in Sammlung v. Heyden. -

18. E. florea Er. - Fr. - Bingen. - Rüdesheim in Blüthen von Salix caprea. - M. (S.) -

\section{Micruria Reitter.}

1. M. melanocephala Mrsh. - Fr. einmal. -

\section{Omosiphora Reitter.}

1. O. limbata F. - In den Fr. Promenaden n. s. an ausfliessen-. dem Saft von Bäumen. - Dlb. (Sch.) - M. (S.) -

Var. unicolor Reitt. - Zweimal von Sch. bei Wlb. gef. [Reitt. vid.] - Auch von L. H. mit der Stammart bei Fr. -

\section{Nitidula Fabricius.}

1. N. bipustulata F. - Fr. s. Die 3 Arten leben an Aas und alten Knochen. - B. fand im April 1861 im Röderwald bei Fr. 1 Stück an einer todten Krähe. - Wlb. (Sch.) - Fr. (St.) - M. (S.) -

2. N. rufipes L. = obscura L. - Es gibt Exemplare von doppelter Grösse wie die gewöhnlichen. Im ganzen Gebiet s. h. Griesheim. - Mo. - Bei Wlb. grusse und kleine Stücke (Sch.). Fr. 29. April 1860 (B.). - M. (S.) -

3. N. quadripustulata F. - Fr. n. s. - M. (S.) -

\section{Omosita Erichson.}

1. O. depressa L. - Fr. s. von C. H. gef. -

2. O. discoidea F. - Fr. von C. H. und B. unter Steinen bei Mist gef. - Wlh. (Sch.) - M. (S.) - G. (L.) - 
3. O. colon L. - Fr. von L. H. und St. gef. Lebensweise wio die vorige Gattung, alle drei Arten n. s. - Wlb. (Sch.) - Fr. 8. April 1860 (B.). - M. (S.) - G. (I.) -

\section{Soronia Erichson.}

1. S. punctatissima Illig. - Nach B. wurde von Harer 1 Stück bei Fr. gef., auch von Hg. -

2. S. grisea L. - Fr. Soden n. s. an ausfliessendem Eichensaft. Unter Weidenrinde schon < 3. von B. gef. - Wlb. (Sch.) - Fr. (St.) - M. (S.) - G. (L.) -

\section{Amphotis Erichson.}

1. A. marginata F. - Im ganzen Gebiet h., jedoch nur unter Rinden bei Formica fuliginosa. Von B. von $>4$. bis $=5.1861$ besonders am Fusse alter Weiden gef. - Wlb. (Sch.) - Auch von Hg. und St. h. bei Fr. gef. - M. (S.) - G. (L.) -

\section{Pria Stephens.}

1. P. Dulcamarae Scop. - Fr. Wald. - Rödelheim. - Mo. = 9. auf Solanum nigrum. - Lokal. Auch von Hg. gef. - M. (S.) -

\section{Meligethes Stephens.}

1. M. rufipes Gyll. - Einmal im Wald bei Langenhain im Taunus. - Einmal bei Fr.; auch von Hg. 2 Stück gef. -

2. M. lumbaris Sturm. - Im Mai auf Wiesen bei Falkenstein. - Fr. Wald s. von St. und Hg. h. gef. -

3. M. Försteri Reitter. - Im Taunus bei Falkenstein fand C. H. 1 Exemplar dieser seither nur aus dem Siebengebirg und Aachen bekannten Art. -

4. M. discolor Reitter. - L. H. fand 1872 diese Art einmal bei Königstein an der Oelmühle. - Der Autor beschrieb 18722 Stücke aus Elberfeld. -

5. M. coracinus Sturm. - Fr. - Bieberer Höhe bei Offenbach. - Auf dem Johannisberg bei Friedberg. - Fr. (Hg.) - Wlb. (Sch.) [Reitt. vid.] - 
6. M. caeruleovirens Först. - Im Fr. Wartforst einmal auf Epilobium angustifolium im Juli gef. -

7. M. Brassicae Scop. = aeneus L. - In ganz Europa einer dèr häufigsten Käfer auf Blüthen. Auch bei uns im ganzen Gebiet. Dlb. (Sch.) - M. (S.) - Fr. (Hg., St.) -

8. M. viridescens F. - Im ganzen Gebist in der Ebere und im Gebirg ebenso h. wie Brassicae. - Wlb. (Sch.) - Auch von Hg. h. gesammelt. -

Var. Germanicus Reitter Fr. einmal. Ausgezeichnet durch den Mangel sichtbarel Härchen auf der Oberseite. -

9. M. Symphyti Heer. - Fr. - Mainkur. - Hofheim. Auch von Hg. h. gef. -- Wll. (Sch.) [Reitter vid.] - M. (S.) -

10. M. Ranunculi Reitter. - Fr. eiumal. - 1872 nach Aachener Stücken beschrieben. -

11. M. subrugosus Gyll. -- Fr., Offenbach, Falkenstein, Königstein, Flörsheim, Mo. n. S. - M. (S.) -

Var. substrigosus Er. - Fr. 3 Stück in rothfaulem Eichenholz. -

12. M. serripes Gyll. = exaratus Först. = quadridens Först. - Fr. Wald an Forsthaus dreimal gef. — Wlb. (Sch.) [Reitter vid.] -

13. M. villosus Bris, - Von St. bei Fr. zweimal gesammelt. [Reittel' vid.] -

14. M. obscurus Er. = palmatus Er. - Fr. Unterwald an Camirnula s. s. - Ems. -

15. M. bidens Brisout. - Fr. 2 Stück, einmal bei Bergen. -

16. M. umbrosus Sturm. - Fr. s. - Cronthal. Auch von Hg. im Gebiet gesammelt; ebenso von St. [Reitter vid.] —

17. M. maurus Sturm. - Fr., Enkheim, Nauheim = 4. auf Potentilla verna. - Schmitterhof bei Giessen. - Auch von Hg. bei Fr. gesammelt sowie von St. [Reitter vid.] - Wlb. (Sch.) -

Var. calvus Reitter. - Fr. einmal gef. -

Var. durus Reitter. - 1 Ex. im Mai bei Mo. -

18. M. incanus Sturm. - Fr. - Flörsheim. - Bieberer Höhe bei Offenbach. - Sonst seltene Art, bei uns z. h.; auch von Hg. gef., sowio von St. [Reitter vid.] - M. (S.) -

19. M. fuliginosus Er. - Auf den Wiesen bei Enkheim, $>5$. zweimal und 1 Stück bei Fr. gef.- - 
20. M. ovatus Sturm. - Im Taunus $>$ 6. einmal ron C. H. gef. -

21. M. moestus Er. = nigerrimus Roshr. - Auf Hecken bei Soden s. s., auch bei Fr. je einmal ron C. H. und Hg. gef., doch auch in Süd-Europa: Andalusien. -

22. M. picipes Sturm = funebris Först. - Auf Waldwiesen in Blumen bei Königstein, Falkenstein, Cronthal, Johannisherg tei Friedberg. Im Frühjahr h. - Fr. (Hg.) -

23. M. flavipes Sturm. = flavicornis MHller. - Fr. - Rebstocker Wald. - Soden. - Bergen. - s. - Auch ron Hg. bei Fr. gesammelt. - Wlb. (Sch.) [Reitter rid.] -

24. m. memnonius Er. = parvulus Bris. = niger Bris. Voin Sch. einmal bei WIb. gef. [Reitter rid.] -

25. M. brunnicornis Sturm. - Fr. je 1 Ex. ron C. H. und Hg. gesammelt. -

26. M. difficilis Heer. - Fr. zweimal im April auf Lamium album. - Wlb. (Sch.) [Reitter vid.] -

27. M. blandulus Reitter. - Fr. einmal auf Lamium album. -

28. M. Miller Reitt, - Seither nur ein österreich. Exemplar bekanut. Einmal ron Sch. bei Wlb. gef. [Reitter rid.] -

29. M. morosus Er. - Fr. s. auf L. album. - Wrib. (Sch.) [Reitter vid.] -

30. M. viduatus Sturm. = melanarius Först. - Auf Wiesen im Fr. Wald an Epilobium angustifolium. - Soden. - Wlb. (Sch.) [Reitter vid.] - Bei Fr. auch ron St. gesammelt [Reitter rid.] -

31. M. pedicularius Gyll. = tenebrosus Först. - Wie die rorige Art ron Hg. eimmal gef. - Wlb. (Sch.) [Reitter rid.] -

32. M. luctuosus Först. - Fr. 1 Ex., seither nur 3 Aachener Stücke bekamnt. - Bei Wlb, ron Sch. einmal gef. [Reitter Tid.] --

33. M. assimilis Sturm. - Fr. zTeimal, Enkheimer Torf́rüche 1 Stück gef. -

34. M. tropicus Reirt. - Von St. bei Fr. einmal gesammelt [Reitter vid.] -

35. M. Lepidii Mill. - Ton St. bei Fr. gesammelt [Reitt. vid.]. -

36. M. fristis Sturm. - Fr. 2 Ex. - Ton C. H., auch ron Hg. gef. - 
37. M. murinus Er. = seniculus Er. - Bei Fr. einmal gef.; auch ron Hg. gesammelt, sowie ron St. [Reitter vid.] -

38. M. lugubris Sturm. = ebeninus Först. - Bieberer Höhe bei Offenbach. - Am Entensee bei Rumpenheim im Sommer je einmal gef.; auch von Hg. s. gesammelt. - Wlb. (Sch.) [Reitter vid.] -

39. M. gagatinus Er. = cristatus Först. - Offenbach am Mainufer, Entensee bei Rumpenheim je 1 Stück gef. - Wlb. (Sch.) [Reitter vid.] -

40. M. bidentatus Bris. - Fr. - Taunus auf Wiesenblumen, auch im Rebstockwald. Seltene Art. Das Männchen hat am letzten Hinterleibssegment zwei starke Zähne und wurde von Bach neucrdings aus Württemberg als Gresseri beschrieben. -

41. M. erythropus Gyll. = egenus Först. - Fr. - Hofheim. - Königstein n. s. S. - Auch von Hg. im Gebiet gesammelt. M. (S.) -

42. M. exilis Sturm. = nigrita Luc. - Fr. dreimal gef. Mo. - Auch Hg. und St. sammelten die Art im Gebiet. -

43. (Odontogethes Reitter) hebes Er. - Griesheim am Main. - Fr. Wald in Distelblüthen und auf Himbeersträuchern; auch von Hg. und St. gef. -

44. (Acanthogethes Reitter) solidus Kugel. - Cronberg, Falkenstein, Königstein im Taunus s. -

45. (A.) brevis Sturm. - Fr. einmal < 7. aus HelianthemumBlüthen entrickelt; ein zweites Exemplar $=6$. in Mo. gef. -

\section{Thalyera Erichson.}

1. T. fervida Oliv. = sericea Er. - n. h., aber verbreitet. - Fr. (L. H., St., Hg.) - Wlb. (Sch.) -

\section{Pocadius Erichson.}

1. P. ferrugineus F. - Im ganzen Gebiet in Bovisten h. - Fr. Von B. im Röderwald am 19. April 1861 auch an einer todten Krähe gef. - M. (S.) -

\section{Cychramus Kugelann.}

1. C. fungicola Heer. - Von Hg. bei Fr. gefunden. Wlb. (Sch.) - 
2. C. luteus F. - Fr. in Boristen im October, aber anch im Juni von Himbeerblüthen geklopft; auch von $\mathrm{Hg}$. und St. gef. Wlb. (Sch.) - M. (S.) -

\section{Cryptarcha Shuckard.}

1. C. strigata $\dot{F}$. - Fr. - Im Gebiet n. s. an ausfliessendem Eichensaft. - Auch unter Weidenrinde von B. gef., sowie von Hg. und St. gesammelt. - Wlb. (Sch.) - M. (S.) - Wsb. Nerothal im Juli (K.). -

2. C. imperialis F. - Fr. - Wie die vorige Art. - Nach B. s. s. hei Fr.; auch von Hg. gef. - Bei Wlb. nach Sch. h. -

\section{Ips Fabricius.}

1. I. quadripunctatus Hbst. - Im Gebiet z. s. - Fr. im Mai aus üherwinterten Baumschwämmen entrickelt. - Oberursel (St.). Hg. fand die Art bei Fr. - Dlb. (Sch.) -

2. I. quadriguttatus F. - Bei Obertrsel unter Rinde von St. gef. - Fr. (Hg.) - Dlb. (Scb.) -

3. (Glischrochilus Murray) quadripustulatus L. - Unter saftiger Nadelholzrinde im Gebiet n. s. - Oberursel (St.). - Fr. von L. H. und Hg., bei Dlb. von Sch. gef. -

4. (Pityophagus Shuckard) ferrugineus L. - Wie die vorige Art z. s. - Fr. auch von Hg. und B. gef. - Mo. = 4. - Oberursel (St.) -

\section{Rhizophag'us Herbst.}

1. R. depressus F. - Fr. Wald $<8$. h. mit Larven unter der Rinde ron Pinus sylvestris, - Auch von Hg. und St. gef. - Fr. 6. April 1860 (B.). - M. (S.) - Dlb. (Sch.) -

2. R. parallelocollis Gyll. - Auf dem Fr. Friedhof n. s. an Nauern. Fr. Wald an Eichen. Hierher die Fr. R. cribratus und perforatus in S. V. Auch von Hg. gef. -

3. R. ferrugineus Panz. - Fr. zweimal von C. H. und Hg. gef. -

4. R. politus Hellw. - Fr. Friedhof unter Ulmenrinde $<5$. - Enkheimer Torfbrüche an Pappeln und Weiden. - Fr. Gärtnerei unter Rinden n. s. s.; auch von B. und St. je einmal gef. - M. (S.) -

5. R. dispar Payk. - Von Hg. bei Fr. gesammelt. - 
6. R. bipustulatus F. - Im Fr. Wald mter Eichen und Birkenrinde n. s.; auch von Hg. und St. gef. - Dlb. (Sch.) - Fr. 6. April 1860 (B.). - M. (S.) -

7. R. parvulus Payk. - Wie bipustul. aber s. s. - Oberräder Schiessstände. -

8. R. caeruleus Waltl. - Bei Dlb. einmal unter Eichenrinde von Sch. gef. -

\section{PELTIDAE.}

\section{Nemosoma Latreille.}

1. N. elongatum L. - In grosser Anzahl aus altem Buchenholz aus dem Fr. Wald ron L. H. und St." erzogen. -

\section{Trogosita Olivier.}

1. T. mauritanica L. = caraboides F. - In allen Welttheilen. Ueberall in Mehlvorräthen und Droguen, aber auch im Freien unter Apfel- und Buchenrinde im Fr. Gebiet, von B. auch im Mulm ron Kirschbäumen gef. - In Dlb. in einem Hanse in Brod, auch dis Larve gef. (Sch.) - M. (S.) -

\section{Peltis Geoffroy.}

-1. P. oblonga L. - Fr. von C. H. einnal an einem Pappelbaumschwamm gef. - Im Mai 1864 von St. aus Fr. Waldholz erzogen; von B. am 9. Juni 1860 einmal bei Fr. gef. -

\section{Thymalns Latreille.}

1. T. limbatus F. - Vom verstorbenen Herrn Emil Kirschbaum 11. s. am Neroberg bei Wsb. gesammelt. - M. (S.) - 


\section{XIX.BYTURIDAE。}

\section{Byturus Latreille.}

1. B. tomentosus F. - Die Iarve s. h. als wohlbekanntes Himbeerwürmchen. Der Käfer viel weniger $h$. in Himbecrblüthen im Juni. Fr. - Feldberg. - Bei Dlb. und Wlb. von Sch. gef. -- M. (S.) -

2. B. fumatus F. - In den Blüthen des Löwenzahn Leontodon taraxacum h. - Fr. - Falkenstein im Mai. - M. (S.) -- Dlb. und Wlb. (Sch.) -

\section{MYCE'TOPHAGIDAE.}

\section{Mycetophagus Hellwig.}

1. M. quadripustulatus L. - Im ganzen Gebiet an Boletus n. s. Fr. Im Sommer und Herbst; von Hg., B. und St. s. h. gesammelt. Dlb. Wlb. (Sch.) - M. (S.) - G. (L.) - Mo. an Weidenschwamm 23. April (K.). -

2. M. variabilis Hellwig = piceus F. - An Buchen- and Lichenschwämmen vom August bis Dezember. Fr. n. s. auch von Hg. und St. gef. - M. (S.) - G. (L.) - Dlb. Wlb. (Sch.) -

3. M. decempunctatus F. - Fr. s. an Barmschwämmen von $<$ 8. bis $=10$, ; auch von $\mathrm{Hg}$. gesammelt. -

4. M. atomarius F. - Bei Fr. im Wald in Schwämmen an Pappeln und den schwarzen Krustenschwämmen an Buchen (Sphaeria deusta) vom Mai bis October. - Am Altkönig $<6$. unter Buchenrinde. - Von Hg., St. und B. s. bei Fr. gesammelt. - Dlb. Wlb. (Sch.) - 
5. M. multipunctatus Hellwig. - In Schwämmen an Eichen und Pappeln im Fr. Wald von Juni bis October; auch von Hg. und St. gef. - M. (S.) - G. (L.) -

6. M. fulvicollis F. - In faulem Buchenholz an der Viehtränke neben der Babenbäuser Chaussee im Fr. Wald einst in Anzahl von L. H., Hg., M. S. und St. im Mai gesammelt. -

7. M. Populi F. - In Schwämmen au Pappeln, Eichen, Buchen im Fr. Wald das ganze Jahr hindurch von uns und Hg. gef. M. (S.) -

8. M. quadriguttatus Müll. - C. H. fand ein einzelnes Exemplar bei Fr. im Mai in alten Eichenschwämmen. -

\section{Triphyllus Latreille.}

1. T. punctatus Hellw. - Im Taunus bei Cronthal in Schwämmen und Pilzen im September s. - Königsberg bei Giessen im October. Bei Fr. von Hg. und St. gesammelt. -

\section{Litargus Erichson.}

1. L. bifasciatus F. - Im ganzen Gebiet unter Rinden und in Baumschwämmen n. s. - Fr. vom April bis September auch von Hg. gef. - An Buchen in der Sonnenhitze schwärmend. - M. (S.) -

\section{Typhaea Kirby.}

1. T. fumata L. - Im ganzen Gebiet an Dung, faulenden Pflanzen, des Abends im Flug h. gef. - Fr. Mainkur, Soden, Rödelheim. Von B. 1863 überwintert unter Laub gef. - Wlb. (Sch.) - M. (S.) - 


\section{PHALACRIDAE.}

(Die Sammlung v. Heyden wurde vom Monographen Tournier revidirt.)

\section{Phalacrus Paykull.}

1. P. corruscus Payk. - Im ganzen Gebiet auf Blüthen, in Genist, unter Rinde h. - Fr. - Griesheim am Main, Offenbach, Soden, Rüdesheim. - Ẃlb. (Sch.) - M. (S.) -

2. P. Caricis Sturm. - Fr. einmal gef. -

\section{Olibrus Erichson.}

1. 0. corticalis Panz. - Fr. - Soden. - Griesheim, Mo. auf Senecio. Auch im Winter h. unter Rinden. - Wlb. (Sch.) - M. (S.) -

2. 0. aeneus Illig. - Fr. auf Kamille, am Röderberg in Kirschblüthen, Rödelheimer Wald unter Rinden. - Griesheim. - Auch von B. s. h. gesammelt. - Wlb. (Sch.) - M. (S.) -

3. O. bicolor F. - Fr. - Soden. - Rödelheimer Wald. St. Goarshausen. - Wlb. (Sch.) -

4. O. liquidus Er. - Soden einmal von C. H. gef. - Wlb. (Sch.) -

5. O. affinis Sturm. - Bingen einmal. Hierher der Binger liquidus in S. V. - Wlb. '(Sch.) -

6. O. Millefolii Payk. - Fr. Wald h. - Bieberer Höhe bei Offenbach. - Wlb. (Sch.) - M. (S.) -

7. 0. pygmaeus Sturm. - Bei Griesheim auf Cineraria $<8$. von C. H. gesammelt. -

8. 0. consimilis Marsh. = geminus IIlig. - Fr. und Offenbach unter Schilf und öfter von Juniperns geklopft. - Griesheim. St. Goarshausen < 5. - Fr. von B. h. gef. - M. (S.) - Wlb. (Sch.) -

9. 0. piceus Steph. - Unter Schilf bei den Kettenhöfen bei Fr. - Auch sonst im Gebiet. - Wlb. (Sch.) - M. (S.) -

10. O. oblongus Er. - Unter Schilf bei Offenbach im October. - 


\section{CUCUJIDAE.}

\section{Brontes Fabricius.}

1. B. planatus F. - Im Dezember im Fr. Wald unter Eichenrinde von C. H. gef.; ebenso bei Dlh. von Sch, gesammelt. -

\section{Laemophioeus Erichson.}

1. L. denticulatus PreyssI. = monilis F. - Im Frühjahr im Fr. Wald an gespaltenem Eichen- und Buchenholz von uns gef. Fr. (Hg. und St.) - Nach B. unter Buchenrinde und in der Sonnenhitze sich auf Buchenholz tummelnd. $-<4$. h. - M. (S.) -

2. L. muticus F. - Ein Stück dieser leicht kenntlichen, gailz schwarzen Art fing Hg. an der Isenburger Chaussee im Fr. Wald an alten Eichen. -

3. L. testaceus F. - Fr. - Schlangenbad tinter Buchenrinde $>6$. -

4. L. duplicatus Waitl. - Im Fr. Wald an geschlagenem Buchenholz im Mai s. -

5. L. ferrugineus Steph. - An. Mehlyorräthen, im.Brod eingebacken gef. -- Fr. auch von Hg. gesammelt. - M. (S.) -

6. L. ater Oliv. var. rufus WaltI. $=$ Sturmii Roshr. - Aus đürrem Fr. Waldholz öfter im Mai und Juni erzogen. - Die dunkle Stammart nur im Süden. -

\section{Pediacus Shuckard.}

1. P. depressus Hbst. - Am Fr. Forsthaus an Eichenholz bei Sonnenuntergang $<8$. 2 Stiick, einmal im Zimmer $=7$. gef. -

[P. dermestoides F. - Sch. fand bei Wlb. eine Larve, ohne Käfer, die Rosenhaner für hierher gehörig bestimmte.]

\section{Lathropus Erichson.}

1. L. sepicola Müll. - C. H. fand $1 \mathrm{Ex}$. an einer Gartenplanke auf dem Mühlberg bei Sachsenhausen. - 


\section{Lyctus Fabricius.}

1. L. canaliculatus F. - Im ganzen Gebiet n. s. in Pfählen, Zäunen und Bretterwänden. - Fr. - Wlb. (Sch.) - M. (S.) - Wsb. an Bleicliplatz in den Zäunen im Juni (K.). -

[L. pubescens Panz. überhanpt sehr fragliche Art, kommt nicht bei Fr. vor; die von L. H. in S. V. erwähnten Stücke waren kleine canalic.]

2. L. bicolor Comolli. - C. H. fand 8 Stück im Mai und Juni in altem Weidenholz bei Fr. -

\section{Silvanus Latreille.}

1. S. bidentatus F. - Im Fr. Wald unter Kieferninde $<6$. von L. H. und Hg. gef. -

2. S. similis Er. - Im Rödelheimer Wald =8. eine Anzahl Exemplare gef. -

3. S. unidentatus F. - Im Fr. Wald und anf dem Niederwald bei Rüdeshein unter Eichenrinde. Bei Fr. h. von B. und Hg. gef. MI. (S.) - G. (L.) - Dlb. (Sch.) $-<10$. unter Pappelrinde Fr. -

\section{Airaphilus Redtenbacher.}

1. A. elongatus Gyll. - Im März an der Schwarzen Steinkaut im Fr. Wald unter Riedgräsern s. s. von C. H. gef. Nach seinen Beobachtungen läuft das Thierchen schnell aber schwankend. - Auf fenchten Wiesen bei Schwanheim und auf der Königswiese am 21. April 1861 von B. n. s. S. gesammelt. - Dlb. (Sch.) - M. (S.) G. (L.) - 


\section{COLYDIIDAE.}

A. COLYDIINI.

\section{Aulonium Erichson.}

1. A. sulcatum 0l. - St. erzog 2 Ex. aus Fr. Waldholz. M. (S.) -

1. C. elongatum $F$. und

\section{Colydium Fabricius.}

2. C. filiforme F. - Beide Arten aus dürrem Fr. Waldholz, auch von St. und Hg. erzogen, unter Eichenrinde h. bei Wlb. von Sch. gef. -

\section{Teredus Shuckard.}

1. T. nitidus F. - Im Fr. Gebiet s. unter Rinden, bei Cronthal von C. H. unter Rinde von Castanea vesca gef. - Von Hg. bei Fr. -

\section{Oxylaemus Erichson.}

1. O. cylindricus Panz. - Von Hg. im Isenburger Wald 2 Stück gef. -

- 2. 0. variolosus Dufour $=$ caesus Er. - Von C. H. an der Obersaustiege im Fr. Wald einmal bei Formica fuliginosa in einer alten Eiche gef. - M. (S.) -

\section{Aglenus Erichson.}

1. A. brunneus Gyll. - B. fand 2 Ex. auf verrottetem Mist am Röderberg bei Fr.; auch St. sammelte die Art. -

\section{Ditoma nlliger.}

1. D. crenata F. - Unter feuchter Nadelholz-, Eichen- und Buchenrinde im Fr. Gebiet h., auch die einfarbig gelbe Varietät. Von B. s. h. auch unter Pappelrinde gef. - Dlb. (Sch.) - 


\section{B. SYNCHITINI.}

\section{Synchit a Hellwig.}

1. S. Juglandis F. - Aus dürrem Holz aus dem Fr. Wald $>5$. von C. H. einmal erzogen; auch von Hg. und St. bei Fr. gef. M. (S.) -

\section{Cicones Curtis.}

1. C. variegatus Hellwig. - An Buchenkrustenschwämmen Sphaeria deusta zuerst im Fr. „Wäldchen“ am Forsthaus h. von C. H. gef.; erste Generation $<5$., zweite $<9$. - Nach seinen Beobachtungen geht die weisse Larve zur Verwandlung in die Erde. - Fr. (Hg.) - M. (S.) -

\section{Colobicus Latreille.}

1. C. emarginatus Latr. - Fr. Wald unter Buchen- und Eichenrinde s., von B. an der Grastränke im Fr. Wald am 20. März 1860 einmal gef.; auch von Hg. und St. gesammelt. - M. (S.) -

\section{SARROTRIINI.}

\section{Pycnomerus Erichson.}

1. P. terebrans $\mathbf{0 l}$. - An aufgeschichtetem Holz im Fr. Wald bei Sonnenuntergang $<8$. s.; einmal in einer alten Hainbuche von C. H. gef.; auch von St. aus Fr. Waldholz erzogen und von Hg. gesammelt. -

\section{Orthocerus Latr. = Sarrotrium Illiger.}

1. O. clavicornis L. - In Sandgegenden unter Steinen n. s., z. B. an der Ziegelhütte am Fr. Wald. - Von B. bei Fr. = 5. einmal gestreift und in den Flörsheimer Kalksteinbrüchen h. herumlaufend angetroffen. - Bei Wlb. s. h. von Sch. und Sandherger gef. - M. (S.) -

\section{LATHRIDIINI.}

(Die Sammlungen v. Heyden und $\mathrm{Haag}$ vom Monographen Reitter revidirt.)

\section{Monotoma Herbst.}

1. M. picipes Payk. - Fr. - Soden an Dunghaufen im Flng gef. - Fr. n. s.; anch von Hg. gesammelt. - M. (S.) - 
2. M. angusticollis Gyll. = conicicollis Aubé. (Halsschild nach vorn stark verengt, Vorderwinkel vorspringend.) - Im Fr. Wald in Nestern der Formica rufa oft in Anzahl ausgesiebt. März, September und Dezember. Im Schwanheimer Wald s. h. von B. gesammelt. - Bei Münster im Taunus bei F. rufa 2. April 1876. - Wlb. (Sch.) -

3. M. formicetorum Thoms. = angusticollis Aubé. - Fr. s. in 'den Nestern der Formica rufa. - Im Schwanheimer Wald von B. h. gef. - Bei Münster wie die vorige. -

4. M. brevicollis Aubé = quadridentatus Thoms. - Fr. zweimal gef. -

5. M. spinicollis Aubé. - Ein Exemplar >. 7. von C. H. an vegetabilischen Dunghaufen bei Fr. gef. -

6. M. quadricollis Aubé. - Eimmal von C. H. bei Fr. gef. -

7. M. Iongicollis Gyll. - An vegetabilischen Dunghaufen > 7. bei Fr. einmal gef. - Hierher die Fr. Exemplare der M. brevipennis des S. V. -

8. M. scabra Märk. i. I. Kunze. - Einmal bei Wlb. von Sch. gef. [Reitter vid.] - G. (L.) - Die von Märkel selbst an C. H. gesthickten Exemplare waren picipes. -

\section{Lathridius Illiger.}

1. L. Iardarius Degeer. - Von Juni bis September an feucliten Stellen unter Laub bei Fr. - Offenbach; am Ufer der Nied unter Weidengebüsch. - Von Hg. gesammelt. - M. (S.) -

2. L. angulatus $M \mathrm{hm}$. = angusticollis Thoms. nec Hummel. - An faulem Holz, an schimmeligen Brettern in Gärten bei Fr. Soden und Königstein. - Hierher auch die Frankfurter und Königsteiner rugicollis in S. V. - Bei Fr. auch von B. und Hg. gef. Wlb. (Sch.) [Reitter vid.] -

[NB. L. alternans Mhm. ist mir nicht aus dem Gebiet bekannt und besitze ihn überhaupt gar nicht, er kommt nur im Norden vor. - Die so bezeichneten Stücke in S. V. waren angulatus. - Viele der Mannerheim'schen Arten sind zweifelhaft.] -

\section{Coninomus Thomson.}

1. C. carinatus Gyll. Thoms. = incisus Mhm. - Bei Fr. an schimmelndem Holz s. - Taunus an Schwänmen im September. 1 Stück am 2. Mai 1861 von B. gef.; auch von Hg. gesammelt. - 


\section{Enicmus Thomson.}

1. (Conithassa Thoms.) hirtus Gyll. - s. s. Bei Fr. einmal und bei Biebrich 1 Stück $>7$. an Baumpilzen von C. H. gef. Fr. (Hg.) - Seltene Art. -

2. (C.) minutus L. - Im ganzen Gebiet an Schimmel unter Brettern, an Fässern in Kellern, in Bäumen an Schwämmen. - Fr. Einmal auch aus dem Raupensack von Coleophora siccifolia $<5$. entwickelt. - (L. assimilis Mhm. - anthracinus Mhm. - scitus Mhm. und minutissimus Mhm. sind leichte Varietäten dieser Art). - Fr. (Hg.) - Wlb. (Sch.) [Reitter vid.] -

3. E. rugosus Hbst. - Unter Rinden bei Fr. z. s. gef. (L. rugipennis und planatus Mhm. sind Varietäten.) - Hierher planatus des S. V. - Fr. (Hg.) - Wlb. (Sch.) [Reitter vid.] -

4. E. transversus Oliv. - Im Fr. Gebiet s. h. in Genist, unter Laub. - Soden. - Nauheim auch auf Salzboden. - Auf Buchenklafterholz im Fr. Wald n. s. von B. und Hg. gef. - Wlb. (Sch.) [Reitter vid.] -

5. E. carbonarius Mhm. - An geschlagenem Eichenholz im Fr. Wald im Juni. Bei Fr. h., so am 6. April 1860 von B. gesammelt. - M. (S.) -

\section{Cartodere Thomson.}

1. C. elongata Curtis = angustata Steph. - Aus Fichtenzapfen aus dem Fr. Wald $=5$. entwickelt; an geschlagenem Buchenholz. - [Hierher: clathratus in S. V. aus Fr.] -

2. C. ruficollis Mrsh. $=$ liliputanus Villa $=$ collaris Mhm. (exilis, concinna und nanula Mhm. sind Varietäten). - Fr. und Taunus in Schwämmen s. - G. (L.) - In wenigen Exemplaren an Steinen auf verrottetem Mist am Röderberg von B. gef. -

3. C. filiformis Gyll. = parallela Mhm. - Aus alten Epheuzweigen $>$ 7. von C. H. erzogen. - Fr. auch von Hg. gef. - M. (S.) -

\section{Dasycerus Brongniart.}

1. D. sulcatus Brongn. - Von C. H. s. s. bei Fr. und Ems unter Moos an schimmelndem Holz gef. - In mehreren Exemplaren unter einem Stein im Fr. Wald am Königsbrünnchen von B. gef. Bei Isenburg im Fr. Wald von Hg. aus Moos gesiebt. - M. (S.) - 


\section{Corticaria Marsham.}

1. C. pubescens Gyll. - Fr. im Juni von Epheu geklopft. Schmitterhof bei Giessen. - Fr. (Hg.) - Eichberg im Rheingau (Richter). - Budenheim bei Mz. (C. H.) -

2. C. pilosa Motsch. var. piligera Mnhm. - Einmal bei Wlb. von' Sch. gef. [Reitter vid.] -

3. C. fulva Comolli $=$ crenicollis $M \mathrm{Mm} .=$ hirtella Thoms. - 2 Ex. in einer Kellerruine bei der Ziegelhütte am Fr. Wald von C. H. gef. - Fr. (Hg.) -

4. C. cylindrica Mhm. - Fr. Wald an der Bruchschneisse in sandigen Gräben unter Laub $>4$. - Ems. - Soden im August. Fr. (Hg.) -

5. C. umbilicata Beck = umbilicifera Mhm. -3 Ex. bei Wlb. von Sch. gef. [Reitter vid.] -

6. C. impressa Oliv. = Iongicornis Hbst. - Fr. im März unter Riedgräsern, im April Enkheimer Torfgruben, Soden. - Ueberwintert unter Baummoos. - Fr. (Hg.) - Wlb. (Sch.) [Reitter vid.] -

Var. C. Reitter. (Flügeldecken rothbraun, die Naht und der Seitenrand schwarz. Die Stammart hat ganz schwarze Flügeldecken.) - C. H. fand 3 Stück im Dezember unter Laub und Moos bei Fr. - [Hierher die als bella Redtb. (aus Fr.) in S. V. erwähnten Stücke.] -

Var. badia Mhm. = campicola Mhm. (ganz gelb) von C. $\mathrm{H}$. - einmal bei Fr. im Genist im Dezember gef. -

7. C. serrata Payk. -- Fr. Wald $=6$. an Eichenschwämmen, Rödelheimer Wald $=8$. - Häufige Art. - Fr. (Hg.) -

8. C. bella Redtb. $=$ Heydeni Reitt. ol. in litt. - Einmal $<5$. aus dürrem Fr. Waldholz von L. H. erzogen. Reitter kennt in seiner Monographie, Stettiner Entom. Zeitung 1875, nur dies eine Stück von dieser Art. -

9. C. Mannerheimi Reitter $=$ Iongicollis Mhm. (nec. Zett.) - Zweimal $=5$. von C. H. in überwinterten Fichtenzapfen aus dem Fr. Wald erzogen. - [Hierher longicollis Zett. in S. V., welche (= formicetorum Mhm.) ich nur aus Erlangen besitze. Auch badia aus Fr. in S. V. hierher.] -

10. C. linearis Payk. - Von Sch. zweimal bei Wlb. gef. [Reitter vid.] - 
11. C. obscura Bris. - Bei Bieber (bei Giessen) einmal und 1 Stück bei Fr. von C. H. $>8$. gef. - Sonst nur aus Frankreich, Böhmen und Mähren bekannt. - Fr. (Hg.) -

12. C. elongata Hummel. - An Hecken bei Soden im Juni und Juli. - Rödelheimer Wald. - Frankfurt in der Stadt am Fenster gef. — Auch von Hg. gesammelt. - Wlb. (Sch.) [Reitter vid.] —

\section{Melanophtha.Ima Motschulsky.}

1. M. gibbosa Mhm. = impressa Marsh. - Im Gebiet auf Hecken und Bäumeı s. h. - Fr. - Offenbach. - Soden auf Kiefern. - Torfwiesen bei Enkheim. - Fr. (Hg.) - Wlb. (Sch.) [Reitter vid.] - Einmal im Nest der Formica rufa bei Münster im Taunus 2. April 1876. - M. (S.) -

2. M. transversalis Gyll. = brevicollis Mhm. - Fr. h. Griesheim am Main. - Schmitterhof bei Giessen. - Hierher die in S. V. als crocata benannten Stücke. - M. (S.) -

3. M. distinguenda Com. - Soden und Ems im Gras von C. H., in altem Buchenholz im Fr. Wald von L. H. und Hg. gef. - Wlb. (Sch.) [Reitter vid.] -

4. M. similata Gyll. - Einmal von C. H. auf Fichten bei Königstein $>6$. gef. [parvula und subtilis Mhm. sind Varietäten]. - Die Fr. und Salzhausener Stücke in S. V. gehören zu fuscula; parvula von Fr. sind gibbosa und fuscula. - Die Art wurde bei Fr. auch von Hg. gesammelt. - Wlb. (Sch.) [Reitter vid.] -

5. M. fuscula Hummel. - Fr. h. an vegetabilischen Dunghaufen und unter Moos, von uns und Hg. gef. - Wlb. (Sch.) [Reitter vid.] -

Var. trifoveolata Redth. - Einmal von C. H. bei Fr. gef. -

6. M. truncatella Mhm. - Fr. einzeln an sandigen Orten im Herbst gef., auch von Hg. -

\section{E. CERYLONINI. \\ Cerylon Latreille.}

1. C. histeroides F. - Ueberall unter Buchen- und Eichenrinde. Fr. Wald $>3$. und $=8$. h. Auch h. in Mist und zweimal bei Formica rufa im Fr. Wald von B. gef. - Dlb. (Sch.) - M. (S.) - G. (L.) Wsb. in Buchenstrünken (K.). - 
2. C. deplanatum Gyll. - Aus dürrem Fr. Waldholz $<5$. erzogen, auch von St. im Fr. Wald gesammelt. Das in S. V. erwähnte Fr. Exemplar von angustatum Er. gehört hierher. - M. (S.) - G. (L.) = 5. dürres Aspenholz Fr. (C. H.) -

3. C. Fagi Bris. - Fr. 2 Stück. [Reitter vid.] -

\section{F. MYCETAEINI.}

\section{Symbiotes Redtenbacher.}

1. S. pygmaeus Hampe. - Einmal in grosser. Anzahl im August und September an schimmeligëm Buchenholz aus dem Fr. Wald entwickelt. - Bei Mz. vor dem Raimundithor an alten Linden von M. S. 3 Stück gef. - M. (S.) -

\section{Mycetaea Stephens.}

1. M. hirta Marsh. - In Kellern in Fr. an schimmeligen Fässern h., auch in Königstein im Taunus. - Ein Stück auf verrottetem Mist 21. März 1860 am Röderberg von B. gef. -

\section{Alexia Stephens.}

1. A. pilifera Müll. - Bei Falkenstein $<6$. in Schwämmen an Baumwurzeln. - Am Feldberg unter Steinen $=5$. - Fr. sehr einzeln von C. H. gef. -

\section{G. LEIESTINI.}

\section{Mylmecoxenus Cherrolat.}

1. M. subterraneus Chevr. - Im Fr. Wald das ganze Jahr hindurch in den Nestern der Formica rufa h. Ebenso bei Münster im Taunus 2. April 1876. - 


\section{CRYPTOPHAGIDAE.}

(Die Sammlungen $\mathrm{v}$. $\mathrm{H}$ e yde $\mathrm{n}$ und $\mathrm{H}$ a ag sind vom Monographen $\mathrm{R}$ e it te r revidirt.)

\section{Aspidiphorus Latreille.}

1. A. orbiculatus Gyll. - Griesheim am Main im August, bei Fr. von C. H. und B. einzeln in faulen Schwämmen und mit dem Streifnetz gef. -

\section{Ephistemus Westwood.}

1. E. globulus Payk. = gyrinoides Mrsh. = ovulum Er. $=$ dimidiatus Sturm. - Im ganzen Gebiet unter Genist und vegetabilischen Dunghaufen h. - Fr. - Soden. - Wlb. (Sch.) [Reitter vid.] - M. (S.) -

\section{Atomaria Stephens.}

1. A. linearis Steph. = Bettae Macq. = pygmaea Heer. Fr. h., ebenso bei Biebrich im Rheingeniste. - Die Arten leben alle unter Laub, an Schimmel und in vegetabilischen Dunghaufen. - Fr. (Hg.) - Wlb. (Sch.) [Reitter vid.] - M. (S.) -

2. A. pulchra Er. - Von Hg. bei Fr. gef. -

3. A. Herminea Reitt. - Von Sch. bei Wlb. gef. [Reitter vid.] -

4. A. procerula Er. - Von C. H. einmal bei Fr. gef.; als elongatula in S. V. erwähnt. -

5. A. nigriventris Steph. = nana Er. - Im Gebiet n. s. Griesheim. - Fr. von C. H. und Hg. gef. - Wlb. (Sch.) [Reitter vid.] - M. (S.) -

6. A. plicicollis Mäkl. = umbrina Er. - Von Sch. bei Wlb. gef. [Reitter vid.] -

7. (Anchicera Thoms.) pulchella Heer $=$ munda Er. - Fr. s. von C. H. an Fässern in Kellern gef. -

8. (A.) nigripennis Payk. - Fr. mit der vorigen Art von C. H. häufiger und von v. Twardowski in einem Baumstumpf im Schwanheimer Wald gesammelt. - Friedberg (Fuhr). -

9. (A.) gravidula Er. - Von Hg. bei Fr. gef. - 
10. (A.) fuscata Schh. = rufa Waltl. - Im Gebiet n. s. Fr. - Entensee bei Rumpenheim, Soden, Nauheim. - Hierher salsicola von Fr. in S. V. und ebenso gravidula. - Fr. (Hg.) - Wlb. (Sch.) [Reitter vid.] -

11. (A.) pusilla Payk. - Fr. n. s., auch von Hg. gef. - Soden im Taunus. - Wlb. (Sch.) [Reitter vid.] -

12. (A.) atricapilla Steph. = nigriceps Er. = rufa Heer. Im Fr. Wald an der Oberensaustiege von C. H. gef. -

13. (A.) nitidula Heer = basalis Er. - Fr. 2 Ex. von C. H. gef., einmal auch von Hg. -

Var. Rhenana Krtz. - Einmal von C. H. bei Fr. gef. --

14. (A.) mesomelas Hbst. - Fr. unter Schilf an den Kettenhöfen, Rödelheimer Wald; auch in Anzahl in dem Neste eines Rohrsängers $>8$., jedoch nur einfarbig gelbe Stücke, von C. H. gef. Bei Fr. am 8. April 1861 von B. s. gesammelt, auch von Hg. -

15. (A.) gutta Steph. = sellata Heer. - Zweimal im Dezember unter Baummoos von C. H. bei Fr., auch von Hg. gef. -

16. (A.) fuscipes Gyll. = concolor Märk. - Zweimal bei Fr.; 1 Stück bei Soden.

17. (A.) turgicia Er. - Von Hg. bei Fr. gef. -

18. (A.) analis Er. = atra Steph. = cognata Sturm. - Im ganzen Gebiet s. h. - Fr. - Rődelheim. - Mo. - Soden. - Hierher cognata Er. in S. V. - Fr. (Hg.) - Wlb. (Sch.) [Reitter vid.] -

19. (A.) apicalis Er. - Im Goldsteinforst im Fr. Wald in einem Nest der Formica rufa einmal von C. H. gef., auch von Hg. gesammelt. -

20. (A.) ruficornis Marsh. = terminata Com. - Fr. einmal an aufgeschichtetem Holz und 1 Stück bei Nauheim; auch von Hg. bei Fr. gef. - Wlb. (Sch.) [Reitter vid.] -

\section{Cryptophagus Herbst.}

1. C. Lycoperdi Hbst. - In Boristen h. im Herbst. - Fr. Soden. - Hohe Mark im Taunus. - Wlb. (Sch.) - Fr. (Hg.) M. (S.) -

2. C. pilosus Gyll. - Fr. Wald z. s. - Hierher die von L. H. in S. V. erwähnten labilis. - Wlb. (Sch.) [Reitter vid.] -

Ueber eine bei Wlb. von Sch. gefangene Cryptophagus-Art mit 
stark eingedrücktem Halsschild schreibt Reitter: „Wohl nur eine Monstrosität. Aehnelt der Var. punctipennis Bris., ist aber wohl nur ein pilosus." - Das Exemplar nun in Sammlung v. Heyden. -

3. C. Schmidtii Sturm = pilosus Heer. - Fr. je einmal von uns gef. -

4. C. setulosus Sturm. - Fr. und Soden je zweimal von C. H. im Juni und August gesammelt. - Wlb. (Sch.) [Reitter vid.] von $\mathrm{Hg}$. -

5. C. affinis Sturm. - Fr. 2 Stück von C. H. gef., später auch

6. C. cellaris Scop. = crenatus Sturm. - Fr. unter faulenden Vegetabilien im April s.; auch von $\mathrm{Hg}$. gef. -

7. C. acutangulus Gyll. - Im Fr. Gebiet aus dürrem Waldholz im Juni erzogen, n. s.; auch im Winter unter Rinden. - Mo. - An den Wänden von Häusern h. in Fr., besonders am Grüneburgweg von B. gef. - Auch von Hg. gesammelt. - M. (S.) - Wlb. (Sch.) - [Reitter vid.] -

8. C. fumatus Marsh. - Fr. Wald an den Wänden des Forsthauses $=10$. - Cronthal im Taunus. -

9. C. quercinus Krtz. - Fr. zweimal von L. H., Cronthal $<10$. einmal von C. H. gef. [Hierher affinis von Cronthal in S. V.] -

10. C. badius Sturm = denticollis Reitt. ol. - In der Oelmühle bei Königstein in Anzahl an schimmeligem Holz gef. Seltene Art. - Mehrere Exemplare am 8. April 1860 bei Fr. von B. gesammelt. Auch Hg. fand diese Art bei Fr. -

11. C. Populi Payk. = grandis Krtz. - Grosse Seltenheit. Einmal bei Fr. an einem faulen Lindenstamm $>6$. von C. H. gef. -

12. C. hirtulus Krtz. = badius Reitt. ol. - Am Fr. Forsthaus einmal im August und $>6$. 1 Ex. bei St. Goarshausen von C. H. gef. -

13. C. distinguendus Sturm. - Von Hg. bei Fr. gef. -

14. C. scutellatus Newm. = bicolor Sturm. Er. - Von Sch. bei Wlb. gef. [Reitter vid.] -

15. C. saginatus Sturm. - In Pilzen im Fr. Wald. - Soden. - Cronthal. - Auch bei Formica rufa (Scriba beobachtete dasselbe). - Fr. (Hg.) - Wlb. (Sch.) [Reitter vid.] - 
16. C. dentatus Hbst. = pallidus Sturm. - Fr. Wald in Schwämmen und einmal in unglaublicher Menge in einer Kiste mit alten Zwetschen. - Hofheim in Weidenblüthen. - Sehr häufige Art. — Soden. - Wlb. (Sch.) [Reitter vid.] - Fr. (Hg.) - M. (S.) -

17. C. cylindrus Kiesw. $=$ parallelus Bris. - Dreimal $=5$. aus' altem Fr. Waldholz erzogen und einmal $>6$. am Fenster gef. von L. H. Neu für Deutschland. Seither nur aus Südfrankreich und vom Parnass in Griechenland bekannt. [Hierher subfumatus in S. V.] -

18. C. scanicus L. - Ueberall in Europa h. - Fr. - Soden; unter Rinde, an Pilzen und faulem Holz. - Bei Fr. einmal von B. gef., auch von Hg. gesammelt. - Wlb. h. (Sch.) - Eichberg im Rheingau (Richter). -

Var. patruelis Sturm. - Einmal bei Fr. gef. -

19. C. subdepressus Gyll. = lapponicus Stm. = pubescens Sturm. - Bei Fr. und Soden gef. s. s. - Fr. (Hg.) -

20. C. Heydeni Reitter. - Ein Exemplar (das einzig bekannte) dieser neuen schönen Art fand L. H. auf der ehemaligen Pfingstweide (dem jetzigen zoologischen Garten) in Fr. an alten Linden. - [In S. V. als Paramec. abietis erwähnt.] -

21. C. vini Panz. - 4 Stück von C. H. bei Fr. gef. -

22. C. n. sp.? - Sch. fing bei Wlb. 1 Stück einer wohl neuen Art. - Reitter schreibt darüber: „Jedenfalls ist er zu einer Beschreibung, weil schlecht erhalten, untanglich. - Vielleicht ist es eine Varietät des cellaris, was man beurtheilen könnte, wenn die Behaarung nicht abgerieben wäre." - Das Exemplar nun in Sammlung v. Heyden. -

\section{Micrambe Thomson.}

1. M. villosa Heer = pilosula Er. - Viermal im Fr. Wald (Wartforst) < 7. mit dem Streifnetz von C. H. gef. - Sauerthal am Rhein < 6. zweimal (C. H.). -

\section{Paramecosoma Curtis.}

1.. P. melanocephala Hbst. - Je einmal von C. H. und B. bei Fr., zweimal bei Wlb. von Sch. gef. -

\section{Emphylus Erichson.}

1. E. glaber Gyll. - Im Fr. Wald aus Nestern der Formica rufa von uns und B. n. s. s. gesiebt, - 


\section{Antherophagus Latreille.}

1. A. nigricornis F. - Von C. H. $>6$. bei Hofheim zweimal auf Himbeerblüthen gesammelt. - Fr. (Hg.) - Wlb. 1 Ex. (Sch.) Wsb. (K.) -

2. A. silaceus Hbst. - Fr. zweimal im Juli auf Blüthen und einmal auf der Bieberer Höhe bei Offenbach von C. H. gef. - Bei Wlb. 2 Stück von Sch. gesammelt. - Am 20. Juli bei Wsb. hinter der Walkmühle und am 3. August bei Mo. im Graben am Rhein gef. (K.) -

3. A. pallens 01. - Im Schwanheimer Wald mehrmals von B. mit dem Streifnetz gef. - Bei Wlb. 3 Ex. (Sch.) - G. (L.) -

\section{Engis Fabricius.}

1. (Compocerus Bedel) glabra Schaller $=$ sanguinicollis $\mathbf{F}$. - Bei Fr. sehr vereinzelt von M. S. und v. Twardowski, auf der Höchster Chaussee unter Steinen bei Pferdemist am 3. Juni 1855 von L. H. gef. - Ein Exemplar im Genist an den Röderhöfen bei Fr. am 3. April 1860 von B. gef. - G. (L.) -

2. E. humeralis F. - An Schwämmen an Weiden, Nussbäumen s. h. - Fr. - Soden. - Ems. - Unter Kirschrinde am Sachsenhäuser Berg 12. Mai 1860 von B. gef. - Wlb. (Sch.) - Fr. (Hg.) - M. (S.) - G. (L.) - Mo. an Weidenschwämmen 23. Mai (K.) -

3. E. rufifrons F. - Bei Fr. einigemal von C. H. gef., mehrfach von Hg. -

\section{Sphindus Chevrolat.}

1. S. hispidus Payk. = dubius Gyll. = Gyllenhali Chevr. $=$ humeralis Mink. - Im Juni im Fr. Wald in Staubpilzen an Kiefernstöcken von C. H. gef. Bei Fr. äusserst selten von B. beobachtet. M. (S.) - 


\section{CISSIDAE.}

(Die Sammlung $\nabla$. Heyden ist von dem Monographen Abeille de Perrin revidirt.)

\section{Cis Latreille.}

1. C. Boleti Scop. - In Boletus-Arten an Bäumen h. - Fr. auch > 3. von B. gef. - Wlb. (Sch.) - M. (S.) -

Var. rugulosus Mellié. - Fr. einmal und. 1 Stück auf dem Niederwald bei Rüdeshein $=4$. von C. H. gef. [In S. V. als setiger von Rüdesheim erwähnt.] -

2. C. setiger Mellié $=$ plagiatus Thoms. - Unter modernder Buchenrinde = 3. dreimal von C. H. gef. - Wlb. (Sch.) -

3. C. micans Hbst. - Ein Stück bei Fr. gef.; desgleichen bei Wlb. (Sch.) -

4. C. hispidus Payk. = crenatus Sahlbg. - Im Gebiet n. s. - Fr. - Niederwald bei Rüdesheim = 4. - Wlb. (Sch.) - M. (S.) -

5. C. striatulus Mell. = flavipes Luc. - In faulem Erlenholz zweimal von L. H. im Jnli gef. -

6. C. comptus Gyll. - Von C. H. bei Fr. ges. - Wlb. (Sch.) -

7. C. bidentatus Oliv. - Von L. H. einzeln bei Fr. gesammelt. -

8. C. nitidus Hbst. - Bei Fr. einzeln von C. H. gef. -

9. C. Alni Gyll. - Bei Fr. einige Stück in Baumpilzen $>2$. gef. -

Var. recticollis Abeille. - Fr. einmal in dürrem Waldholz $>$ 7. gef. -

10. C. castaneus Mell. - In Eichen-, Linden- und Nussbaumschwämmen h. - Fr. - Soden. - Wlb. (Sch.) - M. (S.) -

[C. quadridens Mell. aus Fr. in S. V. ist zu streichen, ich besitze die Art nicht.]

11. C. festivus Panz. - Bei Wlb. von Sch. gesammelt. -

\section{Rhopalodontus Mellié.}

1. R. fronticornis Panz. - Wlb. (Sch.) - Bei Fr. Männchen und Weibchen durch Hg. gesammelt. - 


\section{Ennearthron Mellié.}

1. E. affine Gyll. - An Baumschwämmen im Fr. Wald und in der Hohen Mark im Taunus n. s. [Hierher die in S. V. erwähnten R. fronticornis von Frankfurt.] -

2. E. cornutum Gyll. - Schmitterhof bei Giessen $>4$. in dürrem Hainbuchenholz 4 Stück von C. H. gef. - Wlb. (Sch.) -

\section{Octotemnus Mellié.}

1. O. glabriculus Gyll. - Bei Fr. dreimal von C. H. gef. Wlb. (Sch.) -

\section{TELMATOPHILIDAE.}

\section{Diplocoelus Guérin.}

1. D. fagi Guér. - Im Fr. Wald unter Buchenrinde an der Oberräder Landwehr von L. H., M. S. und B. öfter gef. - Auch schon von C. H. im Gebiet gesammelt. - Fr. (Hg.) -

\section{Psammoechus Boudier.}

1. P. bipunctatus F. - An sumpfigen Gegenden unter Schilf s. Kettenhöfe, Enkheimer. Torfhrüche, an letzterem Ort auch von B. gef. - Fr. (Hg.) -

\section{Telmatophilus Heer.}

1. T. Sparganii Ahr. - Im Mai in Sümpfen Abends auf Sparganium n. S., z. B. Enkheimer 'Torfbrüche. - Fr. 8. April 1860 (B.). - M. (S.) -

2. T. Typhae Fallén. - Mit dem vorigen ebenso. - Fr. 8. April 1860 (B.). - M. (S.) — Wsb. an der Tränke im Sommer (K.). -

3. T. Caricis Sturm. - Wie die beiden vorigen. - Hg. fing die drei Arten bei Fr. - 


\section{EROTYLIDAE.}

\section{Triplax Paykull.}

1. T. Russica L. - Im Fr. Wald an Baumschwämmen n. S. im Juni, besonders an Weiden, auch von Hg. gef. - M. (S.) - Wsb. in Buchenstrünken unter der Rentmauer im Juni (K.). -

2. T. aenea Payk. - L. H. besitzt ein von v. Twardowski im Rheingau gef. Exemplar. - Dlb. (Sch.) -

\section{Tritoma Fabricius.}

1. T. bipustulata F. - Im Fr. Wald und im Taunus $>$ 4. an trockenen Baumpilzen ïberwintert. - Hohe Mark und Hofheim im Taunus im Mai und Juni unter Eichenrinde. In Baumpilzen, besonders an Buchen von B. h. gef. - Bei Münster im Taunus in Menge 2. April 1876 (L. H.) - G. (L.) -

\section{ENDOMYCHIDAE.}

\section{Endomychus Panzer.}

1. E. coccineus L. - Im Fr. Wald früher von C. H. unter schimmeligem $\mathrm{Holz}$ und in Bovisten gef. - Im Thal hinter Oberscheld im Westerwald im Mai (K.). -

\section{Mycetina Mulsant.}

1. M. cruciata Schall. - Einmal von C. H. bei Fr., auch 1 Stück ron Hg. im Fr. Wald gef. - 


\section{Lycoperdina Latreille.}

1. (Golgia Muls.) succincta L. - Im Fr. Wald im März überwintert in Bovisten gef. - Die Larve und Puppe findet sich $>5$., der Käfer entwickelt sich $>6$. und hat nach der Beobachtung von C. H. einen eigenthümlichen Knoblauchsgeruch. - Offenbach. -

2. L. Bovistae F. - Fr. Wald und überall, wo Boviste im November und Dezember in dem Staub derselben überwinternd. - Von B. bei Homburg gesiebt. - G. (Prof. Rossmann). -

\section{COCCINELLIDAE.}

\section{Hippodamia Mulsant.}

1. H. tredecimpunctata L. - Fr. s. s. - Cronthal $<10$. von C. H. gef. Von B. nur einmal bei Fr., von Hg. und St. mehrfach gef. - Dlb. (Sch.) - G. (L.) - Wsb. Kieskaut 2. August (K.). -

Anisosticta Redtenbacher.

1. A. novemdecimpunctata L. - Fr. s. im Juli, auch von Hg. und St. gesammelt. - G. (L.) - Wsb. im Juni (K.) -

\section{Coccinella Linné.}

1. (Adonia Muls.) mutabilis Scriba. - Fr. - Hofheim. Fischbach. - Soden. - Mo. h. auf Pflanzen im Sand. Auch 1 Ex. ohne Flecken auf den Flügeldecken. - Fr. (Hg.) - Wlb. (Sch.) G. (L.) - M. (S.) - Einmal am Grossen Mannstein (Stauffen) im Taunus 2. April 1876 mit nur je 2 Flecken an der Spitze der Flügeldecken (L. H.). -

2. (Adalia Muls.) obliterata L. $=$ livida Degeer $=$ M. nigrum Illig. - Im Fr. Wald unter Nadelholzrinde, meist Pinus picea, im Winter, aber auch am Johannisberg bei Nauheim in Kirschblüthen $=5$., wo keine Fichten in der Nähe waren. - Variirt von hellgelber bis fast schwarzer Oberseite. - Fr. (Hg. und St.) - G. (L.) - Dlb. (Sch.) - 
3. (A.) Bothnica Payk. - Einmal von C. H. auf Pinus picea $>$ 7. bei Fr. gef. -

4. (A.) bipunctata L. - Sowohl die Form mit je einem schwarzen Fleck auf ziegelrother Flügeldecke, als auch die andere extreme Form, mit schwarzen Flügeldecken und je 2 rothen Flecken, gleich h. und stets zusammengefunden, oft auch in Begattung; dann ist die helle Form meist das Männchen. Uebergänge in der Farbe sind seltener. - Fast alle Coccinellen variiren unglaublich in der Farbe der Oberseite; wegen der speciellen Aufzählung verweise ich auch in der Folge bei den Coccinellen auf die verdienstvolle Arbeit Bach's im 3. Bande seiner Käferfauna. Von bipunctata beschreibt er zwölf Varietäten. Die Art überwintert oft in den Häusern oder im Freien unter Rinden. - Im ganzen Gebiet. - Fr. - Soden. - Dlb. Wlb. (Sch.) - G. (L.) - Wsb. an der Wellritz 4. Juli 1851 (K.). - Schierstein am Rhein 17. Mai (K.). -

5. (A.) undecimnotata Schneider. - Fr. s. von C. H. gef., bei Mo. von L. H. im Juli; bei Fr. einmal von B. gesammelt. - M. (S.) -

6. (Harmonia Muls.) marginepunctata Schaller. - Unter Fichtenrinde z. s. im Winter. - Fr. - Variirt von einfarbigen Flügeldecken zu 1. 3. 3. 1. Punkten auf einer Decke. - Fr. (St.) - G. (L.) - Mo. an Kiefern 20. Juli (K.). -

7. (H.) impustulata L. - Variirt von 2. 3. 3. schwarzen Flecken auf gelben Grund, die auf die verschiedenste Weise mit einander verbunden sein können, bis zu solcher Ueberhandnahme der schwarzen Farbe, dass auf der ganzen Oberseite nur noch die Seitenränder des Halsschildes schmal gelb bleiben. Lebt auf Eichen im Fr. Wald. Mainkur. - Soden. - Bingen. - Bei Fr. s. h. von Hg., St. und B. gef. - Wlb. (Sch.) - M. (S.) - G. (L.) - Wsb. 8. August (K.). -

8. (Coccinella Muls.) quatuordecimpustulata L. - Wald von Soden nach Altenhain. - Wsb. - s. h. bei Fr., z. B. 21. März 1860 von B. gef., auch von Hg. - Dlb. Wlb. (Sch.) - M. (S.) - G. (L.) -

9. (C.) variabilis L. - Variirt von einfach gelb bis ganz schwarz durch unzählige Varietäten. Bach führt 7 Hauptfarbenvarietäten an. Durch alle erdenklichen Verbindungen und Ineinanderfliessen von 1-6 Punkten oder Fehlen von einem oder mehreren derselben ist der Variationsfähigkeit dieser Art der grösste Spielraum gegeben; so führt z. B. Mulsant in seiner Bearbeitung der Coccinellen der französischen Fauna allein von dieser Art 47 verschiedene Varietäten an. Die Art ist leicht kenntlich (wenigstens bei uns, im Süden kann dieses Merkmal ver- 
schwinden) an einer zusammengekniffenen Querfalte vor der Spitze der Flügeldecken. - Ueberall s. h. auf Eichen, auch in vielen Varietäten auf Pflaumenbäumen, in Soden gef., wo die Käfer eifrig den Blattläusen nachgingen. - Fr. - Falkenstein, Hohe Mark im Taunus. - Fr. (Hg.) - M. (S.) - G. (L.) - Dlb. Wlb. (Sch.) - Mo. vom 11. Juli bis 3. October (K.). -

10. (C.) hieroglyphica L. - Fr. je einmal von C. H., Hg. und B. gef. - Wlb. (Sch.) - M. (S.) - G. (L.) - Gonsenheim bei Mz. an Kiefern 5. September (K.). -

Var. areata Panz. - Wlb. (Sch.) -

11. (C.) quinquepunctata L. - Ueberall h. - Variirt s. durch Fehlen eines oder mehrerer Punkte. - Fr. auch von Hg. gef. - Dlb. Wlb. (Sch.) - M. (S.) - G. (L.) -

12. (C.) septempunctata L. - In ganz Europa g. - Die Punkte werden oft kleiner und verschwinden zum Theil ganz. - Fr. - Bieberer Höhe bei Offenbach. - Taunus. - Fr. (Hg.) - M. (S.) - G. (L.) - Dlb. Wlb. (Sch.) -

\section{Halycia Mulsant.}

1. (Anatis Muls.) ocellata L. - Diese schön gezeichnete, grösste europäische Coccinellide findet sich s. bei uns auf Kiefern. - Mo. Fr. Wald. - Königstein auf Fichten im Juni. Punkte 1. 3. 3. 1. auf jeder Decke normal. - In Bockenheim fand L. H. an einem Haus am 20. April 1874 eine Varietät, bei welcher der Naht- und Mittelfleck der 3. Reihe fehlt und der Mittelfleck der 2. Reihe sehr klein ist. Fr. (Hg.) - St. fand 1 Ex. mit Flügeldecken ohne Flecken und 1 Ex., bei welchem nur der Schulterfleck vorhanden ist. - M. (S.) - G. (L.) - Mo. an Kiefern 20. Juli 1851 (K.). - Dlb. (Ko.) -

2. (Mysia Muls.) oblongoguttata L. - Lebt auf Kiefern, an manchen Orten n. s. s., z. B. Mo. - Bieberer Höhe bei Offenbach. Altenhain bei Soden von April bis Juli. - Von B., Harer und Fresenius je einmal im Fr. Wald gef., auch von Hg. und St. mehrmals. Wlb. (Sch.) - M. (S.) - G. (L.) - Mo. an Kiefern 2. Juni (K.). - Wsb. hinter dem Kursaal im Mai (K.). -

3. (Sospita Muls.) tigrina L. - (Die weissgelben Flecken stehen auf schwarzem Grund.) Fr. und kleine Feldbergthal $>5$. je einmal auf Erlen von C. H. gef. - Dlb. (Ko.) - H. Boss streifte bei Fr. 
am Eisenbahndamm am Grindbrunnen. 1 Stück nach Mittheilung von B. - Fr. (Hg., St.) - G. (L.) - Wsb. an der Wellritz auf Erlen 30. Juni (K.). -

Var. vigintiguttata F. - (Die weissgelben Flecken stehen auf rothgelbem Grund.) - Soden $>10$. von Erlen und Cronthal $<9$. von C. H. je einmal von Haseln geklopft. -

4. (Calvia, Muls.) quatuordecimguttata L. - Auf Erlen im Gebiet n. s. - Soden, Falkenstein, Wsb. - Nauheim. - Schmitterhof bei Giessen. - Im Mai und Juni am häufigsten. - Fr. s. (Hg., B. und St.) - Wlb. (Sch.) - M. (S.) - G. (L.) - Bei Schierstein am Rheinufer auf Weiden in Begattung 17. Juni (K.). -

5. (C.) decemguttata L. - s. - Im Fr. Unterwald $=5$, einigemal von C. H. gef., auch von Hg. gesammelt. -

6. (C.) bisseptemguttata Schaller. - $3 \mathrm{Ex}$. bei Mo. gef. (K.) -

7. (Myrrha Muls.) octodecimguttata L. - Unter Fichteurinde im Fr. Wald im Winter s. von C. H., Hg. und St. gef. - G. (L.) Wlb. (Sch.) - Mo. von 19. Juni bis 22. August (K.). -

8. (Halycia Muls.) sedecimguttata L. - Bei Fr. n. s., auch von Hg. und St. gef. - G. (L.) - Wsb. auf Birken an der Platter Chaussee (K.).

9. (Vibidia Muls.) duodecimguttata Poda. - Einmal von C. H. bei Soden . auf Eichen $<$ 9. gef.; auch bei Fr. von Hg. - Wlb. (Sch.) -

10. (Thea Muls.) vigintiduopunctata L. - Ueberall n. s. Fr. - Soden. - Wlb., woselbst auch die Larve h. im Gras gestreift wurde (Sch.). - M. (S.) - G. (L.) - Wsb. auf Evonymus von 20. August bis 18. September (K.). -

11. (Propylaea Muls.) quatuordecimpunctata L. - Die schwarzen Flecken auf gelbem Grund können so ineinander fliessen und grösser werden, dass die Flügeldecken schwarz mit gelben Flecken erscheinen. - Fr. h., auch von Hg., B. und St. gef. - Dlb. Wlb. (Sch.) - G. (L.) - Gonsenheim bei Mz. 11.' Juli in Begattung (K.). -

\section{Micraspis Redtenbacher.}

1. M. duodecimpunctata L. - Fr. h. im Genist. - Im frühesten Frühjahr unter Steinen am Main bei der Gerbermühle von B. gesammelt, auch von Hg. gef. - Dlb. Wlb. (Sch.) - G. (L.) - 


\section{Chilocorus Leach.}

1. C. renipustulatus Scriba. - Fr. z. s. - Ems. - Wlb. (Sch.) - M. (S.) - G. (L.) - Wsb. (K.) -

2. C. bipustulatus L. - Fr. h. auf Kiefern. - Wlb. (Sch.) M. (S.) - G. (L.) - Gonsenheim bei Mz. auf Kiefern 5. September (K.). -

\section{Exochomus Redtenbacher.}

1. E. auritus Scriba. - Fr. n. s. - Auf Nadelholz von B. h. gef., auch von Hg. gesammelt. - M. (S.) - G. (L.) -

2. E. quadripustulatus L. - Fr. Wald auf Wachholder n. s. Bieberer Höhe bei Offenbach. - Bei Fr. auch von Hg. und St., bei Wlb. von Sch. gef. - M. (S.) - G. (L.) - Mo. auf Kiefern von 4. August bis 22. September 1851 (K.). -

\section{Hyperaspis Redtenbacher.}

1. H. campestris Hbst. - Bei Fr. einzeln von C. H. gef. Friedberg (Fuhr). -

2. H. reppensis Hbst. - Fr. s. im Frühjahr; auch von Hg. gef. - Wlb. (Sch.) -

\section{Epilachna Chevrolat.}

1. E. undecimmaculata F. = Argus Fourc. - In manchen Gegenden auf der Zaunrübe (Bryonia dioica) n. s. $=6$. in Begattung gef., z. B. bei Bergen in Hecken. - Fr. auch von Hg. und B. s. s. gef. - Bei Dlb. und Wlb. von Sch. aus der Larve erzogen. - B. (S.) - G. (L.) - Wsb. Mühlweg in Juli auf Bryonia (K.). -

2. (Lasia Muls.) globosa Schneider. - Variirt sehr in der Anzahl der Punkte und Zusammenfliessen der Flecken. - Fr. n. s. auf Eichengebüsch. - Von Hg. und B. s. h. gef. - Von Sch. bei Dlb. h. auf Medicago sativa, von deren Blättern sie sich nebst der Larve nährt, gezogen; auch die ungefleckte Varietät. — G. (L.) -

\section{Cynegetis Redtenbacher.}

1. C. impunctata L. - (Die Flügeldecken glasig durchsichtig, flügellos). - Bei Königstein im Taunus in feuchten Wiesen unter Moos 
$<$ 11. s. und $>$ 5. bei Nauheim von uns gesammelt. - Friedberg (Fuhr). - Von Harer und Hg. bei Fr. h. gef. - Wlb. in feuchtem Gras h. von Sch. gestreift. - Wsb. 30. Mai (K.). -

\section{Platynaspis Redtenbacher.}

1. P. villosa Fourc. - Unter Pappelrinde bei Fr. gef. Von B. s. h. auch unter Weidenrinde gesammelt. Auch $\mathrm{Hg}$. fand die Art im Gebiet. - Wlb. (Sch.) - G. (L.) -

\section{Scymnus Kugelann.}

1. (Nephus Mls.) quadrilunulatus Illig. - Bei Fr. und Schmitterhof bei Giessen auf Epheu von C. H. im Herbst gef. - Von B. bei Fr. s. h., auch von Hg. und St. gesammelt. - Wlb. (Sch.) - G. (L.) -

2. (N.) Redtenbacheri Muls. - 1 stück an einer Bretterwand bei Fr. im Mai von C. H. gef. - Bei Wlb. h. von Sch. gef. -

3. (N.) biverrucatus Panz. - Fr. im October. - Bei Enkheim auf Eichengebüsch im Juli. - Wlb. (Sch.) - G. (L.) - Fr. 6. April 1860 (B.). -

4. (Scymnus Muls.) nigrinus Kug. - Fr. - Griesheim. Von Wiesengebüsch bei Falkenstein im Mai geklopft. - Schmitterhof bei Giessen. - Fr. (Hg.) — Wlb. (Sch.) - Mo. auf Kiefern 9. Mai (K.). -

5. (S.) pygmaeus Fourc. - Fr. Wald von L. H. und Hg. n. s. gef. - Wlb. (Sch.) -

6. (S.) marginalis Rossi. - s. - Fr. einmal = 7., Ems 2 Stück von C. H. gef. - Bei Fr, auch von Hg., bei Wlb. von Sch. ges. -

7. (S.) Apetzii Muls. - Bei Ems 3 Stück von C. H. gef. G. (L.) -

8. (S.) frontalis F. - Fr. im Rebstockwald. - Ems. - Ueberall n. s. - Fr. von Hg. gef. - G. (L.) - Wlb. (Sch.) -

9. (Pullus Muls.) Abietis Payk. - Bei Wlb. von Sch., bei Fr. von Hg. gef. - G. (L.) -

10. (P.) fasciatus F. - In Gesellschaft der Blattlaus Aphis sambuci $>6$. von C. H. und von Hg. bei Fr. gef. -

11. (P.) arcuatus Rossi. - Von Hg. einmal bei Fr. gef. - G. (L.) -

12. (P.) impexus Muls. - , Von Hg. bei Fr. gef. -

13. (P.) scutellaris Muls. - Von Hg. bei Fr. gef. - 
14. (P.) discoideus Illig. - Auf Pinus sylvestris bei Griesheim am Main h. - Offenbach auf Juniperus im April, auch bei Fr. Soden auf Eichen. Die Käfer stellen wie alle Arten der Gattung den Blattläusen nach und sind desshalb nicht immer an dieselbe Pflanze gebunden. - Auch von Hg. bei Fr. gesammelt und von B. am 4. Juni 1860 gef. - M. (S.) - G. (L.) -

15. (P.) analis F. - Fr. einmal von C. H. gef., auch von Hg. - Wlb. (Sch.) -

16. (P.) haemorrhoidalis Hbst. - Fr. an der ehemaligen Pfingstweide, jetzt zoologischer Garten; im Rödelheimer Wald $=8$. öfter gef., auch von Hg. gesammelt. -

17. (P.) capitatus F. - Fr. im Juni von Epheu geklopft. Bingen. - Am Feldberg $<6$. öfter gef. - Fr. je einmal von B. und Hg. gef. - Wlb. (Sch.) - M. (S.) - G. (L.) -

18. (P.) ater Kug. - Fr. und Soden auf Kiefern, auch von Hg. gesammelt. - Wlb. (Sch.) $-<5$. Eppstein. -

19. (P.) minimus Payk. - Im Gebiet h. auf Eichen bei Soden. - Fr. im Genist und unter Rinden; auch von Hg. gef. - Wlb. (Sch.) -

\section{Rhizobius Stephens.}

1. R. litura F. - Unter diesem Namen wurden seither alle Rhizobius unseres Gebietes zusammengefasst, bis Seidlitz in seiner Fauna baltica 1872, p. 193, davon eine zweite Art ausschied. - Bei der ächten litura ist das Halsschild von der Basis nach vorn einfach, ohne Bogen, allmälig verengt; die Unterseite des Körpers ist schwarz mit schmalem gelbem Saum, der Körper ist kürzer und gewölbter. Sie ist bei uns z. s. (häufiger in Süd-Europa). L. H. besitzt aus dem Gebiet 1 Stück, auf der Bieberer Höhe bei Offenbach $=4$. auf Kiefern gef.; 1 Ex. aus Fr. von Fichten (und $1 \mathrm{Ex}$. von der Saline Salzhausen in der Wetterau). - Bei Wlb. h. nach Sch. - Auf der höchsten Spitze des kleinen Mannstein (Staufen) im Taunus an den Felsen 2. April 1876 (L. H.). Wsb. (K.) - > 8. Königstein. -

2. R. subdepressus Seidl. - Bei dieser Art ist das Halsschild von der Basis bis zur Mitte gleichbreit, von hier nach vorn ziemlich stark gerundet verengt; die Unterseite ist roth und die Mitte schwarz. Der Körper ist länglicher und flacher. - Bei der ersten Art hat das Prosternum zwischen den Vorderhüften zwei stark erhabene Randlinien, 
die sich vorn spitzwinkelig vereinigen; bei subdepressus sind diese Randlinien nur sehr fein, nach vorn undeutlich oder sehr fein und sich im Bogen verbindend. - Bei Falkenstein im Taunus $=5$. von Wiesengebüsch geklopft ; $<4$. von Juniperus bei Offenbach und bei Fr. unter Rinde von Pinus picea im November. - Bei Wlb. 4 Ex. von Sch. gef. - Wsb. (K.) -

\section{Coccidula Kugelann.}

1. C. scutellata Hbst. - Unter Rinden bei Fr. im April, unter Schilf bei Offenbach und Fr. im Herbst. - Enkheimer Torfbrüche. Von B. s. h. bei Fr. gesammelt, auch von Hg. und St. - Wlb. (Sch.) - Mo. Wassergraben im Mai (K.). -

2. C. rufa Hbst. - Fr. - Offenbach in Gesellschaft der vorigen. - Nur einmal bei Fr. von B. gesammelt, von Hg. und St. öfter. Nicht bei Wlb. (Sch.) - G. (L.) - Mo. mit der vorigen (K.). - Wsb. an der Wellritz 15. Juni (K.). -

\section{CORYLOPHIDAE.}

\section{Sacium Le Conte.}

1. S. obscurum Sahlb. = corticinum Thoms. - [Hierher die in den meisten Sammlungen sich befindenden S. (Clypeaster 01.) pusillum Gyll., welche nahe verwandte Art v. Heyden und Dr. Kraatz nur aus Schweden kennen.] - Fr. Wald an Buchenholz $=4$. von C. H. gef. - Auch in Süd-Europa. - Von B. s. bei Fr. gesammelt. -

\section{Sericoderus Stephens.}

1. S. lateralis Gyll. - Fr. an Pilzen in den Promenaden im Mai, unter Pflanzen auf der Erde im botanischen Garten, vegetabilische Dunghaufen von Mai bis October. - Soden. - Im frühesten Frühjahr unter Steinen auf verrottetem Mist am Röderberg 20. März 1860 von B. s. gef. - Wlb. (Sch.) - 


\section{Corylophus Stephens.}

1. C. cassidoides Mrsh. - Unter Schilf bei den Kettenhöfen bei Fr. - Enkheimer Torfbrüche. - Mit der vorigen Gattung zusammen nur 1 stück gef. von B. - Wlb. (Sch.) -

\section{Orthoperus Stephens.}

(Von L. v. Heyden wurde die Gattung revidirt. Berlin. Entom. Zeitschr. 1874, p. 116.)

1. O. brunnipes Gyll. = piceus Steph. = Dorcatoma Zusmaehusense Beck. - Im Fr. Wald an geschlagenem Buchenholz $=4$. n. s. -

2. 0. atomus Gyll. - Auf dem Schmitterhof bei Giessen einige Exemplare und im Fr. Wartforst einmal im Nest der Formica rufa. -

[Corticalis Redtb., nach S. V. im ganzen Gebiet, kennt L. H. nur aus Südfrankreich.] -

\section{TRICHOPTERYGIDAE.}

(Die Sammlung v. Heyden wurde vom Monographen Gill meister revidirt.)

\section{Ptenidium Erichson.}

1. P. laevigatum Er. - Schmitterhof bei Giessen. - Bei Fr. von C. H. und Hg. gef. -

2. P. pusillum Gyll. - Ueberall s. h. - Die Arten leben an faulenden Vegetabilien, unter Brettern an Schimmel. - Fr. -

3. P. apicale Er. - Fr. h. von uns und Hg. gef. -

4. P. fuscicorne Er. - Fr. 2 Stück von C. H., mehrmals von Hg. gesammelt. -

5. P. formicetorum Krtz. - L. H. fand 2 Ex. in Gesellschaft der Formica fuliginosa an alten Eichen bei den Oberräder Schiessständen. -

6. P. Gressneri Er. - Von Hg. n. s. bei Fr. gesammelt, - 


\section{$-134-$ \\ Ptilium Erichson.}

1. P. Kunzei Heer. - Fr. von C. H. und Hg. s. h. an faulenden Pflanzen; auch unter Steinen auf verrottetem Mist am Röderberg 21. März 1860 von B. gef. -

2. P. angustatum Er. = oblongum Gillm. - Ems an Eselskoth von C. H. gef., bei Fr. von demselben und Hg. gesammelt. -

3. P. minutissimum Weber. - Fr. bei Formica rufa von C. H. gef., auch von Hg. gesammelt. - M. (S.) -

4. P. canaliculatum Er. - In den Enkheimer Torfbrüchen unter Schilf von uns, von Hg. bei Fr. gesammelt. -

5. P. caesum Er. - Von Hg. bei Fr. gef. -

6. P. inquilinum Er. - Bei Fr. von B. 18. April 1860 gef. -

\section{Neuglenes Thomson (= Ptinella Matthews).}

1. N. apterus Guér. - Im Fr. Wald an der Viehtränke am Beckerweg in faulem Buchenholz einmal in Anzahl von L. H., M. S. und Hg. am 1. August 1855 gef. - Die Art ist augenlos und hat ảie charakteristische gelbe Farbe aller Blindinsekten, die im Verborgenen, ohne Einfluss des Tageslichts auf das Pigment, leben. -

2. N. testaceus Heer = limbatus Heer. - Mit dentlichen Augen. - Fr. einzeln. - Cronthal. -

\section{Aderces Thomson (= Pteryx Matthews).}

1. A. suturalis Heer. - Fr. einmal n. s. von C. H. gef. -

\section{Trichopteryx Kirby.}

1. T. lata Matthews. - Bei Fr. einmal von C. H. gef. als grosse atomaria. - Stimmt mit typischen Stücken aus Crefeld. -

2. T. atomaria Deg. - Fr. s. h. unter faulenden Pflanzen, auch von Hg. gef. -

3. T. grandicollis $M h m .=$ fascicularis Gillm. - Fr. etwas seltener, auch von Hg. gef. -

4. T. fascicularis Hbst. = intermedia Gillm. - Von Hg. bei Fr. gesammelt. - 
5. T. thoracica Gillm. - 2 Ex. von C. H. gef. - Von Hg. bei Fr. gesammelt. -

6. T. pumila Er. = sericans Gillm. - Fr. von C. H. n. s. gef., ebenso von Hg. -

7. T. brevipennis Er. = clavipes Gillm. - Von Hg. bei Fr. gesammelt. -

8. T. pygmaea Er. = parallelogramma Gillm. - Wie die vorige Art. -

9. T. sericans Heer $=$ depressa Gillm. - Wie die vorige Art. -

\section{SCAPHIDIDAE.}

\section{Scaphidium Olivier.}

1. S. quadrimaculatum $\mathbf{0 l}$. - Im Fr. Unterwald an schwammigem Holz s. von C. H. gef. - Mo. (St.) - Fr. Wald (Hg.) Dlb. (Sch.) -

\section{Scaphium Kirby.}

1. S. immaculatum Oliv. - In der Fr. Umgegend in Nadelholzwaldungen an schimmeligen Stellen am Boden in alten Mausgängen. Bei Offenbach in einer Reihe von Jahren oft in Menge von L. H., Hg. und St. gef. - C. H. fand die Art auch im Röderwäldchen, am Sandhof und bei Oberrad. - Bei Mz. von S. und Ludwig gef. [Exemplare in Sammlung v. Heyden.] — Im Rebstockerwald von B. gesammelt. -

\section{Scaphisoma Leach.}

1. S. agaricinum Oliv. - Im Gebiet h. an schimmeligem Holz. - Fr. auch von Hg. und B. gesammelt. - Dlb. (Sch.) - Mz. (S.) -

2. S. boleti Panz. - Von M. S. in einem Garten in Bockenheim gef. [4 Stück in Sammlung v. Heyden.] - Bei Fr. von Hg. gesammelt. 3. S. assimile Er. - Einmal von C. H. bei Fr. gef. - 


\section{CLAMBIDAE.}

\section{Clambus Fischer.}

1. C. pubescens Redtb. - Fr. von uns, Hg. und M. S. in grosser Menge im Maingenist gesammelt. — Friedberg (Fuhr). Wlb. (Sch.) - Fr. 3. April 1860 (B.). -

2. C. Armadillo Deg. - Fr. - Soden. - Wlb. (Sch.) -

3. C. minutus Sturm. - Fr. -

\section{Cybocephalus Erichson.}

1. C. festivus Er. = diadematus Chevr. - Fr. s. s. Zweimal an einem morschen Apfelbaum von C. H. gef. -

2. C. exiguus Er. - Von Sch. bei Wlb. gef. -

\section{ANISOTOMIDAE.}

\section{Triarthron schmidt.}

1. T. Märkeli Schmidt. - Ein Exemplar dieser Seltenheit [nunmehr in Sammlung v. Heyden] fand Dr. C. Gerlach (jetzt in Hongkong) im Fr. Wald mit dem Streifnetz. -

\section{Hydnobius Schmidt.}

1. H. punctatissimus Steph. - C. H. fand 2 Stück bei Fr., L. H. 1 Stück an den Offenbacher Kalkstcinbrüchen. - Fr. von St. einmal gef., von B. 1 Stück in der Sandgrube bei Kleinkarben in der Wetterau gef. 1860. - Mo. (K.) - 


\section{Anisotoma Illiger.}

1. A. cinnamomea Panz. - Von Ko. n. s. in Trüffeln bei Dlb. gef. - Von Hg. und Steitz bei Fr. gesammelt. - Wsb. im Adamsthal 20. August (K.). -

2. A. rugosa Steph. = armata Sturm. - Ein Exemplar der Sammlung v. Heyden fing M. S. an der Babenhäuser Landstrasse im Fr. Wald, auch einmal von B. bei Fr. gestreift. -

3. A. Triepkii Schmidt. - An demselben Orte wie die vorige von L. H., M. S. und Hg. Abends s. s. mit dem Streifnetz auf kurzem Gras gef. -

4. A. obesa Er. - Im Fr. Wald mehrfach im Juni und Juli, sowie bei Offenbach gef., im Isenburger Wald von Hg. gesammelt. -

Var. brunnea Sturm. - Fr. einmal. -

5. A. dubia Kugel. - Eine der häufigsten Artèn. - Fr. Wlb. (Sch.) - G. (L.) -

6. A. flavescens Schmidt. - Bei Fr. von M. S. gesammelt. -

7. A. ovalis Schmidt. - Ein im Fr. Wald von M. S. gefangenes Exemplar in Sammlung v. Heyden. Auch von Hg. bei Fr. gef. -

8. A. nigrita Schmidt. - Fr. einmal. -

9. A. rubiginosa Schmidt. - Im Fr. Wald von L. H., Hg. und B. gef. - Wlb. (Sch.) -

10. A. scita Er. - s. s. Art. - Fr. einmal gef. -

11. A. calcarata Er. = ferruginea Schmidt. - Rödelheim. - Fr. n. s. von L. H. und Hg. gef. - Soden (St.) - Wlb. (Sch.) - M. (S.) -

12. C. badia Sturm. - Von Hg. bei Fr. gef. -

\section{Cyrtusa Erichson.}

1. C. minuta Ahr. - Fr. n. s. [Hierher gehören auch die von L. H. gefundenen Stücke von C. pauxilla, welche in S. V. erwähnt sind.] -

2. C. subtestacea Gyll. - Von Hg. bei Fr. gesammelt. -

Colenis Erichson.

1. C. immunda Stm. = dentipes Gyll. - Soden. - Fr. h., auch von B. und Hg. gef. - Bei Wlb. nach Sch. h. - 


\section{Agaricophagus Schmidt.}

1. A. cephalotes Schmidt. - Ein Exemplar dieser Seltenheit fand M. S. im Fr. Wald; es befindet sich nun in der Sammlung v. Heyden. -

2. A. conformis Er. - Einmal von Sch. bei Wlb. gef. -

\section{Liodes Erichson.}

1. L. humeralis F. - - Im Fr. Wald in Staubpilzen an Kiefernstöcken und an Boletus an Bäumen. - Wald bei Oberursel $>5$. -

Var. picea Panz. (ganz rothbraune Flügeldecken) mit der Stammart, welche auf den schwarzen Flügeldecken eine rothbraune Schultermakel hat.

2. L. axillaris Gyll. - Wie die vorige. Seltener. - Fr. - Neuenhain bei Soden. - Cronthal. - Offenbach. - An der Hofheimer Kapelle von B. gesammelt. - Fr. (Hg.) - Bei Dlb. h. (Sch.) -

3. L. glaber Kug. - Am Fr. Forsthaus s. S. unter faulenden Nadelholzzweigen von L. H. gef. - Bei Dlb. einmal von Sch. gef. -

4. L. castaneus Hbst. - Wie die vorige. - Fr. (St.) -

5. L. orbicularis Hbst. - Bei Fr. von Hg. und St. gef. -

\section{Amphicyllis Erichson.}

1. A. globus F. (mit schwarzen Flügeldecken und rothem Halsschild). - Im Isenburger Wald s. s. von L. H. und Hg. mit dem Streifnetz gef. - Mehrere Stücke (auch die Varietät) in lichten Waldstellen auf Gras gestreift, meist in der Nähe des Forsthauses, z. B. am 24. Mai 1860 (B.). -

Var. staphylaea Gyll. (ganz braunroth). Auf der Bieberer Höhe bei Offenbach von L. H., im Schwanheimer Wald ron St. gesammelt. - Fr. (Hg.) -

2. A. globiformis Sahlbg. - Fr. (Hg.) - G. (L.) -

\section{Agathidium Illiger.}

1. A. nigripenne F. - Von St. 4 Stück bei Fr. gesammelt. Wlb. (Sch.) -

2. A. atrum Payk. - Fr. s. auch von Hg. gesammelt. - An geschlagenen Fichtenstämmen im Fr. Wald 2 Stück von B. gef. G. (L.) - 
3. A. seminulum L. - 2 Stück von Fr., ein drittes 1861 bei Formica rufa im Fr. Wald von B. gef., auch von Hg. gesammelt. G. (L.) -

4. A. laevigatum Er. - Fr. - Königstein. - Schmitterhof bei Giessen. - Bei Fr. von B. und Hg. gef. -

5. A. badium Er. - [Mein Fr. Stück in S. V. ist ein laevigatum.] - Bei Fr. von St. gesammelt. - G. (L.) -

6. A. varians Beck - An einem faulen Brett bei der Oelmühle bei Königstein fand L. H. ein Dutzend Exemplare dieses seltenen Thierchens im Juli 1872.

7. A. rotundatum Gyll. - Von Hg. bei Fr. gef. -

8. A. marginatum Sturm. - Viele Exemplare am 8. April 1860 bei Fr. von B. gef. -

[A. nigrinum Sturm besitze ich nicht aus dem Gebiet, wie in S. V. angegeben ist.]

\section{XXXV.SILPH I DAE.}

\section{A. SILPHINI.}

Agyrtes Fröhlich.

1. A. castaneus Fröhl. - Im ersten Frühjahr im Flug am - Grindbrunnen bei Fr., auch in den Strassen an Häusern. - Nauheim. - Von B. zweimal bei Fr., 1 Ex. davon unter einem Feldstein am Hanauer Bahnhof gef. - Hg. fand 4 Stücke bei Fr., auch St. sammelte die Art. - M. (S.) -

2. A. bicolor Cast. = subniger Fairm. - s. s. - Bei Fr. wurden folgende einzelne Stücke gef.: Von Dr. Siebert unter einer Weide, von C. H. = 12. auf Schnee, von M. S. am 15. April 1855 am MainNeckar-Eisenbahndamm im Wald unter Laub. -

\section{Necrodes Leach.}

1. N. litoralis L. - Fr. - An grösseren Aesern; besonders solchen, die im Wasser liegen. - z. s. - An der Mainkur 1873 von B. ge- 
sammelt. - Dlb. Wlb. (Sch.) - Von Hg. früher h. bei Fr. gef., auch von St. - G. (L.) -

\section{Xylodrepa Thomson.}

1. X. quadripunctata L. - Fr. - Soden. - Friedberg auf dem Johannisberg. Lebt auf jungen Eichbäumen, wo sie auf Insekten Jagd macht. - Fr. Wald (St.) - Wsb. (K.) - Auf der Königswiese bei Fr. am 3. Juni 1860 von B. gef. - Dlb. (Sch.) -- Am Hainerweg in der Nähe vom Fr. Wald früher s. h. von Hg. gesammelt. - M. (S.) - G. (L.) -

\section{Thanatophilus Leach.}

1. Th. thoracicus L. - Im ganzen Gebiet h. an Aas. - Fr. Feldberg = 5. - In der Ebene schon am 18. April 1861 von B. gef. - Dlb. Wlb. (Sch.) - M. (S.) - G. (L.) -

2. Th. rugosus L. - Fr. h. - Feldberg. - Dlb. Wlb. (Sch.) - Fr. auch von Hg. und St. gef. - M. (S.) - Wsb. an der Wellritz 16. April (K.). -

3. Th. sinuatus F. - Fr. h. - M. (S.) - G. (L.) - Dlb. Wlb. (Sch.) -

\section{Oiceoptoma Leach.}

1. 0. opaca L. - Fr., besonders im Schwanheimer Wald, s. Auch von Hg. und St. gef. - Dlb. Wlb. (Sch.) - M. (S.) Had. (K.) -

2. O. reticulata F. - Von Hg. und St. bei Fr. öfter gesammelt, auch von B. h. auf Wegen in der Sonnenhitze. - Wlb. (Sch.) Münster bei Soden 2. April 1876. - Bei Bockenheim 17. April 1876 in Begattung. - M. (S.) - G. (L.) - Wsb. Dotzheimer Weg 24. April (K.). -

\section{Silpha Linné.}

1. S. tristis Illig. - Fr. s. s., früher am Oederweg in Hecken, wo sie den Schnecken nachgeht. - Auch von St. im Gebiet einmal, von Hg. 3 Stück gef. -

2. S. obscura L. - Fr. von C. H. und St., von B. und Hg. h. gef. - Münster bei Soden 2. April 1876. - M. (S.) - G. (L.) Dlb. Wlb. (Sch.) - 


\section{Phosphuga Leach.}

1. Ph. laevigata L. - Fr. z. s., auch von St. gef. Von Hg. bei Fr. n. s. gesammelt. - Wsb. 18. Mai (K.). - Wlb. (Sch.) -

2. Ph. atrata L. - In Wäldern h. in faulem Holz, unter Laub. - Schlangenbad. - Fr. h. (Hg. und B.) - Dlb. Wlb. (Sch.) M. (S.) - G. (L.) - Wsb. in Baumstrünken an der Wellritz im März (K.). -

\section{Necrophorus Fabricius.}

1. N. Germanicus L. - Fr. z. s. an Aas von grösseren Thieren. Röderwald bei Fr. 1874 von B. gef. - Had. Wsb. (K.) - Dlb. Wlb. (Sch.) -

Varietät mit je einem kleinen rothen Punkt auf der Mitte jeder Decke fand August Osterrieth bei Fr. [in Sammlung v. Heyden.] -

Varietät mit je einem grossen rothen Fleck vor den Hinterecken der Flügeldecken fanden Gräf und Hg. bei Fr. -

Die Varietät mit vier rothen Flecken (speciosus Schulze) fand Scheidel bei Fr. - Die Art kommt bei Mz. nach S. n. s. in Pferdekoth vor, wo er den grossen Kothkäfern, besonders Geotrupes, nachgeht. Wirft man einen solchen Kothhaufen auseinander und findet man frisch zerfressene Stücke von Geotrupes, da kann man sicher sein, dass N. Germanicus in der Nähe ist. -

2. N. humator F. - Etwas häufiger wie der vorige. - Fr. (Hg. und St.) - Im Röderwald am 27. April 1860 von B. gef. - M. (S.) - G. (L.) - Wlb. (Sch.) -

3. N. vespillo L. - An todten Mäusen, Maulwürfen, Vögeln s. h. - Fr. auch von Hg. gef. - Variirt von $16^{\mathrm{mm}}$ Grösse bis zu $10^{\mathrm{mm}}$. - M. (S.) - G. (L.) - Dlb. Wlb. (Sch.) -

4. N. vestigator Herschel. - Fr. s. von L. H., Hg. und St. häufiger von B. gesammelt. - Wlb. (Sch.) - M. (S.) - G. (L.) Wsb. im Mai (K.). -

5. N. gallicus Duv. (Die Trochanter der Hinterbeine mit gekrümmtem Hacken.) - Fr. zweimal gef. - Oelmühle. - Von Hg. h. bei Fr. gesammelt. -

Var. interruptus Steph. = fossor Er. - Fr., Oelmühle bei Königstein an todten Vögeln. - Bei Fr. von Hg. und B. gef. Wlb. (Sch.) - M. (S.) - Wsb. (K.) - 


\section{$-142-$}

6. N. investigator Zetterst. = ruspator Er. - Friedberg (Scriba). - Oelmühle bei Königstein an todten Vögeln. - Fr. h. (Hg.) - M. (S.) -

Var. microcephalus Thoms. - Von Hg. h. bei Fr. gesammelt. -

7. N. sepultor Charp. - Fr. einmal von C. H., später auch von Hg., St. und Scheidel gef. - M. (S.) -

8. N. mortuorum F. - Im ganzen Gebiet n. s. - Fr. Oelmühle bei Königstein. - Variirt von 9-13 $\mathrm{mm}$ Länge. - Von B. in faulenden Pilzen gesammelt. - Wlb. (Sch.) - Auch von Hg. bei Fr. s. h. gef. - M. (S.) - Wsb. im Wald an der Tränke an todtem Maulwurf im Juni (K.). -

\section{B. C A T 0 P I N I.}

\section{Colon Herbst.}

Alles seltene Arten, die man einzeln Abends vor Sonnenuntergang, ehe es thaut, im Grase mit dem Streifnetz fangen kann. -

1. C. Viennense Hbst. - Fr. - Soden. - Ein Stück im Fr. Wald von B. gef. -

2. (Myloechus Thoms.) fuscicorne Krtz. - Ein Weibchen dieser Art fing L. H. 1871 in Neuenhain bei Soden [vom Monographen Tournier bestimmt.] -

3. (M.) dentipes Sahlb. - Einmal von Hg. bei Fr. gef. -

4. (M.) Zebei Krtz. - Ein Männchen von Sch. bei Wlb. ges. -

5. (M.) appendiculatum Sahlb. - Fr. einigemal von L. H. und M. S. gef. -

6. (M.) calcaratum Er. - Ein Männchen dieser seltenen Art fing Sch. bei Wlb. -

7. (M.) angulare Er. - Fr. ein Männchen von L. H. und eins von B. 1875 bei Kronberg. - Fr. (Hg.) -

8. (M.) brunneum Latr. - Noch die häufigste Art. - Fr. Soden. - Auch von Hg. gesammelt. - M. (S.) - Wlb. (Sch.) -

\section{Catops Paykul.}

1. (Ptomaphagus Illig.) sericeus Panz. - Fr. - Soden. Falkenstein, Biebrich im Rheingenist. - Seckbach in faulen Schnecken. 
- Im Maingenist oberhalb Fr. h. am 6. April 1860 von B. gesammelt, auch von Hg. gef. - Wlb. (Sch.) -- M. (S.) - G. (L.) - Wsb. an Maulwurf an der Wellritz 14. Juli (K.). -

2. (P.) varicornis Roshr. - Bei Offenbach $<4$. von C. H. einmal, bei Fr. am 3. Juni 1860 von B. einmal, von Hg. einzeln gef. -

3. (P.) colonoides Krtz. - Fr. s. s. von L. H. und Hg. gef. -

4. (Nargus Thoms.) Wilkini Spence $=$ praecox Er. - Ein Stück an der Hofheimer Kapelle unter Moos von B. gef.; auch von Hg. gesammelt. -

5. (N.) anisotomoides Spence. - An den Oberräder Schiessständen unter Moos an Eichen von L. H., von Hg. bei Fr. gef. Wlb. (Sch.) -

6. (N.) badius Sturm. - Im . Taunus s. - Ein Exemplar am 16. Juni 1862 bei Fr. in einem alten Vogelnest von B. gef. -

7. (Sciodrepa Thoms.) umbrinus Er. - Bei der Mainkur am Fusse von alten Eichen $>5$. gef. -

8. (S.) fumatus Spence $=$ scitulus Er. - G. (L.) - Mo. 5. Mai und 6. Juli (K.). -

9. (S.) Watsoni Spence = fumatus Er. - Fr. - Soden. An Aas kleiner Thiere s. h. Nach B. besonders gegen Abend h. Röderwald, Königswiese. Die häufigste Art. April und Mai. - Auch bei Fr. von Hg. gef. - Wlb. (Sch.) -

10. (S.) alpinus Gyll. = subfuscus Kelln. - Von Hg. einmal bei Fr. gef. -

11. C. picipes F. - Von St. bei Fr. gef., von Hg. h. Wlb. (Sch.) -

12. C. nigricans Spence. - Auf nassen Wiesen 1 Stiick auf der Königswiese bei Fr. im April 1861, 1 Stück am Ludwigsbrunnen bei Grosskarben 1860 am 6. Mai von B. gestreift; bei Fr. auch von $\mathrm{Hg}$. gesammelt. -

Var. fuliginosus Er. - Fr. -

13. C. fuscus Panz. - Fr. - Von Waldemar Fuchs h. in Gärten bei Fr. gef. Ein Stück 16. Mai 1860 bei Fr. von B. gef., auch von Hg. gesammelt. - Wlb. (Sch.) -

14. C. morio F. - Fr. s., auch von Hg. gef. - G. (L.) - 
15. C. affinis Steph. = nigrita Er. - Fr. s. An todten Krähen im Röderwald 18. April 1861 ron B. gesammelt. - Auf dem Grossen Mannstein (Staufen) im Taunus einmal 2. April 1876 an Fuchslosung ron B. gef. -

16. C. grandicollis Er. - Bei Fr. von Hg. gef. - Wlb. (Sch.) -

17. C. chrysomeloides Panz. - Fr. - Soden am Fuss ron alten Eichen $>10$. Z Zweimal ron B. 1860 bei Fr. gesammelt, auch von Hg. -

18. C. tristis Panz. - Von Sch. einigemal bei Wlb. gesammelt, einmal ron L. H. 2. April 1876 auf dem Grossen Mannstein (Staufen) im Taunus an Fuchs!osung gef. -

Var. Kirbyi Spence = rotundicollis Kelln. - Röderwald bei Fr. an todten Krähen 18. April 1861 und später noch 2 Ex. von B. gef. - G. (L.) - Wlb. (Sch.) -

19. C. neglectus Krtz. - Von B. bei Fr. einmal gesammelt [früher als coracinus bestimmt, der auch nicht in unserem weiteren Gebiete sondern nur in Thüringen beobachtet rurde.] -

\section{Choleva Latreille.}

1. C. angustata F. - Von Hg. bei Fr. gesammelt. - M. (S.) - G. (L.) -

2. C. spadicea Sturm. - Von Hg. bei Fr. gef. -

3. C. intermedia Krtz. - Hg. fand einige Stïcke dieser seltenen Art bei $\mathrm{Fr}^{\circ}$. -

4. C. cisteloides Fröhl. - Fr. h. an modernden Pflanzen, Bockenheim im Frühjahr in Gärten an Küchenabfällen von Hg., B. und St. einzeln gesammelt. - Wsb. an Aborten 15. Norember (K.). -

5. C. agilis Illig. - Fr. S. - Biebrich. - B. fand 2 Stück bei Fr., auch ron $\mathrm{Hg}$. und St. gesammelt. - M. (S.) - 


\section{SCYDMAENIDAE.}

\section{Scydmaenus Latreille.}

1. S. Godarti Latr. - In Sammlung v. Heyden befindet sich ein von M. S. bei Fr. gefundenes Stück. B. fand diese sonst seltene Art auf verrottetem Mist unter Steinen am Abhange des Röderbergs $>3$. s. h. - Auch von Hg. gef. -

2. S. scutellaris Müll. Kunze. - h. - Soden. - Bei Formica cuniculina im Schwanheimer Wald $>3$. von B. gesammelt. - Fr. von uns und Hg. gef. - Wlb. (Sch.) -

3. S. collaris Müll. Kunze. - Fr. n. s. [Hierher die Fr. Stücke des elongatus in S. V.] - B. fand wenige Stücke bei Formica rufa im Fr. und Schwanheimer Wald. -

4. S. pusillus Müll. Kunze. - Fr. einmal von C. H., am 21. April 1861 auf der Königswiese gestreift und s. auch bei Formica rufa von B. gesammelt. -

\section{Neuraphes Thoms.}

1. N. angulatus Müll. Kunze. - Einmal von B. am 8. April 1860 bei Fr. gef., auch von Hg. gesammelt. -

2. N. elongatulus Müll. Kunze. - Bei Fr. von Hg. gesammelt. -

3. N. Sparshalli Denny. - Fr. 2 Stücke von C. H. gef. -

4. $\boldsymbol{N}$. helvolus Schaum. - Fr. $=4$. und Soden $>6$. je einmal gef. -

\section{Euconnus Thoms.}

1. E. pubicollis Müll. Kunze. - Fr. 3. April 1860 von B. gef., auch von $\mathrm{Hg}$. gesammelt. -

2. E. denticornis Müll. Kunze. - Fr. - Nauheim auf Salzboden. -

3. E. hirticollis Illig. - Fr. (Hg.) Von St. auf dem Röderberg gesammelt. Von B. mit S. Godarti zusammen und ebenso h. gesammelt, auch im Genist des Metzgerbruchs s. h. 8. April 1860. - M. (S.) G. (L.) - Wsb. an der Wellritz im April (K.). -

4. E. claviger Müll. Kunze. - s. s. und nur in den Nestern der Formica rufa 12. Juni 1856 von L. H. nnd M. S. am Fr. Forsthaus gef. - 


\section{$-146-$}

5. E. Mäklini Mhm. - 18. Mär» 18615 Stück von B. im Fr. Wald bei Formica rufa gef.; 1 Stück [in Sammlung v. Heyden] von St. gef. Auch Hg. fand die Art bei Fr. - Wlb. (Sch.) -

6. E. Wetterhali Gyll. - Eiu Stück 8. April 1860 bei Fr. von B. gef. - G. (I.) -

\section{Eumicrus Laporte.}

1. E. tarsatus Müll. Kunze. - Fr. h. unter faulenden Pflanzen; auch von Hg. und St. gesammelt. - Von B. $>3$. mit S. Godarti zusammen, aber noch häufiger gesanmelt. -

2. E. Hellwigi F. - Fr. in einer hohlen Eiche bei Ameisen von C. H., im Schwanheimer Wald von L. H. una St. gesammelt. - In ziemlicher Anzahl bei Formica cuniculina im Schwanheimer Wald $>3$. von B. gef. - Isenburger Wald (Hg.). -

3. E. rufus Müll. Kunze. - Fr. s. unter Baumrinden bei Ameisen. - Schwanheimer Wald. -

\section{Euthia Stephens.}

1. E. scydmaenoides Steph. - Bei Fr. von Hg. zweimal gef. -

2. E. Schaumii Kiesw. - Ein Exemplar dieser seither nur ans Griechenland bekamnten Seltenheit fing C. H. $<$ 8. im Garten des Herrn Commerzienrath Reiss in Soden unter Gras. Später wurde das schöne Thierchen auch von Thomson in Schweden gef., hat also eine sehr weite geographische Verbreitung. Das fragliche Exemplar wurde mit dem Kiesenwetter'schen Typ verglichen und arich vom Monographen Saulcy für diese Art gehalten. -

\section{Cephennium Müller.}

1. C. thoracicum Müll. Kunze. - Fr. an feuchten Stellen im Moos und bei Ameisen. - Neuenhain bei Soden. -

2. C. fulvum Schmidt. - Herr Edmund Reitter bestimmte ein von Sch. bei Wlb. gefangenes Stück als fulvum mit der brieflichen Bemerkung: „Viel kleiner; mit kaum sichtbaren Augen, welche bei thoracicum grösser sind." - Ueber ein vielleicht hierher gehörendes Thier schreibt Dr. Böttger: „Drei Stücke, die ich als fulvum Sch.? bestimmt hatte, im April 1861 an der Hofheimer Capelle im Taunus unter Baummoos und Steinen; eins zu derselben Zeit am Königsbrunnen im Fr. Wald in faulendem Moose unter einem Stein." - 


\section{CLAVIGERIDAE.}

\section{Claviger Preyssler.}

1. C. testaceus Preyssl. = foveolatus Müll. - Fr. n. s. an sonnigen Stellen unter Steinen bei Ameisen, hauptsächlich Lasius flavus. - Bei Bingen 25. April 1832 von C. H. h. gef., auch mit Larve, die aber zu schlecht erhalten ist, um genauer beschrieben werden zu können. Sie ist sehr lang gestreckt, blassgelb-grau; Kopf rund, in der Mitte stark vertieft, in der Mitte dieser Vertiefung eine starke Querwulst, an deren Ende seitlich die sehr kurzen dicken Fühler herausragen, die scheinbar aus einem Glied mit sehr kleinem Endgliedchen bestehen. Ich bemerke an der Basis der Fühler sehr kleine gelbe glänzende Pünktchen, die ich für Ocellen halte. Das Halsschild ist rund, nach hinten etwas verengt. Hinterleib (dessen Gliederung nicht zu erkennen) wohl dreimal so lang als Kopf und Halsschild zusammen. Schenkel dick, die Schienen werden nach der Spitze zu dünner und gehen allmälig in das Tarsenglied über, das vorn eine grosse Kralle trägt. Länge $21 / 2 \mathrm{~mm}$. B. fand die Art auch bei Offenbach auf der Bieberer Höhe und im Lehen. - Nach Sch. s. h. bei Lasius flavus und niger bei Wlb.; die Larve ist roth und weich. - Bei Fr. auch von Hg. gesammelt. Bockenheim bei Formica flava 17. April 1876. -

2. C. Iongicornis Müll. - s. s. - C. H. fing 18201 Ex. in Ems, zwei Jahre nach der Entdeckung des schönen Thieres in Bodenbach in der Pfalz durch Pfarrer Müllel. - Voll Sch. mehrfach bei Wlb. gef., bei Lasius niger und flavus mit testaceus in demselben Nest. - 


\section{PSELAPHIDAE.}

(Die Sammlung $\checkmark$. Heyden ron dem Monographen de Saulcy revidirt.)

\section{Chennium Latreille.}

1. Ch. bituberculatum Latr. - Bei Ameisen, Tetramorium caespitum s. s. - C. H. fand 18:0 bei Ems das erste deutsche Exemplar. - Auf der Bieberer Höhe fand C. H. ann t. April 1846, später L. H. und Hg. einige Exxemplare am 3. Mai 1857. - Friedberg (Renner). Sch. fand bei Wlb. ein ganz hellroth-gelbes Exemplar. - Auf den Sandbrüchen zwischen Gonsenheim und Mo. unter Steinen in den Nestern von Tetramorium caespitum im April und Mai n. s. von S. gef. -

\section{Centrotoma Heyden.}

1. C. lucifuga Heyd. - Von C. H. 1 Ex. am 5. April 1845 und 2 Stück am 1. Juni 1846 auf der Bieberer Höhe bei Offenbach entdeckt. Lange waren dies die einzigen bekamnten Stücke, bis das interessante Thier in Sachsen, Südfrankreich und neuerdings Prag gefunden wurde. - In Nestern des Tetramorium caespitum. -

\section{Tyrus Aubé.}

1. T. mucronatus Panz. - Von C. H. bei Fr. vor langen Jahren und von M. S. und L. H. je eimmal an den alten Eichen bei Schwanheim in Gesellschaft der Formica cunicularia gef. -

\section{Batrisus Aubé.}

1. B. formicarius Aubé. - L. H. fanc all Gräfenbruch im Fr. Wald unter Eichenrinde bei Formica cunicularia 1 Ex. - Von St. anch im Schwanheimer Wald gef., ebendaselbst von B. unter den oben angegebenen Verhältnissen 3 Stück und einmal am 27. März 1871. - Auch von Hg. im Fr. Wald und von Sch. bei Wlb. bei Lasius brunneus unter Eichenrinde gef. -

2. B. venustus Reichb. - Am 5. April 1845 ron C. H. hei den Offenbacher Steinlrüchen in Gesellschaft der Formica coniculina, 
von L. H. an den Oberräder Schiesständen in alten Buchen mit derselben Ameise und bei Soden gef. - Auch von St. im Schwanheimer Wald gesammelt, ebendaselbst von B. 9 Stück am 27. März 1861. - Isenburger Wald (Hg.). -

3. B. Delaportei Aubé $=$ Schwabii Reitter. - Von Hg. am Fuss der alten Linden auf der Pfingstweide in 2 Stücken $<4.1860$, von B. unter einem Stein am Abhange des Röderbergs am 13. April 1860 in 1 Stück gef. -

\section{Trichonyx Chaudoir.}

1. T. sulcicollis Reichb. - Von Hg. und M. S. bei Fr., von L. H. in Neuenhain bei Soden gef. - s. s. -

2. T. Märkeli Aubé. - s. - L. $H_{\text {. }}$ und Hg. fanden ihn am 20. Mai 1856 bei Königstein am Fusse alter Eichen mit Formica cunicularia und in Neuenhain bei Soden 1871. - C. H. sammelte die Art auch bei Nauheim im Juni auf Salzboden. - Wlb. (Sch.) -

\section{Pselaphus Herbst.}

1. P. Heisei Hbst. - Unter Laub und Steinen n. s. - Fr. Soden. - Auch am Feldberg. Bei Formica rufa ein Stück = 4. 1861 im Fr. Wald von B. gef. - M. (S.) - G. (L.) - Wlb. (Sch.) -

2. P. Dresdensis Hbst. - Fr. s. s., nur von C. H. früher im Gebiet gef. -

\section{Tychus Leach.}

1. T. niger Payk. - Fr. n. s. aus abgefallenem Laub gesiebt. [Der in S. V. erwähnte, von L. H. bei Fr. gefundene T. ibericus ist ein T. niger Männchen mit rothen Flügeldecken. T. iber. lebt nur in SüdEuropa in der Nähe des Meeres.] - Röderberg (St.) - Von B. h. auf feuchten Waldwiesen, z. B. auf der Königswiese von $<4$. bis $=5$. gestreift. - Fr. (Hg.) - G. (L.) - Wlb. (Sch.) -

\section{Bryaxis Leach.}

1. (Rybaxis Saulcy) sanguinea F. - Unter feuchtem Laub am Fuss vón Bäumen oder in Gräben n. s. - Fr. von uns und Hg. gef. - Soden. - Bingen. - Auf der Königswiese mit der vorigen Gattung von B. gesammelt; derselbe besass eine ganz schwarze Varietät. - Auch Sch. fand bei Wlb. diese Varietait. - M. (S.) - 
2. (B.) fossulata Reichb. - h. - Fr. - Soden. - Saline Nauheim. - Unter Steinen am Abhang des Röderbergs und auf der Königswiese s. h. von B. gef. - Fr. (Hg.) - Wlh. (Sch.) - Wsh.. 3. November" (K.). -

3. (B.) xanthoptera Reichb. - C. H. fand 2 Stück dieser seltenen Art bei Ems; Sch. auch 1 Exemplar bei Wlb. -

4. (B.) Helferi Schmidt. - Ein Bewohner der Meeresküsten, aber auch bei den Salinen im Binnenlande, z. B. Nauheim n. s. - In der Wetterau auch bei Salzhausen. -

5. (B.) haematica Reichb. - Im ganzen Gebiet n. s. - Fr. Rödelheimer Wald. - Bingen. - Sóden. - Auf der Königswiese h. von $<4$. bis $=5$. von B. gestreift. - Auch von Hg. bei Fr. gesammelt. - Wlb. (Sch.) - Wsh. àm Wellritzufer 14. Juni (K.). -

6. (Reichenbachia Leach) juncorum Leach. - Fr. - Soden s. - Auf Schilf am Deutschherrnweiher bei Offenbach am 2. Nai 1860 und auf der Königswiese bei Fr. am 21. April 1861 je 1 Stück ron B. gestreift, auch von Hg. im Gebiet gef. - Wlb. (Sch.) -

7. (R.) impressa Panz. - Bei Fr. n. s. - Von Hg. und B. h. mit B. haematica gef. -

\section{Bythinus Leach.}

1. B. validus Aubé. - [Hierher meine B. puncticollis in S. V., welche Art ich nur von Erlangen besitze.] - Fr. - Griesheim am Main. - Soden. - [Hierher gehört auch das von L. bei G. gefundene Stück von B. puncticollis Denny.] -

2. B. bulbifer Reichb. - Fr. - Unter altem Laub und Schilf n. s. von uns und Hg. gef. - Wlh. (Sch.) -

3. B. Curtisii Denny. - Bei Wlb. von Sch. gef. -

4. B. nodicornis Aubé. - L. H. sah in der Schenck'sclien Sammlung ein bei Wlb. gefangenes Männchen. -

5. B. securiger Reichb. - Fr. - Soden. - Fr. von B. einmal am 2. Mai 1860 bei Fr. gestreift, auch von St. and .Hg. gef. Wlb. (Sch.) -

6. B. Burellii Denny. - Bei Fr. s. von C. H. und Hg. gesammelt. - 


\section{$-151-$ \\ Euplectus Leach.}

1. E. Tischeri Aubé. - Fr. einmal gef. Anch von Hg. ges. -

2. E. Duponti Aubé. - C. H. fand 1 Stück im Garten des Herrn Commerzienrath Reiss in Soden unter Gras. In S. V. irrthümlich von L. H. als Kunzei Aubé benannt. -

3. E. punctatus Muls. Rey. - Zweimal $<5$. ans dürrem $\mathrm{Holz}$ aus dem Fr. Wald erzogen. - Seither nur aus der Schweiz in wenigen Exemplaren bekannt. -

4. E. signatus Reichb. - Fr. s. - Mr. einmal von C. H. im Flug gef., von Sch. bei Wlb. -

5. E. sanguineus Denny. - Von Frg. bei Fr. gesammelt. -

6. E. Karsteni Reichb. - Fr. h. in Mistbeeterde an Brettern. Einmal bei Formica rufa. - Bei Fr. auch von $\mathrm{Hg}$. und St. und am 21. März 1860 anf verrottetem Mist, am 18. März 18611 Ex. bei Formica rufa im Fr. Wald von B. gef. - Wlb. (Sch.) - Wsb. 27. October nnter Lanh (K.). -

7. E. nanus Reichb. - Bei Fr. eimnal von C. H., mehrfach von Hg. und St. auf verrottetem Mist unter Steinen h. am Abhang des Röderbergs 21. März 1860 gef. -

8. E. ambiguus Reichb. - Fr. n. s. - Biebrich im Rheingenist. - Von St. am Königsbrunnen mit dem Streifnetz gef.; ebendaselbst anch von B. h. von $=4$. bis $<5$. - Isenburger Wald (Hg.) -

9. E. bicolor Denny. - s. - Fr. Wald dreimal ans dürrem. Holz erzogen $>5$. -

\section{Trimium Aubé.}

1. T. brevicorne Reichb. - G. (L.) - 


\section{STAPHYLINIDAE.}

(Die Sammlung v. Heyden ist von Scriba und Fauvel, diejenige von Dr. Ha g durch Kraatz revidirt, die Schenck'sche zum Theil von Dr. Eppelsheim.)

\section{A. PIESTINI.}

\section{Siagonium Kirby $=$ Prognatha Latreille.}

1. S. quadricorne Kirby. - Aeusserst s. Ein Weibchen $=9$. in Fr. an einem Haus angeflogen, von C. H. gef. - Friedberg (Fuhr). -

\section{B. PHLOEOCHARINI.}

\section{Phloeocharis Mannerheim.}

1. P. subtilissima Mnhm. - Unter Rinden von Eichen und Kiefern n. s. im Fr. Wald im Herbst. - Fr. (Hg.) -

\section{PROTEININI.}

\section{Phloeobium Erichson.}

1. P. clypeatum Müll. - In Wäldern an Pilzen S. - Rödelheim, Soden, Offenbach. - Von $>5$. bis October. - Fr. (Hg.) - Wlb. (Sch.) - Wsb. (K.) -

\section{Megarthrus Stephens.}

1. M. depressus Payk. - Von Hg. bei Fr. gef. -

2. M. nitidulus Krtz. - Von Hg. hei Fr. gef. [von Dr. Kraatz bestimmt.] -

3. M. denticollis Beck - Am Fr. Forsthaus unter faulenden Pflanzen $<5$. n. s., auch von Hg. gef. - Wlb. (Sch.) -

4. M. sinuatocollis Lac. - Im botanischen Garten in Fr. am Gewächshaus $>4$. Auch von Hg. gesammelt. - Wlb. einmal (Sch.) -

5. M. hemipterus Illig. - Hofheim $>8$. an Pilzen, - Fr. (Hg.) - 


\section{$-153-$ \\ Proteinus Latreille.}

1. P. ovalis Steph. = brevicollis Er. - Dreimal von Hg. bei Fr. gef.

2. P. brachypterus F. - Königstein, Cronthal, Hohe Mark, im Herbst h. an Pilzen. - Fr. auch von Hg. gef. - Wlb. h. (Sch.) -

3. P. macropterus Gyll. - Im Fr. Wald im Sommer. -

4. P. atomarius Er. - Fr. - Soden. Seltener. - Auch von Hg. im Gebiet gesammelt. -

\section{OMALINI:}

\section{Anthobium Stephens.}

1. A. atrum Heer = nigrum Er. - Rüdesheim im April, Epstein $<5$. in Schlehenblüthen. - Eichberg im Rheingau (Richter). Staufen im Taunus 2. April 1876. -

2. A. florale Panz. = triviale Heer. - Bei Fr. von Hg. gef. - Wlb. (Sch.) -

3. (Eusphalerum Krtz.) abdominale Grav. - Cronthal $=5$. in Kirschblüthen; $=4$. desgleichen bei Bingen; $=5$. auf SorbusBlüthen bei Falkenstein; $>4$. bei Königstein in Stachelbeerblüthen; Schmitterhof bei Giessen ; Rüdesheim in Cotoneaster-Blüthen. - Fr. (Hg.) - Wlb. (Sch.) -

4. (E.) signatum Märk. - Hofheim, Falkenstein, Rüdesheim in Kirschblüthen im April. - Fr., auch von Hg. gef. - Wlb. (Sch.) -

5. (E.) limbatum Er. - Schmitterhof bei Giessen > 4. Enkheim $=5$. -

6. (E.) Primulae Steph. = triviale Er. - Wlb. (Sch.) -

7. A. torquatum Marsh. $=$ montivagum Heer $=$ scutellare Er. - Fr. - Soden. - Bingen. - Fr. (Hg.) -

8. A. opthalmicum Payk. - Ems. - Zwischen Soden und Altenhain h. in Spartium - Blüthen <6. - Fr. von Hg. gef. Wlb. (Sch.) -

9. A. minutum F. - Mainkur. - Enkheim. -- Altkönig im Mai auf Wiesenblumen. - Fr. (Hg.) - Wlb. (Sch.) -

10. A. alpinum Heer = luteipenne Er, - Bei Fr, auf Waldblumen s. s. - 
11. A. Iongipenne Er. - Soden und Altkönig in Sorbus AriáBlüthen h. - Fr. (Hg.) -

12. A. stramineum Krtz. - Im Juni einmal am Altkönig in Sorbus Aria-Blüthen; einmal bei Fr. an Hecken an der Bockenheimer Landstrasse. - Fr. von Hg. gesammelt. -

13. A. Marshami Fauv. = torquatum Krtz. - Cronthal in Kirschblüthen und Falkenstein in Crataegus-Blüthen im Mai. -

14. A. Sorbi Gyll. = silesiacum Letzn. - Falkenstein. Fr. auch von Hg. gef. - Wlb. (Sch.) -

\section{Omalium Gravenhorst.}

1. 0. striatum Grav. verum - Fr. s., auch von Hg. gef. -

[Eine nahe verwandte Art ist sulculum Steph. ohne Eindrücke auf Halsschild, und stumpfen, nicht rechtwinkligen Hinterecken - die ich aber noch nicht aus dem Gebiet kenne.]

2. 0. pygmaeum Payk. - In Baumschwämmen bei Fr. s. -

3. O. rufipes Fourc. $=$ florale Er. = maculicorne Heer $=$ nigrum Grav. - Fr. in fanlen Baumpilzen; Höchst in Ahornblüthen; Bingen in Kirschblüthen. - Ems. - Im Frühjahr. - Fr. (Hg.) Wlb. (Sch.) - Stanfen im 'Tammus 2. April 1876. -

4. O. melanocephalum F. = brunneum Payk. - Aus Fr. dürrem Waldholz erzogen. - Fr. (Hg.) -

5. O. iopterum Steph. = lucidum Er. - Fr. aus dürrem Waldholz h. erzogen, anch von Hg. gesaminelt. - Wlb. (Sch.) -

6. O. testaceum Er. - Bei Fr. von Hg. gef. -.-

7. 0. deplanatum Gyll. - Oberrad bei Fr. - Fr. Wald (Hg.). -

8. O. concinnum Marsh. -- Im ganzen Gebiet h. in altem Holz, an Fassschimmel in Kellern; im Fr. Wald (Ohere Saustiege) in Anzahl bei Forinica fuliginosa. -

9. 0. Iapponicum Zett. = conforme $\mathbf{K r t z} .=$ subtile $\mathrm{Krtz}$. - Fr. Wald $<4$. 1 Ex. unter Kiefernrinde von C. H. gef. -

10. 0. planum Payk. - Fr. n. h. - Wlb. (Sch.) -

11. 0. pusillum Grav. = punctipenne Thoms. = abietinum Thoms. - Im ganzon Gebiet unter Kieferninde, anch von Hg. gef. Wlb. (Sch.) - 
12. 0. caesum Grav. - Königstein, Falkenstein, Fr. Wald unter faulenden Pflanzen. - Ueberall g. - Fr. (Hg.) - Wlb. (Sch.) -

13. 0. rivulare Payk. - Im ganzen Gebiet g. an Pilzen und Aas. - Feldberg. - Fr. (Hg.) - Wlb. (Sch.) -

[Das in S. V. erwähnte O. laticolle Krtz. von Homburg ist nach Fauvel ein 0. rivulare.] -

\section{Coryphium stephens.}

1. C. angusticolle Steph. - Ein Exemplar dieser Seltenheit fing C. H. = 10. bei Fr. im Genist. - .

\section{Arpedium Erichson.}

1. A. quadrum Grav. = Heydeni Heer nec Krtz. - Bei uns kommt nur diese Form der Ebene vor. L. H. fand 1 Ex. bei Fr. [Hierher die in S. V. als A. cruentata von Fr. und dem Taunus erwähnten Stücke.] -

[Die alpine Form alpinum Fanv. = Heydeni Krtz. nec Heer fand C. H. nur in der Schweiz, doch erst nachdem Heer seine Beschreibung gegeben hatte.] -

\section{Acidota Stephens.}

1. A. crenata F. = rufa Grav. - Ein frisch entwickeltes Exemplar $>3$. bei Fr. gef., auch von Hg. gesammelt.

\section{Lathrimaeum Erichson.}

1. L. melanocephalum Illig. - Von B. bei Fr. h. gef. -

2. L. unicolor Marsh. = luteum Er. - Einmal bei Königstein $=10$. an Pilzen von C. H., von B. und Hg. h. bei Fr. gef. -

3. L. atrocephalum Gyll. = melanocephalum Mrsh. - Nauheim. - Falkenstein $>5$. unter dürrem Laub. - Bieberer Höhe bei Offenbach $=10$. Einmal in Fr. an einem Hans angeflogen von B. gef., auch von Hg. gesammelt. -

\section{Olophrụm Erichson.}

1. 0. piceum Gyll. - Im Fr. Wald unter Lanb am Main-NeckarEisenbahndamm s. (I. H. und M. S.) -

2. O. assimile Payk. - Bei Fr. einmal von C. H. gef., auch von $\mathrm{Hg}$. gesammelt. - 


\section{$-156-$ \\ Orochares Kraatz.}

1. 0. angustatus Er. - Ein Exemplar dieses seltenen Thierchens fing C. H. $>10$. im Königsteiner Wald. -

Lesteva Latreille.

1. L. Iongelytrata Göze $=$ bicolor F. - Epstein, Falkenstein, Hohe Mark, Fr. Wald an Bachrändern h. - Auch in grosser Menge zum öfteren todt in den Nauheimer Soolkasten gef. - Fr. (Hg.) -

2. L. punctata Er. - Von Hg. 2 Stück bei Fr. gef. -

Geodromicus Redtenbacher.

1. G. nigrita Müll. - Von Sch. bei Wlb. gesammelt. -

\section{Anthophagus Gravenhorst.}

1. A. testaceus Grv. - Emș an der Lahn h. - Hecken am Ginheimer Weg $>$ 9. s. - Wlb. (Sch.) -

2. A. praeustus Müll. - Wsb, öfter von Erlengebüsch geklopft. - Friedberg (Fuhr). - Fr. (Hg.) - Bei heiden Arten beobachtete C. H., dass sie sich „,freiwillig" in Spinnennestern verbargen. Gehen sie da den Spinneneiern nach? -

3. A. caraboides L. - Falkenstein, Königstein, Feldberg, Cronthal, Soden überall h. auf Gebüsch im Mai und Juni. — Läuft äusserst schnell. - Fr. (Hg.) - Wlb. (Sch.) -

Var. abbreviatus F. - Soden, Schlangenbad. - Fr. (Hg.) - s. -

4. A. bicornis Block = armiger Grav. - Bei Soden, Falkenstein und Altkönig von Eichen und Erlen geklopft. - Rüdesheim. Mai und Juni s., in den Alpen h. - Im Taumus auch von Hg. gesammelt. - Wlb. beide Geschlechter (Sch.) -

5. A. alpinus Payk. - Einmal von Hg. im Taunus gef. (!) -

\section{E. OXYTELINI.}

\section{Deleaster Erichson.}

1. D. dichrous Grav. - Fr. am Main (M. S.) - C. H. fing ihn einmal am neuen Irrenhaus $=5$. im Flug. - s. s. - 


\section{Coprophilus Latreille.}

1. C. striatulus F. - In Fr. an den Häusern auf der Strasse n. s. - Nauheim. - April. Auch von Hg. und B. gef. - Münster bei Soden im Taunus 2. April 1876. - Wsb. an der Wellritz an todtem Maulwurf 15. Juli und hinter dem Turnplatz im April (K,). -

\section{Syntomium Erichson.}

1. S. aeneum Müll. - In Soden in der Villa „Reiss" im Grasgarten an bemooster Erde; Schlucht der Kaltwasseranstalt bei Königstein. - Schmitterhof bei Giessen, die Larve $>6$. - Im Taunus bei Königstein auch von Hg. gesammelt. - Wlb. (Sch.) -

\section{Trogophloeus Mannerheim.}

1. (Thinodromus Kraatz) dilatatus Er. - Im Fr. Wald an der Luderbach von $\mathrm{Hg}$. gef. -

2. T. arcuatus Steph. $=$ scrobiculatus Er, - Fr. einmal gef. - G. (L.) -

3. T. bilineatus Steph. = riparius Lac. - Offenbach, im Fr. Wald und an den Kettenhöfen an mit Schilf bewachsenen feuchten Stellen von März bis September, auch von Hg. gef. -

4. T. rivularis Motsch. = Erichsonis Sharp. = bilineatus Er. - Biebrich im Rheingenist, Enkheimer 'Torfgruben, an den Kettenhöfen bei Fr. an feuchten Stellen; auch von Hg. im Gebiet gesammelt. -

5. T. memnonius Er. = obesus Kiesw. - Von Hg. bei Fr. 3 Stück gef. Juli.

6. T. fuliginosus Grav. - Bei Soden auf Salzboden im Mai und

7. T. corticinus Grav. - Fr., Eṇkheim, Rödelheim, Biebrich unter Rinden, z. B. von Apfelbaum, aber auch an feuchten Stellen. -

8. T. elongatulus Er. - Fr. unter Schilf an den Kettenhöfen. - Biebrich am Rhein. - Fr. (Hg.) -

9. T. punctatellus Er. $=$ pygmaeus Heer $=$ myrmecophilus Scriba. - An trockenen Orten unter Steinen an den alten Steinbrüchen bei Offenbach $<4$; ; auch in Gesellschaft von Ameisen an derselhen Lokalität im Juni. - Fr. im Wald an einem Boletus. - 
10. T. exiguus Er. - Ems eimmal von C. H. gef. - Von Fauvel als exig., von Scriba als haloph. bestimmt. - Fr. (Hg.) -

11. T. halophilus Kiesw. - Einmal auf Salzboden bei Nauheim gef. [von Fauvel und Scriba bestimmt.] -

12. T. pusillus Grav. - Fr. 1 Ex. - Wlb. (Sch.) -

13. T. tenellus Er. - Fr. an Düngerhaufen einmal gef., auch von $\mathrm{Hg}$. gesammelt. -

\section{Haploderus Stephens.}

1. H. caelatus Grav. - Solen, Königstein, Bieberer Höhe meist im Flug gef. - h. - Auch von B. und Hg. gef. ... Wlb. (Sch.) [H. carsus Fr. in S. V. als von L. H. bei Soden gef. ist caelatus.] -

\section{Oxytelus Gravenhorst.}

1. 0. rugosus F. - Im ganzen Gebiet s. g. - Fr. von uns und Hg. gef. - Wlb. Dlb. (Sch.) -

2. 0. insecatus Grav. - Fr. - Soden. - Homburg - s. Auch von Hg. im Gebiet gef. -

3. 0. piceus L. - Fr. - Offenbach. - Auch von Hg. gef. -

4. 0. sculptus Grav. - Im Fr. Wald unter faulenden Pflanzen z. s. - Fr. (Hg.) - Wlb. (Sch.) -

5. 0. inustus Grav. - Im ganzen Gebiet. - Fr. (Hg.) Wlb. (Sch.) -

6. 0. sculpturatus Grav. - Ueberall h. - Fr. (B., Hg.) -

7. O. nitidulus Grav. - Im ganzen Gebiet h. an Mist. Fr. (B., Hg.) - Wlb. (Sch.) -

8. 0. complanatus Er. - Ueberall an faulenden Vegetabilien. Fr. auch von Hg. gesammelt. -

9. 0. pumilus Er. - Von Hg. bei Fr. gesammelt. -

10. 0. clypeonitens Pandellé. - Hierher das in S. V. erwähnte Exemplar von 0. intricatus aus Soden, nach Fauvel'scher Bestimmung; der weiter angegebene Fundort Frankfurt ist ein Irrthum. Vielleiclit gehören Scriba's intricatus auch zu clypeonitens, da intricatus Er. = scaber Roshr. nur in Süd-Europa vorkommt. -

11. O. tetracarinatus Block = depressus Grav. - Ueberall s. g. - Fr. (B., Hg.) - Wlb. (Sch.) - 


\section{Platystethus Mannerheim.}

1. P. arenarius Fourcr. = morsitans Payk. - Im ganzen Gebiet verbreitet, in Mist. Auch bei Fr. von L. H., B. und Hg. 'gef. - Wlb. (Sch.) -

2. P. cornutus Grav. Raçe A. = cornutus Gyll. = scybalarius Runde. - Fr. am Mainufer, Oberursel und auf Salzboden bei Nauheim. - (Glänzend, Flügeldecken wenigstens zum Theil gelb oder ganz hell gefärrbt.) -

[Raçe B. = alutaceus Thoms. = tristis Mls. matt, Flügeldecken schwarz, nicht aus dem Gebiet bekannt.]

3. P. capito Heer. - Bei Cronberg und Neuenhain bei Soden s. an feuchten Waldstellen mit dem Streifnetz gef. - Fr. (Hg.) Wlb. (Sch.) -

[P. nodifrons Sahlb. in S. V. beruht sicher auf einer irrthümlichen Bestimmung. - Fauvel (Faune Gallo-Rhén. T. III, p. 183) zieht die Scriba'schen Stücke zu nitens.] -

4. P. nitens Sahlbg. - Fr. 1 Stück an einem Haus von B. angeflogen gef. (als nodifrons bestimmt). -

\section{Bledius Stephens.}

1. B. tricornis Hbst. - Soden an den früheren Salinen und nicht gefassten Salzbrunnen h. Vom Mai bis September; einigemal bei Cronberg. Die Larve $=7$. Das Thier gräbt, wie alle seine Gattungsgenossen, kleine Gänge in der nassen Erde, aus denen man sie durch Daraufklopfen hervorjagen kann. - Auch bei der Saline Salzhausen in der Wetterau. - Nauheim (Scriba). - Obgleich die Art meistens auf Salzboden vorkommt, so findet sie sich doch einzeln an anderen Lokalitäten. Scriba fand sie bei Mz. (L. H. fing sie einmal in Carlsruhe im Gasthof im Zimmer). -- G. (L.) -

2. B. subterraneus Er. - Bei Offenbach in Lehmgruben $<8$. 2 Stück. -

3. B. opacus Block. - In Soden auf Salzboden einzeln; Fr. am Main n. s.; Offenbach in Lehmgrubeń. - Auch von Hg. ges. - G. (L.) -

4. B. pygmaeus Er. - Fr. 2 Stück. - Mo. einmal sehr weit vom Wasser im Flugsand. [Hierher die in S. V. erwähnten B. pusillus Er., welcher = agricultor Heer ist.] -

5. B. fracticornis Payk. - Von Hg. 5 Stück bei Fr. gef. - 


\section{Oxyporus Fabricius.}

1. 0. rufus L. - Im ganzen Gebiet in Wäldern an Pilzen. Taunus. - Bei Fr. auch von Hg. und B. gef. - Wlb. (Sch.). -

2. 0. maxillosus F. - Im ganzen Gebiet seltener. - Fr. Taunus. - Auch von Hg. im Gebiet gesammelt. -

\section{F. S'TENINI.}

\section{Euaestethus Gravenhorst.}

1. E. bipunctatus Ljungh. = scaber Grav. - Bei Hausen und an den Kettenhöfen am Fuss von Binsengebüsch auf feuchtem Boden = 9. bis Winter. - Bei Fr. von Hg. gef. -

2. E. ruficapillus Lac. - Fr. - Rödelheim. - s. -

\section{Stenus Iatreille.}

1. S. biguttatus L. - Fr. - Soden. - Offenbach. - Auch von Hg. und B. bei Fr. gesammelt. - Wlb. Dlb. s. h. (Sch.) -

2. S. bipunctatus Er. - Fr. am Main; Soden. - Fr. (B., Hg.) -

3. S. guttula Müll. $=$ geminus Heer. - Fr. s. von C. H. und Hg. gef. -

4. S. aterrimus Er. - Einmal in einem Nest der Formica rufa bei Münster im Taunus 2. April 1876 von L. H. gef. -

5. S. fossulatus Er. - Fr. ein Männchen. -

6. S. nanus Steph. = declaratus Er. - Fr. s. auch von Hg. gef., an feuchten Stellen im Sodener Wald; Schmitterhof bei Giessen. - Wlb. (Sch.) -

7. S. bimaculatus Gyll. - Königstein s. - Fr. von L. H. und Hg. gesammelt. -

8. S. speculator Lac. $=$ providus Heer ${ }^{*}$ ), - Nauheim und Soden auf Salzboden; Fr. h. in nassen Gräben, auch von B. gef. - Wlb. (Sch.) -

*) Ich stimme nicht mit $\mathrm{F}$ a uvel überein, wenn er den Staphyl. clavicornis Scop. auf diese Species bezieht. - Nach dem heutigen Standpunkte unserer Wissenschaft zu urtheilen, hat Scopoli unter seinem St. cl. alle unsere gelbbeinigen Stenus-Arten zusammengefasst und heutzutage kann Niemand mit Sicherheit angeben, welche der nahverwandten Arten speciell darunter zu verstehen ist. - Dasselbe gilt für Paederus litoralis Grav., auf welchen Fauvel den Staphylinus gregarius Scop., ferner für den Staphyl. cyaneus Payk., auf welchen er den St. ophthalmicus Scop. bezieht. - 
9. S. providus Er. = Rogeri Krtz. = novator Duval. Fr. im Wald s. von C. H., Hg. und B. gef. - Wlb. (Sch.) Staufen im Taunus 2. April 1876. -

10. S. lustrator Er. - Fr. ein Weibchen. -

11. S. Juno F. - Fr. n. s., auch von Hg. gesammelt. -

12. S. ater Mnhm. - Nauheim auf Salzboden. - Fr. - Cronthal. - Bingen. - Bieberer Höhe bei Offenbach. - Auch von Hg. im Gebiet gef. - n. s. - Wlb. (Sch.) - .

13. S. circularis Grav. - Im Geniste bei Fr. h. - Nauheim an der Saline. - Wlb. (Sch.) -

14. S. pusillus Steph. = coniciventris Fairm. - Soden auf Salzboden. - Fr. s. auch von Hg. gef. -

15. S. ruralis Er. - Cronthal einmal $>10$. gef. -

16. S. incrassatus Er. - Rumpenheim einmal. -

17. S. buphthalmus Grav. - Rumpenheim. - Fr. (Ig.) -

18. S. melanarius Steph. = cinerascens Er. = gracilentus Fairm. = inaequalis Muls. - Ein Exemplar = 5. auf dem Mombacher Sand. -

19. S. canaliculatus Gyll. - Fr. einmal von C. H., auch von Hg. gef. -

20. S. melanopus Mrsh. = nitidus Lac. - Von Hg. bei Fr. gesammelt. -

21. S. vafellus Er. - Fr. im botanischen Garten in Mistbeeterde. - Auch von Hg. bei Fr. gef. -

22. S. fuscipes Grav. - Fr. unter nassem Laub n. s. -

23. S. opticus Grav. - Von Hg. bei Fr. gesammelt. -

24. S. Argus Grav. = decipiens Leprieur. - Rödelheim bei Fr. 2 Ex. - Soden einmal. - Fr. (Hg.) -

25. S. humilis Er. - Im Fr. Wald = 3. drei Stück. -

26. S. nigritulus Gyll. - Von Hg. bei Fr. gesammelt. -

27. S. brunnipes Steph. = unicolor Er. -- Fr. s. von L. H. und Hg. gef. - G. (L.) -

28. S. latifrons Er. - Unter Schilf an den Kettenhöfen von C. H., bei Fr. von Hg. gef. -

29. S. paganus Er. - Fr. einmal im Januar unter Moos von C. H., auch von Hg. gesammelt. - 
30. S. tarsalis Ljungh. - Im ganzen Gebiet von uns und $\mathrm{Hg}$. gesammelt. -

31. S. similis Hbst. = oculatus Grav. - In Lehmgruben bei Offenbach. - Cronthal. - Am Mainufer bei Fr. 4. April 1860 (B.), auch, von Hg. gesammelt. - Wlb. (Sch.) — Staufen im Taunus 2. April 1876. -

32. S. solutus Er. - Fr. an den Kettenhöfen und bei Offenbach in Mehrzahl gesammelt. -

33. S. cicindeloides Grav. - Soden. - Fr. g. - Im Gebiet auch von $\mathrm{Hg}$. und B. gef. - Wlb. (Sch.) -

34. S. pubescens Steph. = subimpressus Er. - Fr. an den Ketteinhöfen s. -

35. S. binotatus Ljungh. - Wlb. (Sch.) -

36. S. pallitarsis Steph. = plantaris Er. = cavifrons Mls. - Wie der vorige. -

37. S. foveicollis $\mathrm{Krtz}$. = bifoveolatus Er. = brevicollis Thoms. - Von Hg. bei Fr. gesammelt. -

38. S. picipennis Er. - Wie der vorige. -

39. S. nitidiusculus Steph. = tempestivus Er. - In den Torfbrüchen bei Enkheim einmal $<4$. -

40. S. flavipes Steph. $=$ filum Er. - Fr. Forsthaus im Juli von C. H., bei Fr. von Hg. gesammelt. -

[S. glacialis Heer, den Scriba vom Taunus anführt, kommt sicher dort nicht vor. Er findet sich nur in den Hochalpen am abfliessenden Gletscherwasser.] -

41. S. subaeneus Er. - L. H. fand ihn einmal in Neuenhain bei soden. - Fr. (Hg.) -

42. S. impressus Germ. = Aceris Lac. $=$ angustulus Heer. - Im Fr. Wald von C. H., auch von Hg. im Gebiet gef. - Wlb. (Sch.) -

43. S. geniculatus Grav. - Fr. und Soden je einmal. - Auch von Hg. gesammelt. - Wlb. (Sch.) -

44. S. fuscicornis Er. - Von Hg. bei Fr. gesammelt. -

45. S. pallipes Grav. - Fr. unter Laub und Moos in Winter. - Wlb. (Sch.) -

46. S. Erichsonis Rye = flavipes Er. - Fr. - Höchst. Soden. - Rödelheim im Geniste. - Wlb. (Sch.) - 


\section{$-163-$ \\ G. P A E D E R I N I.}

\section{Sunius Stephens.}

1. S. filiformis Latr. - Bieberer Höhe bei Offenbach unter Steinen an trockenen Orten. - Fr. Wald. - Falkenstein. - B. fïnd 8. März 18611 Ex. bei Formica rufa, sonst h. bei Fr. Auch Hg. sammelte die Art bei Fr. - Wlb. (Sch.) -

2. S. intermedius Er. Heer. - Ein Stück am Fr. Forsthaus. - G. (L.) -

3. S. gracilis Payk. = angustatus Payk. - Fr. Wald an sandigen Stellen h. - Seckbach. - Königstein. - Soden. - B. fand im Schwanheimer Wald 2 Ex. bei Formica rufa. - Auch von Hg. im Gebiet gesammelt. - Wlb. (Sch.) -

\section{Stilicus Latreille.}

1. S. subtilis Er. - Fr. ein Weibchen. - Soden einmal. - Fr. einmal von B., auch von Hg. gef. - Wlb. (Sch.) -

2. S. rufipes Germ. - Fr. zweimal gef. Auch Hg. sammelte diese Art im Gebiet.

3. S. similis Er. - Fr. Wald. - Unter Schilf an den Kettenhöfen bei Fr., von B. und Hg. gef. - n. s. - Wlb. (Sch.) -

4. S. geniculatus Er. - Fr. ein Weibchen. -

5. S. orbiculatus Payk. $=$ affinis Er. $=$ fuscipes Er. $-A \mathrm{~m}$ Röderberg bei Fr. - B. fand 2 Ex. bei Fr., auch Hg. mehrere Stücke. -

[S. Erichsonis Fauv. = orbiculatus Er. noch nicht aus dem Gebiet bekannt.]

6. S. fragilis Grav. - Bei Fr. von Hg., bei G. von L. ges. -

\section{Domene Fauvel.}

1. D. scabricollis Er. - Von Hg. bei Fr. gef. -

\section{Scopaeus Erichson.}

1. S. gracilis Sperk. = Erichsonis Kolen. = apicalis Muls.

- Fr. 1 Männchen. - Rüdesheim = 8. -

2. S. laevigatus Gyll. - Fr. - Offenbach, Neuenhain und Socien. - Ems. - Auch von Hg. bei Fr. gef. - Wlb. (Sch.) -

3. S. minimus Er. - Von Hg. bei Fr. gef. - 
4. S. cognatus Rey. - Soden. - Königsteiner Burg unter Steinen. -

5. S. sulcicollis Steph. = minutus Er. = pusillus Ksw. = abbreviatus Mls.

d) Forma elytris longis. - Bieberer Höhe unter Steinen. [Hierher die von Scriba als minimus bestimmten Stücke.] — Von Hg. bei Fr. gef. -

b) Forma elytris abbreviatis. - Soden einmal an einer Salzquelle. -

\section{Lithocharis Erichson.}

1. L. castanea Grav. - Fr. ein Weibchen. -

2. L. fuscula Mnhm. = rufa Mls. - C. H. fand 1 Stück bei Bingen, L. H. eins bei Soden. -

3. L. ripicola Krtz. - Fr. (Hg.) - G. (L.) -

4. L. ochracea Grav. - Fr. 2 Ex. von C. H., auch von Hg. gef. -

5. L. obsoleta Nordm. = obscurella Er. = opaca Redtb. Bei Rödelheim einige Exemplare, bei Fr. von Hg. gesammelt. -

6. L. brunnea Er. = monticola Hampe = ferruginea Er. - Fr. s., auch von Hg. gef. -

7. L. rufiventris Nordm. - Von Sch. bei Wlb. gef. -

8. L. melanocephala F. - Im ganzen Gebiet h. - Fr: - Im Taunus. - Biebrich. - St. Goarshausen. - Schon am 20. März 1860 von B. gef. - Bei Fr. auch von Hg. gesammelt. - Wlb. (Sch.) G. (L.) - Münster (Soden) bei Formica congerens 2. April 1876. -

\section{Paederus Gravenhorst.}

1. P. brevipennis Lac. = geniculatus Dietr. - Fr. 2 Ex. von C. H., von Hg. öfter gesammelt. — G. (L.) -

2. P. litoralis Grav. - Fr. - Seckbach. - Soden. - Ems. - Auch von Hg. und B. gesammelt. - Wlb. (Sch.) - G. (L.) -

3. P. riparius L. - Im Gebiet h. - Auch von Hg. und B. gef. -

4. P. caligatus Er. = paludosus Dietr. - Unter Schilf hinter den Kettenhöfen bei Fr. -

5. P. limnophilus Er. - G. (L.) - B. fing 21. April 1861 2 Stïck mit dem Streifnetz auf der Königswiese im Fr. Wald. - 
6. P. fuscipes Curtis = Iongipennis Er. - Im Fr. Gebiet s. h., auch von Hg., von B. einmal gef. - G. (L.) - Gimbacher Hof am Fusse des Staufen 2. April 1876 (B.). -

7. P. ruficollis Er.

[Forma a. = sanguinicollis Steph. = Iongicornis Aubé findet sich im Süden.] Bei ·uns kommt nur

Forma $\mathbf{b}_{\text {. }}=$ ruficollis $\mathrm{F}_{\text {. }}=$ gemellus Krtz. vor. - Fr. Nauheim h. an den Ufern der Us. - Ems. -

\section{Lathrobium Gravenhorst.}

1. L. punctatum Fourcr. = brunnipes F. - Fr. einmal gef., von Hg. öfter. - G. (L.) -

2. L. elongatum L. - Im Fr. Gebiet h., auch von Hg. und B. gesammelt. - Wlb. (Sch.) - G. (L.) -

3. L. fulvipenne Grav. = alpestre Heer = Letzneri Gerhardt. - Fr. - Königstein. - Feldberg. - Ueberall h., auch von Hg. gef. - Wlb. (Sch.) - G. (L.) -

4. L. laevipenne Heer $=$ dentatum Kellner. - G. (L.) -

5. L. filiforme Grav. = impressum Heer. - Torfwiese bei Enkheim $>$ 5. - Bei Fr. von Hg. gef. -

6. L. dilutum Er. - Von Hg. bei Fr. gef. -

7. L. Iongulum Grav. - G. (L.) - Fr. (Hg.) - Rödelheim. Bingen. -

[longipenne Fairm, die langflügelige Form, fand Hg. bei Fr.] -

8. L. multipunctatum Grav. - Im Fr. Gebiet n. s., auch von Hg. gef. - G. (L.) -

9. L. quadratum Payk. = terminatum Grav. - Fr. unter Schilf; am Main im Flug gef. -

10. L. pallidum Nordm. - Fr. - Bingen unter Steinen auf den Bergen $=4$. - Auch von $\mathrm{Hg}$. im Gebiet gesammelt. -

\section{Achenium Stephens.}

1. A. depressum Grav. - Friedberg (Fuhr). -

2. A. humile Nicol. - 3 Stücke im Genist des Maines und des Metzgerbruches; 1 Stück am 27. März 1861 unter einem Stein auf einer nassen Wiese an der Bieberer Höhe (B.). [Eins dieser Stücke nun in Sammlung v. Heyden.] — 


\section{Cryptobium Mannerheim.}

1. C. fracticorne Payk. - Fr. - Im ganzen Gebiet. -

\section{H. STAPHYLININI.}

\section{Othius Stephens.}

1. O. fulvipennis F. - Fr. Wald $=3$. in Begattung. Auch von Hg. bei Fr. gef. - Dlb. Wlb. (Sch.) - G. (L.) -

2. O. myrmecophilus Kiesw. - Bei Fr. von Hg. gesammelt. -

3. 0. melanocephalus Grav. - Bingen. - Fr. $<4$. unter Kieferurinde. -

\section{Baptolinus Kraatz.}

1. B. pilicornis Payk. - Von Hg. bei Fr. gesammelt. -

2. B. affinis Payk. = alternans Grav. - Taunus. - Fr., auch von B. einmal gef. - Wlb. (Sch.) -

\section{Leptacinus Erichson.}

1. L. parumpunctatus Gyll. - G. (Scriba). - Fr. (Hg.) -

2. L. batychrus Gyll. = linearis Grav. - Fr. - Offenbach in Lehmgruben. - Die Art variirt sehr in der Grösse; die seither als zwei Arten angenommenen Thiere sind nur die Endpunkte einer Reihe von Zwischenformen. Auf Mist am Röderberg s. h. von B. 20. März 1860, auch von Hg. bei Fr. gef. -

3. L. formicetorum Märk. - Im Fr. Wald n. s. in den Nestern der Formica rufa. - Königstein. - Von B. s. h. im Schwanheimer Wald gesammelt, auch von Hg. gef. - Münster (Soden) im Nest der F. rufa (vera) 2. April 1876. -

\section{Xantholinus Serville.}

1. X. fulgidus F. - Fr. von C. H., Hg. und B. gef. - G. (L.) -

2. X. punctulatus Payk. $=$ atratus Heer $=$ picipes Thoms. = ochraceus Gyll. = Thomsonis Schw. - Fr. - Oberrad. Offenbach. In den Nestern der Formica fuliginosa. - Schmitterhof bei Giessen. - Am Feldberg bei Formica rufa. - Soden. - Auf Mist am 


\section{$-167-$}

Röderberg 21. März 1860 s. h. (B.) - Fr. (Hg.) - Wlb. (Sch.) G. (L.) - Münster im Taunus bei F. rufa 2. April 1876. - Die Art variirt sehr, doch finden sich alle Uebergänge auch zu der chagrinirten Form ochraceus. - Die Form ochraceus auch bei Wlb. (Sch.) und Münster (Soden) in einem Nest der Formica rufa (vera) 2. April 1876. -

3. X. glabratus Grav. - G. (L.) - [L. H. fand ihn bei Lauterbach in Oberhessen.] -

4. X. glaber Nordm. Er. - Sodener Wald bei Formica rufa. -

5. X. tricolor F. - Fr. - Bei Formica rufa Feldberg, Königstein, Schlangenbad. - Bei Fr. auch von Hg. gef. -

6. X. distans Muls. - Schmitterhof bei Giessen und Fr. je einmal. - Wlb. (Sch.) -

7. X. linearis Oliv. $=$ longiventris Heer $=$ multipunctatus Thoms. - Fr. - Seckbach. — Schmitterhof. — Saline Salzhausen. Soden. - Königstein bei Formica fuliginosa, aber auch in fetter Erde, z. B. im Fr. botanischen Garten. - Auch diese Art ist sehr veränderlich, je nach ihrer Verbreitung über Europa, Nordafrika und Mittelasien. - Bei Fr. auch von Hg. gesammelt. - Wlb. (Sch.) -

\section{Emus Curtis.}

1. E. hirtus L. - Fr. im Wald auf der Babenhäuser Chaussee unter Kuhfladen (v. Twardowski, St.) - Bingen (Bach). - Früher auch auf der Bornheimer Haide unter Kuhmist von B. gef. - Im Fr. Wald an der sogenannten Kuhtränke von Hg. gef. - Dlb. (Sch.) -

2. (Creophilus) maxillosus L. - Fr. h. an Aas. In dem Keiler an der Salpeterhütte am Fr. Wald. - Am Main oberhalb Fr. an der Mainschanze von B. gef. - Auch von Hg. im Gebiet gesammelt. Dlb. (Sch.) - Ernsthausen in Nassau (Lehrer Dörr bei K.). -

\section{Leïstotrophus Perty.}

1. L. nebulosus F. - Praunheim bei Fr. - Röderwald und Königstein (B.). - Fr. (Hg.) - Wlb. (Sch.) - Dlb. im Mai (K.). -

2. L. murinus L. - Fr. in Kuhfladen h. Röderwald und Königswiese im Fr. Wald an Aas, seltener als der vorige von B. gef. - Bei Fr, auch von Hg. gesammelt. - Wlb. (Sch.) - 


\section{Staphylinus Linné.}

1. (Trichoderma Steph.) pubescens Deg. - Am Feldberg an Pferdekoth. - Schlangenbad. - An Pferdemist bei Okriftel, auch bei Fr. im April von B. gef., sowie von Hg. - Wlb. (Sch.) - Staufen im Taunus an Fuchslosung 2. April 1876 (B.). -

2. (Staphylinus) fulvipes Scop. - Fr. (M. S.) - Fussweg von Offenbach nach der Bieberer Höhe unter einem Stein in einem trockenen Graben 1 Stück im April 1875 von B. gef. -

3. St. stercorarius Oliv. - Bieberer Höhe bei Offenbach. Bruchschneisse im Fr. Wald s. - Fr. (Hg.) - Wlb. z. h. (Sch.) -

4. St. chalcocephalus F. - Fr. einmal von B. gef., auch von Hg. gesammelt. - Wlb. (Sch.) -

5. St. fossor Scop. - Bingen. - Einmal bei Cronthal im Flug gef. (B.) - Taunus (Hg.) - Wlb. einmal (Sch.). - Wsb. im Juni an der Tränke und an der Wellritz 14. Juli an Maulwurf (K.). -

6. St. erythropterus L. - Bei Fr. von Hg. gef. -

7. St. caesareus Cederh. - Ueberall h. - Offenbach. Fr. (B. und Hg.) - Dlb. Wlb. (Sch.) -

8. (Georius Steph.) olens Müll. = micropterus Redtb. $=$ brachypterus auctorum (nec Brullé). - Exotische Arten bilden in der Zahnform Uebergänge von der ehemaligen Gattung Ocypus (= Georius ältèrer Name) zu Staphyl. Bei einer grossen Reihe Exemplare aus verschiedenen Fundorten finden sich Uebergänge von der kurz- zur längerflügeligen Form. - Fr. s. von C. H. und Hg. gef. - Wlb. mehrmals unter Steinen und auf Wegen (Sch.). -

9. (G.) brunnipes F. - Fr. Wald im October unter Moos s., auch von $\mathrm{Hg}$. gesammelt.

10. (G.) cyaneus Payk. - Fr. n. s. auf Feldwegen. - Unter Steinen im Röderwald und Bieberer Höhe bei Offenbach im April von B., auch von Hg. im Gebiet gef. - Wlb. Dlb. (Sch.) -

11. (G.) nitens Schrk. = similis F. - Auf den Wiesen bei Hausen. - Bingen $=4$. auf den Bergen. - Fr. auch h. von B. gef., sowie von Hg. - G. (L.) - Wlb. Dlb. (Sch.) -

12. (G.) picipennis F. - Steinbrüche hinter Offenbach. - Von uns und Hg. bei Fr. gesammelt. - Wlb. (Sch.) -

13. (G.) fuscatus Grav. - Von Hg. bei Fr. gef. - G. (L.) Wlb. (Sch.) - 
14. (G.) fulvipennis Er. = confusus Baudi. - Fr. einmal auch von Hg. gef. - Wlb. 3 Stück (Sch.). - G. (L.) -

15. (G.) aeneocephalus Deg. = cupreus Rossi. - 3 Stück von Fr. am 4. und 8. April 1860 und 29. April 1861 von B. gef., auch von Hg. gesammelt. - Wlb. (Sch.) -

16. (Tasgius Steph.) pedator Grav. - Mz. (S.) -

17. (T.) ater Grav. - Von Hg. bei Fr. gesammelt: -

18. (Anodus Nordm.) edentulus Block = morio Grav. - Feldberg. - Saline Salzhausen. - Von C. H. und Hg. auch bei Fr. gef. - G. (L.) - Wlb. zweimal (Sch.). -

\section{Actobius Fauvel = Erichsonius Fauvel ol.} (= Pilonthus ol. pars.).

1. A. cinerascens Grav. - Ein Stück bei Bergen = 5. -

2. A. procerulus Grav. = lathrobioides Baudi. - Soden am Teichufer; Hofheim am Bach, der aus dem Lorsbacherthal kommt. -

3. A. prolixus Er. = dissimilis Baudi. - Soden in Gesellschaft des procer. - Nauheim und Wisselsheim auf Salzboden. - Fr. Friedberg (Fuhr). -

\section{Philonthus Curtis.}

1. (Bisnius Step.) splendens F. - Bei Fr. von C. H. und Hg. gesammelt. - Wlb. (Sch.) -

2. (B.) intermedius Lac. - Fr. s., anch von Hg. gesammelt. - Wsb. an der Wellritz an Maulwurf 19. Juli (K.). -

3. (B.) nitidus F. - Von Hg. bei Fr. gef. - G. (L.) -

4. (B.) proximus $\mathrm{Krtz}$. = carbonarius Er. = succicola Thoms. - In Deutsche Entom. Zeitsch. 1875, p. 386, gab L. H. eine Revision dieser Gruppe mit Angabe der Kennzeichen dieser einander nahe verwandten Arten. - Oelmühle bei Königstein an ausgelegten todten Vögeln. - Wlb. (Sch.) - Die älteste Beschreibung ist von Kraatz nach ostindischen Stücken aufgestellt. -

5. (B.) aeneus Rossi. - Schmitterhof bei Giessen unter Moos. - Fr. n. s. an Aas. - Oelmühle bei Königstein. - Auch in NordAmerika, Kaukasus, Sibirien bis Amur und in Tasmanien. - Bei Fr. auch von Hg. gef. - Wlb. (Sch.) - 
6. (B.) carbonarius Gyll. Thoms. = tenuicornis Muls. = punctiventris Janson. - Fr. am Main im Sonnenschein fliegend. Fr. Forsthaus unter faulenden Pflanzen. - Grosse Feldberg. - Häufige Art. -

7. (B.) punctatus Grav. - Fr. 1 Stück. — Friedberg (Fuhr). s. -

8. (B.) cephalotes Grav. - Fr. an Aas s., auch von Hg. gef. - L. H. besitzt ihn auch aus Chile. - Wlb. (Sch.) -

9. (B.) umbratilis Grav. - Am Fr. Forsthaus unter faulenden Pflanzen, bei Fr. auch von Hg. gef. -

10. (B.) sordidus Grav. = placidus Er. - Fr. 1 Ex. aus dürrem Holz entwickelt, auch von Hg. gef. -

11. (B.) fuscus Grav. - Ein Stück unter Rinde Fr. $>4$. -

12. (B.) sanguinolentus Grav. - Im Oberräder Wald. - Offenbach auf dem Weg laufend. - Fr. (Hg.) -

13. (B.) immundus Gyll. = fumigatus Er. - Fr. - Taunus. - Auch von Hg. im Gebiet gesammelt. - Wlb. (Sch.) -

[Scriba führt corvinus Er. als im Gebiet vorkommend an; was er mir aber so bestimmte, waren Stücke von ebeninus.] -

14. (B.) ventralis Grav. - Fr. einmal von C. H., auch von Hg. gef. --

15. (B.) debilis Grav. - Fr. n. s. an Kuhkoth. - Soden. Ginheimer Wald. - Auch von Hg. gef. -

16. (B.) discoideus Grav. - G. (L.) - Fr. (Hg.) -

17. (Ph.) laminatus Creutz. - Fr. am Main im Sonnenschein im October fliegend. Von B. $>$ 4. gef., auch von $\mathrm{Hg}$. gesammelt. -

18. (Ph.) rotundicollis Ménétr. = scutatus Er. - Fr. einmal von C. H., auch von Hg. gesammelt. -

19. (Ph.) atratus Grav. - Fr. (C. H. und Hg.) - Bei Salzschlirf in Oberhessen beobachtete L. H. diese Art, wie sie bei brennender Mittagshitze im August an der Schliz umherlief und, den Hinterleib in die Höhe gestreckt, Jagd auf kleine Fliegen machte. - Wlb. (Sch.) -

20. (Ph.) ebeninus Grav. Raçe a.= varians Thoms. (mit dunkeln Flügeldecken). - Kalkbrüche bei Offenbach. - Bei Fr. h. von I. H. und Hg." gef. - Wlb. (Sch.) -

Die rothflügelige Raçe corruscus Grav. = turbatus Er. vonl Hg. bei Fr. gesammelt. - 
21. (Ph.) quisquiliarius Gyll.

Form a. (mit schwarzen Flügeldecken) ist im Fr. Gebiet 11. s.; auch von $\mathrm{Hg}$. gef. -

Form $b_{0}=$ inquinatus Steph. = rubidus Er. fand L. H. bei Soden, Hg. auch bei Fr.

22. (Gabrius Steph.) splendidulus Grav. - Fr. unter Moos und bei Formica fuliginosa am Fr. Forsthaus; im»Isenburger Wald von Hg. gef. - Wlb. (Sch.) -

23. (G.) astutus Er. - Von Hg. bei Fr. ges. - Wlb. (Sch.) -

24. (G.) fimetarius Grav. - Fr. an Aas, auch von Hg. gef. -

25. (G.) nigritulus Grav. = trossulus Nordm. - Im ganzen Gebiet s. g. - Fr. Wald. - Wiesen bei Hausen. - Hohe Mark im Taunus. - Biebrich am Rhein. - Fr. (Hg.) - Wlb. (Sch.) -

26. (Cheilocolpus Sol.) decorus Grav. - Fr. s. -

27. (Ch.) politus F. - Am Feldberg öfter. - Fr. Wald. Auf den Wiesen bei Hausen. - Wlb. (Sch.) -

28. (Ch.) Mannerheimi Fauvel = lucens Er. nec Manhm. Fr. zweimal von C. H. und Hg. gef. -

29. (Ch.) lepidus Grav. = gilvipes Er. - (Die rothflügelige Form nicht aus dem Gebiet, doch aus Oberhessen bekannt.)

Die Form mit dunkel erzglänzenden Flügeldecken fand C. H. öfter auf der Bieberer Höhe bei Offenbach. -

30. (Ch.) nitidulus Grav. - 2 Stück von Fr. (auch von Faurel für richtig bestimmt zurückerhalten) von C. H. und Hg. gesammelt. -

31. (Ch.) varius Gyll. (mit einfarbig dunkeln Flügeldecken) Fr. Wald unter Moos; im Ginheimer Wald gesellig. - Rumpenheim. Fr. (Hg.) -

Var. bimaculatus Grav. (Flügeldecken mit je einem rothen Fleck, die so gross werden können, dass sie die ganze Flügeldecke einnehmen) fand L. H. s. s. bei Fr. -

32. (Ch.) pullus Nordm. - Offenbacher Kalkbrüche. - G. (L.) - Fr. (Hg.) -

33. (Ch.) tenuis F. - Fr. - Ems. - Mehrere Exemplare im Genist 8. April 1860 von B., auch von Hg. bei Fr. gef. - Wlb. (Sch.) -

34. (Ch.) marginatus F. - Fr. einmal. - Sehr seltene Art. -

35. (Ch.) cruentatus Gmel. L. = bipustulatus Panz. Fr. (Hg.) - s. s. - 
36. (Ch.) longicornis Steph. = scybalarius Nordm. = promptus Er. - Fr. (Hg.) - Soden. - Wlb. (Sch.) -

37. (Ch.) varians Payk. = punctiventris Steph. nec Krtz. $=$ opacus Thoms. - Ueberall im Gebiet h. (mit rothen Vorderhüften und oft mit rothem Fleck der Flügeldecken). - Fr. (Hg.) -

Die Form b. = agilis Grav. (mit schwarzen Vorderhüften; nie mit rothem Discoidalfleck, aber schmal roth gesäumt, oder ganz braun) fand L. H. bei Soden; Hg. bei Fr.

38. (Ch.) albipes Grav. - Von Hg. bei Fr. gef. -

39. (Ch.) fumarius Grav. - Am Röderberg bei Fr. n. s., auch von Hg. gef. -

40. (Ch.) micans Grav. - An der Bruchschneisse im Fr. Wald; auch von Hg. gef. -

41. (Ch.) fulvipes $\mathbf{F}$. = varipes Mls. = obscuripes Bris. Am Röderberg bei Fr. - Bieberer Höhe bei Offenbach unter Steinen. - Hofheim am Bach im Frühjahr. - In nassen Gräben < 4. von B. gef., anch von Hg. gesammelt. - G. (L.) - Wlb. (Sch.) -

42. (Ch.) vernalis Grav. - Fr. im Wald in den Nestern von Formica fuliginosa. - Auch von B. und Hg. bei Fr. gef. -

\section{Vellejus Mannerheim.}

1. V. dilatatus F. - Am Fr. Forsthaus von C. H. in einem Hornissennest in einer alten Eiche und ebenso von v. Twardowski im Schwanheimer Wald gef. -

\section{Quedius Stephens.}

1. 0. microps Grav. = chrysurus Kiesw. - Lebt s. s. bei Formica fuliginosa; mehrmals auch im Juni und Juli aus überwintertem Eichenmulm, worin Sciara-Larven, entwickelt. Die Art ist interessant wegen ihrer sehr kleinen, flachen Augen. -

2. Q. brevis Er. - Seltene Art. - Im Fr. Wald, District Gehren $<5$. in Nestern der Formica rufa gef. - Ebenso hinter dem Forsthaus 6 Stück am 18. März 1861 (B.). -

3. Q. lateralis Grav. - Lorsbacher Thal, Fr. Wald an Pilzen s. - Schlangenbad. - Fr. (Hg.) -

4. Q. ochripennis Ménétr. = floralis Lac. - Früher mit fulgidus vermengt. - Wlb. (Sch.) - 
5. Q. fulgidus F. = bicolor Redtb. - Flügeldecken stets roth. Auch über diese Art und seine Verwandten gab L. H. eine Uebersicht in der Deutschen Entomol. Zeitschr. 1875, p. 385. - Fr. unter Platanrinde in den Promenaden. - Mz. unter Pappelrinde. Aus Weidengallen $<$ 6. entwickelt. - Königstein. - Flörsheim. - Fr. 2 Stück von B., auch von Hg. gef. - [Hierher auch quadripunctatus Thoms.] -

6. a. mesomelinus Marsh. = temporalis Thoms. (schwarze Flügeldecken, Hinterleibsränder fein roth gerandet). - Soden auf Salzboden. - Biebrich an ausfliessendem Saft von Rosskastanien. - Fr. auf der Strasse an Häuserwänden von L. H. gef. - Wlb. (Sch.) -

7. Q. ventralis Arragona = truncicola Fairm. - Ein Exemplar dieses seltenen, an dem mattrothen Hinterleib leicht kenntlichen Thieres fing L. H. $<7$. in nassem Mulm einer hohlen Buche im Fr. Wald. - Wsb. einmal (K.). -

8. 0. xanthopus Er. - Fr. 1 Ex., wahrscheinlich aus dem Taunus. Gebirgsthier. Auch von Hg. gesammelt. -

9. Q. scitus Grav. - Fr. Wald im District Schwengelbrunnen $>3$. - Am Fusse der alten Eichen bei Schwanheim zweimal von B. gef. 1.0. Q. infuscatus Er. - Von Hg. bei Fr. gesammelt. -

11. Q. cinctus Payk. = impressus Panz. = rufocinctus Mnhm. - Fr. auf den Strassen an Häusern, in Kellern, Mainkur an Baumpilzen. - Ein Stück am 3. Juni 1860 von B., auch von Hg. bei Fr. gef. - Wlb. (Sch.) -

12. (Microsaurus Steph.) tristis Grav. = frontalis Er. [Das von Scriba, erwähnte Ex. „Offenbach v. Heyden" ist nicht diese Art, sondern fuliginosus.] - Fr. von Hg. und B. gesammelt. -

13. (M.) fuliginosus Grav. - Soden unter Gras. - Von Hg. und L. H. bei Fr. gef. -

14. (M.) molochinus Grav. - Soden auf Salzboden. - Feldberg = 5. - Fr. (Hg.) - Kommt auch in Nord-Amerika vor. -

15. (M.) picipes Manhm. = gracilicornis Heer. - Im Ginheimer Wald s. s. $=9$. gef. - Wlb. (Sch.) -

16. (M.) nigriceps Krtz. = praecox Fauv. = pineti Bris. Soden und Oberrad je 1 Ex. von uns, auch von Hg. bei Fr. gesammelt. -

17. (M.) limbatus Heer $=$ marginalis Krtz. = maurorufus Er. nec Grav.*) - Von Sch. bei Wlb. gef. -

*) Maurorufus Grav. = modestus Krtz. 
18. (Raphirus Steph.) attenuatus Gyll. = picipennis Heer $=$ fallaciosus Krtz. - Fr. Wald, District Bruchschneisse, von C. H., von Hg. auch bei Fr. gef. -

[Den mit dieser Art nahe verwandten Q. semiaeneus Steph. = semióbscurus Er. = proximus Krtz. sammelte L. H. in Lauterbach in Oberhessen, findet sich aber auch sicher noch in unserem Gebiet.]

19. (R.) boops Grav. - Fr. - Soden. - Auch von Hg. im Gebiet gesammelt. -

\section{Heterothops Stephens.}

1 Stück. -

1. H. praevia Er. = nigra Krtz. = nidicola Thoms. - Fr.

2. H. quadripunctula Grav. - Von Hg. bei Fr. gef. -

3. H. dissimilis Grav. = praevia Thoms. = binotata Duv. $=$ brunneipennis Kiesw. - Fr. h., auch von Hg. gesammelt. -

\section{Euryporus Erichson.}

1. E. picipes Payk. - Von Hg. bei Fr. gesammelt. -

\section{TACHYPORINI.}

\section{Bolitobius Stephens.}

1. B. lunulatus L. nec Er. = atricapillus F. - An Pilzen s. h. im ganzen Gebiet. - Taunus. - Im Herbst. - Fr. von L. H. und Hg., im Röderwald von B. gef. - Wlb. (Sch.) -

2. (Lordithon Thoms.) trinotatus Er. - In Pilzen h. im April und October. — Offenbach. - Niederwald bei Rüdesheim. — Taunus (B.). - Fr. auch von Hg. gef. - Wlb. (Sch.) -

3. (L.) exoletus Er. - In Pilzen n. h. Fr., auch von Hg. gef. Röderwald einmal (B.). - Wlb. (Sch.) -

4. (L.) pygmaeus F. - In Pilzen h. - Soden. - Offenbach im October. - Fr., auch von Hg. gef. - Wlb. (Sch.) -

Var. biguttatus Steph. $=$ intrusus Hampe. - Bei Fr. 2 Stück von C. H. gef. -

5. (L.) bicolor Grav. - C. H. fand 1 Stück dieser seltenen Art bei Schlangenbad. - 


\section{Megacronus Stephens (= Bryoporus Kraatz).}

1. M. striatus 0 I. - An ausfliessendem Eichensaft von uns $<6$. bei Soden und an den Oberräder Schiessständen gef. - Fr. (Hg.) Wlb. (Sch.) -

2. M. cingulatus Mhm. - Von Hg. bei Fr. gef. -

3. M. analis Payk. - Einmal bei Oberrad $\gg$ 9. von C. H. gef. -

4. M. inclinans Grav. - Bei Fr. von Hg. gef. - G. (Scriba). -

5. M. rufus Er. - Von Hg. bei Fr. gef. - Wlb. dreimal gef. (Sch.) -

\section{Mytecoporus Mannerheim.}

1. M. splendidus Grav. - Im Rödelheimer Wald $=8$. von C. H. gesammelt. - Am Entensee bei Bürgel am Main am 15. April 1872 von L. H. einige Stücke gef. -

2. M. rufescens Steph. = lucidus Er. - Von Hg. bei Fr. gef. -

3. M. nanus Er. - Von Hg. bei Fr. gef. -

4. M. punctatus Gyll. - Einmal von C. H. bei Bingen gef. -

5. M. brunneus Mrsh. = lepidus Grav. - Fr. n. s. s. -

Var. ruficornis Krtz. - Zweimal bei Fr. von C. H. im Mai und einmal von L. H. bei Soden gef.; auch Hg. und M. S. fanden die Varietät bei Fr. -

Var. longulus Mhm. - Einmal bei Fr. von C. H. gef., auch von Hg. gesammelt. -

Var. tristis Grav. - L. H. fand 1 stück $>4$. in Fr. an einem Haus angeflogen. -

6. M. splendens Mrsh. - Fr. und Bieberer Höhe unter Steinen $<$ 5. von L. H. je einmal gef. - Auch von Hg. bei Fr. gesammelt. - G. (L.) - Wlb. (Sch.) -

7. M. clavicornis Stph. = pronus Er. - Von M. S. bei Fr. gef. [Stücke in Sammlung v. Heyden.] Auch von Hg. gesammelt. -

\section{Tachinus Gravenhorst.}

1. T. elongatus Gyll. - Von Hg. bei Fr. gef. -

2. T. fimetarius F. - Im Gebiet h. auf Gesträuch. - Bei Soden auf Cornus albus-Blüthen $>6$, bei Höchst auf Ahornblüthe $>5$. von C. H. und in Erlenblüthen im Hengster bei Offenbach s. h. von L. H. gef. - Fr. (Hg.) - 
3. T. subterraneus L. - Von M. S. bei Fr. unter faulenden Pflanzen gef., auch von Hg. gesammelt. -

4. T. collaris Grav. - Eine häufige Art. - Fr. - Soden unter Gras im Juni. - Auch Hg. fand sie oft bei Fr. -

5. T. marginellus F. - Zweimal von M. S. bei Fr. gef. [nun in Sammlung 'v. Heгden], auch ron Hg. gesammelt. -

6. T. laticollis Grav. - Von Hg. bei Fr. gef. -

7. T. flavipes F. - Wie die vorige Art h. - Fr. Wald. Rumpenheim; auch in Maingenist. - Fr. (Hg. und B.) - Wlb. (Sch.) -

8. T. humeralis Grav. - Fr. in Schwämmen und an Menschenkoth n. s. im Herbst. Auch von Hg. gef. -

9. T. pallipes Grav. - L. H. fand im Gebiet nur 1 Stück bei Fr., Hg. mehrere. -

10. T. scapularis Steph. = palliolatus Krtz. - Nur ein Weibchen dieser seltenen Art bei Fr. von L. H. gef. -

11. T. bipustulatus F. - Zweimal bei Fr. von L. H. gef., auch von Hg. gesammelt. -

12. T. rufipes De Geer. - Im ganzen Gebiet h. an Misthaufen und faulenden Pflanzen. - Fr., auch von Hg. ges. - Wlb. (Sch.) --

\section{Habrocerus Erichson.}

1. H. capillaricornis Grav. - Bei Fr. einzeln unter faulendem Holz, an Schwämmen von C. H. und M. S. gesammelt. - Im Fr. Wald von $\mathrm{Hg}$. und B. n. s. aus Laub gesiebt. -

\section{Cilea Duval (= Erchomus Motsch. = Leuco- paryphus Krtz. = Coproporus Krtz.).}

1. C. silphoides L. - Fr. im Juli an vegetabilischen Dunghaufen z. s. von uns und $\mathrm{Hg}$. gef. -

\section{Tachyporus Gravenhorst.}

1. T. obtusus L. - Fr. im Genist, unter Schilf h.; auch von Hg. gef. - Wlb. (Sch.) -

2. T. formosus Matthews $=$ rufus Er. - Fr. - Rödelheimer Wald. - Soden. - Im August s. - [Hierher die in S. V. erwähnten abdominalis von Soden.] - 
3. T. solutus Er. - Im Fr. Wald unter Laub und bei Fr. unter Schilf hinter den Kettenhöfen. Auch von $\mathrm{Hg}$. und B. im Gebiet ge sammelt. - G. (L.) -

4. T. chrysomelinus L. - Im Gebiet h. - Fr. Wald, auch von Hg. gef. - Wlb. (Sch.) -

5. T. hypnorum F. - Im ganzen Gebiet unter abgefallenem Laub g. - Fr. - Bingen in Kirschblüthen $=4$. - Jon Hg. und B. am 28. März 1860 bei Fr. h. gef. - Wlb. (Sch.). - Münster (Soden) bei Formica congerens 2. April 1876. -

6. T. ruficollis Grav. - Von Hg. bei Fr. gesammelt. -

7. T. atriceps Steph. = humerosus Er. = pulchellus Mhm. - Bei Fr. = 8. von C. H. in Gusellschaft von Formica fuliginosa gef. - Auch von Hg. gesammelt. -

8. T. macropterus Steph. = scitulus Er. - Auf der Königsteiner Ruine $=5$. unter Steinen gef., von Hg. bei Fr. -

9. T. pusillus Grav. - n. s. - Fr. - Nauheim $<6$. Auch von $\mathrm{Hg}$. gef. -

10. T. nitidulus $\mathbf{F}$. = brunneus $\mathbf{F}$. - Im ganzen Gebiet g. Fr. - Offenbach. - Soden. - Bingen. - Das ganze Jahr hindurch unter Laub, auch in Schwämmen. Von Hg. überall gef. - Wlb. (Sch.) -

11. T. transversalis Grav. - Von Hg. und B. bei Fr. gef. [1 Ex. in Sammlung v. Heyden.] -

Conurus Stephens (= Conosoma Kraatz).

1. C. bipunctatus Grav. - Von C. H. 2 Stück bei Fr. gef., auch von $\mathrm{Hg}$. gesammelt. -

2. C. bipustulatus Grav. - Von Hg. bei Fr. gesammelt. -

3. C. litoreus L. - Unter feuchtem Holz am Forsthaus im Fr. Wald $=7$. einmal von C. H. gef., auch von Hg. gesammelt. Wlb. (Sch.) -

4. C. pubescens Grav. - An feuchtem Holz und an Baumpilzen s. h. - Fr. Wald. - Oberrad. - Bergen. - Auch von Hg. gef. Wlb. (Sch.) -

Var. immaculatus Steph. = fusculus Grav. - z. s. - Fr. — Soden unter Waldmoos im Sommer. - Auch von Hg. gef. - 
5. C. pedicularius Grav. - In Soden 2 Stück unter Gras von C. H. gef. - Friedberg 'Fuhr). -

Var. truncatellus Grav. - Bei Fr. einmal gef. -

\section{Hypocyptus Mannerheim.}

1. H. longicornis Payk. - Bei Soden im Juni und Juli in Anzahl von Dornzäunen geklopft. - Fr. Wald. - Griesheim am Main. - Enkheimer Torfbrüche. - Epstein im Taunus. - Fr. (Hg.) Wlb. (Sch.) -

2. H. seminulum Er. = pulicarius Er. - Je einmal im Rebstockwald bei Fr. und unter Weidengebüsch von C. H. gef., auch von Hg. gesammelt. - Offenbach unter Schilf im October. - Wlb. (Sch.) -

3. H. ovulum Heer $=$ nigripes. Heer $=$ pygmaeus Krtz. Einmal im Gewächshaus im Fr. botanischen Garten $=5$. von C. H. gef. - Fr. (Hg.) -

4. H. discoideus Er. - Bei Fr. sehr einzeln von C. H. gef., im Herbst unter Schilf hinter den Kettenhöfen. -

5. H. laeviusculus Mhm. - Zweimal bei Fr. im November gef.; im April einmal aus dürrem Fr. Waldholz entwickelt. - Auch von Hg. bei Fr. gesammelt. -

\section{Trichophya Mannerheim.}

1. T. pilicornis Gyll. - Von M. S. bei Fr. gesammelt. - s. s. -

\section{K. A L E O C HAR I N I.}

Dinopsis Matthews.

1. D. erosa Steph. = fuscata Matth. = laticollis Er. Einmal von C. H. bei Fr. gef. -

\section{Myllaena Erichson.}

- 1. M. intermedia Er. - Bei Fr. einmal von C. H. gef., auch von Hg. gesammelt. -

2. M. minuta Grav. - Im Rödelheimer Wald und bei Fr., sowie bei Ems von C. H. gef. - Fr. (Hg.) -

3. M. brevicornis Matth. = gracilis Heer = grandicollis Kiesw. - Bei Ems von C. H. gesammelt. - Fr. (Hg.) - 


\section{Encephalus Westwood.}

1. E. complicans Westw. - In wenigen Exemplaren von C. H. bei Offenbach Abends zwischen den Weiden am Main nach Bürgel zu $=7$. mit dem Streifnetz gef. - Schmitterhof bei Giessen unter Moos im August. - Sehr seltenes Thier. - Fr. - G. (L.) -

\section{Gyrophaena Mannerheim (= Agarieochara Kraatz).}

1. G. pulchella Heer. - Einmal in Mehrzahl in Schwämmen im September von C. H. gef. - Fr. (Hg.) -

2. G. affinis Sahlb. - An Boletus im Fr. Wald h. im Juni. Soden im Mai an faulen Pilzen. - Hengster bei Offenbach. - Fr. (Hg.) - Wlb. (Sch.) -

3. G. nana Payk. - Wie die vorige im Fr. und Rödelheimer Wald im Juni und August. — Soden im Mai. - Fr. (Hg.) -

4. G. fasciata Mrsh. = congrua Er. - An Pilzen bei Hofheim und in der Hohen Mark im Taunus, Fr. Wald, Oberrad, Kesselbruch im Offenbacher Wald. - Fr. (Hg.) -

5. (Agaricochara Krtz.) strictula Er. = laevigata Heer. Bei Bingen von C. H. gef. -

6. (A.) polita Grav. - Von Hg. bei Fr. gesammelt. -

\section{Brachida Muls. Rey.}

1. B. notha Er. (Homalota olim.) - Bei Friedberg von Fuhr einzeln gesammelt, bei Fr. von $\mathrm{Hg}$. - Wlb. (Sch.) -

\section{Oligota Mannerheim.}

1. 0. pusillima Grav. - Im Gebiet n. s. - An vegetabilischen Dunghaufen bei Fr. im Juli, aus alten faulen Fichtenzapfen $=6$. entwickelt; auch im Fr. Unterwald bei Formica rufa gef. - Fr. (Hg.) Wlb. (Sch.) - [Hierher nach Fauvel 0. pygmaea in S. V.] -

2. O. pumilio Ksw. [Hierher die in S. V. erwähnten O. atomaria von Fr. (C. H.)] -

3. O. inflata Mhm. = subtilis Er. - Von Hg. bei Fr. ges. -

4. O. flavicornis Lac. - Je einmal bei Soden und Rüdesheim von C. H. gef., auch von $\mathrm{Hg}$. gesammelt. - 


\section{Placusa Erichson.}

1. P. pumilio Grav. - Unter Birkenrinde im Fr. Wald $>4$. einmal von C. H. gef. -

2. P. infima Er. - Unter Birkenrinde bei Fr. = 2. zwei Stück gef., auch von Hg. gesammelt. -

3. P. adscita Er. - Einmal unter Kiefernrinde $>$ 8. von C. H. gef. -

\section{Homalota Mannerheim.}

1. H. sordida Mrsh. = melanaria Thoms. = lividipennis Er. - Im Offenbacher Wald an Pilzen. - Bingen. - Fr. im September, auch von Hg. gef. -

2. H. parva Sahlbg. = stercoraria Krtz. - Bei Fr. zweimal gef., auch von Hg. gesammelt. -

3. H. orphana Er. - Bei Fr. hinter den Kettenhöfen unter Schilf im Dezember mehrfach gesammelt von C. H.; auch Hg. fand die Art bei Fr. -

4. H. aterrima Grav. - Im Fr. Wald unter faulenden Pflanzen beim Forsthans im Mai, im Rebstockwald bei Fr. im Juni. - Fr. (Hg.) -

5. H. laticollis Steph. = vernacula Er. - Soden unter Gras im Juni. — Bingen. — Offenbach am Mainufer im Juli. — Fr. (Hg.) -

6. H. fungi Grav. - Im ganzen Gebiet unter faulenden Pflanzen und Pilzen g. - Fr. - Enkheimer Torfbrïche. - Bei Oberrad auch in Gesellschaft von Formica fuliginosa im September gef. - Auch von Hg. gesammelt. -

Var. clientula Grav. - Zweimal bei Fr. von C. H. gef., auch von Hg. gesammelt. -

7. H. longicornis Grav. - Fr. an Kuhfladen im Juli und im Februar unter Mloos, auch von Hg. gef. - Wlb. (Sch.) -

8. H. celata Er. = germana Sharp. - Im Fr. Wald an der Oberen Saustiege im Juni bei Formica fuliginosa gef. — Schlangenbad an Pilzen. - Im Dezember unter Laub bei Fr., auch von Hg. gef. -

9. H. sordidula Er. - Einmal bei Offenbach am Mainufer im Juli gef. -

10. H. zosterae Thoms. = vicina Krtz. = nigra Krtz. = hodierna Sharp. - Wie coriaria. - s. - Fr. (Hg.) - 
11. H. laevana Muls. - C. H. fand 1 Stück bei Offenbach am Mainufer im Juli. -

12. H. marcida Er. = livida Mls. - An Pilzen im Wald bei Offenbach und Fr. im October. -

13. H. inquinula Er. - Bei Fr. in Kuhmist im Juli n. s.; bei Ems in Eselskoth von C. H. gef. -

14. H. amicula Steph. $=$ sericea Muls. - C. H. fand 1 Ex. bei Fr., ebenso Hg. -

15. H. palleola Er. - Im Fr. Wald an Boletus im Juni. Fr. Forsthaus. - Ems.

16. H. subterranea Muls. - Von Hg. bei Fr. gef. -

17. H. testaceipes Heer $=$ brevicollis Baudi $=$ varicornis Krtz. - Von C. H. bei Fr. gef. -

18. H. dilaticornis Krtz. - Einige Exemplare > 8. an Schwämmen von C. H. gef. -

19. H. oblita Er. - Im Oberräder Wald an Schwämmen und bei Formica fuliginosa im September. - Auch bei Fr. von L. H. und Hg. gef. -

20. H. nigritula Grav. = bolitobia Thoms. - Im Fr. Wald sowie im ganzen Gebiet h. an Boletus und Pilzen: Cronthal, Hofheim und Hohe Mark im Taunus. - Im Sodener Wald in Nestern der Formica rufa. - Wlb. (Sch.) -

21. H. coriaria Krtz. - Aus altem Fr. Waldholz zweimal im Mai von C. H. erzogen; von Hg. bei Fr. gef. -

22. H. trinotata Krtz. - Fr. im März einmal an altem Menschenkoth. - Fr. (Hg.) -

23. H. Sodalis Er. - Im ganzen Gebiet an Baumschwämmen im September. - Fr. Oberräder Wald bei Formica fuliginosa. - Bingen. — Fr. (Hg.) — Münster (Soden) bei F. rufa (vera) 2. April 1876 gef. -

24. H. sublinearis Krtz. - Von Hg. bei Fr. gef. -

25. H. sericans Grav. = fungicola Krtz. - Im Fr. Wald an Boletus n. s. im Juni; auch von Ḣg. gesammelt. - Wlb. (Sch.) -

26. H. gagatina Baudi. - An Pilzen h. von Juni bis August. - Fr. - Schlangenbad und Hofheim im Taunus. - Fr. (Hg.) -

27. H. divisa Märk. - Bei Fr. von Hg. gef。 - 
28. H. palustris Kiesw. - An nassen Stellen im Fr. Wald. Bei Ems am Ufer der Lahn von C. H. gef. -

29. H. nigricornis Thoms. - Bei Fr. von Hg. gesammelt. -

30. H. angusticollis Thoms. = cavilla Krtz. nec Er. - An Pilzen im Wald bei Offenbach im October. - Bei Bingen'zweimal von C. H. gef. -

31. H. corvina Thoms. = lepida Krtz. - G. (L.) Wlb. (Sch.) -

32. H. brunnea $\mathbf{F}$. = depressa Grav. - In Neuenhain bei Soden von L. H., auf dem Johannisberg bei Friedberg von C. H. gef. - Fr. (Hg.) - Wlb. (Sch.) -

33. H. castanoptera Mhm. = merdaria Thoms. - Einmal bei Fr. gef. von C. H., auch von Hg. -

34. H. succicola Thomson = validicornis Märkl. - Bei Soden an ausfliessendem Saft von Eichen n. s. im Mai und Juni. Fr. (Hg.) -

35. H. gemina Er. - Fr. 2 Stück. -

36. H. hepatica Er. - C. H. fand im April ein Weibchen bei Königstein im Taunus. -

37. H. orbata Er. - Hg. fand die ziemlich seltene Art bei Fr. -

38. H. circellaris Grav. - Auf dem grossen Feldberg $=5$. unter Steinen zweimal von C. H. gef. - Fr., auch einmal von B. gef. -

39. H. parallela $\mathrm{Mhm}$. = talpa Heer. - Von I. H. in Anzahl im Fr. Wald in Nestern der Formica rufa gef. - Ausschliesslicher Ameisengast. -

40. H. exilis Er. - Bei Fr. mehrfach gesammelt von C. H. und Hg. -

41. H. analis Er. - Im ganzen Gebiet s. h. - Bei Offenbach und Cronthal im Taunns unter Steinen; bei Biebrich im Rheingenist; im Fr. Wald bei Formica fuliginosa. - Falkenstein im Taunus. Fr. (Hg.) - Wlb. (Sch.) - Staufen, Taunus 2. April 1876. -

Var. contempta Heer. - Fr. im Flug gef. im Juli, sowie unter Kiefernrinde im April und im Maingenist. - Ems. -

42. H. rufotestacea Krtz. - Einmal von Sch. bei Wlb. gef. - S. S. -

43. H. debilis Er. - Soden im August einmal von C. H. gef. - 
44. H. pilicornis Thoms. = pilosa Krtz. - L. H. besitzt durch H. Fuss 1 Ex. mit der Vaterlandsangabe „Nassau“. -

45. H. velata Er. - Von Hg. bei Fr. gesammelt. -

46. H. fluviatilis Krtz. = gagatina Muls. - Bei Fr. einmal gef. (von Fauvel bestimmt). von $\mathrm{Hg}$.

47. H. Iuridipennis Mhm. - Bei Soden von L. H. gef., bei Fr.

48. H. hygrobia Thoms. = hygrotopora Krtz. - Unter Steinen $>$ 9. am Main bei Fr. (nicht bei Oberrad, wie S. V. angibt). -

49. H. terminalis Gyll. - Fr. einmal gef. -

50. H. elongatula Grav. - Biebrich im Rheingenist. - Fr. Taunus. - Ems. - Ueberall n. s. - Auch von Hg. bei Fr. gef. -

51. H. gregaria Er. - Bei Fr. einmal von C. H. gef., auch von Hg. gesammelt. -

52. H. insecta Thoms. - L. H. fand im Winter 2 Stück am Fusse von alten Weiden bei Bockenheim. -

53. H. nitidula Krtz. - Fr. Wald unter Kiefernrinde im Mai, bei Oberrad im September gesammelt. — Fr. (Hg.) -

Alaobia Thomson.

(Homalota Er. ex parte.)

1. A. scapularis Sahlbg. = ochracea Er. - Bei Fr. und Soden je einmal an vegetabilischen Dunghaufen im Juni und Juli von C. H. gef. - Auch von Hg. gesammelt. -

\section{Aleuonota Thomson. (Homalota Er. ex parte.)}

1. A. pallens Redtb. - An den Offenbacher Kalksteinbrüchen unter Steinen in den Nestern von Tetramorium caespitum im April s. s. -

\section{Callicerus Gravenhorst.}

1. C. rigidicornis Er. - Bei Friedberg von Fuhr gesammelt. -

2. C. obscurus Grav. - Von Hg. bei Fr. gef. - Wlb. öfter (Sch.) - 


\section{Ilyobates Kraatz.}

1. I. nigricollis Payk. - Bei Fr. auf der Höchster Chaussee unter einem Stein einmal ron L. H., 1 Stück von C. H. bei Nauheim auf Salzboden $>5$. und bei Fr. gef., auch von Hg. gesammelt. -

2. I. rufus Krtz. - L. H. fand 2 Stück bei Neuenhain (bei Soden) unter alter Kastanienbaumrinde. -

3. I. forticornis Lac. - C. H. sammelte im Februar 1 Stück im Rheingenist bei Biebrich. -

Calodera Mannerheim (= Chilopora Kraatz).

1. C. nigrita Mnhm. - Einmal bei Ems von C. H. gef. -

2. C. umbrosa Er. - Von C. H. einmal bei Fr. im Juli gef. -

3. C. rufescens Krtz. - Von Hg. bei Fr. gef. -

4. C. aethiops Grav. - 2 Ex, aus dem Rödelheimer Wald unter Laub im März. -

5. (Chilopora) longitarsis Er. - Von Hg. bei Fr. gesammelt. - Wlb. (Sch.) -

Pronomaea Erichson.

1. P. rostrata Er. - Einmal von C. H. = 3. im Fr. Wald beim Forstbaus gef., auch von Hg. gesammelt. -

\section{Ocalea Erichson.}

1. O. castanea Er. - Von Hg. bei Fr. gef. -

2. O. badia Er. - Einmal ron C. H. $>$ 2. auf Schnee gef., 1 Stïck bei Fr. von L. H., auch von Hg. gesammelt. - Wlb. (Sch.) -

\section{Tachyusa Erichson.}

1. T. constricta Er. - Am Lahnufer bei Ems im Juli h. von C. H. gef. - Fr. (Hg.) -

2. T. coarctata Er. - Soden an Teichufern $<8$. - Ems, mit der vorigen. - Fr. (Hg.) -

3. T. scitula Er. - Von Hg. bei Fr. gesammelt. -

4. T. atra Grav. - Fr. - Biebrich im Februar im Rheingenist von C. H. gef. - 


\section{$-185-$}

Ginyeta Thomson.

(Homalota Er. ex parte.)

1. G. labilis Er. - Bei Biebrich im Februar im. Rheingenist einmal von C. H. gef. -

[Alianta Thomson. (Homalota Er. ex parte.) A. incana Er. (Nach Scriba im ganzen hessischen Gebiet; L. H. besitzt sie aus Seligenstadt und wäre auch bei uns noch zu finden.)] -

\section{Oxypoda Mannerheim.}

1. O. lividipennis Mhm. = luteipennis Er. - Im Gebiet h. Bei Fr. auf Hecken am Diebsweg $<5$. - Friedberg. - Wsb. Mo. - Soden $=5$. Abends $6 \mathrm{Uhr}$ h. über Sandwegen in den Anlagen fliegend gef. - Fr. (Hg.) - Wlb. (Sch.) -

2. 0. vittata Märk. - Im ganzen Gebiet in den Nestern der Formica fuliginosa. - Bei Fr., Oberrad und Soden von Juli bis September h. von L. H. gef. - Fr. Wald (Hg.) -

3. 0. opaca Grav. - Fr. h. - Soden, Bingen, Ems. $->6$. bis November. - Auch von Hg. bei Fr. gesammelt. -

4. 0. umbrata Gyll. = cuniculina Er. - Im Fr. Wald von Juni bis August am Forsthaus; im Januar an altem Aas. - Bingen. - Fr. (Hg.) -

5. O. Ientula Er. - C. H. fing 1 Ex. im September bei Oberrad in dem Nest von Formica fuliginosa. - Fr. (Hg.) -

6. 0. togata Er. = hospita Grimmer. - Im Fr. Wald (Bruchschneisse) $>$ 9. einmal von C. H. gef., auch von Hg. gesammelt. -

7. 0. bicolor Muls. - Fr. einmal von C: H. gef. -

8. 0. exigua Er. - Fr. Wald (Bruchschneisse) $=4$. im Sand und bei Nied auf einer Sandfäche $=6$. je einmal von C. H. gesammelt. Auch von Hg. gef. -

9. O. formosa Krtz. - Von Hg. bei Fr. gef. -

10. 0. alternans Grav. - In Pilzen h.. - Fr. - Bingen. Cronthal im Taunus, besonders im September. - Auch von Hg. ges. Wlb. (Sch.) - 


\section{$-186-$}

11. 0. exoleta Er. = riparia Thoms. - Einmal bei den Salinen von Nauheim $=8$. von C. H. gef.; [auch 1 Stück von der Saline Salzhausen in der Wetterau als solitaria in S. V. erwähnt]. - Fr. (Hg.) -

12. 0. rugulosa Krtz. - Ein Exemplar aus Friedberg [in Sammlung v. Heyden] ron Scriba als rufescens Krtz. erhalten. -

13. O. formiceticola Märk. - Im Fr. Wald (Goldsteinforst) zweimal im Nest von Formica rufa $<7$. gef. - Münster (Soden) bei F. rufa (vera) 2. April 1876 gef. -

14. 0. haemorrhoa Manh. - Fr. von L. H. bei Formica rufa. - Ems. - Bei Mo. = 5. von C. H. gef. - Bei Münster wie formicet. gef. -

15. O. amoena Fairm. = flavicornis Krtz. - C. H. fand je 1 Stück an der Saline Nauheim $>8$. und bei Fr. -

16. O. annularis Mnhm. - Bei Fr. in Anzahl ron C. H., bei Soden $=6$. einmal gef. -

[0. soror Thoms. = flava Krtz. - Ein hochalpines Thier, soll nach Scriba ron Katheder bei Fr. gefunden sein. Diese Angabe ist sicher falsch. Der verstorbene Herr Katheder hat öfter Verwechselung: mit der Vaterlandsangabe gemacht.] -

\section{Ocyusa Kraatz.}

1. O. maura Er. - Bei Fr. einmal von C. H. < 3. gef. -

\section{Tomoglossa Kraatz.}

1. T. luteipes Er. - Einmal ron C. H. bei Fr. gef. -

\section{Phloeopora Erichson (= Phloeodroma Kraatz).}

1. P. reptans Grav. - Unter Rinde von Eichen und Kiefern im Gebiet n. s. - Fr. Wald. - Bingen. - Fr. (Hg.) - Wlb. (Sch.) -

2. P. corticalis Grav. - Unter Rinde von Birken und aus altem Fr. Waldholz im Mai erzogen. - Im Dezember in Wsb. unter Platanenrinde von C. H. gef. - Fr. (Hg.) - Wlb. (Sch.) -

Hygronoma Erichson.

1. H. dimidiata Grav. - Fr. - Im Winter unter Laub, im Genist, im März unter Schilf bei den Kettenhöfen. - 


\section{$-187-$ \\ Dinaraea Thomson. \\ (Homalota Er. ex parte.)}

1. D. aequata Er. - Fr. im März unter Rinden; Soden unter Eichenrinde $<$ 7. - Fr. (Hg.) - Wlb. (Sch.) -

2. D. angustula Gyll. - Bei Fr. einzeln von C. H., Hg. und B. gesammelt. - Biebrich am Rhein. -

3. D. linearis Grav. - Einmal < 8. von C. H. bei Soden gef. -

4. D. immersa Er. - Fr. im Februar unter Kiefernrinde und = 7. in Buchenwurmmehl. - s. -

5. D. cuspidata Er. - Fr. $=4$. unter Kiefernrinde, $<5$. aus dürrem Fr. Waldholz erzogen. - Bei Cronthal im Taunus $=5$. unter Kiefernrinde. - Fr. (Hg.) -

6. D. deplanata Grav. - Von Hg. bei Fr. gesammelt. -

\section{Notothecta Thomson. \\ (Homalota Er. ex parte.)}

1. N. flavipes Grav. - Im Fr. Wald n. s. von uns in Nestern der Formica rufa gesammelt $<5$. und November. - Von B. im Fr. Wald hinter dem Forsthaus s. h. am 18. März 1861 gef. Wlb. (Sch.) - Bei Münster wie die folgende gesammelt (nur bei Formica rufa). -

2. N. anceps Er. - Im Fr. Wald im März und Mai wie N. flavipes gesammelt. $-=5$. bei Neuenhain (Soden) an feuchten Waldstellen. - Münster (Soden) bei F. rufa (vera) 2. April 1876. Einmal auch bei congerens. -

\section{Thamiaraea Thomson.}

(Homalota Er. ex parte.)

1. T. cinnamomea Grav. — Von Hg. bei Fr. gef. — Wlb. (Sch.) -

2. T. hospita Märk. - Von Hg. bei Fr. gesammelt. -

\section{Myrmedonia Erichson.}

1. M. collaris Payk. - Im Fr. Wald unter Moos = 4., ebenso bei Oberrad im März einzeln von C. H. gef., auch von Hg. gesammelt. - 
2. M. humeralis Grav. - Im Gebiet bei Formica fuliginosa n. s. - Fr. Wald, Soden, Enkheimer Torfbrüche, Königstein im Taunus im Mai. - Fr. (Hg.) - Am Staufen 2. April 1876 von B. gef. -

3: M. cognata Märk. - Wie die vorige Art, aber häufiger. Fr. Wald, Oberrad, Soden. - Juli bis September. - Fr. (Hg.) Wsb. hinter dem Turnplatz an Eichen bei Formica fuliginosa im Mai (K.). -

4. M. funesta Grav. - Wie die vorige n. s. - Soden im Juli. - Im Fr. Wald schon = 3.; auch von Hg. gef. - Wlb. (Sch.) Wsb. wie die vorige (K.). -

5. M. similis Märk. - Fr. Wald und Oberrad in den Nestern der Formica fuliginosa im August und September s. - G. (L.) -

6. M. limbata Payk. - Fr. - Enkheim. - Königstein im Taunus. - Im April. - Meist nicht bei Ameisen. - Bei Fr. auch von Hg. und B. h. im Genist am 3. April 1860 gesammelt. - G. (L.) -

7. M. lugens Grav. - Fr. Wald unả Königstein im Juli einzeln von uns wie die anderen Arten gef., im Juli. - Fr. (Hg.) -

8. M. Iaticollis Märk. - Wie die vorigen h. bei Fr., Oberrad, Soden , von Juli bis September. - Auch von Hg. gesammelt. - Wlb. (Sch.) -

\section{Drusilla Mannerheim.}

1. D. canaliculata F. - Ueberall s. h. unter Laub. - Fr. Nauheim. - Ausnahmsweise bei Ameisen, z. B. Formica flava. - Auch von $\mathrm{Hg}$. und B. schon am 20. März 1860 gesammelt. - Wlb. s. h. (Sch.) - G. (L.) - Am Gimbacher Hof (Staufen) im Taunus 2. April 1876. -

\section{Lomechusa Gravenhorst (= Atemeles Kraatz).}

1. L. strumosa F. - Bei Formica congerens. s. - Fr. - Am Feldberg von L. H. gef. - Bei Bingen am 25. April 1823 von C. H. einmal n. s. auch mit der Larve gef. - Ein Exemplar der letzteren in Sammlung v. Heyden ist eingetrocknet und daher nicht genau zu beschreiben. Die Larve ist $6 \frac{1}{1} / \mathrm{mm}$ lang, schwarzbraun, asselförmig, halb so brëit wie lang; Kopf gross, über $1 \frac{1}{1} 2^{\mathrm{mm}}$ breit und eben so lang; Fühler dick, 2gliedrig; Halsschild breit kurz, Vorderecken im Bogen abgerundet, Hinterecken spitz abgerundet, nach einwärts gebogen. Der übrige Körper besteht aus 12 sehr schmalen Segmenten, der Hinterrand 
eines jeden in der Mitte gerade, seitlich in stumpfem Winkel nach hinten gezogen, so dass der Körper Aehnlichkeit mit dem Hinterleib von Lampyris hat. Westwood bildet in Introduction to the modern classification of insects 1839. p. 166. fig. 13. die Larve von Aleochara fuscipes ab, welche viel Aehnlichkeit mit dieser Larve hat, doch fehlen dem Binger Thier die zwei Borsten am letz̃ten Segment. -

2. (Atemeles) paradoxa Grav. - Fr. s. - L. H. fand bei Bergen $=5$. $1 \mathrm{Ex}$., welches von einer Ameise in den Mandibeln getragen wurde. - Wlb. (Sch.) -

3. (A.) emarginata Grav. - In Nestern der Myrmica rubra im Fr. Wald im April und Mai unter Steinen n. s. im Wartforst und Bruchschneisse. - Schmitterhof bei Giessen. - Mo. = 5. im Sand. - Fr. (Hg.) - Bei Wlb. von Sch. in den Nestern der Myrmica laevinodis Nyl. und der verwandten Arten, welche unter M. rubra L. begriffen sind, gef. - Im Lehen bei Oberrad 14. Mai (B.). -

Var. nigricollis Krtz. - $5 \mathrm{Ex}$. beim Gimbacher Hof am Fusse des Staufen im Taunus, in einem Nest der M. scabrinodis von L. H. und B. 2. April 1876 gef. -

\section{Dinarda Lacordaire.}

1. D. Märkeli Kiesw. - In den Nestern der Formica rufa im Fr. Wald (Gehren) s. $<$ 5. - Fr. (Hg.) - Münster bei Soden im Taunus 2. April 1876 bei F. rufa gef. -

2. D. dentata Grav. - Fr. Wald, Königstein, Feldberg und Schlangenbad im Taunus, Bingen. - Von April bis Juli in Nestern von Formica (nach Scriba nur congerens) rufa. Doch ist der Name rufa hier noch als Collectivbegriff behandelt und cong. noch nicht als Art abgeschieden. - Uebrigens sicher einmal auch in demselben Nest mit der vorigen Art gef. - Fr. (Hg.) — Sch. fand die Art bei Wlb. in den Nestern der Formica sanguinea Latr. -

\section{Thiasophila Kraatz.}

1. T. angulata Er. - In den Nestern der Formica rufa n. s. - Fr. Wald. - Soden = 3., dann von Juni bis November. - Von B. im März hinter dem Fr. Forsthaus gef. - Im Wald bei Münster (Soden) bei F. rufa (vera) 2. April 1876. - 
2. T. inquilina Märk. - Einmal bei Fr. von C. H. im Juli in einem Nest von Lasius fuliginosus gef. -

\section{Euryusa Erichson.}

1. E. sinuata Er. = coarctata Märk. - C. H. fing im Fr. Wald $>6$. unter loser Baumrinde 1 Stück bei Formica rufibarbis (cunicularia). -

2. E. laticollis Heer. - Fr. Wald unter Rinde $=5$. s., aber auch im Goldsteinforst $<$ 7. bei Formica rufa. - Fr. (Hg.) -

\section{Homoeusa Kraatz.}

1. H. acuminata Märk. - Auf dem Röderberg bei Fr. im Mai bei Lasius niger, und im April 1832 von C. H. bei Bingen in den Nestern von Formica rufibarbis in Anzahl gef. - Wlb. (Sch.) -

\section{Silusa Erichson (='Stenusa Kraatz).}

1. S. rubiginosa Er. - Bei Mo. $=9$. im Sand und bei Fr. je einmal gef. - Fr. (Hg.) -

2. S. rubra Er. - L. H. fand bei Neuenhain (bei Soden) 2 Ex. im Juli 1871. -

\section{Stichoglossa Fairmaire (= Stenoglossa et Ischnoglossa Krtz.).}

1. S. corticina Er. - Zweimal bei Cronthal im Taunus unter Kiefernrinde $<9$. und einmal auf dem Schmitterhof bei Giessen $>8$. von C. H. gef. - Wlb. (Sch.) -

\section{Leptusa Kraatz.}

1. L. ruficollis Er. - Fr. von C. H. und Hg. gef. - Bingen. - Im April. -

2. L. fumida Er. - Von Hg. bei Fr. gesammelt. -

Aleochara Gravenhorst.

1. (Ceranota Steph.) ruficornis Grav. - Bei G. ein Männchen dieser seltenen Art von L., auch einmal bei Wsb. unterhalb der Rentmaver 30. Juni von K. gef. - 
2. A. fuscipes Grav. - Im ganzen Gebiet an Aas h. - Fr. Königstein im Taunus. - Auch von Hg. und B. bei Fr. gef. Wlb. (Sch.) -

Var. lata Grav. (mit dunkeln Flügeldecken) s. - Nach B. am Röderwald h., am Main oberhalb 'Fr. -

3. A. brevipennis Grav. - Im Rödelheimer Wald im März und bei Fr. je einmal von C. H. gef. -

[A. fumata Grav. - Nach Scriba im ganzen hessischen Gebiet s.; aber noch nicht bei Fr. und in Nassau beobachtet.]

4. (Baryodma Thoms.) rufipennis Er. - Bei Fr. von uns und Hg. gef. - Rumpenheim am Main $=7$. - Bei Nauheim auf Salzboden > 6. - G. (L.) -

5. (B.) nigripes Miller. - Bei Fr. von Hg. gesammelt. -

6. (B.) bipunctata Grav. - Fr. - Königstein im Taunus. Bei Fr. auch von Hg. n. s. gef. - G. (L.) -

7. (B.) lanuginosa Grav. - Rödelheim. - Fr. n. s. an Aas, auch von Hg. gef. - Wlb. (Sch.) -

8. (B.) moesta Grav. - An ausfliessendem Saft von Bäumen, z. B. von Aesculus bei Biebrich $>6$. - Fr. unter faulenden Pflanzen, auch von Hg. gesammelt. - Wlb. (Sch.) - G. (L.) -

9. (B.) moerens Gyll. = brunneipennis Krtz. - Einmal bei Fr. von C. H. gef., auch von Hg. gesammelt. -

10. (B.) haemoptera Kraatz. - Friedberg (Fuhr). - G. (L.) -

11. (B.) mycetophaga Krtz. - An Boletus im Fr. Wald 3 Stück $<$ 6. von C. H. gef. - Fr. (Hg.) -

12. (B.) lugubris Aubé = moerens Er. - Zweimal an Pilzen bei Cronthal im Taunus $>10$. und einmal bei Fr. im Juli von C. H. gef., auch von Hg. gesammelt. -

13. (B.) bisignata Er. - Auf dem Johannisberg bei Friedberg von C. H. = 5. gef. -

14. (B.) bilineata Gyll. - Bei Fr. von Hg. gefundene Stücke in Sammlung v. Heyden. -

15. (B.) nitida Grav. - Im Gebiet s. h. - Fr. - Rumpenheim am Main. - Mo. = 5. - Bingen. - Auch von Hg. gesammelt. Wlb. (Sch.) - G. (L.) -

16. (B.) morion Grav. - Im Fr. Wald am Forsthaus unter faulenden Pflanzen < 7.2 Stück, auch von Hg. bei Fr. gef. - Wlb. (Sch.) - 
17. (Dyschara Mls. Rey) inconspicua Aubé. - Bei der Oelmühle bei Königstein an ansgelegtem Aas kleiner Vögel in Anzahl von L. H. gef. -

\section{Microglossa Kraatz (= Haploglossa Krtz. olim).}

1. M. gentilis Lünem. - Bei Soden einmal von L. H., bei Fr. von Hg. gef. -

2. M. pulla Gyll. - Bei Soden auf Salzboden =7. von C. H. gef., bei Fr. von Hg.

3. M. marginalis Grav. = rufipennis Krtz. - Von Ḧ. bei Fr. gesammelt. -

4. M. praetexta Er. - Von Hg. bei Fr. gef. -

\section{Bolitochara Mannerheim.}

1. B. lucida Grav. - Aus überwinterten Schwämmen aus dem Fr. Wald im Mai öfter entwickelt. - Enkheimer Torfbrüche einmal (nicht Offenbach wie in S. V.) - Fr. (Hg.) -

2. B. lunulata Payk. = bella Märk. (sec. Fauv.) - Im Gebiet h. an Pilzen. - Fr. Wald, Offenbach, Hofheim, Königstein im Taunus. - Fr. (Hg.) - Wlb. (Sch.) -

3. B. obliqua Er. - Von Hg. bei Fr. gesammelt. -

\section{Falagria Stephens.}

1. F. thoracica Curtis. - Von C. H. bei Ems gesammelt, von Hg. bei Fr. -

2. F. sulcata Payk. - Unter faulenden Pflanzen im Gebiet n. s. - Fr. Wald. - Im Fr. botanischen Garten im August. - Von B. schon vom 20.-28. März gef. - Fr. (Hg.) -

3. F. sulcatula Grav. - Unter Laub h. - Bingen. - Rüdesheim. - Ems. - Fr. auch von Hg. gef. -

-4. F. obscura Curtis. - h. - Fr. Wald. - Offenbach an Pilzen < 9. - Soden und Nauheim auf Salzboden. - Fr. (Hg.) Von B. $>3$. und < 4. gesammelt. - Fr. $($ Hg. $)-$ G. $\left(\mathrm{L}_{\text {. }}\right)-$ Wlb. (Sch.) - Münster bei Soden im Taunus bei Formica congerens 2. April 1876. - 
5. F. nigra Grav. - Bei Ameisen: Formica rufibarbis und rufa von L. H. h. gef. - Von C. H. auf Salzboden bei Soden und Nauheim (sowie den Wetterauer Salinen Wisselsheim und Salzhausen) gesammelt. - Schmitterhof bei Giessen. - Bei Münster wie die vorige Art h. -

\section{Autalia Stephens.}

1. A. impressa Oliv. - An Pilzen im ganzen Gebiet im September und October. - Fr. - Cronthal im Taunus. - Offenbach. Auch von Hg. gesammelt. -

2. A. rivularis Grav. - Von Hg. bei Fr. gef. -

\section{DASCILLIDAE.}

(Die Sammlung v. Heyden wurde vom Monographen Tournier revidirt.)

\section{A. DASCILLINI \\ Dascillus Latreille.}

1. D. cervinus $\mathbf{L}$. = cinereus $\mathbf{F}$. - Im Juni im Fr. Wald am Forsthaus offter auf Salix caprea von uns gef.; daselbst von B. auf Kräutern h. gestreift. -

\section{B. CYPHONINI. \\ He 10 dl es Latreille.}

1. H. minutus L. - Auf nassen Wiesen $=6$. bei Fr., im Rebstocker Wald, Neuenhain bei Soden im Taunus. - Auch von Hg. bei Fr. gesammelt. - G. (L.) - Mo. in Wiesen (S.). - Wsb. an der Wellritz 16. Juni (K.). -

Var. laetus Panz. (Mit schwarzer Naht und Spitze der Flügeldecken.) - Fr. von L. H. und Hg. s. gef. - Friedberg (Renner). -

2. H. marginatus F. - Auf Wiesen $=5$. am Altkönig im Taunus einmal von C. H. von Erlengebüsch geklopft. - Fr. (Hg.) Wlb. s. (Sch.) - G. (L.) - Wsb. an der Wellritz 18. Mai (K.). - 


\section{Microcara Thomson.}

1. M. testacea L. = livida F. - Bei Fr. s. von C. H. und Hg. gef. - Wlb. (Sch.) -

\section{Prionocyphon Redtenbacher.}

1. P. serricornis Müll. - Bei Fr. von M. S. und C. H. im Biebricher Schlossgarten $>6$. unter der Rinde einer alten Rosskastanie einmal gef. - Hallgarten im Rheingau (Hg.). - Mz. unter Platanenrinde (S.). -

\section{Cyphon Paykull.}

1. C. coarctatus Payk. (Das Weibchen als fuscicornis Thoms. beschrieben.) - Fr. n. s. auf Gesträuch. - Nauheim > 5. - Cronberg. - An Steinen am Schwarzbach bei Hofheim = 5. von C. H. gef. - Auch von Hg. bei Fr. gesammelt. - Wlb. (Sch.) - Mz. (S.) -

2. C. nitidulus Thoms. (Das Weibchen ist pallidiventris Thoms.) - Bei Soden > 6. an Eichen gef., ein Männchen. - Wlb. s. s. (Sch.) - Mr. (S.) -

3. C. Padi L. - An den Enkheimer Torfgruben $<4$. an Graswurzeln. - Königstein an Wiesengebüsch $=5$. $-\mathrm{Fr}$. von C. H. und Hg. gesammelt. -

4. C. variabilis Thunbg. - Unter Schilf n. s., schon im März, bei Fr. an den Kettenhöfen, auch von Hg. und B. gef. - Wlb. (Sch.) - Mz. (S.) - Limburg am Weiher 6. Juni (K.). -

5. C. suturalis Tourn. - Ein Exemplar von C. H. am Hellerhof bei Fr. im Mai am Sumpf. Neu für Deutschland. -

\section{Hydrocyphon Redtenbacher.}

1. H. deflexicollis Müll. - Im Schwarzbach bei Epstein unter und an Steinen im Juli mit Larven, die im Wasser leben, von C. H. gef. - Wald an den Wiesen bei Falkenstein. - Ems am Lahnufer im Juli n. s. von C. H. gesammelt. - St. Goarshausen (Hg.). Wlb. h. (Sch.) -

\section{Scirtes Illiger.}

1. S. hemisphaericus L. - An der Tränke im Fr. Warthforst = 7. auf Crataegus gesellig von C. H. gef. - Auf Disteln am Diębsweg 
bei Fr. $<7$., am Entensee bei Rumpenheim $<$ 8. - Auch von Hg. bei Fr. gesammelt. - Wlb. (Sch.) - Mz. (S.) - Had. Teich unter dem Haidenhäuschen, bei Wsb. an der Tränke, Limburg am Teich 6 Juखi (K.). -

2. S. orbicularis Panz. - Von Hg. 2 Stück bei Fr. gef. -

C. EUCINETINI.

\section{Eucin etus Germar.}

1. E. haemorrhoidalis Germ. - L.' H. fand 1 Stück an einem alten Kiefernstrunke in einem Staubpilz beim Sandhof im Fr. Wald und C. H. 1 Stück $=4$. bei Griesheim im Kiefernwald unter Moos. -

\section{TELEPHORIDAE.}

\section{A. LYCINI. \\ Lygistopterus Mulsant.}

1. L. sanguineus F. - Im Fr. Wald auf Umbellen n. s. von $=6$. bis $>7$. von uns gef. - Aus dürrem Erlenholz entwickelt. Von St. h. aus Fr. Waldholz erzogen. - Auch bei Isenburg im Wald von Hg. gesammelt. - Bei Wlb. h. auf den Blüthen von Ligustrum vulgare (Sch.). - Mz. (S.) - Im Sauerthal auf Schirmblumen 22. Juni, nach K. von Sandberger gesammelt. -

\section{Dictyoptera Latreille (= Eros Newman).}

1. D. Coccinea L. = Aurora Hbst. - Einmal von C. H. bei Fr. und einmal bei G. von S. gef. -

2. D. minuta F. - Bei Fr. von C. H. $>8$. in Strünken von Pinus picea gef. - Friedberg (Fuhr). -

3. D. Cosnardi Chevr. $=$ Merckii Muls. = flavescens Redtb. - Von C. H. bei Fr. und in alten Buchenstöcken im Lorsbacher Thal $=5$. s. s. gef. - 


\section{Homalisus Geoffroy.}

1. H. suturalis Villers. - Im ganzen Gebiet auf Waldwiesen n. s., z. B. im Fr. Wald an der Babenhäuser Landstrasse im Juni und Juli. - Hofheim. - Fr. Wald auch von Hg. gef. — Im Schwanheimer und Rebstocker Wald, im rothen Graben an der Mainkur von B. gesammelt. - Wlb. n. s. in Wäldern (Sch.) - Wsb. Dambach im Juni, Dotzheim, Fasanerie 12. Juli (K.). — Mz. (S.) - +

\section{B. LAMPYRINI.}

\section{Phosphaenus Laporte.}

1. P. hemipterus Geoffr. Var: brachypterus Motsch. - Bei uns kommt nur diese kurzflügelige Form vor; die sehr kurzen Flügeldecken reichen bis zur Mitte des 2. Hinterleibsringes, während sie bei der langflügeligeren Stammart bis in die Mitte des 3. Segments hineinragen. - Die Weibchen haben gar keine Flügeldecken; es sind keine Stücke aus dem Gebiet bekannt. - Das Männchen findet sich einzeln an schattigen Orten, z. B. unter einem Eisenbahnviadukt bei Höchst am Main. - Dr. Steitz fand die Art an einer Lattenwand bei Oberrad; L. H. bei Neuenhain bei Soden auf dem Weg laufend. - Wsb. im Juni von Heynemann in den Anlagen beim Kursaal, nahe beim Weiher öfter auf dem Sand über den Weg laufend. gef. - Von C. H. am Mühlberg bei Sachsenhausen gef. - Bei Fr. von Hg. gesammelt. - Bei Wlb. von Sch. h. am Gras an Hecken gestrichen, aber nur Männchen. Im Grase fand Sch. leuchtende Larven, ganz mit den Merkmalen der Lampyrislarven, aber sehr breit, braun mit gelblichem Rande, vielleicht Larve des Weibchens; sehr schmale, oben ganz schwarzbraune runzelige, wohl die männlichen Larven, unten gelb, oben auch die zwei letzten Segmente. Vorletztes Bauchsegment sehr hell, leuchtend. - Mz. (S.) -

\section{Lamprorhiza Motschulsky.}

1. L. splendidula L. - Im ganzen Gebiet 11. So, zumal in feuchten Gegenden, am Abend leuchtend herumfliegend, besonders im Sommer. Fr. - Soden. - Das Weibchen ist ungeflügelt und leuchtet stärker und anhaltender. Allgemein unter dem Namen „Johanniswürmchen“ 
bekannt. - Bei Wlb. und Dlb. beide Geschlechter, besonders die Männchen S. h., auch die Larven (Sch.). - G. (L.) - Mz. (S.) - Wsb. am Weg nach der Fasanerie 8. Juli (K.). -

\section{Lampyris Geoffroy.}

1. L. noctiluca L. - Viel seltener bei uns als die vorige Art, mehr in gebirgigen Gegenden.: - Von C. H. auf dem Mühlberg bei Sachsenhausen früher gesammelt, im Juli. - Auch von Hg. s. gef. Oefter unter Steinen bei Dlb. beide Geschlechter und Larven ges. (Sch.) - G. (L.) - Wsb. im Chausseegraben am Turnplatz 11. Juli (K.).

- Neuenhain bei Soden (L. H.). -

\section{THELEPHORINI.}

\section{Th elephorus Schäffer.}

1. (Podabrus Westw.) alpinus Payk. (Stammart hat gelbe Flügeldecken und Halsschild, die Mitte des letzteren schwarz.) - Von C. H. $<6$. 3 Stück am grossen Feldberg gef., sonst nicht aus dem Gebiet bekannt. -

Var. lateralis Er. nec L. (Flügeldecken mit schmalem, gelbem Saum, Halsschild ohne dunkeln Mittelfleck) einmal mit der Stammart am Feldberg von C. H. gef. -

\section{2. (Ancistronycha Märk.) abdominalis F. Var. cyanipennis} Bach. = cyaneus Dietr. - Bei uns kommt nur diese breitere Form vor. Bis jetzt im Gebiet nur ein Weibchen von C. H. an feuchten Stellen in der Hohen Mark im Taunus $>6$. gef. -

3. (A.) violaceus Payk. (Normale Stücke haben ganz rothe, Var. tigurinus Dietr. zum Theil dunkle Beine.) Einmal von C. H. am Feldbergthal, Südabhang $=5$. ein normales Männchen gef. - [Hierher das als tigurinus in S. V. erwähnte Exemplar.] - Bei Wlb. nur Weibchen mit ganz rothen Beinen von Sch. gesammelt. -

4. (A.) Erichsonii Bach. = rotundicollis Dietr. - L. H. fand 2 Stück dieser schönen Art im Thal bei der Oelmühle bei Königstein. Die schwarze Farbe der Flügeldeckenspitze hält bei diesen Stücken, ihrer Ausdehnung nach, die Mitte zwischen den Thüringer und Stücken aus dem Riesengebirg einer- und Schwarzwälder Stücken andererseits. Bei den Thüringern ist die hintere Hälfte der Flügeldecken, bei den Taunus- 
stücken das hintere Viertel und bei den Schwarzwäldern mur die äusserste Spitze schwarz gefärot. - Wsb. bei der Wellritzmühle 14. Juli auf Schlehen (K.). -

5. T. annularis Ménétr. = illyricus Mls. = oculatus Var. Kiesw. - Bei Fr. von C. H. und Hg. gef. - [Hierher die in S. V. als oculatus Gebl. erwähnten Stücke (annul. hat schwarze, ocul. rothe Hinterschenkel)]. - Die Art ist kenntlich an den 2 schwarzen Halsschildpunkten auf schwarzem Grunde. -

6. T. fuscus L. - Im Gebiet h. - Fr. im Mai; auch von Hg. und B. gef. - Dlb. und Wlb. s. h.; die Larven im Januar in Menge auf schneeigen Wiesen gef. (Sch.) - Die Larve bei den Landwirthen unter dem Namen „Schneewurm" bekannt (L. H.). - G. (L.) Mz. (S.) -

7. T. rusticus Fall. - Wie die vorige Art im Mai. - Fr. von L. H., Hg. und B. gef. - Bei Wlb. wie fusca gef. (Sch.) - G. (L.) - Mz. (S.) -

8. T. obscurus L. - Fr. im Mai. - Auf Kiefern h. - Falkensteiner Wald im Taunus. - Fr. Wald (Hg.) - Nach B. im Taunus häufiger als bei Fr. - Bei Wlb. wie rustica gef. (Sch.) - G. (L.) Wsb. an Eichen hinter der Walkmühle 24. Mai (K.). - Mo. auf Kiefern 20. Mai mit Eiern (K.). -

9. T. pulicarius F. - Von B. s. hei Fr. am 14. Mai 1861 gesammelt. -

10. T. nigricans Müll. - Fr. - Soden. - Im Juni. - Auch von Hg. gef. - Wlb. (Sch.) - Mz. (S.) -

11. T. pellucidus F. - Fr. und Sodener Wald h. auf Fichten von $=5$. bis $=6$. - Bei Fr. auch von $\mathrm{Hg}$. gef. und einmal ron B. - Wlb. (Sch.) -

12. T. lividus L. (mit gelben Flügeldecken). - Fr. h. von L. H., Hg. und B. gef. - G. (L.) - Wlb. (Sch.) - Mz. (S.) -

Var. dispar F. (mit schwarzen Flügeldecken). - Fr. wie die Stammart h. im Juni; auch von Hg. gef. - Wlb. (Sch.) - G. (L.) - Mz. (S.) -

13. T. Sudeticus Letzn. - Im Hofheimer und Sodener Wald im Taunus im Mai von C. H. gef. [Das Hofheimer Exemplar von haemorrh. in S. V. gehört hierher]. - Fr. (Hg.) - Wlb. (Sch.) - Auf Weissdornblüthen im Adamsthạ hei Wsb. 28. Mai (K.). - 
14. T. haemorrhoidalis $\mathbf{F}$. = clypeatus Illig. - Im kleinen Feldbergthal $>5$. - Bei Falkenstein $<6$. - St. Goarshausen im Mai von C. H. gef. - Fr. (Hg.) - Wlb. (Sch.) -

15. T. rufus L. - Rumpenheim am Entensee $<$ 7. und im Hengster bei Offenbach $>6$. - Fr. (Hg., B.) - Wlb. (Sch.) -

Var. lituratus Fall. - Von Hg. hei Fr. gesammelt. -

16. T. bicolor Hbst. - Im Mai und Juni. - Fr. - Soden im Taunus. - Feldberg, Lorsbacher Thal. - Bei Fr. auch von Hg. gef. -

17. T. fulvicollis $\mathbf{F}$. = nivalis Germ. = thoracicus Redtb. - Im Hengster, einer grossen sumpfigen Fläche bei Offenbach, im Juli von uns h. gef. - Zahlreich bei Fr. von B. gesammelt. -

18. T. bicolor Hbst. = thoracicus Oliv. = fulvicollis Illig. - An sumpfigen Stellen am ehemaligen Rüstersee bei Oberrad im Fr. Wald im Juli von C. H. gef. - Fr. (Hg.) - Wlb. (Sch.) - G. (L.) - Mo. auf Kiefern 8. Juli (K.). -

19. T. paludosus Fallen. - Am Feldberg im Mai und Juni sehr einzeln von C. H. gef. -

20. T. Iateralis L. = oralis Germ. - An Sumpfstellen n. s. - Am Mainufer $=6$., am Entensee bei Rumpenheim = 7., im Hengster bei Offenbach, Soden im Taunus. - Fr. (Hg.) - Zweimal am 17. Mai 1860 bei Fr. von B. gef. - Wsb. an der Wellritz 22. Juni und an der Kiesgrube 26. Juli h. gef. (K.) -

21. T. discoideus Ahrens. - Fr. (Hg.) - G. (L.) -

22. (Absidia Muls.) pilosus Payk. - L. H. fand ein Exemplar dieses Gebirgthieres bei der Oelmühle bei Königstein im Taunus im Juli 1872. - Im Taunus auch von Hg. gesammelt. -

23. (Rhagonycha Eschscholtz) fulvus Scop. = melanurus Oliv. - Auf Blüthen überall g., auf Wiesenblumen, besonders Umbelliferen im Sommer. - Fr. - Soden. - Wlb. s. h. (Sch.) - G. (L.) - Mz. (S.) - Wsb. 14. Juli (K.). -

24. (R.) fuscicornis Oliv. - Bei Soden s. an Eichen $>6$., auf dem Niederwald bei Rüdesheim und im Fr. Unterwald $<7$. von C. H. gesammelt; bei Fr. von Hg. - Wlb. h. (Sch.) - Im Oberolmer Wald bei Mz. (S.) - Wsb. rechts am Fusspfad nach der Platte auf Kiefern im Juni (K.). -

25. (R.) testaceus L. - Auf Wiesengebüsch bei Falkenstein $=5$., im Wald bei der Station Mainkur $>5$. - Fr. (Hg.) - Wlb. h. (Sch.) - G. (L.) - Wsb. auf Saalweiden an der Wellritz 13. Juni (K.). - 
26. (R.) pallidus Göze. - Fr. Wald an Eichen $>5$. - Im Taunus bei Soden und Falkenstein von $>5$. bis $<6$., Johannisberg bei Friedberg = 5. gef. - Fr. (Hg.) - Wlb. (Sch.) - G. (L.) -

27. (R.) femoralis Brullé. - Bei Wlb. von Sch. gesammelt. Mo. auf den Blössen im Kiefernwald von 21. Juni bis 2. Juli (K.). -

28. (R.) ater L. - Im Wald bei Falkenstein und am Feldberg einigemal in einem Nest der Formica rufa im Mai. - Fr. (Hg.) Wlb. (Sch.) - G. (L.) -

29. (R.) elongatus Fallen = paludosus Redtb. - Bei $\mathrm{Fr}$. zweimal auf Pinus picea $<6$. von C. H. gef., auch von Hg. ges. -

30. (Pygidia Muls.) denticollis Schummel = Redtenbacheri Märk. = nivalis Redtb. - Oberrevisor Jännicke fand 1 Stück im Urselthal im Taunus. -

\section{Malthinus Latreille.}

(Diese und die folgende Gattung sind in Sammlung $\nabla$. Heyden von v. Kiesenwetter revidirt.)

1. M. fasciatus Oliv. - Fr. Wald $>$ 5. - Auf Hecken in Soden $>6$. - Königstein im Taunus $>$ 8. - Ems. - Fr. (Hg.) - Von B. n. s. auf der Königswiese im Fr. Wald gestreift. Wlb. (Sch.) - Mz. (S.) -

[M. seriepunctatus Ksw. und balteatus Suffr. kommen nicht im Gebiet vor.]

2. M. glabellus Kiesw. - Auf Hecken in Soden $>6$. in Begattung, ein Weibchen legte, angespiesst auf Silberdraht, noch Eier ab. - Auf Eichen im Falkensteiner Wald. - Mz. (S.) -

3. M. punctatus Fourcr. = flaveolus Hbst. - Königstein $>6$. in Begattung. - Soden auf Hecken. - Bei Bergen im Mai. Fr. Wald $=6$. aus überwinterten Fichtenzapfen entwickelt. - St. Goarshausen von C. H. gef. - Fr. (Hg.) - Wlb. beide Geschlechter (Sch.). - G. (L.) -

4. M. biguttatus $\mathrm{L}$. = biguttulus Payk. - Voll Hg. bei Fr. gesammelt. - Wlb. (Sch.) -

5. M. frontalis Marsh. - Aus Fr. dürrem Waldholz $<$ 5. gezogen. - Auf Ulmen am Affenstein beim neuen Fr. Irrenhaus $=6$. - Fr. (Hg.) - Wlb. (Sch.) - 


\section{Malthodes Kiesenwetter.}

1. M. minimus $L$. = sanguinolentus Fall. - Soden auf Eichen $>$ 6. - Enkheimer 'Torfgruben $=5$. - Fr. von Hg. gef. - G. (L.) - Mz. (S.) -

2. M. marginatus Latr. - Fr. im Mai auf Eichen, ebenso bei Soden und am Altkönig $<6$. - Nauheim. - Bei Fr. auch von Hg. gesammelt. - Mz. (S.) -

3. M. pellucidus Kiesw. - Einmal in der Hohen Mark im Taunus $>$ 7. von C. H. gef. -

4. M. dispar Germ. - Fr. mehrfach gef., auch von $\mathrm{Hg}$. St. Goarshausen $>6$. von C. H. gesammelt. - Wlb. (Sch.) -

5. M. maurus Casteln. - Bei Fr. von Hg. gesammelt. Wlb. h. (Sch.) -

6. M. misellus Kiesw. - G. (L.) -

7. M. hexacanthus Kiesw. - Im Falkensteiner Wald $<6$. auf Eichen und am Feldberg auf Buchengebüsch $>6$. Ein Weibchen legte, aufgespiesst, Eier an der Nadel ab. - Wlb. (Sch.) -

8. M. brevicollis Kiesw. (nec Payk.) - Fr. $<6$. von Epheu geklopft. - Sodener und Rebstocker Wald bei Fr. auf Hecken $>5$. und im Juni. - St. Goarshausen von C. H. gef. - Bei Fr. anch von Hg. gef. - Wlb. (Sch.) -

9. M. spathifer Ksw. - Im Taunus bei Soden und Falkenstein auf Eichen mehrfach gesammelt im Juni. -

10. M. pulicarius Redtb. = dimidiatocollis Roshr. - Einmal $<$ 5. auf Eichen bei Fr. von C. H. gef., auch von Hg. gesammelt. -

\section{DRILINI.}

\section{Drilus olivier.}

1. D. flavescens Rossi. - (Das flügellose, larvenförmige Weibchen wurde von Mielzinsky als besondere Gattung Cochleoctenus vorax beschrieben). - Das Thier lebt im Larvenzustand in den Gehäusen verschiedener. Schnecken, z. B. Helix nemoralis und lapicida, kleine Larven auch in Clausilien. - Man findet sie öfter rasch über den Weg laufen; begegnet sie einer Schnecke, so beisst sie sich mit ihren starken Mandibeln am Kopfe fest und lässt sich von der Schnecke in das Innere 
des Gehäuses ziehen, dessen Insassen sie bald verzehrt und eine andere als Beute aufsucht; sie bleibt zur Verwandlung in dem Gehäuse. Schnecken mit lebenden Driluslarven sind n. s. auf der Ruine Königstein; die vollkommenen Thiere viel seltener. - Mittelgrosse Larve $=8$. gefunden, überwinterte und starb dann aus Hunger. $>5$. in den Schnecken gefundene Larven entwickelten sich $=6$. und lieferten Weibchen $;<5$. 1856 fand L. H. eine männliche Puppe, die am 26. den Käfer lieferte, $1872 \mathrm{im}$ Juli fing L. H. dort ror dem langen Durchgang ein einzelnes Männchen auf Umbellen. Die Weibchen leben rerborgen unter Steinen und in leeren Schneckengeliäusen. - Bei Wlb. das Männchen und die Larve h.; das Weibchen erzog Sch. aus einem Gehäuse der Helix nemoralis, beobachtete auch die Paarung in einem Garten auf dem Erdboden. Bei Had. am Abhang hinter dem Kirchhof im Mai ein Männchen (K.), bei Wsb. im Juni ein Weibchen von Sandberger gef. -

2. D. concolor Ahrens. = pectinatus Schh. - C. H. fand 3 Männchen in Hecken bei Sachsenlausen, L. H. eins in Frankfurt auf der Hochstrasse an einem Haus angeflogen am 24. Juni 1870. - Auf der Bieberer Höhe bei Offenbach fand C. H. $=10$. in Helix nemoralis eine Larve, die sich $<6$, in ein Weibchen verwandelte, das wohl zu dieser Art gehört. - Es ist viel kleiner, dunkler und das Halsschild scheint nicht so breit und kurz wie bei flavescens zu sein. Die Fühlerglieder sind rundlicher und nicht nach der Basis schwach verschmälert wie bei der vorigen Art. - Bei Fr. auch von Hg. gef. - Wsb. am Haus 5. Juni (K.). -

\section{E. MALACHIINI.}

\section{Malachius Fabricius.}

1. M. aeneus L. - Wlb. S. (Sch.) - Mz. (S.) -

2. M. scutellaris Er. - Fr. ron C. H. und Hg. im Mai, auch in Wsb. gef. - L. H. fand bei Mo. am Artillerieschiessstand $<5$. eine Puppe. im Nest einer Mauerbiene an einem Grenzsteine; der Käfer entwickelte sich $=5$. -

3. M. rubidus Er. = fallax Strübing. - Mai und Juni auf Blüthen im Wald. - Mo. = 5. - Wsb. (nach Bach). - Fr. (Hg. and B.) - Wlb. s. h. (Sch.) - Mz. (S.) - Mo. h. auf Euphorhia von $=5$. lis $=7$. (L. H. und K.) - 
4. M. bipustulatus L. - Aus altem Fr. Waldholz h. erzogen im Mai. - St. Goarshausen am Rhein auf Blüthen. - Bei Fr. auch von Hg. und B. gef. - Wlb. (Sch.) - G. (Li) - Mz. (S.) -

5. M. viridis F. - Auf Waldwiesenblumen n. s. von Mai bis August. - Fr. hinter den Kettenhöfen. - Soden. - Lorsbach. Mainkur. - Offenbach. - Bëi Fr. auch von Hg. und B. gef. - Wlb. (Sch.) -- Mr. (S.) - Wsb. 16. Juni, Mo. von 20. Juli bis 15. August (K.). -

6. M. marginellus Oliv. - Aus Fr. Waldholz erzogen. - Von L. H. im Fr. Wald im Mai gesammelt, auch von Hg. und B. gef. Wlb. (Sch.) - Mz. (S.) - Mo. Damm 19. Juni (K.). -

7. M. elegans Oliv. - Ems h. (Bach). - Fr. (Hg.) - Lahnstein 10. Juni (K.). - Sauerthal $<6$. (C. H.) -

\section{Axinotarsus Motschulsky.}

1. A. pulicarius F. - Fr. - Soden im Juni und Juli auf Hecken. - Auch von Hg. und B. gef. - Wlb. (Sch.) - G. (L.) -

2. A. marginalis Er. - Soden auf Hecken im Juni. - Aus altem Buchenholz aus dem Fr. Wald in Anzahl erzogen. - Im August im Rödelheimer Wald gesammelt; bei Fr. auch von $\mathrm{Hg}$. und 1875 von B. gef. - Wlb. (Sch.) - G. (L.) - Wsb. 21. Juni (K.) - Mz. (S.) -

3. A. ruficollis $\mathbf{0 I}$. = rubricollis Marsh. - Fr. Wald am Forsthaus im Juli; im Juni aus dürrem Waldholz erzogen. - Bei Fr. auch von Hg. und B. gesammelt. - Wlb. (Sch.) - G. (L.) -

\section{Ebaeus Erichson.}

1. E. praeoccupatus Gemming. = pedicularius Schrank. Bei Fr. von B. und Hg. gef. - Wlb. (Sch.) - G. (L.) - Mz. '(S.) - Wsb. 11. Juni (K.). - Schierstein am Rhein 25. Juli (K.). -

2. E. flavicornis Er. - Von Hg. bei Fr. gef. -

3. E. appendiculatus Er. - Von C. H. in den Kalksteinbrüchen von Flörsheim $>6$. öfter gesammelt. - Bei Fr. von Hg. gef. -

4. E. thoracicus Fourc. - Flörsheim mit dem vorigen. Soden auf Hecken und Dornzäunen im Juni und Juli. - Bei Fr. auch von Hg. gef. - Wlb. (Sch.) - Mr. (S.) - 


\section{Charopus Erichson.}

1. C. flavipes Payk. = pallipes Er. - Königstein auf Geranium sanguineum $>6$. - Bieberer Höhe bei Offenbach $=6$. - Schmitterhof bei Giessen $>$ 8. - Soden auf Hecken $>6$. - Ems. - Im Fr. Wald fanden I. H. und Hg. diese Art im Hochsommer s. h. mit dem Streifnetz. - Wlb. s. h. (Sch.) - G. (L.) - Mz. (S.) - Mo. 22. Mai (K.). -

\section{Hypebaeus Kiesenwetter.}

1. H. flavipes F. - Aus altem Carpinus-Holz aus dem Fr. Wald eimmal in Anzahl erzogen. - Bei Fr, auch von Hg. gef. Wlb. (Sch.) -

2. H. flavicollis Er. - Bei Fr. einmal von Hg. gef. - Sonst nur in Süd-Europa. -

\section{Anthocomus Erichson.}

1. A. rufus Hbst. $=$ sanguinolentus $\boldsymbol{F}$. - Auf Arundo in den Lehmkauten bei Offenbach auf dem Weg nach Bürgel $>10$. in Anzahl von C. H. gesammelt, ebenso $<11$. bei Fr. hinter den Kettenhöfen. - Mz. (S.) -

2. A. equestris F. - Fr. h. - Soden. - Ueberwintert öfter in den Häusern und findet sich dann an warmen Tagen am Fenster. Fr. (Hg., B.) - Wlb. (Sch.) - M\%. (S.) - Wsb. 10. Mai am Fenster (K.). -

3. A. fasciatus L. - Wie die vorige Art. - Soden auf Hecken $>$ 6. - Bei Fr. von uns, Hg. und B. gef. - Wlb. (Sch.) - G. (L.) - Wsb. 15. Juli am Fenster, auch an der Wellritz 16. Juni (K.). -

4. A. regalis Charp. (Seither als Var. des vorigen betrachtet, wird jetzt für eigene Art gehalten.) - Von C. H. ein Stück bei Soden, von B. eins bei Fr., sowie von Hg. gef. -

\section{Troglops Erichson.}

1. T. albicans L. - Je einmal bei Fr. und $>$ 8. bei dem Schmitterhof bei Giessen gef. - Fr. (Hg.) - Wlb. (Sch.) - 


\section{F. DASYTINI.}

\section{Dasytes Paykul.}

1. D. niger L. - Im Fr. Unterwald auf Blumen von $=5$. bis Juli gef., von B. h. gesammelt. - Wlb. (Sch.) -

2. (Hypodasytes Muls.) obscurus Gyll. - Von M. S. bei Fr. gef. [1 Stück in Sammlung v. Heyden]; auch von Hg. gesammelt. G. (L.) -

3. (Metadasytes Muls.) caeruleus De Geer. - Aus altem Waldholz s. h. erzogen, stets einer der ersten Käfer im Jahr, der sich in der Holzkammer entwickelt. Schon im April im Wald an Buchen. Fr. auch von Hg. und B. gef. - Wlb. (Sch.) - G. (L.) - Niederwald. -

4. (Mesodasytes Muls.) plumbeus Müll. = flavipes F. = coxalis Muls. - Aus altem Buchenholz aus dem Fr. Wald s. h. im Mai und aus üherwinterten Holunderzweigen entwickelt. - Soden. Fr. (Hg.) - Von B. früher öfter, das Männchen 1875 bei Fr. gef. Wlb. h. (Sch.) - G. (L.) - Mz. (S.) -

5. (M.) aerosus Kiesw. = plumbeus Muls. - Fr. von C. H. gef.; auch bei St. Goarshausen. -

6. (Pseudodasytes Muls.) fusculus Illig. - Aus dürrem Fr. Waldholz im Mai erzogen; zu Anfang des Monats im Wald auf EuphorbiaBlüthen. - G. (L:) - Mz. (S.) -

7. (P.) subaeneus Schh. $=$ aeneus 01 . $=$ scaber Suffr. Fr. - Bei Altenhain im Taunus im Juni auf Spartium. - Flörsheim am Main. - Bei Fr. auch von Hg. gesammelt. - Wlb. (Sch.) Unterhalb Bingen auf Spartium (S.) -

\section{Julistus Kiesenwetter.}

1. J. floralis Oliv. - Aus dürrem Fr. Waldholz $=$ und $>4$. 3 Stück erzogen. -

2. J. fulvohirtus Bris. - Aus abgefallenen Fichtenzapfen $<5$. aus dem Fr. Wald einmal und ein zweites Stück $=5$. aus dürrem Fr. Waldholz erzogen. - War seither nur aus Frankreich bekannt. - 


\section{Dolichosoma Stephens.}

1. D. lineare Rossi = filiforme Panz. - Im Juni und Juli auf trockenen Grasplätzen im Fr. Wadd n. s. von L. H. gef. - Bieberer Höhe bei Offenbach. - Rebstockwald bei Rödelheim. - Bei Fr. auch ron Hg. und B. am 25. Mai 1860 gef. - Mz. (S.) - Wsb. zwischen Dotzheim und Chausseehaus im Juni (K.). -

\section{Haplocnemus Stephens.}

1. H. Pini Redtb. = serratus Redtb. - Auf blühenden Kiefern n. s. s. - Fr. - Offenbach am 4. Juni 1856 ron L. H. gef. - Mo. $=5$. - Auch $>4$. aus altem Eichenholz von Soden erzogen (hat vielleicht da nur überwintert). - Fr. (Hg. und B.) - Wlb. (Sch.) Mz. (S.) -

2. H. nigricornis $\mathbf{F}$. - Bei Homburg $<4$. auf Fichten. Bei Fr. noch $=10$. gef. -

3. H. ahenus Kiesw. - Von C. H. einmal $<6$. im Santerthal (bei Lorch am Rhein) gef. -

\section{Danacaea Laporte.}

1. D. pallipes Panz. - Falkensteiner Wald im Taunus $=5$. - St. Goarshausen $<5$. in den Blüthen von Heleborns viridis. Fr. (Hg.) - Wlb. h. (Sch.) - G. (L.) -

2. D. nigritarsis Küst. = tomentosa Panz. (pars.) - Soden. - Ems. - Flörsheimer Steinbrüche auf Thymus. - Fr. auch ron Hg. gesammelt. - Wlb. (Sch.) - 


\section{LYMEXYLIDAE.}

\section{Hylecoetus Latreille.}

1. H. dermestoides L. - L. H. besitzt ein am grossen Feldberg im Taunus gefundenes weibliches Exemplar. - Auch Hg. fand die Art im Taunus. - Wlb. ein Pärchen gef., fliegend in einer .Pappelallee (Sch.). - Wetzlar von Lieutenant Giebeler gef. -

\section{Lymexylon Fabricius.}

1. L. navale L. - Im Taunus, z. B. bei Cronthal, Neuenhain an alten Kastanien, besonders auch an geschlagenen Stämmen im Juli angeflogen, doch nur Weibchen, die sehr schnell umherlaufen, leicht fortfliegen und stets bemüht sind, ihren langen, beweglichen Hinterleib in eine Ritze zu stecken, um die Eier darin abzulegen. Das grösste Weibchen der Sammlung v. Heyden hat $14^{\mathrm{mm}}$ Länge, 2 Männchen nur $4^{1 / 2}-5^{\mathrm{mm}}$. Die Letzteren scheinen selten zu sein, eins fing C. H. im damaligen Neubau des Senckenberg'schen Museums in Frankfurt $=5$. 1830, gerade als die Schreiner. den Fussboden legten. - Taunus (Hg. und B.). Wlb. ein Pärchen an einer alten Eiche (Sch.). - Mz. (nach Bach). - 


\section{C LERIDAE.}

A. CLERINI.

\section{Din o ps steven.}

1. D. albofasciata Charp. — Von S. einmal bei Mrz. aufgefunden. -

\section{Tillus Olivier.}

1. T. elongatus $L$. (Männchen $=$ ambulans $F$. mit schwarzem Halsschild.) - Aus altem Buchenholz aus dem Fr. Wald n. s. im Mai gezogen, später h. von St., auch von Hg. im Wald gef. - B. fand 18601 Stück auf einer alten Buche an der "Louisa" im Fr. Wald, 1 Stück in Fr. an einem Haus angeflogen. - Wlb. 1 Stück (Sch.). Mz. an alten Weiden (S.). -

Var. hyalinus Sturm. (Form des Männchens: am Rand der Flügeldecken mit einem weissen, glasartig durchscheinenden Fleck; die Furche, die sich da findet und von Bach erwähnt wird, bildet sich erst nach dem Tod des Thieres). - Ein Stück aus zweimal überwintertem dürrem Buchenholz aus dem Fr. Wald $>$ 5. entwickelt. -

Var. bimaculatus Donovan. (Form des Männchens mit einer schwachen, glasartigen Querbinde hinter der Mitte der Flügeldecken). Einmal $<5$. aus altem Buchenholz aus dem Fr. Wald entwickelt. -

2. T. unifasciatus F. - Bei Mz. von Dr. Zitz gesammelt [1 Stück in Sammlung v. Heyden]. - Im Ort Mombach auf Hecken von Hg. gef., später auch von S. gesammelt. -

\section{Opilus Latreille.}

1. 0. mollis L. - Aus alter Epheurinde $>3$. und aus altem Buchenholz entwickelt; aus zweimal überwintertem Buchenholz auch noch $>$ 5. - Fr. s., auch von Hg. gef. - 3 Ex. an der Wand eines Hauses in Dlb. gef. (Sch.) - Mz. (S.) -

Var. domesticus Sturm. - s. - Fr. < 7. gef., auch von Hg. gesammelt. - Dlb. einmal (Sch.). - 


\section{Thanasimus Latreille.}

1. (Pseudoclerops Duv.) 'mutillarius F. - Auf den Zimmerplätzen an Eichenholz, früher häufiger bei Fr. im Mai und Juni. - Bei Isenburg von Hg. gesammelt. - Ein Stück auf einer alten Buche am 27. Mai 1874 an der "Louisa“ von B. gef. - Mz. (S.) -

2. T. formicarius L. - An gefälltem Nadelholz s. h. - Fr. Im Röderwald und an der Gehspitz (Fr. Wald) von B. gef. - Dlb. Wlb. s. h. (rothe Brust) (Sch.). - G. (L.) - Mz. (S.) - Dotzheim an gefällten Kiefern im Frühjahr (K.). - Mo. 15. Juli an Kiefernholz (K.). -

3. T. rufipes Brahm. = substriatus Gbł. - Durch sehwarze Brust von der vorigen Art verschieden. - Die Art variirt in der Ausdehnung der rothen Farbe. Typische Stücke des rufipes von $5 \mathrm{~mm}$ Länge (L. H. kennt solche nur aus unserer Gegend) haben ganz gelbe Beine und die erste weisse Binde der Flügeldecke steht auf gelbrothem Grund. Solcher Stücke besitzt L. H. vier aus dem Fr. Wald, im Juli und August aus dürrem Holz erzogen und einmal $<5$. an Kiefernholz bei Fr. gef. — Grössere Stücke von 71/2_-9 $\mathrm{mm}$ Länge aus den Alpen und Schweden haben theilweise schwarze Beine, die erste weisse Binde steht auf schwarzem Grund. Bei beiden Formen sind die eingestochenen Punkte auf dem rothen vorderen Theil der Flügeldecken in Punktreihen geordnet, während diese Stelle bei formicarius grob verworren punktirt ist. - Die dunkleren grösseren Stücke bilden den substriatus Gebl. = femoralis Zett. Mz. (S.) -

4. (Allonyx Duv.) quadrimaculatus Schaller. - An einer alten Linde (jetzt gefällt) hinter dem Bockenheimer Friedhof $<$ 5. von C. H. gef. - Bei Fr. von M. S., Hg. und v. Twardowski gef. - Oberursel im Taunus von Gremmers gef. - Im März̀ 1860 n. s. auf alten Buchen am Forsthaus und auf Kirschen am Röderwald umherlaufend; 1 Ex. an einem Haus im Westend angeflogen, von B. gef. - Wlb. (Sch.) Gaualgesheimerkopf, eine Stunde oberhalb Bingen 20. Mai 1867 (K.). - Mr. (S.) -

\section{Trichodes Herbst.}

1. T. alvearius F. - Fr. auf Blumen in Gärten und Wiesen. Soden. - Die Larve lebt nach Bach in den Nestern der Mauerbienen. - Isenburg (Hg.). - Nach B. bei Fr. häufiger als apiarius. - Bei 
Wlb. s. h. Sch. erzog ihn, aus dem Neste von Polistes gallica. G. (L.) - Had. am Abhang hinter dem Kirchhof (K.). - Mz. (S.) -

2. T. apiarius L. - Die Larve lebt in den Nestern der Honigbiene. - Bei Isenburg von Hg. gesammelt. - Von B. h. bei Fr. gef. - Wlb. s. h. auf Blumen (Sch.). - Mz. (S.) - Auf den Blössen im Wald bei Mo. von 25. Mai bis 22. Juli 1857 (K.). -

3. T. favarius III. - In Isenburg im Garten von $\mathrm{Hg}$, auf Blumen öfter gef. -

\section{B. CORXNETINI.}

\section{Orthopleura Spinola.}

1. O. sanguinicollis F. - Bei Fr. von Steitz einzeln an Eichenholz gesammelt. - Mz. (nach Bach). -

\section{Corynetes Herbst.}

1. C. caeruleus De Geer. - Fr. im Mai in Blüthen n. s.; auch von Hg. gef. - Dlb. in Häusern an Fenstern z. s. (Sch.) - Eichberg im Rheingau (Richter). - Mz. (S.) -

2. C. ruficornis Sturm. - Bei Lorsbach im Taunus in einer hohlen Eiche $>4$. in Begattung gef. - Fr. (Hg.) -

3. (Necrobia Latr.) violaceus L. - Bei Griesheim am Main im August an alten Cadavern. - Fr. $<9$. auch von Hg. gef. Von B. an todten Katzen am Main bei Fr. gef. - Dlb. Wlb. s. h. an Aas, auf Blüthen, in Häusern an den Fenstern (Sch.). - Mz. (S.) -

4. (N.) ruficollis F. - Leht an Naturalien und ist durch den Fellhandel über die ganze Erde verbreitet. - Im Freien einmal unter dem Abhange des Lenabergs gegen Budenheim zu 2. August 1873 auf einer Waldblösse gestreift (K.). — Sonst kein Stück aus dem Gebiet bekannt. (Häufiger bei Darmstadt.) -

5. (Agonolia Muls.) rufipes De Geer. - Fr. 2 Stück von C. H. gef. [L. H. besitzt Exemplare vom Cap, Cuba, Paraguay.] - Auch Hg. sammelte die Art bei Fr. - 


\title{
XLIV. ANOBIIDAE.
}

\author{
A. APATINI.
}

Apate Fabricius.

1. A. capucina L. - Bei Fr. an Eichenholz im Mai, früher auf Zimmerplätzen von C. H. n. s. gesammelt. - Wsb. am Bleichplatz an Pfosten $>5$. (K.). -

2. A. varia Illig. $=$ Dufouri Latr. - Aus Fr. dürrem Buchenholz von uns und St. oft erzogen; im Juni. -

\section{Xylopertha Guérin.}

1. X. sinuata F. - Im Fr. Wald 1 Ex. von Steitz an Eichenklafterholz schwärmend gef.; auch bei Wlb. einmal von Sch. ges. -

\section{B. ANOBIINI.}

\section{Dryophilus Chevrolat.}

1. D. pusillus Gyll. - Lebt auf blühenden Kiefern und Fichten. - Aus Fichtenknospen $=5$. entwickelt. - Auf Pinus picea $>6$. im Altenhainer Wald n. s. von C. H., bei den Offenbacher Kalkbrüchen von L. H. gesammelt. - Von B. 1 Stück bei Fr. gef. - Wlb. ein Weibchen (Sch.). - Mz. (S.) -

\section{Gastrallus Duval.}

1. G. laevigatus $01 .=$ immarginatus Müll. = exilis Gyll. Aus dürrem Fr. Waldholz von L. H. $<6$, öfter erzogen. - Von B. bei Fr. 1875 gesammelt. - Wlb. (Sch.) -

\section{Oligomerus Redtenbacher.}

1. O. brunneus Oliv. - Aus dürrem Fr. Waldholz von $=6$. bis $>$ 7. ofter erzogen; auch in einem alten Apfelbaum gef. - Fr. (Hg.) - 


\section{Anobium Fabricius.}

1. (Sitodrepa Thỏms.) paniceum L. - In einer Insektenschachtel aus dem südlichen Deutschland $=12$. - Fr. in Herbarien oft schädlich im Juni und Juli; auch in altem Brod; von $\mathrm{Hg}$. gesammelt, auch von B. h. bei Fr. - Bei Wlb. s. h. im Hause an Fenstern, in Herbarien, in altem Backwerk (Sch.). - Mz. (S.) -

Var. minutum Sturm. (Kleiner, schmäler.) - Fr. n. s. im Juli. Lebensweise wie die Stammart. - Bei Wlb. oft mit der grossen Form gef. (Sch.) -

2. (Hadrobregmus Thoms.) emarginatum Duft. - In altem Weidenholz bei $\mathrm{Fr} .=6$. zweimal von C. H. gef. - Mz. (S.) -

3. (H.) nitidum Hbst. - Aus dürrem Fr. Waldholz (Buchen) öfter erzogen. - Fr. an Hecken hinter der Oede auch von Hg. ges. Wlb. (Sch.) - Mz. (S.) -

4. (H.) rufipes F. - Fr. - Aus zweimal, fünfmal und neunmal überwinter tem Carpinus-Holz $=5$. erzogen; auch sonst in alten CarpinusStämmen h. gef., einmal auch in einem alten Nussbaum im Juni. Mainkur. - Fr. (Hg. und B.) - Wlb. (Sch.) -

5. A. punctatum De Geer = fulvicorne Sturm. - Bei Soden $>$ 6. auf Dornzäunen von C. H. öfter gef. -

6. A. fagicola Muls. - Im Mai s. h. aus altem Buchenholz aus dem Fr. Wald errogen. - Mz. (S.) -

7. A. domesticum Fourcr. = striatum Oliv. - Aus altem Fr. Waldholz h. er'zogen im Juni; in alten Nussbaummöbeln. - Aus Epheuzweigen, die im April 1860 bei Rüdesheim von C. H. eingesammelt wurden, entwickelte sich der Käfer oft $<6.1861$. - Ebenso bei Mz. von S. gef. - Ems. - Fr. aus dürren Zweigen von Salix alba. Auch bei Fr. von Hg. und B. gesammelt. - Wlb. Dlb. s. h. in Häusern in alten Möbeln. -

8. A. pertinax $L$. = fagi Hbst. = striatum $F$. - Von $\mathrm{Hg}$. bei Fr. gef, - In altem Kirschbaumholz im Röderwald h. bei Fr. von B. gesammelt. - Bèi Wlb. noch nicht beobachtet (Sch.). - Mz. (S.) -

9. A. denticolle Panz. - Selten. - Einmal im Januar unter Buchenrinde; $>10$. am Fr. Forsthaus unter Rinde von Pinus picea. - Nur 1 Stück von B. bei Fr. gef. - Mz. (S. Bach). -

10. (Neobium Muls.) hirtum III. - Einmal von Hg. bei Fr. gef. - 


\section{Xestobium Motschulsky.}

\section{X. rufo-villosum De Geer = pulsator Schall. $=$ tesse-} latum Oliv. - In alten Eichen, Hainbuchen und Weiden bei Fr. und Soden von April bis Juni; Cronthal im Taunus in alten Castania vescaStämmen. - Einmal auch aus einer Schachtel mit Insekten aus Ungarn $<4$. entwickelt. - L. H. hat das Männchen dieser Art oft pochend gesehen; es schlägt mit der Stirn wider das morsche Holz, der laute Ton lockt das Weibchen herbei. - Von B. und Hg. auch bei Fr. gesammelt. - Wlb. b. (Sch.) - Mz. an alten Bäumen in der Rheinallee (S.). - Ernsthausen in Nassau (Lehrer Dörr bei K.). -

2. X. plumbeum Illig. = politum Duft. (Die Stammart hat dunkelerzbraune Flügeldecken). - Aus altem Buchenholz ans dem Fr. Wald h. erzogen von $>4$. bis $<6$. - Auch von Salweiden $>6$. geklopft. - Von Hg. auch bei Fr. gesammelt. - Wlb. (Sch.) - Mz. (S.) - Wsb. Waldweg im Adamsthal im Gras gestreift (K.).

Var. variabile Muls. (Mit rostrothen, wenig metallischen Decken.) - Fr. mit der Stammart n. s. -

\section{Ernobius Thomson (= Liozoum Muls.).}

1. E. Abietinum Gyll. - Einmal bei Cronthal = 5. von C. H. gef. - [Das in S. V. erwähnte Stück aus Fichtenzapfen gehört zur folgenden Art.] - Friedberg (Fuhr). -

2. E. Abietis F. - Aus Fichtenzapfen in vielen Jahren h. von C. H. erzogen. - Im August 1835 eingesammelte Fichtenzapfen lieferten den Käfer im März 1838. - In mehreren Jahren $>4$. aus zweimal überwinterten Zapfen, $=6$. auch aus dreijährigen. - Fr. - Taunus. - Bei Homburg $<4$. von Fichten geklopft. - Auf Kiefern $=6$. bei Mo. [Hierher auch Anobium Pini des S. V.] - Wlb. zweimal (Sch.). -

3. E. mollis L. - Fr. $<7$. zweimal gef., auch von Hg. gesammelt. - Wlb. dreimal gef. (Sch.) -

4. E. Consimilis Muls. - Fr. öfter von L. H. gef. Seither mit mollis verwechselt. - Wlb. einmal (Sch.) -

5. E. parens Muls. - Von $>4$. bis Juli öfter aus überwinterten Fichtenzapfen aus dem Fr. Wald entwickelt. Die Larve lebt nach den Beobachtungen von $\mathrm{C}$. $\mathrm{H}$. in den durch Tortrix Buoliana zerstörten vertrockneten Kiefersprossen. - 
6. E. parvicollis Muls. - Im April und Mai aus unreif abgefallenen Fichtenzapfen entwickelt. - Fr. [Hierher angusticollis des S. V.]

7. E. Iongicornis Sturm. - Einmal $=5$. im Cronthaler Wald im Taunus von C. H. gef. -

8. E. Pini Sturm. [Das in S. V. erwähnte Stück gehört zu Abietis.] - Hg. fand die Art bei Fr. -

9. E. nigrinus Sturm. - Fr. Wald $>4$. einmal auf Kiefern von C. H. gef. -

\section{XYLETININI. \\ Ptilinus Geoffroy.}

1. P. pectinicornis L. - In dürrem Holz und alten Bäumen s. h. im Juni. - Die Weibchen bleiben mehr. im Innern des Holzes, während die Männchen, mit ihren langgekämmten Fühlern stets wedelnd, sehr eilig an den Bohrlöchern umherlaufen. - Fr. - An Weiden, Pappeln, Ulmen, Buchen. - Auch von Hg. gef. - Oberrad in Weiden (B.): - An alten Pappeln in beiden Geschlechtern bei Dlb. (Sch.) Mz. (S.) -

2. P. costatus Gyll. - In hohlen Weiden und Pappeln im Mai und Juni seltener wie die vorige Art. - Fr., anch von $\mathrm{Hg}$. gesammelt, von B. einmal am 25. Mai 1861. - Dlb. wie die vorige Art (Sch.) Mz. (S.) -

\section{Ochina Stephens.}

1. 0. Hederae Müll. - Auf Epheu h., z: B. am Eschenheimerthurm in Fr. von L. H. gef. - Aus einmal überwinterten Epheuzweigen von Rüdesheim = 5. entwickelt. - Bei Fr. auch von Hg. gesammelt. - Mz. aus Epheuzweigen (S.). -

\section{Trypopitys Redtenbacher.}

1. T. Carpini Hbst. = serricornis Duft. - Aus dürrem Fr. Waldholz im Juni und Juli öfter erzogen. - Auch von Hg. gesammelt. -

\section{Xyletinus Latreille.}

1. X. pectinatus F. - Fr. $<6$. an alten Planken. - An alten Apfelbäumen beim Hellerhof. - Bei Wlb. h. an alten Planken und Gartenthüren (Sch.). - Mz. (S.) - 
2. X. laticollis Duft. - Von C. H. eimmal bei Fr. und von Hauptmann Fuhr bei Friedberg 1 Stï̈ck gef. - Auch von Hg. bei Fr. gesammelt. - Wlb. 5 Ex. wie die vorige Art (Sch.). -

3. X. ater Panz. = serratus F. - L. H. hat diese Art im Fr. Wald an der Bahenhäuser Chaussee öfter Abends mit dem Streifnetz gef., z. B. 30. Juni 1856. - Auch von Hg. und B. gesammelt. Wlb. h. wie die vorhergehenden Arten (Sch.). -

\section{Lasioderma Stephens (= Pseudochina Duv.).}

1. L. laeve Illig. = testaceum Redtb. nec Duft. = Redtenbacheri Bach. - Bei Mz. auf Disteln von S. aufgefunden. St. Goarshausen einmal von C. H. $>6$. gef. - Viel grösser wie die folgende Art. -

2. L. serricorne F. = testaceum Dft. Sturm. - Lebt in Tabak und durch diesen überall hin verbreitet. - Fr. in amerikanischem Rollentabak und in ostindischen Cigarren aus Calicut bei Bombay von L. H. lebend gef. - C. H. fand den Käfer auch im Museum in Fr, an getrockneten abessynischen Pflanzen. -

[NB. Die Namen in S. V. müssen umgekehrt gesetzt werden, die erste Art ist serricorne, die zweite laeve. Scriba richtete sich hierbei nach einer Angabe von L. H. Erst später 1864 gab L. H. in der Berliner Entomologischen Zeitschrift eine Auseinandersetzung beider Arten.]

\section{DORCATOMINI.}

\section{Dorcatoma Herbst.}

1. D. Dresdensis Hbst. - Im Fr. Wald am Forsthaus $<7$. von C. H., sowie einmal bei Ems gef. - Mz. (S.) -

2. D. chrysomelina Sturm. - Einmal aus Baumschwämmeni $=5$. von Fr. erzogen. - Seither oft mit der folgenden Art vermengt; erst Dr. Kraatz machte 1871, pag. 146, in Berl. Entom. Zeitschr. auf die Unterschiede aufmerksam. - Bei serra sind die Flügeldecken glänzender und ziemlich gleichmässig punktirt, bei chrysom. matter, fein gerunzelt, dazwischen mit grösseren, aber seichten Punkten besetzt. - Auch von Hg. bei Fr. gef. -

3. D. serra Panz. - Aus Baumpilzen $>4$. erzogen, öfter in Begattung beobachtet. - Fr. - Mz. (S.) - 
4. D. flavicornis F: - In rothfaulem Eichenholz im Fr. Wald von $>6$. bis $<7$. öfter gef. - Fr - Königstein. - Auch ron Hg. gesammelt. -

\section{Anitys Thomson (= Amblytoma Muls.).}

1. A. rubens Ent. Hft. - In rothfaulem Eichenholz im Fr. Wald bei Isenburg von C. H., bei Königstein im Taunus $<6$. von L. H. gef. - Auch ron Hg. bei Fr. gesammelt. — G. (L.) -

\section{Caenocara Thomson (= Enneatoma Muls.).}

1. C. Bovistae Ent. Hefte. - In Bovisten im Gebiet s. - Fr. $<$ 7. vorl C. H. und in Neuenhain bei Soden im Taunus von L. H. gef. - NLz. (S.) -

\section{Mesocoelopus Duval.}

1. M. niger Müll. $=$ murinus Stm. = pubescens Duft. Von altem Epheu bei Rüdesheim $=6$. von C. H. abgeklopft. - Bei Fr. aus dünnen abgestorbenen Epheuzweigen s. von B. erzogen. -

\section{E. PTININI.}

\section{Hedobia Latreille.}

1. (Ptinomorphus Muls.) imperialis L. - Aus dürrem Fr. Waldholz (Buchen) im April und Mai oft erzogen; auch aus zweimal üherwintertem Carpinus-Holz. - Von Hg. bei Fr. gef. - Von B. von Hecken geklopft, einmal auch an einem Hause in Fr. angeflogen gef. - Nz. (S.) -

2. (P.) regalis Duft. - Bei Soden zweimal von C. H. $>$ 6. aus Hecken geklopft. - Fr. (Hg. B.) - Seltene Art. -

\section{Ptinus Linné.}

1. (Gynopterus Muls.) sexpunctatus Panz. - Fr. Wald und Fr. Promenaden unter Rinde von Buchen und Platanen im Winter. Larve $>3$. in Coccus auf Eichen; Käfer $>$ 9. entwickelt. (Beobachtung von C. H.) - Auch von B. bei Fr. s. an Häusern gef. - Wlb. einmal (Sch.). - 
2. (G.) dubius Sturm. = crenatus Payk. - Bei Fr. und den Offenbacher Kalkbrüchen n. s. von männlichen Blüthen der Kiefern von $=4$. bis $<6$. geschüttelt. -

3. P. rufipes Oliv. (Weibchen ist elegans Illig.) - Aus altem Holz oft erzogen: an Buchen, besonders Carpinus im April und Mai. - Weibchen noch im Juni und Juli. - Fr. - Soden. - Auch von Hg. im Gebiet gesammelt. - Wlb. beide Geschlechter h. (Sch.) Mz. (S.) -

4. P. ornatus Müll. = fuscus Sturm. - Von C. H. bei Fr. und Rüdesheim s. gef.; im April 1860 gesammelte Zweige lieferten den Käfer $<6$. 1861. - Bei Soden auch aus dürren Pflaumenzweigen $>$ 6. erzogen. - Mz. (S.) -

5. P. fur L. - Im Winter unter Rinden von Buchen im Fr. Wald und von Platanen in Wsb. - In Häusern h. in Fr., auch den Insektensammlungen schädlich, sowie den Herbarien und ausgestopften Thieren. - Dlb. Wlb. h. in Häusern und Herbarien, noch nie in den Insektenkasten gef. (Sch.) - Mz. (S.) -

Eine nur halb so grosse Varietät ist bei Fr. n. s. [Hierher subpilosus des S. V. aus Fr, auch die von M. S. gefundenen, wovon Stücke in Sammlung v. Heyden.] - Wlb. zwei Weibchen (Sch.). -

[P. bicinctus Sturm: - Die Fr. Stücke in S. V. sind fur, Lorscher Stïck richtig.]

6. P. latro F. - In Häusern n. s. - Fr. = 5. - Auch in Insektensammlungen. Das Männchen recht selten, L. H. besitzt nur 1 Stück. - Auch von Hg. bei Fr. gef. - Wlb. h. mit fur (Sch.). Mz. (S.) -

7. P. brunneus Duft. - Fr. und Mr. s. von C. H. gef., doch nur Weibchen. - Bingen. - Bei Fr. auch von Hg. gef. - Bei Mz. auch von S. gesammelt. -

8. P. testaceus Oliv. = hirtellus Sturm. - Einmal von C. H. bei Fr. gef. -

9. P. pilosus Müll. - Fr. ein Pärchen von C. H. gef. - Einmal eine helle Varietät im Fr. Wald in Eichenmulm bei Formica fuliginosa $>4$. - Mulsant zieht zu dieser Art den pallipes Duft., den Kiesenwetter davon trennt; manche Autoren halten sie für die beiden Geschlechter einer Art. -

10. (Cyphoderes Muls.) bidens Oliv. = sycophanta Illig. = raptor Sturm. - Fr. einmal unter Rinde $=4$. gef. - Ueber die 
Synonymie dieser Art siehe Berliner Entonı. Zeit. 1867, p. 132. (raptor bei Muls. = quercus Ksw. noch nicht in Deutschland gef.) Bei Fr. auch von Hg. gef. -

11. (Heteroplus Muls.) pusillus Sturm. - Bei Fr. ein Männchen von C. H., öfter von Hg. gef. - Die Angabe von Scriba, dass die Art nach C. H. in Bucheln h. sei, beruht auf einem Irrthum, er fand einen kleinen fur in einer Buchel. -

\section{Niptus Boieldieu.}

1. (Epauloecus Muls. = Tipnus Thoms.) griseofuscus De Geer $=$ crenatus $\mathbf{F}$. = globosus Ahr. = minutus Panz. $-\mathrm{Fr}$. einmal. - C. H. fand auf der Ruine Königstein im Taunus $>$ 9. eine Anzahl Exemplare in Schneckenhäusern mit fast vertrockneten Thieren. - Bei Fr. auch von Hg. gef. -

\section{Gibbium Scopoli.}

1. G. scotias F. - Ein von Dr. Zitz in Mz. gefundenes Stück in Sammlung v. Heyden. - Von Scheidel lebend im Senckenberg'schen Museum in Fr. beobachtet, wo es mit Naturalien in Baumwolle aus Australien eingeschleppt war. - Bei Mz. auch noch später von S. ges. -

\section{TENEBRIONIDAE.}

\section{[A s i d i n i.}

1. Asida sabulosa Goeze = grisea F. - Diese süd-europäische Gattung ist nördlich der Alpen nur von dem einen, von Bach angegebenen Fundorte bekannt, nämlich Boppard, Laacher See, Coblenz und wabrscheinlich zwischen Bingen und Bonn am ganzen Rhein. Auf meine Anfrage schrieb mir H. Bach, mit welchem ich im Jahre 1875 vergeblich nach dem Thier suchte, folgendes: „Ich habe sie noch nicht auf nassauischem Gebiet (d. h. rechte Rheinseite) gefunden, da ich aber diesen Käfer auf den sonnigen Seiten der Berge oberhalb sowohl wie unterhalb 
der Stadt fand, so zweifle ich nicht daran, dass er auch jenseits des Rheines, Boppard gegenüber, vorkommt. Sie scheint auch hier zu den grössten Seltenheiten zu gehören; trotzdem ich zu verschiedenen Zeiten an den Orten ihres Aufenthaltes war, habe ich sie im vorigen Jahre (1875) nicht zu Gesicht bekommen."]

\section{A. BLAPTINI.}

\section{Blaps Fabricius.}

1. B. mucronata Latr. - In Fr. in der Stadt h. im Juli in Pferdeställen gef. - Mz. (Scriba). - In Kellern und Magazinen in Fr. gesammelt (B.). -

2. B. mortisaga L. - Bei uns s. s. - Zwei bei Fr. von M. S. gef. Stücke in Sammlung v. Heyden. - Ebenfalls von Fr. (B.) Mz. (S.) -

3. B. similis Latr. = fatidica Sturm. - Fr. zusammen mit mucronata. - In den Steinbrüchen auf den Bergen bei Rüdesheim $=4$. von C. H. gef. - Alle 3 Arten auch von Hg. bei Fr. gef. - Die letzte Art bei Fr. schon 13. April 1861 von B. "gẹf. - Wlb. (Sch.) - G. (L.) -

\section{B. CRYPTICINI.}

\section{Crypticus Linné.}

1. C. quisquilius L. = glaber F. - In Sandgegenden unter Steinen h. - Fr. am Forsthaus. - Offenbach. - Mo. - Fr. auch von $\mathrm{Hg}$. und B. gef. - Mz. (S.) -

\section{OPATRINI.}

\section{0 patrum Fabricius.}

1. 0. sabulosum L. - In Sandgegenden s. h. unter Steinen und auf den Wegen laufend. - Fr. - Bockenheim 17. April 1876. - An sonnigen Rainen im Gebiet h. von B. gef. - Wlb. h. nebst der Larve unter Steinen (Sch.). — Am Staufen im Taunus 2. April 1876. Mr. (S.) - 


\section{Microzoum Redtenbacher.}

1. M. tibiale F. - In Sandgegenden h. - Fr. - Unter Steinen auf Sanaboden $=5$., zur selben Zeit auch auf der Königswiese gestreift (B.). - Wlb. (Sch.) - Mo. im Sand h. von 17. Mai bis August (K.). - Mz. (S.) -

\section{BOLITOPHAGINI. \\ Bolitophagus Illiger.}

1. B. armatus Panz. - In trockenen Eichenschwämmen im Fr. Wald von C. H., Hg. und M. S. gesammelt. -

\section{Eledona Latreille.}

1. E. agricola Hbst. - In trockenen Agaricus an Bäumen oft in grosser Menge. - Fr., auch von Hg. gef. und von B. h. gesammelt. - Wlb. h. (Sch.) - G. (L.) - Mz. (S.) -

\section{E. DIAPERINI.}

\section{Diaperis Geoffroy.}

1. D. Boleti L. - In trockenen Baumschwämmen, besonders Boletus h. - Fr., auch von Hg. gef. - An Weiden in Boletus bei Enkheim und im Taunus von B. gesammelt. - Mo. im Juni (K.). Mz. (S.) -

\section{Scaphidema Redtenbacher.}

1. S. metallicum $F_{\text {. }}=$ bicolor $F$. = aeneum Panz. - Von M. S. einmal in Sachsenhausen in einer Hecke an der Oppenheimer Landstrasse gef. - Wlb. einmal (Sch.). - G. (L.) -

\section{Platydema Laporte.}

1. P. dytiscoides Rossi $=$ violaceum F. - Aus altem Buchenholz aus dem Fr. Wald $<7$. einzeln entwickelt, auch von L. H. an der Babenhäuser Landstrasse im Fr. Wald in alten Buchen gef., von Hg. bei Isenburg gesammelt. — G. (L.) - 


\section{Pentaphyllus Redtenbacher.}

1. P. testaceus Hellwig. - Im April und Mai in rothfaulem Eichenholz im Fr. Wald und bei Königstein im Taunus von C. H. n. s. gef. - Bei Fr. von Hg. gesammelt; am Röderwald s. h. von B. im Mulm alter Kirschbäume. - G. (L.) -

\section{F. ULOMINI.}

\section{Uloma Redtenbacher.}

1. U. culinaris L. - Bei Fr. recht selten. - Von L. H. im Schwanheimer Wald unter Eichenrinde am 17. Mai 1856, von $\mathrm{Hg}$. bei Fr. am Hellerhof in alten Weidenbäumen gef. -

\section{Tribolium Mac Leay.}

1. T. ferrugineum F. - Von C. H. im Fr. Wald unter Rinden einigemal gef., auch von Hg. bei Fr. gesammelt. - Wlb. (Sch.) -

\section{Palorus Mulsant.}

1. P. melinus Hbst. $=$ depressus F. - Unter Rinde von Eichen =.5. und von Apfelbäumen = 4. bei Fr. gef. - Soden unter Eichenrinde > 9. - Bei Fr. auch von Hg. gesammelt. - Mz. (S.) -

2. P. bifoveolatus Duft. = Ratzeburgi Wissm. - Fr. einmal im Brod eingebacken gef. (C. H.) - Seither oft zu dem vorigen gestellt, aber kleiner, Kopf und Halsschild weniger stark und weitläufiger punktirt, das letztere breiter als lang, Flügeldecken weniger tief punktirt gestreift, Zwischenräume mit einer feinen Punktreihe. -

\section{Hypophloeus Hellwig.}

1. H. cimeterius Hbst. = castaneus F. - Unter Rinden bei Fr. - Schlangenbad und Königstein gef. - Auch von Hg. bei Fr. gesammelt. - Dlb. (Sch.) - Mz. (S.) -

2. H. Fraxini Kug. = ferrugineus Creutz. - Zweimal unter Kiefernrinde im Fr. Wald $=4$. und $<12$. am Forsthaus gef. -

3. H. bicolor Oliv. - Aus dürrem Fr. Waldholz s. h. entwickelt von Mai bis Juli, im September und October unter Apfelrinde gef., auch von $\mathrm{Hg}$. und B. gesammelt. - 
4. H. fasciatus F. - Zweimal bei Fr. $<5$. gef. an Eichenholz, in dem er Löcher gräbt. -

5. H. linearis F. - Einmal h. aus dürrem Fr. Waldholz $<6$. entwickelt, unter Kiefernrinde $>8$. gef. -

\section{G. TENEBRIONINI.}

\section{Tenebrio Linné.}

1. T. opacus Duft. - Bei Altenhain bei Soden im Taunus $<6$. einmal von C. H. in einer alten Eiche gef. -

2. T. obscurus Harrer. F. - In Fr. von dem verstorbenen Katheder in Pferdeställen im October oft gef. [eine Anzahl Exemplare in Sammlung v. Heyden], auch von Hg. gesammelt. - An Häusern in Fr. s. (B.) - Wlb. (Sch.) Die Larve in einem Wandkorb, wo sie das Papier, womit er ausgeklebt ist, abschabte. In Gesellschaft war Prionychus ater, doch erklärte Rosenhauer die Larve als zu T. obscurus gehörig.

3. T. molitor L. - Im ganzen Gebiet s. h. in Mehlvorräthen, unter dem Namen „Mehlwurm“ ist die Larve allbekannt. - Käfer $>7$. - Fr. - Wlb. Dlb. (Sch.) - Wsb. im Juni (K.). - Mz. (S.) -

4. T. picipes Hbst. = transversalis Duft. = noctivagus Muls. - Oefter in Baumpilzen bei Fr. gef. $>5$. und $<9$. - Der Käfer ist entwickelt $>8$, die Larve in faulem Holz. - Auch von St. aus Fr. Waldholz erzogen und von Hg. gesammelt. -

\section{H. HELOPINI.}

\section{Stenomax Allard.}

1. S. incurvus Küst. - Bei Fr. z. s. in hohlen Bäumen von uns und Hg. gef. - Friedberg (Fuhr) in Mehrzahl an alten Apfelstämmen. - B. fand ihn in alten Kirschbäumen in der Nähe des Königsbrunnens am 27. Mai 1874. - Mz. (S.) -

[Den naheverwandten S. lanipes L., noch nicht aus dem Gebiet bekannt, besitze ich aus dem Schwarzwald. Die Spitzen an den Flügeldeckenden sind sehr wenig entwickelt.] 


\section{Nalassus Mulsant.}

1. N. striatus Foucr. = caraboides Panz. -- Im Fr. Wald n. s. am Fusse von Bäumen < 3. unter Moos. - Auch von Hg. gef. - Wlb. (Sch.) - Mz. (S.) - Die Larve $>6$. bei Fr. unter Moos (C. H.). -

2. N. quisquilius F. - Auf dem Niederwald bei Rüdesheim von C. H. unter Eichenrinde, bei Fr. von Hg. öfter gef. im Wald; B. h. an alten Bäumen gef. - Wlb. (Sch.) - Die Larve bei Schlangenbad unter Moos $>7$., der Käfer entwickelte sich $<8$. -

\section{CIS TELIDAE.}

\section{Allecula Mulsant.}

1. A. morio F. - Im Juli in hohlen Eichei und Buchen n. s. - Fr. auch von B. gesammelt. - Mz. (S.) -

2. A. Rhenana Bach. - Seither nur 1 Ex. vom Niederwald bei Rüdesheim bekannt, welches H. v. Hagens fing. - L. H. erzog $>6$. ein Pärchen aus Fr. Waldholz und hat die Art ausführlicher beschrieben in Deutsche Entomol. Zeitschr. (früher Berliner) 1875, p. 389. -

\section{Eryx Stephens (= Prionychus Solier).}

1. E. ater F. - Fr. in hohlen Linden gesellig $=6$. gef. Auch von Hg. gesammelt, einmal von B. - Wlb. 2 Stück. -

\section{Hymenalia Mulsant.}

1. H. rufipes $\mathbf{F}$. = fusca Illig. - Im Fr. Wald am Forsthaus auf Kiefern =6. von C. H. gef. - Auch von Hg. öfter gesammelt. - Mo. im Kiefernwald (K.). - 


\section{Cistela Fabricius.}

1. (Isomira Muls.) murina L. (Stammform mit dunklem Halsschild und helleren Flügeldecken). - Fr. auf Spargeln $>6$. - Auf Gebüsch bei Fischbach im Taunus $=5$. - Bei $\mathbf{M}_{0}:=6$. öfter. Bei Fr. auch ron Hg. gef., eimmal ron B. - Wlb. h. (Sch.) -

Var. maura F. (Ganz dunkle Oberseite.) - Fr. viermal gef. -

2. C. ceramboides L. - Der Käfer im Mai bei Fr. und Schwanheim an alten Eichen; die mehlmurmartige Larve $>11$. im Eichenwurmmehl. - Von uns, Hg. und M. S. wurde der Käfer öfter gef. Wlb. einmal (Sch.). - Mrz. (S.) -

3. (Gonodera Muls.) luperus Hbst. = fulvipes F. (Stammart mit schwarz metallischen Flügeldecken.) - Im Fr. Wald im Unterwald und Obersaustiege im Mai; bei Isenburg auch ron Hg. gef.; zweimal bei Fr. von B. gesammelt. -

Var. ferruginea F. (Flügeldecken rothbraun.) - Fr. Wald im Unterwald einzeln, auch ron Hg. gesammelt. -

\section{Mycetochares Latreille.}

1. (Ernocharis Thoms.) barbata Latr. (Das Männchen = linearis Panz., das Weibchen = brevis Panz.) - In alten Bäumen h.; aus Fr. Waldholz: Eichen, Buchen und Hainbuchen von April bis Juli oft erzogen. - Soden. - Schmitterhof bei Giessen. - Dlb. Wlb. (Sch.) - Wsb. in Buchenstrunk an der Rentmauer 30. Mai bis 5. Juni (K.). - Mrz. (S.) -

2. (E.) bipustulata Illig. (Mann) = scapularis Gyll. (Weib). Von Hg. zwei Männchen und Weibchen bei Fr. gef. - Dlb. einmal (Sch.). - G. (L.) -

3. M. flavipes $\mathbf{F}$. = humeralis $\mathbf{F}$. - Von Hg. zweimal bei Fr. gef. - Dlb. einmal (Sch.). -

4. M. axillaris Payk. - Aus altem Buchenholz aus dem Fr. Wald $>$ 5. einmal in Anzahl ron C. H. erzogen. -

Var. morio Redtb. = linearis Redtb. = maurina Muls. Von Hg. bei Fr. einmal gef. -

\section{Cteniopus Solier.}

1. C. flavus Scop. = sulphureus L. - Fr. s., anch bei $\mathrm{M}_{0}$. auf Umbellen $<7$. von S., uns und Sch. gesammelt. - Auch ron $\mathrm{Hg}$. bei Fr. gef., zweimal von B. - 
Var. bicolor F. (des Münnchens mit dunklem Kopf und Halsschild). - Von K. mit der Stammart bei Mo. h. gesammelt. -

\section{Omophlus Solier.}

1. O. Amerinae Curtis $=$ pubescens Muls. $=$ lividipes Muls. $=$ pinicola Redtb. - Bei Mo. auf Euphorbia > 5. von S. und C. H. gesammelt, anch von K. gef. von 2.-19. Juni bei Gonsenheim im Gras. Mz. (S.) - Wlb. 4 Ex. (Sch.) -

\section{LAGRIIDAE.}

\section{Lagria Fabricius.}

1. L. hirta L. - Auf Hecken im ganzen Gebiet s. h. - Fr. Soden $>6$. Larve und Puppe, der Käfer $<7$. von C. H. beobachtet. - Von B. h. auf blühenden Labiaten, z. B. Mentha gef. - Wlb. s. h. (Sch.) - Wsb. auf Hecken 24. August (K.). - Mz. (S.) -

\section{MELANDRYIDAE.}

\section{A. TETRATOMINI. \\ Tetratoma Fabricius.}

1. T. fungorum F. - In Baumschwämmen h. an Eichen, Buchen, Apfel- und Nussbäumen von September bis in den Winter. - Fr. Soden. - Beim Fr. Forsthaus. - Bei Dlb. und Wlb. (Sch.) - 
- B. ORCHESIINI.

Eustrophus Latreille.

1. E. dermestoides F. - Bei Fr. von Hg. ges. - Mz. (S.) -

\section{Hallomenus Panzer.}

1. H. binotatus Quens. = humeralis Panz. - Einmal von C. H. bei Fr. gef. - Mz. (S.) -

\section{Orchesia Latreille.}

1. O. micans Panz. - Fr. aus dürrem Waldholz im Juni einzeln, oft aus überwinterten Baumschwämmen im Mai und Juni erzogen. Soden von Dornhecken geklopft. - Von B. im Isenburger. Wald s. im Herbst aus faulendem Laub gesiebt. - Mz. (S.) --

2. 0. fasciata Payk. - Von St. in Mehrzahl im Juli 1876 in der Holzkammer erzogen, worin nur Buclienholz aus dem Fr. Wald. -

\section{DIRCAEINI.}

\section{Abdera stephens.}

1. A. quadrifasciata Curtis. - Von Hg. bei Hallgarten im Rheingau auf blühenden Kastanien gesammelt. -

2. A. triguttata Gyll. Var. scutellaris Muls. - C. H. erzog $=5$. ein Stück, das sich aus schwammiger Kiefernrinde entwickelte, welche $=3$. im Fr. Wald eingesammelt wurde. -

3. (Carida Muls.) affinis Payk. - Aus Fr. dürrem Waldholz und aus alten überwinterten harten Eichenschwämmen im Mai oft erzogen. - Hallgarten im Rheingau (H.). -

4. (C.) flexuosa Payk. - Aus dürrem Fr. Waldholz von $>4$. bis $=5$. öfter entwickelt. -

\section{Anisoxya Mulsant.}

1. A. fuscula Illig. = tenuis Roshr. - Bei Hallgarten im Rheingau auf blühenden Kastanien von Hg. gef. - 


\section{Dircaen Fabricius.}

1. (Phloeotrya Steph.) Vaudoueri Muls. - Bei Fr. zweimal von C. H., bei Cronthal $=7$. in Holz der Castanea vesca von C. H. und M. S. je einmal gef. -

\section{Xylita Paykul.}

1. X. laevigata Hellen $=$ discolor F. - Einmal von C. H. im Fr. Gebiet gef. -

\section{HYPULINI.}

\section{Hypulus Paykul.}

1. H. quercinus Quens. - L. H. fand 2 Ex. in einer hohlen Eiche im Fr. Wald an den Schiessständen am Schwengelbrumnen. -

\section{E. MELANDRYINI.}

\section{Melandro $\mathbf{y}$ Fabricius.}

1. M. caraboides F. - Aus dürrem Fr. Waldholz h. erzogen im Mai. - In der Hohen Mark im Taunus in Buchstöcken $>5$., bei Cronthal in alten Kastanien $=5$. - Fr. (Hg.) - Von B. in alten Buchenstämmen hinter dem Fr. Forsthaus am 19. Mai 1861 gesammelt. - Dlb. l. (Sch.) - G. (L.) - Mz. (S.) -

2. M. flavicornis Duft. = barbata Sturm. - Bei Dlb. einmal von Sch. gef. -

\section{F. CONOPALPINI. \\ Conopalpus Gyllenhal.}

1. C. testaceus Ol. (Stammart mit gelben Flügeldecken.) - Aus dürrem Fr. Buchenholz im Mai von uns und St. manchmal n. s. erzogen. -

Var. flavicollis Gyll. = Vigorsii Steph. (Nit schwarzen Flügeldecken.) — Seltener mit der Stammart voin Fr. elzogen. - 


\section{MORDELLIDAE.}

\section{A. SCRAPTIINI.}

\section{Scraptia Latreille.}

1. S. fuscula Müll. - Aus dürrem Fr. Waldholz von $>5$. bis $<7$. n. s. erzogen, an geschlagenem Eichenholz beim Fr. Forsthaus bei Sonnenuntergang $<8$. gef.; voll alten Hainbuchen bei der Mainkur $=7$. geklopft. - G. (L.) - Mz. (S.) -

2. S. dubia Oliv. = fusca Latr. - Hg. fand diese sonst im Süden lebende Art einmal bei Fr. -

\section{B. MORDELLINI.}

(Die Sammlung v. Heyden wurde von dem Monographen Fmery in Neapel revidirt.)

\section{Pentaria Mulsant.}

1. P. badia Roshr. = sericaria Muls. - C. H. fand 1 stück dieses seltenen Thierchens bei Rüdesheim $=8$. -

\section{Anaspis Geoffroy.}

1. A. Geoffroyi Müll. - Bei Fr. 2 Ex. von C. H., einzeln auch von St. und Hg. gesammelt. -

Var. c. Emery. (Flügeldecken gelb, an der Basis schwarz, ebensowie eine Nahtbinde und eine seitliche Randmakel; die Stammart hat schwarze Decken mit gelbem Humeralfleck.) - Fr. einmal. -

2. A. maculata Fourc. = obscura Marsh. - Bei Fr. von C. H., Hg., M. S. und B. gesammelt. Ungefleckte Stücke von subtestacea durch schwarze Brust, von fläva durch nicht perlschnurförmige Fühler zu unterscheiden. -

3. A. frontalis L. - Aus altem Holz $>$ 4. entwickelt. - Ems. - Fr. anch von Hg. und B. gef. - Von Wiesengebüsch bei Falkenstein geklopft $=5 .-$ Nauheim $>$ 5. (C. H.) - Wlb. (Sch.) - 
Var. Iateralis F. (Vorderecken des Halsschildes roth.) - Fr. (Hg.) - Wlb. (Sch.) -

4. A. confusa Emery = thoracica Muls. = Iateralis Thoms. - Aus diirrem Fr. Waldholz im April und Mai erzogen. - Soden an Dornzäunen im Juli. - Bei Fr. anch von Hg. und B. gesammelt. Von A. ruficollis durch gan\% rothen Kopf unterschieden. Diese Art ist in den Sammlungen meist als thoracica L. bestimmt, diese gehört aber in die Gruppe mit perlschnurförmigen Fühlern (Untergattung Nassipa) und ist eine schwarzflügelige Form der flava. -

5. A. ruficollis F. - Fr. zweimal aus dürrem Waldholz $=4$. erzogen, auch von Hg. gef. - Wlb. (Sch.) - (Halsschild ganz roth, Kopf schwarz, oder wenigstens auf dem Scheitel.) -

6. A. pulicaria Costa = forcipata Muls. - Fr. einmal gef.; ich hielt sie früher für labiata Costa, die in S. V. zu streichen ist, da sie sich nur in Süd-Italien findet. Mit frontalis verwandt, aber nur der Mund ist roth, 3 und 4 Bauchsegment beim Männchen mit stielförmigen Anllängen, bei frontalis ist dies nur auf Segment 3 der Fall. -

7. A. subtestacea Steph. (Durch die ganz gelbe Färbung der Ober- und Unterseite von allen Arten zu unterscheiden.) - Soden an Dornzäunen im Juni. - Aus dürrem Fr. Waldholz = 5. erzogen. Wlb. (Sch.) -

8. (Nassipa Emery) flava L. - - Aus dürrem Fr. Waldholz = 4 . erzogen, auch von $\mathrm{Hg}$. gesammelt und von B. 17. Mai 1860 gef. Cronthal im Taunus $=5$. auf Blüthen. - Soden $=6$. an Dornzäunen. - Wlb. (Sch.) - Die einzige gelbflügelige Art mit perlschnurförmigen Fühlern,

9. (N.) melanostoma Costa $=$ monilicornis Muls. - Mit rufilabris verwandt, aber durch das letzte Fühlerglied, welches viel länger als das vorhergehende ist und durch den Mangel der Anhänge beim Mäunchen verschieden. - Aus Buchenholz aus dem 'Fr. Wald von $=4$. bis $<5$. erzogen. - Schmitterhof bei Giessen. -

10. (N.) rufilabris Gyll. - Gebirgsthier (z. B. h. im Schwarzwald). - Fr. einmal (L. H.). - Die meisten Stücke der Sammlungen im Gebiet werden wohl zur vorigen Art gehören. -

11. (Silaria Muls.) varians Muls. - (Die Stammart mit schwarzem Halsschild.) - In Soden im Taunus an Dornzäunen $>6$. von C. H. gef. - Mz. (S.) - 
Var. b. Emery = collaris Muls. (Nit rothem Halsschild.) Bei Königstein im Taunus $=5$. auf Crataegus-Blüthen, auch mit der Stammart in Begattung von C. H. gef.; auch noch < 8. - Ems. -

12. (S.) quadrimaculata Gyll. - Variirt mit schwarzem und rothem Halsschild. - Ems. - Rüdeshein auf Blüthen = 8. von C. H. gesammelt. - Bei Fr. ron L. H. und Hg. gef. - Von B. am Mühllberg bei Fr. und bei St. Goarshausen am Rhein gesammelt. — Wlb. (Sch.) - Mz. (S.) -

\section{Tomoxia Costa.}

1. T. biguttata Gyll. - Fr. aus dürrem Waldholz oft erzogen. Begattete sich $>5$. - Auf alten Weiden bei Oberrad s., anf Eichen bei Schwanheim 1875, bei Hattenheim im Rheingau 1873 von B. gesammelt. - Wlb. zweimal (Sch.). -

\section{Mordella Linné.}

1. M. maculosa Naezen $=$ atomaria F. - In Sammlung v. Heyden befindet sich ein ron MI. S. bei Fr. gesammeltes Stück; es sass an einer Plankenwand. - Auch ron Hg. s. 's. gef. -

2. M. fasciata F. - Fr. s. - In den Torfstichen bei Enkheim $>$ 7. - Bei Isenburg von Hg. gesammelt. - W1h. (Scli.) - M[z. (S.) -

Variirt sehr. - Bei einer Varietät Var. c. Emery = sericeoguttata Muls. sind fast die ganzen Flügeldecken oben grau seidenartig behaart. - Stücke mit ganz geschwuidenen Binden (die Spitze der Decken bleibt immer unbehaart) bilden die Var. villosa Muls. nec Sclurk. - Ich sal noch kein Stïck aus dem Gebiet. - Die ächte villosa Schrk. scheint nur in Oesterreich vorzukommen und unterscheidet sich durch andere Form des letzten Gliedes der Maxillarpalpen, welches bei villosa seine grösste Breite an der Basis hat, suboral und ein- und einhalbmal so lang wie breit ist, während bei aculeata die grösste Breite in der Nitte liegt und es fast dreieckig ist. - [Villosa in S. V. gehört zu MLordellistena micans Germ.] -

3. M. aculeata L. (Schwarz behaart.) - Bieberer Höhe $=7$. - Hohe Mark im Taunus > 7. - Fr. ans übermintertem alten Buchenholz $=6$. - Wlb. s. h. (Sch.) - ML. (S.) -

Var. brevicauda Costa $=$ brachyura Muls. (Kleiner, braun behaart, Pygidium kürzer.) - Soden im Taunus $=8$. auf Umbellen in Begattung. - Ems. - Königstein = 9. - 


\section{Mordellistena Costa.}

1. (Mordellochroa Emery) abdominalis F. - C. H. fand zwei Weibchen bei Fr. - Die Art auch von Hg. gesammelt. - Wlb. (Sch.) - M\%. (S.) -

2. (Mordellistena) brunnea F. - Fr. Wald am Forsthaus $<8$. - Griesheim am Main im August. - Soden auf Umbellen in Begattung $=$ 8. - Fr. (Hg. B.) - Wlb. (Sch.) - Kopf und Halsschild sind bei dieser Art immer ganz gelb. -

3. M. humeralis F. - S. S. - Wlb. einmal (Sch.). - Mz. (S.) -

4. M. lateralis Oliv. - Aus dürrem Fr. Waldholz im Juni und Juli öfter erzogen. - Hofheim im Taunus in Rubus-Blüthen $>6$. Soden > 8. - Fr. einzeln (B.). - Wlb. (Sch.) - Kleiner wie die vorhergehende Art, der gelbe Fleck weiter nach hinten verlängert, Seitenund Nahtrand immer schwarz. -

5. M. parvula Gyll. = pusilla Redtb. - Bei dieser Art ist eine der Querleisten auf den Hinterschienen länger als die anderen. Die Stammart ist etwas kleiner, die Hinterecken des Halsschildes stumpf zugerundet; das Pygidium conisch, überragt das Hypopygidium um die Hälfte der Länge. - Bieberer Höhe $=6$. - Soden < 7 . Wlb. (Sch.) -

Var. inaequalis Muls. (Grösser, Hinterecken kaum gerundet, Pygidium dünn, zweimal so lang als das Hypopygidium. - Die Larve überwintert in den alten Stengeln von Artemisia und Valeriana dioica im Mark; der Käfer entwickelt sich in der zweiten Hälfte Mai. - F1. hinter der Grüneburg. - Hengster bei Offenbach $>6$. -

Var. picipes Costa. - Vorderbeine und Fühlerbasis rothgelb. Fr. mit inaequ. in Artemisia. - Noch im Juni gef. -

6. M. brevicauda Bohem. = subtruncata Muls. - Alle Querleisten gleichlang. - Das Thier ist ,elongata“; das Pygidium abgestutzt, die Hinterecken des Halsschildes abgerundet; die Oberseite mit schwarzer Behaarung. - Bei Soden im Taunus auf Blüthen $<6$. s. - Fr. einmal. - 12. Mai 1860 von B. gesammelt. --

7. M. micans Germ. = grisea Muls. - Pygidium zugespitzt, Hinterecken des Halsschildes rechtwinkelig oder spitzig; Vorderschienen beim Münnchen verbreitert. Leicht kenntlich durch die seidenartige, branne oder graue Behaarung der Oberseite. - Im Fr. Wald und bei Mo. auf Euphorbia cyparissias im August. - Bei Fr. auch von Hg. und B. gef. - Mr. (S.) - 
8. M. pumila Gyll. = stricta Costa. - Schmäler als die briden vorigen Arten: ,angustata“, mit brevicauda nahe verwandt, doch sind die Hinterecken des Halsschildes spitzer und das erste Glied der Hintertarsen ist dünner. - Fr. 11. h., anch von Hg. gef. - Wlb. (Sch.) -

9. M. stenidea Muls. - Durch die sehr schmale Gestalt un die geringe Grösse $(3 \mathrm{~mm})$ leicht kenntlich. Die Hinterecken des Halsschildes sind stumpf. - Wlb. (Sch.) - Fr. Wald am Forsthaus und bei Soden $<7$. - Bieberer Höhe bei Offenbach. - [Hierher die Stücke von pumila in S. V. aus Oberstein.] -

\section{RHIPIPHORIDAE.}

\section{Metoecus Gerstïcker.}

1. M. paradoxus L. - In Sammlnng v. Heyden befindet sicj ein ron C. Schneider am 5. October 1862 an der Iseuburger Chaussee gegenüber dem Brückchen an einer Buche gefundents Exemplar. Es ist ein Weibchen (Flügeldecken gelb mit schwar\%em Spitzenlängswisch). Die Art lebt parasitisch in Wespemnestern in der Frde. - Friedberg (Remner). - Von Herrn Dr. Fr. Sandberger vor langen Jahren bei Wlb. gesammelt. - 


\section{M E L O I D A E.}

\section{A. MELOINI.}

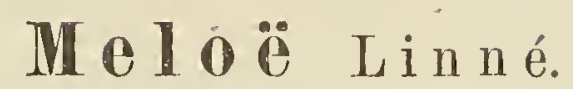

(Jede Art besitzt eine kurzflügelige Form A. und eine langflügelige Form B.)

1. M. proscarabaeus L. - Auf Gras und Kleeäckern h.; die Larven in Nestern von bienenartigen Thieren in der Erde. - Der Käfer in April und Mai. - Fr. - Johannisberg bei Nauheim. - Hofheim in Taunus. - Bieberer Höhe bei Offenbach. - Dlb. Wlb. (Sch.) Fr. (Hg.) - G. (L.) - Wsb. (K.) - Mz. (S.) -

Form B. - Bei Hofheim; bei Fr. von Hg. gef. - Wlb. 2 Ex. (Scilı.) - G. (L.) - Wsb. (K.) -

Eine Form mit sehr kleinem Halsschild (angusticollis milhi i. 1.) fing C. H. bei Eschborn und Sch. bei Wlb. -

Das grösste Stück der Sammlung v. Heyden ist 23, das kleinste nur $5 \mathrm{~mm}$ lang. -

2. M. violaceus Marsh. - Fr. $=5$. auf dem Johannisberg bei Nauheim. - Am Feldberg < 6. ein Weibchen mit sehr glänzendem Kopf und Halsschild. Länge $10-33 \mathrm{~mm}$. - Fr. (Hg.) - Von B. schon am 15. April 1861 h. gef. - Wsb. (K.) -

Form B. - Fr. - = 4. s. - Auch von Hg. ges. - Wlb. (Sch.) -

3. M. autumnalis Oliv. - C. H. fand bei Fr. 2 Stück der Form B. von $5^{\mathrm{mm}}$ Länge im October. Sonst $12 \mathrm{~mm}$. - Auch von $\mathrm{Hg}$. s. s. gesammelt. - Wlb. 3 Ex. (Sch.) - Wsb. (K.) - Mz. (S.) -

4. M. Hungarus Schrk. = limbatus F. - Ein Stück in Sammlung v. Heyden (Form B.) fand der verstorbene Gremmers bei Isenburg. -

5. M. decorus Brandt = pygmaeus Redtb. - Von Lieutenant Giebeler bei Wetzlar gef., von I. H. bestimmt. -

6. M. cicatricosus Leach. - C. H. fand 2 Stück (Form A.) bei Fr. - (Form B. kennt L. H. von dieser Art nicht.) -

7. M. coriarius Brandt Er. - Zweimal von C. H. bei Fr. gef. (Form B.) - Variirt von 30-21 mm. - Form A. sammelte Sch. bei Wlb. - 
8. M. variegatus Donov. - Sulzbach bei Soden auf Aeckern $>$ 5. von C. H. gef. - Auf dem Johannisberg bei Nauheim von L. H. $<$ 5. - Fr. (Anton Schmid.) - Variirt von 23-11 mm. - Bei der Grüneburg bei Fr. einzeln von Hg. gesammelt. - Berger Höhe einmal (B.). Wsb. (Gymnasiallehrer Dr. Schenck.) - Wsh. (K.) -

Form B. - Fr. einmal von L. H. gef. - Fr. (Hg.) - Wsb. (K.) -

9. M. rugosus Marsh. - Im October z. s. - Fr. - Budenheim bei Mz. - Friedberg (Renner). - Variirt von 14-5 mm. - Wlb. 1 Ex. (Sch.) - Mz. (S.) -

Form B. - Fr. einmal von C. H. gef. -

10. M. scabriusculus Brandt Er. $->4$. und $<5$. - Friedberg (Renner). - Johannisberg bei Nauheim h. - Fr. - Hofheim im Taunus. - Bieberer Höhe bei Offenbach. - Variirt von 19-6 $\mathrm{mm}$. - Auch von Hg. bei Fr. gef. und einzeln von B. - Wlb. (Sch.) G. (L.) -

Form B. - Fr. Bieberer Höhe. -- Wlb. (Sch.) -

11. M. brevicollis Panz. - Fr. im Mai s. - Variirt von 20-5mm. - Auch ron Hg. gesammelt. - Wlb. (Sch.) - G. (L.) -

Form B. - Wlb. (Sch.) -

\section{B. LYTTINI.}

\section{Sitaris Latreille.}

1. S. muralis Forst. = humeralis F. - Ueber dieses seltene Thier veröffentlichte L. H. in der Deutschen Entomol. Zeitschr. 1875, p. 390, folgendes:

„Das Thier wurde von Schuljungen beim Abbruch eines alten Hauses auf der Altgasse zu Frankfurt im IIerbst 1874 in grosser Anzahl an den Mauern sitzend gefunden. Dr. Oscar Böttger salı davon 75 Stück, von denen ich 30 besass. Alle Exemplare, die ich vergleichen konnte, waren Männchen. Die Art lebt bekanntlich in den Nestern einer Mauerbiene (nach Mulsant in Lyon bei Anthophora hirsuta und acervorum); die Weibchen, an den dicken Hinterleibern und den viel kürzeren Fühlern kenntlich, leben verborgener; meine in Scriba's Verzeichniss ,die Käfer im Grossherzngthum Hessen etc." erwähnten Exemplare aus Mamnheim wurden 1829 von Vogt aus einem Bienemest hinter dem Fensterladen genommen; es sind nur Weibchen. - Dr. med. Moritz Schmidt fand 
vol einigen Jahren ein lebendes Männchen auf dem Götheplatz in Frankfurt und Professor Dr. Kirschbaum eil Stïck auf der Strasse in Wiesbaden im Auguist." -

\section{Cerocoma Geoffroy.}

1. C. Schäfferi L. - Auf Camillen frülıcr an der Höchster Chaussee von C. H. gef. - Bockenheim. - Isenburger Wald > 5. Im Hengster bei Offenbach $>6$. von L. H. und B. n. s. gef. G. (L.) - Mz. (S.) -

\section{Cantharis $\mathrm{L} .=$ Lytta $\mathrm{F}$.}

1. C. vesicatoria L. - Unter dem Namen "spanische Fliege“ allbekannt und officinell. - Von L. H. bei Fr. cinzeln auf Eschen an der Oede gef., auch früher von C. H. gesammelt. - Hg. beobachtete, dass die Art in Bad Soden am Brunnen No. 6 zwei grosse Eschen total abgefressen hatte; schon von weitem bemerkte man den eigenthümlichen Gernch; alle Wege lagen voll zertretener Thiere. - Homburg und Bonames (B.). - Dlb. Wlb. s. h. (Sch.) - Wsb. auf Eschen und Liguster in Gärten zeitweise h. im Mai und Juni (K.). — Mz. (S.) -

\section{PYROCHROIDAE.}

\section{Pyiochroa Geoftroy.}

1. P. coccinea L. = rubra De Geer = purpurata Schrk. Fr. s. - Im Gebiet h. - Cronthal in Kiefernstöcken $=5$. von uns, im Taunus auch von Hg. gef. - Altkönig und Oberursel (St.). Friedberg (Renner). - Die Larven h. auch unter Eichenrinde sind sehr gefrässig, fressen sich in Gefangenschaft leicht einander auf. In der Ebene im Rebstöcker Wald s. s., im Taunus h. von B. gesammelt, z. B. an der Gundelhardt bei Epstein. - Bei Wlb. und Dlb. Käfer und Larve h. unter Rinde, der Käfer anch auf Blüthen (Sch.). - Wsb, hinter dem Turnplatz in Buchenstrünken (K.). — Mz. (S.) - 
2. P. serraticornis Scop. = purpurata Müll. = satrapa Schrk. = rubens Schall. - Von Hg. und St. im Taunus am Altkönig gef. - Walluf im Rheingau in Pappelstäminen (K.). - Im Oberolmer Wald bei Mz. (S.) -

3. P. pectinicornis L. - Am Feldberg auf Himbeeren von $=5$. bis $<6$. von C. H., am Altkönig auf jungen Fichten von Hg., L. H. und St. gef. - Im Oberolmer Wald bei Mz. (S.) -

\section{ANTHICIDAE.}

\section{A. EUGLENINI.}

Euglenes Westw. (= Xylophilus Latr.).

1. (Anidorus Muls.) nigrinus Germ. - Bei Fr. von C. H. und Hg. s. gef.; von B. 3 Stïck. -

2. E. oculatus Gyll. = pygmaeus Muls. - Von C. H. $<8$. am Fr. Forsthaus bei Sonnenuntergang beide Geschlechter oft an Eichenholz gef. - Fr. (Hg.) -

3. (Aderus Westw.) boleti Marsh. = populneus Panz. Im Fr. botanischen Garten im März unter Rinden und im Dezember in faulem Holz im Fr. Wald von C. H. gef.; eimmal von B. in Fr. an einem Hause der Praunheimer Strasse. - Fr. (Hg.) -

\section{B. ANTHICINI.}

\section{Notoxus Geoffroy.}

1. N. monoceros L. - Bei Fr. n. s. an faulenden Pfanzen im August und September, bei Mo. $=6$. gef. - Von B. und Hg. s. h. bei Fr. gesammelt. - Bei Wlb. h. im Gras abgestreift (Sch.). G. (L.) - Wsb. an der Wellritz 28. Juni, Rüdesheim im Mai, Mo. Damm 20. August (K.). - Mz. (S.) - 
2. N. trifasciatus Rossi = cornutus F. - C. H. fing bei Rüdesheim $=8.2$ Stück auf Centaurea calcitrapa. -

\section{Anthicus Paykul.}

1. (Cyclodinus Muls.) humilis Germ. - Im Gebiet nur auf Salzboden, besonder's bei Nauheim h. von $=4$. bis $>8$, von uns und $B$. z. B. 8. April 1860 gesammelt. -

2. A. floralis F. - Bei Soden an Dunghaufen fliegend oft $=8$. von C. H. gef. - G. (L.) - Sch. kennt nur diese Art (nicht quisq.) von Wlb. - Fr. (Hg.) - Wsb. (K.) -

3. A. quisquilius Thoms. - Bei dieser auch stärker punktirten Art fehlen die beiden kleinen T'ubercehn vorn auf der Mitte des Halsschildes, welche den ächten floralis auszeichnen. - Fast häufiger als floralis. - Am Mainufer an trockenen Kiesstellen $=6$. - Cronthal im Taunus noch $>10$. - Wsb. (K.) -

4. A. bifasciatus Rossi. - Von v. Twardowski bei Fr. öfter gesammelt. - Von B. nur ein Stück an einem Hause der Praunheimer Strasse gef. - G. (L.) - Fr. Hg. einmal. -

5. A. antherinus L. - Soden im Wald an Eichen und im Flug gef. von $>5$. bis in August. - Nauheim auf Salzboden = 8.; zu Anfang desselben Monats bei Rüdesheim. - Bei Fr. von C. H. und Hg. gef. - Nach B. bei Fr. ungemein h. - Wlb. s. h. (Sch.) G. (L.) - Mz. (S.) -

[A. Iuteicornis Schmidt wird in S. V. angeführt als in Giessen von Laubenheimer gef., diese Art ist in der Laubenh. Sammlung im Giessener Museum nicht vorhanden.] —

6. A. flavipes Panz. - In trockenem Kies an der Lahn bei Ems im Juli n. s. ebenso $<9$. bei Fr. am Mainufer. - In Sandgruben $>$ 5. zwischen Enkheim und Bischoffsheim (B.). - G. (L.) - Fr. (Hg.) - Mo. 4. Mai (K.). -

[A. nectarinus Panz. - Bei Mz. nach Bach gef.; Schmitt, der so lange dort sammelt, kennt die Art, die in Ost-Europa vorkommt, nicht von Mz.] - 


\section{OEDEMERIDAE.}

\section{Dryops Fabricius.}

1. D. femorata F. - Ein Weib dieses seltenen Thieres fing Lientenant Giebeler auf blühendem Hollunder im Wispertlal. -

\section{Nacerdes Schmidt (= Anoncodes Schmidt).}

1. N. rufiventris Scop. - C. H. fing vor langen Jalren zwei Männchen an einem nassen Graben bei Sachsenhansen zwischen der Oppenheimer Landstrasse und dem Frankfurt-Bebraer Bahnhof. - Mz. (S.) -

2. N. ustulata F. - Mit der vorigen Art, gleichfalls zwei Männchen bei Sachsenhausen. - Mz. (Bach). - Friedberg (Fuhr). -

Ischnomera Steph. (= Asclera Schmidt).

1. I. sanguinicollis F. - Von dem verstorbenen Riese im Fr. Wald $>4$. von Kiefern geklopft. - Von St. ans Fr. Waldholz $<4$. erzogen. - Mz. (S., Bach). - Wlb. einmal (Sch.). - Neroberg bei Wsb. 14. Juni (K.). -

2. I. caerulea L. - Aus Fr. dürrem Waldholz $>4$. oft erzogen. - Im Fr. Wald an Buchenstämmen < 6. - Wlb. h. (Sch.) - G. (L.) - Fr. (Hg.) - Fischanstalt bei Wsb. 21. Juni (K.). Auf Weissdornblüthen zwischen Platter Clanussee und Adamsthal 25. Mai (K.). -

\section{Oedemera Olivier.}

1. O. Podagrariae L. - Auf Waldblumen h. - Fr. - Soden < 8. - Auch von B. und Hg. gesammelt. - Dlb. Wlb. h. (Scli.) G. (L.) - Mo. Wsb. (K.) - Mz. (S.) -

2. 0. femorata Scop. $=$ flavescens L. - Bei Wlb. ein Weibchen von Sch. gef. - Mo. beide Geschlechter (K.). - Mz. (S.) -

3. O. subulata Oliv. = marginata F. - Bei Falkenstein im Taunus im Mai und Juni von C. H. gef. - Fr. (Hg.) - Mo. (K.) - 
4. 0. nobilis Scop. = caerulea L. - Fr. - Soden von Mai bis Juli auf Waldblumen. - Ems von C. H. gef. - Von Hg. und B. bei Fr. s. gesammelt. - Wlb. (Sch.) - Waldwiese hinter Adamsthal bei Wsb. 30. Mai (K.). --

5. O. flavipes F. - Fr. Wald im Hinkelsteinforst auf Blumen $>$ 6., bei Ems von C. H. gef. - Wlb. (Sch.) - Fr. (Hg.) - Raunheim am Main 10. Juli (K.). -

6. 0. virescens L. - Fr. Wald im Wartforst $=6$. - Bei Falkenstein im Taunus $=5$. - Nach B. und Hg. bei Fr. s. h.; ebenso bei Wlb. (Sch.) - G. (I.) -

7. 0. lurida Marsh. - Fr. Wald im Unterwald < 7. - Nach B. und Hg. h. bei Fr., auch bei Wlb. (Sch.) - Ein kupferfarbiges Exemplar fand $K$. bei Wsb. an der Kohlhecke 29. Juli. -

\section{Chrysanthia Schmidt.}

1. C. viridissima $\mathbf{L}$. $=$ thalassina Gyll. - Von $>6$. bis $<$ 8. auf Waldblumen s. - Von B. und Hg. bei Fr. n. s., aber weniger h. als die folgende Art, gesammelt. - - Von B. auch im Schwanheimer Wald gef. - Mz. (S.) -

2. C. viridis Schmidt. = viridissima F. Oliv. - Fr. auf Waldblumen. In Begattung < 9. gef. - Fr. h. nach B. und Hg. - Wlb. (Sch.) - G. (L.) - Beide Arten von K. bei Raunheim am Main 10. Juli ges. -

Var. geniculata Sturm. (Die Beine, Mund, Taster und Fühler dunkler.) - Fr. einmal von C. H. gef. -

\section{PYTHIDAE.}

\section{A. SALPINGINI.}

\section{Salpingus Illiger.}

1. S. castaneus Panz. = Piceae Germ. - Im Fr. Wald unter Kiefernrinde von Hg. und Dr. Siebert öfter gesammelt. - Auch von C. H. $<9$. einmal und auf der Bieberer Höhe bei Offenbach $>3$. auf Kiefern von L. H. gef. - [Hierher aeratus Muls. des S. V.] - Mz. (S.) - 
2. S. ruficollis Panz. = Roboris $\mathbf{F}$. = viridipennis Steph. $=$ ruficeps Bose. - G. (L.) -

\section{Lissodema Curtis.}

1. L. cursor Gyll. = Heyanum Curtis. - In Sammlung v. Heyden befindet sich das einzige von v. 'Twardowski beim Fr. Forsthaus gefangene Exemplar. -

2. L. denticolle Gyll. = quadriguttatum Lap. - Bei Soden an Dornzämen n. s. $>$ 6. von C. H. gef. - Wlb. (Sch.) -

\section{Rhinosimus Latreille.}

1. R. ruficollis $L$. = Roboris Payk. - Aus dürrem Fr. Waldholz im April und Juni öfter erzogen; ; anch unter Birkenrinde im Januar von C. H. gef. - G. (L.) - Mr. (S.) - Wsb. Platanenrinde im Februar (C. H.). -

2. R. planirostris F. = Spinolae Costa. - Aus Fr. dürrem Waldholz im Mai erzogen. - Soden an Dornzäunen $<6$. und bei Wsb. unter Platanenrinde $>12$. von C. H. gesammelt. - G. (L.) - Wsb. an der Wellritz in Baumstrünken im April (K.). -

\section{B. MYCTERINI. \\ Mycterus Olivier.}

1. M. curculionoides F. - Bei Fr. von C. H. gef. - Bei Mo. auf einer Blöse des Kiefernwaldes auf Disteln von K. und L. H. öfter gesammelt von 21. Mai bis 20. Juni. - Mz. (S.) - 


\section{BOSTRYCHIDAE.}

(Die Sammlung $\nabla$. Heyden ist von dem Monographen E ich h of f revidirt.)

\section{A. PLATYPINI. \\ Platypus Herbst.}

1. P. cylindrus F. - Von C. H. bei Fr. gesammelt. - L. H. fand das interessante Thier einmal n. s. im Wald von Soden nach Cronthal in frisch abgeholzten Eichenstrünken; der Käfer sitzt tief im Splint. Bei Wlb. h. an Eichen (Sch.). - Auf der Platte bei Wsb. von $\mathrm{Hg}$. 'gef. - Mo. (K.) - An Eichenstrünken hinter dem Schiessplatz bei Wsb. s. h. 24. April (K.) - Mz. (S.) -

\section{B. SCOLYTINI.}

\section{Scolytus Geoffroy (= Eccoptogaster Herbst).}

1. S. destructor Oliv. Ratzb. = scolytus Gyll. - Von Hg. bei Fr. gef. - Wlb. (K.) einmal. -

2. S. Ratzeburgi Janson = scolytus F. Ratzb. = destructor Redtb. - Aus Birkenholz aus dem Fr. Wald im Juni s. h. erzogen. Früher mit dem destr. 01. verwechselt, von dem er sich dadurch unterscheidet, dass der Hinterrand des ersten Abdominalsegmentes in der Mitte stumpf (nicht scharf wie bei dest.) ist; das 3. Segment des Männchens hat einen grossen knopfförmigen (bei dest. klein) Höcker in der Mitte; das Weibchen von Ratzeb. auf dem 3. und 4. Segment einen kleinen Höcker, der bei dest. fehlt u. s. w. - Auch von Hg. bei Fr. gesammelt. -

3. S. intricatus Ratzb. - Aus dürrem Fr. Waldholz von $=\mathbf{5}$. bis $>6$. entwickelt. - Nach Beobachtungen von C. H. findet sich die Larve im März in grosser Menge unter der Rinde abgängiger junger Eichen und in den Aesten älterer Bäume. -

4. S. Pruni Ratzb. - Im Juni und August bei Fr. in Sorbus von C. H., bei Mo. $=5$. in Menge in Prunus domestica von uns, auch die Larve da $=$ 3. gef. - Fr. h. (B.) - Mo. (Hg.) - 
Var. Pyri Ratzb. - Die Larven $>$ 9., der Käfer im folgenden Jahr $=6$. unter Apfelrinde bei Fr. von C. H. gef. - Bei Wlb. in alten Apfelbäumen (Sch.). -

5. S. rugulosus Ratzb. - Bei Soden in fingerdicken Aesten von wilden Birnbäumen $<6$.; bei Oberursel in Prunus Spinosa $>6$.; in Mo. von C. H. $>$ 8. gesammelt. - Auch Zimmer fand die Art bei G. in Prunus. - Wlb. (Sch.) - Fr. (Hg.) - Mz. (S.) -

6. S. Carpini Er. - Unter Rinde von Carpinus betula (Hainbuche) einmal von C. H. bei Fr. gef. -

7. S. castaneus Ratzb. - Die Sammlung v. Heyden besitzt zwei von Zimmer bei G. in Prunus gesammelte Stücke. - Wlb. 2 Ex. (Sch.) -

\section{HYLESININI.}

\section{Hylastes Erichson.}

1. H. ater Payk. - Unter Kiefernrinde h. von $=5$. bis $<8$. im Fr. Wald. - Von B. bei Fr. am 6. April 1860 gesammelt. Wlb. (Sch.) - G. (L.) - Fr. (Hg.) - Mz. (S.) -

2. H. cunicularius Er. - Bei Fr. s. in Fichten von C. H. gesammelt. - Wlb. (Sch.) - G. (L.) -

3. H. linearis Er. = variolosus Perris. - Bei Wlb. einmal von Sch. gef. -

4. H. opacus Er. - Lebt unter Kiefernrinde $>4$. bei Fr.; $=5$. bei Cronthal; $>10$. bei Königstein und Rumpenheim am Main. - Wlb. (Sch.) - Fr. (Hg.) -

5. H. attenuatus Er. - Unter der Rinde junger Kiefern $>8$. öfter gef. - Fr. -

6. H. angustatus Hbst. - Aus jungen Kiefernpflanzen $>8$. gesammelt. - Fr., auch von B. gef. - Wlb. (Sch.) - G. (L.) Fr. (Hg.) -

7. H. palliatus Gyll. $-\mathrm{Fr}$. $=4$. von. Weissdorn geklopft; bei Nauheim und Cronthal $=5$. gesammelt. - Soden. - B. fand die Art viermal bei Fr. - Wlb. (Sch.) - G. (L.) - Fr. (Hg.) - Wsb. auf Lärchen zwischen der Fasanerie und dem Holzhackerhäuschen im Mai (K.). - 
8. H. Trifolii Müll. - Bei Mz. von S. auf Klee gesammelt und aus Kleestengeln und Wurzeln erzogen. - C. H. fing ein Stück $>5$. bei Soden. - Bei Wlb. zweimal von Sch. gef. - G. (L.) - Fr. (Hg.) -

\section{Hylurgus Latreille.}

1. H. ligniperda F. - Im Fr. Wald öfter von uns gesammelt. - Dlb. (Sch.) - Fr. (Hg.) -

\section{Blastophagus Eichhoff.}

1. B. Hederae Schmitt. - Von S. bei Mz. aus dürren Ephenästen erzogen. -

2. B. piniperda F. - Ueberall s. h. in Kiefern und den Trieben schädlich, selbst an hochstämmigen Bäumen $>6$. und $<7$. - Offenbach. - Budenheim am Rhein. - Mo. $<5$. - Der Käfer bleibt oft gelblich. - Wlb. Dlb. s. h. (Sch.) - G. (L.) - Fr. (L. H., Hg.) - Mz. (S.) - Wsb. bei Dotzheim h. im Frühjahr an Kiefern (K.). -

Ueber B. piniperda finde ich eine alte Notiz meines Vaters aus dem Jahre 1841. — „Er zerstörte eine Anzahl hochstämmiger Kiefern; sie waren Ende Juni völlig dürr und wurden gefällt. Unter der Rinde fanden sich in grosser Anzahl, der Verwandlung nahe, die Larven und Puppen, sowie noch weiche, hellgelbliche Käfer. - In den dünneren Zweigen der Krone derselben Bäume hauste in grosser Anzahl Carphoborus minimus F. - Der Käfer hatte jetzt seine Eier zu beiden Seiten des Hauptganges abgesetzt und eine Anzahl selir kleiner Larven auch schon den Anfang der Seitengänge begonnen. - Pityophthorus bidens fand sich in geringerer Anzahl unter ihm vor und schien gleiche Lebensweise zu führen. Andere erwachsene, vom Wind umgeworfene Kiefern waren als Fangbäume liegen geblieben und zeigte sich jetzt um dieselbe Zeit (Ende Juni) unter ihrer Rinde B. piniperda als vollkommener Käfer, frisch eingebohrt, in grosser Anzahl. - An den vorerwähnten Stämmen fanden sich keine alten Käfer und an diesen Fangbäumen durchaus keine Larven und Puppen."

3. B. minor Hartig. - In Kiefernholz bei Fr. z. s. $<$ 5. und im Juni gef. -

\section{Dendroctonus Erichson.}

1. D. micans Kug. - Von Steitz im Taunus einmal am Fuchstanz am Fusse des grossen Feldberg an Pinus sylvestris gef. — G. (Klingelhöffer). - 


\section{Carphoborus Eichhoff.}

1. C. minimus F. - Von C. H. im Fr. Wald in Menge $>6$. und $<7$. in den dünnen Aesten der Kiefern, die von Blast. piniperda zerstört wurden, gesammelt. - Fr. (Hg.) -

\section{Phloeophthorus Wollaston.}

1. P. tarsalis Först. = Spartii Nördlgr. - Im April unter der Rinde von Spartium scoparium an manchen Orten s. h., z. B. auf der Bieberer Höhe, im Fr. Wald von uns gesammelt. - St. Goarshausen (Hg.). -

\section{Hylesinus Fabricins.}

1. H. crenatus F. $-\mathrm{L}$. H. fand diese schöne Art $<4$. an der Gerbermühle am Main bei Oberrad in Eschen, auch unter Eschenrinde $>1$. - Aus Fr. dürrem Waldholz $=6$. öfter entwickelt. - Wlb. (Sch.)

- Fr. (Hg.) - Mr. (S.) -

2. H. Oleiperda F. = suturalis Redtb. - Diese sonst nur in Süd-Europa in Oelbäumen beobachtete Art sammelte St. einmal in grosser Anzahl aus Buchenholz aus dem Fr. Wald. -

3. H. Fraxini F. - Bei Fr. unter Eschenrinde $>1$. und $>8$. von C. H. öfter ges. - G. (Zimmer). - Dlb. (Sch.) - Mz. (S.) -

\section{Polygraphus Erichson.}

1. P. pubescens F. - Aus dürrem Fr. Waldholz $=5$. und Juni öfter entwickelt. Bei Fr. unter Fichtenrinde von C. H. $>6$. öfter gef. Am 10. März 1860 an der "Louisa" unter der Rinde junger Fichten h. von B. gesammelt. - Fr. (Hg.) -

\section{BOSTRYCHINI.}

\section{Xyloterus Erichson.}

1. X. lineatus Oliv. - Aus dürrem Fr. Waldholz $>4$. und $<6$. öfter von C. H. erzogen. - G. (Zimmer). - Dlb. (Sch.) Fr. (Hg.) - 
2. (Trypodendron Steph.) domesticus L. - Aus dürrem Waldholz $=4$. öfter erzogen. - Dr. Julius Ziegler fand den Käfer in Fr. öfter in Robinien. - Fr. (Hg.) -

\section{Crypturgus Erichson.}

1. C. pusillus Gyll. - Unter Fichtenrinde im Fr. Wald $=4$. - Die Stämme waren schon vor zwei Winter gefällt. - Unter Rinde von Pinus strobus im Februar. -

\section{Cryphalus Erichson.}

1. C. Ratzeburgi Ferrari = Tiliae Ratzb. - Von C. H. bei Fr. einmal unter der Rinde abgefallener Lindenzweige gef. - Wlb. (Sch.) - Fr. (Hg.) -

2. C. binodulus Ratzb. - Aus dürrem Fr. Waldholz einmal von C. H. $<$ 6. oft erzogen. - Wlb. (Sch.) -

3. C. Tiliae Gyll. = Abietis Ratzb. - Einmal aus Fr. Waldholz erzogen. - Mz. (S.) -

4. (Ernoporus Thoms.) Thomsoni Ferrari = Fagi Thoms. - In Anzahl < 5. aus Buchenholz aus dem Fr. Wald erzogen. -

\section{Pityophthorus Erichson.}

1. P. Lichtensteinii Ratzb. - In abgestorbenen Zweigen junger Kiefern <.6. einzeln von C. H. bei Fr. gef.; im August auch in Pinus picea. -

2. P. bidens F. - Aus Kiefernstangenholz n. s. erzogen von $=6$. bis $>8$. - Fr. Wald. - Fr. (Hg.) -

\section{Thamnurgus Eichhoff:}

1. T. Kaltenbachii Bach. - Von C. H. aus den Stengeln von Betonica officinalis aus dem Fr. Wald (Gehren) $<9$. erzogen; die Larve $=7$. - Bei Boppard lebt die Art in Teucrium scorodonia und Origanum vulgare; von Kaltenbach auch in Lamium album gef. Dr. Bach fand die Art, nach brieflicher Mittheilung an L. H., auf nassauischem Gebiet, Boppard gegenüber zwischen den Ortschaften Filzen und Kamp. - B. fing ein Stück bei Fr. am 3. Juni 1860. - G. (L.) - 


\section{$-246$}

Bostrychus Fabricius.

1. (Cumatotomicus Ferrari) stenographus Dft. - Bei Fr. n. s. von C. H. gef., in Kiefernstämmen unter der Rinde. -

[(C.) typographus L. - Scheint im Gebiet zu fehlen. Von Sch. weder bei Dlb. noch bei Wlb. beobachtet.]

2. (Onthotomicus Ferrari) curvidens Germ. - Am 6. April 1860 bei Fr. 2 Stück ron B. gef. -

3. (0.) Laricis F. - Unter Kiefernrinde im Fr. Wald von Juni bis $=9$. h. - Lorsbacher Thal $>4$. - Von B. am 6. April 1860 bei Fr. h. gesammelt. - Bei Dlb. und Wlb. die häufigste Art nach Sch. -

Oberförster Eichhoff bestimmte eine Anzahl Stücke, $=2$. unter Fichtenrinde bei Fr. gef., unter laricis, als Uebergänge zur folgenden Art (suturalis). -

4. (0.) nigritus Gyll. (das Männchen) = suturalis Gyll. (das Weibchen). - Männchen nicht aus unserem Gebiet bekannt. - Weibchen n. s. im Fr. Wald $<$ 5. unter Fichtenrinde von uns gef. Cronthal im Taunus in Pinus sylvestris $<6$. - Auch Hg. fand bei Fr. nur Weibchen. -

\section{Xylocleptes Ferrari.}

1. X. bispinus Ratzb. - Im Februar h. in den Zweigen von Clematis vitalba gesellig im Mark. - Bei Fr. und auf der Bieberer Höhe bei Offenbach von uns und Hg. gesammelt. — Wlb. ein Männchen und zwei Weibchen (Sch.). -

\section{Xylebor'us Eichhoff.}

1. X. monographus $\mathbf{F}$. - Das Männchen, überall äusserst s., ist viel kleiner als das Weibchen, hat anf dem abschüssigen Halsschild vorn ein Zähnchen. - Bei dieser Gattung, sowie bei Anisandrus sind die Männchen s. s. - Sollten hier nicht parthenogenetische Zustände (jungfräuliche Fortpflanzung) obwalten und die Männchen nur zeitweise, sozusagen zur Auffrischung der Zucht nöthig sein? Von monogr. kennt L. H. nur zwei Männchen, eins als cornutus F. aus Tyrol erhalten, ein anderes fing C. H. bei dem Fr. Forsthaus < 7. an Eichenklafterholz. Weibchen unter Buchenrinde bei $\mathrm{Fr}$. $=5$. n. s. - Bei Wlb. ein Stück (Sch.). - G. (L.) - Fr. (Hg.) - 
2. X. dryographus Er. - Männchen kennt L. H. nicht. - Ein Weibchen fing C. H. $<7$, beim Fr. Forsthaus. - Bei Dlb. und Wlb. die Weibchen h. (Sch.) - Fr. (Hg.) -

3. X. Saxeseni Ratzbg. - L. H. besitzt zwei Männchen, eins aus Westfalen, das andere fand C. H. bei Fr. (es ist kleiner und etwas kürzer als dâs Weibchen). - Das Weibchen $=2$. aus Zweigen junger Fichten, $=3$. todt unter Birkenrinde, $>5$. beim Fr. Forsthaus oft im Flug gef. - Bei Soden im Taunus im Mai auch unter der Rinde eines Eichenstrunkes. - Weibchen bei Wlb. (Sch.) - G. (L.) - An einer alten Rüster (Ulmus) an den Kettenhöfen 27. April 1876 öfter. -

\section{Anisandrus Ferrari.}

1. A. dispar F. (Weibchen = tachygraphus Shlb.) - Die Männchen sind kurz rundlich, halb so lang als die Weibchen. Die Sammlung v. Heyden besitzt von Groos bei Wsb. gefangene Stücke. - Weibchen aus dürrem Fr. Waldholz $<4$. öfter erzogen; bei Cronthal $=5$. im Flug gef. - Bei Dlb. und Wlb. beide Geschlechter in Baumschulen sehr schädlich, zerstören die Bäumchen, bohren sich tief ins Holz; , auch unter Eichenrinde (Sch.). - Auch im Pflanzgarten der Fasanerie bei Wsb. den jungen Apfelbäumchen schädlich (K.). - Mz. (S.) -

\section{Dryocoetes Eichhoff.}

1. D. villosus F. - C. H. fing ein Weibchen $<7$, in einem Nest der Formica rufa am Fuss einer alten Eiche (wohl zufällig?). Von B. einmal gef. - Bei Wlb. n. s. (Sch.) - Fr. (Hg.) -

2. D. cryptographus Ratzb. - Aus dürrem Aspenholz aus dem Fr. Wald in Menge $=5$. entwickelt. - Fr. (Hg.) -

3. D. Coryli Perris. - Lebt in der Haselstaude. - C. H. fing ein Stück $>4$. in den Fr. Promenaden im Flug. - Neu für unsere Fauna. -

4. D. bicolor Hbst. - Aus dürrem Buchenholz aus dem Fr. Wald $=6$. in Menge, einzeln aus Populus tremula $=4$. erzogen; im Februar unter Carpinus-Rinde gef. - Von B. am 20. März 1860 bei Fr. h. gesammelt, auch von $\mathrm{Hg}$. gef. - 


\section{CURCULIONIDAE.}

\section{A. OTIORHYNCHINI. \\ Mylacus Schönherr.}

1. M. rotundatus F. - Bei Fr., Friedberg $=5$. und Mo, einzeln $=4$. von C. H. gesammelt. (Früher zu Omias gestellt.) - Fr. einmal (Fresenius). - Am Schiersteiner Hafen am Rhein 13. April h. von K. gef. - Mz. (S.) -

\section{Otiorhynchus Germar.}

1. O. scabripennis Schh. - C. H. fing ein einzelnes weibliches Stück $=4$. auf dem Rochusberg bei Bingen (Bestimmung vom Monographen Stierlin). Sonst in den Alpen und Vogesen. - [Hierher wahrscheinlich der von Bach erwähnte armadillo von Boppard, welcher hochalpin ist.] -

2. O. fuscipes Oliv. - Von Hg. und St. im Taunus gef. -

3. O. niger F. - Von Hg. bei Fr. einmal gef. - Götheruhe, Fr. Wald (Metzler) 1876. -

4. O. morio F. = unicolor Hbst. - Von Sch. bei Dlb. aufgef. an alten Pappeln n. s. -

5. 0. raucus F. - Ueberall h. - Falkenstein $>8$. - Auf dem Röderberg bei Fr. $<5$., zerstörte zu derselben Zeit in der Fr. Gärtnerei das Laub von Rubus vitis idaea. - Wlb. (Sch.) - G. (L.) - Mz. (S.) -

6. 0. porcatus Hbst. - Fr. n. h. - Auf dem Feldberg von Scheidel gef. - Wlb. (Sch.) - Bei Fr. unter Steinen, h. im Taunus von B. gef. - Fr. (Hg.) - Mz. (S.) -

7. O. septentrionis Hbst. - Je einmal bei Fr. von L. H., Hg. und Fresenius (nach B.) gef. -

8. O. singularis $\mathbf{L}$. = picipes F. - Ueberall s. h. - Bei Cronberg im Taunus $<6$. auf wilden Rosen, $<4$. bei Homburg auf Fichten; am Feldberg $<6$.; bei Fr. $=6$. auf Rubus vitis idaea. - 
Friedberg (Renner). - Bei Fr. im Frühjahr unter Steinen und im Maingenist (B.). - Wlb. (Sch.) - G. (L.) - Mz. (S.) - Mo. auf Kiefern 29. Mai (K.). -

Var. Chevrolati Schh. - Mit der Stammart bei Fr. auf Rubus. - Auf Pappeln beim neuen Irrenhaus am Affenstein $=5$. gef. (L. H.) -

9. O. sulcatus F. - Bei Fr. von C. H. und St. sehr einzeln gesammelt. - Mz. (S.) -

10. 0. Ligustici L. = Bruckii Bach. (Die Stammart einförmig, nicht fleckig beschuppt.) - G. (L.) - Wsb. (K.) - Mz. (S.) -

Var. collaris F. (Deutlich scheckig beschuppt.) - Bei Fr. n. s. h. von C. H. gef.; nach B. ungemein h. unter Steinen und im Maingenist. - G. (L.) - Wlb. (Śch.) - Wsb. (K.) - Mo. Rheinufer im Sand unter Weiden 5. Mai in Menge (K.). - Bockenheim auf Wegen 7. April 1877. -

11. 0. ovatus L. - Fr. h. unter Moos. - Königstein $<6$.; Soden $>6$. bei Formica rufa. - Wlb. (Sch.) - Mz. (S.) $-\mathrm{M} 0$. 5. Mai (K.). -

\section{Peritelus Germar.}

1. P. hirticornis Hbst. - Bei Soden auf Hecken $>6$. von C. H. einzeln gesammelt. - Mz. (S.) -

2. P. griseus Oliv. - Im Fr. Wald $>4$. im Wartforst auf Juniperus auf der Bieberer Höhe $>6$. und bei Griesheim am Main von C. H. oft gef. - Fr. h. (B.) - Mz. (S.) -

\section{Phyllobius Schönherr.}

1. P. maculicornis Germ. - Je einmal von C. H. bei Königstein und Falkenstein im Taunus im Mai gef. - Fr. (Hg.) -

2. $\mathbf{P}$. glaucus Scop. $=$ calcaratus $\mathbf{F}$. $=$ alneti $\mathbf{F}$. $=$ Pyri Schh. - Falkenstein im T'aunus $=5$. auf Erlen, Fr. $>$ 5. auf Ahorn in Begattung. - Fr. in der Gärtnerei vor dem Eschenheimerthor auf Urtica dioica im Juni h. von L. H. gesammelt; die Weibchen oft mit je zwei kahlen Flecken auf den grünbeschuppten Flügeldecken, welche während der Begattung durch das Sichfesthalten des Männchens am Weibchen durch die vier Vorderfüsse des ersteren entstehen. - Fr. h. (B.) - Wlb. (Sch.) - G. (L.) - Mz. (S.) -

Var. atrovirens Schh. - Von Hg. bei Fr. gef. - 
3. P. Betulae F. - Fr. $=5$. auf Pappeln, $>6$. bei Soden auf Hecken. - Bei Nauheim > 5. von C. H. gef. - G. (L.) Dlb. Wlb. g. auf Waldbäumen (Sch.). - Fr. (Hg.) - Mrz. (S.) -

4. P. argentatus L. - Bei Fr. $>$ 4. auf Eichen, im Juni am kleinen Feldbergthal, am grossen Feldberg und bei Königstein gesammelt. - G. (L.) - Dlb. Wlb. auf Obstbäumen s. g. - Fr. (Hg.) Nz. (S.) -

Var. viridans Schh. - Einmal von C. H. $>$ 5. bei Nauheim gef. -

5. P. oblongus L. - Fr. h. - Von Hg. und B. s. h. auf jungen Buchen, von Sch. bei Wlb. h. an Obstbäumen gef. - Mz. (S.) -

6. P. Pyri L. = vespertinus F. Schh. - Bei Enkheim $=5$. auf Eichen, auf Erlen im Taunus bei Soden, Falkenstein und am Feldberg. - B. fand ein Weibchen in Begattung mit einem Männchen von Metallites atomarius. - G. (L.) - Wlb. (Sch.) - Fr. (Hg.) Nz. (S.) -

7. P. Pomonae Oliv. - Bei Fr. von uns und Hg. gef., nach B. h. - Wlb. (Sch.) - G. (L.) -

Var. cinereipennis Schh. - Zwei Stück auf Schlehen und Weiden im April und Mai bei Fr. von C. H. gef. -

8. P. viridiaereus Laich. $=$ uniformis Mrsh. - Im Taunus bei Falkenstein im Mai und Juni n. s., auch bei Nauheim öfter gef. Fr. Wald auf Spiraea ulmaria. - Wlb. (Sch.) - G. (L.) - Fr. (Hg.) - NIz. (S.) -

9. (Pseudomyllocerus Desbrochers) sinuatus F. - Bei Fr. von C. H. $>6$. öfter auf Potentilla gesammelt. -

\section{B. BRACHYDERINI. \\ Polydrosus Germar.}

1. P. undatus F. - Auf Birken bei der Mainkur $>5$., zu derselben Zeit und bis $<6$. am Fusse des Feldberg. - Bingen schon $=4$. von uns gesammelt. - Wlb. h. (Sch.) - G. (L.) - Fr. (Hg.) - Mr. (S.) -

2. P. impressifrons Schh. - Bei Wlb. von Sch. gef. -

[P. flavipes Deg. - Nach S. V. im ganzen Gebiet n. S. L. H. kennt die Art nur aus Mittelfrankreich; Stücke, die er unter diesem Namen von Stierlin als den ächten flavipes erhielt, waren corruscus.] - 
3. P. pterygomalis Schh. - Ein Weibchen $=7$. im Sodener Wald und einmal bei Fr. von C. H. gef. - Wlb. 4 Stück (Sch.). Fr. (Hg.) -

4. P. cervinus Gyll. - Im Fr. Wald an der unteren Saustiege $>8$. ; im rothen Graben an der Mainkur auf Eichen > 7. - Im Taunus von $=5$. bis $=6$. im Thal am kleinen Feldberg sowie bei Falkenstein und Königstein. - Rüdesheim am Rhein $<5$. - Fr. h. (Hg. und B.) - Wlb. h. (Sch.) - Mz. (S.) -

5. P. Arvernicus Desbrochers. - C. H. fand diese wenig bekannte Art zweimal im Taunus bei Homburg $<4$. auf Fichten und $>6$. am Feldberg. - (Mit cervinus verwandt, aber mit kupfrigen gescheckten Flecken.) -

[P. chrysomela Oliv. = salsicola Fairm. soll nach Scriba im ganzen Gebiet s. sein; L. H. kennt ihn nur von der französischen Westküste und aus Portugal.] -

6. P. confluens Steph. $=$ perplexus Schh. - Bei Fr. $=7$., bei Mo. $=6$. von C. H. gef. - G. (L.) -

7. P. sparsus Schh. - Bei Fr. von C. H. zweimal aufgef. -

8. P. sericeus Schaller. - Bei Fr. von uns und Hg. auf. Erlen, Haseln und Schlehen im Juni und Juli h. gesammelt. - Soden $>6$. - Dlb. Wlb. (Sch.) - Mz. (S.) -

9. (Eudipnus Thoms.) micans F. - Fr. $<5$. auf frischem jungem Buchenlaub s., auch im Juli auf Eichen, doch nur abgeriebene, alte Stücke; auch von Hg., St. und B. s. gef. - Dlb. Wlb. h. (Sch.) - G. (L.) - Mz. (S.) -

10. (Metallites Schh.) mollis Germ. - In Soden $>6$. auf Hecken von C. H. gef., mit dem Vermerk ,keine Pinus in der Nähe". - Hengster bei Offenbach von L. H. gesammelt. - Kleine Feldbergthal =6. - Dlb. Wlb. (Sch.) - Fr. (Hg.) - Mo. auf Kiefern im Juni in Begattung (K.). -

11. (M.) atomarius Ol. - Fr. n. s. von B. gef. - Wlb. 2 Ex. auf Eichen (Sch.). - Wsb. an der Platte 20. Juni auf Kiefern (K.). - Mz. (S.) -

12. (M.) Iris Oliv. = marginatus Steph. = ambiguus Schh. - Im Fr. Wald $<6$. oft sehr schädlich an jungen Eichen, indem er Knospen und Blätter zerfrisst. - Im Hofheimer Wald schon > $>$. Johannisberg bei Friedberg $=5$. - Bei Fr. von Hg. und B., bei Wlb. von Sch. s. h. gef. - Dotzheim auf Kiefern im April (K.). - Mz. (S.) - 


\section{$-252-$ \\ Scythropus Schönherr.}

1. S. mustela Hbst. - Auf der Bieberer Höhe an den Steinbrüchen auf blühenden Kiefern $=4$. n. s. von uns gesammelt. - Fr. s. (Hg. B.) - G. (L.) - Mz. (S.) - Mo. auf Kiefern 29. Mai (K.). - Dotzheim ebenso im April (K.). - Limburg am Schafberg $>$ 5. (K.). -

\section{Sciaphilus Schönherr.}

1. S. muricatus F. - Im Taunus in der Seulberger Mark $=9$., Wsb. $=10$., bei Rüdesheim $<5$. unter Steinen. - Ems. - G. (L.) - Fr. von uns und Hg. gef. - Mz. (S.) -

\section{Platytarsus schönherr.}

1. P. echinatus Bonsdorff = hirsutulus F. (Omias olim.) Im Sodener Wald auf Caprifolium $<6$. von C. H. gesammelt. Schwarze Steinkaut in der „Louisa“, Fr. Wald im März unter Riedgräsern. - Wlb. in Anzahl mit dem Streifnetz gef. (Sch.) - Fr. (Hg.) -

\section{Foucartia Duval.}

1. F. squamulata Hbst. (Strophosomus 0l.) - Bei Seckbach auf Wiesen $=5$. und bei Bergen auf Carduus. - Wlb. h. (Sch.) -

\section{Barypeithes Duval.}

1. B. pellucidus Schh. - Von Sch. bei Wlb. aufgef. - Fr. (Hg.) -

2. B. araneiformis Schrk. $=$ brunnipes Oliv. $=$ ebeninus Schh. - Unter Steinen auf dem Plateau des grossen Feldberg $>6$. von C. H. und am 4. September 1871 von L. H. gef. - Soden $=7$. - In Rüdesheim < 5. bei Ameisen. - Wlb. (Sch.) - Fr. (Hg.) Mz. (S.) -

3. B. mollicomus Ahr. = punctirostris Sch. - Von Hg. bei Fr. gef. -

4. B. tenex Schh. - Unser Gebiet ist der Hauptfundort des sonst seltenen Thieres. - Im Fr. Wald $=6$. an Klafterholz, Falkenstein; in der Hohen Mark an Schwämmen; Schlangenbad unter Moos; C. H. fand die Art auch n. s. bei Ameisen im Fr. Wald und L. H. bei Soden und Königstein $h$. in den Nestern von Formica cunicularia. - [Hierher Omias concinnus in S. V., der nur in Süd-Europa vorkommt.] - Fr. s. (Hg. B.) - Mz. (S.) - 


\section{Omias Germar.}

1. 0. mollinus Schh. $=$ Bohemanni Schh. - In Sammlung v. Heyden befindet sich ein von M. S. bei Fr. gefundenes Stück, das in S. V. als pruinosus erwähnt ist. - Mz. (S.) -

2. 0. gracilipes Panz. $=$ forticornis Schh. $=$ validicornis Märk. - Fr. von Hg. gesammelt. -

\section{Strophosomus Schönherr.}

1. S. Coryli F. = illibatus Schh. - Fr. h. von uns und Hg. gef. - Mz. (S.) -

2. S. obesus Mirsh. = Coryli Schh. - Fr. h. Den jungen Eichen $<6$. schädlich. - Nach B. seltener als die vorige Art. Wlb. s. (Sch.) - G. (L.) - Fr. (Hg.) -

3. (Neliocarus Thoms.) faber Hbst. - Auf trockenen Triften bei Rumpenheim = 7., auf Wiesen bei Hausen $=9$. - Fr. einige Stücke (Hg. und B.). - Wlb. (Sch.) - G. (L.) - Mo. auf den Blössen an Kiefern 30. Juni (K.). - [S. retusus Mrsh. in S. V. ist zu streichen.] -

\section{Eusomus Germar.}

1. E. ovulum Illig. - Fr. im April und Mai auf Schlehen. Bergen. - In Menge bei Bockenheim 3. Juni 1876 auf Achillea millefolium, dessen Blätter von dem Käfer ganz zerfressen waren. - Mz. (S.) - Mo. vom 23. Mai bis 20. Juli (K.). -

\section{Brachyderes Schönherr.}

1. B. incanus L. = lepidopterus Schh. - Im Fr. Wald an Baumstämmen <3. - Bei Mo. im Sand in der Nähe vom Kiefernwald $>$ 8. - Fr. (Hg.) - Mz. (S.) - Gonsenheim bei Mz. an Këfern $>$ 8. (K.) -

\section{Sitones Schönherr.}

(Die Sammlungen $\nabla$. He yde und Ha $\mathrm{H}$ sind vom Monographen Allard bestimmt.)

1. S. griseus F. - Bei Fr. von C. H. und Hg., bei Mo. auf Spartium scoparium $>4$. von L. H. s. gesammelt. - Mz. (S.) -

2. S. longicollis Schh. - Einmal von C. H. bei Fr. gef. - 
3. S. flavescens Mrsh. $=$ octopunctatus Sch. - Von C. H., Hg. und B. eine Anzahl Stücke bei Fr. gef. - Mz. (S.) -

4. S. suturalis Steph. - Einige Exemplare von C. H. und Hg. bei Fr. aufgef.; zweimal bei Wlb. (Sch.) -

5. S. sulcifrons Thunbg. = Medicaginis Redtb. - Fr. Taunus. - h. - Wlb. (Sch.) - Fr. (Hg.) - Mz. (S.) -

Var. argutulus Schh. - Im Rödelheimer Wald $=8$. und bei Rüdesheim $<5$. gesammelt. - Fr. (Hg.) -

6. S. tibialis Hbst. (Männchen $=$ chloropus Mrsh. Weibchen $=$ striatellus Schh.) - Schmitterhof bei Giessen $>4$. - Ems. Königstein im Taunus $=9$. - Striatellus bei Fr. - Wlb. (Sch.) Fr. (Hg.) - Mz. (S.) -

Var. ambiguus Schh. - Fr. im April öfter. gef. - Ems. Königstein mit der Stammart. - Mz. (S.) -

7. S. Waterhousei Walt. $=$ setosus Redtb. - Einmal bei Wlb. von Sch. gef. -

8. S. crinitus Oliv. - Im Fr. Wald $=3$. im August bei Griesheim am Main. - Fr. (Hg.) - Mo. (St. S.) -

9. S. Regensteinensis Hbst. - Auf Spartium scoparium h.; überwintert, schon $=3$. in Begattung bis $>4$.; dann wieder $=9$. - Schmitterhof bei Giessen. - Fr. s. h. (B.) - Am Staufen -im Taunus 2. April 1876 (L. H. und B.) - - Wlb. (Sch.) - Fr. (Hg.) - Mz. (S.) -

10. S. Cambricus Steph. $=$ cribricollis Sch. - Von Hg. bei Fr. gef. -

11. S. puncticollis Steph. - In trockenen Wiesen auf der Erde bei Hausen = 9. - Wlb. (Sch.) - Fr. (Hg.) - Mz. (S.) -

12. S. lineatus L. - Fr. h. auf Fusswegen im Sand und an Häuser angeflogen. - Wlb. (Sch.) - Fr. (Hg., B.) - Mz. (S.) -

Var. geniculatus Schh. - Zweimal von C. H. im November am Fuss von Weiden bei Fr. gef. - Hz. (S.) -

13. S. discoideus Schh. - Von Sch. bei Wlb. gef. -

14. S. hispidulus F. - Fr. h. im Juli. - Bei Falkenstein im Taunus $>4$. - Bei Budenheim am Rhein = 10. - Wlb. h. (Sch.) - Fr. (Hg.) -

15. S. humeralis Steph. $=$ promptus Schh. - Bei Soden im Gras < 8. und bei Griesheim am Main. - Wlb. (Sch.) - Fr. (Hg.) - 
16. S. inops Schh. - Einigemal bei Fr. von C. H. und Hg. gef. -

\section{Trachyphloeus Germar.}

(Die Sammlungen v. Heyden und Haag von Seidlitz revidirt.)

1. T. alternans Schh. - Bei Fr. einmal von C. H. gef. (öfter bei der Saline Wisselsheim in der Wetterau).

2. T. spinimanus Germ. - Einmal $=5$. bei Friedberg von C. H. gef. -

3. T. scabriculus L. - An den Kalkbrüchen hinter Offenbach $<6$.; im Fr. Wald auf der Bruchschneisse in Sandgräben $>4$.; bei Fr. auf thonigen Triften $=5$. - Im Juni bei Mo. auf dem Sand unter Steinen. - Wlb. (Sch.) - Fr. h. (Hg. B.) - Mz. (S.) -

4. T. scaber L. - Bei Hausen $=9$. auf trockenen Wiesen auf der Erde. - Zu derselben Zeit und im Juni bei Königstein, $>4$. an Häusern der Stadt angeflogen. - Nauheim auf Salzboden $=8$. [Hierher squamosus des S. V.] - Wlb. (Sch.) - Fr. (Hg.) Mz. (S.) -

5. T. aristatus Gyll. - Oefter bei Ems und Fr. von C. H. gesammelt. - Wlb. (Sch.) -

6. T. squamulatus Oliv. - Bei Hausen zusammen mit scaber, bei Offenbach mit scabriculus. - Bingen im April. - Wlb. (Sch.) Mz. (S.) -

\section{CNEORHININI.}

\section{Cneorhinus schönherr.}

1. (Dactylorhinus Tourn.) globatus Hbst. = geminatus Hbst. - Bei Mo. im Sand $>5$. und $<6$. h. unter Euphorbia von uns, S., Hg. und K. gesammelt. - Nach Wagner bei Bingen dem Weinstock schädlich. - Ingelheim 1875 (B.). - Wlb. (Sch.) - G. (L.) -

\section{Barynotus Germar.}

1. B. obscurus F. - Auf dem grossen Feldberg von L. H. gesammelt. - G. (L.) - Dlb. (Sch.) - Fr. von Hg. h., auch von B. gef. - Mz. (S.) - 


\section{Liophloeus Germar.}

1. L. nubilus $F$. - Bei Bingen $>4$, bei Homburg $=5$, bei Soden $>5$. gesammelt. - Dlb. Wlb. s. h. (Sch.) - G. (L.) Mz. (S.) -

\section{TANYMECINI.}

\section{Chlorophanus Dalman.}

1. C. viridis L. - Bei Fr. je zweimal von Hg., C. H. und B. gesammelt. - Die Arten leben auf Weiden. - G. (L.) - Wlb. (Sch.) - Mz. (S.) -

2. C. pollinosus F. - Je ein Stück bei Fr. von C. H. und Hg. gef. - Mz. (Dr. Zitz), in S. V. als graminicola erwähnt, später dort auch von S. gesammelt. -

3. C. graminicola Schh. - Am Rhein bei Mo. $>$ 8. von L. H. gef. - [Hierher das als salicicola in S. V. erwähnte Stück, welche Art nur in Südost-Europa rorkommt.] - Fr. (B.) - Mz. (S.) -

\section{Tanymecus Germar.}

1. T. palliatus F. - Fr. öfter gef., besonder's $=7$. beim Hellerhof auf Arctium lappa. - Wlb. (Sch.) - Fr. (Hg. B.) - Mz. (S.) - Wsb. am Ufer der Wellritz 26. Mai (K.). -

\section{E. TROPIPHORINI.}

Tropiphorus Schönherr.

1. T. elevatus Hbst. $=$ Mercurialis F. - Im Taunus auf dem Feldberg von L. H. zweimal gesammelt. - s. - G. (L.) - Am Hafen von Schierstein am Rhein 13. April (K.). - Ein Exemplar mit fast verloschenen Rippen fand Sch. bei Wlb. -

\section{F. RHYTIRHININI.}

Gronops Scbönherr.

1. G. lunata F. - Von C. H. einmal bei Fr., von L. H. einmal bei Nauheim $=5$. in Begattung gef. - G. (L.) - Mz. (S.) - 
Var. rubrica Ahrens. (Rothe Flügeldecken mit wenig markirter Zeichnung.) - L. H. fand ein Stück $<5$. im. Fr. Wald an der Maver am Forsthaus, C. H. ein anderes $=8$. bei Nauheim auf Salzboden. -

Var. seminiger Allard. (Die schwarzen Flecken so sehr entwickelt, dass nur zwei schmale weisse Querbänder auf den Flügeldecken übrig bleiben.) - Einmal bei Fr. von C. H. gef. -

\section{G. HYPERINI.}

\section{Alophus Schönherr.}

1. A. triguttatus F. = nictitans Schh. - Bei Fr. von uns und Hg. öfter gesammelt; auch von H. Harer gef. (siehe S. V.), sowie von B. h. im Feuchten unter Steinen. - Dlb. Wlb. h. (Sch.) Mz. (S.) - Bockenheim 7. April 1877 auf sandigen Wegen (L. H.). -

\section{Hypera Germar.}

1. H. tesselata Hbst. = maculata Redtb. - C. H. fand im Sauerthal bei Lorch am Rhein $<6$. einen Cocon, aus welchem sich Ende des Monats der seither nicht aus unserer Gegend bekannte Käfer entwickelte. [Die Bestimmung ist von dem Monographen Capi o mont.] - St. fand 2 Ex. in den Torfbrüchen bei Enkheim. - Mz. (S.) -

\section{Phytonomus Schönherr.}

(Die Sammlungen v. Heyden und $\mathrm{Ha}$ a g vom Monographen Capiomont bestimmt.)

1. (Donus Capt.) punctatus F. - Bei Fr. und im Taunus von uns und $\mathrm{Hg}$. gesammelt. - Von B. s. an Sonnenblumen im Garten, auch unter Steinen gef. - Wlb. (Sch.) - G. (L.) - Mz. (S.) -

2. (D.) fasciculatus Hbst. $=$ variegatus Bach. - Von C. H. einmal bei Fr. gef. -

3. (Erirhinomorphus Cap.) Julini Sahlb. = alternans Steph. - Einmal in Anzahl von C. H. bei Fr. gesammelt. - G. (L.) -

4. (E.) Pollux F. - Bei Fr. n. s. von uns und Hg. gesammelt. - G. (L.) -

5. (E.) Rumicis L. - C. H. fand in Gonsenheim bei Mz. $=9$. auf der oberen Blattseite von Polygonum amphibium einen ovalen, erbsengrossen, weitmaschigen, ziemlich festen, gelblichen Cocon, aus welchem sich Ende des Monats der Käfer entwickelte. - Fr. 1875 (B.). Wlb. (Sch.) - G. (L.) - Fr. (Hg.) - Mz. (S.) - 
6. (E.) tigrinus Schh. - Nen für Mitteldeutschland. - Am Nürmbergerhof im Rheingan einmal 11. September von K. gef. -

7. (Dapalinus Cap.) Meles F. = Trifolii Hbst. - Bei Fr. von Hg. gesammelt. -

8. P. Polygoni F. - Im Fr. Wald $>$ 4. auf der Bruchschneisse in Sandgräben n. S. - Wlb. (Sch.) - G. (L.) - Fr. (Hg., B.) Mz. (S.) -

9. P. elongatus Payk. = mutabilis Germ. - C. H. fand hinter Offenbach auf den Wiesen mit dem Streifnetz $>5$. die grüne Larve mit weisser Rückenlinie, welche sich $=6$. „ohne" Gespinnst verwandelte. - De Geer bildet T. V. tab. 7. fig. 17-21. die Larro ron P. Plantaginis ab; nach der Beobachtung von C. H. hat die Larre ron elongatus mit der ron Plantag. die grösste Aehnlichkeit. De Geer sagt darüber schon 1781 in der Uebersetzung ron Goeze: „Im Juli häufig die Larven auf Wegerich. Klein, munter, mit einer weissen Längsstreife auf dem Rücken. Beim ersten Anblick sollte man sie für die Blattlansfresser mit dem spitzigen betreglichen Kopfe (larra Muscae Pyrastri L.) ansehen, die eben so grün sind und auch eben dergleichen lange Rückenstreifen haben. Bei genauerer Beobachtung aber zeigt sich's, dass jene einen kleinen schwarzen, runden, hornartigen, unbetreglichen Kopf haben. Die Ringe des Körpers sind wegen der vielen Runzeln undeutlich. Unten ron einem Ende zum andern die Fleischwarzen, die ihnen statt der Füsse dienen. Durch die Lupe sieht man auf der Haut in Querlinien viele kleine, schwarze Pünktchen und in jedem ein kurzes Härchen. Am 11. hatten sie sich auf den Blumen schon eingesponnen. Die Gespinnste grüngelblich, langkuglicht, mit dünnen Wänden elastisch, wie Pergament. Vor Ende des Monats erschienen die Käfer." - G. (L.) - Fr. (Hg.) - Mrz. (S.) -

10. P. suspiciosus Hbst. - Bei Fr. n. s. - Bei Mo. $>8$. gef. - Wlb. (Sch.) - Fr. (Hg.) - Enkheim (St.). -

11. P. murinus F. - Einige Stücke ron C. H. und Hg. bei Fr. gef. - Wlb. (Sch.) -

12. P. variabilis Hbst. = suturalis Redtb. - Die Larre auf exrigem Klee. - Soden = 5. in Begattung. - Fr. h. (Hg.) - Mro. $<$ 6. - Wlb. (Sch.) - G. (L.) -

13. P. Plantaginis De Geer. - Beschreibung der Larve siehe bei P. elongatus. - Der Cocon ist nur halb so gross rie bei rumicis, wenigstens bei dem einen Stück, das sich in Fr. entrickelte. - Fr. z. s. - Von B. am 3. Juni 1860 gef. - Wlb. (Sch.) - G. (L.) - Fr. (Hg.) - 
14. P. trilineatus Mrsh. = plagiatus Redtb. - C. H. fand ein Stück bei Fr. - Wlb. (Șch.) -

15. P. nigrirostris F. - Schon $>4$. gef. - Die Larve minirt $<6$. in den Blättern von Ononis, der Käfer Ende des Monats entwickelt. - Fr. - Concons länglich, farblos. Länge $5 \mathrm{~mm}$. - Auf Kleefeldern s. h. von B. gestreift. - G. (L.) - Wlb. (Sch.) - Fr. (Hg.) - Mz. (S.) -

16. P. Viciae Gyll. - Einmal bei Fr. gef. - G. (L.) , -

\section{Limobius Schönherr.}

1. L. dissimilis Hbst. - L. H. und Hg. fingen die Art einmal h. am 7. Mai 1867 bei Mo. auf Geranium im Wald an den Schiessständen. - Mz. (S.) -

\section{H. CLEONINI.}

Cleonus Schönherr.

1. (Plagiographus Chevrolat) obliquus F. - Bei Fr. von St. gesammelt. -

$[(P$.$) nebulosus \mathbf{L} .=$ carinatus De Geer $=$ glaucus Panz. kenne ich nur aus dem Schwarzwald und von Cleve.]

2. (P.) turbatus Schh. = nebulosus Steph. = glaucus Schh. - Bei Fr. von uns, Hg. und St. gesammelt. -

[Chromosomus Fabricii Gemminger. = roridus F. - Nicht bei Fr. sonḋern Üngarn, in S. V. zu streichen.]

3. (Chromoderus Motsch.) affinis Schranck. = albidus $F_{0}==$ candidus Hbst. - Bei Fr. von uns, Hg. und B. einzeln gef. Mz. (S.) - Budenheim (C. H.). -

4. (Leucosomus Motsch.) quadripunctatus Schrank. = ophthalmicus Rossi. - Fr. einzeln in Kalkgegenden. - Mo. $=5$. und bei Hochheim von L. H. gef. - Offenbach an den Kalkbrüchen und auf der Bieberer Höhe $<6$. - Fr. nur einmal' gef. (B.) - Fr. (Hg.) - Mr. (S.) -

5. (Pachycerus Schönh.) segnis Germ. = scabrosus Schh. - Einmal von C. H. bei Fr. gef. [Bestimmung vom Monographen Chevrolat.].-

6. (P.) albarius Schh. - Fr. einmal wie segnis. - 
7. (Megaspis Schh.) caesus Schh. = cunctus Schh. - Bei Fr. von uns gef. - Rüdesheim $=4$. - [Hierher alternans Oliv. des S. V., welche Art nur in Süd-Europa vorkommt. - Wlb. (Sch.) -

8. (Pseudocleonus Chevr.) costatus $\mathbf{F}$. = cinereus F. [Das in S. V. erwähnte Fr. Stück gehört nicht hierher.] - Fr. (Hg.) - Mz. (S.) -

9. (P.) grammicus Panz. = bilineatus $\mathbf{0 I}$. - L. H. fand diese schöne Art bei Mo. - Fr. (Hg., St.) - Mz. (S.) -

10. (Cyphocleonus Motsch.) trisulcatus Hbst. - Bei Fr. von uns und Hg. gesammelt. - Wlb. (Sch.) -

11. (C.) tigrinus Panz. $=$ dealbatus Gmel. $=$ marmoratus $\mathbf{F}$. - Bei Fr. s. - An den Hochheimer Steinbrüchen > 7. - Auf sandigen Wegen in der Sonnenhitze, so 25. Mai 1860 an der Isenburger Warte bei Fr. von B. gef. - Dlb. Wlb. (Sch.) - G..(L.) - Fr. (Hg.) - Mz. (S.) -

12. C. sulcirostris L. - Auf Disteln n. s. - Mz. - Rüdesheim = 4. - Am Mainufer bei Fr. unter Steinen z. h. von B. gesammelt. - Dlb. Wlb. (Sch.) - Fr. (Hg.) - Mz. (S.) -

\section{Lixus Fabricius.}

[NB. Meine sämmtlichen Lixus und Larinus waren vom Monographen Capiomont bestimmt, aber nach dessen Tod in Frankreich verloren gegangen, so dass ich selbst gar nichts mehr aus dieser Gruppe besitze; ich kann desshalb nur nach dem Verzeichniss der Sendung, welches mir Capiomont noch zuschickte, und nach alten Notizen unsere Arten aufzählen. L. v. H. - Auch Sammlung Hag von Capiomont revidirt.] -

1. L. paraplecticus L. - Am Langen See an der Mainzer Landstrasse $<6$. von C. H. gef. - Von St. h. in den Enkheimer T'orfbrüchen gesammelt; auch von Hg. und B. gestreift. -

2. L. Iridis Oliv. = turbatus Gyll. = gemellatus Schh. Bei Wlb. von Sch., bei Fr. von Hg. gef. -

3. L. Ascanii L. - Bei Budenheim am Rhein von L. H. gef., im Sauerthal bei Lorch $<6$. von C. H. - Fr. - Soden. - Nach B. bei Fr. s. h. bei Sonnenhitze auf fenchten Grasstellen im Spätsommer. Wlb. einigemal (Sch.). - Sachsenhausen (St.). - Fr. (Hg.) - Mz. (S.) - Mo. 10. August (K.). - 
4. L. Myagri Oliv. = marginemaculatus Bach. - Von C. H. bei Fr. und Ems gesammelt. - G. (L.) -

5. L. Junci Schh. - Von Hg. bei Fr. gef. -

6. L. Algirus L. $=$ angustatus F. - Bei Wlb. einmal von Sch. gef. -

7. L. Bardanae F. - Bei Fr. und auf der Bieberer Höhe von C. H. gef. - In den Enkheimer Torfbrüchen von St. gesammelt. Fr. (Hg.) - Mz. (S.) -

8. L. bicolor Oliv, - Von C. H. und St. einzèln bei Mo. gesammelt. - Mz。. (S.) -

9. L. filiformis F. - Bei Fr. und Mo. n. s. von uns, Hg. und St. auf Disteln gesammelt. - Mz. (S.) -

\section{Larinus Germar.}

1. L. senilis F. = pollinis Laich. - Die Larve fand C. H. bei Soden in dem Fruchtboden von Carlina acaulis $<9$. und die Puppe $>$ 9.; der Käfer entwickelte sich $<10$. - Wlb. (Sch.) -

2. L. turbinatus Schh. - Im 'l'aunus einmal von C. H. gef. -

3. L. Sturnus Schall. - Im Hengster bei Offenbach $>6$, bei Fr. nur einmal von C. H. gef. [Die Stücke von Fr. und Mz. in S. V. gehören zu conspersus.] - Wlb. (Sch.) - Von St. beim Fr. Forsthaus auf Disteln gesammelt. -

4. L. conspersus Schh. - Bei Fr. oft gesammelt, bei Mo. $=6$. auf Disteln. - Fr. (Hg., B.) -

5 L. Carlinae Oliv. - Bei Fr. h. von L. H., St. und Hg. auf Disteln. [Hierher die seither für planus F. gehaltene Stücke, welche Art nicht zu deuten ist.] - Wlb. (Sch.) - Auf der Spitze des Kleinen Mannstein (Staufen) im Taunus unter Moos 2. April 1876 (L. H.). Mz. (S.) -

6. L. Jaceae F. - Bei Hofheim und Königstein von C. H. gesammelt. - Fr. h. (B., St.) - Wlb. (Sch.) -

\section{Rhinocyllus Germar.}

1. R. antiodontalgicus Gerbi. - Die Stammart $=8$. bei Mo. auf Carduus. - Auch bei Fr. oft von C. H. und Hg. gesammelt. Mz. (S.) - 
Var. latirostris Latr. - Bei Mo. =6. auf Disteln (S.) Fr. eimmal gef. auch von B. - Wlb. z. h. (Sch.) - Mz. (S.) -

Var. Olivieri Schh. - Von C. H. in Anzahl bei Fr. gesammelt. -

\section{MOLITINI.}

\section{Liparus Oliv. = Molytes Schönh.}

1. L. coronatus Goeze. - Taunus. - Nach B. h. - Dlb. (Sch.) - Fr. (C. H., Hg.) - Mz. (S.) -

2. L. Germanus L. - Bei uns s. s. - C. H. fing $>$ 8. ein Stück auf der Ruine Königstein im Taunus. - Dlb. z. h. (Sch.) Traunus (St., Hg.) - Mz. (S.) -

\section{Liosoma Stephens.}

1. L. ovatulum Clairv. = deflexum Panz. - Wlb. z. h. (Sch.) - G. (L.) -

\section{Plinthus Germar.}

1. P. caliginosus F. - Bei Fr. auf dem Röderberg $<5$. und bei Bergen in den Weinbergen unter Steinen n. s. von uns und $\mathrm{Hg}$. gesammelt. - Im Maingenist und am Main unter Steinen, auch am Metzgerbruch (B.). - Wlb. (Sch.) - Mz. (S.) -

\section{Styphlus Schönherr.}

1. (Orthochaetes Germ.) setiger Beck. - C. H. fing je ein Stück bei Ems und $>7$. im Taunts zwischen Soden und Neuenhain unter Geniste an Weinbergen. - Fr. (Hg.) -

\section{K. HYLOBIINI. \\ Lepy i us Germar.}

1. L. capucinus Schall. $=$ binotatus F. - Bei Fr. am Röderberg im April von C. H. öfter gesammelt, auch in Begattung. - Von B. h. unter Steinen im Frühjahr an der Mainschanze gef. - Wlb. (Sch.) - G. (L.) - Fr. (Hg.) - Mz. (S.) - 
2. L. colon F. - Fr. - Ems. - Von Hg. und B. h. auf Weiden an den Röderhöfen bei Fr. gef. - Dlb. Wlb. (Sch.) - G. (L.) - Mz. (S.) - Wsb. in Begattung 17. Mai (K.). -

\section{Hylobius Schönherr.}

1. H. Abietis L. - In Wäldern an Fichtenstämmen h. - Fr. Die Larve unter Fichtenrinde, der Käfer entwickelt sich schon in Herbst und überwintert. - Friedberg (Renner). - Fr. s. h. (B., Hg.) Dlb. Wlb. (Sch.) - G. (L.) - Mz. (S.) -

2. H. fatuus Rossi. - Bei Fr. von C. H. und Hg., in den Torfbrüchen bei Enkheim in der Nähe alter Weiden und Pappeln von L. H. gef. - Friedberg (Fuhr). - Ein Stück an der Nied bei Bonames an einer Pappel. - G. (L.) - Mz. (S.) - Mo. 13. September (K.). -

\section{ERIRHININI.}

\section{Pissodes Germar.}

1. P. Pini L. - Aus Kiefernholz aus dem Fr. Wald einzeln entwickelt $=5$. uni $<7$. - Fr. (Hg., St.) - Ernsthausen in Nassau (Dörr bei K.) - Mz. (S.) -

2. P. notatus F. - S. h. aus dürrem Kiefernholz aus dem Fr. Wald $=5$. entwickelt; die Larve November 1828 von C. H. gef. Der Käfer noch $<10$. im Freien. - Mo. $=5$, am Feldberg $<6$. Fr. 11. Mai 1861 (B.). - Wlb. (Sch.) -

\section{Grypidius Schönherr.}

1. G. Equiseti F. - Fr. - Tamus. - In den Enkheimer Torfbrüchen s., so 17. Mai 1860 von B. gef. - Wlb. (Sch.) - G. (L.) Fr. (Hg.) - Mz. h. (S.) -

2. G. brunnirostris F. - Bei Fr. nur einzeln von C. H. und Hg. gesammelt. -

\section{Notaris Germar (Erirhinus olim).}

1. N. bimaculatus F. - Von Hg. einmal oft bei Fr. gef. - 


\section{- $264-$ \\ Pachytychius Jekel.}

1. P. sparsutus $\mathbf{0 l}$. - Bei Fr. und Ems sehr einzeln in den Schoten von Genista pilosa im Juni und Juli von C. H. gesammelt. -

\section{Erycus Tournier.}

1. E. Scirpi F. - In Anzahl bei Fr. von C. H., Hg. und St. gesammelt. -

2. E. acridulus L. - Fr. n. s. in sumpfigen Stellen von uns und Hg. gesammelt. - Von B. s. h. im Frühjahr an der Mainschanze gef. - Wlb. (Sch.) - Mz. (S.) -

\section{Erirhinus Schönherr.}

(Diese und die folgende Gattung sind in Sammlung v. Hey den durch die Specialisten We n cker und Tournier revidirt.)

1. E. Festucae Hbst. - Drei Stück von C. H. gef. $>$ 6. Fr. (Hg.) -

2. E. Nereis Payk. - Von Hg. bei Fr. gesammelt. - Wsb. an der Tränkwiese 20. Juni 1852 (K.). -

3. E. scirrhosus Schh. - Von C. H. an Maiabenden bei Fr. auf Sparganium gesammelt. [Die Seligenstädter festucae in S. V. gehören hierher.] - Fr. (Hg.) -

\section{Dorytomus Stephens.}

1. D. vorax F. = macropus Redtb. - Larve $>4$.; der Käfer von Mai bis August bei Fr., Offenbach und Mz. auf Pappeln n. s. von uns und S. gesammelt. - Friedberg (Fuhr). - Fr. einmal am 22. Mai 1860 (B.), auch von Hg. gef. - Had. im Juni (K.). - Ingelheim (B.). -

Var. ventralis Steph. (Ganz blassgelb). - Bei Fr. von C. H. und St. gef. -

2. D. filirostris Schh. = Riehlii Bach. - An der Gerbermühle am Main bei Oberrad h. von L. H. und M. S. gef.; von C. H. und S. bei Mo. $>$ 8. an den Fischteichen. Lebt auf Pappeln. - Fr. 22. Mai 1860 (B.). - Wlb. (Sch.) - Auch von Hg. im Gebiet gesammelt. -

3. D. costirostris Schh. - Auf Pappeln bei Mz. = 8. und $<6$. bei Fr. auf Silberpappel gef. - Von Hg. und B. einigemal bei Fr. gesammelt, - Mo. (S.) - 
4. D. Silbermanni Wenck. - L. H. fand je ein Stück bei Fr. und Neuenhain bei Soden im Taunus.

5. D. taeniatus F. - Die Larve $=5$. in den männlichen Kätzchen von Salix caprea; den Käfer bei Fr. und Soden im Juni gef. Fr. (B.) - Mz. (S.) -

6. D. agnathus Schh. - Bei Fr. zweimal von C. H. gef. -

7. D. tortrix L. - Fr. einzeln. - Falkenstein im Taunus $<9$, - Wlb. (Sch.) - Fr. (Hg.) -

8. D. bituberculatus Zettst. - Bei Fr. zweimal gef. - Wlb. einmal (Sch.). -

9. D. flavipes Panz. - Von Hg. bei Fr. gesammelt. -

10. D. salicinus Gyll. - L. 'H. fand ein Stück bei Fr. -

[Die Bestimmung von affinis Payk. in S. V. scheint fraglich, den Käfer kennt L. H. nur aus Oesterreich und Ungarn.]

11. D. validirostris Schh. - Bei Wlb. ron Sch. gef. -

12. D. occalescens Schh. - Bei Fr, je einmal $=4$. und $<6$. von L. H. auf Silberpappeln in der Promenade gef. -

13: D. minutus Schh. - Zweimal von C. H. in Fr. im botanischen Garten unter Rinden $>3$. gef.

14. D. majalis Payk. - Von C. H. bei Fr. <4. in den Knospen von Salix cinerea und Mitte des Monats bei Bingen auf Salix caprea gesammelt. - Fr. (Hg.) -- Mz. (S.) -

15. D. punctator Hbst. = fructuum Marsh. - Im Fr. Wald $<7$. und September auf Salix caprea. -

16. D. dorsalis L. - Von Hg. einmal bei Fr. gef. --

\section{Pseudostyphlus Tournier (Dorytomus ol, pars).}

1. P. pilumnus Schh. - Einmal im Rebstockwald bei Fr. von C. H. gef. - Wlb. (Sch.) - Fr. (Hg.) -

2. P. infirmus Hbst. - Von C. H. bei Fr. im Dezember unter Baummoos gef. -

\section{Smicronyx schönherr.}

1. S. cicur Schh. = variegatus Schh. - Soden im Juni und Juli auf Dornzäunen. - Mo. - Flörsheim. - Taunus im Königsteiner Wald. - Von C. H. n, s. im Juli auf der Flachsseide, Cuscuta Europaea 
gesammelt, besonders bei Fr. auf der Eschenheimer Landstrasse. - Ems. - Wlb. h. (Sch.) - Fr. (Hg.) -

2. S. coecus Reich (Mit fast kahler Oberseite und sehr kleinen Augen.) - Einmal von C. H. gef. bei Fr. [Bestimmung ron Wencker.] -

\section{Brachonyx Schönherr.}

1. B. pineti Payk. = indigena Hbst. - Auf Kiefern ron $=4$. bis August n. S. - Fr. - Offenbach. - Griesheim am Main. Wlb. (Sch.) - Von B. s. h. im Röderwald und am Königsbrunnen im Fr. Wald gef., anch (zufällig?) in Menge 18. März 1861 bei Formica rufa, hinter dem Fr. Forsthaus. - Mz. (S.) - Mo', auf Kiefern von Mai bis August (K.). -

\section{Tanysphyr'us Germar.}

1. T. Lemnae F. - Bei Fr. und Ems n. s. von C. H. am Ufer von Gewässern gef. - Fr. h. (B., Hg.) -

\section{Anoplus Schönherr.}

1. A. plantaris Naezen (Mann kleiner, kürzeren Rüssel = plantaris Suffr. = depilis Thoms. - Weib $=$ Roboris Suffr. = plantaris Thoms). - Siehe Deutsche entom. Zeitschr. 1876, pag. 190. — Das Männchen auf Birken bei Altenhain im Taunus $>9$. - Fr. - Das Weibchen auf Alnus glutinosa (seltener auf alba) bei Fr. von C. H. $>$ 5. gef. - Schon $>$ 4. bei Falkenstein im Taunus. — Ems (Bach). -

[Der nahe verwandte A. setulosus Kirsch (kenntlich an den weissen aufstehenden Borstenreihen der Flügeldecken, nicht weissen niederliegenden Haaren wie bei plantaris) ist noch nicht im Gebiet beobachtet.]

\section{Bagouls Germar.}

(Die Arten in Sammlung $\nabla$. Heyden rom Monographen Brisout revidirt.)

1. (Hydronomus Schönh.) Alismatis Mrsh. - G. (L.) - Nur einmal bei Fr. von C. H. gef. - Wlb. (Sch.) - Im Genist 11. s., auch an Wasserpflanzen im Mai 1860 bei Enkheim gestreift (B.). - Mz. (S.) - Wsb. Kieskaut 11. Juli h. (K.) -

2. (Lyprus Schh.) cylindrus F. - Drei Stïck von C. H. bei Fr. gef., einmal ron B. - 
3. B. subcarinatus Schh. - Bei Fr. die weniger seltene Art. Von C. H. schon 1812 gef. im Mai am Sumpf hinter dem Hellerhof und im November am ehemaligen Rüstersee. -

[B. frit Gyll. nicht bei Fr. wie in S. V. angeführt.]

4. B. Collignensis Hbst. = lutulentus Schh. = puncticollis Schh. - Bei Fr. von Hg. gesammelt. -

5. B. nigritarsis Thomson. (Seither mit Collignensis verwechselt, Kraatz gab Berl. Entom. Zeitschr. 1871, pag. 169 die Unterschiede.) Bei Fr. einmal von C. H., gef. -

\section{CRYPTORHYNCHINI. \\ Camptorhinus Schönherr.}

1. C. statua F. - Im Gebiet von C. H. in dem Eichenwald zwischen Königstein und Soden unter der alten Rinde noch lebender Eichen im October gef., wo er überwintert. - Wenn er beunruhigt wird, so stellt er sich todt und bleibt lange unbeweglich liegen. Sclrwanheim unter der Rinde alter Eichen (Hg., St.) - Bei Mz. nur einmal in einem Holzmagazin an einem Stïck Buchenholz gesammelt (S.) -

\section{Gasterocercuis Laporte.}

1. G. depressirostris F. - Von St. oft aus Fr. Waldholz im August erzogen. Er variirt in der Grösse (ohne Rüssel) von $8-3^{1 / 2} \mathrm{~mm}$. - Mz. (S.) -

\section{Cryptorhynchus nlliger.}

1. C. Lapathi L. - Bei Fr. an Weiden h. = 8. in Begattung. -Am Metzgerbruch bei Fr. von B., bei Wlb. von Sch. und bei G. von L. gef. - Fr. (Hg.) - Mz. (S.) - Hinter Dotzheim an jungen Eichen 25. Mai (K.). -

\section{Acalles Schönherr.}

1. A. abstersus Schh. - C. H. fand $=6$. ein Stück an Dornzäunen bei Soden im Taunus. - G. (L.) -

2. A. turbatus Schh. - Von Hg. bei Fr. gef. -

Var. misellus Schh. - Von Hg. bei St. Goarshausen gef. -

3. A. ptinoides Mrsh. - Bei Fr. einzeln von C. H. und Hg. gef. - G. (L.) - 
4. A. hypocrita Schh. - Unter Rubus-Gesträuch, unter einem Stein im Juli bei Ems einmal und 1 Stück $<6$. bei Lorsbach im Taunus an einem Buchenstumpf im dunklen Wald von C. H. gef. Mz. (S.) - Wsb. hinter dem Adamsthal an einem Buchenstrunk (K.) -

\section{N. MAGDALININI.}

(Die Sammlung v. Heyden von dem Monographen Desbrochers revidirt.)

\section{Magdalinus Schönherr.}

Mz. (S.) -

1. M. Memnonius Gyll. = carbonarius F. - Fr. (Hg., St.) -

2. M. linearis Gyll. - Auf Kiefern = 6. von C. H., Hg. und B. bei Fr. gef. -

3. M. nitidus Gyll. - Fr. einmal (B.). -

4. M. phlegmaticus Hbst. - Auf Kiefern bei Fr. = 4. gesammelt von uns und Hg. - Mz. (S.) -

5. M. violaceus L. Gyll. = Heydenii Desbroch. - Larve und Käfer im Mai in Menge unter der Rinde und im Holz junger Fichten im Er. Wald im Kesselbruch von uns gef.; aber einmal auch häufig auf Betula alba. - Fr. (Hg.) -

6. M. frontalis Gyll. = violaceus Desbr. nec L. - Von Hg. bei Fr. gef. -

7. M. duplicatus Germ. - Von. C. H. und Hg. bei Fr. gef. $h$. im Kesselbruch $<6$. an Kiefern. - Mo. =6. - Wlb. (Sch.) -

8. M. rufus Germ. - Aus dürrem Fr. Waldholz zweimal erzogen. - L. H. fand die Art bei Mo. auf Kiefern. - Schwanheim (St.). - Wsb. am Schiessplatz 19. April und an der Tränkwiese 20. Juni 1852 (K.). -

9. M. carbonarius $\mathrm{L}$. = atramentarius Germ. = atratus Gyll. - s. - Bei Fr. je einmal von C. H. und 9. Juni 1860 von B. gef., auch von St. gesammelt. -

10. M. Cerasi L. - Fr. h. - Am Feldberg $<6$. auf Eichen, bei Soden bis $=7$. - Fr. im Holzstall, wo nur Buchenholz. Wlb. (Sch.) - Mz. (S.) -

11. M. aterrimus $\mathrm{L}$. = stygius Gyll. = asphaltinus Germ. Bei Fr. und Mo. im Mai h. auf Ulmen von uns, Hg. und St. gesammelt. - Fr. am 16. Mai 1860 (B.). - Wlb. (Sch.) - Mz. (S.) - 
12. M. barbicornis Latr. - Bei Fr. einzeln. - Königstein im Taunus $<6$. - Fr. zweimal (B.). - Enkheim (St.). -

13. M. flavicornis Schh. - s. - Fr., Königstein, Soden $>6$. auf Pflaumenbäumen. - Mz. (S.) -

Var. fuscicornis Desbr. - Einmal von C. H. bei Fr. gef. -

14. M. Pruni L. - Fr. h. $>$ 5. auf Apfelbäumen und $=5$. im Taunus bei Falkenstein anf Sorbus aucuparia. - Wlb. (Sch.) Fr. (Hg.) - Mz. (S.) -

15. M. nitidipennis Schh. - Auf Pappeln am Main zwischen Bürgel und Offenbach $>5$. - Bei Fr. von C. H. einmal und $<6$. von B. gef. -

\section{TYCHIINI.}

\section{Balaninus Germar.}

(Die Arten in Sammlung v. Heyden vom Monographen Desbrochers bestimmt.)

1. B. Elephas Schh. - Beim Fr. Forsthaus im Wald $<9$. s. - Im August aus überwinterten Eicheln erzogen. - Fr. s. (B.) Wlb. (Sch.) - Mz. (S.) -

2. B. pellitus Schh. - Ein Männchen bei Fr. von C. H. gef. -

3. B. glandium Mrsh. = venosus Germ. - Aus Eicheln aus dem Fr. Wald = 5. erzogen. - Wlb. (Sch.) - Fr. (Hg.) - Mz. (S.) -

Var. cinereus Desbr. - Einmal bei Soden auf Eichen $<8$. von C. H. gef. -

4. B. tesselatus Fourcr. = turbatus Schh. - Im Mai aus zweimal überwinterten Eicheln aus dem Fr. Wald oft erzogen, bei Soden $=5$. auf Eichen; im Juli bei Ems von C. H. gef. - Fr. (Hg.) Wlb. (Sch.) -

5. B. nucum L. - Lebt in Haselnüssen. - s. - Ems. - Wlb. Dlb. - Käfer nur fünfmal an Haselnussstauden im August (B.) Fr. von uns und Hg. gesammelt. - Mz. (S.) -

6. B. villosus F. - Die Larve lebt bis $>6$. in den frischen Gallen der Teras terminalis auf Eichen; sie geht zur Verwandlung in die Erde. Der Käfer entwickelt sich schon im März. - Im Taunus bei Soden und Altenhain, sowie bei der Mainkur auf Eichengebüsch h. im Mai. - Dlb. Wlb. h. auf Eichen, auch die Larve, wie angegeben, beobachtet (Sch.) - Fr. (Hg., B.) - Mz. (S.) - 
7. B. Cerasorum Hbst. - C. H. fand ein Weibchen bei Fr. Auch von Hg. einzeln gesammelt. - Wlb. (Sch.) -

8. B. rubidus Gyll. - Ein Männchen von C. H. und einmal von Hg. bei Fr. gesammelt. -

9. (Balanobius Jekel.) crux F. - Bei Fr. n. s. - Hofheim im Taunus $>$ 4. - Fr. auf Weiden (B.). - Wlb. (Sch.) - Fr. (Hg.) -

10. (B.) Brassicae F. - Aus Weidenblattgallen von Fr. erzogen; der Käfer überwintert; im August h. - Wlb. (Sch.) - Fr. (Hg., B.) - Mz. (S.) -

11. (B.) pyrrhoceras Mrsh. - Auf Eichen h. bei Soden im Juli. - Johannisberg bei Friedberg schon $=5$. - Wlb. (Sch.) Fr. (Hg., B.) - Mz. (S.) -

\section{Anthonomus Germar.}

(Die Arten in Sammlung $\nabla$. Heyden vom Monographen Desbrochers bestimmt.)

1. A. rectirostris $\mathrm{L}$. = druparum L. - Lebt auf Kirschbäumen, besonders dem verwilderten und Sauerkirschbaum von $=4$. bis $=6$. - Johannisberg bei Friedberg. - Hofheim im Taunus. Fr. (Hg. und B.) - Wlb. (Sch.) - Mz. (S.) -

2. A. Rubi Hbst. - Fr. Wald, Offenbach, Griesheim im Juli und August. - Bei Bingen von Wagner aus Esparsette-Klee erzogenl. [Exemplar in Sammlung v. Heyden.] - Wlb. (Sch.) - Fr. (Hg., B.) - Mz. (S.) -

3. A. pubescens Payk. - Fr. - Friedberg (Fuhr). - Fr. (L. H. und Hg.) s. -

4. A. varians Payk. - Auf blühenden Kiefern s. h., besonders im April, aber auch noch $>6$. - Bei Fr. $<6$. auf Pinus sylvestris, frisch entwickelt zwischen den männlichen trockenen Blüthen. - Auch bei Mo. und auf der Bieberer Höhe bei Offenbach h. - Auf der Königswiese im Fr. Wald h. gestreift von B., z. B. 21. April 1861. Fr. (Hg.) - Mz. (S.) -

5. A. Pyri Schh. $=$ cinctus Redt. - Von Wagner in Bingen gef. [in Sammlung s. Heyden]. - Mz. (S.) -

6. A. Ulmi De Geer. - Auf Ulmen $=5$. bei Fr. zweimal von C. H. gef. - Wlb. einmal (Sch.), ebenso bei Fr. (B.). -

7. A. rufus Schh. $=$ nitidirostris Desbr. - Einmal von C. H. bei Fr. gef. - 
8. A. Pruni Desbr. - Einmal von Sch. bei Wlb. gef.; seither nur aus Lothringen bekannt, z. B. Metz. -

9. A. pedicularius $L$. $=$ Schönherri Desbr. - Bei Fr. einmal auf blühendem Weissdorn gef. - Auch von Hg. gesammelt. - St. Goarshausen (K.). -

10. A. conspersus Desbr. - L. H. fand einmal eine Anzahl Exemplare auf blühendem Sorbus aucuparia auf der Chaussee von Reifenberg nach dem Feldberg im Taunus. - [Hierher cinctus in S. V. ans dem Taunus.] -

11. A. Pomorum L. - Aus Áepfelblüthen $<6$. entwickelt, aber auch $=5$. bei Falkenstein von Schlehen und bei Königstein von Sorbus aucuparia geklopft. - Ueberall h.; ïberwintert unter Laub und Baumrinden, z. B. bei Soden im October. gef. - Enkheim auf dürrem Eichengebüsch im April (Obstbäume in der Nähe). - Fr. (Hg., B.) - Wlb. Dlb. an Apfelbäumen (Sch.) - Mz. (S.) -

\section{Acalyptus Schönherr.}

1. A. Carpini Hbst. - Von St. bei Fr. gesammelt. -

2. A. rufipennis Schh. = alpinus Comolli. - Einmal $=8$. von C. H. im Fr. Wald (Bruchschneisse) von Populus tremula geklopft. -

\section{Elleschus Schönherr.}

1. E. scanicus Payk. - Einmal $=5$. an der Mauer eines Hauses in Fr. von C. H. gef. - G. (L.) - Fr. (Hg.) -

2. E. bipunctatus L. - Im April und Mai in den Blüthen von Salix caprea bei Fr., Falkenstein und Königstein n. s. s. - G. (L.) — Fr. (Hg., St.) - Mz. (S.) -

\section{Lignyodes Schönherr.}

1. L. enucleator Panz. - Von M. S. 1856 an der Gerbermühle am Main bei Oberrad einmal gestreift, später an demselben Orte 1860 von B. gef. - An den Rödelheimer Dämmen zwischen Bockenheim und dem Rebstockwald von v. T'wardowsky h. von blühenden Eschen geklopft von Juni bis Juli 1860. - L. H. fand an demselben Orte einige Stücke 28. Mai 1875. - Mz. (S.) - 


\section{Tychius Germar.}

(Die Arten in Sammlung v. Heyden von C. Brisout und Tournier bestimmt.)

1. T. quinquepunctatus L. - Bei Fr. im Juni und Juli auf Wiesen n. s. - Rebstockwald. - Ems. - Rüdesheim = 8. - Fr. Wald h. in der Sonnenhitze gestreift (B.). - Wlb. (Sch.) - Fr. (Hg.) -

Eine Varietät, bei welcher die Flecken, sowie die ganze Oberseite durch eine seidenglänzende graue Behaarung verdeckt sind, fand $\mathrm{L}$. $\mathrm{H}$. einmal bei Soden. -

2. T. polylineatus Germ. - Von Hg. bei Fr. gef. -

3. T. Schneideri Hbst. = lineatulus Steph. Bris. - Im. Falkensteiner Wald $>$ 8. einmal von C. H. gef. - Fr. (Hg.) -

4. T. venustus $\mathbf{F}$. - Lebt auf Spartium scoparium. $=4$. in Begattung. - Fr. - Bieberer Höhe bei Offenbach. - Falkenstein im Taunus = 5. - Friedberg (Fuhr). - Wlb. (Sch.) - Staufen im Taunus 2. April 1876. - Fr. h. (Hg., B.) - Mz. (S.) -

Var. genistae Schh. (Einfarbig grau ohne Längsbinden.) Zweimal von C. H. bei Fr. gef. - Wlb. (Sch.) -

5. T. flavicollis Schh. = curtus Bris. - Zweimal bei Fr. im Rebstockwald im Juni von C. H. gef. -

6. T. junceus Reich. - Nauheim einmal $>5$., bei Budenheim am Rhein $>$ 7. oft auf Medicago officinalis von C. H. gesammelt. Wlb. (Sch.) - Mz. (S.) -

7. T. Meliloti Steph. - Ein Weibchen < 7. am Forsthaus im Fr. Wald von C. H. gef. -

8. T. tomentosus Hbst. - Im Juni und Juli bei Fr. im Rebstockwald und Soden n. s. - Wlb. (Sch.) - Fr. (Hg.) -

9. (Miccotrogus Schönh.) picirostris F. - Fr. im Juni h. auf Wiesen mit dem Streifnetz gef. - Ems. - Wlb. (Sch.) - Fr. (Hg.) - Mz. (S.) -

\section{Sibinia Germar (= Sibynes Schönh.).}

1. S. primita Hbst. - Bei Fr. von C. H. und Hg. öfter gesammelt $>$ 7. - Wlb. (Sch.) - Nürnbergerhof im Rheingau 11. September (K.). -

Var. phalerata Stev. - Einmal bei Fr. gef. - Mz. (S.) -

2. S. cana Hbst. - Bei Fr. = 6. auf Silene nutans von C. H. gesammelt. - 
3. S. Viscariae L. - Bei Fr. s. gesammelt. - Bei Rüdesheim am Rhein fand C. H. die Art $<6$. auf Isatis tinctoria. -

4. S. fugax Germ. - Auf dem jetzt zum Theil urbar gemachten Lerchesberg über Sachsenhausen, früher oft von C. H. auf Cucubalus Behen gesammelt im Juli. -

5. S. Potentillae Germ. - C. H. fand die Art einzeln bei Fr. im Mai auf Erica, auch von Hg. und B. bei Fr. gesammelt. -

\section{Mecinus Germar.}

1. M. pyraster Hbst. - Im. Fr. Wald von uns und Hg. bei der Ziegelhütte gesammelt. - Ueberwintert unter Rinde. - Soden. Rüdesheim $<5 . \quad$ Flörsheim $=8$. - Fr. 3 Stück (B.). Wlb. (Sch.) -

2. M. collaris Germ. - L. H. fing 1 Ex. 27. Juni 1856 mit dem Streifnetz an der Gerbermühle bei Oberrad am Main. -

3. M. janthinus Germ. - In Sammlung v. Heyden befindet sich ein von B. bei Fr. gesammeltes Stück. - Fr. (Hg.) -

4. M. Heydeni Wencker. - C. H. fand vier Exemplare (sonst nur zwei Stück aus Hagenau im Elsass bekannt) $>5$. im Fr. Wald an der Bruchschneisse in Sandgräben, eins $<.5$. auf Linaria und $z$ wei $<$ 7. im Fr. Wald am Forsthaus mit dem Streifnetz. -

\section{Gymnetron Schönherr.}

(Sammlung $\nabla$. Heyden vom Monographen Brisout revidirt.)

1. G. pascuorum Gyll. Var. bicolor Schh. - C. H. fand $=9$. ein Stück bei Königstein im Taunus. - Fr. von Hg. und öfter von St. gesammelt. -

2. G. Beccabungae L. = concinnus Schh. - Bei Fr. im Mai und Juni an Gräben mit dem Streifnetz gef. - Bei Soden = 5. auf Veronica Beccabunga von C. H. gesammelt. - Mz. (S.) -

Var. Veronicae Germ. - Wlb. (Sch.) - Fr. (Hg.) -

3. G. labilis Hbst. - Auf kahlen Hügeln $>8$. bei Griesheim am Main und im Fr. Wald an der Louisa im Mai mit dem Streifnetz einzeln gesammelt. - Fr. (Hg.) - Ems (Bach). -

4. G. stimulosus Germ. - Einmal von C. H. bei Flörsheim am Main gef. - 
5. G. melanarius Germ. = perparvulus Schh. - Bei Bergen $>$ 5. ron uns n. s. auf Veronica teucrium gesammelt. - Wlb. (Sch.) -

6. G. asellus Grav. - Auf Verbascum im Fr. Wald (Waldan) $=5$. und bei Rumpenheim < 7. gesammelt. - Fr. (Hg.) - Mr. (S.) -

7. G. netus Germ. - Von C. H. bei Rïdesheim = 8. gef. Ems (Bach). -

8. G. spilotus Germ. - Von Dr. Richter einmal bei der Irrenanstalt Eichberg im Rheingau gef. -

9. G. Linariae Panz. - Fr. $<5$. in Blüthen von Linaria, auch in Anemone nemoralis. - Bei Wlb. gezogen ans Gallen ron Linaria vulgaris (Sch.). - Fr. (Hg., St.) -

10. G. teter F. - Bei Ems von C. H. gesammelt. - Wlb. (Sch.) - Fr. (Hg., St.) -

11. G. Antirrhini Germ. - Auf Verbascum Thapsus im Juni und Juli im Fr. Wald; im Angust bei Griesheim; = 5. auf Carlina bei Offenbach. - Fr. (Hg., St.) - Mz. (S.) -

12. G. noctis Hbst. - Bei Fr. im Juli auf Linaria vulgaris und $=$ 8. bei Rüdesheim am Rhein. - Wlb. (Sch.) - Fr. (Hg.) -

13. G. pilosus Schönh. - Fr. $=7$. in haselnussgrossen Gallen des Stengels von Linaria vulgaris gef. Die schon entwickelten Käfer blieben bis zum nächsten Jahr in den vertrockneten Gallen sitzen. Fr. (B.) -

\section{Miarus Stephens.}

1. M. graminis Schh. - Von $>6$. bis $=8$. in Blüthen ron Campanula Fr. Wald. - Ems. - Wlb. (Sch.) - Fr. (Hg.) -

2. M. Campanulae L. - Bei $\mathrm{Fl}$. auf Lychnis viscaria, bei MIo. =6. auf Cerastium und bei Falkenstein auf Campanula. - Wlb. (Sch.) - Fr. (Hg., B.) - Mr. (S.) -

3. M. micros Germ. - Im August bei Fr. eimmal auf Erica gef., auch von Hg. gesammelt. -

\section{Cionus Clairville.}

1. C. Scrophulariae L. - Fr. h. anf Scrophutaria nodosa ron uns, Hg., St. und B. gef. - Wlb. (Sch.) - G. (L.) - Mz., (S.) - 
2. C. Verbasci F. - Fr. n. s. auf Verbascum. - Wlb. (Sch.) G. (L.) - Fr. (Hg., St.) — Mz. (S.) -

3. C. Olivieri Rosh. - Bei Fr. S. - Wlb. einmal gef. (Sch.) -

4. C. Thapsus F. - Fr. n. h. - Wlb. s. (Sch.) - G. (L.) Lebt auf Verbascum thapsus. - Fr. (Hg., St.) - Mz. (S.) -

5. C. hortulanus Mrsh. - Fr. im Juli h. - Wlb. (Sch.) G. (L.) - Fr. (Hg., St. und B.) -

6. C. olens F. - Ems. - Fr. Larve $<6$, Käfer $>6$. auf Verbascum h. - Mz. (S.) -

7. C. Blattariae F. - Auf Scrophularia nodosa n. s. von $=4$. bis $<$ 7. - Enkheim. - Bingen. - G. (L.) - Fr. (wir, St., Hg., B.) - Mz. (S.) -

8. C. pulchellus Hbst. - Wlb. s. (Sch.) - Fr. von St. oft gef., auch von $\mathrm{Hg}$. und B. -

9. C. Solani F. - Fr. Wald an der Grastränke = 5. auf Scrophularia. - > 6. bei St. Goarshausen von C. H. gef. -

10. (Stereonychus Suffr.) Fraxini De Geer. - C. H. fand $=5$. im Fr. Wald an der Babenhäuser Landstrasse an einem Grasstengel einen Cocon, aus welchem sich $<6$. der Käfer entwickelte. Fr. (Hg.) -

\section{Nanophyes Schönherr.}

1. (Sphaerula Kiesw.) hemisphaericus Oliv. - Einmal von C. H. bei Fr. im Rödellheimerwald gef. = 8. -

2. (S.) Lythri F. - Ueberall h. anf Lythrum salicaria; besonders $>$ 9. bei Soden und Fr. - Enkheim (B.) - Wlb. (Sch.) - Fr. (Hg.) - Schwanheim (St.) - Mz. (S.) -

\section{Orchestes Illiger.}

1. O. Quercus L. - Von $=7$. bis $=9$. auf Eichen h. bei Soden im Taunus; die schwarze Larve in Eichenblätter $=6$. minirend von C. H. gef. - Fr. n. s. (Hg., B.) - Wlb. (Sch.) - Mz. (S.) -

2. O. rufus Oliv. (Mit rothem Hinterleib.) - Stammart nicht aus dem Gebiet bekannt. -

Var. haematicus Germ. - Bei Mo. und Rüdesheim im August auf Ulmen. - Wlb. (Sch.) - Fr. (Hg.) - 
3. 0. scutellaris F. = carnifex Germ. - Aus Erlenblätter aus dem Isenburger Wald entwickelt einmal $>6$. - Ein Stück auf dem Johannisbèrg bei Friedberg $=5$. von C. H. gef. -

4. 0. Alni L. (Stammart mit schwarzem Fleck auf jeder. Flügeldecke.) - Bei Fr. auf Ulmen s. von C. H. beim Hellerhof gesammelt. - Fr. (Hg., B.) - Wsb. an der Wellritz am Bleichplatz auf Erlen 29. Juni 1852 (K.). -

[Var. ferrugineus Mrsh. (Oberseite einfarbig roth.) - Nicht aus dem Gebiet bekannt.] -

Var. atricapillus Mrsh. = melanocephalus Oliv. (Oberseite einfarbig gelb.) - Einmal bei Fr. gef. $<6$. -

5. O. Ilicis F. - Auf Eichen bei Enkheim, Fr. und Soden von $=5$. bis $>8$. s. h. - Die Larve minirt $>5$. in Eichenblättern, entwickelt sich $=6$. - Wlb. (Sch.) - Fr. $\left(\mathrm{Hg}_{.}\right)-\mathrm{Mz}$. (S.) -

6. O. Jota F. = Rosae Hbst. - Bei Fr. s. von uns und Hg. gef. -

7. O. Fagi L. - Auf Buchen s. h., überwintert unter Rinden und Laub. - Fr. - Feldberg. - Von April bis Juni. - Aus Weidenblättern $>9$. entwickelt, worin die Larve minirte. - Wlb. (Sch.) Fr. (Hg. B.) - Mz. (S.) -

8. 0. pratensis Germ. = Waltoni Curtis. - Einmal von C. H. bei Fr. mit dem Streifnetz gef. auf Wiesen hinter Rödelheim $<6$. -

9. O. erythropus Germ. - Auf Eichen bei Soden von $<7$. bis $>$ 9. öfter von uns gesammelt. - Fr. (Hg.) -

10. 0. Lonicerae Herbst. - Lebt auf Lonicera xylosteum, besonders von $=4$. bis $=5$. - Bei Fr., Falkenstein, Bingeni und Rüdesheim von uns n. s. gesammelt. - Schmitterhof bei Giessen noch $>8$. - Wlb. (Sch.) - Fr. (Hg.) - Mz. (S.) -

11. O. Populi F. - Auf Pappeln und Weiden h. von Mai bis August. - Fr., Rödelheim schon im frühesten Frühjahr von B. gef. - Wlb. (Sch.) - Fr. (Hg.) - Mz. (S.) -

12. 0. Rusci Hbst. = bifasciatus Illig. - Fr. s. s. - Auf Birken bei Königstein im Taunus =6. von C. H. gef. - Fr. (Hg., B.) -

13. 0. Avellanae Donovan $=$ signifer Crtz. - Auf Eichen von $>$ 5. bis $=8$. - Fr. Wald am Forsthaus. - Soden. - Enkheim. - Rödelheim. - Am Feldberg. - >11. abgeschuppte Exemplare gef. - Wilb. (Sch.) - Fr. (Hg.) - Mr. (S.) - 


\section{$-277-$}

14. (Tachyerges Schönh.) stigma Germ. = Jota Payk. Lebt auf Weiden von $=5$. bis Juli. - Fr. - Offenbach. - Hofheim im Taunus. - Wlb. h. (Sch.) - Fr. (Hg.) -

15. (T.) saliceti F. - Auf Weiden im Juni und Juli. - Offenbach. - Rebstockwald bei Fr. - Mr. (S.) - Ems (Hg.). -

16. (T.) decoratus Germ. - Auf Weiden von $<5$. bis $>8$. - Hofheim im Taunus. - Mo. - Wlb. (Sch.) - Fr. (L. H. und Hg.) - Mz. (S.) -

17. (T.) Salicis L. - Auf Weiden vom April bis = 5., dann wieder im August. - Die Larve $>6$. in Blättern von Salix caprea minirend, entwickelt $=7$. - Fr, auch von B. und Hg. gef. - Hofheim. - Falkenstein. - Wlb. (Sch.) - Mz. (S.) -

Var. minor (nur halb so gross wie normale Stücke). - Auf Weiden bei Offenbach einzeln $=5$. -

18. (T.) rufitarsis Germ. = confundatus Steph. - s. s. Ems einmal von C. H. gef. -

\section{Rhamphus Clairville.}

1. R. flavicornis Clairv. - Die Larve (von C. H. beschrieben Berl. Entom. Zeitschr. 1862, pag. 63) minirt in den Blättern von Kirschen, Schlehen, Birnen, Aepfeln, Birken, Sahlweiden und Pappelı $>10$.; der Käfer entwickelt sich $>4$., zu welcher Zeit man ihn h. findet. - Fr. - Soden. - Königstein. - Bingen von Wagner in Kirschblättern gef. - Einige Exemplare zeigen einen grünlichen Schimmer; zweifelhaft ob sie zu aeneus zu stellen sind. - Wlb. einmal (Sch.). Mz. (S.) -

\section{P. CEUTORHYNCHINI.}

Mononychus Germar.

1. M. Pseudacori F. - Von Scheidel im Schwanheimer Wald gesammelt. - Auch von Hg. oft gef., sowie von St. - Mr. (S.) -

2. M. Salviae Germ. - Hg. fand 1 Ex. dieses südlichen Thieres bei Fr. -

\section{Coeliodes Stephens.}

1. C. Epilobii Payk. - Auf Epilobium angustifolium im Fr. Wald h. im Juli von uns gesammelt. - Fr. h. (Hg., B.) - Mz. (S.) - 
2. C. Geranii Payk. - Bei Fr. = 6. auf Geranium pratense von L. H. und im Sanerthal bei Lorch am Rhein auf Geranium sanguineum <6. von C. H. 1. s. gesammelt. - Mr. (S.) - Offenbach. Fr. (Hg., B.) -

Var. exiguus Oliv. - Fr. ron Hg. und einmal nach B. von Fresenius gef. - Wlb. h. (Sch.) -

3. (Stenocarus Thoms.) guttula F. Gyll. = fuliginosus Thoms. - Bei Fr. einmal von C. H. gef. - Fr. Wald h. (Hg., B.) - $\mathrm{Mz}$. (S.) -

4. (St.) fuliginosus Mrsh. - Im Närz und April an Hänsern und im Flug gef. - Bei Hausen auf der Erde in trockenen Wiesen $=9$. - Fr. zweimal 20. März 1860 (B.), auch von Hg. gesammelt. Mz. (S.) -

5. (Cidnorhinus Thoms.) quadrimaculatus $L$. $=$ didymus F. Im Juni und Juli im Fr. und Rebstocker Wald; Offenbach. - Fr. h. (Hg., B.) - Wlb. (Sch.) - Mz. (S.) -

6. (Megacetes Thoms.) Quercus F. - Auf Eichen $>6$. bei Soden n. s.; im Fr. Wald am Forsthaus $<7$. im April bei Bingeu und $=5$. auf dem Johannisberg bei Friedberg gesammelt. - Wlb. (Sch.) - Fr. (Hg.) - Mz. (S.) -

7. (M.) ruber Marsh. - Bei Fr. von Hg. gesammelt. -

8. (M.) rubicundus Payk. - Einmal $=5$. im Fr. Wald von C. H. gef.; auch von Hg. gesammelt. -

9. (M.) subrufus Hbst. = trifasciatus Bach. - Bei Soden im Juni und Juli n. s. auf Eichen. - Wlb. (Sch.) - Fr. (Hg.) Mz. (S.) -

10. (M.) Lamii Hbst. - Bei Soden im Juli auf Hecken und im Gras. - Wlb. h. (Sch.) - Fr. (Hg.) - Mz. (S.) -

\section{Scleropterus Schönherr.}

1. (Rhytidosomus Schh.) globulus Payk. - Von C. H. auf Populus tremula bei Fr. einmal $=5$. gef. - Fr. einmal (B.), ebenso Hg. - Mz. (S.) -

\section{Marmaropus Schönherr.}

1. M. topiarius Germ. - Bei Fr. je einmal gef. von C. H., St. und B. - Mz. (S.) - 


\section{Rhinoneus schönherr.}

1. R. Castor F. - Vor $>3$. bis August bei Fr. gesammelt in sandigen Gegenden. - Fr. Wald, Griesheim, Bürgel am Main. Wlb. (Sch.) - Fr. (Hg.) - Mz. (S.) -

2. R. guttalis Schh. = subfasciatus Schh. - Auf Polygonum avicularia $=7$. von C. H. gesammelt; Frankfurter, Rebstöcker und Rödelheimer Wald von Juni bis August; im Sand am Mainufer im Mai. - Wlb. (Sch.) - Fr. (Hg.) -

3. R. pericarpius F. - Auf Rumex $=5$. von C. H. gef. Von April bis Juli. - Fr., Soden, Offenbach. - Fr. (Hg., B.) Wlb. (Sch.) - Mz. (S.) -

4. R. inconspectius Hbst. - Bei Fr. im März unter Riedgräsern; $<$ 8. am Entensee zwischen Bürgel und Rumpenheim. - Wlb. (Sch.) Fr. (Hg., St.) - Mz. (S.) -

5. R. bruchoides Hbst. - Im Fr. Wald (Bruchschneisse) in Sandgraben $>4$. - Hüpft nach der Beobachtung von C. H. zollweit. - Wlb. (Sch.) - Fr. (Hg.) - Nr. (S.) --

\section{Phytobius Schönherr.}

(Dr. Kirsch gab in der Deutschen Entom. Zeitschr. 1875, pag. 398, eine vortreffliche Auseinandersetzung der Gattung.)

1. P. Comari Hbst. - Je einmal von C. H. und Hg. bei Fr. gef. -

2. P. quadrituberculatus F. - Im Fr. Wald beim Forsthaus $=3$., $>4$. bei Bergen und $>$ 8. auf dem Schmitterhof bei Giessen gesammelt.

3. P. velaris Schh. - Einmal von C. H. bei Fr. gef. -

4. P. Waltoni Schh. - Wie der vorige. -

5. P. quadrinodosus Schh. - Im Fr. Wald auf der Bruchschneisse in Sandgräben $>4$. zweimal von C. H. gesammelt. -

6. P. quadricornis Gyll. - Bei Rödelheim im März einmal von C. H. gef. -

7. (Eubrychius Thoms.) velatus Beck - Von M. S. einmal bei Fr. gef. - [In Sammlung v. Heyden.] -

\section{Amalus Schönherr.}

1. A. scortillum Hbst. - Im Mai auf Erica bei Fr. und $<6$. bei Altenhain bei Soden im Taunus gesammelt. - Fr. (B.) - Mz. (S.) - 


\section{Orobitis Germar.}

1. O. cyaneus L. - Fr. 2 Ex. von C. H. gesammelt, L. H. fand bei Homburg ein Stück in dem. Magen einer Kröte. - Im Schwanheimer Wald s. gestreift (B.) - Wlb. 2 Ex. (Sch.) - Wsb. im Gras am Waldrand beim Geisberg 20. Juni 1852 (K.). -

\section{Ceutorhynchidius Duval.}

1. C. troglodytes F. - Ueberall h. vom April bis September. Offenbach. - Bieberer Höhe. - Ems (1833). — Wlb. (Sch.) Fr. (Hg., B.) - Mz. (S.) -

2. C. horridus Panz. - Von Hg. zweimal bei Fr. gef. -

3. C. floralis Payk. - Ueberall auf Pflanzen; von April bis Juni z. B. auf Kiefern, Disteln, Lonicera, Capsella otc., auf welchen aber die Larve nicht lebt. - Johannisberg bei Nauheim. — Soden. - Hofheim. - Mo. - Bieberer Höhe bei Offenbach. - Wlb. (Sch.) Fr. (Hg.) - Mz. (S.) -

4. C. pyrrorhynchus Marsh. - Bei Fr. von Hg. gesammelt. -

5. C. nigrinus Mrsh. $=$ depressicollis Schönh. - Zweimal bei Fr. gef, von C. H. und Hg. -

6. C. pulvinatus Scḥh. - Bei Fr. einigemal auf Sysimbrinm gef.; St. Goarshausen $>6$. - Mz. (S.) -

7. C. pumilio Gyll. - Im Gebiet h. von $=4$. bis $>6$. Fr. Wald. - Soden. - Bei Rödelheim öfter auf Capsella bursa pastoris gef. im Juni. - Fr. (Hg.) -

8. C. terminatus Hbst. = apicalis Gyll. - Bei Fr. einzeln gesammelt von C. H., einmal von B. - Wlb. (Sch.) -

\section{Ceutorhynchus Germar.}

1. C. macula alba Hbst. - Bei Fr. öfter gesammelt von uns und Hg. - Mz. (S.) -

2. C. suturalis F. - Bei Fr. einmal von B. am 13. März 1860 gef., auch von Hg. - Mz. (S.) -

3. C. syrites Germ. - Im Rebstockwald bei Fr. im Juni einmal. - Wlb. (Sch.) - Fr. (Hg.) -

4. C. assimilis Payk. - Von $=4$. bis $=8$. h. - Offenbach. - Soden. - Bingen. - Bei Rüdesheim auf Sysimbrium Sophia $=8$. 
- Griesheim am Main, Rödelheim. - In Menge auf den Blüthen von Brassica Rapae bei Wlb. (Sch.) - Fr. (Hg.) - Mz. (S.) -

5. C. Erysimi F. - Im Rödelheimer Wald h. im Juni. - Enkheimer Torfbrüche im Mai auf Capsella. $-<6$. bei Nauheim auf Lepidium graminifolium. - Bei Fr. schon 29. April 1860 von B. gef. - Wlb. h. (Sch.) - Fr. (Hg.) - Mz. (S.) -

6. C. contractus Mrsh. - Von $=5$. bis August n. s. - Enkheim auf Bursella, Nauheim auf Lepidium graminifolium. - Fr. und Rebstöcker Wald. - Wlb. (Sch.) - Fr. (Hg., B.) - Mz. (S.) -

7. C. setosus Schh. - Fr. öfter gef. - Beim Forsthaus $=3$. - Schmitterhof bei Giessen > 8. - Wlb. (Sch.) -

8. C. Cochleariae Gyll. - Soden auf Wiesen $>4$. - Wlb. (Sch.) - Fr. (Hg.) - Mz. (S.) -

Var. atratulus Gyll. - In der sogen. „Hölle“ bei Gronau in der Wetterau = 8. auf Xylosteum von C. H. gef. - Fr. (Hg.) -

9. C. constrictus Mrsh. - Soden im Gras $=6$. s. - Wlb. (Sch.) Fr. (C. H., Hg.) -

10. C. versicolor Bris. (Vom Autor bestimmt.) - Einmal auf den Rödelheimer Wiesen $=8$. und ein Stück bei Fr. gef. - Mz. (S.) -

11. C. nanus Schh. - Bei Bergen auf Alyssum calicinum von C. H. $>5$. bei Mo. im Mai und $<6$. von L. H. gesammelt. Fr. (Hg.) - Mz. (S.) -

12. Ericae Schh. - Auf Haidekrant h. Fr. Wald im August; bei Cronberg im Taunus < 6. - Wlb. (Sch.) - Fr. (Hg.) - Mz. (S.) -

13. C. Echii F. - Auf Echium überall h. = 7. - Bieberer Höhe bei Offenbach. - Kleinkarben (B.). - Wlb. (Sch.) - Fr. (Hg.) - Mz. (S.) -

14. C. Raphani F. - Fr. n. s., in den Enkheimer Torfbrüchen $<$ 8. - Mz. (S.) -

15. C. abbreviatulus F. - Auf Echium und Cynoglossum bei Fr. und Enkheim $<$ 5. und $<$ 8. gesammelt. - Fr. (Hg., St., B.) - Mz. (S.) -

16. C. crucifer Oliv. - Auf Cynoglossum officinale im Fr. Wald von Mai bis Juli öfter gesammelt, auch von $\mathrm{Hg}$. und St. gef. -

17. C. litura F. - 4 Ex, bei Fr. von C. H. gesammelt. Mz. (S.) - 
18. C. trimaculatus F. - Auf Disteln bei Fr. (Bornheim) öfter gesammelt. - Friedberg (Fuhr). - Mz. (S.) -

19. C. asperifoliarum Schh. - Fr. im Mai auf Crnoglossum. Ueberwintert unter Moos. - Wlh. (Sch.) - Fr. (Hg., B.) - Mz. (S.) -

20. C. campestris Schh. - Ems. - Schmitterhof bei Giessen $>$ t. - Wlb. (Sch.) - Fr. (Hg.) - Mz. (S.) -

21. C. signatus Schh. - Auf der Bieberer Höhe bei Offenbach $=6$. einmal von C. H. gef. - Mz. (S.) -

22. C. albosignatus Schh. - Von Hg. bei Fr. gef. -

23. C. molitor Schh. - 3 stïck bei Fr. von C. H. gesammelt. -

24. C. rugulosus Hbst. - Im Juli und August auf Achillea millefolium bei Fr. - Rödelheimer Wald. - Wlb. (Sch.) -

25. C. melanostictus Marsh. - Im Fr. Wald an der Obersaustieg'e am Sumpf im August. - Bergen > 5. - Fr. (Hg.) - Mz. (S.) -

26. C. Euphorbiae Bris. (Vom Autor bestimmt.) - Einmal bei Fr. von C. H. gef. -

27. C. quadridens Panz. - Auf der Bieberer Höhe $>3$. auf Fichten von L. H., bei Soden auf Hecken im Juli ron C. H. gef. -

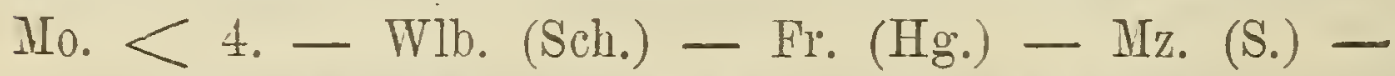

28. C. marginatus Payk. = punctiger Schh. - Fr. Wald auf der Bruchschneisse in Sandgräben $<4$. Rebstockwald im Juni. - Bei Wib. von Sch. gesammelt. - Fr. (Hg.) -

29. C. denticulatus Schrk. - Fr. s. - Fr. (Hg., B.) Mz. (S.) -

30. C. rusticus Schh. - Einmal von C. H. bei Fr. gef. -

31. C. pollinarius Forst. - Bei Fr. sehr einzeln gef. G. (L.) -

32. C. angulosus Schh. - Bei Fr. einmal von C. H. gef. -

33. C. picitarsis Sch. = tarsalis Schh. - 4 Ex. bei Fr. gesammelt. - Auch ron Hg. gef. -

34. C. sulcicollis Schh. - Nach der Beobachtung ron C. H. zerstört er bei Fr. in Nenge die Blätter des Rapses. - Bei Wlb. auch von Sch. unter denselben Umständen beobachtet. - St. Goarshausen $>6$. - Auch auf Sysimbrium amphibium im Nai. - Budenheim am Rhein.

- Wlb. (Sch.) - Fr. (Hg., B.) - Mz. (S.) -

35. C. Rapae Schh. - Bei Fr. von C. H. auf Erysimum gesammelt; auch von Hg. gef. - Mz. (S.) - 
36. C. Napi Schh. - Fr, h. in angeschwemmtem Genist. Mz. (S.) -

37. C. aeneicollis Germ. - Bei Nauheim auf Lepidium graminifolium $<6$. von C. H. zweimal gef. -

38. C. cyanipennis Germ. - Bei Fr. gef. von C. H., Hg. und B. - Wlb. ebenso (Sch.). --

39. C. chalybaeus Germ. - Im Juli bei Fr. von C. H. und Hg. gesammelt. - Mz. (S.) -

40. C. pubicollis Schh. - Im Fr. Wald öfter gef., $<7$. am Forsthaus. -

[Der von Fuhr bei Friedberg gesammelte quercicola F., in S. V. erwähnt, ist eine nicht zu enträthselnde Art.]

\section{Poophagus Schönherr.}

1. P. Sisymbrii F. - Im Mai auf Sisymbrium amphibium bei Fr. von C. H. gef., von $\mathrm{Hg}$., L. H. und $\mathrm{B}_{0}=4$. in den Enkheimer Torfgruben. - G. (L.) - Mz. (S.) -

\section{Tapinotus Schönherr.}

1. T. sellatus F. - Im Fr. Wald an der Grastränke, Kesselbruch und Hainerbruch von $=5$. bis $<7$. auf Lysimachia vulgaris s. von uns, Hg. und B. gesammelt; am Rumpenheimer Tümpel am Schiessstand noch $=9 .-$ G. (L.) - Mz. (S.) -

\section{Coryssomerus Schönherr.}

1. C. capucinus Beck = Ardea Germ. - Bei Fr. von C. H. und $\mathrm{Hg}$. öfter gesammelt. - Griesheim am Main < 8. - Nach B. beinahe s. h. zu nennen; in der Sonnenhitze an Wegrändern abgestreift, auch im Genist; am häufigsten $<5 .-$ G. (L.) - Wlb. (Sch.) Mz. (S.) -

\section{Baridius Schönherr.}

(Die Sammlung v. Heyden von dem Monographen Brisout revidirt.)

1. B. morio Schh. = Resedae Bach. - Einmal von C. H. bei Fr. gesammelt.

2. B. Artemisiae Hbst. - Bei Fr. n. s. von C. H., Hg., St. und B. gesammelt. - Bei Näuheim $>6$. auf Artemisia vulgaris, bei Enkheim < 8. gef. - Wlb. (Sch.) - G. (L.) - 
3. B. sulcipennis Bris. - Das einzig bekannte Exemplar dieses merkwürdigen 'Thieres wurde von C. H. bei Fr. gesammelt. Es ist schwarz, sehr kurz und breit, die Flügeldecken mit sehr tiefen Furchen, die Zwischenräume mit einer Reihe sehr grosser Punkte. -

4. B. laticollis Mrsh. = picinus Germ. - Bei Fr. von C. H. öfter $>5$. in Sandgruben, von L. H. bei Mo. zu derselben Zeit bis $=6$. im Sand unter Steinen gesammelt. - Fr. (Hg., St.) - Mz. (S.) -

5. B. caerulescens Scop. - An Häusern und Mauern bei Fr. im April n. s. auf dem Lerchesberg über Sachsenhausen unter Steinen $=5$. von C. H. gesammelt. - G. (L.) - Mz. (S.) - Fr. (Hg., B.) -

Var. chloris F. - Von C. H., Hg. und B. einzeln bei Fr. gesammelt. -

6. B. fallax Bris. - Einmal bei Fr. von C. H. gef. - Sonst nur wenige Stücke aus Frankreich bekannt; von L. H. schon Berliner Entom. Zeitung 1867, pag. 380 erwähnt.

7. B. Lepidii Germ. - Bèi Fr. n. s. gesammelt, auch von Hg., St. und B. - G. (L.) -

8. B. picicornis Mrsh. = Abrotani Germ. - Im Rebstöckerwald bei Fr. und bei Flörsheim im Juni je einmal von C. H. gef. Fr. (St.) - Mz. (S.) -

9. B. T-album L. - Bei Fr. öfter gesammelt. - L. H. fing 1 Ex. auf den Torfwiesen bei Enkheim $>5$. - [Bei Hannover lebt die Art auf Cladium germanicum.] - Wlb. 1 Stück (Sch.). - Fr. (Hg., St., B.) - Mz. (S.) - Wsb. an der Wellritz auf Wiesen im Mai (K.). -

Var. pusio Schh. - Fr. zweimal von C. H. gef. -

\section{Q. CALANDRINI.}

\section{Sphenophorus Schönherr.}

1. S. mutillatus Laicharting. - [Hierher der abbreviatus unseres Gebietes, welche Art nur in Süd-Europa vorkommt.] - Bei mutillatus sind die abwechselnden Zwischenräume schmäler und enger punktirt, die Flügeldecken kürzer. - Mz. (Dr. Zitz). - L. H. fand den Käfer n. s. $=5$. auf sandigen Wegen laufend zwischen Mo. und Budenheim, bei Rüdesheim und Flörsheim am Main; bei Ingelheim 1875 zahlreich von B. gesammelt. - Bei Fr. dreimal am Metzgerbruch 1874 von B, und 
einmal in den Bockenheimer Wiesen nach dem Hellerhof zu nach einer Ueberschwemmung am 30. März 1875, am Kettenhof 27. April 1876. - Wsb. am Nerothal 18. April (K.). -

\section{Calandra Clairville (= Sitophilus Schönh.).}

1. C. granarius L. - Ueberall h. im Gebiet an Häusern; auf Kornspeichern dem Getreide schädlich, als „schwarzer Kornwurm“ allgemein bekannt. - Fr. - Soden. - Nach B. in Fr. 1870 massenhaft in Gerste gef., auch Offenbach. - Dlb. Wlb. (Sch.) - Mz. (S.) Bei Wsb. an einem Rain im Nerothal unter einem Stein im April (K.). -

2. C. Oryzae L. - Besonders in Reis. Durch den Handel über die ganze Erde verbreitet; L. H. besitzt Stücke aus Kamtschatka. Fr. von L. H., Hg. und B. gesammelt. - Dlb. Wlb. (Sch.) - Mz. (S.) -

\section{Dryophthorus Schönherr.}

1. D. Iymexylon $\mathbf{F}$. - In faulem nassem Holz von Weiden, Buchen, Eichen. - Fr. Wald. - Soden. - Von $>4$. bis $<7$. - Zweimal in Fr. am Haus angeflogen (B.). - Wlb. (Sch.) Fr. (Hg.) - Mz. (S.) -

\section{Cossonus Clairville.}

1. C. linearis F. - Unter Rinde und im. Holz von Pappeln und Weiden h. das ganze Jahr hindurch. - Soden. - Nauheim. - Fr. von uns, Hg. und B. gef. - Dlb. in altem Pappelholz (Sch.). - Mr. (C. H., S.) -

2. C. parallelepipedus Hbst. = ferrugineus Clairv. - Soden zweimal an alten Eichen und Pappeln $>5$. und $<6$. von C. H. gef. - Von B. 4 Stiick am Haus gef. - Fr. (Hg.) -

3. C. cylindricus Sahlberg. - Im Holz von Pappeln und Weiden n. s. von Februar bis $=6$. beobachtet. - Fr. (I. H., Hg., B.) Wsb. (C. H.) -

\section{Rhyncolus Creutzer.}

(Die Sammlung v. Heyden von Des b r ochers revidirt.)

1. R. porcatus Germ. - Im Fr. Wald einigemal unter Kiefernrinde von C. H. und Hg. gef. - Mz. (S.) -

2. R. culinaris Reich = exiguus Schh. - Von C. H. bei Soden zweimal im Juni und Juli gef. - Fr. (Hg.) - 
3. R. cylindrirostris Oliv. - Bei Fr. $>4$. an altem Buchenholz und in Biebrich an Aesculusholz $>6$. je eimmal von C. H. gef. - Fr. $\left(\mathrm{Hg}_{\circ}\right)$ - Wlb. zweimal (Sch.). -

4. R. punctulatus Schh. - Aus dürrem Fr. Waldholz von $<4$. bis $>$ 6. öfter erzogen. - Von B. bei Fr. 1875 gef. - Mz. (S.) -

5. R. reflexus Schönh. - Bei Fr. zweimal von C. H. gef. -

\section{Stereocorynes Wollaston.}

1. S. truncorum Germ. - Bei Fr. $=5$. in Pappelholz, auch bei Mz. $=8$. - Fr. Wald $<6$. in dürrem Erlenholz. - Fr. (Hg., B.) - Wlb. 1 Strïck (Sch.). - Bei Mz. anch von S. gef. '-

\section{R. APIONINI.}

\section{Apion Herbst.}

(Die Sammlung $\nabla$. Heyden vom Monographen Wencker revidirt.)

1. A. Pomonae F. - Von Juniperus $=4$. und von Pinus picea $>$ 5. geklopft*), von Eichen bei Offenbach = 10. - Fr. - Soden. - Rüdesheim. - Schmitterhof bei Giessen. - Wlb. (Sch.) Fr. (Hg., B., St.) - Mz. (S.) -

2. A. Craccae L. - Ueberall h. von Mai bis August auf Nadelholz und Eichen, anf Hecken und Wiesen. - Fr. - Soden. - Bieberer Höhe bei Offenbach. - Auf der Königswiese im Fr. Wald 21. April 1861 von B. gestreift. - Wlb. (Sch.) - Fr. (Hg., St.) - Mz. (S.) -

3. A. cerdo Gerstäcker. - s. - Im Juni im Rebstocker Wald bei Fr., bei Offenbach $=10$. auf Eichen. - Ems. - Fr. zweimal 6. Mai 1860 von B. gef., auch einmal von Hg. gesammelt. -

4. A. subulatum Kirby (Weibchen ist Marshami Schh.) Ein weibliches Exemplar bei Fr. von C. H. gef. - Wlb. beide Geschlechter (Sch.). - Auch Hg. fand die seltene Art einmal bei Fr. Mz. (S.) -

5. A. opeticum Bach. - Einmal bei Fr. und ein Männclen bei Ems ron C. H. gef. -

*) Besonders im ersten Frühling tindet man die mannigfaltigsten Käfer auf jungen Nadelholztrieben, wohin sie wahrscheinlich durch den Harzgeruch gelockt werden. 
6. A. ochropus Schh. - Im Fr. Wald <.9. s. - Aus Vicia cracca von Fr. von Mühlig $>$ 7. erzogen. - Wlb. ein Männchen gef. (Sch.) - Fr. (Hg., B.) - Mz. (S.) -

7. A. confluens Kirby. - Bei Fr. 3 Stück gef. im Juli. - s. -

8. A. stolidum Germ. - Bei Fr. dreimal von C. H., auch von B. und Hg. einzeln gesammelt. - Ems. - Wlb. (Sch.) -

9. A. vicinum Kirby. - Je einmal bei Fr. und $=10$. auf Schilf bei Offenbach gef. - Fr. (Hg.) - Wsb. bei Dotzheim 12. Juli in Begattung (K.). -

10. A. atomarium Kirby. - n. s. - Im Fr. Wald von April bis August. - Rumpenheim am Main. - Königstein = 8. - Bei Ems auf Cuscuta europaea. - Lebt auf Serpyllum (Thymus). - Wlb. s. h. (Sch.) - Fr. (Hg.) - Mz. (S.) -

11. A. parvulum Muls. = Serpyllicola Wenck. - Bei Fr. 3 Ex. und 1 Stück bei Offenbach $=7$. von C. H. gef. [Wenckers Typen.] - Mz. (S.) -

12. A. Hoockeri Kirby - Fr. Wald zweimal im März, in Soden < 8. im Gras gesammelt. - Friedberg (Fuhr). - Fr. (Hg.) -

13. A: penetrans Germ. - Von St. einmal bei Fr. gef. und 1 Stück 4. April 1861 von B. - Wlb. z. h. (Sch.) -

14. A. tenue Kirby. - n. s. im Juli und August. - Soden. - Offenbach. - Nauheim. - Wlb. (Sch.) - Mz. (S.) - Fr. von uns und St. gesammelt. -

15. A. pubescens Kirby = civicum Germ. - Im August. Rödelheimer Wald'; an Schilf bei Offenbach. - Schmitterhof bei Giessen. - Fr. (Hg. und B.) - Wlb. (Sch.) -

16. A. aeneum F. - Auf wilden und Garten-Malven h. - Ems. - Rüdesheim < 8. - Taunus. - Wlb. (Sch.) - Bergen (L. H.) Fr. (C. H., Hg., St.) - Mz. (S.) -

17. A. radiolus Kirby. - Auf Malven noch häufiger wie die vorige Art. Im Juli und $>10$. - Soden. - Ems. - Rüdesheim. Wlb. (Sch.) - Fr. (C. H., Hg., St., B.) -

18. A. Onopordi Kirby. - Bei Fr. im Rebstockwald im Juni; bei Soden im Gras öfter $<8$. - Offenbach $=7$. - Bei Nauheim auf Calcitrapa < 6. - Fr. (Hg., B.) - Wlb. (Sch.) - Mr. (S.) -

19. A. Carduorum Kirby = gibbirostre Gyll. - Bei Fr. einigemal gesammelt (C. H., St.). - Mr. (S.) - 
20. A. Caullei Wenck. - C. H. fand 1 Stück bei Flörsheim am Main $=8$. -

21. A. laevigatum Kirby. - s. - Einmal bei Fr. von C. H. gef. -

22. A. brevirostre Hbst. - Fr. Wald im Juli, Bieberer Höhe bei Offenbach. - Im August auf Hypericum. - Ems. - Fr. (Hg., St.) - Mz. (S.) -

23. A. pallipes Kirby. - Auf der Burg Falkenstein auf Mercurialis perennis und Vinca minor $=5$. von uns gesammelt. - Ems. Fr. (St.) - Mz. (S.) -

24. A. flavimanum Schh. = millum Bach. - Von C. H. dreimal bei Fr. gef. -

25. A. fuscirostre F. - Auf Spartium scoparium im Mai und Juni auf der Bieberer Höhe und im Wald bei Neuenhain im Taunus von uns gesammelt. - Fr. von B. gef. 20. Mai 1860. - Wlb. (Sch.) - Fr. (Hg., St.) - Mz. (S.) -

26. A. difficile Hbst. - Aus Schoten von Genista erzogen. Fr. Wald, Försterwiese. - Soden. - Von Juli bis = 9. bei Königstein. - Schmitterhof bei Giessen $>$ 8. - Bei Fr. auf Veronica chamaedrys gesammelt. - Wlb. (Sch.) - Fr. (Hg., St.) -

27. A. Genistae Kirby. - Fr. Wald auf Genista tinctoria, von Mai bis Juli. - Ems. - Mo. > 5. - Von B. bei Fr. gef. 17. Mai 1860, auch von Hg. und St. - Mz. (S.) -

28. A. rufirostre F. - Auf Malva rotundifolia n. s. s. am Röderberg bei Fr. - Bei Soden $=6$. und $<8$. von C. H. und bei Bergen von L. H. gesammelt. - Ems. - Fr. (Hg., St.) -

29. A. flavofemoratum Hbst. - C. H. fand $>6$. die Larve minirend in den Blättern von Medicago sativa bei Soden; der Käfer entwickelte sich $<7$. - Schmitterhof bei Giessen $>8$. - Königstein im Taunus $=9$.

30. A. Malvae F. - In Gesellschaft des A. rufirostre auf Malva rotundifolia, Röderberg. - Bergen. - Ems. - Fr. von B. gef. 22. Mai 1860 ; auch von Hg. und St. gesammelt. -

31. A. vernale F. - Bei Fr. n. s., am Forsthaus im Juli. Friedberg (Fuhr). - Fr. h., z. B. 29. April 1860 (B.). - Wlb. (Sch.) - Bei Fr. auch von Hg. und St. gef. - Mr. (S.) -

32. A. Viciae Payk. - Bei Fr. n. s. - Rödelheimer Wald $=8$. - Offenbach $=7$. - Wlb. (Sch.) - Fr. (Hg., St.) - Mz. (S.) - 
33. A. dissimile Germ. - Seltene Art. - Bei Fr. ein Männchen und im Rebstockwald im Juni ein Weibchen von C. H. gef. -

34. A. varipes Germ. - Von Juli bis September n. s. Rödelheim. - Cronberg. - Soden. - Schmitterhof bei Giessen. Wlb. (Sch.) - Fr. (Hg., St.) -

35. A. Fagi L. = apricans Hbst. - Von Juni bis $>8$. n. s. - Griesheim. - Soden. - Offenbach. - Am Feldberg im Taunus. Ems. - Wlb. (Sch.) - Fr. (L. H., Hg., B.) - Mz. (S.) -

36. A. Ononidis Gyll. - Bei Fr. zweimal von C. H. gesammelt, einzeln von Hg. - Mz. (S.) -

37. A. flavipes F. = elegans Dietr. - Von Juni bis August h. - Rödelheimer und Rebstocker Wald hei Fr. - Soden auf Eichen. Wlb. (Sch.) - Fr. (Hg., St.) -

38. A. Trifolii F. = aestivum Germ. - Von Juni bis August h. - Rödelheimer Wald. - Soden. - Offenbach. - Ems. - Bei St. Goarshausen sclion $<5$. - Wlb. (Sch.) - Fr. (Hg., St., B.) -

39. A. ruficrus Germ. - Von Hg. bei Fr. gef. -

40. A. assimile Kirby. - Bei Soden im Taunus $<8$. Fr. Wald am Forsthaus. - Ems. - Wlb. (Sch.) - Fr. (Hg., St., B.) - Mz. (S.) -

41. A. nigritarse Kirby. - Im Fr. Wald sclion im März, häufiger im Juli und August. - Offenbach. - Soden. - Schmitterhof bei Giessen. - Wlb. (Sch.) - Fr. (Hg., St., B.) -

42. A. miniatum Schh. - Von L. H. in Anzahl auf Rumex im ehemaligen Finkenhof bei Fr. gesammelt. - Fr. (Hg., St. B.) Wlb. (Sch.) -- Mz. (S.) -

43. A. haematodes Kirby = frumentarium Payk. - Bei Griesheim im August; $<$ 10. bei Cronthal. - [In Spanien, besonders Asturien, fand L. H. diese Art in zahlloser Menge auf Erica arborea, vielleicht lebt sie bei uns auf Calluna vulgaris.] - Bei Fr. n. s. gestreift (B.). - Wlb. (Sch.) - Auch von Hg. und St. im Fr. Gebiet gesammelt. -

44. A. rubens Walton. $-<6$. auf Rumex acetosella h. Soden im Juli. $-=9$. bei Bürgel am Main und bei Königstein im Taunus. - Ems. - Wlb. (Sch.) - Fr. (Hg., St.) - Mr. (S.) -

45. A. sanguineum De Geer. - Bei Fr. s. von C. H. gesammelt. - Fr. (Hg., B.) einmal. - Wlb. (Sch.) - 
46. A. cruentatum Walton. - Bei Wlb. von Sch. h. gef. Fr. (Hg., St.) -

47. A. Gyllenhali Kirby. - Bei Fr. von Hg. gef. -

48. A. elongatum Germ. = millum Schh. - Lebt auf Salvia pratensis. - Von C. H. s. bei Fr. gesammelt. -

49. A. seniculus Kirby $=$ pubescens Schh. - Häufige Art. - Im Fr. Wald auf der Bruchschneisse $>4$. - Angust bis October g. auf Weiden, Erlen, Eichen bei Soden, Cronthal, Hofheim. - Offenbach, Rumpenheim. - Lebt auf Ononis. - Ems. - Wlb. s. h. (Sch.) - Fr. (Hg., St.) - Mz. (S.) -

50. A. columbinum Germ. - Bei Fr. einzeln von Hg. und B. gef. -

51. A. simile Kirby = superciliosum Gyll. - Sehr seltene Art. - Bei Fr. 2 Stück $>8$. und einmal bei Cronthal $<10$. ron C. H. gef. -

52. A. ebeninum Kirby = Kunzei Schh. - Fr. einzeln. Falkenstein im Taunus $>$ 8. - Wlb. h. (Sch.) - Fr. (Hg.) Mz. (S.) -

53. A. platalea Germ. (Weib = validirostre Schh.) - Bei Fr. wenige Exemplare von C. H. und Hg. gesammelt. - Taunus. Mz. (S.) -

54. A. Ononis Kirby = glaucinum Schh. (Maun = perplexum Schh.) - Taunus. - Griesheim am Main im August auf Ononis spinosa. - Fr. (Hg.) - Mr. (S.) -

55. A. Ervi Kirby. - Bei Sulzbach bei Soden auf Coronilla varia. - Cronthal $<9$. - Bei Offenbach $=7$. - Bei St. Goarshausen $<$ 5. - Ems. - Fr. (Hg., St.) - Mz. (S.) -

56. A. Loti Kirby $=$ angustatum Kirby $=$ glabratum Germ. - Im Juli und August bei Soden und Griesheim am Main. - Ems. Fr. (Hg.) -

57. A. filirostre. Kirby $=$ morio Germ. - Im Fr. Wald und bei Griesheim im Juli und August s. - Wlb. (Sch.) - Fr. (Hg., St.) - Mz. (S.) -

58. A. Meliloti Kirby. - Bei Offenbach $=7$.; bei Soden und Griesheim im Angust. - Fr. (St.) - 
59. A. virens Hbst. - Bei Offenbach im Juli. - Im Rödelheimer Wald und bei Nauheim im Auguist. - Wlb. (Sch.) - Fr. (Hg., St., B.) - Mz. (S.) -

60. A. punctigerum Germ. - Bei Fr. und im Taunus im Mai auf Astragalus. - Fr. (Hg., St.) -

61. A. Spencei Kirby $=$ foveolatum Kirby $=$ intrusum Gyll. - Je einmal bei Fr. und Königstein $=7$. von C. H. gef. Wlb. (Sch.) -

62. A. sulcifrons Hbst. - Sehr seltene Art. - C. H. fand 3 Stück auf Statice armeria $<8$. bei Griesheim am Main. -

63. A. Aethiops Hbst. - Im Fr. Wald schon = 3. - Offenbach = 7. - Schmitterhof bei Giessen im August. - Wlb. (Sch.) Fr. (Hg., B.) - Mz. (S.) -

64. A. livescerum Schh. - Bei Fr. und Rüdesheim $<$ 7. je einmal von C. H. gesammelt. - Friedberg (Fuhr). - Fr. (Hg.) -

65. A. Astragali Payk. - Bei Fr. auf Astragalus öfter gesammelt im Mai. — Griesheim im August. - Fr. (Hg., St., B.) -

66. A. elegantulum Payk. = coracinum Sch. - Bei Bingen $=5$. einmal von C. H. gef. -

67. A. vorax Hbst. = pallicorne Schh. - Bei Cronberg im Taunus von $<6$. bis $>9$. - Schmitterhof bei Giessen $>8$. Fr. (Hg., St.) - Mr. (S.) -

68. A. pavidum Germ. = plumbeum Schh. - Bei Sulzbach bei Soden im Juni. auf Coronilla varia in Anzahl von C. H. gesammelt. - Rödelheimer Wald. - Bieberer Höhe bei Offenbach. - Wlb. (Sch.) - Fr. (B., St.) - Mz. (S.) -

69. A. Pisi F. - s. h. - Rebstocker Wald. - Griesheim im August. - Mo. $=5$. - Wlb. (Sch.) - Fr. von uns, Hg., B. und St. gef. - Mz. (S.) -

70. A. Sorbi Hbst. = Sahlbergi Schh. - L. H. fand bei Fr. ein Weibchen, C. H. ein Pärchen. - Friedberg (Fuhr). - Wlb. beide Geschlechter von Sch. gef. in dem Fruchtboden von Anthemis arvensis. Fr. (Hg., St.) -

71. A. dispar Germ. - C. II. fand bei Soden im Gras $>7$. 1 Stück. -

72. A. striatum Marsh. = atratum Germ. - Auf der Bieberer Höhe bei Offenbach $=5$. h. auf Spartium scoparium gesammelt. - 
Schmitterhof bei Giessen $>4$. - Wlb. (Sch.) - Fr. (Hg.) Mz. (S.) -

73. A. immune Kirby $=$ Betulae Schh. - Bei Fr. and Ems gesammelt, sowie im Taunus; auch von $\mathrm{Hg}$. gef.

74. A. humile Germ. - Im Fr. Wald am Forsthaus $<7$., Rebstocker Wald. - Wlb. (Sch.) - Fr. (Hg., St., B.) - Mz. (S.) -

75. A. Sedi Germ. - Epstein im Taunus $<$ 5. auf Secum reflexum, Rüdesheim $=4$. an S. album, Soden an S. acre, bei Fr. an S. telephium (purpurascens). - Wlb. s. (Sch.) - Fr. (Hg.) -

76. A. simum Germ. - Leht $>$ 9. auf Hypericum perforatum, aber auch bei Soden im Juli auf Sedum gef. - Falkenstein und Ems im August. - Bei Rumpenheim am Main >10. - Wlb. s. (Sch.) Fr. (Hg.)

77. A. minimum Hbst. = foraminosum Schh. - Von Juni bis August besonders bei Offenbach gesammelt an Schilf. - [In Holland, bei Scheveningen erzog C. H. diese Art aus Nematus-Gallen auf Salix vitellina.] - Wlb. (Sch.) - Fr. (Hg., St.) -

78. A. violaceum Kirby. - Im Gebiet n. s. von Juni bis August. - Rödelheimer und Rebstocker Wald. - Griesheim. - Offenbach. - Taunus. - Wlb. (Sch.) - Fr. (Hg., St.) -

79. A. Marchicum Hbst. = Spartii Kirby. - Fr. Wald von Juli bis September. - Ems. - Cronberg im Taunus. - WJb. (Sch.) - Fr. (Hg.) -

80. A. affine Kirby. - Einmal von C. H. bei Fr. auf Spartium scoparium, auch von Hg. gef. -

\section{Rhynchites Herbst.}

(Die Sammlung $\nabla$. Heyden vom Monographen Desbrochers revidirt.)

1. R. auratus Scop. - Auf Schlehen bei Königstein $>6$., bei Rumpenheim = 7. - Fr. s. - Ems. - Wlb. seltener als der folgende (Sch.). - Fr. s. (Hg., B.) - Mz. (S.) - Mo. auf Schlehen an jungen Früchten im Mai (K.). -

2. R. Bacchus L. - An den Knospen der Apfelbäume $>9$, auch auf Schlehen $=5$. bei Königstein. - An manchen Orten h. und oft schädlich. - Bei Fr. n. h. (Hg., B.) - Wlb. (Sch.) - Nir. (S.)

- Wsb. im April auf Weissdorn und Apfelblüthe (K.). - 
3. R. caeruleocephalus Schaller. - Lebt auf Birken. - Auf der Bieberer Höhe $=7$., im Fr. Wald bei der unteren Saustiege $>8$; Rebstockwald. - s. - Am Goldstein im Fr. Wald h. (B.) - Fr. (Hg.) -

4. R. aequatus L. - Auf Crataegus bei Fr. n. s. $=5$. Schmitterhof bei Giessen $>4$. - Auf Schirmblumen am Forsthaus rom 2. bis 23. Mai 1860 s. (B.) - Wlb. (G.) - Fr. (Hg.) Mz. (S.) - Wsb. auf Weissdorn im Mai und auf blühenden Schlehen im April (Ko). -

5. R. cupreus L. - Bei Fr. s. auf Pflaumenbäumen. - Nach B. s. vom 7. bis 22. Mai 1860 gef. - Dlb. Wlb. l. auf Pflaumenbäumen (Sch.). - Fr. (Hg.) - Mz. (S.) -

6. R. aeneovirens Mrsh. = Iongirostris Bach. - Bei Cronthal auf Eichen $=5$; im $\Lambda$ pril bei Rüdesheim und bei Fr. auf Salix caprea. - Falkenstein im Taunus. - Fr. einmal 3. Juni 1860 (B.). Wlb. auf Eichen (Sch.). — Staufen im Taunus 2. April 1876. -

Var. Fragariae Schh. - Bei Falkenstein $=5$. und Fr. je einmal von C. H. gef. - Nauheim. - [Hierher longir. in S. V.] -

7. R. interpunctatus Steph. = Alliariae Gyll. (= megacephalus Schh. tom. V. nec I.) - Bei Rüdesheim im April zweimal und einmal bei Fr. von C. H. gef. - [Hierher Alliariae Payk. in S. V., welche Art L. H. nur aus England besitzt.] - Fr. (Hg.) -

8. R. conicus Illig. - Auf Eichen, Birnbäumen, Schlehen s. h.; auf Sorbus aucuparia bei dem Schmitterhof bei Giessen $>4$. - Soden. - Königstein, August bis October. - Wlb. (Sch.) - Fr. (Hg., B.) -

9. R. pauxillus Germ. - Auf Schlehen h. im April und Mai. -Falkenstein. - Rüdesheim. - Auch n. s. auf Sambucus gef. Wlb. (Sch.) - Fr. (Hg.) - Mz.'(S.) -

10. R. Germanicus Hbst. $=$ minutus Schh. - Auf Eichen n. s. von April bis Juli. - Fr. Wald, Enkheim, Nauheim. - Wlb. (Sch.) - Fr. (Hg.) - Mz. (S.) -

11. R. uncinatus Thoms = planirostris Desbr. nec F. Im Fr. Wald $=6$. auf Birken in Begattung, auch auf Sahlweiden, Erlen, Zitterpappel bis $<$ 8. - Enkheim. - Am Feldberg im Taunus. - h. - (Bei uncinatus sind die Vorderschienen immer zweimal leicht gebogen, an der Spitze mit einem Haken, die Augen springen mehr vor.) - Fr. (B.) - 
12. R. planirostris F. verus = nanus Payk. - Auf Betula alba s. im Mai. - (Planirostr. hat einfache Vorderschienen, ohne Haken.) - Wlb. z. h. (Sch.) - Fr. (Hg.) - MIz. (S.) -

13. (Bytiscus Thoms.) Betuleti F. - Als „,Rebsticher" den Weinbergen oft schädlich; den Käfer $<6$. auf Weinstöcken gefunden. Er rollt wie seine Gattungsgenossen das Blatt zu einer langen Düte, um darin ein Ei abzulegen. - Im Lorsbacherthal $=5$. h. auf Linden, deren Blätter er gleichfalls rollt; ebenso bei Fr. auf Populus canadensis. - Bei Wlb. auf Weinstöcken und rerschiedenen Waldbäumen gef. (Sch.) - Fr. (Hg., B.) - Mrz. (S.) - Wsb. an der Tränke auf Sahlmeiden im Juni, Weinreben im Rheingau (goldglänzende Stücke); bei Dotzheim auf Rosen 17. September (blaue Stücke) (K.). -

14. (B.) Populi L. - Auf der Bieberer Höhe = 7. auf Aspen; auf Pappeln bei Mo. = 5. - h. - Fr. 20. Nai 1860 (B.). Wlb. (Sch.) - Mz. (S.) - Wsb. auf Zitterpappel im Juni (K.). -

15. R. sericeus Hbst. = ophthalmicus Steph. - An jungen Eichenschösslingen an der Wiese der Hohen Mark im Taunus $>6$. und bei Falkenstein zu Anfang des Monats. - Fr. h. (Hg., B.) - MIz. (S.) -

16. R. pubescens F. = cavifrons Schh. = cyanicolor Schh. - Bei der Mainkur $>$ 5. und im April auf dem Niederwald bei Rüdesheim auf Eichen; Soden im Taunus. - s. - Fr. n. h. (Hg., B.) - Wlb. (Sch.) -

17. R. comatus Schh. = ophthalmicus Redtb. (der Mann ist olivaceus Schh.). - Auf Eichen im April auf dem Niederwald; $=5$. bei Soden und Falkenstein zu Ende des Monats auf Birken im Rebstockwald bei Fr. - Olivaceus $<6$. bei Falkenstein. - [Hierher ophthalmicus in S. V.] - Wlb. (Sch.) - Fr. (Hg.) - Mrz. (S.) -

18. R. megacephalus Germ. = constrictus Schh. - Bei Fr. einmal ron $\mathrm{Hg}$, und bei Mo, von K. gesammelt. -

19. R. Betulae F. - Auf Erlen h. - Fr. - Falkenstein $=5$. - Enkheimer Ried 28. April 1860 (B.). - Wlb. (Sch.) - G. (L.) - Fr. (Hg.) - N.z. (S.) -

\section{Attelabus Linné.}

1. A. curculionoides L. - Ueberall h. auf jungen Eichentrieben. - Ems. - Fr. von uns, Hg., St. und B. gesammelt. - G. (L.) Dlb. Wlb. (Sch.) - Ml\%. (S.) - 


\section{Apodlerus Olivier.}

[1. Coryli L. (Die Stammart mit ganz schwarzen Beinen und zum grössten Theil dunklem Halsschild nur in Nord-Europa.)]

Var. Avellanae L. (rothes Halsschild und Schenkel) h. auf Haseln und Erlen. - Taunus. - Ems. - Wlb. Dlb. h. (Sch.) - Offenbach (B.). - G. (L.) - Fr. von uns, Hg., St. und B. gef. - Mz. (S.) -

Var. morio Bon. - Hg. fing ein ganz schwarzes Exemplar bei Isenburg. -

\section{RHINOMACERIDAE.}

\section{Nemonyx Redtenbacher.}

1. N. lepturoides F. - Am Fr. Wald bei der Ziegelhütte von C. H. vor langen Jahren auf Rittersporn (Delphinium consolidum) gesammelt. - Hg. fand 3 Stiick bei Fr. -

\section{Rhinomacer Fabricius.}

1. R. attelaboides F. - s. - Im Schwanheimer Wald $=5$. auf Eichenblüthen von C. H. gesammelt. - Im Röderwald im Gras von April bis 19. Mai nicht gerade s. gestreift (B.). - G. (L.) — Fr. (Hg.) - Mz. (S.) - Mo. 24. April (K.). -

\section{Diodyrhynchus Schönherr.}

1. D. Austriacus Schh. - Bei Mo. gesammelt $=4$. (C.. H.) - Dotzheim bei Wsb. auf Kiefern im April (K.). - Mz. (S.) -

Var. pallidus Meg. - Fr. auf Kiefern h.; =4. in Begattung. - Falkenstein $=5$. - Röderwald, Königsbrünnchen 21. April 1861 h. (B.) - Fr. (Hg.) - 


\section{ANTHRIBIDAE.}

\section{Platyrhinus Clairville.}

1. P. latirostris F. - An Weissbuchen bei der MIainkur $>5$. öfter von L. H. gef. - Fr. (v. Twardowski, Hg., St.) - Unter Bucheurinde an der Götheruhe im Fr. Wald s. (B.) - Dlb. (Sch.) - Wsb. in Buchenstrünken h. im Juni, auch 14. September (K.). -

\section{Tropideres Schönherr.}

1. T. albirostris Hbst. - Fr. s. - Nur einmal von B. unter Buchenrinde an der Götheruhe 11. Mai 1860 gef. (B.) - Hg. 2 Stïck. - Von St. aus der Holzkammer erzogen. - Wlb. (Sch.) - Mz. (S.) -

2. T. sepicola Hbst. - Aus altem Eichenholz aus dem Fr. Wald $<$ 5. öfter erzogen; bei Fr. auch von Mühlig gef. - Mz. (S.) Offenbach (L. H. in Sammlung Haag). -

3. T. niveirostris F. - Von Linden- und Buchenhecken $=8$. beim Fr. Forsthaus abgeklopft. - Bei Soden $<6$. an Dornzäunen. An den Zimmerwiesen an alten Weiden ( hof bei Giessen $>8$. - - Einmal an der Götheruhe unter Buchenrinde 27. Mai 1860 von B. gef. - G. (L.) - Fr. (Hg., St.) - Mr. (S.) -

4. T. cinctus Payk. - Einigemal von C. H. und Hg. bei Fr. gef. - Friedberg (Fuhr). -

\section{Anthribus Geoffroy.}

1. A. albinus L. - Am Fr. Forsthaus an einem alten Eichenbaum $=5$. ein Männchen gef. und $=7$. aus altem überwintertem Carpinusholz ein Weibchen erzogen (C. H.). - Ein Stück im Fr. Wald am Goldstein auf Birkenstammausschlag gestreift 1861 (B.). - Fr. (Hg.), ron St. aus der Holzkammer entwickelt. - Dlb. (Sch.) - Ernsthausen in Nassau (Dörr bei K.). - 


\section{Brachytarsus Schönherr.}

1. B. scabrosus F. - Fr. einmal. - Wald bei der Mainkur $>$ 5. auf Hainbuchen. - Cronthal im Taunus $=5$. an einem alten Apfelbáum. - s. - Die Larve lebt unter dem Schild der Coccus-Art: Lecanium carpini, welche von ihr ganz aufgezehrt wird. - Fr. (St., Hg. zweimal). - Wetzlar (Lieutenant Giebeler). -

2. B. varius F. - Im Fr. Wald an der Försterwiese auf Fichten. - C. H. erzog den Käfer $<8$. aus Coccus racemosus. - Sodener Wald $=5$. - Mehrfach am Fr. Forsthaus $<6$. gestreift (B.). Fr. (Hg., St.) -

\section{Urodon Schönherr.}

1. U. rufipes Oliv. - Flörsheim am Main auf Reseda lutea $>6$. - Offenbach = 7. - Ems. - Mo. (Hg.) - Mz. (S.) -

2. U. suturalis F. - Bei Soden $=7$. - Flörsheim $>6$. auf Reseda lutea. - Mo. (K., Hg.) - Mz. (S.) -

3. U. conformis Suffr. - Besitzt L. H. durch Prof. Rosenhauer aus Ems (als pygmaeus bestimmt; die Urodon in Sammlung v. Heyden sind von Allard revidirt.) - [Hierher pygmaeus in S. V.] - Wsb. (Bach). -

\section{B R U C H I D A E.}

\section{Spermophagus Steven.}

1. S. Cardui Bohem. - Fr. n. s. $>5$. in den Blüthen von Crataegus oxyacantha. - Bei Rüdesheim =8. - Im Sand bei Mo. $<$ 6. - Bei Fr. n. s. auch von B. gef. - Mo. (Hg., K.) Mz. (S.) - Wlb. (Sch.) -

\section{Bruchus Linné.}

(Die Sammlung v. Heyden ist vom Monographen Allard revidirt. - Ueber die deutschen Arten der Sammlung vide Berlin. Entom. Zeit. 1867, pag. 381 und 1868, pag. 324.)

1. B. marginalis F. - Fr. aus Schoten von Astragalus im September oft erzogen. - Am Goldstein im Fr. Wald s. gestreift (B.). Fr. (Hg.) - 
2. B. picipes Germ. - [Hierher naclı Kraatz das einzelne Frankfurter Stück, das Allard für obsoletus Blanch. hielt und ron L. H. unter diesem Namen Berl. Entom. Zeit. 1867 erwähnt wird.] - Auch Hg. fand die Art bei Fr. -

3. B. pusillus Germ. - Einmal bei Fr. von C. H. gef. - Neu. für Deutschland, in Sammlung v. Heyden auch aus Stettin, vide Krtz. Berl. Entom. Zeit. 1868, pag. 328. -

4. B. canus Germ. (nec. = cisti F.) - Bei IIo. $<6$. einzeln gef. - Bieberer Höhe =6. - Fr. (Hg.) - Mz. (S.) -

5. B. debilis Schh. - Ueberall h. aber mit canus vermengt. Bieberer Höhe $=6$. und $<9$. - Hinter Offenbach $<6$. auf Cynoglossum. - Mo. im Juni. - Bergen <6. -

6. B. olivaceus Germ. - [Hierher die 1. c. von L. H. erwähnten Stuicke von virescens nach Krtz.] - Sauerthal bei Lorch am Rhein von C. H. $<$ 6. gef. $-\mathrm{MLz}$. (S.) -

7. B. pisorum-L. = Pisi L. - Ueberall in Erbsen h. - Fr. - Im Hofheimer Wald $<6$. aur Rubus-Blüthen. - Fr. schon $>3$. gef. (B.) - Mr. (S.) -

8. B. rufimanus Schh. - Bei Fr. und Ems je einmal ron C. $H$. gef., auch von Hg. gesammelt. - Dlb. Wlb. h. auf Vicia faba (Sch.). -

9. B. affinis Fröhl. = flavimanus Schh. - Bei Wlb. zweimal gef. (Sch.) - Fr. (Hg.) -

10. B. signaticornis Schh. -- Von B. in Fr. gef. - [Stücke in Sammlung v. Heyden.] -

11. B. granarius L. - [Hierher seminarius L. in S. V., welche Art noch nicht gedeutet ist.] - Ueberall g. - Aus Samen von Vicia pisiformis = 3. - Ealkenstein im Taunus im Mai auf Orobus vernus. - Fr. Wald auf Lathyrus $=5$. - Cronberg im Taunus auf Wiesen $<6$. - St. Goarshausen $>$ 6. - Wlb. Dlb. s. h. (Sch.) - Fr. (Hg.) - NIz. (S.) -

12. B. luteicornis Illig. (nubilis ist n i cht das Männchen dieser Art nach Kraatz's genanester Untersuchung dieser sehr verwickelten Gruppe. - Siehe Berl. Entom. Zeit. 1868, pag. 313.) - L. H. besitzt nur Männchen. - Bieberer Höhe abgeklopft $=10$. - Fr. $=5$. Soden. - Bei Fr. s. h. (B., Hg.) - Wlb. s. h. (Sch.) - Eichberg im Rheingau, beide Geschlechter von Richter gef. -

13. B. nubilus Schh. - Wald bei der Mainkur $>$ 5. auf Vicia angustifolia. - Fr. - Wsb. ein Weibchen (K.). - 
14. B. Loti Payk. = Lathyri Steph. - Bei Falkenstein im Taunus $>$ 8. einmal von C. H. gef. -

15. B. pubescens Germ. - Bieberer Höhe $=5$. auf Spartium. - Auch im Fr. Wald. - Friedberg (Fuhr). - Mz. (S.) -

16. B. ater Marsh. = Cisti Payk: - Fr. h. in Blüthen das ganze Jahr hindurch von Schlehen, Epilobinm, Asclepias vincetoxicum (Bieberer Höhe < 7.). - Euphorbia, Genista. - Aber besonders h. $>6$. an den Schoten von Spartium scoparium. - Wlb. s. h. (Sch.) Mz. (S.) -

\section{CHRYSOMELIDAE.}

\section{A. CLYTHRINI. \\ Clythra Laicharting.}

1. (Labidostomis Redtb.) tridentata F. - Bei der Mainkur auf Birken $>5$. in Begattung. - Mitte des Monats am Feldberg. Mz. (S.) -

2. (L.) humeralis Schneid. - Fr. (Hg.) - Dlb. anf ewigem Klee (Sch.). - Wsb. (K.) - Lahnstein 9. Juni (K.). -

3. (L.) Iongimana L. - Fr. einmal von C. H. gef. - Einige Stücke bei Fr. (Hg., B.) - G. (L.) - Dlb. wie die vorige (Sch.) Mz. (S.) -

4. (Lachnaea Lacord.) sexpunctata Scop. = Iongipes L. .Von St. auf dem Johannisberg bei Nauheim gesammelt. - St. Goarshausen (Anton Schmid). - Bei Bingen mehrmals (S.); Sauerthal $<6$. -

5. Clythra quadripunctata L. = quadrisignata Märk. - Die Cocons $<5$. in den Nestern von Formica rufa. Der Käfer entwickelte sich $=5$. - Auf Gesträuch h. - Fr. - Soden. - Man hielt seither die Märkel'sche Art, die speciell aus Ameisen-Nestern erzogen wurde, für eine eigene Art, bei welcher gewöhnlich der Fleck in der Mitte der Flügeldecken grösser als bei Stücken ist, die man für die ïchte quadripunctata hielt. Bei grösseren Reihen aus den verschiedensten Gegenden Europa's verschwinden aber auch die von Suffrian angegebenen feinen 
Unterschiede. L. H. bęsitzt zwei Stücke von quadripunctata aus Ungarn und dem Algäu, bei welchen dieser Fleck kaum noch ịn einer Spur vorhanden ist. - Fr. (Hg.) - Dlb. Wlb. (Sch.) - Mo. (K.) - Mz. (S.) -

6. C. laeviuscula Ratzbg. - Fr. $>$ 6. auf Weiden. - Nach Hg. und B. s. h. - Dlb. Wlb. (Sch.) - Mo. von 21. Juni bis 12. August (K.). - Mz. (S.) -

7. (Gynandrophthalma Lac.) salicina Scop. = cyanea F. Bei Königstein im Taunus $=5$. h. auf Haseln. - Ems. - Bei Fr. n. S. gestreift, so am 12. Mai 1860 (B.)。 - G. (L.) - Dlb. Wlb. (Sch.) - Wsb. Waldwiese hinter dem Adamsthal im Juni (K.). -

8. (G.) affinis Hellw. - Bei Königstein wie die vorige Art. G. (L.) - Fr. (Hg.) - Wlb. Dlb. (Sch.) - Wsb. im Wellritzthal im Sommer (K.). -

9. (G.) aurita L. - G. (L.) - Fr. (Hg.) - Dlb., nicht bei Wlb. (Sch.) -

[G. flavicollis Charp., die Bach als bei Ems gefunden aufführt, ist eine irrige Bestimmung.]

10. (Cheilotoma Redtb.) bucephala F. - Bei Friedberg von Fuhr gesammelt. -

11. (Coptocephala Redtb.) - Die Gruppe von Dr. Kraatz besprochen Berl. Entom. Zeit. 1872, pag. 219 - scopolina F. vera. Die in S. V. erwähnten Stücke gehören zur folgenden Art; ein ächtes Weibchen fand Hg. bei Fr. -

12. (C.) quadrimaculata F. - Bei Mo. h. auf Peucedanum orioselinum $=7$; der Sack $>6$. von C. H. gef. - Fr. (L. H.) Unter den Mo. Stücken ein Exemplar mit ,schwarzer" Oberlippe, von Kraatz mit dieser Bemerkung bestimmt, aber in seiner Arbeit nicht erwähnt. - Fr. h. (B.) - Hg. fand ein ächtes Männchen. - Mz. (S.) -

\section{B. CRYPTOCEPHALINI.}

(Die Sammlung v. He yde $\mathrm{n}$ ist vom Monographen Suf f $\mathrm{r}$ ia $u$ revidirt.)

1. C. Coryli L. - Bei Cronthal auf jungen Birken und im Schwanheimer Wald von v. Twardowski gef. - Bei Fr. zweimal am 19. Mai 1860 gef. (B.) - Mz. (S.) - Neroberg bei Wsb. 9. Juni auf Birken (K.). -

2. C. cordiger L. - Bei Fr. einmal von C. H. gef. -

3. C. variegatus F. - Bei Friedberg von Fuhr gef. - 
4. C. octopunctatus Scop. = variabilis Schneid. - Fr. zweimal von C. H. gef. - Friedberg (Fuhr). -

5. C. sexpunctatus L. - Einmal $<6$. bei Falkenstein im Taunus auf Salix caprea von C. H: gef. - C. (L. in Sammlung als variegatus.) - Fr. einmal am 11. Mai 1861 (B.), auch von St. gef. - Mz. (S.) - Königstein $>5$.

6. C. violaceus Laich. - Im Wald bei Langenhain (bei Epstein) im Taunus $<6$. auf Blüthen, besonders von Hieracium n. s. - Im Sodener Wald auf derselben Pflanze $=6$. in Begattung. - Fr. (Hg., B.) - Königstein (L. H.). - Friedberg (Fuhr). - G. (L.) - Mz. (S.) - Dlb. Wlb. (Sch.) - Lorch am Rhein 5. Juni (K.). -

7. C. sericeus L. (verus teste Suffr.) - Einmal bei Fr. gef. - Mz. (S.) -

8. C. aureolus Suffr. - Ems im Juli s. - Fr. (C. H., Hg.) Dlb. Wlb. (Sch.) - Mz. (S.) -

9. C. Hypochaeridis L. - Im Wald bei Langenhain $<6$. auf Blumen. - Fr. n. s. (Hg.) - Dlb. Wlb. (Sch.) - Mr. (S.) -

10. (Proctophysus Redtb.) lobatus F: - Ein Weibchen fing K. bei Lorch am Rhein auf Crataegus 5. Juni. -

11. (Disopus Redtb.) Pini L. = abietis Suffr. - Fr. $<10$. - Mo. > 8. - Bicberer Höhe bei Offenbach = 10. in Begattung auf Pinus sylvestris n. S. - Im Kiefernwald auf dem grossen Sandbruch bei Mz. h. (S.) - Oestrich am Rhein 16. Mai (K.). -

12. C. nitidus $\mathbf{L}$. $=$ nitens $\mathbf{L}$. $-\mathrm{Fr}$. von $>5$. bis $<7$. auf Birken. - Königstein $<6$. - Fr. (Hg.) -- Wlb. (Sch.) - Mz. (S.) - Lorch 5. Juni (K.). - Wsb. am Neroberg 7. Juli (K.). - Burg -Lahneck 9. Juni (K.). -

13. C. ochrostoma Harold. = C. nitidulus Gyll. nec F. Einmal bei Ems von C. H. $<7$. auf Corylus avellana gef. - Mz. (S.) -

14. C. Wydleri Faldrm. = quadripustulatus Gyll. - Fr. einmal $<6$. auf Pinus sylvestris von C. H. gef. - Mz. (S.) -

15. C. Moraei L. - Fr. n. s. auf Hypericum perforatum. $>6$. auf Galium luteum. - Ems im Juli auf Spartium scoparium. G. (L.) - Von Hg. and B. bei Fr. s. h. gef. - Mz. (S.) - Dlb. Wlb. (Sch.) -

Var. vittiger Heyden. (Halsschild schwarz, Vorder- und Seitenrand, sowie eine in der Mitte am breitesten und da fein getheilte Querbinde 
roth.) - Fr. einmal von C. H. gef. - Von Marseul in seincr Monographie Abeille XIII, 1874, pag. 165, erwähnt. - Wsb. 1 Stück (K.). -

16. C. flavipes F. - Fr. - Ems $<7$. - Falkenstein $<6$. - Fr. (Hg., B.) - Mz. (S.) - Dlb. Wlb. (Sch.) - Sauerthal <6. -

17. C. decemmaculatus $L$. = decempunctatus $L$. - Fr. von C. H. s. s. im Enkheimer Torfbruch, von L. H., St. und M. S. einigemal mit dem Streifnetz gef. - (Stammart Flügeldecken gelb, jede mit 5 schwarzen Punkten: 2, 2, 1.) -

Var. Bothnicus L. (Flügeldecken ganz schwarz.) - Fr. eimmal von C. H. gef. -

18. [C. flavescens Schneid. (Stammart ganz gelbe Oberseite mit schwarzer Schulterbeule.) - Nicht aus dem Gebiet bekannt.] -

Var. frenatus F. (Flügeldecken schwarz, Halsschild in der Mitte mit einer gelben Längslinie.) - Bei Schwanlheim einmal von v. Twardowski gef. [in Sammlung v. Heyden]. -

19. C. flavilabris F. = fulcratus Germ. - Fr. Wald im Kesselbruchdistrict auf Erlen $<9$. - Im Schwanheimer Wald von St. und auf Rhamnus frangula $<6$. von L. H. s. s. gesammelt. Fr. (Hg., B.) - G. (L.) -

20. C. marginatus F. (Männchen ganz blaue Flügeldecken, Weibchen jede mit einẹr breiten gelbeı ausgebuchteten Längsbinde.) Bei Soden ein Weibchen $>6$. auf Eichen von C. H. gef. - Desgleichen bei Fr. von Hg. - St. fand beide Geschlechter. - Mz. (S.) -

21. C. vittatus F. $-\mathrm{Fr}$. - Soden $<6$. auf Waldblumen. Ems n. s. auf Spartium scoparium im Juni und Juli. - Fr. h. (Hg., B.) - G. (L.) - Mz. (S.) -

22. C. bilineatus L. - Bei Fr. einzeln und im Hof der Falkensteiner Ruine $>8$. von C. H. gef. - G. (L.) - Fr. (Hg.) $-\mathrm{Mz}$. (S.) - Mo. auf Kiefern 8. Juli (K.). -

23. C. amoenus Drap. = vittula Suffr. - Bei Fr. zweimal von C. H., auch von Hg. gef. -

24. C. pygmaeus F. - Bei Ems in Anzahl im Juli auf Thymus serpyllum von C. H. gesammelt. - Fr. s. von Hg. und B. gef. Wlb. (Sch.) - Mz. (S.) -

25. C. fulvus Goeze $=$ minutus F. - Auf Wiesen bei Offenbach < 8. - Fr. Wald an der Ziegelhütte = 7. auf Artemisia. Fr. h. auf Weiden (Hg., B.). - G. (L.) - Wlb. (Scl.) -

26. C. Populi Suffr. - Ems (S.). - Mz. (S.) - 
27. C. pusillus F. - (Variirt mit ganz gelben, gefleckten bis ganz schwarzen Flügeldecken.) - Lebt auf Eichen. - Fr. - Soden von $<$ bis $=7$. - Ems $=7$. - Auch auf Betula $=8$. gef. Fr. h. auf Erlen (B.). - Mz. (S.) -

28. C. rufipes Goeze = gracilis F. - Lebt auf Weiden. Fr. $>6$. - Mz. $=6$. - s. - Fr. Wald am Goldstein, an den Röderhöfen, überhaupt h. (B.) - Wlb. (Sch.) - Wetzlar (Lieut. Giebeler). -

29. C. chrysopus Gmel. = Hübneri F. - Fr. in Anzahl von C. H. und Hg. gef. - Wlb. (Sch.) - Mz. (S.) - Lorch 5. Juni (K.). Auf der Waldeck bei Oberingelheim 1877 (B.). -

30. C. labiatus L. - Fr. - Im Taunus < 7. auf Birken. Wald bei Enkheim $>7$. - Ems. - Fr. einmal von Hg. und B. gef. - G. (L.) - Wlb. (Sch.) - Mz. (S.) -

Var. digrammus Suffre - Bei Ems einmal < 7. von C. H. gef. - G. (L.) - Mo. 17. Mai (K.). -

31. C. exiguus Schneid. = Wasastjernii Gyll. - Fr. je einmal von C. H. und Hg. gef. - G. (I.) --

32. C. ocellatus Drap. = geminus Gyll. - Fr. $-\operatorname{Ems}<7$. - Auf Weiden bei Hofheim $>6$. und bei Mz. $=6$. - G. (L.) Fr. h. (Hg., B.) - Wlb. (Sch.) -

33. C. querceti Suffr. - Fr. ein Weibchen von C. H. gef. -

34. C. imperialis Laich. = bistripunctatus Germ. - Ein Männchen im Juli bei Ems von C. H. gef. - Von Suffrian 1842 ebenda auf Corylus gesammelt. -

35. C. bipunctatus L. (Stammart mit je einem kleinen schwarzen Fleck hinter der Mitte jeder Flügeldecke.) - Fr. - Ems im Juli. Auf Eichen und Birken. - Fr. (B.) - Taunus (Hg.). - Dlb. Wlb. (Sch.) - Mrz. (S.) - Wsb. Neroberg auf Birken von Mai bis Juni (K.). $-<6$. Schwanheim auf Rhamnus frangula. Sauerthal $<6$. -

Var. limbatus Laich. = lineola F. - (Der Punkt zu je einem langen schwarzen Längswisch erweitert.) - Fr. s. -

36. C. biguttatus Scop. = bipustulatus F. - Fr. - Soden, auf Waldblumen $>6$. - G. (L.) - Fr. h. (Hg.) - Wlb. (Sch.) - Mz. (S.) - Sicher gute Art, Uebergänge fehlen. Flügeldecken stärker punktirt. -

\section{Pachybrachys Suffian.}

1. P. hieroglyphicus $\mathbf{F}$. = histrio F. - Auf Weiden bei Fr. $>$ 8.; bei Hofheim im Taunus $>6$. - Friedberg (Fuhr). - Fr.

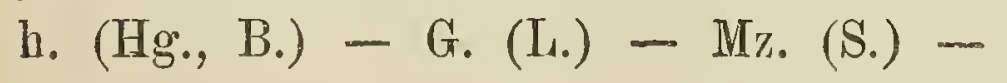


2. P. histrio Oliv. - Bei Ems von C. H. gef. (Pygidium schwarz.) - Fr. einmal (B.). - Mo. am Damm am Rhein auf Weiden im Juni (K.). - Mz. (S.) -

Var. bisignatus Redtb. (Pygidim mit zwei gelben Flecken.) Mit der Stammart bei Mo. (K.) -

\section{EUMOLPINI.}

Oomorphus Curtis.

1. 0. concolor Sturm. - (Die Gattung Lamprosoma, zu welcher diese Art gestellt wurde, kommt nur in Amerika vor.) - Wlb. s. h. auf feuchten Wiesen, auch die Larve da gef. (Sch.) -

\section{Pachnephorus Redtenbacher.}

1. P. pilosus Rossi = arenarius Panz. - Bei der Mainkur und Bergen an thonigen feuchten Stellen $=5$. - Auch am Main im Sand unter Weidengebüsch (C. H.). - Nz. (S.) -

\section{Chrysochus Redtenbacher.}

1. C. pretiosus F. - L. H. besitzt ein von Zitz bei Mz. auf Asclepias vincetoxicum gefundenes Stück; auch S. fand noch später daselbst die Art. -

\section{Adoxus Kirby.}

1. A. obscurus L. - Auf Epilobium s. - Fr. - Ems. Nach Hg. und B. bej Fr. s. - G. (L.) - Mz. (S.) - Wlb. s. h. (Sch.) - Had. auf nassen Wiesen am Haidenhäuschen (K.). -

Var. vitis F. - In Süd-Europa dem Weinstock schädlich. - Fr. s. im Mai, aber nicht an Wein (auch Bach fand ihn bei Boppard h., aber nie in den Weinbergen). - B. fand bei Fr. nur 1 Stück 12. Mai 1861. - Had. mit der Stammart (K.). - Fr. (Hg.) s. - Ausser der Farbe der Flügeldecken finden sich keine Form- und Sculpturunterschiede, welche die Trennung beider Arten rechtfertigte. -

\section{CHRISOMELINI.}

\section{Timarcha Latreille.}

1. T. tenebricosa L. - [Hierher laerigata L. in S. V., die nur im änssersten Süden ron Europa und Algier vorkommt.] - Fr. Wald 
h. von uns und Hg. gef. - Die Larve auf Galium. - Wlb. (Sch.) - Mz. (S.) -

2. T. violaceonigra De Geer $=$ coriaria Laich. - Fr. Friedberg. - Falkenstein. - Ueberall g. - G. (L.) - Wlb. (Sch.) - Mz. (S.) -

Var. Ein lebhaft gefärbtes Stück fand $\mathrm{Hg}$. auf dem Feldberg im Taunus; das Halsschild ist blau, die Flügeldecken metallisch. -- Von Fairmaire in seiner Monographie erwähnt. -

3. T. metallica Laich. - Zweimal auf dem Altkönig im Taunus von C. H. gef. - [Hierher globosa vom Feldberg in S. V. und auch wohl die von Fuhr bei Friedberg gefundenen Stücke. - T. globosa H. Sch. besitzt L. H. nur aus Krain, Banat und Illyrien.] - G. (L.) - Auf der Hohen Wurzel im Taunus unter Steinen am 29. Mai gef. (K.) -

\section{Chrysomela Linné.}

(Die Sammlung $\nabla$. Heyden ist von Suffrian revidirt.)

1. C. staphylea L. - Fr. n. s. - nach Hg. und B. h. unter Steinen. - G. (L.) - Dlb. h. auf Gebüschen (Sch.). - Mz. (S.) -

2. C. purpurascens Germ. - Von C. H. einmal bei Falkenstein im Taunus gef., von Hg. ebenfalls im Taunus gesammelt. -

3. C. rufa Duft. -- Mannhäuser Thal bei Wetzlar einmal (Lieut. Giebeler). Von L. H. bestimmt. -

4. C. varians Schall. $=$ Hyperici De Geer. - Fr. - Schlangenbad. - Variirt messinggelb, kupferroth, blau, grün, violett, schwarz. - Auf Hypericum perforatum überall h. - Oberursel und Hohe Mark im Taunus. - Fr. Wald am Goldstein (B.). - G. (L.) - Dlb. Wlb. (Sch.) - Mz. (S.) -

5. C. Goettingensis L. - An feuchten Orten h. $>7$. unter Steinen. - Mo. > 7. auf der grossen Sandfläche. - G. (L.) Dlb. Wlb. (Sch.) - Mz. (S.) -

6. C. haemoptera L. - Fr. - Soden unter Steinen. - In ganz Europa l. - G. (L.) - Dlb. (Sch.) - Mz. (S.) -

7. C. Molluginis Suffr. - Dlb. (Sch.) einmal. - Gaualgesheimer Kopf bei Bingen 20. Mai (K.). -

8. C. sanguinolenta L. - Fr. nach Hg. und B. h. - G. (L.) - Dlb. (Sch.) - Mz.' (S.) -

9. C. Gypsophilae Küst. - 3 Ex. bei Fr. von C. H. gef. Mz. (S.) - 
10. C. marginalis Dft. -- Fr. n. s. - Ems. - An der Grüneburg bei Fr. auf Wiesenblumen dreimal gef. (B.) - Dlb. (Sch.) - Mz. (S.) -

11. C. limbata F. - In Sandgegenden. - Fr. Wald auf der Sandschneisse. - Beim Fr. Forsthaus > 8. - Im Maingenist 8. April 1860 einmal gef. (B.) - Fr. (Hg.) - Mz. (S.) -

12. C. carnifex F. - Fr. z. s. - Friedberg (Fuhr). Mz. (Hg., S.) -

13. C. cáerulescens Suffr. - Bei Mo, von S., Hg. und L. H. n. s. gef. - Eine der mittleren Rheingegend eigentlümliche Art. Fr. s. s. - Mo. ein Weib $=10$. gebar 18 lebende Larven; dieselben verliéssen bei der Geburt die Eiliaut. -

14. C. marginata F. - Je einnial bei Fr. von C. H. und Hg. gef. - G. (L.) - Wlb. (Sch.) -

15. C. analis F. = Schach F. - Bei Fr. zweimal von C. H. gef. - Am Goldstein im Fr. Wald s. s. gestreift, auch von Hg. ges. -

16. C. caerulans Scrb. = violacea Panz. - Auf Mentha g. - Fr. auch von Hg. gef. - Dlb. Wlb. (Sch.) -

17. C. graminis L. - Die Larve und Käfer auf Tanacetum. Fr. - Friedberg (C. H.). - Bonames (B.). - Mz. (S.) - Wsb. (K.) -

18. C. fastuosa Scop. - Schlangenbad. - Auf Urtica dioica früher in der Frankfurter Gärtnerei (jetzt Trutz) h. - Fr. (Hg.) Mz. (S.) - Dlb. S. h. auf Galeopsis Ladanum (Scl..). -

19. C. cerealis L. - Ueberall h. - Fr. - Auf Spartium von L. H. und Hg. gef. - In Gräben an der Babenhäuser Landstrasse. G. (L.) - Dlb. auf Weiden und unter Steinen (Sch.). -

20. C. polita L. - Fr. 11. s. an Weiden. - G. (L.) - Dlb. Wlb. (Sch.) -

21. C. oricalcia Müll. = lamina F. - G. zweimal (L.). Fr. (Hg.) - Dlb. (Sch.) -

22. C. Hyperici Forst. = fucata F. -- Bieberer Höhe bei Offenbach. - Fr. sehr einzeln (L. H., Hg.). - G. (L.) - Wlb. (Sch.) -

Var. quadrigemina Suffr. - Gonsenheim bei Mz. von Ludwig gesammelt. [In Sammlung von Heyden.] - Fr. einzeln; auch von Hg. gesammelt. -

23. C. geminata Gyll. Payk. - Fr. öfter von C. H. und Hg. gef. - Mz. (S.) - Dlb. Wlb. (Sch.) -

[Eine Oreina ist aus dem Gebiet nicht bekannt.] 


\section{Phytodecta Kirby $=$ Gonioctena Redtenbacher.}

1. P. rufipes De Geer. - Fr. einzeln auf Weiden; auch von Hg. und B. h. gef. - Wlb. h. (Sch.) - Mr. (S.) -

2. P. viminalis L. - Fr. h. auf Salix cinerea. - (Variirt von hellgelber Oberseite mit schwarzem Kopf bis ganz schwarz.) - G. (L.) - Mz. (S.) - Wlb. in allen Varietäten, ganz schwarze Stücke h., auch ganz gelbroth bis auf den Kopf und die Augen, auch Kopf und Thorax schwarz, Decken roth (Sch.).

3. P. flavicornis Suffr. - Hofheim im Taunus auf Salix caprea. - Stücke mit einfarbig gelben und einfarbig schwarzen Flügeldecken. - G. (L.) - Fr. (Hg.) - Mz. (S.) Suffr. - Fr. (Hg.) -

4. P. Linnaeana Schrk. = decempunctata Schrk. = Triandrae

5. P. olivacea Forst. $=$ litura F. - Auf Spartium scoparium h. - Fr. Wald. - Taunus. - Am Buchrain bei Offenbach n. h. (B.) G. (L.) - Mz. (S.) - Wsb. auf der Waldwiese vor der Rentmauer 20. Mai (K.). -

6. P. quinquepunctata F. - Lebt auf Sorbus aucuparia. Fr. - Weg von Oberursel nach dem Feldberg h. - Fr. (Hg.) -

7. P. pallida L. - Am Feldberg auf Salix caprea. - Fr. einmal (B.), auch von Hg. gef. -

\section{Melasoma Steph. = Lina Redtenbacher.}

1. M. aenea L. - Auf Erlen überall g. - (Goldgrün, blaugrün, blau, violet, schwar\%) - Fr. - Königstein. - G. (L.) - Wlb. Dlb. (Sch.) - Mr。 (S.) - Wsb. hinter der Walkmühle 11. August 1851 (K.). -

2. M. collaris L. - Bei Fr. von Hg. in 6 Ex. aufgef. -

3. M. ruficaudis De Geer $=$ cuprea F. - Auf Erlen S. Hofheim im Taunus, - Fr. (Hg., B.) - G. (L.) - Mz. (S.) - Dlb. Wlb. (Sch.) -

4. M. Lapponica L. - Friedberg (Scriba). -

Var. Bulgarensis F. (Einfarbig blau.) - Von Hg. einmal bei Fr. gef.

5. M. Populi L. - Auf Pappeln überall g. - Larve $>8$. auf Populus nigra gef. $->4$. bei Falkenstein. - Fr. = 6. - G. (L.) - M\%. (S.) - Dlb. Wlb. (Sch.) - 
6. M. Tremulae F. - Mo, auf Populus nigra. - Fr. seltener als die rorige (B.). - Mz. (S.) - Dlb. Wlb. (Sch.) -

7. M. Iongicollis Suffr. - Fr. - Ems. - Bei Falkenstein $<9$. s. h. auf Aspen. Zu dieser Jahreszeit sind die Flügeldecken mehr fahlgelb. - L. H. besitzt 1 Stück (Weibchen), das ron $=10$. bis $<2$., an der Nadel angespiesst, weiter lebte und erst starb, als die Schachtel aus einem ungeheizten in ein geheiztes Zimmer gestellt wurde. Wahrscheinlich können unbefruchtete Weibchen, die also ihren Lebenszweck noch nicht erreicht haben, wenn günstig ang'cspiesst, d. h. wenn keine edlen Theile verletzt sind, recht lange noch an der Nadel leben. Sie scheinen in einen Art Schlaf zu verfallen, denn sie bewegen die Gliedmaassen nur, wenn man sie berührt. - Fr. (Hg., B.) -

\section{Plagiodera Redtenb.}

1. P. versicolora Laich. = Armoraciae L. - Im ganzen Gebiet g. auf Weiden und Pappeln. - Fr. - Cronberg im Taunus. Offenbach. - Bei Fr. auch ron Hg. und B. gef. - G. (L.) - - Dlb. Wlb. (Sch.) - Mz. (S.) -

\section{Gastroidea Hope = Gastrophysa Redtenb.}

1. G. Polygoni L. - Fr. h. auf Polygonum, besonders auch auf Buchweizen im Mai. - Nach B. bei Fr. g., selbst in den Gärten innerhalb des Stadtgebietes. - G. (L.) - Dlb. Wlb. (Sch.) - Mr. (S.) -

\section{Phaedon Latreille.}

1. P. pyritosus Oliv. - Bei Bergen auf Ranunculus von C. H., bei Fr. in der Gärtnerei (Lindau) von L. H. gef. - Von B. h. auf Weiden (wohl zufällig) gesammelt bei Fr. - Wlb. (Sch.) -

2. P. Armoraciae L. Thoms. = Cochleariae Gyll. Redth. $=$ Betulae Suffr. - Fr. je einmal ron C. H. und Hg. gef. - Wlb. 2 Ex. (Sch.) - Mrz. (S.) -

3. P. Cochleariae F. = grammicus Dft. Suffr. - Fr. auf Cochlearia armoracia n. s. - Enkheim. - Auch von B. und Hg. gef. - G. (L.) - Wlb. (Sch.) - Mz. (S.) -

4. P. concinnus Steph. = Triglochinis Schaum. - Von Dr. Bose bei Selters in Nassau gef., von C. H. an den Wetteraner Salinen auf Triglochin maritimum z. B. bei Wisselsheim bei Friedberg. - 


\section{$-309-$ \\ Prasocuris Latreille.}

1. (Hydrothassa Thoms.) aucta F. - Königstein. - An den Röderhöfen bei Fr. s. h., auch im Genist 4. April 1860 von B. gef. - G. (L.) - Fr. (Hg.) - Mz. (S.) -

Var. egena (Ziegl.) Suffr. (Ohne rothen Saum der Flügeldecken.) - Von Hg. bei Fr. aufgef. -

2. (H.) marginella F. - Lebt auf Ranunculns aquaticus. - Auf der Königswiese im Fr. Wald n. s. am 16. April 1861. - G. (L.) - Fr. (Hg.) - Wlb. (Scl.). - Dlb. im Eichbachthal im Mai (K.). -

3. (H.) Hannoverana F. - Fr. von Hg. und B. n. s. gef. G. (L.) - Wlb. (Sch.) -

4. P. Phellandrii L. - Fr. n. s. auf Wasserpflanzen. - Im Genist g., Röderhöfe bei Fr. am 4. April 1860. - G. (L.) Wlb. (Sch.) - Mz. (S.) - Had. Teich am Haidenhäuschen im Frülljahr (K.). -

5. P. Junci Brahm = violacea $F$. $=$ Beccabungae Illig. Auf Veronica Beccabunga in Wassergräben bei Fr. n. h. - Aus Genist bei Fr. einmal (B.). - Wlb. (Seh.) -

\section{Phratora Redtenbacher.}

1. P. vulgatissima L. - Fr. - Auf Pappeln und Weiden h. Auch von B. und Hg. h. gef. - G. (L.) - Dlb. Wlb. (Sch.) - Mzz. (S.) -

Var. tibialis Suffr. - Fr. n. s.' auf Weiden. - G. (L.) Dlb. Wlb. (Sch.) -

2. P. Vitellinae L. - Fr. h., auch von Hg. und B. gef. G. (L.) -

3. P. laticollis Suffr. = atrovirens Cornel. - Ein von Suffrian selbst bestimmtes Exemplar fand C. H. bei Fr. -

\section{E. CASSIDINI. \\ Cassida Linné. \\ (Die Sammlung v. Heyden ist von Suffrian revidirt.)}

1. C. equestris F. - Fr. n. s. - Schmitterhof bei Giessen $=8$. - Mz. (S.) - Fr. 16. Mai 1860 (B.). - Wlb. in Menge auf Mentha sylvestris (Sch.). - Wsb. auf Mentha und Lycopus europaeus zwischen Walkmühle und Adamsthal 28. August (K.). - Mz。 (S.) - 
2. C. hemisphaerica Hbst. - Fr. n. s. noch $=10$. - Mz. (S.) - Fr. s. auch von Hg. und B. gef. - G. (L.) - Wlb. s. h. (Sch.) -

Var. $\beta$. Suffr. (nigriventris Heyden) - (Der Hinterleib bis auf einen schmalen gelben Saum schwarz, bei der Stammart ist er ganz gelb.) - Fr. zweimal von C. H. gef. - Wlb. s. (Sch.) -

3. C. canaliculata Laich. $=$ Austriaca F. - Fr. je einmal von C. H. und L. H. $>$ 4. an der Eschenheimer Landstrasse auf Salvia pratensis; auch von v. Twardowski und von Wiegand int Taunus in Nehrzahl gef. - Im Ieben sind Halsschild und Decken oben silbergrün und braun gefleckt, die Ränder braun. - Mz. (S. 1843 auf Arthemisia absinthium.) - Einmal von B. am 20. Mai 1861 bei Fr. gef., auch von $\mathrm{Hg}$. gesammelt. -

4. C. Murraea L. - Die Larve und Käfer auf Inula salicina $<$ 7. bei Offenbach von L. H. und B. gef. - Braune Stïcke bei Fr. - Mz. (S.) - G. (L.) - Wsb. (Sch.) - Mz. (S. Nur grüne Stücke.) - Fr. (Hg.) -

Var. maculata L. (Grün mit schwarzen Flecken.) - Fr. G. (L.) - Auf Inula salicina am Rheinufer zwischen Schierstein und Biebrich im Juli (Sandberger bei K.). -

5. C. sanguinosa Suffr. - Fr. einzeln von C. H. und 1 Stïck bei St. Goarshausen $<5$. gef. - Mz. (S.) - Wlb. (Sch.) - Soden (L. H.) - Fr. = 7. auf Achillea millefolium. - Im Hengster bei Offenbach $>$ 6. in Begattung gef. (C. H.) -

6. C. rubiginosa Illig. = viridis $\mathbf{F}$. - Fr. auf Kletten (Arctia lappa) $>5$. in Begattung. - Soden $<8$. auf Serratula. - Ems von C. H. gef. - Mz. (S.) - G. (L.) - Fr. (Hg., B.) - Dlb. Wlb. (Sch.) - Wsb. auf Disteln im Sommer mit Larven und Puppen (K.). Mz. (S.) -

7. C. seladonia Gyll. = filaginis Perris = rotundicollis Bris. - Bei Mo. zweimal mit Larve $>5$. auf Gnaphalium arenarium gef.; der Käfer entwickelte sich <6. - Mz. (S.) -

8. C. ferruginea Goeze = thoracica Fourc. nec Kugel. G. (L.) -

9. C. vibex L. - Fr. n. s. - Von C. H. mit Larve = 7. auf Jurinea gef. - Mr. (S.) - G. (L.) - Wlb. (Sch.) - Wsb. (K.) Soden (L. H.). -

10. C. denticollis Suffr. - Einigemal bei Fr. von C. H. und Hg. gef. - Mz. (S.) - Wlb. (Sch.) - 
11. C. chloris Suffr. - languida Cornel. - Fr. einmal gef. - Im Wald von Montabaur in Nassau (Suffrian). - Mz. (S.) Wlb. (Sch.) - Homburg (v. Kraatz bei Hg.). -

12. C. stigmatica Suffr. = chloris Cornel. nec Suffr. Fr. dreimal gef. - Seltene Art. - G. (L.) - Auch Hg. sammelte die Art im Gebiet. -

13. C. sanguinolenta F. = prasina Illig. - Fr. n. s. auf Achillea millefolium. - G. (L.) - Bewahrt mitunter noch nach dem Tode ilıre schốne Färbung (B.). - Fr. (Hg., B.) - Wlb. (Sch.) -

14. C. azurea F. = lucida Suffr. - Fr. (Hg.) - Ems auf Cucubalus behen $=7$. und $=8$. von C. H. und Suffrian gef. -

15. C. margaritacea Schall. - Bei Fr. n. s. - Auf Clematis $=10$. in Begattung gef. - Mz. (S.) - Soden auf Eichengebüsch h. - Fr. (B.) - Wlb. z. h. (Scli.) -

Var. albiventris Germ. Suffr. (Unterseite bis auf den Kopf grünlichgelb.) - Fr. zweimal von C. H. gef. -

16. C. nobilis L. - Fr. auf Spergula arvensis von C. H. gef. - Fr. < 11. unter Moos zum Ueberwintern versteckt, öfter gef. Nauheim =8. auf Salzboden. - Mz. (S.) - Fr. s. (Hg., B.) G. (L.) -

17. C. oblonga Illig. - Fr. h. - Am Feldberg im Taunus $<6$. — Ganz hellgefärbte Stücke (vide Suffrian, Stettiner Zeit. 1844, pag. 254) fand C. H. zweimal bei Fr. - Mz. (S.) - Fr. (Hg., B.) -- Wlb. (Sch.) -

18. C. obsoleta Illig. - Ems (Suffrian). - Mz. (S.) - Von C. H. auf Stellaria holostea gef. - Fr. S. (L. H., Hg. und B.) G. (L.) - Wlb. (Sch.) - Wsb. (K.) -

19. C. subferruginea Schrk. = ferruginea F. - Fr. n. s. - Mz. (S.) - Bei Fr. auch von Hg. und B. gef. - G. (L.) Wlb. (Sch.) -

20. C. nebulosa L. - Fr. h. - Am Feldberg < 6. - Der Käfer hat im Juli 1858 um Fr. grossen Schaden gethan; im Gutleuthoffeld zerstörte er 40 Morgen Runkelrüben; bei Bonames die Dickwurzpflanzen. - Auch im Sommer 1876 fand er sich in einigen Gegenden Nassaus, 7. B. in der Gemarkung Kostheirn, als Runkelrübenverderber (K.). - Fr. (Hg., B.) - G. (I.) - Wlb. die grüne Varietät seltener als die andere (Sch.). - 


\section{F. HISPINI.}

\section{His pa L i nné.}

1. H. atra L. (Ungeflügelte Stücke = aptera L. nur in SüdEuropa.) - Ueberall h. - Fr. - G. (L.) - Auf feuchten Wiesen gegen Abend mit dem Streifnetz am Goldstein im Fr. Wald 14.-25. Mai 1861 gef. (B.) - Wlb. s. h. (Sch.) - Mz. (S.) - Mo. auf dem Damm am Rhein (K.). - Waldwiese hinter Adamsthal bei Wsb. <6. (K.) Wsb. an der Tränke 15. October (K.). - Mo. auf Hecken 1. August (K.). -

\section{G. GALLERUCINI.}

Adimonia Laicharting.

1. A. Tanaceti L. - Ueberall h. im Gras von $=7$. bis $<12$. - Fr. - Offenbach. - Soden. - Ems. - Das kleinste Exemplar der Sammlung v. Heyden ist 5, das grösste $9^{\mathrm{mm}}$ lang. - Fr. Wald (B.). - G. (L.) - Wlb. (Sch.) - Mz. (S.) -

2. A. rustica Schall. - Fr. Wald an der Bruchschneisse $>9$ : - Mo. $=6$. ein Weibchen Eier legend. - Von Fr. 3 Stück (B.). G. (L.) - Mz. (S.) - Wlb. (Sch.) -

3. A. interrupta Oliv. - Nur in Sandgegenden. - Fr. Wald hinter der Ziegelhütte in den Sandgruben $<11$. - Mo. $=6$. h. von S., K. und uns gef. -

4. A. sanguinea F. - Fr. einmal (Hg.). - Wlb. zweimal; Halsschild nur neben eingedrückt, blutroth (Sch.). -

5. A. Capreae L. - Ueberall g. auf Weiden $>4$. bis $=10$. - Auch < 9. auf Birken. - Fr. (B.) - G. (L.) - Mz. (S.) Wlb. (Sch.) - [Stirn dicht runzelig punktirt, Decken einfarbig, Schenkel schwarz.] -

Die nahe verwandte Art A. suturalis Thoms. - (Stirn sparsam punktirt, Flügeldeckennaht schwarzbraun, Schenkel gelb) - noch nicht im Gebiet gefunden. -

\section{Galleruca Fabricius.}

1. G. Viburni Payk. - Auf Viburnum opulus n. s. $>9$. bei Fr. - Mz. (S.) - Wlb. (Sch.) - Wsb. an der Wellritz im Herbst (K.). Königstein $>8$. - 
2. G. Crataegi Forst. $=$ Calmariensis F. $=$ xanthomelaena Schrk. - Hinter dem Ort Mombách $>5$. zahllos auf Ulmenhecken, jedes Blatt war durch die Larven scelletirt. Die Farbe ist im Leben grünlichgelb mit schwarzer Längsbinde, im Tod bräunlichgelb. - Bei Fr. nur einmal $=6$. am Affenstein beim neuen Irrenhaus von C. H. auf Ulmen. - Eier und Larven bei Mo. = 6. - Die länglichen, zitzenförmigen, grüngelblichen Eier werden in zwei Streifen verbunden nebeneinander gelegt. - Wlb. s. h. (Sch.) - Mz. (S.) - Schierstein am Rhein auf Ulmen h. von 4. bis 20. Juni (K.). - Rüdesheim auf Ulmen $<8$. -

3. G. Nymphaeae L. - G. 3 Stück (L.). - Bei Fr. im Netzgerbruch von Hg. gef. - Mo. im Graben am Rhein auf Nymphaea alba 20. September (K.). -

4. G. Sagittariae Gyll. - Fr. anf der ehemaligen Zinmerwiese $>$ 9. mit Larve auf Polyganum amphibium einigemal von C. H. gef. Fr. (Hg.) -

5. G. lineola F. - Auf Weiden h. bei Fr. = 5. - Anfang des Monats bei Hofheim; $=4$. auf Kiefern einigemal (zufällig) im Fr. Wald, Bruchschneisse. — Schmitterhof bei Giessen = 8. - Fr. (Hg., B.) - G. (L.) -

6. G. Calmariensis L. = Lythri Gyll. - Fr. auf Lythrum im Mai in Begattung öfter gef. - Auf der Königswiese im Fr. Wald n. s. (B.) - G. (L.) - Waldmannshausen in Nassau 4. Juni (K.). Wlb. s. h. (Sch.) - Mz. (S.) -

7. G. tenella L. - Fr. einigemal von C. H. und Hg. gef. Wlb. h. an der Lahn auf Wasserpflanzen (Sch.). - Mz. (S.) - Wșb. h. 5. Juni (K.). -

\section{Phyllobrotica Redtenbacher.}

1. P. quadrimaculata L. - Am Entensee bei Rumpenheim $=7$. auf Scutellaria. - Am Sumpf beim Hellerhof bei Fr. < 7. - Von L. H. und M. S. in den Enkheimer Torfbrüchen gef. - An letzterem Ort anch von B. auf Scutellaria gef. - Mz. (S.) -

\section{Agelastica Redtenbacher.}

1. A. Alni L. -- Ueberall in grosser Menge auf Erlen. - Fr. Königstein. - Cronthal. - Bonames (B.). - G. (L.) - Dlb. Wlb. (Sch.) - Mr. (S.) - 
2. A. Halensis L: = nigricornis F. - Ueberall 11 . s. auf Galiun. Bei Soden $>8$. - Zu derselben Zeit am Schmitterhof bei Giessenl. - Fr. s. (B.) - Bei Wlb. einer der gemeinsten Käfer (Sch.). - Mz. (S.) - Wsb. 10. August in Begattung gef. (K.) - MIo. 12. August 1851 (K.). -

\section{Luperus Geoffroy.}

1. (Calomicrus Steph.) circumfusus Mrsh. = Spartii Ent. Hft. - Ems von C. H. auf Spartium scoparium gesammelt. - Fr. s. (Hg.) - Wlb. s. h. (Sch., K.) -

2. (C.) pinicola Duft. - Auf Kiefern im ganzen Gebiet h. Soden > 7. - Fr. (Hg., B.) - Wlb. s. (Sch.) - Mr. (S.) - Mo. auf Kiefern von 5. bis 15. Juli (K.). -

3. L. niger Geoffr. = dispar Ksw. i. I. (Weibchen mit rothem, Männchen mit schwarzem HaIsschild.) - Wsb. ein Pärcheıl (K.). -

4. L. xanthopoda Schrk. = rufipes Joan. (Beide Geschlechter mit schwarzem Halsschild, Beine ganz gelb.) - Wsb. unter der Rentmauer an der Fischzuchtanstalt 10. Juni ein Weibchen gef. -

5. L. longicornis F. = rufipes Gyll. Kiesw. = betulinus Fourcr. (Beide Geschlechter mit schwarzem Halsschild, Hinterschenkel an der Basis schwarz.) - Auf Birken =6. bei Königstein. - Fr. > 5. auf Erlen. - G. (L.) - Dlb. an Obstbärumen (Sch.). - Gonsenheimer Capelle bei Mz. 11. Juni (K.). -

6. L. flavipes L. Joannis. (Männchen und Weibchen mit rothem Halsschild.) - Königstein > 6. - Fr. (Hg.) - G. (L.) - Gustavsburg an der Mainmündung 12. Juni (K.). - Wlb. (Sch.) -

\section{H. HALTICINI.}

(Die Sammlungen $\nabla$. Heyde $\mathrm{n}$ und $\mathrm{H}$ a a g vom Monographen Allard revidirt.)

\section{Haltica Geoffroy.}

1. (Graptodera Allard.) Lythri Aubé. - Fr. auf jungen Birkenausschlägen $>6$. h. - Im Fr. Wald (Bruchschneisse) $=5$. auf Birken. - Mz. (S.) -

2. (G.) ampelophaga Guér. - In der Frankfurter Gärtnerei vor dem Eschenheimer Thor $<5$. h. auf Rosenhecken. - Im Fr. Wald auf Epilobium =6. und > 7. - Fr. (Hg.) - 
3. (G.) Erucae Oliv. = quercetorum Foudr. - Im August und September 1858 so häufig im Fr. Wald auf Eichen, dass Bäume und Gesträuch ganz kahl gefressen waren. - Die Eier werden $>6$. bis zu zwölf Stück auf der Unterseite der Eichenblätter abgelegt. Fr. g. (Hg., B.) - Wlb. (Sch.) - Mz. (S.) -

4. (G.) Coryli Allard = brevicollis Foudr. - Auf Haseln s. s. $=6$. bei Fr. von C. H. gesammelt. - Mz. (S.) -

5. (G.) Oleracea L. - Ueberall g. auf Epilobium, Oenethera. Fr. - Soden. - Mo. $>$ 8. - Dlb. Wlb. noch nie auf Gemüse, meist auf Gebüschen gef. - Mz. (S.) -

6. (G.) pusilla Dft. = Potentillae Allard. - Bei Königstein = 9. auf Epilobium. - Soden auf Wiesen. - Fr. (Hg.) - Wlb. (Sch.) - Mz. (S.) -

7. (G.) cognata Kutsch. = montana Foudr. - L. H. besitzt ein von Vicomte de Bonvouloir bei Ems gefangenes Exemplar. -

8. (Hermaeophaga Foudr.) Mercurialis F. - Bei Falkenstein $>$ 5. auf Mercurialis perennis. -

9. (Chalcoides Foudr.) nitidula L. - Nur einmal bei Nauheim $>$ 5. von C. H. gef. - Wlb. z. h. auf Bachweiden mit der folgenden (Sch.). - Dlb. h. auf Weiden an Bächen (K.). -

10. (C.) aurata Mrsh. Foudr. = versicolor Kutsch. - Auf Weiden. - Eins. - Offenbach = 7. h. - Fr. (Hg.) - Wlb. auf Bachweiden s. (Sch.) -

11. (C.) smaragdina Foudr. - Im Lorsbacher Thal $<$ 5. auf Weiden. - Fr. auf Salix caprea, auch von Hg. gesammelt. -

12. (C.) chloris Foudr. - Fr. einzeln. -

13. (C.) helxines L. Allard. - Falkenstein = 5. auf Aspen. - Fr. auf Salix caprea h., auch von Hg. gef. - Dlb. Wlb. auf Bachweiden ungemein $\mathrm{h}$. (Sch.) -

14. (Epitrix Foudr.) pubescens Ent. Heft. - Auf Belladonna. - Fr. (Hg., B.) -

15. (E.) intermedia Foudr, - Von Ig. bei Fr. gef. -

16. (E.) Atropae Märk. Kutsch. - Rumpenheim auf Atropa Belladonna h. gef. (L. H., Hg.) - Fr. (C. H.) - Mo. (v. Bodemeyer). -

17. (Crepidodera All.) transversa Mrsh. = impressa Duft. - Offenbach am Mainufer = 7. - Ems. - Fr. s. h. (Hg., B.) Wlb. s. (Sch.) - Mz. (S.) - 
18. (C.) impressa F. = rufa Küst. - Ein Exemplar dieser südlichen Art fand Hg. bei Fr. -

19. (C.) exoleta L. = ferruginea Scop. - Offenbach wie die vorige Art, häufiger. - Ems $=8$. auf Cucubalus behen h. Fr. (Hg., B.) - Wlb. h. (Sch.) - Mz. (S.) -

20. (C.) rufipes L. - Falkenstein $=5$. auf Vicia; auf Wiesen bei Cronberg < 6. - Am Altkönig im Mai. -- Nauheim > 5. St. Goarshausen. -- Fr. n. s. (Hg., B.) - Wlb. (Sch.) - Mlz. (S.) -

21. (C.) nigritula Gyll. - Fr. Wald auf der Bruchschneisse in Sandgräben > 4. - Offenbach, Griesheim am Main. -

22. (Hippuriphila Foudr.) Modeeri L. - Fr. - Bieberer Höhe bei Offenbach = 7. - Schmitterhof bei Giessen $>8$. - Fr. s. (Hg., B.) - Wlb. (Sch.) -

23. (Ochrosis Foudr.) Salicariae Payk. - Am Entensee zwischen Rumpenheim und Bürgel $<8$. auf Lysimachia vulgaris von L. H. und B. gef. - Fr. Wald ebenso $>5$. - N\%. (S.) -

24. (0.) ventralis Illig. = abdominalis Küst. $=$ nigriventris Bach. - Fr. sehr einzeln. - Cronthal im Taunus < 10. - Fr. (Hg.) -

25. (Balanomorpha Foudr.) rustica $\mathrm{L} .=$ semiaenea F. Im Rebstockwald bei Fr. s. h. im Juni. - Sodenerwald an feuchten Grasstellen $=5$. - Nauheim $>5$. auf Salzboden an Lepidium graminifolium. - (Variirt ganz dunkelerzgrün, sowie mit rothbrauner Flügeldeckenspitze, welche Farbe so überhand nehmen kann, dass nur die Naht grün bleibt; ron dieser letzten Varietät ein Nauheimer Stück. Fr. (Hg., B.) - Wlb. (Sch.) -

26. (B.) obtusata Gyll. - Von Hg. bei Fr. gef. -

27. (B.) Chrysanthemi Ent. Heft. - Fr. Wald in der Bruchschneisse > 4. - Griesheim am Main im August. - Ems. - Fr. (Hg.) -

28. (Batophila Foudr.) Rubi Payk. - Auf Brombeeren (Rubus fruticosus) h. im Taunus bei Hofheim $>6$, bei Cronberg $<9$. St. Goarshausen $<5$. - Wlb. (Sch.) -

29. (Podagrica Allard) fuscipes F. - Fr. einmal von Hg. gef. -

30. (P.) Malvae Illig. - Von Hg. bei Fr. gef. -

31. (P.) fuscicornis L. - Auf Malven überall g: - Soden > 7. - Fr. s. von uns, Hg. und B. gef. - Wlb. (Sch.) - 
32. (Phyllotreta Foudr.) Armoraciae Ent. Heft. - Leht auf Meerrettig (Cochlearia armoracia). - Fr. nur einigemal von C. H., Hg. und B. gef. - Mz. (S.) -

33. (Ph.) tetrastigma Comolli. - An der Gerbermühle bei Oberrad am Main von L. H. und M. S. - s. s. mit dem Streifnetz gef. - Fr. (Hg.) -

34. (Ph.) flexuosa Illig. Kutsch. = fallax All. - Einmal bei Fr. von L. H. und Hg. gef. - Mz. (S.) -

35. (Ph.) Brassicae $\mathrm{F}$. = quadripustulata Marsh. - Fr. n. s. - Im Winter unter Moos. - Auch in Gärten in der Stadt g. (B.) - Fr. (Hg.) - Wlb. (Sch.) - Mz. (S.) -

36. (Ph.) ochripes Curtis = excisa Redth. - Fr. z. s. Im Wald auf der Bruchschneisse im Sandgraben > 4. - Auch auf Sisymbrium alliaria. - Fr. (Hg:) -

37. (Ph.) sinuata Redtb. - Auf Gemüsen von April bis Juni: - Fr. (Hg.) - Wlb. (Sch.) -

38. $(\mathbf{P h}$.$) undulata Kutsch. = flexuosa Allard. - Bei Fr.$ einzeln gesammelt von uns und Hg. - Wlb. (Sch.) -

39. (Ph.) nemorum L. - Fr. n. s., auch von Hg. und B. gef. - Dlb. Wlb. s: h. auf Brassica oleracea im April und Mai (Sch.). Mz. (S.) -

40. (Ph.) vittula Redth. - Unter Schilf an den Kettenlöfen bei Fr. = 3. - Rödelheimer Wald $=8 .-$ Fr. auf Pinus picea $<8$. - Mo. = 5. - Er. (Hg.) - Wlb. einmal (Sch.). -

41. (Ph.) atra Ent. Heft. - Griesheim am Main = 8. - Auf Hecken bei Soden $>6$. - Bieberer Höhe bei Offenbach $=6$. Fr. (Hg., B.) -

42. (Ph.) obscurella Illig. = poeciloceras Comolli. - Auf Brassica Erysimum, Sisymbrium im Frühjahr bei Fr. — Soden $>6$. auf Hecken und $=7$. im Gras, auch auf Pflaumenbäumen. - Hofheimer Wald < 6. - Rüdesheim $=8$. - Dlb. Wlb. auf Brassica oleracea mit nigripes (Sch.). - Mz. (S.) -

43. (Ph.) melaena Foudr. - Bei Fr. zweimal von C. H. und Hg. gef. -

44. (Ph.) diademata Foudr. -- Von Hg. bei Fr. gef. -

45. $(\mathbf{P h}$.) nigripes Panz. = Lepidii Ent. Heft. - Offenbach am Main = 7. - Soden < 8. auf Reseda lutea. — Fr. und Rebstock- 
wald, Griesheim h. - Bei Fr. g., am 20. März 1860 z. B. (B.) Dlb. Wlb. die gemeinste Art auf kleinen Pflanzen von Brassica oleracea, die sie oft ganz zerstört, auch Lepidium sativum und Radieschen (Sch.). -

46. (Ph.) antennata Ent. Heft. = nodicornis Allard. - Auf Reseda lutea und luteola im April und Mai. - Fr., Rumpenheim, Mo. auf der Futterpflanze n. s. - Bei Fr. auch ron Hg. und B. gef, -

47. (Aphthona Allard) Cyparissiae Ent. Heft. - Auf Euphorbia Cyparissias h. - Fr. Wald $<$ 7. - Bieberer Höhe bei Offenbach < 9. - Ems. - Fr. s. (Hg., B.) - Wlb. (Sclı.) - Mz. (S.) -

48. (A.) lutescens Gyll. - Am Entensee zwischen Bürgel und Rumpenheim = 9. auf Lythrum. - Fr. $\left(\mathrm{Hg}_{\circ}\right)$ -

49. (A.) caerulea Payk. - Lebt auf Schwertlilie (Iris piseudacorus) in Wassergräben an der Ziegelhütte am Fr. Wald, schon im Februar; dann von $>4$. bis $>7$. - Fr. - Mo. - Enkheimer Torfbrüche. - Rumpenheim am See beim Schiessstand. - Fr. (Hg., B.) -

50. (A.) hilaris Steph. Allard. = virescens Foudr. - Fr. n. s. s. - An feuchten Stellen im April. - Von Fichten geklopft = 11 . und $<$ 12. - Schmitterhof bei Giessen $=$. 8. - Fr. (Hg.) -

51. (A.) venustula Kutsch. = Euphorbiae All. = cyanella Foudr. - Fr. s. $=9$. im Wald bei der Unteren Saustiege. - Bei Altenhain bei Soden $>$ 9. auf Birken. - Ems. - Fr. (Hg.) Mz. (S.) -

52. (A.) atrocaerulea Steph. All. = cyanella Redtb. $=$ Euphorbiae Foudr. - Fr. n. s. - Falkensteiner Wald $>8$. St. Goarshausen $<5$. auf Euphorbia C5parissias. - Rüdesheim. Fr. (Hg.) -

53. (A.) violacea Ent. Heft. = Pseudacori Redtb. = sublaevis Bohem. - Bei Fr. zweimal von L. H. gef. -

54. (A.) herbigrada Curtis = Campanulae Redtb. - Auf Campanula n. s. zwischen Soden und Cronthal im Taunus ron uns gef. - Wlb. (Sch.) -

Longitarsus Latreille 1829 (= Thyamis Steph. 1831 = Teinodactyla Chevr. 1837).

1. L. Echii Ent. Heft. = tibialis Dft. - Eimmal bei Fr. $>6$. von C. H. gef. - Mz. (S.) - 
2. L. obliteratus Rosh. = consociatus Först. = pulex Foudr. - Bei Bergen $>$ 5. auf Origanum n. s. - Fr. auf Thymus serpyllum h. - Wlb. (S.) - Mz. (S.) -

3. L. Absinthii Bach. Kutsch. - Von Bach zuerst auf nassauischem Gebiet zwischen Filzen und Kamp am Rhein auf Wermuth entdeckt. - Wlb. (Sch.) -

4. L. Anchusae Payk. - Fr. Wald im Wartforst $=5$. auf Cynoglossum, ebenso bei der Ziegelhütte; Bieberer Höhe bei Offenbach auf Echium $>5$. his $<6$.; bei Enkheim auf Pulmonaria $<4$. Auf Symphytum amplibium h. - Fr. (Hg.) - Wlb. (Sch.) - Mz. (S.) - Wsb. an der Walkmühle auf Pulmonaria im April (K.). Fr. (Hg.) -

5. L. niger Ent. Heft. - Bei Ems einmal von C. H. gef. -

6. L. parvulus Payk. = pumilus Illig. - Soden $>6$. auf Rubus. - Fr. $>$ 5. - Ems. - Fr. (Hg.) - Wlb. (Sch.) -

7. L. apicalis Beck = analis Dft. - Wsb. im Wald hinter Adamsthal 14. September (K.). -

8. L. Holsaticus L. = pulicarius L. - Rumpenheim am Teich beim Schiessstand $>10$. - Fr. einigemal gef.; auch von Hg. Mo. 6. August (K.). -

9. L. castaneus Foudr. - Einmal bei Fr. von C. H. gef. -

10. L. Iuridus Scop. - Königstein = 9. - Entensee bei Rumpenheim zu derselben Zeit je einmal gef. - Fr. auf Scabiosa. Ems. - Fr. (Hg.) -

11. L. brunneus Dft. - Wlb. (Sch.) -

12. L. dorsalis F. - Auf Senecio. - Bei Fr. einmal öfter von C. H. gef., auch von Hg. - Wlb. h. (Sch.) -

13. L. Nasturtii F. - Auf Anchusa im Mai. - Im Fr. botanischen Garten auf Cynoglossum. - Ems. - Fr. (Hg.) - Wlb. (Sch.) -

14. L. suturalis Mrsh. - Von Hg. bei Fr. gef. -

15. L. thoracicus Allard. - Im Rebstockwald im Juni. - Fr. -

16. L. Verbasci Panz. - Bei Fr. von uns und Hg. gef. Auf Verbascum n. S. - Dlb. Wlb. h. (Sch.) - Mz. (S.) -

Var. Thlapsi Marsh. All. (Mit schwarzer Naht und Fleck hinter der Schulter.) - Fr. einmal von C. H. gef. - Mz. (S.) - 
17. L. Iateralis Illig. - Auf Verbascum im April und Mai bei Fr. n. h. -

18. L. atricillus Gyll. = fuscicollis Foudr. - Fr. - Sodener Wald $=6$. je einmal von C. H. gef. - Auch $\mathrm{Hg}$. sammelte die Art in Gebiet. -

19. L. melanocephalus Gyll. - Rüdesheim $<$ 5. auf Plantago. - Ems. - Fr. (Hg.) -

20. L. piciceps Steph. = picipes Allard. - Soden einmal $<$ 8. von C. H. gef. - Wlb. (Sch.) -

21. L. cerinus All. - Rödelheimer Wald $=8$. - Soden $=7$. im Gras. - Rüdesheim < 8. auf Malven. -

22. L. Ballotae Mrsh. (Früher mit cerinus vermengt.) - Bei Fr. von Hg. gef. -

23. L. pusillus Gyll. - Soden $=7$. im Gras. - Saline Nauheim > 8. - Ems. - Fr. (Hg.) - Wlb. (Sch.) -

24. L. femoralis Mrsh. = Boppardiensis Bach. = pratensis Foudr. - Fr. im Juli auf Echium s. - Wlb. (Sch.) -

25. L. tabidus F. - Ems. - Enkheim $>$ 7. - Fr. (Hg.) Wlb. (Sch.) -

26. L. rufulus Foudr. - Bei Fr. ein Pärchen gef. -

27. L. flavicornis Steph. = rubiginosus Foudr. - Unter Weidengebüsch am Main bei Offenbach $>8$. $<9$. h. - Soden am Teich hinter dem Curhaus. - Wlb. (Sch.) -

28. L. ferrugineus Foudr. - Soden im Gras = 7. - Schmitterhof bei Giessen $>8$. je einmal gef. -

29. L. Ochroleucus Mrsh. - Rüdesheim auf Malven (C. H.). Fr. (Hg.) - Mz. (S.) -

30. L. pellucidus Foudr. $=$ testaceus All. - Soden $<9$. auf Eichen. - Fr. s., auch vol $\mathrm{Hg}$. gesammelt. -

31. L. Teucrii All. - Am ehemaligen Rüstersee im Juli an Graswurzehn. - s. s. geflügelt. - Lebt auf Teucrium chamaedrys. -

32. L. subquadratus All. - Fr. einmal von C. H. gef. -

33. L. laevis Duft. = succineus Foudr. - Fr. auf Jacea $<7$, auf Achillea millefolium $=7$. - Königstein $=9$. $-\mathrm{Fr}$. auf 
Senecio Jacobaea. - Ems. - Auch auf Symphytum h. - Fr. (Hg.) - Wlb. (Sch.) -

34. L. ordinatus Foudr. - Fr. einmal gef. - [Hierher und zu subquadr. die abdominalis in S. V.] -

\section{Plectroscelis Redtenbacher.}

1. P. semicaerulea Ent. Heft. - Bei Fr. auf Hecken hinter der Oede > 6. - Ems. - Wlb. (Sch.) - Mz. (S.) -

2. P. concinna Mrsh. = dentipes Ent. Heft. - Ueberall h. - Fr. Wald = 4. von Juniperus geklopft; Forsthaus $<8$. - Rödelheimer Wald $=8$. - Königstein $>6$. auf Origanum. - Soden $=8$. auf Inula conyza. - Am Entensee bei Rumpenheim = 9. - Homburg auf Fichten h. <4. - Ems. - Fr. g. (Hg., B.) - Wlb. (Sch.) Mz. (S.) -

3. (Chaetocnema Foudr.) meridionalis Foudr. = obesa Boield. - Enkheimer Torfgruben = 5. - Fr. - Seltene Art. -

4. (Ch.) aridella Payk. - Fr. Wald am Forsthaus < 7 . Griesheim am Main im August. - Bieberer Höhe =6. - Königstein $=9 .-\mathrm{Ems}=8$. - Fr. s. h. $(\mathrm{Hg} .$, B. $)-$ Wlb. (Sch.) - Mz. (S.) -

5. (Ch.) Sahlbergi Gyll. - Bei Griesheim am Main im August. - s. s. - Einmal bei Fr. von Hg. gef. -

6. (Ch.) Mannerheimii Gyll. - Einmal bei Fr. von C. H. gef. -

7. (Ch.) aridula Gyll. - Im Rebstockwald bei Fr. im Juni. Im Fr. Wald am Forsthaus im August. - Nauheim = 8. auf Salzboden. - Auf Hecken und im Gras h. - Mo. - Fr. (Hg.) -Wlb. (Sch.) -

8. (Ch.) confusa Bohem. - Bei Fr. von C. H. einzeln ges. -

\section{Psylliodes Latreille.}

1. P. Dulcamarae Ent. Heft. $\rightarrow$ Fr. $>$ 8. auf Solanum dulcamare. - Auch von B. n. s. gef. - Mz. (S.) -

2. P. chalcomera Illig. - Lebt auf Disteln (Carduus). - Fr. Wald an der Kühruhe $<9$. - Hofheim im Taunus $<6$. - Griesheim im August. - Fr. (Hg.) -

3. P. Hyoscyami L. - Bei Wlb. von Sch. gesammelt. - 
4. P. chrysocephala L. - Auf Hecken überall h. - Soden $>6$; Rödelheimer Wald $=8$. - Flörsheim am Main $>6$. - Einmal $<10$. auf Hyoscyamus niger und Solanum dulcamara. - Ems. - Fr. (Hg.) - Wsb. an der Wellritz im Juni (K.).. - Wlb. (Sch.) - Mz. (S.) - Königstein > 8. -

Var. nigricollis Mrsh. (Flügeldecken gelb, anstatt grün wie bei der Stammart.) - Einmal bei Offenbach am Main am Leinpfad $=7$. mit dem Streifnetz von C. H. gef. -

5. P. nucea Illig. - Sehr seltene Art. — Einmal bei Hochheim in den Kalkbrüchen $=6$. von C. H. gef. -

6. P. Thlaspis Foudr. - Im Sauerthal bei Lorch am Rhein auf Isatis tinctoria $<6$. einmal von C. H. gef. -

7. P. instabilis Foudr. - Bei St. Goarshausen auf Cheiranthus cheiri $>6$. h., ebenso Anfang des Monats mit der vorigen Art bei Lorch auf Isatis h. von C. H. gef. -

8. P. Napi Ent. Heft. = Rapae Illig. - Einzeln bei Fr. von C. H. und Hg. gef. - Ems. - Wlb. z. h. (Sch.) - Mz. (S.) -

9. P. herbacea Foudr. = cupronitens Först. = cuprea Kutsch. - Fr. Wald am Forsthaus $<7$. und einmal $>7$. an Hanf gef. - [Hierher cuprea in S. V.] -

10. P. attenuata Ent. Heft. - Sulzbach bei Soden im Juni s. h. auf Hanf, dessen Blätter Käfer und. Larve durchlöchern; ebenso bei Hofheim. - Fr. auf Hopfen (Humulus) $<6$. - Lorsbacher Wald = 5. - Rebstockwald bei Fr. im Juni. - Fr. (Hg.) - Wlb. (Sch.)

- Mz. (S.) -

11. P. Kunzei Foudr. - Bei Fr. einmal von M. S. (in Sammlung v. Heyden) und 1 Stück $>6$. auf Hecken in Soden von C. H. gef. -

12. P. affinis Payk. = exoleta Illig. - Fr. h. - Ueberwintert unter Moos. - St. Goarshausen $<5$. - Fr. n. s. (Hg., B.) Wlb. (Sch.) -

13. P. picina Mrsh. = picea Redtb. - Von Hg. bei Fr. gef. -

14. P. Iuteola Müll. = propinqua Redtb. - Am Rödelheimer Damm $=8$. - Offenbach am Main am Leinpfad = 7. - Ems. Fr. (Hg.) -

15. P. cucullata Illig. - Von Hg. einmal bei Fr. gef. - 


\section{Dibolia Latreille.}

1. D. occultans Ent. Heft. - Bei Königstein $>5$. einmal auf Origanum von C. H. gef. -

2. D. Försteri Bach = Buglossi Foudr. - Bei Falkenstein auf Stachys sylvatica $<6$. s. gef. (C. H.) - Feldbergthal $=5$. Wlb. (Sch.) -

3. D. cryptocephala Ent. Heft. - Fr. Wald auf der Bruchschneisse im Sandgraben $>4$. - Bieberer Höhe von $=7$. bis $<9$. - Fr. Wald am Forsthans < 7. - Mo. $<$ 5. - Ems. -

4. D. Cynoglossi Ent. Heft. - Bei Fr. von Hg. s. gef. -

5. D. rugulosa Redtb. - Griesheim am Main = 8. - Bergen $<$ 6. auf Origanum. - Mo. auf dem Sand an Gnaphalium arenarium $<6$. - Lebt auch auf Stachys hirta n. s. bei Mo. - Fr. (Hg.) -

6. D. timida Illig. = Eryngii Bach. - Fr. und Ems je einmal von C. H. gế. -

Apteropoda Redtenbacher.

1. A. globosa Illig. = conglomerata Illig. = globus Dft. Fr. (Hg.) - Wlb. (Sch.) -

2. A. graminis Ent. Heft. = Hederae Illig. = ciliata Oliv. Im Fr. Wald an der Babenhäuser Landstrasse von L. H., Hg. und M. S. gef. - An feuchten Stellen bei Fr. am Diebsweg im April. Falkenstein $<5$. - Schlangenbad von C. H. gef. - Ems. - Nur in wenigen Stücken von B. bei Fr. gesammelt. - Wlb. (Sch.) -

3. A. splendida All. - Viermal bei Fr. von Hg. gef. - [Von Allard bestimmt.] -

\section{Mniophila Stephens.}

1. M. muscorum E. H. - Bei Ems von C. H. gesammelt. [Bei Heidelberg von Hg. oft gef.] — Wlb. h. auf feuchten moosigen Wiesen (Sch.) -

\section{Sphaeroderma Stephens.}

1. S. testacea F. - Offenbach am Mainufer $=7$. - Rüdesheim < 8. - Fr. (Hg.) - Wlb. (Sch.) - 
2. S. Cardui Gyll. - Im Rebstockwald bei Fr. $=6$. - Bei Soden auf Disteln $<8$. - Fr. n. s., auch von B. gef. - Wlb. (Sch.) - Mz. (S.) -

\section{Argopus Fischer.}

1. A. hemisphaericus Duft. - Bei Fr. von Hg. einmal gef. -

\section{CRIOCERINI.}

\section{Orsodacna Iatreille.}

1. O. Cerasi L. - Zwischen Althönig und Feldberg im Taunus $>6$. von L. H. h., doch nur in hellen Varietäten auf Sorbus aucuparia. - [Hierher nigriceps in S. V.] - Cronthal (M. S.) - Wiese bei Grosskarben 6. Mai 1860 gestreift (B). - Wlb. (Sch.) - Mz. (S.) Langenschwalbach auf Blumen in Wiesen (Hg.). -

\section{Zeugophora Kunze.}

1. Z. subspinosa F. - Fr. auf Populus tremula $=5$. - Bei Hofheim $<5$. auf Weiden. - Im Taunus $<7$. auf Birken. - Hinter dem Forsthaus im Fr. Wald am 12. Mai 1861 von B. von Weiden abgestreift. - Fr. (Hg.) - Wlb. (Sch.) - Mz. (S.) -

2. Z. scutellaris Suffr. - Bei Fr. am Diebsweg (Eschenheimer Landstrasse) $>9$. und 10. auf Populus italica, Salix und Alnus. s. - Auch von Hg. und B. bei Fr. gef. - Mo. im August (K.). Mz. (S.) -

3. Z. flavicollis Marsh. - Fr. einzeln gef., einmal von B. und Hg. - Mo. (K.) -

4. Z. frontalis Suffr. - Von Hg. bei Fr. einmal gef. -

Lema Fabricius.

1. L. Erichsonii Suffr. - Fr. Wald beim Forsthaus, Bruchschneisse auf Kiefern von $=3$. bis $=4$. - Seckbach $=5$. - s. s. - Fr. (Hg.) -

2. L. cyanella L. - Ueberall h. - Rebstockwald bei Fr. Griesheim am Main. - Nauheim. - Bieberer Höhe auf Eichen von $>$ 5. bis < 9. - Fr. (Hg., B.) - G. (L.) - Dlb. Wlb. (Sch.) Mz. (S.) -

Var. puncticollis Curt. - Fr. s. s. - Königsteiner Wald im Taunus $>$ 4. - Dlb. Wlb. (Sch.) - 
3. L. flavipes Suffr. - Aeusserst selten. - Bei Isenburg von Hg. im Garten gef. - Wlb. einmal (Sch.) - Mo. 9. August zweimal (K.). -

4. L. melanopa L. - Ueberall h. - Fr. Wald $=5$. - Rebstockwald bei Fr. im Juni. - Nauheim > 8. - Fr. (Hg., B.) G. (L.) - Mz. (S.) - Wlb. h. an Getreidehalmen (Sch.). -

\section{Crioceris Geoffroy.}

1. C. Lilii Scop. = merdigera F. - Ueberall h. in Gärten auf lilienartigen Pflanzen. - Am Feldberg auf Lilium martagon von I. H. gef. - Fr. (Hg.) - Offenbach (B.) - G. (L.) - Dlb. Wlb. (Sch.) - Mz. (S.) -

2. C. merdigera L. = brunnea F. - Lebt auf Convallaria. Fr. Wald < 8. - Bieberer Höhe = 5. - Fr. (Hg., B.) - Wlb. auch auf Lilien (Sch.) - Mo. 3. August (K.). - Auf Spargel bei Mo. am Graben am Rhein 25. Juni und am Damm 12. August 1851 (K.). -

3. C. duodecimpunctata L. - Ueberall h. auf Spargeln (Asparagus) $=6$. in Begattung. (Normalform mit 12 Punkten 1.2.1. 1. 1. oder bei einer Varietät: 1. 2. 0. 0. 1.) - Beide Formen auch von B. gesammelt. - G. (L.) - Wlb. (Sch.) - Mz. (S.) -

4. C. Asparagi L. - Ueberall g. auf Spargeln. - (Die drei weissgelben Flecken auf jeder Flügeldecke sind meist gross und verbinden sich untereinander, so dass die Grundfarbe als drei schmale mit der Naht zusammenhängende dunkle Bänder erscheinen, von denen das mittelste Band sehr breit werden kann und mit der breiten Naht dann ein dunkles Kreuz bildet, oder es kann auch bis auf zwei kleine Punkte ganz verschwinden, so dass dann eine breite weisse Binde mit zwei schwarzen Pünktchen in der Mitte der Flügeldecken entsteht (Var. pupillata Ahr.). - Fr. = 6. in Begattung. - Die Var. pupill. $>$ 4. bei Fr. Wlb. h. sehr variabel (Sch.). - Mz. (S.) -

\section{KK. DONACIINI.}

\section{Donacia Fabricius.}

1. D. crassipes F. - Fr. (Hg.) - G. (L.) -

2. D. versicolorea Brahm = bidens Oliv. = cincta Germ. Am Entensee zwischen Bürgel und Rumpenheim $=7$. einmal von C. $\mathrm{H}$. gef. - Fr. je einmal (Hg., B.) - Wlb. (Sch.) - 
3. D. dentata Hoppe. - Bei Mo. im Graben am Rhein zwischen Nymphaea alba 20. September (K.). -

4. D. aquatica L. = dentipes F. - Ueberall h. auf Wasserpflanzen. - Fr., auch von Hg. und B. gef. - Wlb. (Sch.) - G. (L.) - Wsb. 30. August Teich am Haidenhäuschen (K.). - NIz. (S.) -

5. D. bicolora Zschasch = Sagittariae F. - Ueberall h. wie die vorige. - Am Entensee bei Bürgel =6. in Begattnng. - Fr. $<$ 6., auch von Hg. und B. gef. - G. (L.) - Wlb. (Sch.) - Had. am Elbbach (K.). - Mz. (S.) -

6. D. limbata Panz. = Iateralis Bon. = Lemnae F. Wie die vorige am Entensee h. in Begattung $=6 .-$ G. (L.) Wlb. (Sch.) - Fr. (Hg., B.) - Mr. (S.) - Wsb. an der Tränke 30. August (K.). -

7. D. impressa Payk. = brevicornis Ahrens. - Ein sicher bestimmtes Stück dieser oft falsch gedeuteten Art fand Sch. bei Wlb. -

8. D. thalassina Germ. - Fr. s. s. von C. H. gef. - [Hierher v. Heyden's brevicornis in S. V. als grosses Weib.] - Wsb. (K.) -

9. D. sericea L. - (Die Stiicke aus unserem Gebiete, die L. H. sah und auch von Dr. Kraatz revidirt wurden, gehören zu dieser Art; die nahe verwandte D. Proteus Kunze ist noch nicht bei uns gef. Ueber die Unterschiede beider vid. Kraatz, Berl. Entom. Zeitschr. 1869, pag. 267.) - Die Art variirt durch broncefarben, kupferroth, grün, blau, violett. - Fr. (L. H., Hg.) - Hengstersumpf bei Offenbach = 5; Friedberg (Renner). - Wlb. (Sch.) - Wsb. (K.). - G. mit ziemlich langen Fühlern (L.). - Zu einem grossen breiten weiblichen Stück, das C. H. in der Hohen Mark im Taunus $>6$. am Wasser fand, schrieb Dr. Kraatz: „Es hat einfarbige Beine und Fühler, Glied 2. und 4. der letzteren ist eher kürzer als bei Comari Suffr." - B. fand bei Fr. zwei typische Stücke 22. Mai 1861. -

10. D. braccata Scop. = nigra F. - Bei Fr. einmal von C. H. - Bei Friedberg von Fuhr [in Sammlung v. Heyden] gesammelt. Bei Fr. nur ein Stück 4. April 1860 von B. gef. -

11. D. consimilis Schrk. = discolor Hoppe. - Fr. $=6 .-$ Taunus n. S. - G. (L.) - Dlb. Wlb. auf Caltha palustris (Sch.). Fr. s. (B., Hg.) -

12. D. affinis Kunze. - Fr., auch von B. s. gef. - G. (L.) Var. rustica Kunze. $-\mathrm{Fr}$. - Kleines Feldbergthal $=6$. - 
13. D. semicuprea Panz. = simplex F. pars. - Am Entensee bei Rumpenheim $=6$. in Begattung öfter gef. - Fr. s. (Hg. B.) G. (L.) - Mz. (S.) - Lahn bei Wetzlar (Giebeler). -

14. D. clavipes F. = Menyanthidis F. - Fr. s. - Soden $>5$. - Fr. einmal 9. Juni 1860 von B. gef., auch von Hg. gesammelt. Wlb. 1 Ex. (Sch.) - Mz. (S.) -

15. D. simplex F. = linearis Hoppe. - Fr. - s. - G. (L.) - Wlb. h. (Sch:) -

16. D. vulgaris Zschach = Typhae Ahrens. - Am Entensee wie semicuprea. -

17. D. Fennica Payk. - Einmal $>4.1828$ von C. H. am Mainufer an der Mauer des Untermainquais gef., als noch die Insel vorhanden war. - G. 4 Stück (L.). -

18. D. Malinovskyi Ahr. - Bei G. zweimal von L. gef. -

19. D. cinerea Hbst. = Hydrocharis F. - Fr. s. -

\section{CERAMBYCIDAE.}

\section{A. PRIONINI.}

\section{Prionus Geoffroy.}

1. P. coriarius L. - Schon von Brahm 1790 aus Fr. erwähnt. - Im Fr. Wald h. an Buchenstämmen. - Königstein im Taunus (L. H.). - Offenbach (B.). - Von C. H. < 8. bei Fr. gef., das Männchen flog im Sonnenschein um das am Baumstamme sitzende Weibchen. In Mz. in einem Holzhof gef. (S.) - Had. (K.) - Dlb. Wlb. (Sch.) -

\section{Aegosoma Serville.}

1. A. scabricorne Scop. - s. s. im Fr. Wald von Steitz und M. S. gef. (Babenhäuser Landstrasse) an alten Buchen. - Von C. H. $=6$. und von St. aus dürrem Fr. Waldholz (Buchen) erzogen. - Im Fr. Wald am Schwengelbrunnen 2 Stück, an der Götheruhe und in Offenbach $<$ 8. mit Buchenbrennholz verschleppt von B. gef. - Wsb. (K.) - 


\section{B. CERAMBYCINI. \\ Spondylis Fabricius.}

1. S. buprestoides L. - Im ganzen Gebiet n. s. - Fr. Wald am Forsthaus $>6$. und $=7$. an Kiefernholz. - Fr. Wald am Schwengelbrunnen (B.). - Mz. (S.) - Dlb. (Sch., K.) - Wlb. (K.) -

\section{Asemum Eschscholtz.}

1. A. striatum F. - Ueberall h. an Kiefernstämmen von $<5$. bis < 6. im Fr. Wald. - G. (I.) - Dlb. (Sch.) - Mz. (S.) -

Var. agreste F. (Mit gelbbraunen Flügeldecken, die Stammart schwarzbraun.) - n. h. - Sodener Wald $>$ 5. an Birkenwurzeln; bei Cronthal $=5$. in Kiefernstöcken von C. H. gef. -

\section{Criocephalus Mulsant.}

1. C. rusticus L. - Bei Wsb. ein todtes Stück von Lientenant Giebeler gef. -

\section{Hesperophanes Mulsant.}

1. H. pallidus Oliv. = mixtus F. - Fr. aus Eichenholz von St. erzogen. -

\section{Tetropium Kirby (= Criomorphus Muls.).}

\section{T. luridum $L$.}

a. aulicum F. (Oberseite schwarz, Beine schwarz.) - Fr. einmal (B.). - Fr. Wald, Forsthaus (St.). -

ß. fulcratum F. (Oberseite schwarz, Beine roth.) - Am Fr. Forsthaus mit der Stammart (St.). -

$\gamma$. luridum verum. (Flügeldecken gelbbraun.) - G. (L.) - Wie die Stammart bei Fr. (St.) -

2. T. fuscum F. - Bei Fr. zweimal von C. H. gef. $>$ 5. am Forsthaus an Fichtenscheitholz. -

\section{Nothorhina Redtenbacher.}

1. N. muricata Dalm. - Aeusserst selten. - Von Gremmers einmal im Isenburger Wald an einer Buche gef. [früher in Sammlung. v. Heyden, jetzt Grenier in Paris]. - 
Hylotrupes Serville.

1. H. bajulus L. - Ueberall h. in Häusern an altem Gebälk. $>6$. bis = 7. - Fr. (B.) - Wlb. (Sch.) - Dlb. (Sch., Ko.) Mz. (S.) --

Var. lividus Muls. (Mit blassgelben Flügeldecken.) - Fr. s. Dlb. Wlb. (Sch.) -

\section{Callidium Fabricius.}

1. (Rhopalopus Muls.) femoratum L. - Soden $<6$. an Holzstössen. - Fr. Wald zu derselben Zeit von L. H. gef. - G. (L.) Am Forsthaus 24. Mai 1860 zweimal gef. (B.) - Mz. (S.) -

2. C. violaceum L. - Fr. $<$ 6. s. an Kiefern. - G. (L.) Fr. einmal (B.). - Dlb. Wlb. g., auch in Häusern (Sch.). - Mz. (S.) -

3. C. sanguineum L. - Auf Zimmerplätzen an Eichenstämmen. Fr. s. $>$ 4. - Im Fr. Wald $=5$. an Eichenklafterholz. - Fr, einmal (B.). - Dlb. (Sch.) - Mz. (S.) -

4. C. Alni L. - An der Mainkur von L. H., Fr. öfter von C. H. gef. - Fr. Wald (Hinkelsteinforst) $=5$. an Eichenholz. - Fr. 12. Mai 1860 (B.). - Mz. (S.) - Wlb. (Sch.) -

5. C. rufipes F. - Soden $>4$. aus überwinterten Pflaumenzweigen von C. H. erzogen. - Wlb. (Sch.) - Mr. (S.) - Wsb. an einem Bäckerhaus (K.). - Ernsthausen in Nassau (Dörr bei K.). -

6. (Phymatodes Muls.) variabile L. - Unendlich variabel. -

o. Nigrinum Mls. (Flügeldecken schieferblaugrau, Halsschild roth, mit mehr oder weniger dunkleren Flecken und Schattirungen; Schenkel dunkel, selten hellroth.) -

ß. Fennicum F. (Wie die vorige Form, aber Halsschild einfarbig rothgelb, Schenkel dunkel oder hell.) - Mz. (S.) -

$\gamma$. praeustum F. (Flügeldecken lehmgelb, nach hinten zu mehr oder weniger dunkel gefärbt.) - Mz. (S.) -

$\delta$. nigricolle Muls. (Halsschild schwarz, Flügeldecken lehmgelb.) -

ع. testaceum L. (Der ganze Käfer röthlichgelb.) - Ernsthausen in Nassau (Dörr bei K.). - Limburg 6. Juni (K.). -

Alle Varietäten zusammen ans Eichenholz in der 4. Generation $=5$. h. im Zimmer erzogen. - Fr. Wald. - Friedberg (Renner). - G. (L.) - Von B. $=$ und $>5$. gef. - Dlb. s. g. in allen Varietäten (Sch.). - 
7. (Ph.) melancholicum F. - Ein von Jännicke in Niederrad gefundenes Stück in der Sammlung Stern. -

\section{Clytus Fabricius.}

1. (Plagiographus Muls.) detritus L. - An Eichenstämmen auf Zimmerplätzen und im Fr. Wald h. im Mai und Juni, Hofheim im Taunus (B.). - Mz. (S.) - Wsb. an gefällten Eichen (Sandberger bei K.). - Fr. (St.) - Ernsthausen in Nassau (Dörr bei K.). -

2. (P.) arcuatus L. - Wie der rorige h. - G. (L.) - Dlb. (Ko.) - Hofheim und Ehlhalten im Taunus (B.). - Erscheint nach C. H. früher als detritus = 5. - Fr. (St., L. H.) - Mz. (S.) - Wlb. s. h. auf Zimmerplätzen (Sch.). -

3. (Xylotrechus Chevr.) rusticus L. = liciatus L. - Aus Fr. dürrem Waldholz $<6$. öfter erzogen von uns und St. - Fr. in einem Stadtgraben von B. gef. - Im Freien auch schon $=5$. an alten Buchenstämmen. - Mz. (S.) -

4. C. tropicus Panz. - Aus Fr. Waldholz s. erzogen $<6$. von uns und St. - Lebt in Eichen. - Vier Stück von Boss im Freien bei Fr. gef. (B.) -

5. C. Arietis L. = Gazella F. - Fr. $>$ 5. von C. H., St. und B. gef. - Soden $=6$. an Klafterholz. - G. (L.) - Dlb. Wlb. g. (Sch.) - Mz. (S.) - Mo. auf blühendem Weissdorn 13. Juli und auf Hecken 23. Mai 1852 gef. (K.) -

6. C. Antilope Zetterst. $=$ Arietis F. - Fr. einzeln von St. 1869 in der Holzkammer gezogen. - Mz. (Bach, S.) - 1877 mehrfach. -

7. C. Duponti Muls. (Col. France 1839, pag. 84 ) = C. Sternii Krtz. (Berl. Entom. Zeitschr. 1870, pag. 219) = Auboueri Desbr. (Ann. France 1872, pag. 429.) - Von Kraatz nach 2 Ex. beschrieben und abgebildet, welche St. aus Fr. Waldholz im Frühsommer 1868 erzog, von denen eins nun in Sammlung v. Heyden. [Diese Art gab zu einem heftigen Federkrieg Veranlassung, besonders in den Ann. de France, indem er von einigen Forschern für den schon 1841 von Gory beschriebenen C. cinereus aus Paris gehalten wird. Ich schliesse mich der Ansicht der Letzteren an, die Beschreibungen von Gory und von Mulsant (edit. II, 1862 - in edit. I, 1839 als Duponti beschrieben), sowie die zwar wenig gelungene Abbildung bei ersterem sind dieser Annahme nicht entgegen. - Der älteste Name ist Duponti. L. v. H.] - 1877 einmal aus Eichenholz gezogen. - 
8. C. Verbasci L. = ornatus Herbst. - Nach Brahm auf Wollkrautbliuthen bei $\mathrm{Mz}$. im Juni h., später noch von S. gef. - Die Sammlung v. Heyden besitzt Stïcke aus dem Odenwald und von Speyer. - Von Harer bei Fr. zweimal gef. (B.) - Auch St. sammelte die Art im Gebiet und Sch. erhielt sie aus Nassau. - Ernsthausen in Nassau (Dörr bei K.). - Franenstein im Rheingau (Sandberger bei K.). -

9. C. Massiliensis L. - Mehr im Süden des Gebiets. - Mo. $<$ 7. auf Umbellen von C. H. gef. - Wlb. s. (Sch.) - Mz. (S.) Mo. im Juli am Rheindamm auf Dolden 12. August 1851 (K.). Eltville im Rheingau (St.). -

10. C. figuratus Scop. = plebejus F. - Bei Fr. und Bergen $<6$. von uns gesammelt. - An der Götheruhe im Fr. Wald 1875 h. von B. gesammelt. - Dlb. Wlb. s. (Sch.) -

11. C. mysticus L. - Bei Fr. von L. H. offer gef. - G. (L.) - Auf blühenden Schlehen am Buchrain bei Offenbach 1871, an der Götheruhe im Fr. Waid 1874 gef. (B.) - Dlb. Wlb. (Sch.) - Die Larve nach C. H. im $\mathrm{H}_{0 l z}$ von Acer campestris. - Aus der Holzkanmer öfter erzogen und im Schwanheimer Wald gef. (St.) - Mz. (S.) -

[Dass Cartallum ebulinum L. von General Klingelhöfer bei Butzbach gef. worden sein soll, beruht sicher auf Irrthum; die Gattung ist auch nach Mulsant ausschliesslich ,meridional“". In Spanien und bei Marseille h.]

\section{Gracilia Serville.}

1. G. minuta $\mathbf{F}$. = pygmaea $\mathbf{F}$. - In ungeheurer Menge entwickelt aus alten Weidenkörben in einem Magazin auf dem Domplatz in Fr. - Zwei Generationen im Jahr. - Von B. auch aus Fassreifei in Fr. erzogen. - Wlb. in Häusern (Sch.). - Mz. (S.) -

\section{Obrium Latreille.}

1. 0 . cantharinum $L$. = ferrugineum $F$. - s. - Aus dürrem Fr. Eichenholz im Juni öfter erzogen (I. H., St.). - Mz. (S.) -

2. 0. brunneum F. - Im Fr. Wald am Forsthaus $=6$. h. auf Umbellen an schattig ${ }^{\prime}$ Orten. - Aus dürrem Waldholz schoń $>4$. erzogen. - Im Altenhainer Wald bei Soden $>6$. auf Pinus picea. Am Forsthaus viermal (B.). - Wlb. (Sch.) - Mr. (S.) - Nauheim (v. Harold) Juni 1876. - 


\section{Anisarthron Redtenbacher.}

1. A. barbipes Schrk. - s. s. im Gebiet. - Fr. (Gremmers, B.) - Bockenheim (M. S.). - Friedberg (Fuhr). - Mz. in alten Rüstern öfter gef. (S.) -

\section{Callimus Mulsant.}

1. C. angulatus Schrk. $=$ cyaneus $\mathbf{F}$. - Fr. s. s. - Aus dürrem Waldholz $=5$. entwickelt. (Bei dem Weibchen ist der zweite Hinterleibsring mit einem dicken orangegelben Haarkranz versehen.) -

\section{Stenopterus Olivier.}

1. S. rufus L. - Auf dem Gaualgesheimerkopf eine Stunde oberhalb Bingen, 1874 auf Blumen h. von B. gef.; auch einmal bei $\mathrm{Fr}$. (St., B.) - Mz. (S.) - Mo. am Damm 20. Juli 1851 (K.). -

\section{Molorchus Fabricius.}

1. M. minimus Scop. = umbellatarum L. - Fr. n. s. auf Blüthen, besonders Cornus alba im Juni am neuen Irrenhaus. - Soden $>$ 6. auf Hecken. - G. (L.) - Fr. h. (B.) - Wlb. s. h. auf Blüthen, z. B. Weissdorn (Sch.). - Eichberg im Rheingau (Richter). - Mz. (S.) - Wsb. auf Cornus sanguinea 12. Juni (K.). -

2. M. minor L. = dimidiatus F. - Aus zweimal überwintertem Fr. Waldholz $=5$. n. s. erzogen. - Altenhainer Wald bei Soden $>5$. - C. H. fand den Käfer in dem Puppennest unter Fichtenrinde $<5$. - Auf Umbelliferen an der Sachsenhäuser Warte 14. Mai 1860 h. (B.) - Wlb. mit dem vorigen (Sch.). - Mz. (S.) - Wsb. bei der Kapelle an einem Gartenhaus h. (Giebeler). -

3. M. discicollis Heyd. (Deutsche Entom. Zeit. 1876, pag. 383.) - Das typische Weibchen [nun in Sammlung v. Heyden] wurde von Dr. Richter im Rheingau bei der Irrenanstalt Eichberg auf Spiraea gef. - Von allen Arten durch das breite, fast kreisrunde Halsschild und die breiten kurzen Flügeldecken verschieden. - K. fand ein Männchen bei $\mathrm{MIO}$. -

\section{Necydalis Linné.}

1. N. abbreviatus Panz. - Ueber diese und die verwandte folgende Art sprach L. H. ausführlich Berlin. Entom. Zeit. 1864, pag. 329, und führte zugleich an, dass der Linnésche major nach der 
Beschreibung nicht sicher zu deuten ist, aber eher zur folgenden Art gehört. Diese Art lebt in Eichen, Buchen, Ulmen. - Fr. s. s. im Juni aus dürrem Waldholz entwickelt (L. H. und St.). -

2. N. Salicis Muls. = major L.? = abbreviatus F.? = Populi Büttner. - Viel kleiner und zierlicher wie die vorige Art. Sie lebt in Aspen bei Fr. (St.), meist aber in Weiden und ist stellenweise n. s. - L. H. fand sie h. $>6$. im Sonnenschein um Mittag an alten Weiden am Main zwischen Sachsenhausen und Oberrad fliegend. - Auch von M. S., St. und Steitz gesammelt. - Von B. in Kirschbäumen am Röderwald und an der Mainkur n. s. gef. - Wsb. an alten Weiden 5. Juli (K.). - Biebrich am Rhein an der Schwimmschule 7. August (K.). -

\section{Purpuricenus Serville.}

1. P. Koehleri L. - Von Schṇeider 1874 bei Fr. am Metzgerbruch an alten Weiden gef. - Nach Brahm bei Mz. s. am Weinstock. - Bei Rüdesheim von Ko. gef. - Zwischen Mo und Militärschiessstand 15. Juni 1876 an einem Aprikosenbaum von Major Alex. von Homeyer gef. - An dem Mo. Damm auf Weiden, auch unter dem Hartenberg bei Mz. auf Weissdorn öfter gef. (S.) - Caub (F. Sandberger bei Sch.); auch K. fand da ein Männchen mit rothem Halsschildfleck. -

\section{Rosalia Serville.}

1. R. alpina L. - Ein Alpenthier, doch auch in Hohenwittlingen bei Urach in Württemberg n. s. von B. beobachtet. - Gelegentlich durch Schiffs-Bauholz verschleppt, hat sich die schöne Art an passenden Lokalitäten eine Zieitlang erhalten. So war sie nach Notizen von C. H. einmal vor vielen Jahren $n$. s. an einer alten Eiche am .Rhein bei Biebrich, er selbst fing ein Stück im Flug in Fr. am Mainufer. Dr. med. Fr. Stiebel fing ein Exemplar an der Hohen Wurzel bei Schlangenbad im Taunus, also weit im Innern des Landes. - Sch. erhielt ein Exemplar von der Platte bei Wsb. -

\section{Aromia Serville.}

1. A. moschata L. - An Weiden überall n. s. - Fr. am Mainufer $>$ 7. - Königstein. - Wlb. s. g. (Sch.) - Mz. (S.) Wsb. (K.) - 


\section{Cerambyx Linné (= Hammaticherus Serv.).}

1. C. cerdo L. = heros Scop. F. - Die grosse A.rt. - $\Lambda n$ alten Eichen im Gebiet n. s. wie Lucanus cerrus. - Schwanheimer Wald. - Mainkur = 7. - An der Gerbermühle bei Oberrad am Mainufer (B.). - Den Käfer fand C. H. in bedeutender Anzahl $<6$. entwickelt in einer gefällten kernfaulen Eiche, er erschien im Freien $=7$. - Had. (Sch.) - An zwei alten Eichen unterhalb Mo. öfter ges. (S.) Ein Exemplar (Weib) nur $28 \mathrm{~mm}$ lang, fand Giebeler am Neroberg bei Wsb. -

2. C. Scopolii Füssly = cerdo Scop. nec L. - Die bekannte kleine Art. - In Cronthal im Taunus $=5$. an alten Kastanienstämmen s. h. - Fr. Wald auf Blüthen, besonders Spiraea ulmaria und Viburnum opulus. -- G. (L.) - Fr. in Buchen (B.). - Dlb. Wlb. s. g., auch eine sehr kleine Varietät (Sch.). - Mz. (S.) - Wsb. (K.) -

\section{LAMIINI.}

\section{Dorcadion Dalman.}

1. D. fuliginator L. - Im Gebiet nur längs dem Laufe des Main und Rhein auf Cyrenenmergel (und hier ein ächtes charakteristisches Thier dieser Formation des alten Mainzer Beckens), daher nicht bei Fr. selbst, sondern erst bei Hochheim (Anton Schmid und Dickin, B. <6.) - Bei Wsb. h. - [Mainaufwärts bei Würzburg. - Creuznach. - Rheinabwärts bis Coblenz.] — Die Stücke gehörẹn alle der einfarbig grauen Grundform an. - Mz. im Mai n. s. an Mauern und Baumstämmen. (Brahm, später S.). - Am Weg von Wsb. nach Schierstein im Juni (K.). -

Var. atrum Bach. (Fast jedes Dorcadion, das sonst die schönsten Zeichnungen durch anliegende helle Haare auf den Decken. besitzt, bildet eine schwarze, oft glänzende, durchaus haarlose - nicht abgeschuppte Varietät - so auch hier.) - Von B. 1874 auf dem Gaualgesheimer Berg gef. - [Die Sammlung v. Heyden besitzt 1 Stück aus Mosbach bei Heidelberg.] —

\section{Lamia Fabricius.}

1. L. textor L. - Im Gebiet an Weiden n. s. - Fr. $<6$. - Mz. < 10. - G. (L.) - Mainkur (B.). - Dlb. Wlb. (Sch.) -

\section{Monochammus Latreille.}

1. M. galloprovincialis Oliv. = pistor Germ. - Eine Anzahl Exemplare erzogen wir aus Kiefernholz (Aeste von der Krone der Bäume), 
das aus dem Fr. Wald stammte, $>6.1860$. - C. Schneider fand 1874 ein Exemplar im Flug in Sachsenhausen und B. ein Stück auf dem Ziem'schen Zimmerplatz vor dem Obermainthor. Das Schildchen hat an der Basis einen dreieckigen nackten Längsfleck, die Flügeldecken ohne Quereindruck hinter dem ersten Drittel der Naht. Fühler und Beine rothbraun. Es ist sicher eine gute Art, die hoch oben in den Gipfeln der Bäume lebt, nur gegen Abend schwärmt und desshalb seither bei uns übersehen wurde. -

\section{Acanthocinus Stephens (= Astynomus Steph.).}

1. A. aedilis L. - An gefällten Kiefernstämmen überall g. Die Puppe $<$ 8. von C. H. gef. unter Kiefernrinde im Fr. Wald, der Käfer entwickelte sich $<9$. - Im Freien im Mai und $<6$. gef. G. (L.) - Von B. im Gehspitzwald, Röderwald, Offenbach gef. Dlb. (Sch., Ko.) - Wlb. s. g. auf Zimmerplätzen, auch in Häusern. - Wsb. bei Dotzheim auf Kiefern im Frühjahr (K.). - Mz. (S.) -

\section{Leiopus Serville.}

1. L. nebulosus L. - Aus dürrem Buchenholz aus dem Fr. Wald s. h. im Mai erzogen. - Auch $=6$. aus Carpinus betula Holz entwickelt; die Larve überwintert unter der Rinde, der Käfer bohrt ovale Löcher durch dieselbe. - Am Fr. Forsthans 20. Mai 1860 dreimal abgestreift (B.). - Dlb. s. g. (Sch.) - Mz. s. (S.) - 1877 aus Eichenholz (St.). -

2. (Oplosia Muls.) fennicus Payk. - Ein Exemplar, nun im Besitze von L. H., fand Dr. Richter bei der Irrenanstalt Eichberg im Rheingau. - In Deutschland grosse Seltenheit. -

\section{Exocentrus Mulsant.}

1. E. Lusitanus L. - [Balteus L. = balteatus F. ist nach Harold Cat. = Parmena unifasciata Vill. - nach Seidl. gehören sie hierher. Nach der Linné'schen Diagnose, Syst. nat. XII, pag. 1067, gehört balteus eher zu Parmena. Sie lautet: thorace spinoso, corpore ferrugineo, abdomine ovato, elytris fascia nigricante. Auch die Fabricius'sclie Diagnose fascia lata fusca passt besser auf Parmena.] - Von C. H. aus Lindenholz von Fr. erzogen; $>$ 8. aus Birkenreisern entwickelt. Mo. = 10. an Ulmen. - Einmal am Fr. Forsthaus Mai 1860 gestreift (B.). - Mz. (S.) - 


\section{Pogonocherus Latreille.}

1. P. fasciculatus Deg. $=$ fascicularis Panz. $=$ hispidus $L$. pars. - Im Juli aus dürrem Fr. Waldholz einzeln entwickelt. G. (L.) - Fr. einmal 6. Mai 1860 (B.). - Fr. (St.) -

2. P. ovatus Goetze $=$ ovalis Gmelin. $-\mathrm{Fr}$. $>2$. unter Fichtenrinde. - Aus dürrem Kiefernholz aus dem Fr. Wald von $>6$. bis = 7. öfter erzogen. - s. - Auf Fichtenklafterholz 5. April 1860 zweimal gef. (B.); auch von St. gesammelt. - Mz. (S.) -

3. P. hispidus $\mathrm{L} .=$ dentatus Fourcr. $(1775)=$ pilosus $\mathrm{F}$. Fr. $<8$. aus überwinterten Epheuästchen entwickelt. $-<6$. Soden auf Dornzäunen. - Königstein =9. auf Sambucusblüthen. - G. (L.) - Bei Fr. 6. Mai 1860 zweimal von B. gef., auch von St. gesammelt. - Wlb. n. s. (S.) - Mz. (S.) - Oberscheld in Nassau im Mai an Kiefern (K.). - Wsb. Dotzheim im April (K.). -

4. P. bidentatus Thoms. = hispidus Laich. Gyll. - Die Art mit je zwei Zähnchen an der Flügeldeckspitze. - Friedberg (Fuhr). Fr. von St. und B. gef. - Bei der Irrenanstalt Eichberg im Rheingau (Dr. Richter). - Wlb. (Sch.) -

\section{Acanthoderes Serville.}

1. A. clavipes Schrk. = varius F. - Erst in der letzten Zeit von St. aus Fr. Eichenholz erzogen. - Im Rebstöcker Wald von Harer, bei Fr. von Boss gef. - Bei Mz. im Holzhof öfter gesammelt (S.). -

\section{Mesosa Serville.}

1. M. curculionoides L. - Aus dürrem Buchenholz $>9$. von uns aus dem Fr. Wald n. s. erzogen; auch schon im Juli. - Bei Fr. 1. September 1862, im Rebstöcker Wald und am Grafenbruch bei Offenbach von B. gef. - Fr. Wald Eichen. Oberstlientenant Saalmüller 1877 Juni.

2. M. nebulosa F. = nubila Oliv. - Aus dürrem Buchenholz aus dem Fr. Wald $<4$. und 5. oft von uns erzogen. $->5$. auf Carpinus-Hecken. - Am Grafenbruch bei Offenbach auf Espen s. (B.) - Béi der Irrenanstalt Eichberg im Rheingau von Eichen geklopft (Dr. Richter). - Mz. (Dr. Zitz, S.) - Wlb. (Sch.) -

\section{Agapanthia Serville.}

1. A. lineatocollis Donov. = Cardui F. nec L. - Von St. im Isenburger Wald einzeln gef. - 
2. A. angusticollis Gyll. - Friedberg (Fuhr). - G. (L.) Bei Isenburg auf Disteln (Hg.). - Fr. (B.) - Mz. (S.) - Wsb. einmal (K.). - Eichberg im Rheingau (Richter). -

3. A. violacea F. Muls. - Durch schmälere Decken, fast gleichbreites schwarzes Halsschild, schwarze Beine und ganz schwarze Fühler von micans verschieden, bei welcher das erste Fühlerglied glänzend blau ist. - Aeusserst selten; nur ein bei Friedberg von Dr. Renner gefundenes Stück in Sammlung v. Heyden. -

\section{Anaestethis Mulsant.}

1. A. testacea $\mathbf{F}$. - Soden im Taunus $<7$. auf Eichen. Fr. Wald hinter dem Forsthaus auf Rubus-Gebüsch $=6$. - Ems (C. H.). - Fr. (St., B. 1876). -

\section{Saperda Fabricius.}

1. (Anaerea Muls.) carcharias L. - In Pappeln. - Auf dem Wege von Offenbach nach Bürgel auf Pappelgebüsch in manchen Jahren n. s. von L. H. gesammelt. - Fr. n. s. (B.) - Dlb. (Sch., Ko.) an Pappeln s. g. - Ebenso bei Mz. n. s. (S.) -

2. (Amilia Muls.) similis Laich. = phoca Fröhl. - Dr. Steitz fand 1 Ex. dieser Seltenheit in Fr. in einem Garten im Sachsenlager (nun in Sammlung Stern). -

3. S. scalaris L. - Aus Buchenholz aus dem Fr. Wald $<5$. oft erzogen von C. H. und St. - L. H. fand Puppen, die sich entwickelten, unter der Rinde alter Apfelbäume hinter der „Louisa“ am Fr. Wald. - In Kirschbäumen n. s. am Röderwald und an der Mainkur, auch bei Vilbel (B.). - Von St. auch in Aspen und Kirschbäumen gef. - Mz. von blühenden Apfelbäumen geklopft (S.). -

4. S. perforata Pall. = Seydlii Fröhl. -- Aus Aspenholz aus dem Fr. Wald einmal in grosser Menge $=5$. erzogen; ebenso $h$. von St. - Mz. (S.) -

5. (Argalia Muls.) octopunctata Scop. = Tremulae F. - Auf geschlagenem Aspenholz am Grafenbruch bei Offenbach s., aber mehrfach von B. gef. - (Die Stücke sind in der Offenbacher Sammlung.) -

6. (Compsidia Muls.)' populnea L. - In Anschwellungen junger Aspenzweige n. s. - Fr. Soden $>$ 5. - Schwanheimer Wald, Schwengelbrumnen 9. Mai 1860 (B.). - Bei Dlb. s. g., auch bei Wlb. (Sch.) - Had. (K.) - 


\section{Menesia Mulsant.}

1. M. bipunctata Zoubk. - Von Dr. Gerlach, B. und v. Twardowski im Schwanheimer Wald 1860 n. s. $>6$. auf der Blattunterseite von Rhamnus frangula gef.; nahe am Goldstein. -

Var. quadripustulata Muls. (Mit je zwei kleinen, weissen Flecken auf den Flügeldecken.) Mit der Stammart. -

\section{Oberea Mulsant.}

1. O. oculata L. - Auf Weiden s. im Gebiet. - L. H. fand sie früher bei Fr. in der Gärtnerei. - Am Metrgerbruch bei Fr. g. (B.) - Am Mainufer auf Weiden (St.). — Mr. (S.) -

2. O. pupillata Schh. - G. (Zimmer, L.) [in Sammlung v. Heyden]; von L. 23. Mai 1862 auf Lonicera xylosteum gef. -

3. 0. erythrocephala Schrk. - Bei Mo. im Mai auf Euphorbia cyparissias von L. H. und St. h. gef. - Friedberg (Fuhr). - Fr. einmal (B.). - Bei Mo. auch von K. gesammelt vom 8. - 20. Juli. -

4. O. linearis L. - Am Röderberg bei Fr. h. an der Unterseite der Blätter von Haselstauden gesammelt von L. H., M. S., St. und Jännicke. - Wlb. (Sch.) -

\section{Stenostola Redtenbacher.}

1. S. ferrea Schrk. $=$ nigripes F. $=$ Tiliae Küst. - Im Fr. Wald bei Isenburg $>4$. und $=5$. auf Urtica dioica gef. von C. H. - Fr. (Lindheimer). - Wlb. g. auf Linden (Sch.). - Altkönig im Taunus (St.). -

\section{Phytoecia Mulsant.}

1. P. Argus F. Var. Jourdani Muls. - Sonst nur ans der Gegend von Lyon bekannt. - S. entdeckte die Art bei Mz., woher in Sammlung v. Heyden einige Stücke. - St. fand 1 Stück bei Mo. bei den Schiessständen auf dem grossen Sand. - Nach den Beobachtungen von S. fliegt das interessante Thier nach Art der Cicindelen vom Boden auf. Er fand sie gar nicht selten am Lenaberg auf Potentilla. -

2. P. pustulata Schrk. = lineola F. - Mz. (Brahm). - s. s. an Weiden im Mai. - [Herrstein an der Nahe, Tischbein.] - Mo, auf Wiesen in Gräben im Juni (K.). - Mz. (S.) - 
3. P. ephippium F. - Fr. Wald von uns, St., M. S. und B. gef. - Sodener Wald =6. - G. (L.) - Fr. 16. Mai 1860 gestreift (B.). - Zwischen Fr. Forsthaus und Isenburg $=6$. ; auch schon $=5$. in der Stadt Fr. von C. H. gef. - Mz. (S.) -

4. P. nigricornis F. = Solidaginis Bach. - Auf Tanacetum von uns öfter gesammelt. - Fr. von uns, St. und B. gef. - Bergen $<6$. - Friedberg (Renner und Fuhr). - Fr. von Mühlig aus Artemisia erzogen. - (Die Augen hinten scharf ausgerandet, nicht getheilt wie bei virescens.) - G. (L.) - Mz. s. im Mai auf Wolfsmilch (Brahm). - Dlb. Wlb. h. (Sch.) - Die Bach'schen Originale sind auf nassauischem Gebiet, Boppard gegenüber, zwischen Filsen und Camp gef. (Briefliche Mittheilung an L. H.) -

5. P. cylindrica L. - Fr. Wald (L. H., B. 25. Mai 1860). Ems. - Sodener Wald $=5$. - Fr. an der Unteren Saustiege $<5$. - G. (L.) - Wlb. h. (Sch.) -

6. P. virescens F. (Durch die hinten vollständig getheilten Augen leicht kenntlich.) - Auf Echium und Cynoglossum n. s. - Bieberer Höhe $>$ 5. in Begattung, auch $=7$. - Taunus. - Fr. s. (B.) Gonsenheimer Capelle bei Mz. (K.) - Mz. (S.) - Dlb. Wlb. (Sch.) -

\section{Tetrops Stephens (= Polyopsia Muls.).}

1. T. praeusta L. $-\mathrm{Fr}$, Wald bei Schwanheim $=5$. auf Rhamnus frangula. - Fr. $=6$. auf Schlehenhecken. - Soden $>5$. auf Sahlweiden. - G. (L.) - Fr. h. - Enkheim (B.). - Dlb. Wlb. g. auf Obstbäumen (Sch.). - Mz. (S.) - $\mathrm{M}_{0}$. auf Hecken (K.). -

\section{LEPTURINI.}

Stenocortus Geoffroy (= Rhagium F. pars).

1. S. sycophanta Schrk. = scrutator Oliv. $=\operatorname{mordax}$ F. - An alten Eichen n. s. = 5. - Unter der Rinde entwickelt schon $=11$. - Fr. - Soden. - G. (L.) - Auch in Birken bei Offenbach April 1870 von B. beobachtet. - Dlb. Wlb. s. g. (Sch.) - Mz. (S.) -

Var. cephalotes Mils. = grandiceps Thoms. - Mit der Stammart. $->4$. im Lorsbacher Thal im Taunus. -

2. S. Linnei Laich. $=$ mordax De Geer $=$ inquisitor $F$. Cronthal $>10$, unter der Rinde von Castanea vesca. - Als Käfer 
zum Ueberwintern schon völlig entwickelt $=9 .-$ Fr. h. (B.) Dlb. Wlb. s. g. (Sch.) - MIz. (S.) -

3. S. bifasciatus F. - In Cronthal $=5$. öfter an rindenloser Castanea vesca von C. H. gef. - G. (Zimmer) in Pinus sslrestris. In Fichten bei Offenbach im April 1870 von B. s. beobachtet. Taunus (St.). - Wlb. s. (Sch.) - Engenhahn im Taunus im Juni (K.). -

\section{Rhagium Fabricius.}

1. R. inquisitor $\mathbf{L}$. = indagator $\mathbf{F}$. - Variirt in dér Grösse von $16-8 \mathrm{~mm}$. - Fr. $>4$. unter Rinde von Pinus sylrestris. - Rebstöcker Wald h. (B.) - Mz. (S.) - Taunus (St.) - Dlb. Wlb. g., auch in Häusern (Sch.). -

\section{Rhamnusium Latreille.}

1. R. bicolor Schrk. = Salicis F. - L. H. fand die schöne Art in grosser Anzahl und vielen Varietäten in den hohlen „Drei Linden“ bei Soden. - Fr. von C. H. und M. S. $<5$. in Buclien gef. G. (L.) - B. fand die Art in Ahorn an der Höchster Landstrasse; in Pappeln zwischen Offenbach und Bürgel; in Nussbäumen an der Nainkur, auf Eichen im Rebstöcker Wald. - Ausserdem in Hattenheim im Rheingau; im T'aunus einzeln. - Dlb. n. s. an Pappeln (Sch.). - S. fand den Käfer h. in alten anbrüchigen Rüstern einmal bei Wörrstadt bei Mz. in grosser Menge.

Var. glaucopterum Schall. (Flügeldecken roth, bei bicolor blau.) - Mit der Stammart seltener. „Drei Linden“. - Fr. in Buchen im Mai. - Wsb. (K.) -

Var. ambustum Heyd. (Vorderhälfte der Flügeldecken dunkelbraun mit violettem Schimmer, die hintere Hälfte gelblich braun, Spitze der Decken schmal schwarz. - Nur einmal mit den vorigen in den „Drei Linden". -

\section{Toxotus Serville.}

1. T. Quercus Götze. (Männchen, schwarze Decken mit rotheî Schultern ist $=$ humeralis F. - Weibchen mit braungelben Declen ist = dispar Panz.) - Bei Friedberg beide Geschlechter von Renner und Fuhr gef. - Ein Weibchen von L. bei G. gef. 1. Juni 1862. Ein Männchen bei Dlb. (Koch). - Rebstöcker Wald 2 Stück (B.). Mz. (S.) - 
2. T. meridianus L. - Variirt in der Farbe der Flügeldecken, die schwarzgrau oder mit braundurchscheinenden Schultern oder ganz gelbbraun sind; die Beine sind gelblich, die Kniee, Schienen und Tarsen zum Theil schwarz, helle Stücke bei uns s. - Fr. B. einmal. - Ein Stück mit dunkler Flügeldeckenspitze, Oberseite fein silbergrau behaart von L. bei G. gef. - Dlb. (Koch). - Dlb. Wlb. (Sch.) - Mz. (S.) - Dunkle: Königstein <6. - Friedberg (Renner, Fuhr). - Dlb. Wlb. (Sch.) - Die Art wurde von B. bei Fr. h. gef. -

Var. chrysogaster Schrk. (Ganz schwarz.) - Dlb. (Sch.) Wsb. (K.) -

\section{Oxymirus Mulsant.}

1. 0 . cursor L. (Weib = noctis L.) - Eine weibliche Flügeldecke fand Lieutenant Giebeler bei Wsb. -

\section{Acmacops Le Conte.}

1. (Dinoptera Muls.) collaris L. - Fr. Wald auf Blumen $=5$. n. h. - G. (L.) - Fr. h. (B.) - Had. hinter dem Kirchhof (K.). - Wsb. an der Wellritz 1. Juni (K.). -

\section{Judolia Mulsant.}

1. J. cerambyciformis Schrk. = quadrimaculata Scop. $=$ octomaculata Schall. = decempunctata Oliv - - Auf der Vorderhälfte der Flügeldecken stehen in einer Reihe sechs (auf jeder Decke drei) schwarze Punkte, die ganz verschwinden können [wie 3 Ex. in Sammlung v. Heyden], sich aber auch unter einander verbinden und oft so zusammenfliessen, dass sie eine gezackte Binde bilden. - Fr. Wald auf Blumen $=5 .-$ Hohe Mark im Taunus $>7 . \mathrm{h} .-$ Dlb. Wlb. s. g. (Sch.) -

\section{Grammoptera Serville.}

(Diese und die folgende Gattung revidirt von I. v. Heyden in Deutsche Entom. Zeitschr. 1876, pag. 317.)

1. G. femorata $F$. = variegata Germ. - An einem Kiefernklotz $=5$. im Goldsteinforst im Fr. Wald, auch sonst sehr einzeln gef. - G. (L.) - Fr. h. 16. Mai 1860 von B. gef. - Wlb. (Sch.) -

2. G. tabacicolor De Geer $=$ chrysomeloides Schrk. = laevis F. - Fr. Wald auf Blumen = 5. h. - G. (L.) - Fr. (B.) - Wlb. (Sch.) - Wsb. an der Wellritz (K.). - Lahnstein 9. Juni (K.). - 
3. G. ustulata Schall. = splendida Hbst. = praeusta F. Im Fr. Wald am Forsthaus auf Eichen $>5$. von C. H. s. s. gef. - Auch aus Waldholz = 4. erzogen. - Bei der Irrenanstalt Eichberg im Rheingau (Dr. Richter). - Mr. (S.) -

4. G. ruficornis F. - Fr. im Wald auf Blüthen. - G. (L.) Fr. h. (B.) - Dlb. Wlb. (Sch.) - Mr. (S.) - Lahnstein 9. Juni und Wsb. an der Wellritz 25. Juni (K.).

5. G. analis Panz. - Aeusserst s. - Aus dürrem Fr. Waldholz $=4$. entwickelt. - Cronthal $=5$. in Begattung. - (Bei dem Mämnchen ist der ganze Hinterleib schwarz, bei dem Weibchen die 2-3 letzten Segmente roth.) - 1877 aus Eichenholz (St.). -

\section{Cortodera Mulsant.}

1. C. humeralis Schall. = quadriguttata $F$. (Flügeldecken je mit zwei hellrothen Flecken an der Basis, welche auch fehlen können.) - Fr. einmal $>5$. (ohne Flecken) von C. H. gef.; mit normader Färbung von Sch. bei Wlb. gesammelt. -

Var. suturalis F. (Flügeldecken gelbbraun, die Naht oft schwarz.) - Am Fr. Forsthaus im Wald s. auf Eichen $>5$. - Friedberg (Renner, Fulrr). - Fr. h. im Mai 1860 (B.). - Wlb. (Sch.) -

\section{Leptura Linné.}

1. (Anoplodera Muls.) rufipes Schall. - Bei Ems einmal gef. von St. - Am Fr. Forsthaus einmal 3. Juni 1826 von C. H. auf Blüthen gef. - MIz. (S.) - Natheim (v. Harold) Juni 1871. -

2. (A.) sexguttata $\mathbf{F}$. (Jede Flügeldecke mit 3 rothen Flecken hinter einander.) - Wlb. auf Chaerophyllum temul. (Sch.) - Mz. (S.) -

Var. exclamationis F. (Die Flecken 2. und 3. fliessen der Länge nach zusammen.) - Cronthal $=5$. und Königstein $=6$. auf Wiesenblumen je einmal von C. H. gef. - G. (L.) - Wlb. (Sch.) - Nauheim (v. Harold) Juni 1871. -

3. (Vadonia Muls.) livida F. - Fr. im Wald auf Blumen. Fr. einmal (B.). - Dlb. Wlb. ho (Sch.) - Mo. auf Waldblössen am 30. Juni, 22. Juli und 7. August (K.). -

4. L. maculicornis De Geer. - Friedberg von Remer gef. [Exemplar in Sammlung v. Heyden.] — Wlb. einmal (Sch.). -

5. L. fulva De Geer = tomentosa F. - Bei Fr. in Oberrad in Gärten auf Rosen von L. H. und M. S. gef. - Schlangenbad (C. H.). 
- Fr. und Taunus mehrmals gef. (B.) - Wlb. s. (Sch.) - Mo. auf Blössen im Kiefernwald 8. Juli (K.). -

6. L. sanguinolenta $L$. = variabilis De Geer. - Dlb. (Sch.) -

7. L. scutellata F. - Aus Buchenholz aus dem Fr. Wald s. h. entwickelt im Mai. - Fr. auf Blumen einzeln im Wald (B.). - In Buchen aur der „Louisa“, am Buchrain bei Offenbach (B.). - Mz. (S.) -

8. L. testacea L. (Weib ist = rubra L.) = rubrotestacea Illig. - In alten Kiefernstrünken s. h. im Juli. - Fr. Wald. Cronthal im Taunus. - Auf Blüthen < 8. - Rebstöcker Wald, auch im Taunus von B. gef. - Dlb. Wlb. (Sch.) - Wsb. auf Eichen hinter der Walkmühle 11. August 1851 (K.). -

9. L. erythroptera Hagenb. = rufipennis Muls. - Acusserst s. - Nach C. H. bei Wsb. gef. - Hg. fand im Wald bei Isenburg an einer alten Eiche ein Stück und erzog ans derselben, als der Baum gefällt wurde, noch mehrere Exemplare. -

\section{Strangalia Serville.}

1. S. aurulenta F. - Bei Schlangenbad einmal von C. H. gef. -

2. S. quadrifasciata L. - An alten Weiden in Sachsemhausen am Obermain $>6$. von L. H. gef. - Im Wald bei Cronthal $<7$. in Paarung. - In der Hohen Mark im Taunus $>7$, auf Blumen. Königstein und Nerothal bei Wsb. (B.) - Wlb. (Sch.) - Mz. (S.) -

Var. $\beta$. Muls. = interrupta Heyd. (Die vordere Binde in Flecken aufgelöst.) - Mit der Stammart am Obermainufer in Weiden. Dlb. (Koch). - Wlb. (Sch.) -

Var. - K. fand bei Wsb. zwei Weiber, bei welchen an den Fühlern die drei letzten Glieder, sowie die Spitze des vierten gelb sind. -

3. S. aethiops Poda = atra F. - Bei Soden auf Eichen von L. H. gef. - Fr. $=5$. auf Blumen. - Cronthal $<6$. auf EuphorbiaBlüthen. - G. (I.) - Nauheim (v. Harold) Juni 1871. -

4. S. maculata Poda = elongata De Geer = armata Hbst. = calcarata 01. = subspinosa F. - In der Hohen Mark in Taunus $>$ 7. h. auf Blüthen. - Bei Fr. s. h. (B.) - Dlb. Wlb. (Sch.) Mz. (S.) -

5. S. attenuata F. - Fr.'s. s. von C. H. gesammelt. -

6. S. revestita $\mathbf{L}$. = villica $\mathbf{F}$. - Aus dürrem Buchenholz aus dem Fr. Wald $<5$. und $=6$. öfter entwickelt. - An der Gerber- 
mühle bei Oberrad am Main von M. S. gef. - Mo. am Rhein auf Eichen (K., Hg., St.). - (Die Stammart ist roth, mit schwarzen Flügeldecken, Mittel- und Hinterbrust.) - Wlb. (Sch.) - Mo. auf den Blössen 18. Juli (K.). -

Var. ferruginea Muls. ist ganz gelbroth. - Bei Fr. von Hg., St. und Katheder gesammelt.

Var. discicollis (Heyd.) Scriba. - In S. V. ist eine schöne Varietät erwähnt, welche L. H. einmal $>6$. in Fr. im Zimmer fing. Sie lat sich wahrscheinlich aus dem Holzstall im Hause entrickelt, worin nur Buchenholz aus dem Fr. Wald war. Die Flügeldecken, der Kopf, mit Ausnahme des rothen Mundes, die Fühler, die Mitte der Vorderbrust, die ganze Mittel- und Hinterbrust, der Bauch bis auf die zwei letzten Segmente, welche wie die Beine dunkel-rothbraun sind, schwarz. Das Halsschild ist roth, der mittlere Theat schwarz, der Vorderrand roth. -

7. S. nigra L. - Fr. auf Waldblumen n. s. - Ems. - Dlb. Wlb. (Sch.) -

8. S. melanura $\mathrm{L}$. = sutura nigra De Geer $=$ similis Hbst. - Ueberall h. auf Waldblumen $=7$. - Fr. - Wald nach Langenhain im Taunus $<6$. in Begattung. - G. (L.) - Fr. (B.) - Dlb. Wlb. (Sch.) -

9. S. bifasciata Müll. = quadrifasciata Poda $=$ cruciata Oliv. - Wie die vorige. - Fr. Wald $<6$. - Ems. - Fr. (B.) - Dlb. Wlb. (Sch.) - 


\section{Nachträge.}

p. 17. 2. Cymindis axillaris $\mathbf{F}$. = homagrica Dft. von Lientenant Giebeler bei Wetzlar einige Stücke, die ich gesehen labe, gef. - Neu für unsere Gegend. -

p. 26. Amara lucida Dft. - Fr. $>4$. -

Celia fusca Dej. - Kleines Männchen bei Budenheim $<9$. von C. H. gef. -

Celia cursitans Zmrm. fand Lieutenant Giebeler bei Wetzlar. p. 28, 29. Lientenant Giebeler fand bei Wetzlar die Harpalus-Arten honestus Dft., rubripes Dft., melancholicus Dej., anxius Dft., flavitarsis Dej. und picipennis Dft.

p. 34. Haliplus impressus F. von Lieutenant Giebeler bei Wetzlar gef. -

p. 37. Graphoderes zonatus Hoppe. - Von B. 1877 bei Bockenheim ein Weibchen gef. -

p. 42. 6. Philydrus maritimus Thoms. - Von Lieutenant Giebeler einmal bei Wetzlar an der Lahn gef. - Neu für Mitteldeutschland. -

p. 48. Heterocerus laevigatus Panz. fand Lientenant Giebeler bei Wetzlar. -

p. 54. Aphodius pusillus Hbst. - Feldberg $=5$. - Nauheim $=5$. - Soden $<8$. -

A. tristis Panz. - Fr. von $>4$. bis $>$ 5. s. -

p. 55. Der eingeklammerte Passus nach A. pecari ist zu streichen und dafür zu setzen

\section{3. (Plagiogonus Muls.) rhododactylus Marsh. $=$ arenarius} Oliv. - Fr. - Wsb. = 6.

p. 68. Lieutenant Giebeler fand bei Wetzlar Cetonia marmorata $\sigma^{7}$ mit C. aurata $\&$ und am 22. Juni 1877 ebenso marmorata Mann mit metallica Weib in copula. - 
p. 77. Dromaeolus barmabita Villa. - Von St. 1876 ans Buchenholz, $>6.1877$ aus Eichenholz oft gezogen. -

p. 79. Megapenthes tibialis Boisd. fand Lientenant Giebeler bei Wetzlar. -

p. 81. Hinter Athous niger I. einzuschalten:

8. Athous deflexus Thoms. - Frïher mit A. niger vermengt, aber kleiner, schmäleres Halsschild, erhabenere Flïgeldeckenzwischenräume. $3 \mathrm{Fr}$. einzeln (L. H.). -

p. 8t. Agriotes sputator L. und Var. rufulns Lacord. fand Lieutenant Giebeler bei Wetzlar. -

p. 92. Hister corvinus Germ. - Auf der Waldeck bei Oberingelheim von B. gef。-

p. 94. Abraeus:

1. globulus richtig,

2. globosus richtig,

3. parvulus ein Stück richtig,

4. granulum Er. ist das andere Stück von parvulus (dem punctatissimus Reitt. täuschend ähnlich, aber prosternum verschieden). -

\section{p. 95. Acritus:}

1. fulvus richtig,

[atomarius fällt weg, 2 der B.'schen Stücke der Sammlung v. Heyden sind nach Reitter Orthoperus brunnipes],

2. 3. minutus und nigricornis kommen beide im Gebiet vor. - nigricornis unterscheidet sich von minutus durch etwas kleinere Gestalt, dunklere Fühler und die Bildung der Brust. -

p. 105. Byturus. Für den Namen tomentosus hat als älterer Name Sambuci Scop. und für fumatus F. Rosae Scop. einzutreten. - Die letztere hat grosse vorgequollene, Sambuci flache Augen.

- Sambuci Var. flavescens Mrsh. (gelbbehaart). Fr. einmal $<5$.

p. 109. 4. Silvanus frumentarius F. - Von M. S. bei Bonames gef. -

p. 111. Monotoma picipes Payk. - Bei Fr. von B. 8. April 1877 gef. -

p. 115. Corticaria elongata Hummel. - Bei Fr. von B. 8. April 1877 gef. - 


\section{$-347-$}

p. 124. Triplax Russica L. fand Lieutenant Giebeler bei Wetzlar in einom Apfelbaum. -

p. 133. Orthoperus brunnipes richtig. Das Synonym Dorcatoma Zusmaehus. lässt man am besten unberücksichtigt, da es sich nicht eruiren lässt, welche Orthoperus-Art Beck vor sich hatte. Das alte Stück in Sammlung v. Heyden aus Fr., das speciell diese Bezeichnung trägt, ist nach Reitter ?= punctatus Wankow., stimmt auch ziemlich mit seinem punctulatus. - corticalis aus Südfrankreich ist pilosiusculns. - corticalis ist synonym von:

2. atomus, richtig.

p. 140. 3. Silpha carinata Illig. - 17. Mai 1877 von L. H. zweimal auf der Feldbergkuppe gef. - Neu für das Gebiet. Gebirgsthier. - Vor S. tristis einzuschalten. -

p. 143. Catops anisotomoides Spence. - Von B. bei Budenheim gef. -

p. 145. 5. Neuraphes rubicundus Schaum. - Von B. einmal bei Fr. gef. -

p. 152. Euplectus Duponti Aubé. - Ein zweites Stück fand B. in seinem Garten in Fr. auf der Bleichstrasse im Flug. -

p. 170. Nach Philonthus sordidus einzuschalten:

43. (B.) rufimanus Er. - Bei Fr. von M. S. gef. -

p. 171. Philonthus Mannerheimi Fauvel. - Fr. von B. gef. 7. April 1877. -

p. 175. Mycetoporus rufescens Stephens. - Von B. bei Fr. 1877 gef. -

p. 190. Euryusa sinuata Er. - Von B. 29. April 1877 bei Fr. bei Formica cunicularia gef. -

p. 195. Telephoridae muss Thelephor. heissen. -

Lygistopterus sanguineus F. - Von St. in Menge aus Eichenholz $<$ 7. erzogen. -

p. 198. Thelephorus pulicarius F. - Von B. auf der Waldeck bei Oberingelheim gesammelt. -

p. 206. Nach Dolichosoma als zweite Art einzuschalten:

2. (Psilothrix Redtb.) viridi-coeruleum Geoffr. = nobile Illig. - Von B. auf der Waldeck bei Oberingelheim 1877 entdeckt. - Neu für das Gebiet. - 


\section{$-348$}

p. 211. Apate varia Illig. - 1877 von St. auch aus Eichenholz erzogen. Oligomerus brumeus 01. - 1877 von St. auch aus Eichenholz erzogen. -

p. 227. Conopalpus testaceus. - 1877 von St. anch aus Eichenholz erzogen; es war nur solches in der Holzkammer. -

p. 235. Cantharis vesicatoria L. - In Menge 28. Juni 1877 in der Villa Reiss bei Cronberg auf Eschen von L. H. gef. -

p. 255. Dactylorhinus globatus Hbst. - Juli 1877 von Oberstlieutenant Saalmüller am Bebraer Bahndamị bei der „Lonisa“ gef. - Neu für das Frankfurter Gebiet. -

p. 264. Dorytomus filirostris Schh. - Bei Wetzlar dreimal von Lieutenant Giebeler auf Brennnesseln am Scheibenstand Brückebon gefunden. -

p. 300. Nach Cryptocephalini fehlt = Cryptocephalus Geoffr. 


\section{Zusammenstellung der Familien.}

\begin{tabular}{|c|c|c|c|c|}
\hline & Arten. & & & Arten. \\
\hline I. Carabicidae & 272 & Uebertrag & & 1171 \\
\hline II. Dytiscidae & 81 & XXXIII. Clambidae . & & \\
\hline III. Gyrinidae. & 6 & XXXIV. Anisotomidae & & 34 \\
\hline IV. Palpicornia & 62 & XXXV. Silphidae . & & 54 \\
\hline V. Heterocidae & 7 & XXXVI. Scydmaenidae & & 22 \\
\hline VI. Parnidae . & 13 & XXXVII. Clavigeridae . & & \\
\hline VII. Georyssidae & 1 & XXXVIII. Pselaphidae . & & 34 \\
\hline VIII. Lucanidae . & 4 & XXXIX. Staphylinidae & & .524 \\
\hline IX. Scarabaeidae & .108 & XL. Dascillidae & & 14 \\
\hline X. Bupr & . 46 & XLI. Thelephoridae & & 93 \\
\hline XI. Eucnemidae & .11 & XLII. Lymexylidae & & \\
\hline XII. Elateridae & 89 & XLIII. Cleridae . . & & 17 \\
\hline XIII. Dermestidae & 22 & XLIV. Anobiidae & & 58 \\
\hline XIV. By & 13 & XLV. Tenel & & 28 \\
\hline $\mathrm{XV} . \mathrm{Hi}$ & 53 & XLVI. Cistel & & 13 \\
\hline XVI. Micı & 1 & XLVII. Lagriidae. & & \\
\hline XVII. Nitidulidae . & .105 & XLVIII. Melan & & 16 \\
\hline XVIII. Peltidae . & 4 & XLIX. Mordellidae & & 28 \\
\hline XIX. Byt & 2 & L. Rhil & & \\
\hline XX. My & 11 & LI. Meloidae & & 14 \\
\hline XXI. Phalacridae. & 12 & LII. Pyrochroidae & & \\
\hline XXII. Cucujidae & 16 & LIII. Anthicidae . & & 11 \\
\hline XXIII. Colydiidae & 58 & LIV. Oedemeridae & & 14 \\
\hline XXIV. Cryptophagidae & 54 & LV. Pythid & & \\
\hline XXV. Ciss & 15 & LVI. Bostrychidae & & 50 \\
\hline XXVI. Telmatophilidae & 5 & LVII. Curculionidae & & 493 \\
\hline XXVII. Erotylidae . & 3 & LVIII. Rhinomaceridae & & \\
\hline XVIII. Endomychidae & 4 & LIX. Anthribidae . & & 11 \\
\hline XXIX. Coccinellidae & 59 & LX. Bruchidae . & & 17 \\
\hline XXX. Coryl & 5 & LXI. Chrysomelidae & & 306 \\
\hline XXXI. Trichopt & 24 & LXII. Cerambycidae & & 115 \\
\hline XXXII. Scaphidiidae. & & Zusammen im Gebiet & & 3161 \\
\hline Zu übertrage & 1171 & & & \\
\hline
\end{tabular}




\section{Verzeichniss der Gattungen.}

\begin{tabular}{|c|c|c|c|c|c|}
\hline & & & & & \\
\hline x Bonell . & - 24 & glenus Er. . & 110 & sticta Redtb. & .125 \\
\hline era Steph. . & .226 & gonolia Muls. & 210 & isotoma Illig. . & 137 \\
\hline leus Leach & & gonum Bon. & & Anisoxya Muls. & \\
\hline dia Muls. . & . 199 & grilus Sol. . & & Anitys Thoms. & 216 \\
\hline s Schönh. & .267 & griotes Esch. & & Anobium F.. & \\
\hline calyptus Schönh. & .271 & gyrtes Fröhl.. . & . 139 & Anodus Nordm. & 169 \\
\hline canthocinus Steph & 335 & iraphilus Redtb.. & .109 & Anomaeocera Shuck. & \\
\hline oderes Serv. & .336 & a Thoms. & .183 & la Sam. . . & \\
\hline gethes Reitt & 102 & ara Gras. . & .190 & les Schmidt & 238 \\
\hline m Steph. . & .165 & a Thoms. & .183 & a Muls. & 342 \\
\hline Steph. . . & .155 & Steph. . . & .116 & pes Jekel. & 57 \\
\hline Ieach. & . 37 & la F. . & & 1 & 266 \\
\hline dera Esch. & 72 & x Duv. . & . 209 & Lap. & 58 \\
\hline ps Le Cont & e 341 & s Schh. . & . 257 & ia Esch. . & \\
\hline Le Conte. & . 95 & Schb. & .279 & phagus Lat & 16 \\
\hline s Muls. . & .55 & Bon.. . & & $D \sim-1$ & \\
\hline rus Kiesw. & . 82 & Le Conte & . 96 & & 15 \\
\hline & .169 & ma Muls.. & .216 & Er. & 204 \\
\hline us Latr. & . 29 & & .337 & Germ. & 27 \\
\hline & .125 & is Er. . & .138 & $a v$. & 15 \\
\hline ra Latr. & . 78 & is Muls. & . 66 & & \\
\hline a Steph. & . 23 & Er. . . & & us Geoffr. & 29 \\
\hline s Thoms. & .134 & Thoms. . & & & 211 \\
\hline Westw. & .236 & & .337 & us Latr. & \\
\hline aia Laich. . & .312 & s Muls. & .337 & s Illig. & \\
\hline ' & .125 & yeoff. . & .228 & a All. . & \\
\hline s Kirby . & 304 & uls. . & .127 & ost. . . & $28+2>3$ \\
\hline s Lsc1. & 85 & 1 & . 117 & s Oliv. & \\
\hline na Serv. . & .327 & us Er. . & & Redtb. & \\
\hline G S C & & & 197 & & \\
\hline & .336 & dls. . & .236 & Is Fisch. & \\
\hline ra Krtz. & .179 & s leil. & .247 & 1. Meg. . & \\
\hline & & n Redtb. & .332 & Serv. & \\
\hline & .138 & tylus Dej. & & um Er. . & \\
\hline & & & & Schmidt. & \\
\hline
\end{tabular}




\begin{tabular}{|c|c|c|c|c|}
\hline semum Esch.. . & $\begin{array}{r}\text { Seite. } \\
.328\end{array}$ & Bradytus Zmim. & $\begin{array}{r}\text { Seite. } \\
-\quad 26\end{array}$ & $\begin{array}{r}\text { Seite. } \\
\text { Ceutorhynchus Schh. } 280\end{array}$ \\
\hline siobates Thoms. & . 46 & Brontes F. . . & .108 & Chaetarthria Steph. . 44 \\
\hline spidiphorus Latr. & .117 & Broscus Panz. . & . 19 & Chaetocnema Steph. . 321 \\
\hline aomus Steph. & .335 & ruchus L. . & .297 & Chalcoides Foudr. $\quad .315$ \\
\hline eles Steph. . & $\cdot 188$ & ryaxis Leach. & .149 & Chalcophora Sol. . \\
\hline tholus Thoms. & . 92 & porus Krtz. & .175 & Charopus Er. . \\
\hline thous Esch. . & 81 & us L. . . & & Cheilocolpus Sol. . \\
\hline tomaria Steph. & .117 & us Leach & .150 & ma Redtb. \\
\hline tagenus Latr. & . 86 & ytiscus Thoms. & . 294 & um Latr. . \\
\hline ttelabus L. . & .294 & Byturus Latr. . & 105 & Chilocorus Leach . \\
\hline ulonium Er. . & . 110 & & & Chilopora Krtz. . \\
\hline utalia Steph. . . & .193 & jius Thoms. & . 51 & Chlaenius Bon. . \\
\hline Ixinotarsus Motsc & 203 & ara Thoms. & .216 & Chlorophanus Germ. 256 \\
\hline & & Ara Clair'v. . & .285 & Latr. . . . 144 \\
\hline ster Clairv. & . 19 & Ius Bon. . & & derus Motsch. 259 \\
\hline Germ. . & . 266 & us Grav. & .183 & hia Schmidt 239 \\
\hline us Germ. & . 269 & $\mathrm{n}$ F. & .329 & othrys Esch. 72 \\
\hline ius Jekel . & . 270 & s Muls. . & .332 & us Redtb. 304 \\
\hline orpha Fou & $\therefore 316$ & is Bon. . & & mela L. . \\
\hline chiödte & . 29 & ra Mhm. . & .184 & la L.. . \\
\hline us Krtz. & . 166 & us Steph. & .314 & Curtis . . 111 \\
\hline & .283 & 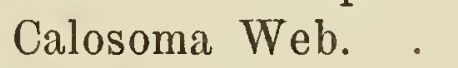 & & inus Thoms. . 278 \\
\hline us Germ. . & . 255 & uls. . . & .128 & iv. . . . 176 \\
\hline a Thoms. & . 191 & inus Schh. & .267 & s Clairv. . \\
\hline s Duv. . & . 252 & Fisch. : & & Cis Latr. . . \\
\hline Foudr. & 316 & L. . . & . 235 & .224 \\
\hline & . 148 & L. . . . & & us Fisch. \\
\hline n Latr. . & . 31 & lorus Esch. & & Preyssl. \\
\hline each. . & . 44 & & .226 & Schh. \\
\hline NLEpH. & .169 & as Eichh. & .244 & Latr. . \\
\hline F. . . . & .219 & s Leach & & ster Latr. \\
\hline phagus Eichb & 1. 243 & Thoms. & .113 & a Laich. . \\
\hline Is Motsch. . & . 16 & & . 309 & F.. . . \\
\hline & .159 & ayk. & . 142 & tus Illig. . \\
\hline Bon. . . & . 10 & & & s Schh. . \\
\hline us Steph. . & . 174 & a Heyd. & .148 & Kug. . . \\
\hline ra Mhm. & . 192 & m Müll. & .146 & L. . \\
\hline gus Illig. & . 220 & & . 334 & s 'Thoms. \\
\hline$\pi$ & .246 & Kirby & . 190 & Schh. \\
\hline a Muls. & .179 & & . 96 & Er. . . \\
\hline uus Web. & . 14 & on Leach. & & us Latr. . \\
\hline x Schh. . & .266 & & . 235 & erus Muls. \\
\hline leres Schh. & .253 & tum Latr. & & Hbst. . . . \\
\hline & . 96 & & .115 & $F$ \\
\hline & . 297 & & - 68 & Colymbetes Clairv. \\
\hline & . 30 & & & rus Bedel \\
\hline
\end{tabular}


Seite

Compsidia Muls. .

Coninomus Thoms.

Conithassa Thoms.

Conopalpus Gyll. .

Conosoma Krtz.

Conurus Steph.

Copris Geoffr. .

Coprophilus Latr.

Coproporus Krtz. .

Coptocephala Redtb. 300

Coraebus Lap. . . . 72

Corticaria Illig. . . 114

Cortodera Muls. . 342

Corylophus Steph. . 133

Corymbites Latr.

Corynetes Hbst.

Coryphium Steph.

Coryssomerus Schh. . 283

Cossonus Clairv. . . 285

Creophilus Steph. . 167

Crepidodera All. .

Criocephalus Muls. . 328

Crioceris Geoffr. . . 325

Criomorphus Muls. . 328

.Cryphalus Er. . . . 245

Cryptarcha Shuck. . 103

Crypticus Latr. . . 219

Cryptobium Mhm. . 166

Cryptocephalus Geoff. 300

Cryptohypnus Esch. . 79

Cryptophagus Hbst. . 118

Cryptopleurum Muls. 47

Cryptorhynchus Illig. 267

Crypturgus Er. . . 245

Cteniopus Sol. . . 224

Ctenonychus Steph. . 85

Cumatotomicus Ferr. 246

Curtonotus Steph. . 26

Cybister Curtis . . 40

Cybocephalus Fr.. . 136

Cychramus Kug. . . 102

Cychrus F. . . . 10

Cyclodinus Muls. . 237
Cyclonotum Er.

Cylindromorphus Ksw.

Cymatopterus Esch. .

Cymindis Latr.

Cynegetis Redtb.

Cyphocleonus Motsch.

Cyphoderes Muls.

Cyphon Payk.

Cyrtusa Er. .

Cytilus Er.

Dactylorhinus Tourn. 255

Dadopora Thoms.

Danacaea Lap.

Dapalinus Cap.

Dascillus Latr.

Dasycerus Brongn. . 113

Dasytes Payk.

Deleaster Er.

Demetrias Bon.

Dendroctonus Er.

Dendrophilus Leach.

Dermestes L.

Diacanthus Latr. .

Diachromus Er.

Diaperis Geoffr.

Dibolia Latr.

Dicerca Esch

Dictyoptera Latr. .

Dinaraea Thoms.

Dinarda Lac.

Dinops Ster.

Dinopsis Matth.

Dinoptera Muls.

Diodyrhpochus Schn

Diplocoelus Guér. . 123

Dircaea F. . . . 227

Disopus Redtb. . . 301

Ditoma Illig. . . . 110

Dolichosoma Steph. . 206

Dolopius Redtb.

Domene Faur. .

Donacia F.

217

194

137

89

97

206

258

193

205

156

15

243

93

86

82

26

220

323

70

195

187

189

208

178

341
46 Donus Cap.

Seite.

75

Dorcadion Dalm. . 334

38 Dorcatoma Hbst. . . 215

17 Dorcus Mac Leay . 50

129 Dorytomus Germ. . 264

Drapetes Redtb.

76

Drilus Oliv.

201

Dromaeolus Ksw.

77

Dromius Bon. . . 15

Drusilla Mhm. . . 188

Dryocetes Eichh.*) . 247

Dryophilus Chevr. . 211

Dryophthorus Schh. 285

Dryops Fabr. . . . 238

Dyschara Muls. . . 192

Dyschirius Bon. . . 14

Dytiscus L. . . . 40

Ebaeus Er.. . . 203

Eccoptogaster Hbst. . 241

Elaphrus F. . . 10

Elater L. . . . 78

Eledona Latr. . . 220

Elleschus Schh. . . 271

Elmis Latr. . . . . 49

Emphylus Er. . . 120

Emus Curtis . . . 167

Encephalus Westw. . 179

Endomychus Panz. . 124

Engis F. . . . . 121

Enicmus Thoms. . 113

Ennearthron Mell. . 123

Enneatoma Muls. . 216

Enochrus Thoms. . 43

Epaphius Redtb. . . 30

Epauloecus Muls. . 218

Ephistemus Westw. 117

Epilachna Chevr. . 129

Epitrix Foudr. . . 315

Epuraea Er. . . . 97

85 Erchomus Motsch. . 176

163 Erichsonius Fauv. ol. 169

325 Eriglenus Thoms. . 39

*) Nicht Dryocoetes, wie im Text steht. 
Seite.

Erirhinomorphus Cap. 257

Erirhinus Schh. . . 264

Ernobius Thoms. . 213

Ernocharis Thoms. . 224

Ernoporus Thoms. . 245

Eros Newm. . . 195

Erycus Tourn. . . 264

Eryx Steph. . . . 223

Euaesthetus Grav. . 160

Eubrychius Thoms. . 279

Eucinetus Germ. . 195

Eucnemis Ahr. . . 77

Euconnus Thoms. . 145

Eudipnus Thoms. . 251

Euglenes Westw. . 236

Eumicrus Lap. . . 146

Euplectus Leach . . 151

Eupleurus Muls.

Euryporus Er. .

Eurythyrea Sol.

Euryusa Er.

Eusomus Germ.

Eusphalerum Krtz.

Eustrophus Latr. . 226

Euthia Steph. . . 146

Exocentrus Muls. . 335

Exochomus Redtb. . 129

Falagria Steph.

Foucartia Duv.

Gabrius Steph.

Galleruca F.

312

Gasterocercus Lap. . 267

Gastrallus Duv. . . 211

Gastroidea Hoppe . 308

Gastrophysa Redtb. 308

Gaurodytes Thoms. . 39

Geodromicus Redtb. . 156

Georius Steph. . . 168

Georyssus Latr.

Geotrupes Latr.

Glischrochilus Murray 103

Glyptodactylus Gaut. 23
Seite

Gnathoncus Duv. . . 94

Gnorimus Lepel.

Gnypeta Thoms.

Golgia Muls.

Gonioctena Redtb.

Gonodera Muls.

Gracilia Serv.

Grammoptera Serv.

Graphoderes Esch.

Graptodera All.

Gronops Schh.

Grypidius Schh.

Gymnetron Schh.

Gymnopleurus Illig.

Gynandrophthalma

Lac. .

Gynopterus Muls.

Gyrinus Geoffr.

Gyrophaena Mhm.

Hillabrocerus Er.

HadrobregmusThoms. 212

Hadrotoma Er.

Haliplus Latr. :

Hallomenus Panz.

Haltica Geoffr. .

Halycia Muls.

.127

Hammaticherus Serv. 334

Haplocnemus Steph. 206

Haploderus Steph. . 158

Haploglossa Krtz. . 192

Haptoderus Chaud. . 24

Harmonia Muls. . . 126

Harpalus Latr.

27

Hedobia Sturm . . 216

Helochares Muls. . 43

Helodes Payk. . . 193

Helophorus F. .

45

Hermaeophaga

Foudr.

315

Hesperophanes Muls. 328

Hetaerius Er. . . , 93

Heterocerus F. . . 48

Heteroplus Muls. . . 218

Heterostomus Muls. . 96

Heterothops Steph. . 174
Seito.

Hippodamia Muls. 125

Hippuriphila Foudr. 316

Hispa L. . . . . . 312

Hister L. . . . . . 91

Homalisus Geoffr. . 196

Homaloplia Steph. . 66

Homalota Mhm. . 180

Homoeusa Krtz. . . 190

Hoplia Illig. . . . 67

Hydaticus Leach . . 37

Hydnobius Schmidt . 136

Hydrachna F. . . 35

Hydraena Kug. . . 46

Hydrobius Leach . . 42

Hydrochus Germ. 45

Hydrocyphon Redtb. 194

Hydronomus Schh. .266

Hydrophilus Geoffr. . 42

Hydroporus Clairv. . 35

Hydrothassa Thoms. 309

Hydrous Brullé . . 42

Hygronoma Er. . . 186

Hygrotus Thoms. . 35

Hylastes Er. . . 242

Hylecoetus Latr. . . 207

Hylesinus F. . . 244

Hylobius Schh. . . 263

Hylotrupes Serv. . . 329

Hylurgus Latr. . . 243

Hymenalia Muls. . 223

Hypebaeus Ksw. . 204

Hypera Germ. . . 257

Hyperaspis Redtb. . 129

Hyphydrus Illig. . . 35

Hypocyptus Mhm. . 178

Hypodasytes Muls. . 205

Hypoganus Ksw. . . 83

Hypophloeus Hellw. . 221

Hypulus Payk. . . 227

Tlybius Er. . . . 38

Ilyobates Krtz. . . 184

Ips F. . . . . . 103

Ischnodes Germ. . . 79

Ischnoglossa Krtz. . 190

Ischnomera Steph. . 238 


\begin{tabular}{|c|c|c|c|c|c|}
\hline omira Muls. & $\begin{array}{l}\text { Serte. } \\
.224\end{array}$ & Lina Redtb. . & $\begin{array}{c}\text { Seite. } \\
.307\end{array}$ & Melanophthalma & \\
\hline dolia Muls. & . 341 & Liodes Latr. . & .138 & & \\
\hline \multirow[t]{2}{*}{ Julistus Ksw. } & 205 & Lionychus Wissm. & & Melanotus Esch. & \\
\hline & & loeus Germ. & 256 & sis Oliv. . & \\
\hline dostomis Redt & 299 & Liopterus Esch. . & 39 & asoma Steph. . & .307 \\
\hline ius Er. . & 43 & Liosoma Steph. & 262 & igethes Kirby. & 99 \\
\hline lus Leach & . 37 & Liotrichus Ksw. & & opterus Muls. & \\
\hline a Lac. . & . 299 & Liozoum Muls. & . 213 & L. . . . & 233 \\
\hline sap. . . & . 78 & Liparus Oliv. . & . 262 & Melolontha L. . & 59 \\
\hline oens Er. & . 108 & Lissodema Curt. & . 240 & sia Muls. . . & \\
\hline us Chaud. . & - 23 & rgus Er. . & . 106 & us Duv. & .216 \\
\hline a F. . . & .225 & ocharis Er. & .164 & S Muls. & \\
\hline F. & .334 & F. . . . & 260 & rv. . & .336 \\
\hline ra Spin. & 70 & chusa Grav. . & .188 & Schmidt & \\
\hline s Bon. & . 16 & tarsus Latr. & .318 & s Muls. & .205 \\
\hline hiza Duv. & .196 & Meg. . . & & Schh. . & .251 \\
\hline L. . . & .197 & on Thoms. & .174 & us Gerst. & \\
\hline Germ. & .261 & 'a Latr. . & & & . 274 \\
\hline s. . . . & .129 & us L. . & 50 & Schh. & 27 \\
\hline a Steph. & .215 & s Latr. & & & .120 \\
\hline Illig. . & .112 & as Geoffr. . & .314 & th. . & . 12 \\
\hline m Er. & .155 & rdina Latr. & $\cdot 125$ & & . \\
\hline & .165 & F. $\quad \therefore$ & . 109 & rtz. & . 192 \\
\hline s Er.. & .108 & pterus Muls. & .195 & is Latr. & \\
\hline bia Latr. . & - 17 & Ion F. . & .207 & is Esch. & \\
\hline eja Meg. . . & . 31 & us Chaud. & & ph. & .17 \\
\hline eiopus Serv. . & .335 & rus Schl. . & .266 & $\mathrm{dtb}$ & .22 \\
\hline phus Pert & .167 & Lytta L. . . & 235 & & \\
\hline Fröhl. . & & & & Iuls. & \\
\hline 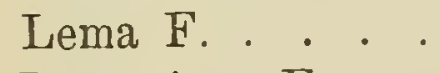 & .324 & drtos Thoms & & h. . & .323 \\
\hline cinus Er. & .166 & us Müll. & & & \\
\hline & .342 & s Schh. & .268 & Muls. . & .3 \\
\hline des Hbst. & . 85 & S F. . . & . 202 & ah. . . & 26 \\
\hline rtz. & .190 & s Latr. & .200 & nus Latr & \\
\hline Germ. & . 262 & es Ksw. . & . 201 & s Schh. & \\
\hline Grav. . & .156 & us Schh. & .278 & Hbst. . & \\
\hline cyphus Kr & Z. 176 & eus Dej. . . & & - & \\
\hline aus Motsc & h. 259 & us Germ. . & .273 & na Costa & \\
\hline Latr. & . 19 & es Thoms. & .278 & aroa & \\
\hline des Schh. & .271 & us Steph. & .175 & & \\
\hline is Leach & 44 & es $\mathrm{Ksw}$. & & us Er. . & \\
\hline I Iate & 89 & is Steph. & .152 & ea Steph. . & \\
\hline & . 49 & & .260 & ina Muls. & \\
\hline & . 259 & sternum Muls. & . 47 & ochares Latr. & \\
\hline & · 81 & 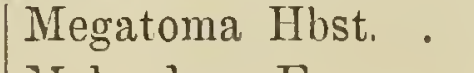 & . 87 & & \\
\hline imoxenus Motsch. & 42 & Melandrya F. & .227 & Hellw. . & \\
\hline
\end{tabular}


Mycetopolus Mhm.*) 175

Mycterus Oliv. . . 240

MÍlacus Schh. . . 248

Myllacua Er. . . . 178

Myloechus Thoms. . 142

Myrmecoxenus

Chevr.

Myrmedonia Er.

Myrmetes Mars.

Myrrha Muls.

Mysia Muls.

Nacerdes Schmidt

Nalassus Muls.

Nanophyes Schh. .

Nargus Thoms.

Nassipa Emery

Nebria Latr.

Necrobia Latr. .

Necrodes Leach

Necrophorus F.

Necydalis L.

Neliocarus Thoms.

Nemonyx Redtb. .

Nemosoma Latr.

Neobium Muls.

Nephus Muls. .

Neuglenes Thoms.

Neuraphes Thoms.

Niptus Boield. . 218

Nitidula F. . . . 98

Nosodendron Latr. . 90

Notaphus Meg. . . a

Notaris Germ. . . 263

Noterus Clairv. . . 37

Notiophilus Dumér. . 9

Nothorhina Dalm. . 328

Notothecta Thoms.

Notoxus Geoffr.

Dberea Muls.

Obrium Latr.

Ocalea Er.
238

223

275

143

229

13

210

141

332

253

295

104

212

130

134

45

18 Oomorphus Curtis

Opatrum F. . .

0 Ophonus Ziegl.

Opilus Latr.

Oplosia Muls.

Orchesia Latr.

Orchestes Illig.

Orectochilus Lac. .

Orithales Ksw. .

Orobitis Germ. .

Orochares Krtz.

Orsodacna Latr.

331

184
Seite.

Orthoperus Steph seito.

Orthopleura Spin. . 183

56 Orthopleura Spin. . 210

316 Orjctes Illig. . . . 58

46 Osmoderma Lepell. . 69

123 Othius Steph. . . 166

31 Otiorhynchus Germ. . 248

186 Otophorus Muls. . 53

15 Oxylaemus Er: . . 110

56 Oxymirus Muls. . . 341

102 Oxyomus Muls. . . 55

238 Oxypoda Mhm. . 185

140 Oxyporus F. . . 160

107 Oxytelus Grav. . . 158

211 Oxythyrea Muls. . 68

179

22 achnephorus

155 Redtb. . . . .304

154 Pacliybrachys Suffr. . 303

23 Pachycerus Gyll. . . 259

253 Pachytychius Jekel . 264

225 Paederus Grav. . . 164

9 Palorus Muls. . . 221

98 Panagaeus Latr. . 18

98 Paramecosoma Curtis 120

52 Parmus F. . . . . 48

51 Paromalus Er. . . 93

94 Patrobus Dej. . . . 20

246 Pediacus Shuck. . . 108

19 Pedilophorus Steff. . 89

304 Pelobius Schh. . . 35

219 Peltis Geoffr. . . 104

27 Pentaphyllus Latr. . 221

208 Pentaria Muls. . . 228

335 Percosia Zmrm. . . 26

226 Perileptus Schaum. . 30

275 Peritelus Germ. . . 249

41 Peryphus Meg. . . 32

82 Phaed on Lac. • . 308

280 Phaenops Lac. . . 71

156 Phalacrus Payk. . . 107

324 Pheletes Ksw. . . . 81

111 Philochthus Steph. . 31

262 Philonthus Curtis . 169

*) Nicht Mytecoporus, wie im Text steht. 


\begin{tabular}{|c|c|c|c|c|}
\hline Philydrus Sol. . & $\begin{array}{r}\text { Seite. } \\
\cdot \quad 42\end{array}$ & Pogonocherus Latr. . & Seite. & Pygidia Muls. \\
\hline oeobium Er. . & .152 & olydrosus Germ. . . & 250 & Pyrochroa F. \\
\hline loeocharis Mhm. & .152 & lygraphus Er. . & 244 & \\
\hline :oma Krtz. . & . 186 & psia Muls. & .339 & Quedius Steph. \\
\hline thorus Woll. & . 244 & olyphylla Har. & & \\
\hline Er. & .186 & Poophagus Schh. . & 283 & Rantus Esch. . \\
\hline a Steph. & .227 & Prasocuris Latr. . & 309 & Raphirus Steph. . \\
\hline aus Lap. & .196 & Pria Steph. . . . & 99 & Reichenbachia Leach \\
\hline a Leach & 141 & cyphon Redtb. & .194 & $\mathrm{~F}$ \\
\hline Redtb. . & 309 & s Geoffr. . & .327 & ha Esch. \\
\hline us Schh. . & .249 & hus Sol. & 223 & um Latr. \\
\hline otica Redtb. & 313 & hus Dej. & 20 & Clairv. \\
\hline a Kirby. & 67 & es Bon. . & 10 & as $\mathrm{Ge}$ \\
\hline Foudr. & $\cdot 317$ & tysus Redtb. & 301 & cer F. . \\
\hline es Muls. & 329 & ha Latr. . & .152 & as Schh. . \\
\hline Schh. . & .279 & tea Er. & 184 & \\
\hline a Kirby & 307 & a Muls. & .128 & Steph. . \\
\hline & 338 & Latr. & 153 & jus Hbst. \\
\hline is Schh. & .257 & s Heer & 56 & Latr. \\
\hline erm. . & .263 & us Latr. . & .123 & tus Mell. \\
\hline as Shuck. . & .103 & Hbst. . & . 149 & Muls. \\
\hline rus Eichh. & 245 & a Duv. & 215 & \\
\hline & .180 & & & itz. \\
\hline era Redtb. . & .308 & r..... . . & .260 & \\
\hline onus Mulsant & & Pseudoclerops Duv. . & . 209 & us Schh. \\
\hline & & Pseudodasytes Muls. & & \\
\hline phus Chevr. & .259 & myllocerus & & xis Saulcy \\
\hline phus Muls. & . 330 & & .250 & \\
\hline homs. . & . 39 & Pseudostyphlus Tour- & & \\
\hline offri. & . 50 & & .265 & as Illig. . \\
\hline $\mathrm{L}$ & .220 & Psilothrix Redtenb. & & $\mathrm{F}$ \\
\hline Redtb. & . 130 & (Nachtrag) . . & .347 & s Er. \\
\hline & 241 & les Latr. & .321 & im Illig. . \\
\hline Clairv. & $\cdot 296$ & um Er. . & 133 & dtb. \\
\hline & . 24 & ichus Er. & . 22 & V. . \\
\hline Leach. & • 91 & Matth. & 134 & $\operatorname{ach}$ \\
\hline & .159 & Gonffy. & 214 & \\
\hline & .252 & $\Pi \pi$ & 134 & Is Schh \\
\hline & 321 & a $M$ & 134 & a Thoms. \\
\hline Frr & . 94 & orphus Muls. & .216 & Illig. . . \\
\hline & 262 & & & terus Schh. \\
\hline s Er. . & $\cdot 102$ & phagus Illig. & - 142 & s Geoffr. . \\
\hline us Westw. & 197 & 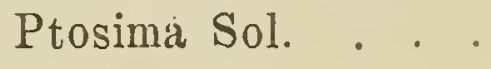 & & $\mathrm{Fr}$ \\
\hline ica All. . . & .316 & Pullus Muls. . & . 130 & atia Latr \\
\hline (2) & & & & \\
\hline oecilus Bon. & & Pycnomerus Er. & .111 & scymis ing. \\
\hline
\end{tabular}




\begin{tabular}{|c|c|c|c|c|c|}
\hline & Seite. & & & & \\
\hline ytropus Schh.*) & 52 & Sternotrupes Jekel & 57 & Tharops Lap. & \\
\hline Mac Leay. & 66 & teropus Meg. . . & 24 & Thea Muls. . . & 128 \\
\hline oderus Steph. & 132 & ichoglossa Fairm. & 190 & Thelephorus Schäff. & 197 \\
\hline cosomus Redtb. & - 85 & tilicus Latr. . . & .163 & Thiasophila Krtz. & 189 \\
\hline cus Esch. . . & 85 & tomis Clairv. . & . 22 & Thinodromus Krtz. & 157 \\
\hline nium Kirby & . 152 & trangalia Serv. . & $\cdot 343$ & Throscus Latr. & \\
\hline a Germ. . & .272 & trophosomus Schh. & .253 & Thyamis Steph. & 318 \\
\hline ynes Schh. . & .272 & Schh. . & 262 & Thymalus Latr. & 104 \\
\hline ia Muls. . & .229 & teph. . . & .163 & Tillus Oliv. . . & \\
\hline la L. . . & .140 & s Redtb. . & .116 & Timarcha L. . & 304 \\
\hline a Er. & .190 & ta Dillw. . & & is Thoms. . & \\
\hline us Latr. . & .109 & Hellw. & . 111 & as Steph. . & \\
\hline caria Marsh. & . 89 & um Er. & . 157 & lossa Krtz. & \\
\hline enaron $\mathrm{F}$. & . 50 & Synaptus Esch. & . 85 & ia Costa. . & $\cdot 230$ \\
\hline ius Latr. . & & & & Toxotus Serv. . & \\
\hline Isatr. . & 234 & us Grav. . & 175 & Trachycellus Moraw & \\
\hline epa Thoms. . & .212 & es Schh. & .277 & roblom & 25 \\
\hline Schh. . & . 253 & us Grav. & . 176 & s F. . & \\
\hline us Schh. & .285 & Lac. & & is Clairv. . & \\
\hline $\mathrm{yx}$ Schh. & $\cdot 265$ & Schaum & 31 & Le Conte & \\
\hline Er. . . . & • 99 & a Kirby & & chmidt & \\
\hline Iuls. . & .127 & Er. & 184 & m Mac Leay & у 221 \\
\hline s Kug. . & 44 & TT & & & \\
\hline agus Stev. & 297 & Germ. & 256 & a Steph. & 168 \\
\hline & . 46 & s' Germ. & 266 & s 1 & 209 \\
\hline a Steph & .323 & on. . & 20 & ud. & 149 \\
\hline & 275 & Schh. & 283 & $\mathrm{hm}$. & 178 \\
\hline s Schh. & 284 & ph. . . & .169 & Kirby & $1:$ \\
\hline hevr. . & 121 & Foudr. & .318 & $\mathrm{~T}_{1}$ & 151 \\
\hline is Clairv. & 20 & ilus Heer & .123 & s Latr. . & \\
\hline s F. . & 328 & & .222 & llus Latr. & \\
\hline us L. . . & 168 & huck. & 110 & Payk. . & 124 \\
\hline is 'Thoms. & 278 & Er. . & & F. . & 124 \\
\hline as Geoffr. & 339 & a F. & 225 & as Kug. . & \\
\hline sa Krtz. . & 190 & Kirby . & 328 & s Er. . . & \\
\hline (2) & 2 & & 339 & a Latr. & \\
\hline All. & 222 & Muls. & & s Mhm. & 157 \\
\hline us Oliv. & 332 & & 102 & $\operatorname{Tr}$ & 104 \\
\hline la Redtb. & 338 & ea Thoms. & . 187 & & \\
\hline & 160 & rus Eichh. & .245 & chh. & \\
\hline usa Krtz. . : & $\cdot 190$ & us Latr. & . 209 & $\operatorname{Tro}$ & \\
\hline & & & & Tropiphorus Schh. & \\
\hline nychus Suffr. & & Tonol- & & Trox F. . & 57 \\
\hline
\end{tabular}

*) Nicht Scythropus, wie im Text steht. 


\section{- 358}

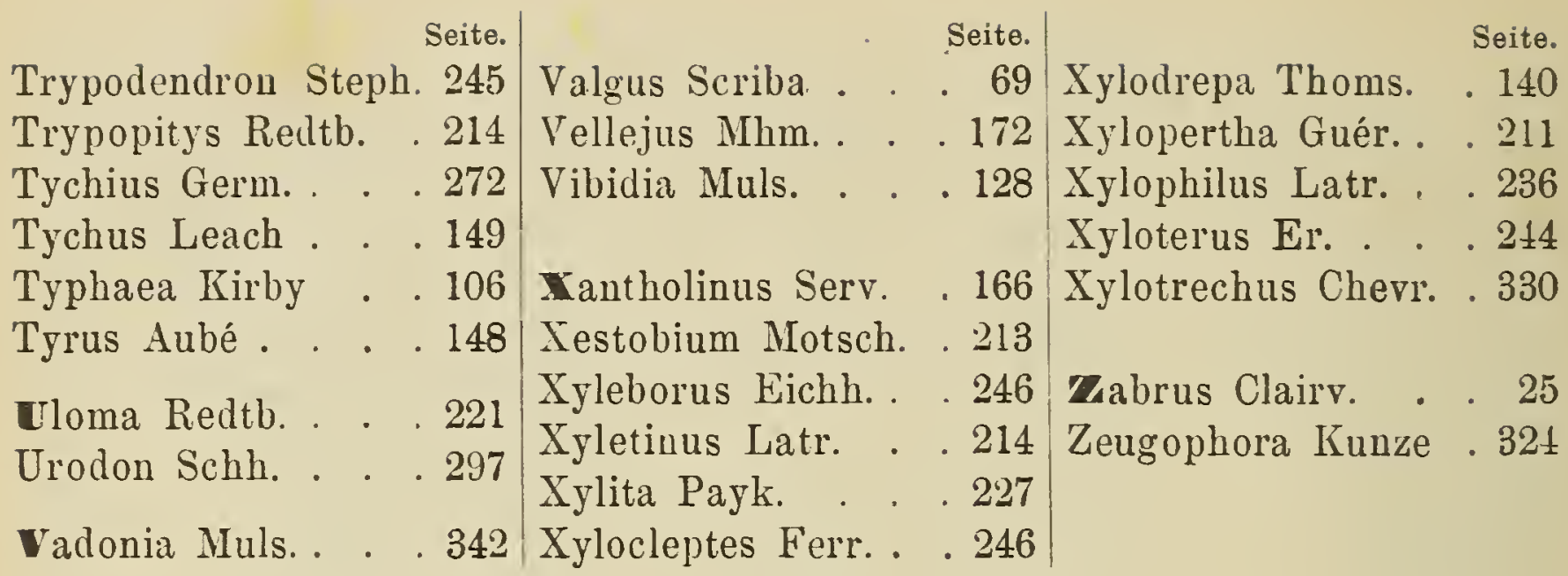

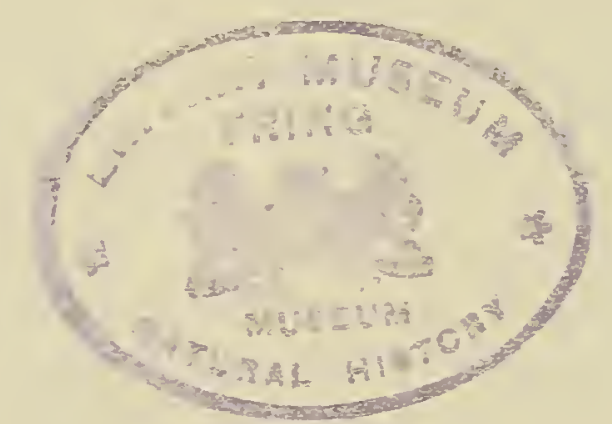

Wiesbaden. L. Schellenberg'sche Hof-Buchdruckerei. 







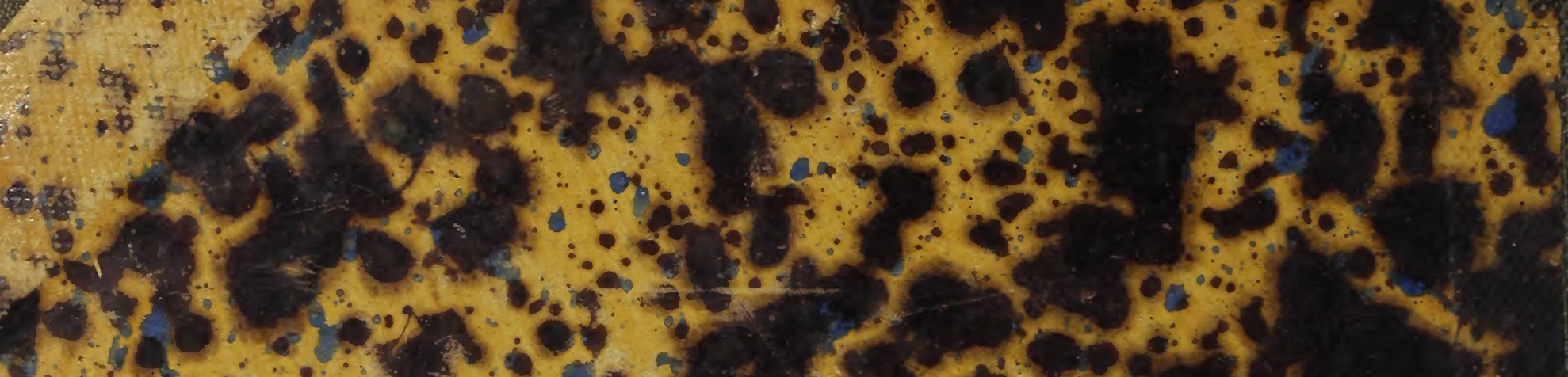

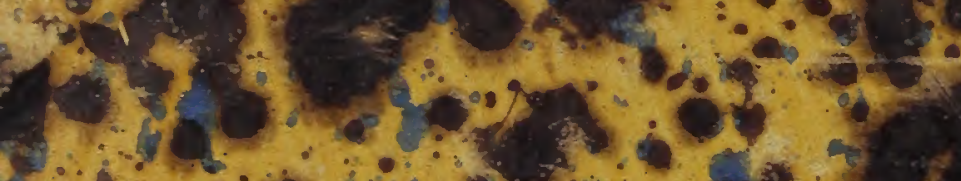

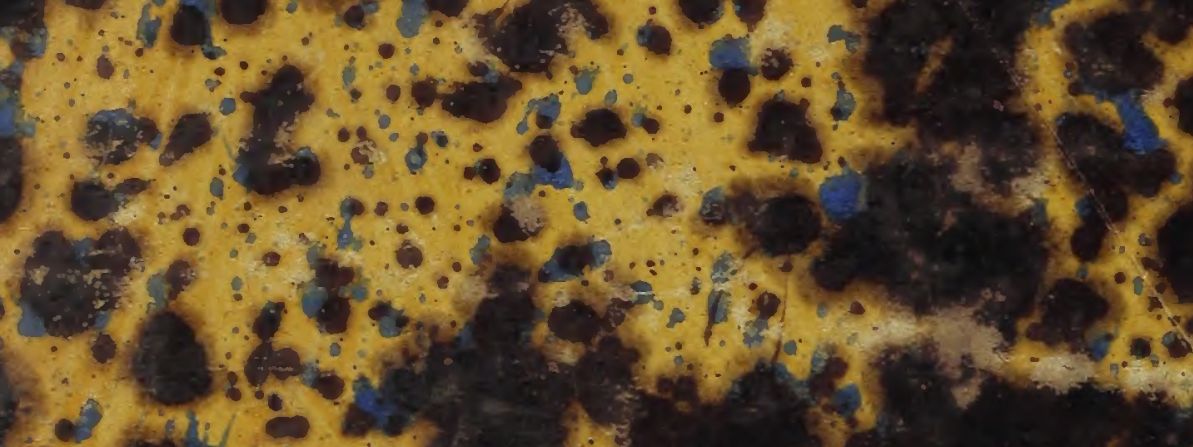

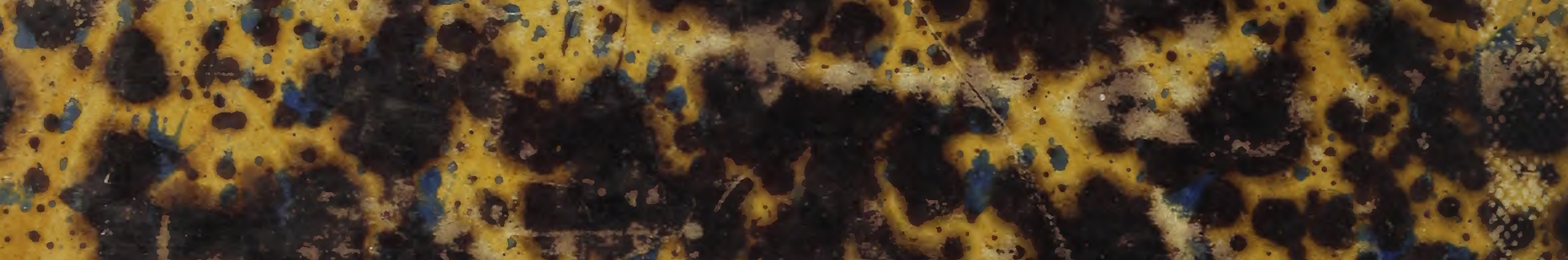
ita kstagt thans?

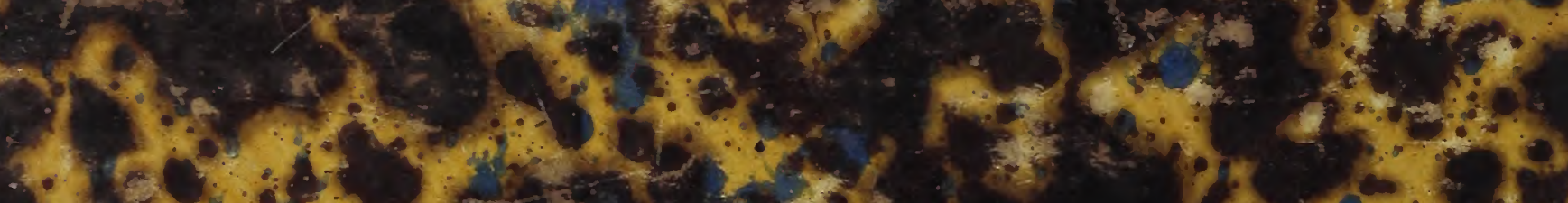

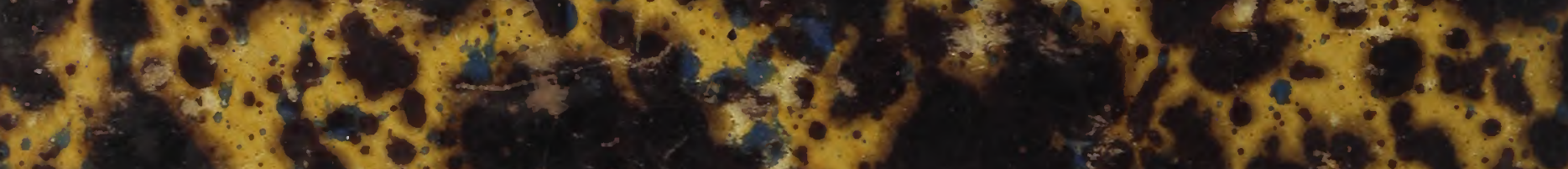

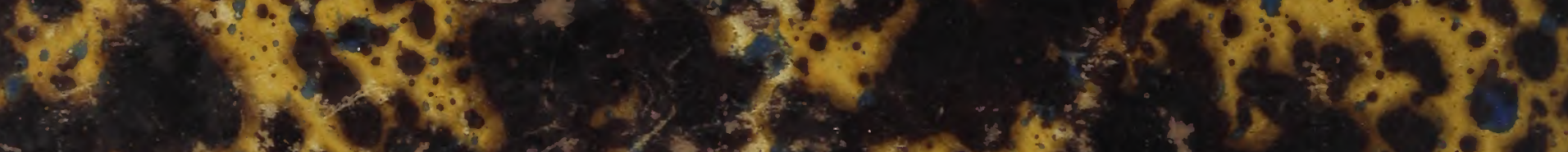

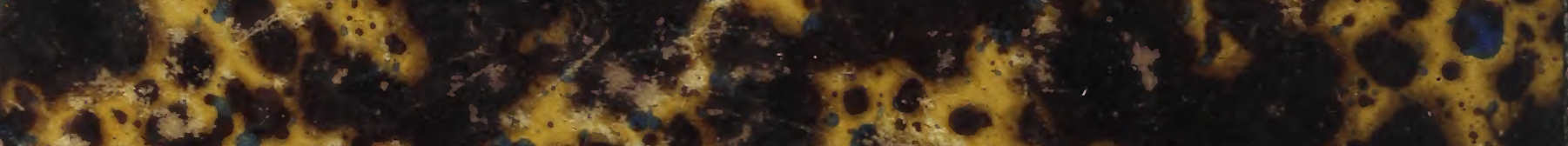
sta. $+2 x$

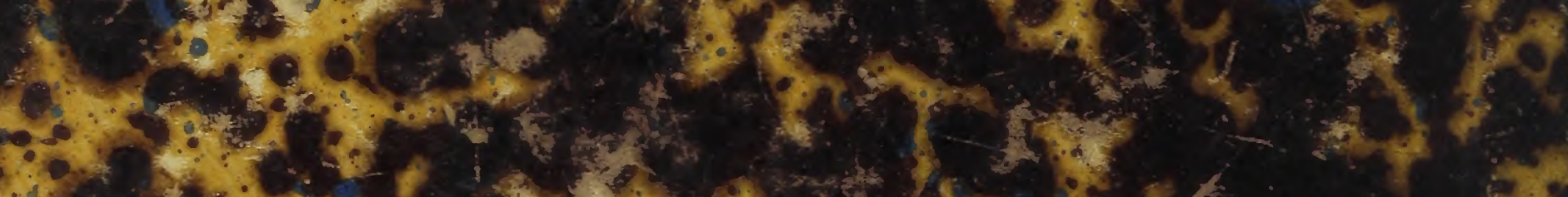

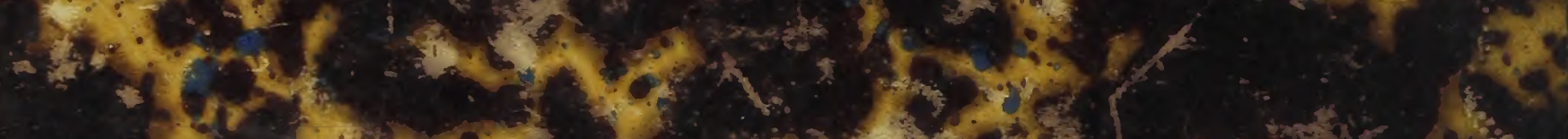

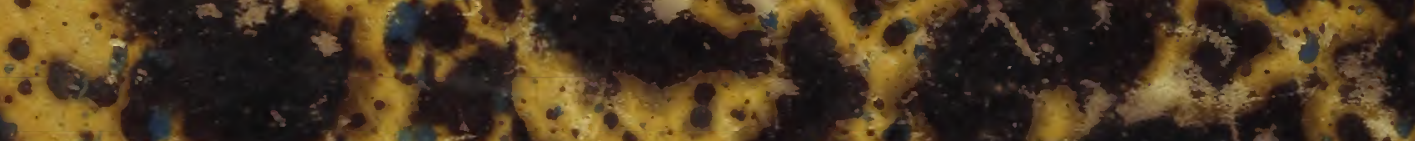

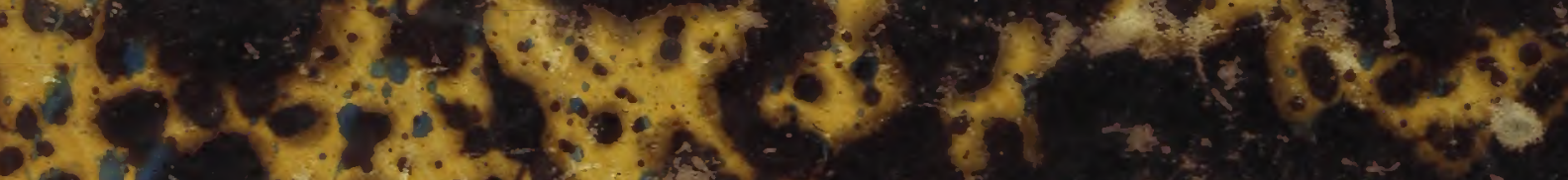

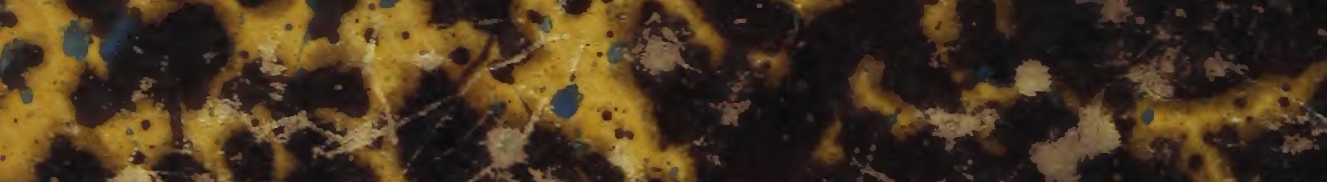
45 h the

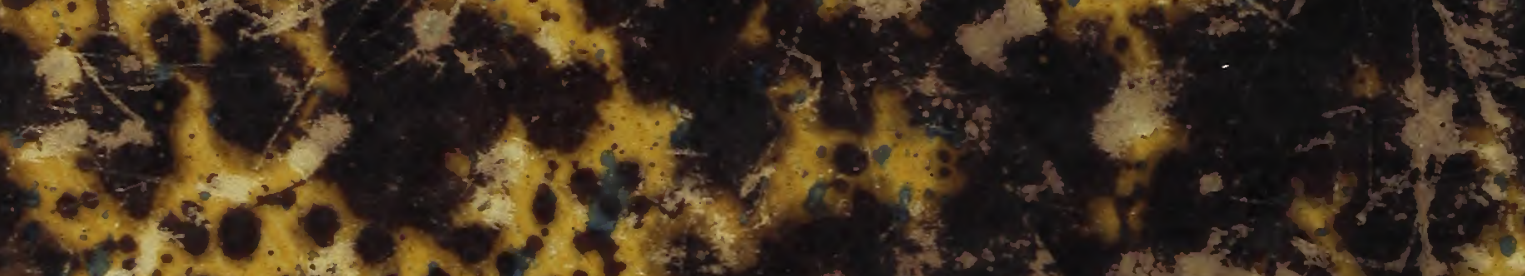

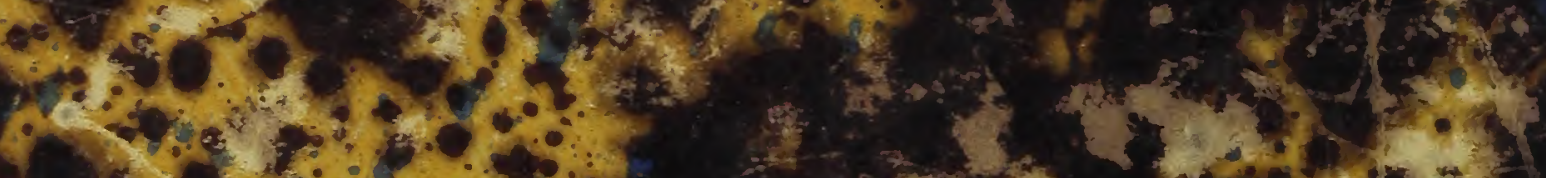

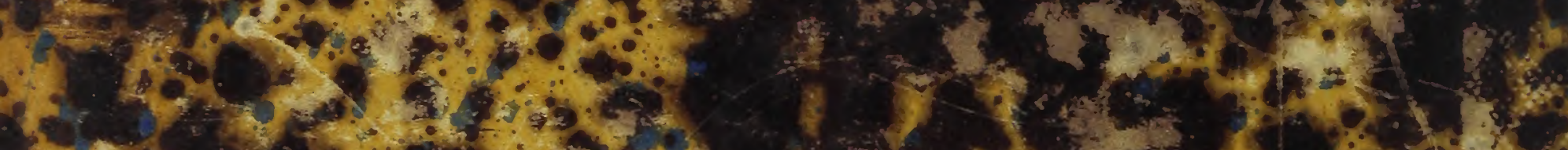

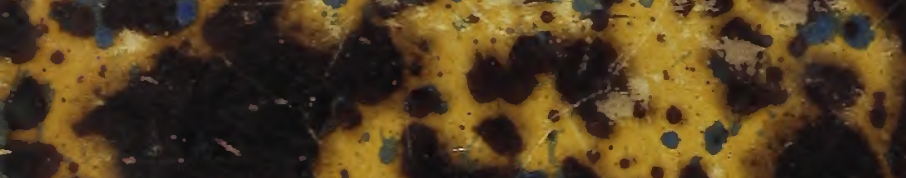

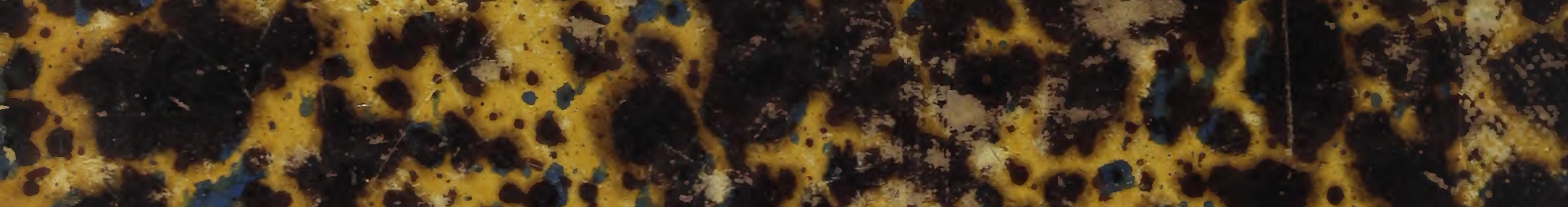

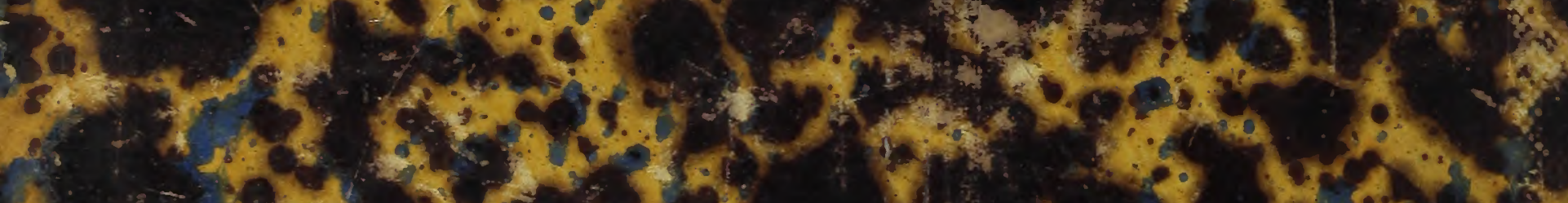

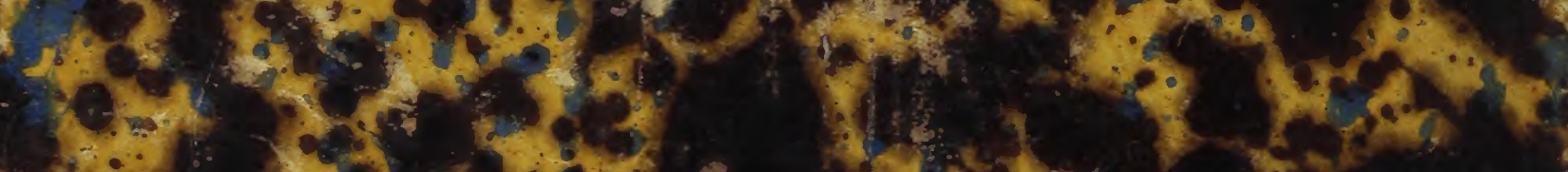

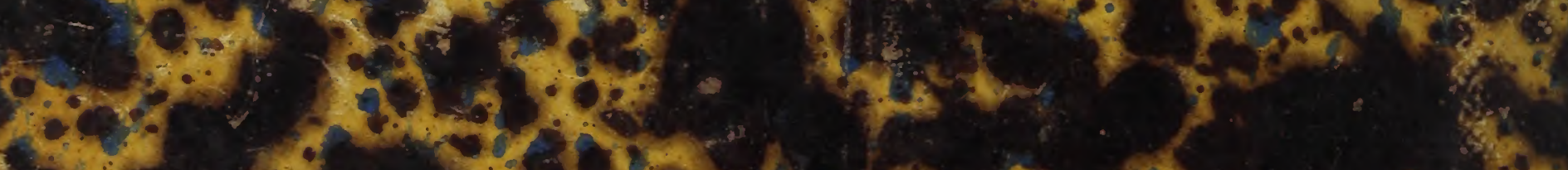

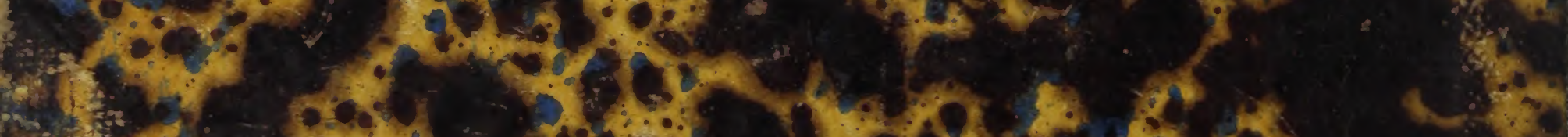

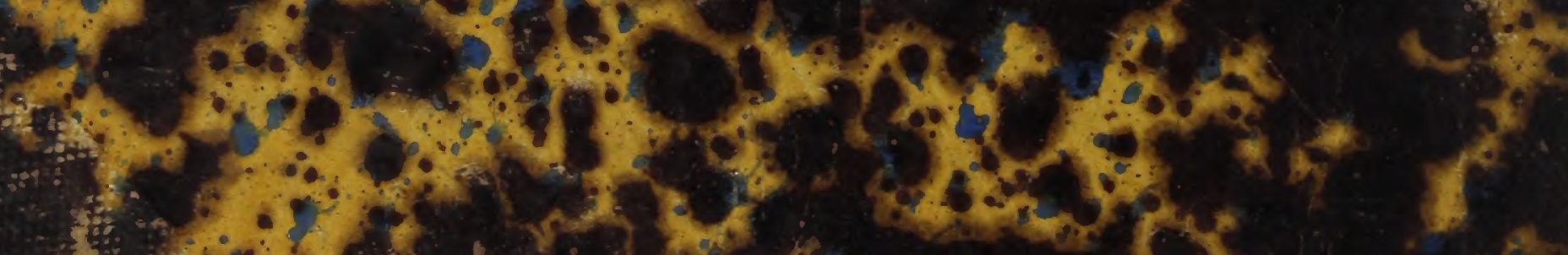

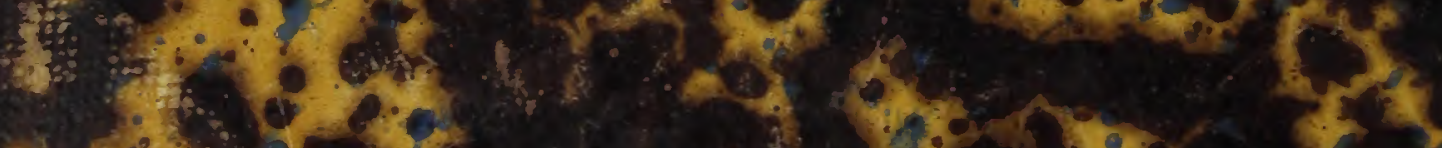

